Distribution Category:

General Physics (UC-410)

\title{
ANL-96/15
}

ARGONNE NATIONAL LABORATORY

9700 S. Cass Avenue

Argonne, Illinois 60439-4801

\section{PHYSICS DIVISION ANNUAL REPORT}

April 1, 1995 -- March 31, 1996

Walter F. Henning

Director

November 1996

Preceding Annual Reports

ANL-93/12 1992-1993

ANL-94/31 1993-1994

ANL-95/14 1994-1995

DISTAEUTION OF THS DOCUNENT IS URLMTED 


\section{Edited by Karen J. Thayer}


DISCLAIMER

Portions of this document may be illegible in electronic image products. Images are produced from the best available original document. 


\section{DISCLAIMER}

This report was prepared as an account of work sponsored by an agency of the United States Government. Neither the United States Government nor any agency thereof, nor any of their employees, makes any warranty, express or implied, of assumes any legal liability or responsibility for the accuracy, completeness, or usefulness of any information, apparatus, product, or process disclosed, or represents that its use would not infringe privately owned rights. Reference herein to any specific commercial product, process, or service by trade name, trademark, manufacturer, or otherwise does not necessarily constitute or imply its endorsement, recommendation, or favoring by the United States Government or any agency thereof. The views and opinions of authors expressed herein do not necessarily state or reflect those of the United States Government or any agency thereof. 


\section{FOREWORD}

The past year has seen several major advances in the Division's research programs.

In heavy-ion physics these include experiments with radioactive beams of interest to nuclear astrophysics, a first exploration of the structure of nuclei situated beyond the proton drip line, the discovery of new proton emitters - the heaviest known, the first unambiguous detection of discrete linking transitions between superdeformed and normal deformed states, and the impact of the APEX results which were the first to report, conclusively, no sign of the previously reported sharp electron positron sum lines.

The medium energy nuclear physics program of the Division has led the first round of experiments at the CEBAF accelerator at the Thomas Jefferson National Accelerator Facility and the study of color transparency in rho meson propagation at the HERMES experiment at DESY, and it has established nuclear polarization in a laser driven polarized hydrogen target.

In atomic physics, the non-dipolar contribution to photoionization has been quantitatively established for the first time, the atomic physics beamline at the Argonne 7 GeV Advanced Photon Source was constructed and, by now, first experiments have been successfully performed.

The theory program has pushed exact many-body calculations with fully realistic interactions (the Argonne $v_{18}$ potential) to the seven-nucleon system, and interesting results have been obtained for the structure of deformed nuclei through meanfield calculations and for the structure of baryons with QCD calculations based on the Dyson-Schwinger approach.

Yet one event stands out: 1996 was the 50th Anniversary of Argonne!

The Physics Division (including its predecessors) has been part of Argonne from the beginning and looks back to a history full of exciting science. Some of this is captured in a brochure prepared by the Division on the occasion of the Open House for the 50th Anniversary, which is reproduced on the following pages. Also the cover of this Annual Report reminds us of some of the history of the Physics Division.

Against the background of Argonne's 50th Anniversary and the history of the Physics Division it is sad that we have to report the death this May of Alexander Langsdorf, one of the original and longtime members of the Physics Division. Alex played an important role in the developments of the Physics Division as outlined in the memorial on the following page.

Walter F. Henning

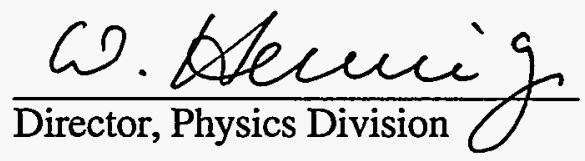


Alexander Langsdorf, Jr.

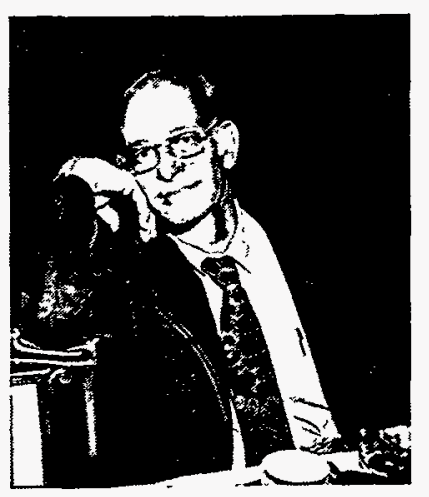

Alexander Langsdorf, Jr. died May 24, 1996, after a long and distinguished career as an experimental nuclear physicist. He was 83 . Alex was a member of the Argonne Physics Division from its very beginnings. He was a physicist in every sense of the word - well educated, highly motivated, very inventive, interested in many aspects of life and nature, and an independent thinker.

Alex Langsdorf was a native of St. Louis. He earned his bachelors degree in 1932 from Washington University in St. Louis, his Ph.D. degree in 1937 from MIT, then spent a year at Berkeley as a Research Fellow, and then back to Washington University as an Instructor in Physics. In 1943 he joined the staff of the Metallurgical Laboratory at the University of Chicago. There he worked on the development of nuclear technology with Enrico Fermi and many other prominent scientists. When Argonne National Laboratory was formed in 1946, Alex joined its staff.

Alex Langsdorf has many notable achievements to his credit, but there is room to mention only a few. While still a graduate student at MIT he invented the diffusion cloud chamber, an instrument that allows the path of charged particles such as cosmic rays to be tracked continuously. This device became one of the principal tools for research in nuclear and high-energy physics during the 1950's. At Argonne's Site A, where Alex worked at two of the world's first nuclear reactors, he invented a device called a "pile oscillator" which enabled him to measure accurately the capture cross section for slow neutrons. The extensive data acquired with this system was important both for the design of nuclear reactors and for the experimental verification of the nuclear shell model formulated at Argonne by Maria Goeppert-Mayer, who in 1963 shared the Nobel Prize in Physics for this achievement. Alex Langsdorf played a central role in the design, construction, and development of several accelerators during his career: a historically early cyclotron at Washington University and two electrostatic accelerators at Argonne. The latter included one of the first pressurized Van de Graaffs and was the major facility for research in the Physics Division in the 1950s. Not only did he build accelerators but he also used them as tools for important physics research for much of his career. During the twenty-year span 1955-1975, Alex and his talented team of physicists initiated a pioneering program of fast-neutron interactions with light nuclei. Using new large shielded neutron detectors conceived and designed by Langsdorf, he and his group acquired a massive and refined body of data that clarified many aspects of the shell model.

In everything that Langsdorf did he was exceptionally inventive, a perfectionist, and insisted on having a thorough, quantitative understanding of everything he did. He was generous in giving credit to others. Without exception, those who knew him have expressed admiration for his abilities and achievements and have fond memories of the period when they worked with Alex. Alex retired in 1977 but continued his interests in accelerator development in a non-paid appointment with the Argonne Physics Division, working closely with the accelerator group. He finished his working career by serving as Associate Editor of the journal of Applied Physics Letters until very recently. 
THE

\section{ARGONNE \\ PHYSICS \\ DIVISION}
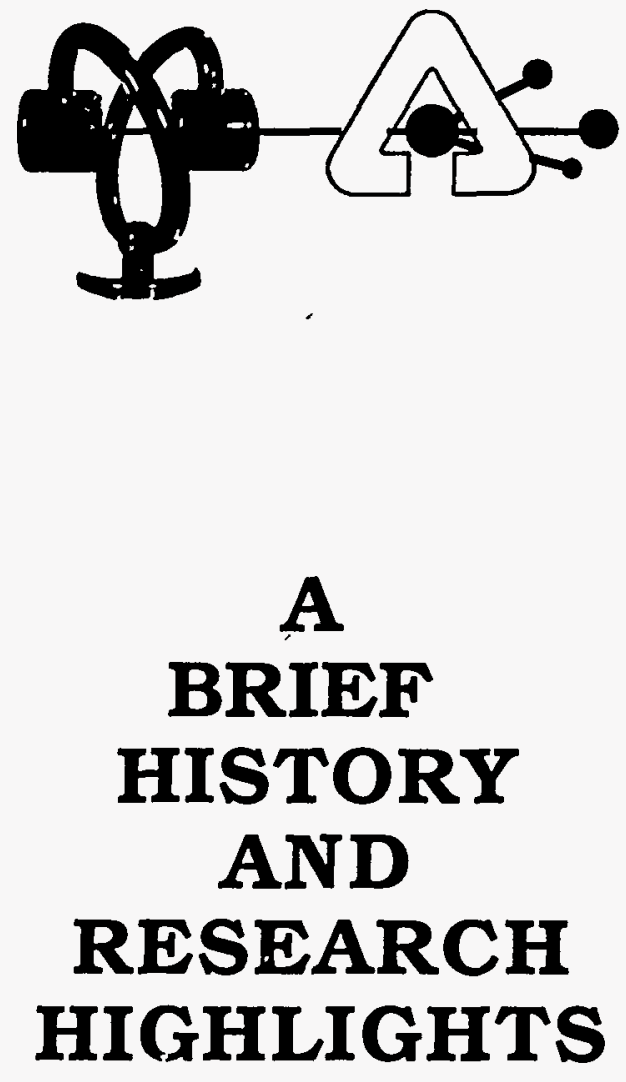

History

The work of the Physics Division started in 1943 when the world's first nuclear reactor, built by a team lead by Nobel-prize winner Enrico Fermi (shown below), was moved from Stagg Field at the University of Chicago to Site A in the Argonne Forest Preserve, about 5 miles east of the present site of Argonne National Laboratory (ANL).

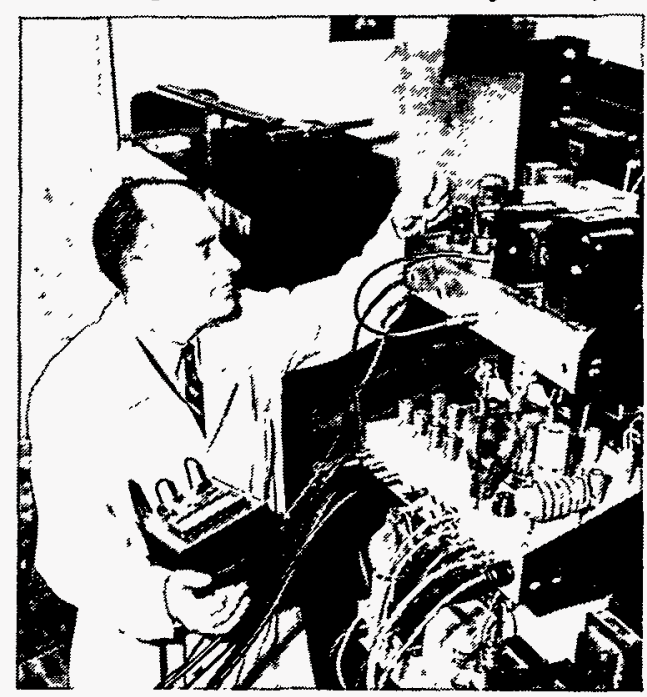

The reinstalled reactor (termed CP-2) was, at the time, a uniquely powerful tool for research with neutrons. Several years later a second and much more powerful reactor CP-3 was built at Site A. These two intense sources of neutrons made it possible for Fermi and others to establish the fundamental properties of the neutron and to initiate a group of pioneering experiments of major importance for future nuclear, solid-state, and reactor physics. This work continued into the 1950's and was advanced by the still greater neutron intensity provided by construction of the reactor $\mathrm{CP}-5$ on the present ANL site.

In 1951, the Physics Division (PHY) began moving into its present home in Building 203. At that time the scope of activities in PHY and in ANL as a whole began to expand greatly, and an impressive range of specialized areas of work initiated in PHY were gradually spun off into new divisions (see figure). The wide range of spin-off activities is impressive.

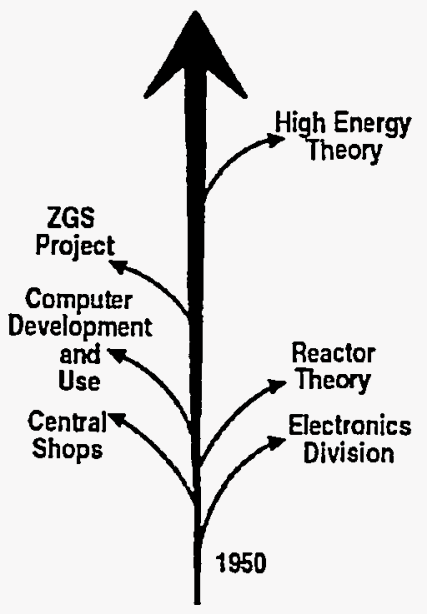


During the 1950's the emphasis in experimental physics began to shift from experiments with low-energy neutrons provided by reactors to those with energetic charged particles from accelerators. An Argonne-built Van de Graaff accelerator went into operation in 1953, and this modest beginning was followed by a series of accelerators with different and/or enhanced characteristics. The latest of these machines is the superconducting accelerator ATLAS in PHY and the immensely powerful X-ray source APS in another part of Argonne. Since the early 1980's heavy-ion beams from ATLAS have been the primary probes for studies of heavy, complex nuclei and, to a lesser extent, atoms; and the new APS will soon be supporting a wide variety of investigations, including atomic-physics research of PHY.

Although the accelerators at ANL are forefront machines they cannot possibly have all of the characteristics needed for our broad experimental program, which includes all aspects of nuclear physics and much of atomic physics. Consequently, other accelerators scattered around the world are used by our staff when appropriate and, conversely, about 200 scientists from universities and other laboratories in the USA and abroad are users of our accelerators each year. The scope of this activity can be judged from the attached map which shows the home institutions of our USA users.

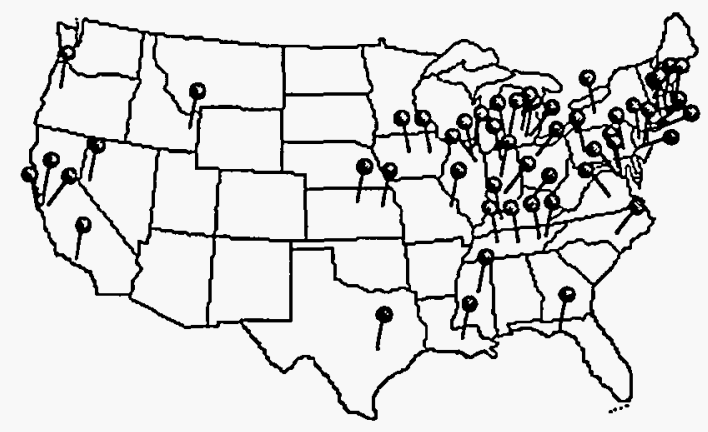

The work with reactors and accelerators was pioneering research in the structure of the nucleus of the atom and on the force between nucleons, the protons and neutrons which make up the nucleus. Although most of the experiments now use large machines, historically, some important work in PHY has been carried out with small equipment. This work includes pioneering research on the Mössbauer effect, photoionization, $\beta$-decay, nuclear spectroscopy, and atomic beams.

To be meaningful, the results of physics experiments must be interpreted in terms of physical theory. Sometimes theory guides the experiment and other times new experimental results lead to new or modified theory. From its beginning PHY has had an exceptionally strong theoretical program, with emphasis on nuclear theory. Maria Goeppert Mayer's work in the Division eamed her the Nobel Prize (1963) for the demonstration that many nuclear properties can be explained in terms of a shell model in which the neutrons and protons in a nucleus are arranged in shells. Maria Goeppert Mayer is shown with a group of theorists at one of a series of workshops which were important for the development of the shell model. A plaque of her is displayed at the main entrance of the Physics Building.

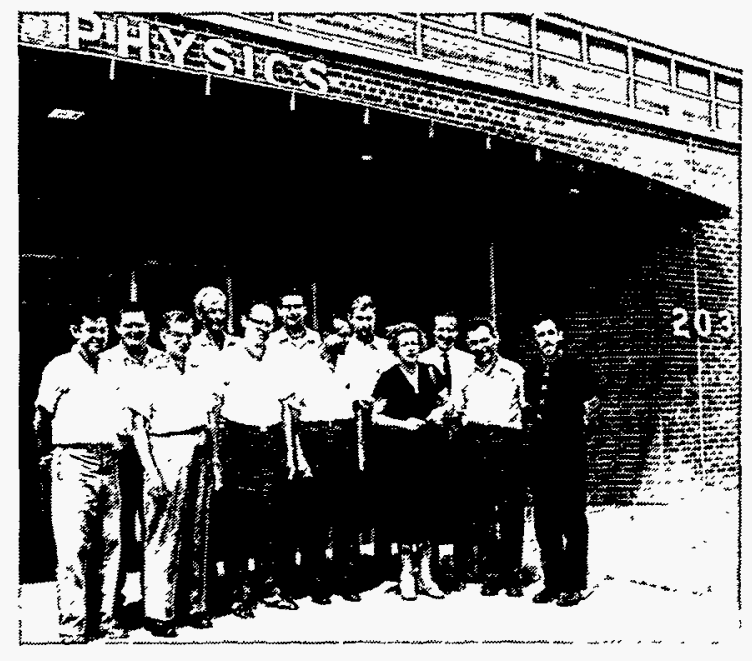

Research Highlights

\section{Nuclear Theory}

The nuclear shell model proposed by Maria Goeppert Mayer became one of the central organizing principles of nuclear physics and presented a comprehensive interpretation of a large number of experimental observations. The model has been extended and developed at Argonne from the 1940's up to the present. That effort has entailed the pioneering development of computational software for nuclear theory, beginning with utilization of the primitive first computers of the 1950's. Some of this software spun off into a commercial venture and is now widely used by economists. Today the underpinnings of the shell model are being studied by starting from the interaction between free nucleons.

In addition to theory closely tied to complementary experiments, such as the shell model and the theory of the Mössbauer effect, appreciable effort has gone into investigating phenomena that are difficult or impossible to study experimentally in the laboratory. These include ground-breaking work on nuclear matter, an infinite system of nucleons that is effectively realized in neutron stars; on calculating reactions important in astrophysics; and on understanding the fundamental structure of the nucleon in terms of quarks, the microscopic building blocks of the nucleon.

\section{Neutron Physics}

The study of neutron properties and their interactions with nuclei extended from 1943 to about 1980 . In the initial period, thermal (low velocity) neutrons from the reactor CP-2 and CP-3 were used in a wide range of pioneering experiments. The experiments of primary importance to physics were those that defined the fundamental behavior of free neutrons: properties such as the wave-like behavior of neutrons and proof that the nuclear forces for protons and neutrons are equal.

By 1954 all reactor-based research moved to the more powerful reactor CP-5. When a neutron from the reactor is captured by a nucleus, a highly-excited nucleus is formed, which then decays by emitting high-energy gamma rays to 
the lower-energy states that the nucleus can exist in. The extensive study of these gamma rays at $\mathrm{CP}-5$ played a significant role in the formulation of a so-called randommatrix theory of Wigner, which has found widespread application in many fields.

An entirely different investigation at CP-5 was one in which the decay of polarized free neutrons was studied. This experiment gave the fundamental character of the weak force, which is responsible for radioactive decay by, for example, changing neutrons into protons and electrons. Symmetry has been a strong theme in physics since the forties and the weak interaction has played the greatest role in developing that theme. This pioneering Argonne experiment played a role in confirming that a left-right symmetry called parity was violated, but that time-reversal symmetry was obeyed by the weak interaction.

\section{Heavy-ion Nuclear Physics}

Most nuclei are either spherical or slightly distorted, but superdeformed nuclei, which are shaped like footballs, are very exotically different. Argonne research revealed a new region of superdeformation in the periodic table and provided the first definition of the essential quantum-mechanical properties of superdeformed nuclei. This work also showed that in the creation of superdeformed nuclei there is an unusual series of events, with a change from initial chaos to order, back to chaos and then to order again.

ATLAS research elucidated how colliding heavy nuclei interact; in gentle collisions a few nucleons are exchanged, but with increasing impact more nucleons are traded until the ions actually fuse together.

Whereas almost all radioactive nuclei decay by emitting electrons or alpha particles, work at ATLAS has found an exotic class of proton radioactivity in heavy nuclei. Protons "drip" out from these loosely bound nuclei.

A very sensitive technique, Accelerator Mass Spectrometry, for detecting minute amounts of heavy elements (less than one part in a trillion) was developed for ATLAS, and was applied to research in other fields, such as the dating of fossils and minerals (millions of years old) and the study of meteorites.

\section{Light-ion Nuclear Physics}

In the fifties and sixties, Argonne provided a large body of data for establishing the validity of the nuclear shell model. At the FM Tandem Electrostatic Accelerator, experiments where particles are exchanged between light-ion beams and target nuclei showed how the shells were filled. These experiments also provided vital information on the force acting between nucleons inside the nucleus; for this work, John Schiffer was awarded the Bonner Prize for outstanding nuclear physics research.

The fundamental properties of many stable and unstable nuclei were measured in 1950-1960 by passing.low-energy beams (from an oven) through radio-frequency fields. The results were vital for confirming the (then) newly proposed shell model.

In nuclei the protons can vibrate collectively against the neutrons, a mode referred to as a giant dipole resonance. Experiments in which protons (from the FM Tandem) are captured, followed by the decay of this resonance through the emission of very high-energy gamma rays, shed light on this important nuclear vibration.
Medium-energy Nuclear Physics

Medium-energy nuclear physics concentrates on understanding the force and particles which hold nuclei together. This is the strongest force known in the universe. A special role in this force is provided by the lightest of the strongly interacting particles, the pion, which serves a similar role to photons (particles of light) in electric interactions. Argonne led a series of measurements at the Los Alamos Meson Physics Facility to learn how pions interact with nuclei and demonstrated that pions often shared their energy with several protons when they completely disappear.

Our deepest understanding of nuclei and the strong force is in terms of the point-like quarks and gluons which make up all strongly interacting matter. In high-energy photon reactions, we were able to directly count the six valence quarks that make up deuterium, which consists of a proton bound to a neutron. At Fermilab, measurements with the highest energy probes, which can directly knock quarks from a nucleus (though for only a very brief time), showed that the distributions of quarks in the proton is different from that in a nucleus such as carbon or lead.

\section{Mössbauer Effect}

In 1958 a German graduate student found a strange effect about the way gamma rays were absorbed in certain materials. This result was widely doubted, but physicists at Argonne were the first to reproduce the experiment and the graduate student, Rudolf Mössbauer, received the Nobel Prize two years later. The Argonne physicists went on to discover the best material in which this type of absorption can take place, an isotope of iron $\left({ }^{57} \mathrm{Fe}\right)$ and the Mössbauer effect, and especially ${ }^{57} \mathrm{Fe}$, now provide a major technique in materials research with applications in biology and other fields.

\section{Atomic Physics}

The electronic structure of atoms and molecules was studied at Argonne with much higher precision than elsewhere, through the use of light to knock out their electrons. This led to important new knowledge of the strengths of the bonds between atoms in molecules and of chemical reactions. This work provided vital information for understanding the interaction between sunlight and molecules in the atmosphere, which is one of the main causes of atmospheric pollution. Argonne physicists also pioneered another method for studying the structure of molecules by passing molecular beams though a thin foil, causing them to blow apart (explode). The resulting pattern of this Coulomb explosion gave a magnified, life-sized "picture", which reveals how the atoms in the molecule bond together.

\section{Accelerator Development}

In 1975 the Physics Division, in collaboration with the Chemistry Division, undertook the task of building ATLAS, the world's first superconducting accelerator for particles heavier than the electron. The major technical challenges included: (1) design and fabrication of new types of superconducting radiofrequency accelerating structures and (2) unprecedented beam quality (well-defined energy and a very narrow pulse). A highly successful superconducting linear accelerator (linac), which boosts the energy of heavyion projectiles coming from the existing tandem 
electrostatic accelerator, was developed. It has been the mainstay of the nuclear physics program at Argonne and its concept has been copied all over the world. Lowell Bollinger (seen working on a linac structure) was awarded the Bonner Prize in nuclear physics for his work and leadership in this pioneering project.

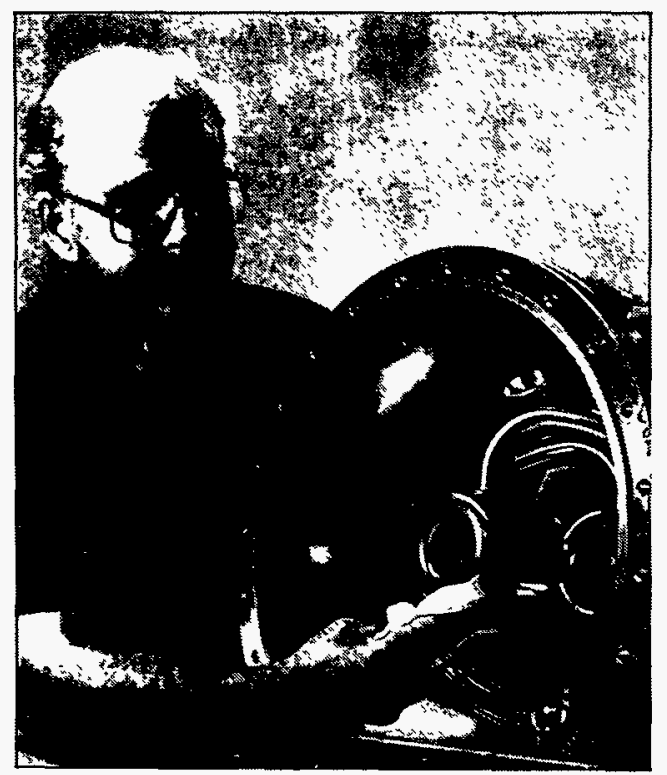

After completion of the planned tandem-linac system in 1985, a demanding upgrade was undertaken: the design and construction of a positive-ion source and a superconducting linac that could replace the original tandem. Here the major challenge was to design and build structures that could accelerate very slow-moving ions. This goal was achieved, and the new system, completed in 1992, is now able to accelerate ions effectively from throughout the entire periodic table, from hydrogen (protons) to uranium.

\section{Present Research Programs}

At present research in the Physics Division studies the properties of nuclei and atoms. In nuclear physics, experiments at the Division's heavy-ion accelerator ATLAS aim to study the structure of the nucleus, e.g. the interplay between its tendency to act like a classical liquid drop and its propensity to behave like a microscopic object consisting of a few interacting nucleons (protons and neutrons), or how the nucleus is affected by spinning or heating it. Other experiments at higher-energy accelerators, using more violent collisions with projectiles such as electrons or protons, attempt to study the quarks (which are constituents of protons and neutrons) inside the nucleus.

The theory group calculates properties of nuclei, e.g. by testing if their properties can be described as the summation of the many interactions between two free nucleons, and whether, when nucleons interact inside the nucleus, their behavior is altered from that of free nucleons.

Electrons circulating around the nucleus govern the properties of atoms. Atomic physics experiments study the consequences of the electron-nucleus and electron-electron interactions, either by stripping electrons from fast accelerated atoms at ATLAS or by knocking out the electrons with high- intensity light from the new Advanced Photon Source (APS) at Argonne.

New designs for accelerators are being developed in PHY to make it possible to produce exotic radioactive atoms, which do not normally occur in nature, and then to accelerate them for energetic collisions to make and study yet more exotic radioactive nuclei. Such studies explore new horizons in nuclei and also study how all the elements are formed in stars.

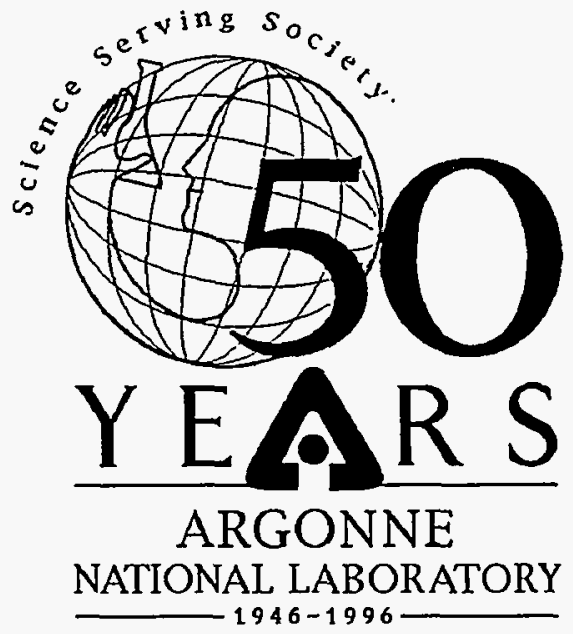

Argonne National Laboratory operated by The University of Chicago for the U.S. Department of Energy under Contract W-31-109-Eng-38 


\section{TABLE OF CONTENTS}

Page

\section{HEAVY-ION NUCLEAR PHYSICS RESEARCH ......1}

\section{A. EXOTIC NUCLEI AND EXPERIMENTS WITH} SECONDARY BEAMS .................................................

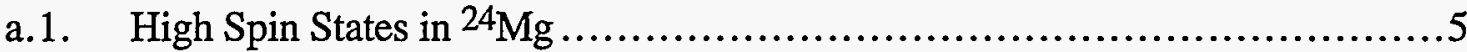

a.2. Studies of Very Neutron Deficient $N \approx Z$ Nuclei Using AYE-Ball ...............6

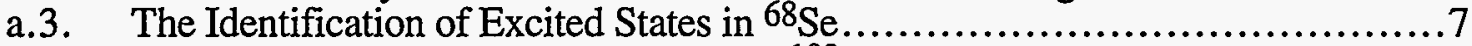

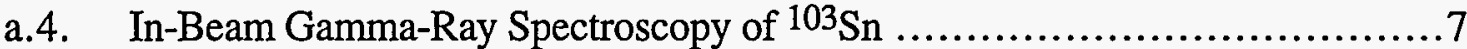

a.5. In-Beam Gamma-Ray Spectroscopy Along the Proton Drip Line using the Recoil-Decay Tagging Method ......................................8

a.6. Study of ${ }^{176-179} \mathrm{Hg}$ using the Recoil Decay Tagging Method ..................9

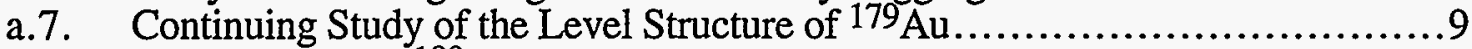

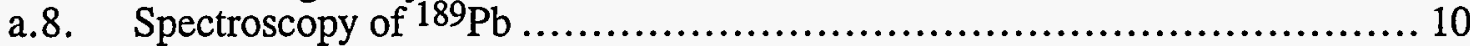

a.9. Collective Motion in Light Polonium Isotopes .................................. 10

a.10. Onset of Deformation Effects in Radon Isotopes ............................ 11

a.11. Nuclide Production Near ${ }^{100}$ Sn using a Radioactive Beam $\ldots \ldots \ldots \ldots \ldots \ldots \ldots \ldots . \ldots \ldots \ldots$

a.12. Study of Proton Radioactivities .......................................... 13

a.13. Identification of $166 \mathrm{Pt}$ and ${ }^{167} \mathrm{Pt}$ and the

Determination of the ${ }^{168} \mathrm{Pt}$ Half-Life............................................ 13

a.14. Discovery of the New Isotope $178 \mathrm{Tl}$ from its Alpha Decay ................... 14

a.15. The Study of Fine Structure in the Alpha Decay of Nuclei near

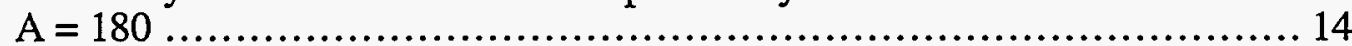

a.16. Search for Fine Structure in 192 Po $\alpha$ Decay ................................. 15

a.17. Level Structure of ${ }^{226} \mathrm{U}$ and Its Alpha Decay.............................. 15

a.18. Conversion Electron Spectroscopy at the Fragment Mass Analyzer Focal Plane: Studies of Isomeric Decays Near the Proton Drip-Line .................................................... 16

a.19. The Astrophysical Reaction Rate for the $18 \mathrm{~F}(\mathrm{p}, \alpha){ }^{15} \mathrm{O}$ Reaction ................ 17

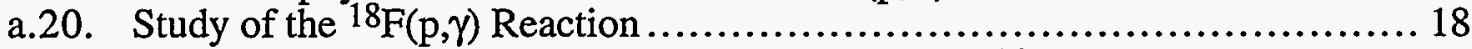

a.21. Measurement of Spectroscopic Factors around $A=56$ with a ${ }^{56 \mathrm{Ni}}$ Beam ....

B. GAMMA-RAY SPECTROSCOPY STUDIES ......................... 20

B.a. States in the Superdeformed Secondary Minimum and Their Coupling with States in the Normal Well

a.1. Spins, Parity and Excitation Energies of a Superdeformed Band in ${ }^{194} \mathrm{Hg}$ from One-Step Discrete Decays to the Yrast Line..................... 22

a.2. Search for One-Step Decay Gamma Rays Connecting

Superdeformed and Normal States in ${ }^{192} \mathrm{Hg}$................................ 24

a.3. The ${ }^{194} \mathrm{~Pb}$ Decay-Out Quasi-Continuum of Gamma Rays................... 24 
a.4. 2nd and 3rd Moments of Low Statistics (High-Energy)

$\gamma$-ray. Spectra.

a.5. A Technique for the Measurement of the Spin of Superdeformed

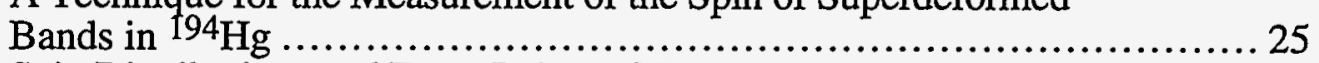

a.6. Spin Distributions and Entry Points of Super- and Normal-Deformed

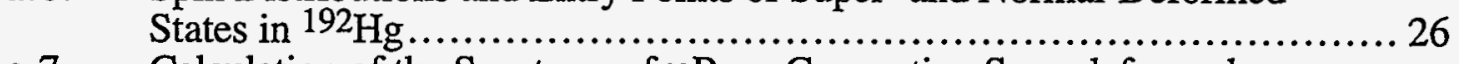

a.7. Calculation of the Spectrum of $\gamma$ Rays Connecting Superdeformed and Normally Deformed Nuclear States ............................... 26

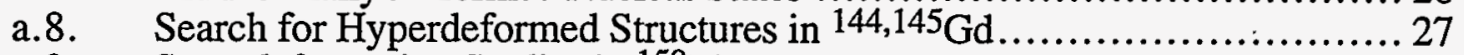

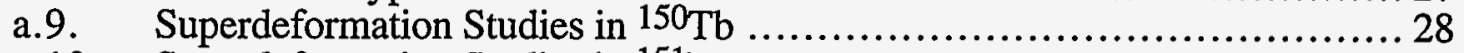

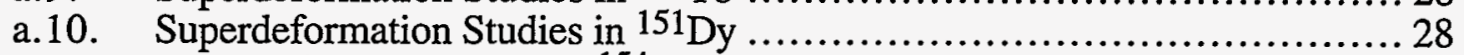

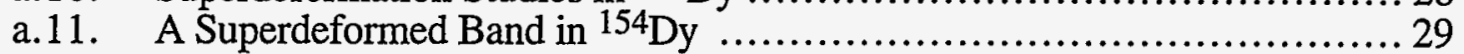

a.12. Superdeformation, New Shape Minima and Band Termination

Spectroscopy in 155 Dy ............................................... 30

a.13. Experimental Search for Very Extended Shapes in the $A \sim 180$

Region............................................................. 31

a.14. Evidence for Octupole Vibration in the Superdeformed Well of

$190 \mathrm{Hg}$ from Eurogam.................................................... 31

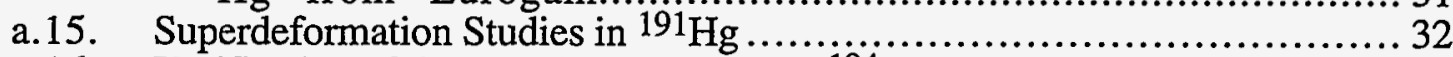

a.16. Verification of the $\Delta \mathrm{I}=4$ Oscillations in ${ }^{194} \mathrm{Hg}$ Superdeformed

Band s............................................................... 32

a.17. Differential Quadrupole Moments of Identical Superdeformed

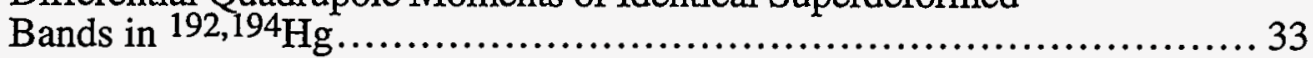

a.18. Alignment Additivity in the Two-Quasiparticle Superdeformed

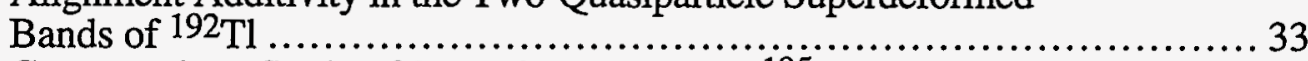

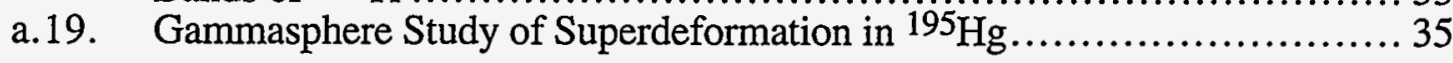

B.b. Other Nuclear Structure Studies.................................... 35

b.1. "Anatomy" of a Phase Transition Along the Yrast Line at ${ }^{154}$ Dy ........... 36

b.2. Probing the Phase Transition Boundary of Hot States in ${ }^{154}$ Dy ............... 37

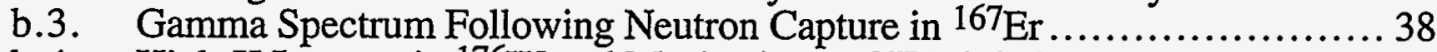

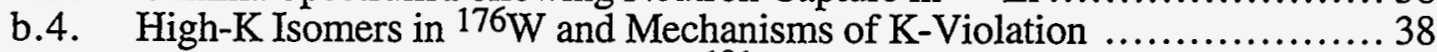

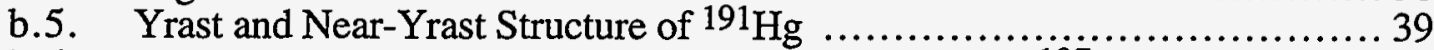

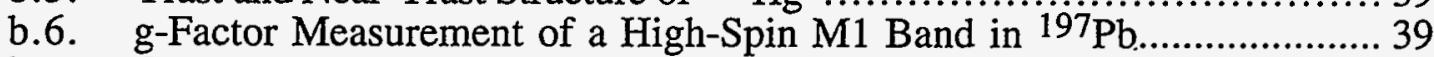

b.7. Gamma Rays from Fission Fragments................................... 40

b.8. Harmonic Two-phonon Gamma Vibrational State in

Neutron-Rich ${ }^{106 \mathrm{Mo}}$................................................ 40

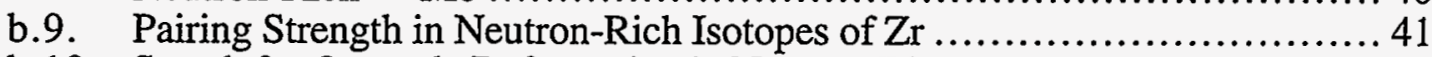

b.10. Search for Octupole Deformation in Neutron-Rich Cs Nuclei ................ 42

b.11. Search for the Two-Phonon Octupole Vibrational States in ${ }^{208} \mathrm{~Pb}$.

b.12. Level Structures at High Spins in Nuclei Near and at $N=52 \ldots \ldots \ldots \ldots \ldots . . .43$

b.13. Search of Collectivity at $N>52$ via Lifetime Measurements in $96-98 \mathrm{Ru}$

b.14. Lifetime Measurements in $180,182,184 \mathrm{Pt}$ Beyond the Backbend ............... 44

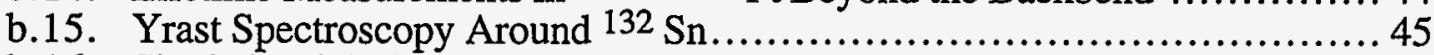

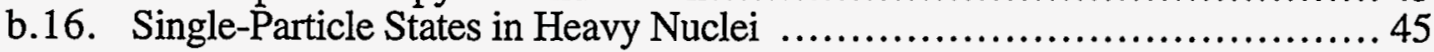


a.1. Angular Correlation Measurements for ${ }^{12} \mathrm{C}\left({ }^{12} \mathrm{C},{ }^{12} \mathrm{C}\right){ }^{12} \mathrm{C}\left(3^{-}\right)$

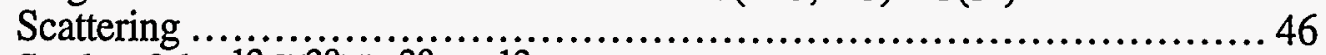

a.2. Study of the ${ }^{12} \mathrm{C}\left({ }^{20} \mathrm{Ne},{ }^{20} \mathrm{Ne}\right){ }^{12} \mathrm{C} *$ Reaction ................................. 48

a.3. Studies of Fission Hindrance in Hot Nuclei ............................. 48

a.4. Evaporation Residue Cross Sections for ${ }^{32} \mathrm{~S}+184 \mathrm{~W}$............................. 49

a.5. Evaporation Residue Cross Sections for the ${ }^{100} \mathrm{Mo}+{ }^{116} \mathrm{Cd}$

Reaction .............................................................. 49

a.6. Time-Scale in Quasi-Fission Reactions ....................................... 49

a.7. Evaporation Residue Cross Sections for the ${ }^{64} \mathrm{Ni}+144,154 \mathrm{Sm}$

Reaction .............................................................. 50

a.8. Survival Probability of Th-like Nuclei as a Function of Excitation

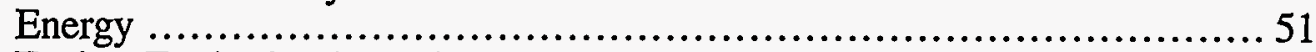

a.9. Fusion Excitation Functions Involving Transitional Nuclei .......................55 52

a.10. Multi-Neutron Transfer Reaction in the System ${ }^{58} \mathrm{Ni}+{ }^{124} \mathrm{Sn}$ at

Subbarrier Energies............................................................. 52

a.11. Deep-Inelastic Scattering near the Coulomb Barrier ............................5 53

a.12. Production of Nuclei in the $\mathrm{N}<126$ and $\mathrm{Z}>82$ Region .................... 54

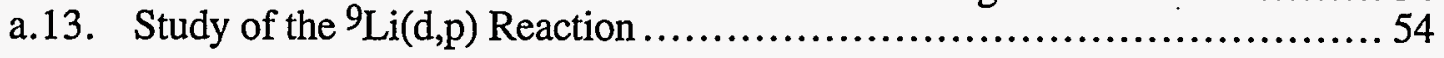

D. SUPERCRITICAL FIELDS AND OTHER TOPICS ...................... 55

a.1. Studies of Positron Electron Pair Production in $238 \mathrm{U}+{ }^{181} \mathrm{Ta} \ldots \ldots \ldots \ldots \ldots . \ldots 5$

a.2. Studies of Positron Electron Pair Production in $238 \mathrm{U}+232 \mathrm{Th}$....................5 56

a.3. Dynamic Positron Production in ${ }^{238} \mathrm{U}+{ }^{181} \mathrm{Ta}$ and

${ }^{238 \mathrm{U}}+{ }^{232} \mathrm{Th}$ Collisions ................................................. 57

a.4. Monte Carlo Studies of APEX ............................................ 58

a.5. Silicon Detector Timing Measurements at APEX …........................ 59

a.6. Measurements of Internal Pairs from ${ }^{206} \mathrm{~Pb}$.................................. 59

a.7. Spectroscopy of High-Lying States in Actinide Nuclei ...................... 60

a.8. Nature of Ordering in Confined Crystalline Ionic Systems ...................61 61

a.9. The Influence of Repeating Cells on Simulations of

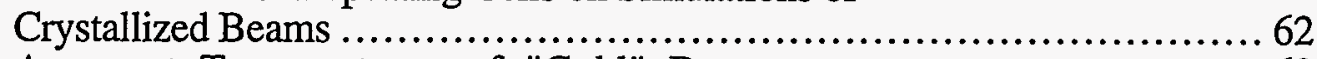

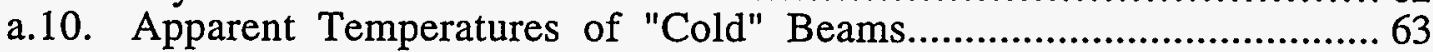

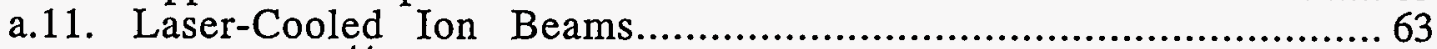

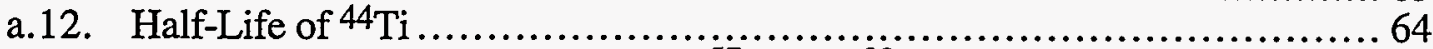

a.13. Lifetimes of the Isomeric States in ${ }^{57} \mathrm{Fe}$ and ${ }^{83} \mathrm{Kr}$............................... 64

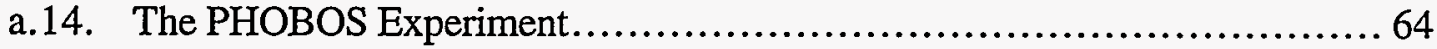

E. EQUIPMENT DEVELOPMENT AT THE ATLAS FACILITY................ 66

a.1. The AYE-Ball (Argonne-Yale-European Gamma-Ray Spectrometer) .........66 66

a.2. Fragment Mass Analyzer Project ......................................... 67

a.3. Development of an Ion Chamber for Use with the FMA.........................6 67

a.4. A Position-Sensitive Channel Plate Detector System for the FMA …....... 68

a.5. High-Efficiency Silicon Box Detector Array for the FMA

Focal Plane .......................................................... 68

a.6. Determination of a Mass Spectrum with the FMA from

Time-of-Flight Measurements 
a.7. Using the FMA for Radiative Capture Cross-Section Measurements of Interest to Astrophysics....

a.8. First In-Beam Test of the Vanderbilt-ANL-LSU Array of

Neutron Detectors...................................................... 70

a.9. Development of a Radioactive Beam Coulomb Excitation Chamber

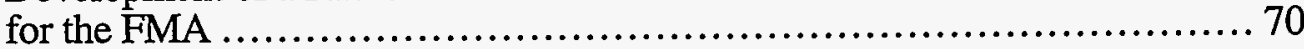

a.10. Measurements of the Transport Efficiency of the Fragment

Mass Analyzer ........................................................ 71

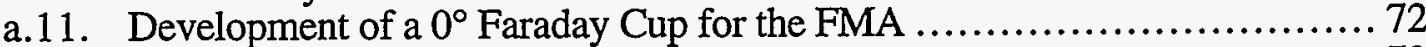

a.12. $\mathrm{BaF}_{2}$ High-Energy Gamma Array - LEPPEX............................... 72

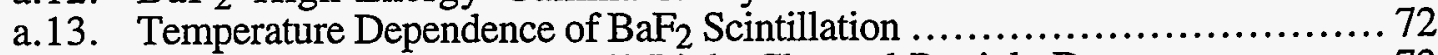

a.14. Development of Si-CsI $\triangle E-E$ Light Charged Particle Detectors................. 73

a.15. Use of the Gas-Filled-Magnet Technique for Particle Identification at Low Energies...................................................... 73

a.16. Production of $18 \mathrm{~F}$ Beams from a SNICS Ion Source ............................. 74

a.17. Test of the Beam Transport System at Low Velocities ..................... 74

a.18. Status of the Argonne-Notre Dame BGO Gamma-Ray Facility

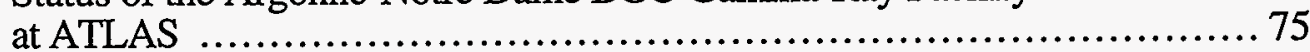

a.19. Completion of Testing and Procurement of BGO

Compton-Suppression Detectors for Gammasphere.......................... 75

a.20. Software Developments for Gammasphere ............................. 75

a.21. High-Spin Gamma-Ray Spectroscopy Software Developments ............ 76

a.22. Towards a One-Cube DCO Method with Gammasphere........................ 76

a.23. Triple Angular Correlations of $\gamma$ Rays Following Spontaneous

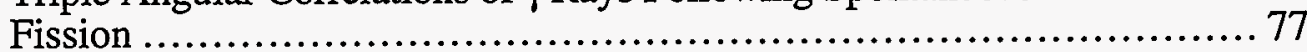

a.24. Neutron Detectors for Operation with Gammasphere ......................... 77

a.25. Development of a Plan to Move Gammasphere to ANL in 1997 ............... 77

a.26. Nuclear Target Development ............................................. 78

a.27. Physics Computing Facilities............................................. 79

a.28. Data-Acquisition Systems......................................... 80

F. ASSISTANCE TO OUTSIDE USERS OF ATLAS $\ldots \ldots \ldots \ldots \ldots \ldots \ldots \ldots 1$

a. Experiments Involving Outside Users..................................... 82

b. Outside Users of ATLAS and of ATLAS Technology --

c. Summaries of the Continuing User Programs for FY $1995 \ldots \ldots \ldots \ldots \ldots \ldots . \ldots 8$

c.a.1. Nuclear Physics ................................................ 88

c.a.2. Atomic Physics.................................................. 88

d. ATLAS - Technology Transfer ........................................ 90

\section{OPERATION AND DEVELOPMENT}

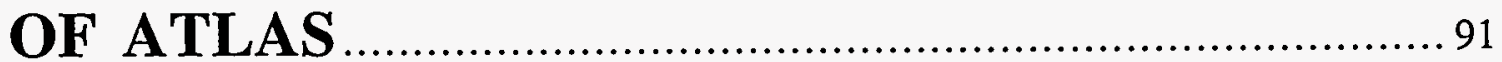

A. OPERATION OF THE ACCELERATOR............................ 93

a. Upgrade of the Linac Control System.................................. 94

b. Linac Beam-phase Monitoring at the Entrance to Each Section ............... 94

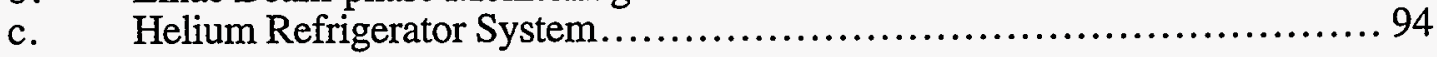


B. ACCELERATOR PHYSICS AND LINAC DEVELOPMENT ............96

a. High-Charge-State Ion Sources ...................................... 97

b. Superconducting Accelerating Structures for Low-Beta Heavy-Ions........... 97

C. ACCELERATOR PHYSICS RELATED TO THE ACCELERATION

OF RADIOACTIVE BEAMS ............................................. 97

a. Acceleration of Very Low Velocity and $\mathrm{q} / \mathrm{m}$ Radioactive Ions .................. 98

b. Superconducting Quadrupoles for Focussing in Low-beta, Low $-\mathrm{q} / \mathrm{m}$ Linacs........................................................ 98

c. Ion Sources and Targetry for Use in an Advanced Radioactive Beam Facility ............................................................ 99

d. Charge-State Enhancement Concepts for Radioactive Beam Accelerators.

e. Concepts for Remote Handling and Shielding at an Advanced Radioactive Facility

\section{MEDIUM-ENERGY NUCLEAR PHYSICS} RESEARCH

A. SUBNUCLEONIC EFFECTS IN NUCLEI

a. Deep-Inelastic Muon Scattering from Nuclei with Hadron

Detection .............................................................. 103

b. Electron-Deuteron Scattering With a Polarized Deuterium Gas

Target in the VEPP-3 Electron Storage Ring ................................105

c. Laser-Driven Polarized Hydrogen and Deuterium Internal Targets...........105

d. Nuclear and $Q^{2}$ Dependence of Quasielastic (e,e'p) Scattering at

Large Momentum Transfer ....................................................106

e. Polarized Tritium Target Development ...................................106

f. Electroproduction of Kaons and Light Hypernuclei …….......................107

g. Two-Body Photodisintegration of the Deuteron at Forward Angles and Photon Energies Between 1.5 and $4.0 \mathrm{GeV}$.................... 107

h. A Study of Longitudinal Charged-Pion Electroproduction in D,

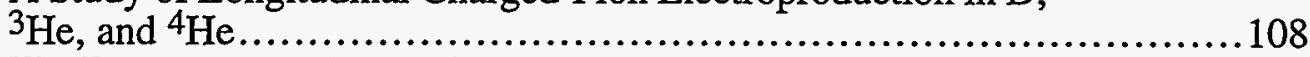

i. The Energy Dependence of Nucleon Propagation in Nuclei as Measured in the (e,e'p) Reaction.................................................109

j. $\quad$ Measurement of Proton Polarization in the $d(\gamma, p) n$ Reaction ................110

k. Short-Orbit Spectrometer for Hall C at CEBAF...................................110

1. $\quad 3 \mathrm{He}$ Target for Hall $\mathrm{C}$ at CEBAF ..................................... 110

m. HERMES, Measurements of Spin-Structure Functions and Semi-Inclusive Asymmetries for the Proton and Neutron at HERA..........111

n. Nuclear Transparency and Longitudinal Asymmetry in $A\left(e, e^{\prime} \rho^{0}\right)$ at High Energies .....................................................113

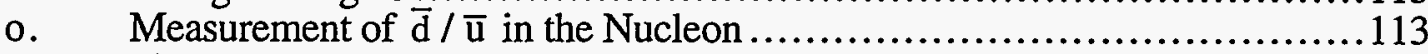

p. First Measurement of the Triton Asymmetry in Muon Capture by Polarized Muonic ${ }^{3} \mathrm{He}$ 


\section{A. NUCLEAR DYNAMICS WITH SUBNUCLEONIC DEGREES OF} FREEDOM

a. Production of Mesons and Nucleon Resonances at

$\mathrm{GeV}$ Energies.

b. Pomeron-Exchange and Exclusive Electroproduction of $\rho$-Mesons

c. Calculation of E2/M1 Ratio of the $\Delta \rightarrow \gamma N$ Within the Chiral

Constituent Quark Model

d. Phenomenological Coupled-Channel Model for $\pi \mathrm{N}$ and $\gamma \mathrm{N}$

Reactions With ${ }^{*}$ Excitations

e. Electroproduction of Pions on the Three-Nucleon Systems

f. Threshold Pion Production from Nucleon-Nucleon Collisions ..............118

g. Production of $\eta$ Meson in Photo-Nucleon and Nucleon-Nucleon

Reactions............................................................. 118

h. Medium Effects on Binary Collisions With the $\Delta$ Resonance ................119

i. The Effect of the $\Delta$ Excitation on Proton:Proton Bremsstrahlung ..........119

j. The Effect of the $\Delta$ Three-Body Force on Effective

Nucleon-Nucleon Interactions of the Nuclear Shell-Model

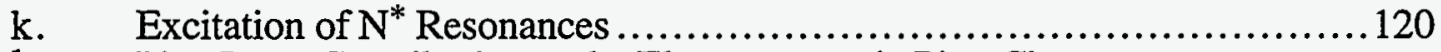

1. Pion Loop Contribution to the Electromagnetic Pion Charge

Radius

m. Analytic Structure of the Quark Propagator in a Model With an

Infrared-Vanishing Gluon Propagator ................................. 121

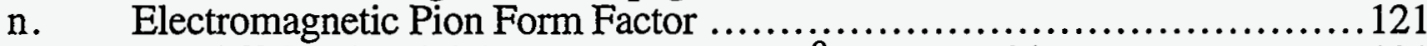

o. The Off-Shell Axial Anomaly via the $\gamma * \pi^{0} \rightarrow \gamma$ Transition................121

p. Model Gluon Propagator and Pion and $\rho$-Meson Observables.................122

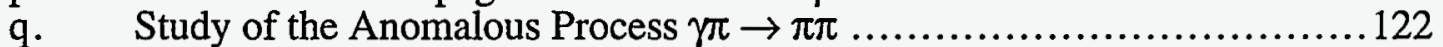

r. Electromagnetic Charged and Neutral Kaon Form Factors .................123

s. Bethe-Salpeter Equation Studies of $\mathrm{SU}_{\mathrm{f}}(3)$ Mesons......................123

t. Electromagnetic Nucleon Form Factors................................. 124

u. Quark and Diquark Confinement Beyond Rainbow/Ladder

Approximation ................................................... 124

v. Dynamical Chiral-Symmetry Breaking and Confinement at

Finite Temperature ................................................... 125

w. Nucleon Faddeev Equation Studies...........................................125

$\mathrm{x}$ QCD Greens Functions in the Infrared ................................ 125

y. Relativistic Approaches to Hadrons and Nuclei at Medium

Energies......................................................... 126

z. Electroweak Transitions in Quark Model Baryons...............................126

aa. Current Operators in Relativistic Few-Body Systems .....................126

bb. Theory of Hadronic Nonperturbative Models ...........................127

cc. Osterwalder-Schrader Positivity of Euclidean Quark Propagators...........127 
B. NUCLEAR FORCES AND NUCLEAR SYSTEMS

a. Three-Body Correlations in Few-Body Nuclei ........................... 128

b. Variational and Green's Function Monte Carlo Calculations of

Light p-Shell Nuclei ............................................... 128

c. Ground States of Larger Nuclei ..................................... 130

d. Short-Range Structure of the Deuteron and Other Nuclei ...................130

e. Charge-Independence Breaking in Light Nuclei ............................131

f. Microscopic Calculation of Elastic and Transition Form Factors

of Lithium.............................................................. 131

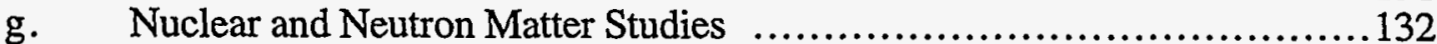

h. Elementary Diagrams in Nuclear and Neutron Matter ...................... 132

i. Spin-Orbit Splitting in Neutron Drops ....................................133

j. $\quad$ Calculation of Effective Interactions of the Nuclear Shell

Model Using the Argonne v18. Potential .....................................134

k. $\quad \Lambda$ Single-Particle Energies .......................................... 134

1. Core-Nucleus Distortion in Hypernuclei ................................ 134

m. Space-Exchange Effects in Light Hypernuclei ............................ 134

n. Charge-Symmetry Breaking $\Lambda$-Nucleon Interaction....................... 135

o. Suppression of the $\Lambda-\Sigma$ Coupling in Nuclear Matter ...................... 135

p. Nuclear Core Dynamics and Rearrangement Energy

for Hypernuclei

C. NUCLEAR STRUCTURE AND HEAVY-ION REACTIONS

a. Coulomb Dissociation of ${ }^{8} \mathrm{~B}$

b. Momentum Distributions in Nuclear-induced Breakup

Reactions

c. Pairing Gap in the Inner Crust of Neutron Stars.

d. Pair Correlations in Heavy, Neutron-rich Nuclei ......................... 138

e. Nuclear Energy Surfaces at High-Spin in the A 180

Mass Region ..................................................... 138

f. Very Extended Shapes in the A 150 Mass Region .........................139

g. Rotational Spacings in Superdeformed Bands of Nuclei .................. 140

h. Experimental Search for Very Extended Shapes in the

$A \sim 180$ Region ..................................................... 141

i. Single-particle States in the Heaviest Elements ......................... 142

j. $\quad$ Many-Body Wave Functions............................................ 142

k. Nuclear Structure Near the Drip Line...................................... 142

1. Survey of Nuclear Energy Surfaces in the A 120 Region .................143

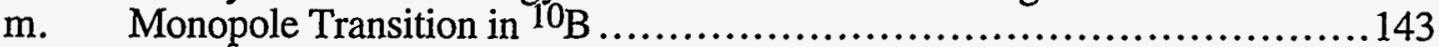

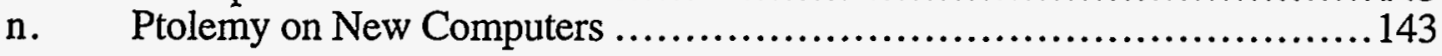

D. FUNDAMENTAL QUANTUM MECHANICS ......................... 143

a. Aharonov-Bohm Effects in Normal Metal Shields..............................143

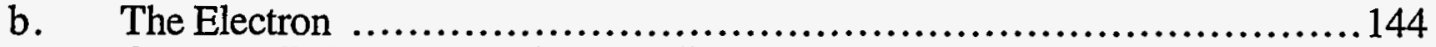

c. Quantum Ballistic Evolution: Application to Quantum

Computation 


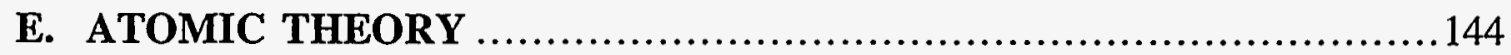

a. Interactions of High-Energy Photons with Matter .........................144

b. Interactions of Fast-Charged Particles with Matter .......................145

c. Beam Polarization and Non-Dipole Effects in X-Ray

Scattering and Electron Ejection.................................................145

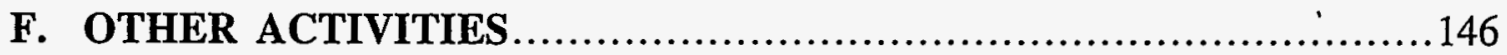

a. Theory Workshop on Pairing Forces ..................................146

b. Eighth Annual Midwest Nuclear Theory Get-Together ..........................146

\section{ATOMIC AND MOLECULAR PHYSICS}

RESEARCH ....................................................... 147

A. ACCELERATOR-BASED ATOMIC PHYSICS ......................... 149

a. Measurement of Short Lifetimes in Highly-Charged Ions

using a Two-Foil Target .....................................................150

b. Spectral Distribution of the Two-Photon Decay of He-Like

Krypton ............................................................ 151

c. Wavelength and Lifetime Measurements in Highly-Ionized

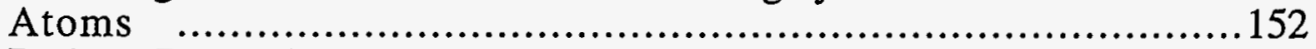

d. Positron Production in Heavy-Ion Collisions .................................153

e. Spectroscopy of H-Like Uranium ....................................153

f. Interactions of High-Energy, Highly-Charged Xe Ions with $\mathrm{C}_{60} \ldots \ldots \ldots \ldots . .154$

g. Precision Lifetime Measurements by Single-Photon Counting..................154

h. Hyperfine Structure Studies of Transition Metals.................................155

B. ATOMIC PHYSICS AT SYNCHROTRON LIGHT SOURCES .........155

a. Non-Dipolar Asymmetries of Photoelectron Angular

Distributions......................................................... 156

b. Double and Single Photoionization of Helium by $60-\mathrm{keV}$

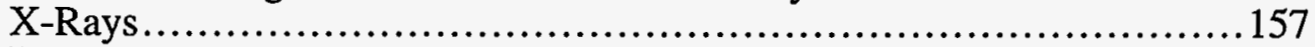

c. Inclusive Recoil Momentum Spectroscopy of Single and

Double Ionization of He by 85-400 eV Photons ........................... 158

d. Auger Resonant Raman Spectroscopy ………..............................158

e. X-Ray Scattering and Fluorescence Studies............................. 159

f. X-Ray Resonance Raman Scattering .......................................159

g. Electron/X-Ray Coincidence Studies of Inner-Shell Processes ...............160

h. Preparations for Atomic Physics at the Advanced Photon Source ............161 


\section{OTHER EDUCATIONAL ACTIVITIES IN THE} PHYSICS DIVISION

a. Enhancement of Minority Involvement in DOE Nuclear Physics Programs

b. Nuclear Physics Award for Faculty in Undergraduate Institutions

c. Scientific Support of SciTech Museum Exhibits and Outreach

d. The "Museum in a School" Program

e. The $\mathrm{E}=\mathrm{mc}^{2}$ Exhibition

Staff List.

Publications 


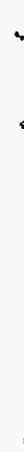

เ

xviii

, 


\section{HEAVY-ION NUCLEAR PHYSICS RESEARCH}

\section{OVERVIEW}

The heavy-ion program in the Argonne Physics Division spans a broad range of activities from the exploration of the multiple facets of nuclear structure at the limits of the valley of stability to the delineation of the properties of superdeformed nuclei; from the characterization of reactions in the vicinity of the Coulomb barrier to investigations of the effects of the strong fields generated in collisions between very heavy nuclei; from the measurement of cross sections of astrophysics interest to the development of the instrumentation and techniques required to perform experiments with radioactive beams of small intensity. This is a versatile program with involvement of many university groups and outside institutions, characterized by extensive collaborations between in-house and outside researchers. Most of these studies are carried out at the ATLAS accelerator, but parts of the program take advantage of world-class instrumentation available elsewhere.

The study of the structure of nuclei at the very limits of stability is currently one of the major thrusts of research at ATLAS. The excellent, high-intensity heavy-ion beams from ATLAS and the high detection efficiency and excellent background suppression of the Fragment Mass Analyzer (FMA) provide the tools required for a wide range of studies encompassing topics such as proton radioactivity, production of very heavy nuclei, and nuclear spectroscopy at and beyond the drip line.

The three heaviest proton emitters known today were discovered at the FMA using the recoil implantation system installed behind the focal plane of the instrument. Nuclei identified according to their mass in the focal plane are implanted in a double-sided Si strip detector where their subsequent decay can be measured and correlated with the information gathered at the time of implantation. The first proton emitter above the $\mathrm{Z}=82$ closed shell was measured in this way in $185 \mathrm{Bi}$. The decay proceeds from a low-lying state based on the $s_{1 / 2}$ intruder configuration with a proton energy of $1.585(9) \mathrm{MeV}$ and a half-life of $44(16) \mu \mathrm{s}$.

Nuclear structure studies of nuclei at the limits of stability, i.e. at the proton drip line and beyond, were greatly facilitated this year by the coupling of the FMA with a more powerful array for gamma-ray detectors than was possible earlier. The AYE-Ball array (Argonne-Yale-European Gamma-ray Spectrometer) was put together as a collaborative effort between scientists from the U.K. and Argonne. Because this array of 18 Compton-suppressed spectrometers contained $9 \mathrm{Ge}$ detectors of large volume, the detection efficiency was increased by an order of magnitude with respect to what was achieved earlier with the 10 spectrometers of the local Argonne-Notre Dame BGO gamma-ray facility.

The experimental program with AYE-Ball was only recently completed and the data analysis of most measurements is still in progress. Nevertheless, it is already possible to summarize briefly some of the results. The region in excitation energy where particle decay and electromagnetic emission compete in ${ }^{24} \mathrm{Mg}$ was 
investigated. All known high-spin states in ${ }^{24} \mathrm{Mg}$ were clearly identified with AYEBall and the FMA and new levels were observed as well, while a $\mathrm{J}=9$ state reported in an earlier measurement was not. Several experiments were devoted to the study of nuclei with equal proton and neutron number in order to search for neutron-proton pairing effects. The lightest even $\mathrm{Hg}$ isotopes known thus far, $176,178 \mathrm{Hg}$, were found to exhibit shape coexistence between an oblate level structure based on the groundstate and an excited band sequence based on a prolate minimum. The excitation energy of the latter increases as one moves away from the $\mathrm{N}=102$ mid-shell. First measurements of excited states in $200 \mathrm{Rn}$ indicate that the onset of ground-state deformation predicted by recent mean field calculations is not borne out by experiment. Prompt gamma-ray sequences were correlated with the protons emitted from the ground state and the isomeric state of ${ }^{147} \mathrm{Tm}$, truly representing in-beam spectroscopy beyond the drip line. Prompt gamma cascades were correlated with the alpha decay of both the ground state and the isomeric excited level in ${ }^{156} \mathrm{Hf}$. The results obtained provide an explanation for the presence of the isomer. This state can be regarded as a classical case of an yrast trap.

A major effort is devoted to gamma-ray experiments with a focus on studies of superdeformation in the mass 150 and 190 regions. The search addresses the physics associated with states within the superdeformed (SD) well and their coupling outside the well.

The long cascades of rotational transitions between SD states are easy to detect with modern arrays such as Gammasphere. It was much harder to localize the SD bands in excitation energy and spin and to link them to the normal-deformed (ND) yrast states. For example, in the mass 150 and 190 regions, the intra-band transitions of more than $100 \mathrm{SD}$ bands were found, yet, despite many attempts, there is no band for which the exact excitation energies, spins and parity was conclusively determined. This problem has been a focus of the Argonne research program for quite some time. It was shown that the decay-out of a SD band occurs when a SD level, embedded in a sea of hot ND states, acquires a small component of the hot compound state, and decays through this component. This description is supported by the measured decay spectrum, which has a statistical quasicontinuous character, and by its agreement with a calculation of the statistical spectrum. This calculation suggested that direct, one-step decay has a branching ratio of up to $\sim 5 \%$ of the total decay-out and may be detectable. In a recent Gammasphere experiment on the ${ }^{194} \mathrm{Hg}$ nucleus, four discrete gamma rays directly connecting levels of the yrast SD band to the yrast states were discovered. Thus, the excitation energies and spins of all members of the lowest SD band were established for the first time, together with their likely parity. This result represents a new, stringent test of the theoretical description of superdeformation.

Many other results on the properties of superdeformed bands were also obtained this year. For example, detailed lifetime measurements on the SD bands in ${ }^{151} \mathrm{Dy}$ and ${ }^{152}$ Dy were performed in the same experiment, hereby eliminating the uncertainties associated with the stopping powers etc. It was found that the "identical" bands in the two nuclei have the same quadrupole transition moment and that the deformation depends strongly on the number of occupied intruder orbitals. The island of superdeformation around 152Dy was extended with the discovery of SD bands in 154Dy and 155Dy. Further insight in the issue of "identical" SD bands was also obtained from a study of the bands in the odd-odd ${ }^{192} \mathrm{Tl}$ nucleus where it was shown that the alignments of the four measured bands relative to the ${ }^{192} \mathrm{Hg} \mathrm{SD}$ 
core can be accounted for from the additive contributions of the assigned quasiproton and quasineutron orbitals derived from bands in the odd-A neighbors.

The APEX project studies the effects of extremely strong electromagnetic fields in close collisions of high- $Z$ atoms at energies close to the Coulomb barrier. This situation was predicted to give rise to qualitatively new phenomena associated with the over-critical binding of the inner electron orbits, such as the spontaneous emission of positrons. Experiments carried out at GSI Darmstadt over the past decade produced some remarkable and unusual results, i.e. sharp lines in sumenergy spectra of $\mathrm{e}^{+}-\mathrm{e}^{-}$pairs. If confirmed, these results would seem to signal the appearance of some interesting and possibly fundamental new physics. The collaboration, which involves scientists from Argonne, Berkeley, Chicago, Florida State, Michigan State, Princeton, Queen's, Rochester, Washington and Yale, has recently completed a study of positron and electron pairs in collisions of ${ }^{238} \mathrm{U}+$ ${ }^{181} \mathrm{Ta}$ at $5.95,6.10$ and $6.30 \mathrm{MeV} / \mathrm{u}$ and $238 \mathrm{U}+{ }^{232} \mathrm{Th}$ at $5.95 \mathrm{MeV} / \mathrm{u}$. In none of the analyses is any evidence found for sharp sum-energy lines. For the specific case of the isolated decay of a neutral particle of mass $1.4-2.1 \mathrm{MeV} / \mathrm{c}^{2}$, the upper limits on cross sections obtained from the present data are less by one to two orders of magnitude than the previously reported cross sections.

The study of the various reaction mechanisms involved in the interaction of two heavy ions at energies in the vicinity of the Coulomb barrier remains a topic of much interest. Over the past years, experiments covered topics such as the dependence of the subbarrier enhancement on the stiffness of the reaction partners, multi-nucleon transfer and deep-inelastic scattering at subbarrier energies, and hindrance of fission in hot nuclei. Experimental studies of the time scale for fission of hot nuclei were recently carried out using the emission rate of neutrons, gamma rays, and charged particles as clocks for the fission process. Strong indications have emerged that fission is strongly retarded relative to expectations based on a statistical description of the process. In order to gain more insight on the dissipation mechanisms responsible for the hindrance, two new types of measurements were initiated at ATLAS: measurements of evaporation residue cross sections and of target-like deeply inelastic products in kinematic coincidence with binary reaction partners.

The acceleration of unstable beams is rapidly becoming one of the highest priorities in low-energy nuclear structure research, as outlined in the recent Long-Range Plan exercise by the Nuclear Science Advisory Committee. A working group within the Physics Division is concentrating on the physics case for radioactive beams and on a novel technical concept for a facility with substantial capabilities incorporating ATLAS. A working document summarizing the current status of the discussions was prepared. The efforts have since continued in several directions. Detailed design studies for several components of the acceleration scheme are on-going. These are described elsewhere in this report. On the experimental side, experience is being gained on the development of the techniques necessary to perform experiments with radioactive beams. Currently, these efforts focus on experiments with ${ }^{18} \mathrm{~F}$ and $56 \mathrm{Ni}$ radioactive beams.

For the first time the $18 \mathrm{~F}(\mathrm{p}, \alpha)^{15} \mathrm{O}$ reaction rate was studied at ATLAS with a radioactive $18 \mathrm{~F}$ beam at energies which are of interest to explosive nucleosynthesis. By utilizing the gas-filled magnet technique, a clean identification of the reaction products was achieved. A resonant state in ${ }^{19} \mathrm{Ne}$ with spin $3 / 2^{+}$was found with an excitation energy of 7.064 (4) MeV, and proton and $\alpha$-particle widths of 5 (1.6) and $8.6(2.5) \mathrm{keV}$, respectively. This $\ell_{\mathrm{p}}=0$ resonance is found to dominate the 
astrophysical reaction rate at temperatures $\mathrm{T} 9>0.5$. The efforts now concentrate on a measurement of the ${ }^{18} \mathrm{~F}(\mathrm{p}, \gamma){ }^{19} \mathrm{Ne}$ reaction and it is felt that it should be possible to carry out the measurement for $\frac{\Gamma \gamma}{\Gamma_{p}} \sim 10^{-3}$.

$56 \mathrm{Ni}$ is the heaviest $\mathrm{N}=\mathrm{Z}$ doubly magic nucleus for which a beam with sufficient intensity can be produced. So far, only an experiment studying inelastic scattering was performed with the ${ }^{56} \mathrm{Ni}$ radioactive ions being produced in a high energy fragmentation reaction. Based on the experience with $18 \mathrm{~F}$, the development of a $56 \mathrm{Ni}$ beam at ATLAS has been started. The physics that will be addressed in the first experiments relates to $56 \mathrm{Ni}$-induced few nuclear transfer reactions and the study of neutron-deficient nuclei near the proton drip line.

\section{A. EXOTIC NUCLEI AND EXPERIMENTS WITH SECONDARY BEAMS}

The study of nuclei at the very limits of stability is currently a subject of renewed interest, as can be seen from the conclusions expressed in the recent Long Range Plan for Nuclear Science prepared by the Nuclear Science Advisory Committee (NSAC). Theoretical interest in nuclei far from stability was also stimulated by the conceptual developments of future Radioactive Beam Facilities. Many interesting aspects emerged and await experimental investigation. With the facilities available at ATLAS, it is possible to address some of the physics issues of interest in a major way.

The Fragment Mass Analyzer (FMA) has emerged as a unique and powerful tool for selecting nuclei at the very limits of stability. The fact that the proton drip line can be reached, even passed, was demonstrated in extensive measurements of groundstate proton decay. To exploit this capability, and to extend it into the domain of prompt spectroscopy of excited nuclear states, a powerful device for gamma-ray spectroscopy is also desirable. Preliminary experiments with the existing Argonne-Notre Dame array of Compton-suppressed Ge detectors at the FMA target location showed the promise of coupled FMA-gamma ray studies, but an array of photon detection with greater efficiency was needed to sustain the progress. The AYE-Ball array of gamma-ray detectors described elsewhere in this report was installed at the target position of the FMA for the second half of calendar year 1995. With an increase of roughly an order of magnitude in efficiency over what was available earlier, it is possible to explore nuclei at the proton drip line and beyond. Furthermore, the research with this instrument can be considered as the precursor to the program to be conducted when Gammasphere and the FMA are coupled.

Work towards the development of a radioactive beam facility to be coupled to the ATLAS accelerator was initiated in the Physics Division last year. The activities culminated with the preparation of a working document summarizing the physics case and a possible design for a facility. Since then the efforts continued in two directions. Substantial R\&D effort towards resolving some of the technical challenges is taking place as described elsewhere in this report. On the other hand, an experimental program utilizing secondary (radioactive) beams was begun. At the present time, the latter focuses on the acceleration of the ${ }^{18} \mathrm{~F}$ and ${ }^{56} \mathrm{Ni}$ isotopes in order to address mainly questions of astrophysical interest. 
a.1. High Spin States in ${ }^{24} \mathrm{Mg}$ (C. J. Lister, A. Wuosmaa, R. R. Betts, D. J. Blumenthal, M. P. Carpenter, S. M. Fischer, G. S. Hackman, R. V. F. Janssens, and J. Schwartz)

Nuclei in the p- and sd- shells remain critical to our understanding of nuclear structure in general, as it is there that many theoretical approaches can be used, and be confronted with a broad and detailed body of experimental data. In particular, nuclei in the middle of shells, like ${ }^{20} \mathrm{Ne}$ and ${ }^{24} \mathrm{Mg}$, allow us to understand the microscopic makeup of the collective behavior, and its evolution with excitation energy and spin. The concepts of loss of collectivity and band termination can be investigated in more detail than elsewhere, as both fully microscopic and phenomenological calculations are available. At the highest energies, above $20 \mathrm{MeV}$ in excitation, highly deformed alpha-cluster configurations are known which mainly decay by alphaparticle breakup, but may relax to more conventional shell-model states by $\gamma$-ray emission. The relationship between these cluster states and more conventional nuclear structures is also of great interest.

Despite a wealth of experimental data, gleaned from transfer reactions, Coulomb excitation, beta decay, light and heavy-ion induced fusion reactions and a variety of other probes, information on states of angular momentum greater than $J=8 \hbar$ is scarce. This is unfortunate, as many of the outstanding issues of structure concern these levels. The reason for the lack of data is that the reactions which populate these states, heavy-ion reactions at more than twice the Coulomb barrier, tend to produce a vast array of other states, both in ${ }^{24} \mathrm{Mg}$ and other nuclei, so identifying the levels of interest through their decays is technically challenging.

In order to try to address these issues, and identify the electromagnetic decays of states of very high spin in ${ }^{24} \mathrm{Mg}$, the AYE-Ball array was employed to isolate radiation from high spin states in $24 \mathrm{Mg}$. A short experiment was performed using the ${ }^{12} \mathrm{C}\left({ }^{16} \mathrm{O}, \alpha\right){ }^{24} \mathrm{Mg}$ reaction at $51.5 \mathrm{MeV}$. This energy was chosen in order to reproduce the conditions under which the highest spin gamma decaying state in ${ }^{24} \mathrm{Mg}$ had been reported. ${ }^{1}$ Our technique involves directly identifying ${ }^{24} \mathrm{Mg}$ recoils near zero degrees in the Fragment Mass Analyzer (FMA) and gamma rays in the AYE-Ball spectrometer. The method worked well and evaporation residues could be identified at the focal plane. Proper time random

\footnotetext{
$\overline{1}$ A. E. Smith et al., Phys. Lett. B176, 292 (1986).

2L. R. Greenwood et al., Phys. Rev. C 6 , 2112 (1972).
}

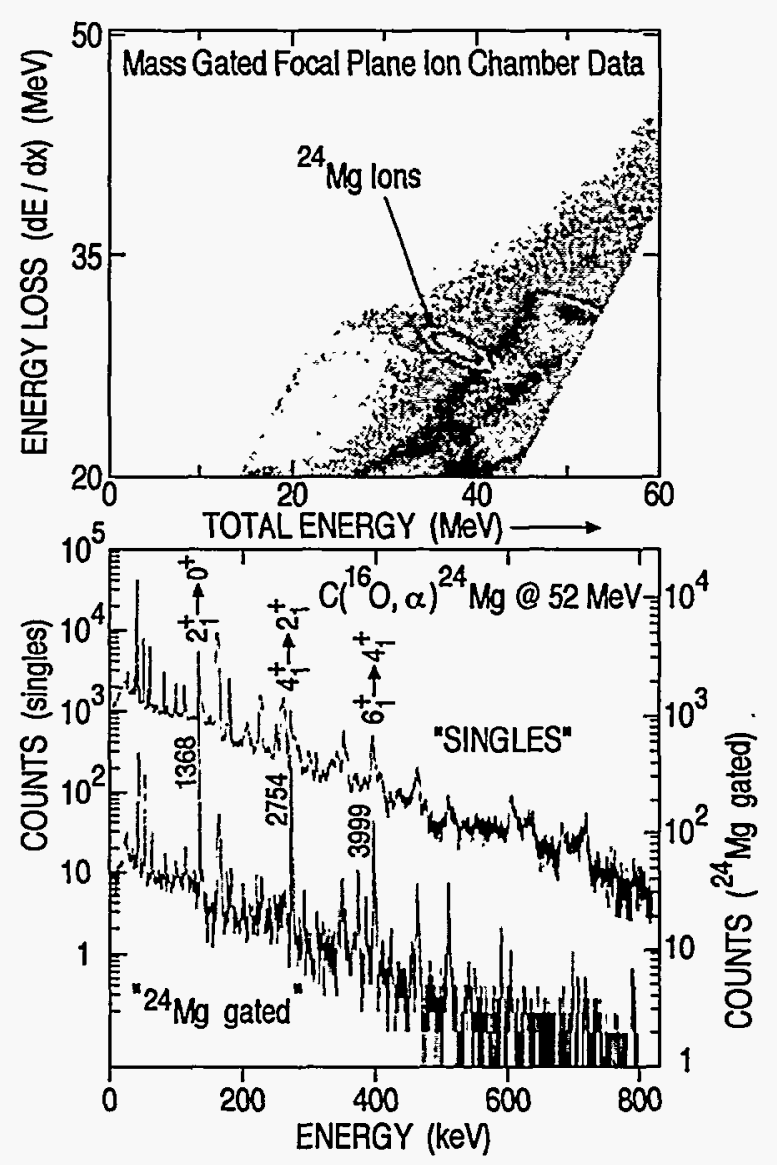

Fig. I-1. Top panel. A portion of the mass-gated energy loss $v$ s. energy map from the ionization chamber behind the focal plane of the FMA, showing a clear feature arising from ${ }^{24} \mathrm{Mg}$ residues. No other mass $M=24$ ion is strongly produced. Lower Panel. A sample of the "total" gamma-ray spectrum and that gated by ${ }^{24} \mathrm{Mg}$.

subtraction allowed for the construction of gamma spectra with only transitions associated with the decay of states in $24 \mathrm{Mg}$. This is illustrated in Fig. I-1. Although all the known $\mathrm{J}=8$ states were observed, the reaction should also have populated states to considerably higher spin, ${ }^{2}$ notably the decay from the proposed ${ }^{1}$ spin $\mathrm{J}=9$ states at $16.40 \mathrm{MeV}$ should have been seen.

Considerable technical advances are still possible, including establishing the energy of the gamma decaying states in ${ }^{24} \mathrm{Mg}$ through measuring the coincident alpha particle and the time-of-flight of the residues. This combination should allow a uniquely sensitive measurement for states which have small 
gamma-decay branches, but which lie far above the particle-emission threshold. The very high spin states with $\mathrm{J}>8$ are predicted to lie in this regime.

The FMA gating method seems very powerful and can be improved considerably. The reaction used is two body, so measurement of either the ${ }^{24} \mathrm{Mg}$ or ${ }^{4} \mathrm{He}$ reaction products uniquely determines the excitation energy of the state. In fact, measuring the ${ }^{24} \mathrm{Mg}$ time of flight appears most appropriate, as it can be done with sufficient precision that the excitation energy of the emitting state can be determined to a few hundred
$\mathrm{keV}$, dependent mainly on target thickness. However, the simultaneous measurement of the partner ${ }^{4} \mathrm{He}$, emitted near $180^{\circ}$, should allow the extraction of an almost background-free spectra of high-lying, gammaemitting states. Even without gamma-ray detection, a search for high-lying states which have electromagnetic decays will be straightforward and should have unprecedented sensitivity. Ultimately, the measurement of these high-spin states through their gamma decay using Gammasphere triggered by the FMA appears an interesting and challenging avenue to explore.

\section{a.2. Studies of Very Neutron Deficient $\mathbf{N} \approx \mathbf{Z}$ Nuclei Using AYE-Ball}

(C. J. Lister, C. Davids, D. Seweryniak, D. J. Blumenthal, M. P. Carpenter, D. Ackermann, D. Henderson, R. V. F. Janssens, J. Schwartz, D. Nisius, S. Mohammadi,* S. Vincent,* P. H. Regan,* W. Gelletly,* C. Pearson,* J. Simpson, $†$ and D. D. Warner†)

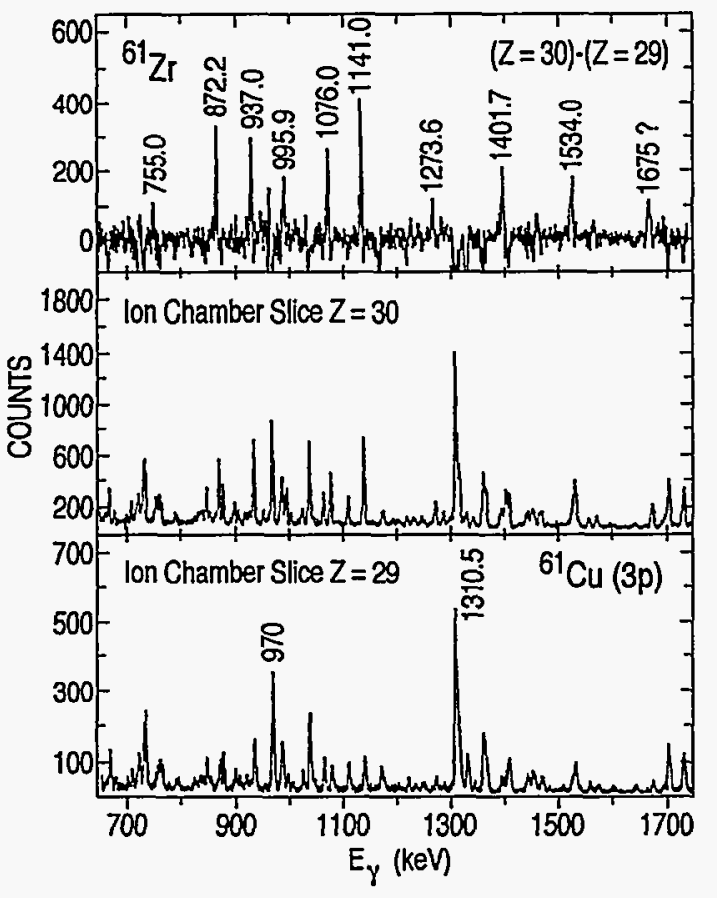

Fig. I-1A. Spectrum showing the $Z$ discrimination from the split anode ionization chamber for the $A=61$ recoils in the ${ }^{24} \mathrm{Mg}+{ }^{40} \mathrm{Ca}$ experiment. The $Z=30$ (Ni) lines from the $2 p n$ channel can be clearly separated from the $3 p$ lines to ${ }_{29}^{61} \mathrm{Cu}$.

Nuclei with equal numbers of protons and neutrons constitute uniquely symmetrical systems in nature. The

*Daresbury Laboratory, United Kingdom †University of Surrey, United Kingdom

${ }^{1}$ C. J. Lister et al., Phys. Rev. C 42, R1191 (1990) effects of deformed shell gaps in the nuclear mean field for certain nucleon numbers is amplified in such systems and even minimal information on the excited states of these nuclei can give a useful insight into the deformed potential. Studies of weakly populated, very neutron-deficient nuclei along the $N=Z$ line via fusion evaporation reactions have been performed using the AYE-Ball array in conjunction with the FMA. Three experiments, led by the Surrey and Daresbury groups, in collaboration with ANL, were performed using the (a) $54 \mathrm{Fe}+{ }^{36} \mathrm{Ar}$ at $120 \mathrm{MeV}$; (b) $58 \mathrm{Ni}+{ }^{36} \mathrm{Ar}$ at 120 $\mathrm{MeV}$, and (c) ${ }^{40} \mathrm{Ca}+{ }^{24} \mathrm{Mg}$ at $65 \mathrm{MeV}$ reactions, respectively. The main aims of these experiments were to identify the hitherto unknown first excited states in the $\mathrm{N}=\mathrm{Z}$ nuclei ${ }_{44}^{88} \mathrm{Mo},{ }_{46}^{92} \mathrm{Pd}$ and ${ }_{31}^{62} \mathrm{Ga}$, respectively.

To detect high efficiency recoil gated $\gamma$ singles and recoil gated $\gamma-\gamma$ coincidences, the germanium detector array AYE-Ball was used in conjunction with the Argonne fragment mass analyzer (FMA). AYE-Ball is an array of 18 Compton-suppressed germanium detectors, including seven high-efficiency Eurogam-type detectors. The array was placed around the target position of the FMA, together with 11 NE213 liquid scintillator neutron detectors and a split anode gas ionization chamber at the end of the FMA. An example of the $\mathrm{Z}$ separation is shown in the mass 61 gated spectra (Fig. I-1A) from the ${ }^{40} \mathrm{Ca}+{ }^{24} \mathrm{Mg}$ reaction which clearly separates the $2 \mathrm{pn}\left({ }^{61} \mathrm{Zn}\right)$ and $3 \mathrm{p}\left({ }^{61} \mathrm{Cu}\right)$ residual channels. For high recoil velocities, this setup allowed both $\mathrm{Z}$ and $\mathrm{N}$ characterization of the reaction recoils in coincidence with prompt $\gamma$ rays. The experiments provided sufficient sensitivity to select very weakly populated, very neutron deficient nuclei. Recoil and neutron gated $\gamma-\gamma$ coincidence matrices have been 
produced to expand the yrast decay schemes of nuclei populated in these reactions.

In addition to the weak $\mathrm{N}=\mathrm{Z}$ nuclei, many other channels have considerable reaction cross sections and the data obtained on these nuclei constitutes a significant increase in the knowledge of their near-yrast states. For example, ${ }^{58} \mathrm{Ni}$ is strongly populated via the $\alpha 2 \mathrm{p}$ channel in the ${ }^{24} \mathrm{Mg}+{ }^{40} \mathrm{Ca}$. The channel selection afforded by the FMA has enabled the identification of many new transitions in this $T_{Z}=1$ nucleus, which is of interest since it can be described as a single neutron pair outside the $\mathrm{N}=\mathrm{Z}=28$ doubly magic closed shell.

The data from all three experiments are currently under analysis.

a.3. The Identification of Excited States in ${ }^{68} \mathrm{Se} \mathrm{(C.} \mathrm{J.} \mathrm{Lister,} \mathrm{D.} \mathrm{J.} \mathrm{Blumenthal,}$ M. P. Carpenter, C. N. Davids, D. Seweryniak, W. Gelletly,* P. Regan, ${ }^{*}$ C. J. Gross, $\dagger$ and D. D. Warner + )

Shape polarization of nuclei was a topic of considerable interest in the last decade, as both theoretical calculations and experimental techniques have evolved to a point where reliable calculations can be made for most nuclei, then tested in experiment. One particularly challenging area for our understanding of shape polarization is in $\mathrm{N}=\mathrm{Z}$ nuclei between masses $\mathrm{A}$ $=58$ and 100. As nucleons fill the fpg shell-model space, the nuclear ground states are predicted to be spherical, oblate, prolate and triaxial in turn, before returning to spherical. $\mathrm{N}=\mathrm{Z}$ nuclei in this region are particularly easily polarized, as the level densities are low, with states of differing shape-driving effect. Both neutrons and protons are occupying the same orbits and have reinforcing shape-driving influence. The correct prediction of this sequence of shape changes, and the

\footnotetext{
*University of Surrey, United Kingdom

†Oak Ridge National Laboratory

†Daresbury Laboratory, United Kingdom

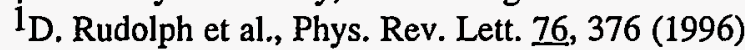

calculation of the mean field deformation and binding energy represents a particularly stringent test of our ability to understand shape polarization in all nuclei, as the changes are uniquely rapid and large.

A series of experiments in the 1980's measured some properties of $\mathrm{N}=\mathrm{Z}$ nuclei from germanium to molybdenum. ${ }^{1}$ However, data on one of the more interesting nuclei, ${ }^{68} \mathrm{Se}$ with $\mathrm{N}=\mathrm{Z}=34$, were too poor to allow a reliable estimate of the shape. The nucleus is of particular interest, as the calculations indicate that it has very large $(\beta=0.3)$ oblate deformation -- a characteristic very unusual in nuclei. Using the inverse ${ }^{12} \mathrm{C}\left({ }^{58} \mathrm{Ni}, 2 \mathrm{n}\right){ }^{68} \mathrm{Se}$ reaction at $210 \mathrm{MeV}$, the AYE-Ball gamma-ray spectrometer, and the FMA, the gamma rays known in ${ }^{68} \mathrm{Se}$ were confirmed and new transitions identified. Data analysis is in progress to establish a decay scheme and extract information on the evolution of the shape of ${ }^{68} \mathrm{Se}$ with spin.

\section{a.4. In-Beam Gamma-Ray Spectroscopy of ${ }^{103} \mathrm{Sn}$ (D. Seweryniak,}

I. Ahmad, H. Amro, D. Blumenthal, L. T. Brown, B. C. Busse, M. P. Carpenter,

L. F. Conticchio, C. N. Davids,'S. M. Fischer, D. J. Henderson, R. V. F. Janssens,

T. L. Khoo, C. J. Lister, D. Nisius, W. B. Walters, ${ }^{*}$ and W. F. Mueller†)

To extend spectroscopic information on nuclei in the vicinity of the very neutron-deficient doubly-magic ${ }^{100} \mathrm{Sn}$ nucleus, an in-beam experiment was performed with the AYE-Ball array using a $58 \mathrm{Ni}$ beam at 205 $\mathrm{MeV}$ on a ${ }^{50} \mathrm{Cr}$ target. The main goal of the experiment was to observe gamma-ray transitions in ${ }^{103} \mathrm{Sn}$ which has only 3 neutrons outside the ${ }^{100} \mathrm{Sn}$ core. The AYE-Ball array was equipped with $16 \mathrm{HPGe}$ detectors and 2 LEP spectrometers. Since excited states in ${ }^{103} \mathrm{Sn}$ have not been seen before and the predicted maximum cross section for the $(1 \alpha, 1 \mathrm{n})$ channel leading to ${ }^{103} \mathrm{Sn}$ is only about 100 microbarns, an emphasis was put on identification of observed gamma-ray lines. The mass-to-charge state ratio of recoiling nuclei was measured at the FMA focal plane. An ion chamber placed behind the focal plane of the FMA supplied some information on the atomic number of the residues. Sixteen neutron detectors placed at the target position were used to assign the number of emitted neutrons to observed gamma rays. The experiment lasted 5 days with the average beam current of about 3 to $5 \mathrm{pnA}$. The data analysis is in progress. 


\section{a.5. In-Beam Gamma-Ray Spectroscopy Along the Proton Drip Line using the}

Recoil-Decay Tagging Method (D. Seweryniak, I. Ahmad, H. Amro,

D. Blumenthal, L. T. Brown, M. P. Carpenter, C. N. Davids, S. M. Fischer,

D. J. Henderson, R. V. F. Janssens, T.L. Khoo, C. J. Lister, D. Nisius,

W. B. Walters, ${ }^{*}$ T. Davinson, $\uparrow$ R. J. Irvine, $\uparrow$ P. J. Woods, $\uparrow$ I. Hibbert, C. Parry, and R. Wadsworth)

In-beam gamma-ray spectroscopy of neutron-deficient nuclei is undergoing a renaissance with the introduction of the so-called Recoil-Decay Tagging (RDT) method. The long-standing interest and the expertise in the experimental proton and alpha decay studies at ATLAS,

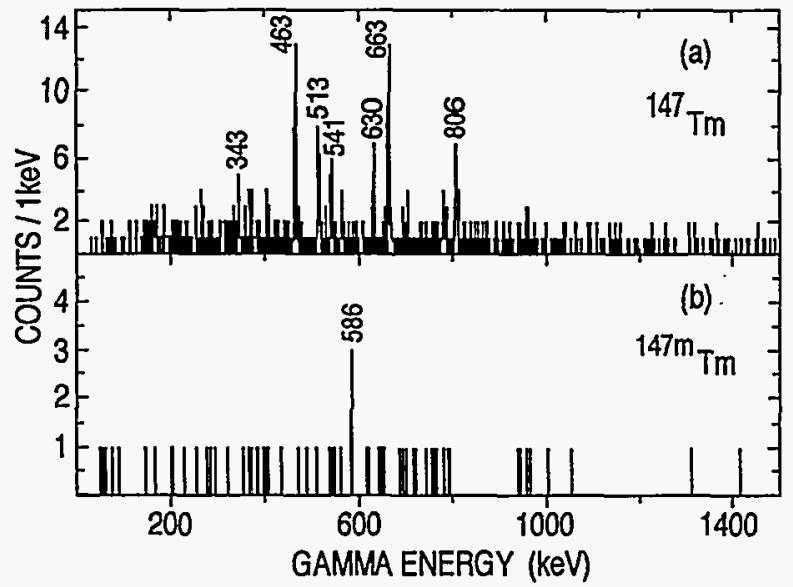

Fig. 1 -2. (a) Gamma rays in 147 Tm leading to the $112^{-}$ground state; (b) gamma rays in 147 Tm leading to the $3 / 2^{+}$isomeric state.

combined with the possibility of using the AYE-Ball array, offered a unique chance to test the limits of the new method and to take a leading role in applying it to studies of nuclear structure at and beyond the proton drip line. Figure I-2(a) shows gamma rays leading to the ${ }^{147} \mathrm{Tm}$ ground state, while Fig. I-2(b) shows the gamma-ray spectrum leading to the isomeric state.

An experiment was performed to reach several very neutron-deficient nuclei along the proton drip line. The experimental setup consisted of the AYE-Ball array in front of the FMA and a Double Sided Silicon Strip Detector (DSSD) placed behind the focal plane of the FMA. The residual nuclei were dispersed in the FMA according to their mass-to-charge-state ratio and implanted into the position-sensitive DSSD placed behind the focal plane of the FMA. The subsequent characteristic alpha decay observed in the same pixel of the DSSD as the implantation allowed complete identification of the implanted residues and thus of the gamma rays detected at the target position.

A $58 \mathrm{Ni}$ beam was used to bombard several targets, namely: ${ }^{58} \mathrm{Ni}\left({ }^{54} \mathrm{Fe}\right),{ }^{92} \mathrm{Mo},{ }^{102} \mathrm{Pd}$ and ${ }^{106} \mathrm{Cd}$, aiming at the island of alpha and proton radioactivity above ${ }^{100} \mathrm{Sn}$, at the proton emitter ${ }^{147} \mathrm{Tm}$, and at the proton drip-line nuclei in the $A=156-164$ region. Each irradiation took about 1 day and provided a vast amount of interesting data. Excited states in a dozen neutrondeficient nuclei were observed for the first time. For example, prompt gamma-ray cascades were correlated with the alpha decay of the ground state and the isomeric state in $156 \mathrm{Hf}$. The $6^{+}-4^{+}-2^{+}-0^{+}$ sequence was identified in another even-even nucleus, $160 \mathrm{~W}$. During an additional one-hour-long test run using a ${ }^{78} \mathrm{Kr}$ beam on a ${ }^{92} \mathrm{Mo}$ target, a set of gamma rays was assigned to ${ }^{166} \mathrm{Os}$. Gamma-ray transitions correlated with protons emitted from the ground state and the isomeric state in ${ }^{147} \mathrm{Tm}$ were also found, truly representing in-beam gamma spectroscopy beyond the proton drip line. Although the yield of another proton emitter, ${ }^{113} \mathrm{Cs}$, proved to be too small, gamma-ray lines in its alpha-decaying neighbor, ${ }^{10} \mathrm{~T} \mathrm{Te}$, were observed.

Detailed data analysis and interpretation of the results is in progress. A follow-up RDT experiment to reach light $\mathrm{Pt}$ and $\mathrm{Os}$ isotopes will be run at ATLAS in spring 1996 . The RDT method will certainly play an important role after Gammasphere is moved to Argonne and coupled to the FMA.

\footnotetext{
*University of Maryland, †University of Tennessee
} 
a.6. Study of ${ }^{176-179} \mathrm{Hg}$ using the Recoil Decay Tagging Method (M. P. Carpenter, D. Ackermann, D. J. Blumenthal, C. N. Davids, S. M. Fischer, G. Hackman, R. V. F. Janssens, T. L. Khoo, T. Lauritsen, C. J. Lister, D. Nisius, D. Seweryniak, P. J. Woods, * H. Amro, T. Brown, J. H. Hamilton, $\uparrow$ A. V. Ramayya, $\uparrow$ W. Reviol, J. Schwartz, and J. Simpson§)

In light $\mathrm{Hg}$ isotopes ( $100 \geq N \geq 108$ ), shape coexistence was established close to the ground state where rotational bands built on collective oblate and prolate shapes are observed. 1 While this shape coexistence feature is predicted to be sustained in lighter $\mathrm{Hg}$ nuclei, such measurements become increasingly difficult due to the fact that the cross-sections for producing these nuclei become very small $(<1 \mathrm{mb})$ and fission dominates $\left(\sigma_{\text {fiss }}>100 \mathrm{mb}\right.$ ).

In an attempt to study this shape-coexistence phenomenon all the way to the proton-drip line, we identified for the first time, $\gamma$-ray transitions in 176${ }^{179} \mathrm{Hg}$ using the AYE-Ball Compton-suppressed Ge array that was setup at the target position of the Fragment Mass Analyzer (FMA). Unambiguous assignment of $\gamma$ transitions to a particular nuclide was accomplished by utilizing the recoil decay tagging method, a technique which correlates $\gamma$ rays emitted from excited residues with the charged-particle radioactivity of their decay. ${ }^{2}$ Preliminary analysis of the data shows the $2^{+}$state in ${ }^{178} \mathrm{Hg}(558 \mathrm{keV})$ and

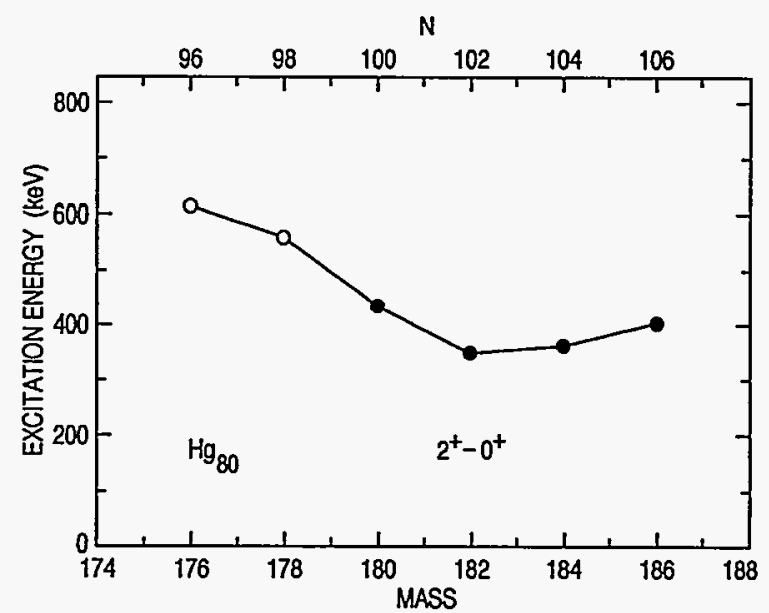

Fig. I-3. Systematics of the $2^{+}$excitation energy in even-even $\mathrm{Hg}$ isotopes centered around $\mathrm{N}=102$. The open circles represent the results from the recent work at the FMA.

${ }^{176} \mathrm{Hg}(615 \mathrm{keV})$ continuing to rise as one moves away from mid-shell at $\mathrm{N}=102\left({ }^{182} \mathrm{Hg}\right)$ (see Fig. I-3). Analysis of the data is continuing.

*University of Edinburgh, United Kingdom, $\uparrow$ Vanderbilt University, łUniversity of Tennessee, §Daresbury Laboratory, United Kingdom

${ }^{1}$ W. Nazarewicz, Phys. Lett. B305, 195 (1993) and references therein.

${ }^{2}$ E. S. Paul et al., Phys. Rev. C 51,78 (1995).

\section{a.7. Continuing Study of the Level Structure of ${ }^{179} \mathrm{Au}$ (M. P. Carpenter,} I. Ahmad, H. Amro, D. J. Blumenthal, B. Crowell, C. N. Davids, S. M. Fischer, G. Hackman, D. J. Henderson, R. V. F. Janssens, T. L. Khoo, T. Lauritsen, C. J. Lister, D. Nisius, D. Seweryniak, W. F. Mueller,* C. R. Bingham,* W. Reviol,* L. L. Riedinger,* B. H. Smith,* J. F. Wauters, ${ }^{*}$ and W. C. Ma†)

Last year several transitions in ${ }^{179} \mathrm{Au}$ were identified from an experiment with the Fragment Mass Analyzer (FMA) and 10 Compton-suppressed Ge detectors positioned around the target. While the data were limited, a tentative level scheme was established. Recently, a new experiment was performed at ATLAS using the AYE-Ball array in conjunction with the FMA and the double-sided silicon strip detector (DSSD). The reaction chosen for this experiment was ${ }^{124} \mathrm{Te}(58 \mathrm{Ni}, \mathrm{p} 2 \mathrm{n})$ at a beam energy of $255 \mathrm{MeV}$. The

*University of Tennessee, †Mississippi State University first part of the experiment used a $0.385-\mathrm{mg} / \mathrm{cm}^{2}{ }^{124} \mathrm{Te}$ target, and residues were allowed to recoil into the FMA. Using the recoil decay tagging method, unambiguous assignments of gamma-rays to ${ }^{179} \mathrm{Au}$ is possible. The second part of the experiment involved a $0.5-\mathrm{mg} / \mathrm{cm}^{2}{ }^{124} \mathrm{Te}$ target with a $60-\mathrm{mg} / \mathrm{cm}^{2} \mathrm{~Pb}$ backing. In this part of the experiment, an array of twelve neutron counters was used with the AYE-Ball array. A total of $\sim 100$ million $\mathrm{Ge}-\mathrm{Ge}$ coincidence events were recorded, and the analysis of these new data is in progress. 
a.8. Spectroscopy of ${ }^{189} \mathrm{~Pb}$ (M. P. Carpenter, D. J. Blumenthal, T. Brown, L. Conticchio, C. N. Davids, D. Gassmann, R. V. F. Janssens, T. L. Khoo, T. Lauritsen, C. J. Lister, D. Nisius, A. Baxter,* A. Byrne,* G. Dracoulis, * B. Kibedi,* and G. Lane*)

Recently, evidence for low-lying, prolate-deformed, rotational bands was found in the very neutron-deficient isotopes ${ }^{186} \mathrm{~Pb}$ and ${ }^{188} \mathrm{~Pb}$. ${ }^{1,2}$ In both these nuclei, the main feature of the decay schemes is a single band with level spacings for the third and higher excited states which are consistent with those of a deformed rotor. In order to determine whether or not low-lying prolate structures are also present in the neighboring odd-mass isotopes, measurements were carried out on $187,189 \mathrm{~Pb}$.

In an initial measurement performed at the 14UD accelerator in Canberra, a cascade of four transitions were tentatively assigned to ${ }^{189} \mathrm{~Pb}$ using the reaction ${ }^{164} \mathrm{Er}\left({ }^{29} \mathrm{Si}, 4 \mathrm{n}\right){ }^{189} \mathrm{~Pb}$ at $140 \mathrm{MeV}$. In order to confirm

*Australian National University, Canberra, Australia

1J. Hease et al., Phys. Lett. B302, 399 (1993)

2 A. M. Baxter et al., Phys. Rev. C 4ㅇ, R2140 (1993) this assignment, a follow-up experiment was performed at ATLAS by bombarding a ${ }^{158} \mathrm{Gd}$ target with a 178$\mathrm{MeV}{ }^{36} \mathrm{Ar}$ beam. To discriminate against competition from fission and to provide mass identification of $\gamma$ radiation, recoiling evaporation residues were detected by the Fragment Mass Analyzer (FMA) in coincidence with $\gamma$ rays detected by 10 Compton-suppressed $\mathrm{Ge}$ detectors positioned around the target. The mass identification supplied by the FMA and the observation of coincidences with lead $\mathrm{x}$-rays, allowed for the cascade of four transitions to be assigned unambiguously to ${ }^{189} \mathrm{~Pb}$. The features of the level schemes observed in $186,188 \mathrm{~Pb}$ are also present in ${ }^{189} \mathrm{~Pb}$, suggesting that a deformed structure co-exists with spherical states in this nucleus as well. A paper is being prepared for publication.

a.9. Collective Motion in Light Polonium Isotopes (M. P. Carpenter, I. Ahmad, D. Blumenthal, C. N. Davids, D. Henderson, R. G. Henry, R. V. F. Janssens, T. L. Khoo, T. Lauritsen, C. J. Lister, H. Penttilä, D. Nisius, W. Younes, * J. A. Cizewski, * H.-Q. Jin, ${ }^{*}$ L. A. Bernstein,* and D. P. McNabb*)

The onset of collective motion and the evolution of nuclear shapes near shell closures are still rather poorly

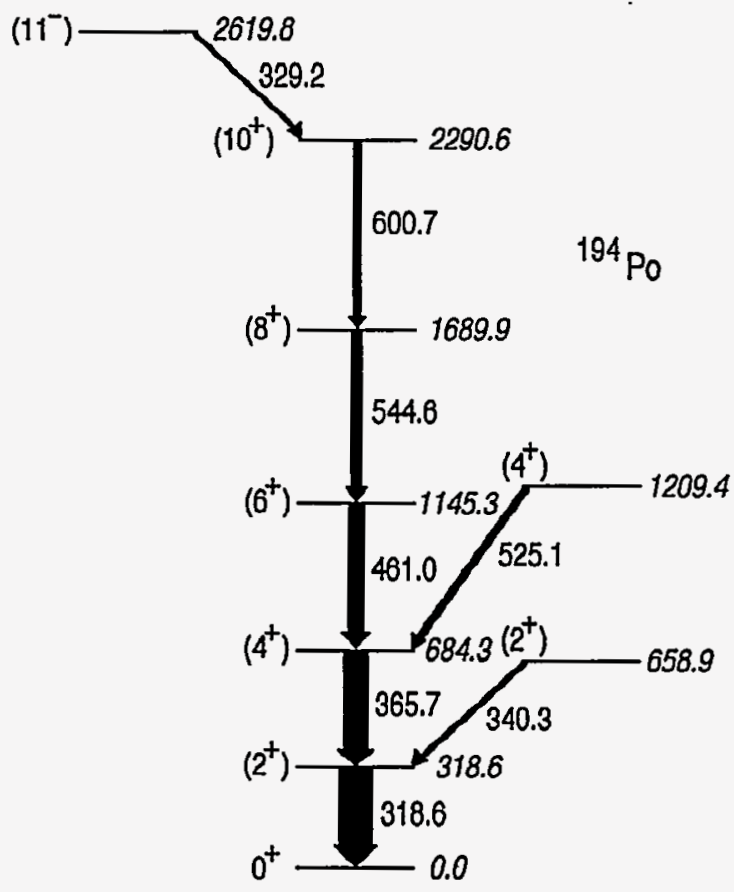

Fig. I-4. Deduced ${ }^{194}$ Po level scheme. Spin assignments are based on systematics. understood. Recent studies of the systematics identified a critical phase transition from spherical to deformed shapes when $\mathrm{E}\left(2^{+} 1\right)<145 \mathrm{keV}$ for nuclei with $38<\dot{Z}$ $<82$. The data for $Z>82$ are sparse and do not extend to the neutron midshell $(\mathrm{N}=104)$ where collective effects are expected to be the largest.

We recently studied excited states in ${ }^{194} \mathrm{Po}$, the most neutron-deficient Po isotope studied to date. The ${ }^{194} \mathrm{Po}$ nucleus was formed by the bombardment of a ${ }^{170} \mathrm{Yb}$ target with $142 \mathrm{MeV}{ }^{28} \mathrm{Si}$ beam. The evaporation residues were identified using the FMA in coincidence with gamma rays detected with the Argonne-Notre Dame BGO gamma-ray facility at the target position. FMA- $\gamma$ and FMA- $\gamma-\gamma$ coincidences were used to identify transitions in ${ }^{194} \mathrm{Po}$ and construct a partial level scheme (see Fig. I-4). For the heavier Po isotopes, the $E\left(4^{+} 1\right) / E\left(2^{+} 1\right)$ ratio is always less than 2.00 , the value expected for a harmonic vibrator.

\footnotetext{
*Rutgers University

${ }_{1}$ L. A. Bernstein et al., Phys: REv. C 52, 621 (1995)

2 W. Younes et al., Phys. Rev. C 52, R1723 (1995).
} 
${ }^{196} \mathrm{Po}$ is the first isotope to have a collective structure and a shape which is transitional between spherical and deformed. ${ }^{1}$ The evolution of this shape is very rapid in these light Po isotopes. Using the $318.6 \mathrm{keV}$ for the energy of the $2^{+}$state in ${ }^{194} \mathrm{Po}$, one can extrapolate the $2^{+}$energy in ${ }^{192}$ Po which comes to be $\sim 170 \mathrm{keV}$. This is very close to the critical value of $145 \mathrm{keV}$ for the transition to a deformed shape. Experiments are planned to identify states in ${ }^{192} \mathrm{Po}$. The results on ${ }^{194}$ Po were published recently. ${ }^{2}$

a.10. Onset of Deformation Effects in Radon Isotopes (D. J. Blumenthal, D. Seweryniak, C. N. Davids, R. V. F. Janssens, C. J. Lister, S. J. Freeman,* J. L. Durell,* M. L. Leddy,* R. B. E. Taylor,* and A. G. Smith*)

The structure of nuclei in the light pre-actinide region $(\mathrm{Z}>82, \mathrm{~N}<126)$ is attracting increasing interest. ${ }^{1}$ Calculations indicate that a stable ground-state deformation should develop away from stability with the deformation parameter reaching a value $\varepsilon_{2}=0.2^{2}$ At this deformation the energy of the $\mathrm{J}=2^{+}$state should fall from a value of $500 \mathrm{keV}$ in the near spherical nuclei to about $125 \mathrm{keV}$ and a collective ground-state rotational band should be present. Although some evidence was claimed for increased collectivity in the more neutron-deficient radon and polonium isotopes, there is no clear evidence for the existence of deformation in this region.
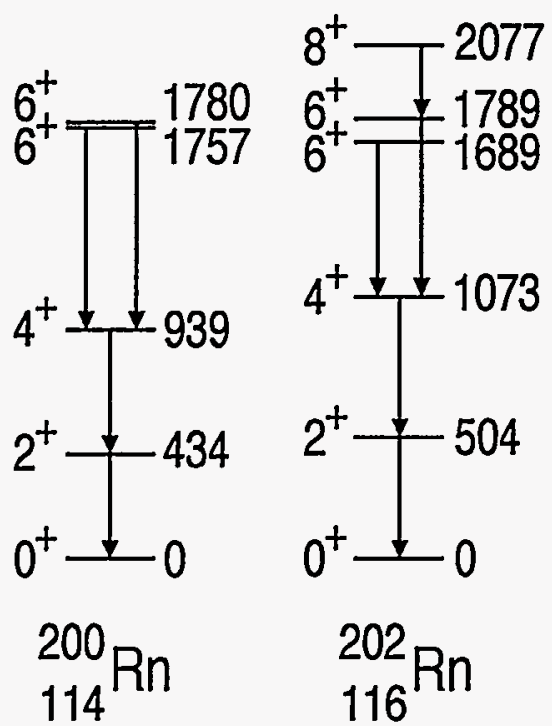
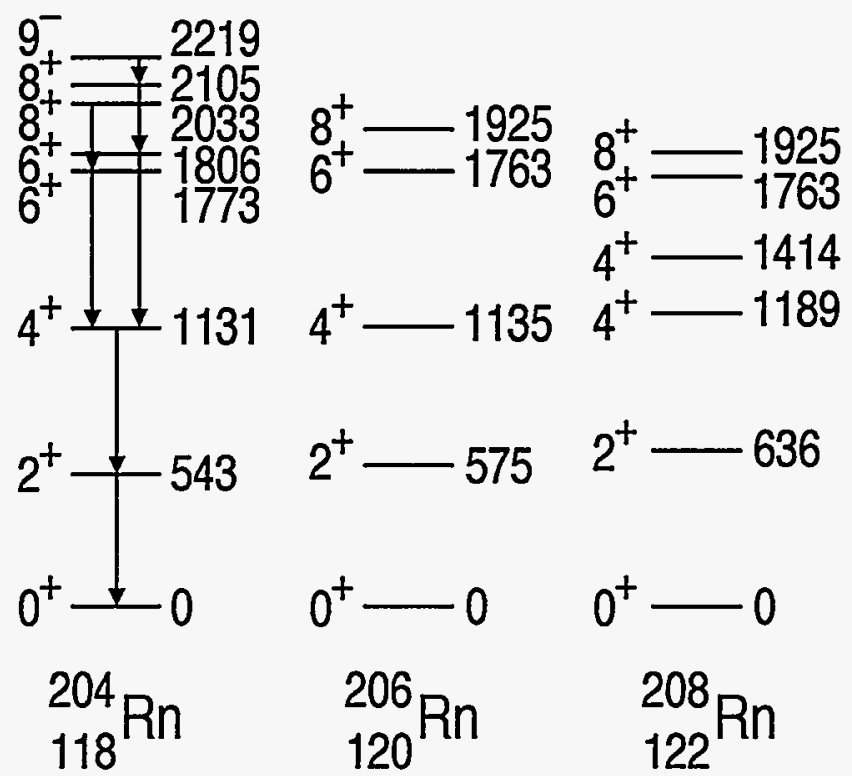

Fig. I-5. The low-lying levels for the lightest radon isotopes. The decay schemes for $200,202,204$ Rn are derived from data taken at ATLAS.

The isotope ${ }^{202} \mathrm{Rn}$ is predicted to be the last of the neutron-deficient species with a near-spherical groundstate shape in the chain of radon isotopes; lighter isotopes are expected to be deformed. The calculated onset of deformation is rather sudden. An experiment was performed using the ${ }^{176} \mathrm{Hf}\left({ }^{28} \mathrm{Si}, 4 \mathrm{n}\right){ }^{200} \mathrm{R} \mathrm{n}$ reaction. The beam was chosen to be at near-barrier energies to produce compound systems as cold as possible, minimizing fission competition. Residue gamma rays were detected using a $1.2 \%$ efficient array of large detectors provided by the AYE-Ball

*University of Manchester, United Kingdom

${ }^{1}$ S. J. Freeman et al., Phys. Rev. C $\underline{50}$, R1754 (1994)

2P. Möller et al., Phys. Scr. 29, 402 (1984) ollaboration. The FMA was used to implant residues into a silicon strip detector placed $40-\mathrm{cm}$ behind the focal plane, where the alpha-decay of the recoil ion and subsequent daughter decays were recorded. Such a system provided extremely sensitive isotopic selection by not only gating on charge-to-mass ratio, but also by selecting gamma-ray transitions in coincidence with recoiling ions which subsequently decay by the emission of $\alpha$ particles.

Using these techniques the gamma-ray transitions between low-lying states in $200 \mathrm{Rn}$ were identified. A full analysis is ongoing, but a spectrum generated online identified transitions in coincidence with $\alpha$ particles corresponding to the decay of ${ }^{200} \mathrm{Rn}$. The $2 \rightarrow 0$ 
transition has an energy of $434 \mathrm{keV}$ and suggests that ${ }^{200} \mathrm{Rn}$ has a near-spherical shape. This can also be seen by comparing the low-lyng levels for $200_{\mathrm{Rn}}$ with the heavier even-even isotones (see Fig. I-5). The lowlying levels in $200 \mathrm{Rn}$ follow the trend of other less neutron-deficient isotopes, i.e., that of a smooth and gradual fall in energy. The analysis of these data continues but it appears that the predicted onset of deformation does not occur, or, that its onset is delayed until lighter isotopes. Such phenomena might be related to inadequacies of the theoretical calculations. For example, imprecise knowledge of the energy of particular shape-driving single-particle orbitals could lead to a poor prediction of the point at which deformation sets in; neglect of triaxiality could lead to the prediction of a large static deformation whereas a low deformation, $\gamma$-soft system might be a better description. Further investigation of more neutrondeficient isotopes will address some of these questions.

\section{a.11. Nuclide Production Near ${ }^{100} \mathrm{Sn}$ using a Radioactive Beam (M. P. Carpenter, C-L. Jiang, C. J. Lister, G. Hackman, D. J. Blumenthal, C. N. Davids, W. F. Henning, R. V. F. Janssens, D. Ackermann, S. M. Fischer, T. L. Khoo, T. Lauritsen, V. Nanal, D. Nisius, D. Seweryniak, H. Amro, and J. Schwartz)}

A program has begun at ATLAS to develop a "double"foil target technique which utilizes a radioactive beam to produce nuclei near the proton-drip line via two successive heavy-ion-induced fusion reactions. The radioactive beam is produced in the first target and the residues have sufficient recoil energy to further induce fusion reactions on a secondary target. It is the goal of this project to identify secondary residues using the Fragment Mass Analyzer (FMA) and an ionization chamber at the focal plane. Recently, we made the first attempts to produce and identify secondary reactions using a ${ }^{12} \mathrm{C}-40 \mathrm{Ca}$ target and a ${ }^{58} \mathrm{Ni}$ beam. The ${ }^{58} \mathrm{Ni}+$ ${ }^{12} \mathrm{C}$ reaction produces residues with $\mathrm{A} \sim 64$ and recoil energies around $230 \mathrm{MeV}$. These primary residues have sufficient recoil energy to induce further fusion reactions in the ${ }^{40} \mathrm{Ca}$ target creating secondary residues with $\mathrm{A}$ 100.

This initial study was distributed into two separate measurements. The goal of the first measurement was to study the production mechanisms and backgrounds associated with primary and secondary reactions, respectively. For primary reactions, ${ }^{58} \mathrm{Ni}+{ }^{12} \mathrm{C}$ and $58 \mathrm{Ni}+40 \mathrm{Ca}$ reactions were examined at the appropriate beam energies, and residues were identified by detecting the emitted $\gamma$ rays with the AYE-Ball array. One of the primary residues produced in the ${ }^{58} \mathrm{Ni}+{ }^{12} \mathrm{C}$ reaction is ${ }^{64} \mathrm{Zn}$. In order to investigate the mass distribution of residues produced by secondary reactions, a study of the reaction ${ }^{64} \mathrm{Zn}$ on $40 \mathrm{Ca}$ was carried out as well. This measurement enabled us to (i) determine relative cross- sections for residues produced in this reaction as a function of bombarding energy, (ii) measure the response of the ion-chamber for Z-separation by gating on gamma-rays associated with particular residues, and (iii) determine the transmission of $\mathrm{A}=100$ recoils through the FMA.

In the second portion of this experiment, a double-foil target which consisted of $0.4 \mathrm{mg} / \mathrm{cm}^{2}$ of enriched ${ }^{40} \mathrm{Ca}$ evaporated onto a $0.6-\mathrm{mg} / \mathrm{cm}^{2}{ }^{12} \mathrm{C}$ was used. Based on our previous studies, a ${ }^{58} \mathrm{Ni}$ beam energy of $310 \mathrm{MeV}$ was chosen in order to minimize fragmentation of the secondary residues. In this portion of the experiment, we performed measurements where the $58 \mathrm{Ni}$ was incident first on the ${ }^{12} \mathrm{C}$ foil as well as measurements where the target was rotated $180^{\circ}$ so that the beam first struck the ${ }^{40} \mathrm{Ca}$. Any production of $\mathrm{A} \sim 100$ residues by secondary reactions can only be made with the target in the first orientation.

Preliminary analysis of the data show peaks in our M/Q spectrum which are consistent with $\mathrm{A}=100$ residues when proper time-of-flight gating is performed. This mass peak is observed when the target is placed in the proper orientation. We have benefited greatly from our initial studies and believe we will improve our technique substantially by utilizing a small Faraday cup for better beam suppression and a channel-plate detector for improved energy and time-of-flight measurements. Recently, four more days of beam time were approved for continuing development of this technique. 
a.12. Study of Proton Radioactivities (C. N. Davids, P. J. Woods,* J. C. Batchelder, $\uparrow$ C. R. Bingham, $\neq$ D. J. Blumenthal, L. T. Brown, B. Busse, L. F. Conticchio, T. Davinson,* M. Freer,** D. J. Henderson, R. J. Irvine*, H. T. Penttilä, $\nmid \dagger$ D. Seweryniak, K. S. Toth,§§ W. B. Walters,II and B. E. Zimmerman $\ddagger$ )

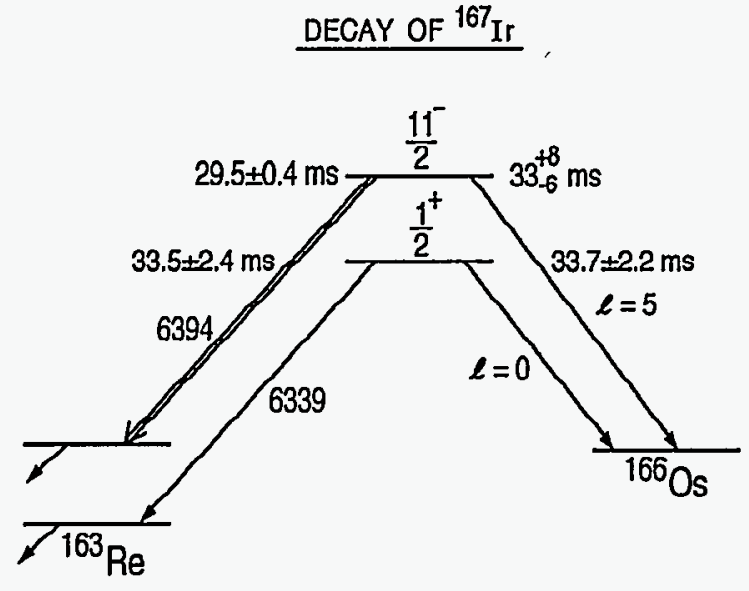

Fig. I-6. Decay scheme for proton emitter ${ }^{167}$ Ir.
In a series of runs using the double-sided Si strip detector implantation system at the FMA, several new heavy proton emitters were discovered. These include ${ }^{161} \operatorname{Re}$, ${ }^{165} \mathrm{Ir}$, and ${ }^{166} \mathrm{Ir}$. Further information was also obtained on the proton emitter ${ }^{167} \mathrm{Ir}$, showing that it has two proton-emitting states. Each of these states also decays by alpha emission. Figure I- 6 shows the deduced decay scheme for ${ }^{167}$ Ir. The alpha decays were followed for 4 generations. The data on the proton emitter ${ }^{185} \mathrm{Bi}$ was published ${ }^{1}$ showing that its decay takes place from a low-lying $J=1 / 2^{+}$intruder state to the ground state of ${ }^{184} \mathrm{~Pb}$. This decay proceeds through a small admixture in the ${ }^{184} \mathrm{~Pb}$ ground state of the $0^{+}$intruder state located at an excitation energy of about $600 \mathrm{keV}$. Further searches for heavy proton emitters with $\mathrm{Z}>84$ will be conducted.

*University of Edinburgh, United Kingdom, †Louisiana State University, $\ddagger$ University of Tennessee, §Vanderbilt University, TOregon State University, IIUniversity of Maryland, **University of Birmingham, $\uparrow \dagger$ University of Jyväskylä, Finland, §§Oak Ridge National Laboratory

${ }^{1}$ C. N. Davids et al., Phys. Rev. Lett. 76, 592 (1996).

\section{a.13. Identification of ${ }^{166} \mathrm{Pt}$ and ${ }^{167} \mathrm{Pt}$ and the Determination}

of the ${ }^{168}$ Pt Half-Life (C. N. Davids, P. J. Woods,* J. C. Batchelder, $\dagger$

C. R. Bingham, D. J. Blumenthal, L. T. Brown, B. Busse, L. F. Conticchio,

T. Davinson,* D. J. Henderson, R. J. Irvine,* D. Seweryniak, K. S. Toth,**

W. B. Walters,Il and B. E. Zimmerman $\ddagger$ )

In a series of ${ }^{78} \mathrm{Kr}$ bombardments of ${ }^{92} \mathrm{Mo}$ the new isotopes ${ }^{166} \mathrm{Pt}$ and ${ }^{167} \mathrm{Pt}$ were identified via their alpha-decay properties. The alpha-decay energies and half-lives of these two nuclides are as follows: 1) ${ }_{166} \mathrm{Pt}, \mathrm{E}(\alpha)=7110(15) \mathrm{keV}, \mathrm{T} 1 / 2=0.3(1) \mathrm{ms}$; and 2$)$ ${ }^{167} \mathrm{Pt}, \mathrm{E}(\alpha)=6988(10) \mathrm{keV}, \mathrm{T}_{1 / 2}=0.7(2) \mathrm{ms}$. Also, the half-life of $168 \mathrm{Pt}$, which was previously unknown, was determined to be $1.8(4) \mathrm{ms}$. In a separate but concurrent experiment involving the ${ }^{78} \mathrm{Kr}+{ }^{96} \mathrm{Ru}$ reaction, ${ }^{170} \mathrm{Pt}$ was made and a half-life of $14.7(5) \mathrm{ms}$ was measured for it; the one published value is $6_{-2}^{+5} \mathrm{~ms}$. Results for ${ }^{162-164}$ Os contained in the same data sets were also analyzed and by using mother-daughter correlations, the alpha branches of $162,163,164$ Os were determined to be near $100 \%$. Comparing these new data with results previously available for platinum and osmium isotopes shows a relatively smooth trend of the alpha-reduced widths for these nuclei, all of which lie in the mass region below $\mathrm{Pb}(\mathrm{Z}=82)$.

*University of Edinburgh, United Kingdom, †Louisiana State University, $\ddagger$ University of Tennessee, IIUniversity of Maryland, **Oak Ridge National Laboratory 


\section{a.14. Discovery of the New Isotope ${ }^{178} \mathrm{Tl}$ from its Alpha Decay}

(M. P. Carpenter, H. Amro, D. Ackermann, D. J Blumenthal, C. N. Davids,

S. M. Fischer, G. Hackman, R. V. F Janssens, T. L. Khoo, T. Lauritsen, C. J. Lister,

D. Nisius, D. Seweryniak, P. J. Woods,* T. Brown, J. H. Hamilton, $\mathbb{T}$

A. V. Ramayya,II W. Reviol,II J. Schwartz, and J. Simpson ††)

There has been a strong program at the FMA studying proton radioactivity using a double-sided silicon strip detector (DSSD). A number of new proton emitters ranging from $Z=75(\mathrm{Re})$ to $Z=83(\mathrm{Bi})$ were discovered at ATLAS. Missing from this group is the discovery of a proton emitter in the $\mathrm{Tl}$ isotopes $(\mathrm{Z}=81) .{ }^{177} \mathrm{Tl}$ is predicted to be the heaviest $\mathrm{Tl}$ proton emitter. The lightest identified $\mathrm{Tl}$ isotope is ${ }^{179} \mathrm{Tl}$ which decays by $\alpha$ emission $\left(E_{\alpha}=6.56\right.$ and $\left.7.20 \mathrm{MeV}\right)$.

In the past year, we performed an experiment at the FMA using the ${ }^{78} \mathrm{Kr}+{ }^{103} \mathrm{Rh}$ reaction at $240 \mathrm{MeV}$ leading to the compound nucleus ${ }^{181} \mathrm{Tl}$. The main purpose of this study was to identify for the first time $\gamma$ transitions in ${ }^{178} \mathrm{Hg}$ via the recoil decay tagging method, a technique which correlates $\gamma$ rays emitted from excited residues with the charged particle radioactivity of their decay. In this experiment, gamma rays were measured at the target with the AYE-Ball array and alpha energies were measured in the DSSD.

In the course of our analysis, we identified three previously unknown alpha decay lines $\left(\mathrm{E}_{\alpha}=6.71,6.79\right.$ and $6.87 \mathrm{MeV}$ ) which are correlated with $\mathrm{A}=178$ residues. In turn, all three alpha lines are correlated with the same daughter decay $\left(\mathrm{E}_{\alpha}=6.54 \mathrm{MeV}\right)$ which corresponds to the energy of the ground-state alpha decay of ${ }^{174} \mathrm{Au}$. Based on these observations, we assigned these alpha lines to ${ }^{178} \mathrm{Tl}$, a previously unknown $\mathrm{Tl}$ isotope. In addition, we found no evidence that ${ }^{178} \mathrm{Tl}$ proton decays. A paper reporting our results is in preparation.

*University of Edinburgh, United Kingdom, TVanderbilt University, IIUniversity of Tennessee, ††Daresbury Laboratory, United Kingdom

a.15. The Study of Fine Structure in the Alpha Decay of Nuclei near $A=180$ (D. J. Blumenthal, M. P. Carpenter, D. Ackermann, C. N. Davids, S. M. Fischer, G. Hackman, R. V. F Janssens, T. L. Khoo, T. Lauritsen, C. J. Lister, D. Nisius, D. Seweryniak, P. J. Woods, * H. Amro, T. Brown, J. H. Hamilton,I

A. V. Ramayya,II W. Reviol,Il J. Schwartz, and J. Simpson††)

An abundance of alpha decay fine structure in several nuclei in the $A=180$ region was observed in recent implantation/decay experiments at the FMA. Residual nuclei from the ${ }^{103} \mathrm{Rh}\left({ }^{78} \mathrm{Kr}\right.$, xpxn) reaction at $380 \mathrm{MeV}$ were transported through the FMA and implanted into a double-sided silicon strip detector (DSSD). The nuclei produced most strongly included $176-178 \mathrm{Hg}, 176-$ ${ }^{178} \mathrm{Au}, 175-177 \mathrm{Pt}$, and their daughters. The pixel nature of the detector allowed the correlation of mass with decay energies over several generations. A $25 \%$ germanium detector was placed behind the DSSD. It recorded energy and time information in events that involved the emission of a gamma ray following the implantation or decay of a nucleus in the DSSD. The sensitivity to and resolution for $\mathrm{x}$-rays provided additional information in those cases where gamma-ray transitions were either forbidden or highly converted. The mass-alpha-gamma correlations clearly identified fine structure. Initial data analysis confirmed known alpha-decay fine structure in ${ }^{172} \mathrm{Ir},{ }^{175} \mathrm{Pt}$, and ${ }^{176} \mathrm{Pt}$. Gamma-ray transitions that were previously assigned to $168 \mathrm{Re}$, but not placed in its level scheme were confirmed as well.

*University of Edinburgh, United Kingdom, qVanderbilt University, IUniversity of Tennessee, $† \dagger$ Daresbury Laboratory, United Kingdom 
a.16. Search for Fine Structure in ${ }^{192} \mathrm{Po} \alpha$ Decay (I. Ahmad, D. J. Blumenthal, C. N. Davids, R. V. F. Janssens, D. Seweryniak, J. Wauters, ${ }^{*}$ C. R. Bingham, ${ }^{*}$ X. S. Chen,* W. Reviol,* B. E. Zimmerman,* N. Bijnens, $\uparrow$ M. Huyse, $\dagger$ L. F. Conticchio, W. B. Walters, $\ddagger$ J. C. Batchelder,§ E. F. Zganjar,§ H. Penttilä,II and B. C. Busse)

In the study of low-lying $0^{+}$states and shape coexistence in the lead region, the ${ }^{188} \mathrm{~Pb}$ nucleus forms an especially interesting case, as a $0^{+}$intruder state is expected at low energy and a prolate deformed band down to $2^{+}$was recently observed in $186,188 \mathrm{~Pb}$. Relative alpha-decay rates to excited $0^{+}$states give information on the particle-hole character of the connecting states, and the mixing between excited $0^{+}$ and ground state in parent and daughter nuclei. In the case of ${ }^{192} \mathrm{Po}$, considerable mixing between the ground state and a $0^{+}$intruder state (not yet observed) is expected, both from level systematics of the neutrondeficient even-even Po isotopes, and from their ground state alpha-decay reduced widths. This will most probably result in a hindrance factor smaller than one, a unique case. We studied the alpha decay of ${ }^{192} \mathrm{Po}$ in January 1995 at the FMA using the DSSD detector, with a $\mathrm{Si}$ (for conversion electrons) and a LEPS detector (for $\mathrm{X}$ rays) utilizing the reaction ${ }^{160} \mathrm{Dy}\left({ }^{36} \mathrm{Ar}, 4 \mathrm{n}\right)(\sim$ $2.5 \mu \mathrm{b})$. We confirmed half-lives for ${ }^{193-196} \mathrm{Po}$ and the alpha-branching ratio of $190 \mathrm{~Pb}$ and of $188 \mathrm{~Pb}$ through parent-daughter alpha-alpha correlations. By gating on mass 192 recoils and a short time window (< $200 \mathrm{~ms}$ ) on the time difference between recoil and decay, we observed a 6610(30)-keV alpha line which we assign as a candidate for alpha feeding to the intruder state in $188 \mathrm{~Pb}$ at $560 \mathrm{keV}$, with a hindrance factor (HF) of 0.9(4). The energy and HF are in agreement with the even-even $\mathrm{Pb}$ systematics. The small $\mathrm{HF}$ indicates large configuration mixing in ${ }^{192} \mathrm{Po}$. The experiment shows that the setup at the FMA is very well suited for fine structure studies of very exotic nuclei. In the near future, with an improved setup and choice of reaction, we will repeat the experiment to firmly identify the fine structure and get good statistics on the HF.

*University of Tennessee, $\nmid$ IKS, Katholieke University, Leuven, Belgium, $\ddagger$ University of Maryland, §Louisiana State University, IUniversity of Jyväskylä, Finland

\section{a.17. Level Structure of 226U and Its Alpha Decay (I. Ahmad, H. Amro, B. B. Back, D. Blumenthal, M. P. Carpenter, C. N. Davids, S. Fischer, G. Hackman, R. V. F. Janssens, T. L. Khoo, T. Lauritsen, C. J. Lister, D. Seweryniak and J. Schwartz)}

It was theoretically predicted and experimentally established that the nuclei with $\mathrm{Z} \sim 90$ and $\mathrm{N} \sim 134$ have the largest octupole octupole correlations. Several theoretical calculations predict ${ }^{224} U$ and ${ }^{226} U$ to be the most octupole deformed nuclei of the actinide region. These nuclei are very neutron-deficient and the cross section for their production is very low. For this reason the structure of these nuclei has not been studied. The FMA is the best instrument to study nuclei with such low cross sections. Production and decay of ${ }^{226} U$ were recently investigated using the FMA. We first tried the ${ }^{208} \mathrm{~Pb}\left({ }^{24} \mathrm{Mg}, \alpha 2 \mathrm{n}\right)$ reaction but did not observe any ${ }^{226} \mathrm{U}$ alpha particles in one 8-hour shift. This indicates that the reaction products formed by $\alpha 2 n$ evaporation have very low cross sections and also have low transmission through the FMA because of the wider angular distribution than those of xn products. Using the ${ }^{208} \mathrm{~Pb}\left({ }^{22} \mathrm{Ne}, 4 \mathrm{n}\right)$ reaction we were able to observe 14 counts of ${ }^{226} \mathrm{U}$ in 7 hours (see Fig. I-7) when a beam current of 7 pna was used. This rate is too low to perform in-beam gamma-ray spectroscopy at the target location in coincidence with alpha particles detected at the focal plane of the FMA. However, with the replacement of the PPAC by a microchannel plate and the addition of the silicon box at the strip detector location, we hope to gain a factor of $\sim 7$. With the improved yield and the availability of Gammasphere, inbeam gamma-ray spectroscopy will be possible on light $\mathrm{U}$ and $\mathrm{Pa}$ nuclei. 


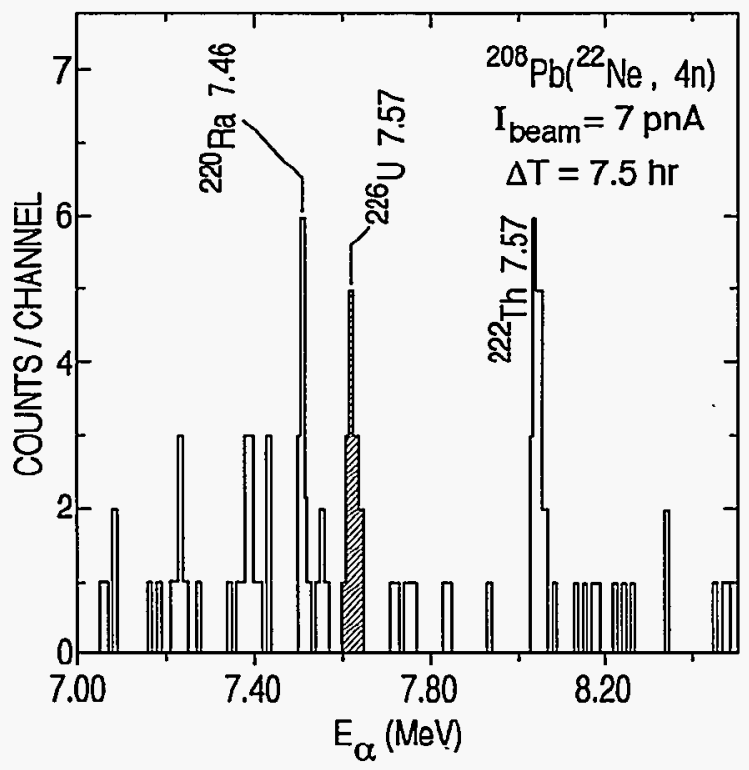

Fig. I-7. Alpha spectrum of mass 226 fraction measured with the double-sided silicon strip detector at the focal plane of FMA. The number next to the peak represents the energy of the peak in $\mathrm{MeV}$.

\section{a.18. Conversion Electron Spectroscopy at the Fragment Mass Analyzer Focal Plane: Studies of Isomeric Decays Near the Proton Drip-Line (D. Nisius, R. V. F. Janssens, I. Ahmad, B. Crowell, M. P. Carpenter, C. N. Davids, D. Henderson, R. G. Henry, R. Hermann, T. L. Khoo, T. Lauritsen, H. Penttilä, P. Bhattacharyya,* L. Ciszewski,* P. J. Daly,* Z. W. Grabowski,* R. H. Mayer,* and C. T. Zhang*)}

The FMA has proven to be an ideal instrument for the detailed study of microsecond isomers behind its focal plane, following mass separation. In nuclei with isomeric lifetimes longer than the flight time through the device, decay gamma rays and conversion electrons can be studied behind the focal plane free from the backgrounds of prompt radiation and delta electrons. Precise determinations of conversion coefficients can then be made and multipolarities assigned to the decay gamma rays.

The decays of seniority-two $\pi\left(\mathrm{h}_{11 / 2}\right)^{n}$ isomers in the $\mathrm{N}$ $=82$ nuclei ${ }^{150} \mathrm{Er}$ and ${ }^{152} \mathrm{Yb}$ and the decay of three quasi-particle isomers in their respective $\mathrm{N}=81$ isotopes ${ }^{149} \mathrm{Er}$ and ${ }^{151} \mathrm{Yb}$ were studied at the FMA focal plane following mass separation. The recoiling ions were stopped on a catcher foil where their subsequent decay gamma rays and conversion electrons were detected with two large $\mathrm{Ge}$ detectors and two roomtemperature Si PIN diodes, respectively. The lowenergy transitions de-exciting the isomers in $151,152 \mathrm{Yb}$ were observed for the first time in the electron spectra. With $\mathrm{K}, \mathrm{L}$ and $\mathrm{M}$ conversion coefficients determined where possible, multipolarity assignments were made for many of the decay $\gamma$ rays of the four nuclei. The results were published. 1

*Purdue University

${ }^{1}$ D. Nisius, Phys. Rev. C $\underline{52}, 1355$ (1995) 


\section{a.19. The Astrophysical Reaction Rate for the ${ }^{18} \mathrm{~F}(\mathrm{p}, \alpha)^{15} \mathrm{O}$ Reaction}

(K. E. Rehm, M. Paul,* A. D. Roberts, C. L. Jiang, D. J. Blumenthal, S. M. Fischer, J. Gehring, D. Henderson, J. Nickles, $\dagger$ J. Nolen, R. C. Pardo, J. P. Schiffer, and R. E. Segel $\ddagger$ )

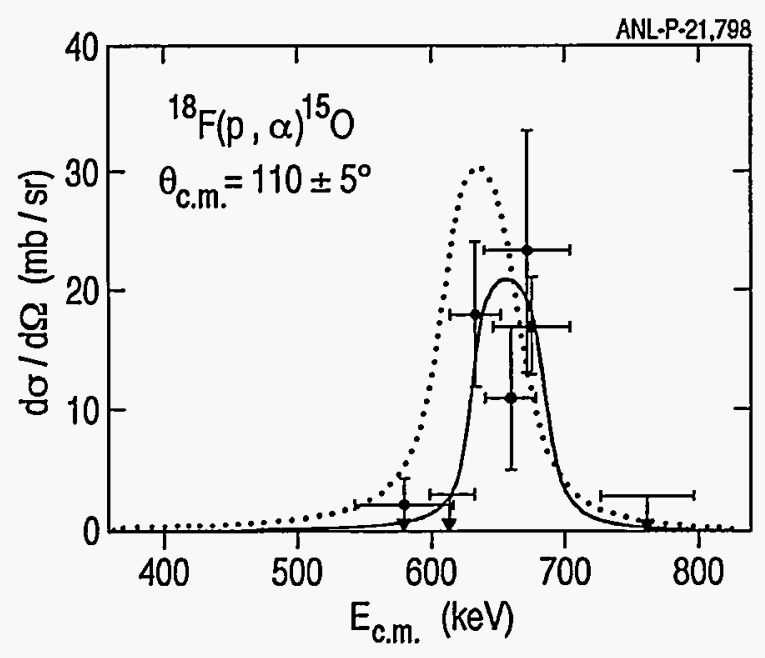

Fig. I-8. Cross sections for the ${ }^{18} F(p, \alpha)^{15} O$ reaction measured at various incident energies in the angular range $\theta_{c . m .}=110^{\circ} \pm 5^{\circ}$. The horizontal error bars represent the respective target thicknesses. The solid line represents the cross section calculated from a resonance with parameters given below averaged over an energy interval of $55 \mathrm{keV}$.

The synthesis of heavier elements in explosive nucleosynthesis in a proton-rich environment is believed to proceed through the nuclide ${ }^{19} \mathrm{Ne}$ which is produced either directly via the ${ }^{15} \mathrm{O}(\alpha, \gamma){ }^{19} \mathrm{Ne}$ reaction or via the ${ }^{14} \mathrm{O}(\alpha, \mathrm{p})^{17} \mathrm{~F}$ reaction followed by the sequence ${ }^{17} \mathrm{~F}(\mathrm{p}, \gamma){ }^{18} \mathrm{Ne}\left(\beta^{+}\right)^{18} \mathrm{~F}(\mathrm{p}, \gamma){ }^{19} \mathrm{Ne} .{ }^{19} \mathrm{Ne}$ is then the starting point for the rp-process producing nuclei up to ${ }^{56} \mathrm{Ni}$ and beyond. In the reaction chain starting with proton capture by $17 \mathrm{~F}$, however, there is a competing reaction, ${ }^{18} \mathrm{~F}(\mathrm{p}, \alpha){ }^{15} \mathrm{O}$, which recycles material back into the $\mathrm{CNO}$ cycle. How ${ }^{18} \mathrm{~F}$ interacts with protons at low energies is therefore of key interest for controlling the breakout from the "hot CNO cycle" into the rp-process. This is evidenced by recent network calculations which show a strong dependence of the reaction flow on the ${ }^{18} \mathrm{~F}(\mathrm{p}, \alpha)^{15} \mathrm{O}$ rate. ${ }^{1}$ For this reason a measurement of the ${ }^{18} \mathrm{~F}(\mathrm{p}, \alpha)$ cross section is needed requiring the development of radioactive ${ }^{18} \mathrm{~F}$ beams.

In collaboration with the Institute of Medical Physics of the University of Wisconsin at Madison we developed a method to produce ${ }^{18} \mathrm{~F}$ beams with intensities of $\sim 1$ ppA on target. Details about this method have been published. ${ }^{2}$ The main difficulty in the experiment was the admixture of the stable ${ }^{18} \mathrm{O}$ isobar in the ${ }^{18} \mathrm{~F}$ beam with an intensity which is $\sim 500-2000$ times stronger. The experiment was performed at the split-pole spectrograph using the method of a gas-filled magnet in order to separate the different reaction products. ${ }^{3}$ An excitation function for the ${ }^{18} \mathrm{~F}(\mathrm{p}, \alpha)^{15} \mathrm{O}$ reaction was measured in the energy range $E_{\mathrm{cm}}=600-800 \mathrm{keV}$ (see Fig. I-8). In this energy range a resonance state with a spin value of $3 / 2^{+}$was found at an energy of $652 \mathrm{keV}$ above threshold which is populated in an $\ell_{p}=0$ transition. Using the previously measured $\Gamma_{\mathrm{p}} / \Gamma_{\alpha}$ ratio of $0.58,4$ proton and alpha widths of $\Gamma_{\mathrm{p}}=5 \pm 1.6$ and $\Gamma_{\alpha}=8.6 \pm 2.5 \mathrm{keV}$ were extracted from the measured cross sections. This $\alpha$-width is in good agreement with the width observed for the analogue state in the mirror nucleus ${ }^{19} \mathrm{~F}$. Due to the absence of a centrifugal barrier for this transition, the $3 / 2^{+}$state dominates the astrophysical reaction rate in the temperature range $\mathrm{T}=$ $(0.5-2) \times 10^{9 \circ} \mathrm{K}$. The data were published. ${ }^{5}$

*Hebrew University, Jerusalem, Israel , †University of Wisconsin, Madison, $\ddagger$ Northwestern University

${ }^{1}$ L. van Wörmer et al., Astrophys. J. 432, 326 (1994).

2 A. Roberts et al., Nucl. Instrum. Methods. B103, 523 (1995).

${ }^{3}$ K. E. Rehm et al., Nucl. Instrum. Methods $\underline{A 370}, 438$ (1996).

4 S. Utten, Ph.D. Thesis, Yale University 1994, unpublished.

5K. E. Rehm et al., Phys. Rev. C $\underline{53}, 1950$ (1996). 
a.20. Study of the $18 \mathrm{~F}(\mathrm{p}, \gamma)$ Reaction (K. E. Rehm, D. Blumenthal, L. Daniel,* C. N. Davids, S. Fischer, C. L. Jiang, C. Lister, J. Nickles,* M. Paul, † R. C. Pardo, J. P. Schiffer, D. Seweryniak, R. E. Segel, $\ddagger$ and F. L. H. Wolfs§)

As outlined in a.20., ${ }^{18} \mathrm{~F}$ is an important nucleus for the breakout from the hot CNO cycle into the rp process. ${ }^{19} \mathrm{Ne}$, which is the starting point for the rpprocess, can be reached either via the ${ }^{15} \mathrm{O}(\alpha, \gamma)$ reaction, with ${ }^{15} \mathrm{O}$ produced partially through the ${ }^{18} \mathrm{~F}(\mathrm{p}, \alpha)^{15} \mathrm{O}$ reaction, or directly through the $18 \mathrm{~F}(\mathrm{p}, \gamma)$ reaction. Since the width $\Gamma_{\gamma}$ for this process is about a factor-of1000 smaller than the proton width $\Gamma \mathrm{p}$, the yield for the $(\mathrm{p}, \gamma)$ reaction is expected to be about three orders of magnitude smaller than the yield for the $(p, \alpha)$ reaction. These small cross sections require, therefore, a corresponding increase in detection efficiency. Since in the inverse reaction $\mathrm{p}\left({ }^{18} \mathrm{~F},{ }^{19} \mathrm{Ne}\right) \gamma$ the recoiling heavy residues are emitted at forward angles within an opening angle of less than one degree, they can be detected with high efficiency with the Fragment Mass Analyzer. Compared to the $(p, \alpha)$ experiments, this gives an improvement in efficiency by a factor of about 200 . Together with a higher ion source extraction efficiency and an improved transmission through the tandem accelerator, we expect to measure yields for the $(p, \gamma)$ reaction corresponding to a width $\Gamma_{\gamma}$ of about $1 \mathrm{ev}$. In order to check the separation between the reaction products and the beam at $0^{\circ}$ we studied in a test experiment the ${ }^{18} \mathrm{O}(\mathrm{p}, \gamma){ }^{19} \mathrm{~F}$ at energies of $\mathrm{E}_{\mathrm{cm}}=700$ $900 \mathrm{keV}$ where a resonance with a strength $\omega \gamma=1.4$ $\mathrm{eV}$ in ${ }^{19} \mathrm{~F}$ was observed. The measured excitation function is shown in Fig. I-9. The shaded area is calculated with resonance parameters from Ref. 1 which were obtained from experiments with a high-intensity proton beam and an ${ }^{18} \mathrm{O}$ target. Good agreement between the two data sets is observed. The measurements indicate that the primary ${ }^{18} \mathrm{O}$ beam is

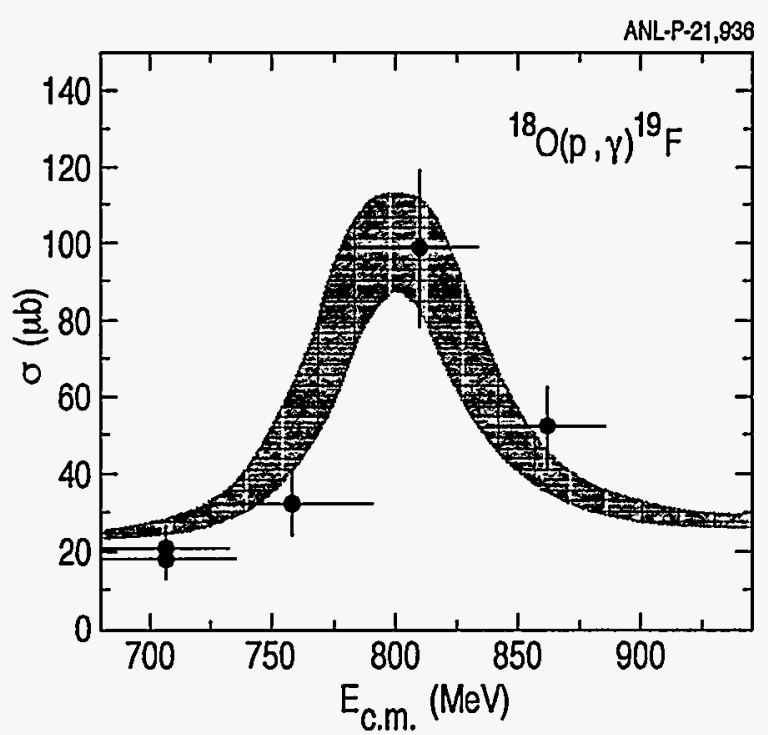

Fig. I-9. Excitation function for the ${ }^{18} O(p, \gamma){ }^{19} \mathrm{~F}$ reaction. The hatched area is calculated with parameters from Ref. 1 including a $20 \mu b$ background caused by a small $F$ contaminant in the $\mathrm{CH}_{2}$ target.

suppressed in the focal plane of the FMA by more than a factor of $10^{8}$. The particle identification was achieved via a $\triangle E-E$-ionization chamber mounted behind the $x-y$ position-sensitive focal-plane detector. Together with the $\triangle \mathrm{E}-\mathrm{E}$ signals, the FMA can provide a suppression factor of better than $10^{13}$, which is sufficient for experiments with weak radioactive ion beams.

\footnotetext{
*University of Wisconsin, Madison, $\uparrow$ Hebrew University, Jerusalem, Israel, $\ddagger$ Northwestern University, §University of Rochester

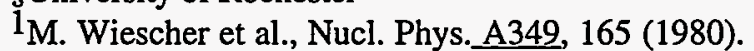




\section{a.21. Measurement of Spectroscopic Factors around $A=56$ with a ${ }^{56} \mathrm{Ni}$ Beam} (K. E. Rehm, D. Ackermann, D. Blumenthal, S. Fischer, J. Görres, * W. Henning, R. V. F. Janssens, C. L. Jiang, C. Lister, V. Nanal, J. Nolen, R. C. Pardo, M. Paul, $†$ J. P. Schiffer, R. E. Segel, $¥$ M. Wiescher,* and A. H. Wuosmaa)

$56 \mathrm{Ni}$ is the heaviest $\mathrm{N}=\mathrm{Z}$ doubly-magic nucleus for which a beam with sufficient intensity can be produced. So far only an experiment studying inelastic scattering has been performed with the ${ }^{56} \mathrm{Ni}$ ions produced in a high-energy fragmentation reaction. Based on the experience with ${ }^{18} \mathrm{~F}$, we started with the development of a ${ }^{56} \mathrm{Ni}$ beam at the ATLAS accelerator. The physics that will be addressed in the first experiments are $56 \mathrm{Ni}$ induced few nuclear transfer reactions and the study of neutron-deficient nuclei near the proton drip-line.

$56 \mathrm{Ni}$ will be produced via the ${ }^{58} \mathrm{Ni}(\mathrm{p}, \mathrm{p} 2 \mathrm{n})^{56} \mathrm{Ni}$ reaction with a $50-\mathrm{MeV}$ proton beam from the IPNS injector at Argonne. The main component in the beam will be from the stable ${ }^{56} \mathrm{Fe}$ isobar. Test measurements with $\mathrm{Ni}$-samples at the SNICS ion source show that the intensity ratio ${ }^{56} \mathrm{Ni} / 56 \mathrm{Fe}$ should be about a factor of 10 higher than observed for the ${ }^{18} \mathrm{~F} /{ }^{18} \mathrm{O}$ case. In the first experiment we plan to study the single-particle states in ${ }^{5} \mathrm{Ni}$ and ${ }^{57} \mathrm{Cu}$ populated in the $\left({ }^{2} \mathrm{C},{ }^{11} \mathrm{C}\right)$ and
$\left(C^{12}, 11_{B}\right)$ reactions, respectively. In order to separate these reactions induced by the $56 \mathrm{Fe}$ and $56 \mathrm{Ni}$ components of the beam, the outgoing particles will be detected in kinematic coincidence using the split-pole spectrograph and a large-area Si-strip detector. The heavy reaction products (e.g. ${ }^{57} \mathrm{Co}$ and ${ }^{57} \mathrm{Cu}$ produced in the one-proton transfer reaction $\left({ }^{12} \mathrm{C},{ }^{11} \mathrm{~B}\right)$ with ${ }^{56} \mathrm{Fe}$ and $56 \mathrm{Ni}$, respectively) will be identified according to mass and $\mathrm{Z}$ with the spectrograph focal-plane detector. The coincident ${ }^{11_{\mathrm{B}}}$ particles will be detected at the appropriate angles with a multi-strip Si-detector. This detection scheme was tested with ${ }^{58} \mathrm{Ni}$ beams at energies of $400 \mathrm{MeV}$. Excellent mass and charge resolution for Ni-like reaction products was observed at scattering angles $\theta_{\mathrm{lab}}=3^{\circ}-6^{\circ}$. Using a $5 \times 5 \mathrm{~cm}^{2} \mathrm{Si}$ detector with fifty $1-\mathrm{mm}$ wide strips, a $\mathrm{Q}$-value resolution of $500 \mathrm{keV}$ was achieved. Further test measurements with a mixed ${ }^{58} \mathrm{Ni}-58 \mathrm{Fe}$ beam are planned for early 1996 with the goal to have the first experiments with $56_{\mathrm{Ni}}$ beams in Summer 1996.

*University of Notre Dame, $\uparrow$ Hebrew University, Jerusalem, Israel, $¥$ Northwestern University 


\section{B. GAMMA-RAY SPECTROSCOPY STUDIES}

The major part of the work at Argonne was on the study of superdeformation, primarily in the mass 190 region, but also in the mass 150 and 80 regions. Our work covers not only the nature of states inside the SD minimum, but also elucidates the physics related to the feeding into and decay from this minimum. We have made a major discovery in identifying the high-energy gamma rays which directly connect SD and normal yrast states in ${ }^{194} \mathrm{Hg}$ and, thereby, have for the first time determined the excitation energies, spins and probable parity of a SD band. There is also a diverse program on non-superdeformed nuclei, which covers aspects such as: quenching of pairing with temperature, a search for double octupole-phonon states in ${ }^{208} \mathrm{~Pb}$, conservation of the $\mathrm{K}$-quantum number at high spin, phase transitions in mesoscopic systems, structure of high-lying states in actinide nuclei, and spectroscopy of fission fragments.

New large $\gamma$-ray detector arrays are currently under construction in the United States (Gammasphere) (GS) and in Europe (Eurogam). These arrays provide new opportunities for nuclear structure research. Argonne is participating vigorously in the construction of Gammasphere and performed experiments with the device in 1993-1995. The group is also collaborating in several experiments at Eurogam. Results from these experiments show clearly the promise and power of these devices. At Argonne we developed a battery of programs to analyze the new high-fold data from these instruments.

The main research tool at ATLAS for this program is the Argonne-Notre Dame BGO gamma-ray facility which consists of 50 hexagonal BGO detectors (used mainly as a sum-energy/multiplicity filter) surrounded by 12 Compton-suppressed Ge detectors. Auxiliary equipment includes: a scattering chamber, constructed by the University of Kansas, for coincidence measurements between $\gamma$ rays and particles; a.plunger apparatus, developed by Notre Dame, for recoil-distance measurements of nuclear lifetimes; and dedicated chambers for special experiments (g-factor measurements, fission-fragment coincidence measurements, etc.). A rare capability exists at ATLAS for performing $\gamma-\gamma$ coincidence experiments with the Fragment Mass Analyzer (FMA). Ten Compton-suppressed Ge spectrometers can be located at the target position of the FMA for this purpose.

Several projects are joint efforts with groups from Lawrence Berkeley and Lawrence Livermore Laboratories, Orsay, the University of Notre Dame, Purdue University, North Carolina State University, INEL-Idaho National Engineering Laboratory, the Australian National University, the University of Manchester, the University of Tennessee, the University of Liverpool and Rutgers University. The work at Eurogam was performed in collaboration with several other European laboratories.

\section{B.a. States in the Superdeformed Secondary Minimum and Their Coupling with States in the Normal Well}

\section{Introduction}

The occurrence of an excited secondary minimum at large deformation provides a rare opportunity to study states which are cold, although highly excited with respect to the normal yrast line. Within the superdeformed well, isolated from normal states with smaller deformation, there is a cold "ground" state, as well as low-lying excited states which can give rise to sharp equally-spaced transitions. With increasing excitation energy, the coupling with states outside the well grows until the separate identity of SD states melts away. When the SD "yrast" state lies high enough above the true (normal) yrast state, then a coupling occurs between a cold system with a hot normal one, causing the SD band to decay.

Research on superdeformation at Argonne addresses the physics associated with states within the SD well and their coupling with states outside the well. Investigation of the decay out of SD states into lowerlying normal states allows us to examine the coupling between a cold, ordered system and a hot, chaotic one. In addition, it is essential for experimental determinations of the spins, parities and excitation energies of states in a SD minimum. Furthermore, this 
investigation has unexpectedly led to a new tool for examining the reduction of pairing in a mesoscopic system. Discrete line spectroscopy investigates the cold SD bands. Study of the feeding of SD bands and of the

\section{Decay of SD Bands}

Over the past few years we have been developing methods to investigate the decay out of SD bands. Our approach is to combine experimental and theoretical studies. In addition, we also investigated the $\gamma$ decay following thermal neutron capture, which has similarities to decay from highly-excited SD states. To find the elusive connection between SD and normal states we used two opposite approaches. In the first, we succeeded in extracting the total quasicontinuous spectrum of $\gamma$ rays connecting the two classes of states. This study led us to the second approach, which is to identify the simplest connections, namely the $\gamma$ rays which, in one step, connect SD levels directly to the normal yrast levels. We achieved a major breakthrough by discovering the 1-step $\gamma$ rays from a SD band in ${ }^{194} \mathrm{Hg}$. Thus, the spins and likely parity for all levels of the yrast SD band in ${ }^{194} \mathrm{Hg}$, as well as their excitation energies, were determined. After a decade of superdeformation studies and the identification of over $150 \mathrm{SD}$ bands in the mass 150 and 190 regions, there is finally one for which the spin and parity quantum numbers are known. With the imminent completion of Gammasphere and Eurogam our simple approach of

\section{Cold States}

We found over 30 SD bands in the mass 190 region from work done at ATLAS, Gammasphere and Eurogam. This large body of data was vital in helping to identify the occurrence of "identical" bands, i.e. SD bands in neighboring nuclei which have transition energies with $\Delta E<1 / 500$, or which have identical dynamic moments of inertia $\mathfrak{g}(2)$. The identical bands, which were not anticipated, are still not explained, but may imply a symmetry which has yet to be identified. Our current work is directed towards answering the most critical questions concerning identical bands: namely, do levels with identical transition energies have the same spins and are the deformations equal?

Another striking observation is a staggering of alternate levels in three SD bands in ${ }^{194} \mathrm{Hg}$, which suggests the presence of a $Y_{44}$ symmetry (i.e. four-fold symmetry in a plane perpendicular to the symmetry axis). In addition, we discovered a band in ${ }^{151} \mathrm{Dy}$, with energies midway between those in $152 \mathrm{Dy}$, which provides associated quasicontinuum $\gamma$ rays probes the nature of excited states and their increasing mixing with normal states.

searching for the highest-energy 1-step $\gamma$ rays should lead to the spins and parities of many more SD bands.

We extracted the complete spectrum of the $\gamma$ rays linking states in two separate wells in a number of mass 190 nuclei. The spectra, which have a quasicontinuous distribution with superimposed broad structures and sharp peaks, establish the decay mechanism as due to mixing of a SD state with some of the sea of normal states in which it is embedded. We propose that a conspicuous clustering of $\gamma$ strength between 1.4 and $2.2 \mathrm{MeV}$ is due to a rearrangement of the level densities by pair correlations. A model was developed to calculate levels from all quasiparticle excitations, as well as the ensuing statistical spectrum from a highly excited state. The calculated statistical spectra reproduce the observed features of the decay spectra, including the differences in even-even and oddeven nuclei. Thus, the decay spectra from SD states are serendipitous probes for the quenching of pairing with temperature. The $\gamma$ spectra associated with the different stages of a $\gamma$ cascade which flows through ST bands show that the nucleus undergoes an unusual double cycle of chaos-to-order transition.

additional evidence for pseudospin symmetry. In ${ }^{154}$ Dy we found a SD band which has energies identical to those of an excited SD band in ${ }^{153}$ Dy and is the first SD band found to decay to prolate collective normal states.

Identification of vibrational states in the SD well can serve to establish the rigidity of the deformation with respect to $\beta, \gamma$ or octupole distortions. Theory has pointed out that SD nuclei may manifest octupole instability. We found the first indications for an octupole vibrational band in $190 \mathrm{Hg}$ (from Gammasphere data) at a surprisingly low energy $(\sim 600$ $\mathrm{keV}$ ) above the yrast SD band.

We measured lifetimes of individual states of SD bands in $192,{ }^{194} \mathrm{Hg}$ which prove that the deformation is indeed large and that it is stable with respect to spin and particle excitation. The quadrupole moments of two identical bands are the same, suggesting that they have the same deformation. 


\section{Excited SD States}

From Eurogam and Gammasphere data we established that excited SD bands give rise to a pronounced E2 bump in the $\gamma$ spectrum. This feature allows us to probe the collective properties of excited SD states. There are preliminary indications that the quadrupole moment and moment of inertia of the excited SD states are larger than those of the yrast SD band in ${ }^{192} \mathrm{Hg}$. We studied the coupling of excited SD and ND states and the mechanism for the unexpectedly large population of SD states by both experiment and theory. We are able to reproduce by Monte Carlo simulations all observables connected with the feeding: band intensities, variation of intensity with spin, entry distribution (in spin and energy) of states leading to trapping in the SD well, and the spectra of feeding $\gamma$ rays.

\section{a.1. Spins, Parity and Excitation Energies of a Superdeformed Band in ${ }^{194} \mathrm{Hg}$ from One-Step Discrete Decays to the Yrast Line (T. L. Khoo, M. P. Carpenter, T. Lauritsen, D. Ackermann, I. Ahmad, D. J. Blumenthal, S. M. Fischer, R. V. F. Janssens, D. Nisius, E. F. Moore, * A. Lopez-Martens, $\ddagger$

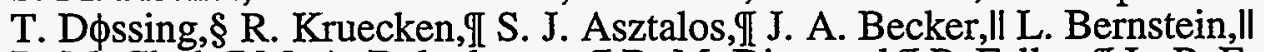 R. M. Clark, TI M. A. Deleplanque, II R. M. Diamond, I P. Fallon, II L. P. Farris,\| F. Hannachi, $\ddagger$ E. A. Henry, Il A. Korichi;** I. Y. Lee,II A. O. Macchiavelli, II and F. S. Stephens DD}

Around $150 \mathrm{SD}$ bands have been found in the $\mathrm{A}=150$ and 190 regions. However, the connections between superdeformed (SD) and normal-deformed (ND) states, the excitation energies and the quantum numbers of these SD bands are not known, despite many attempts to determine them. Thus, a major challenge in the study of superdeformation is to determine these quantum numbers. Our approach to this problem is a coherent program to study the decay out of SD bands. We

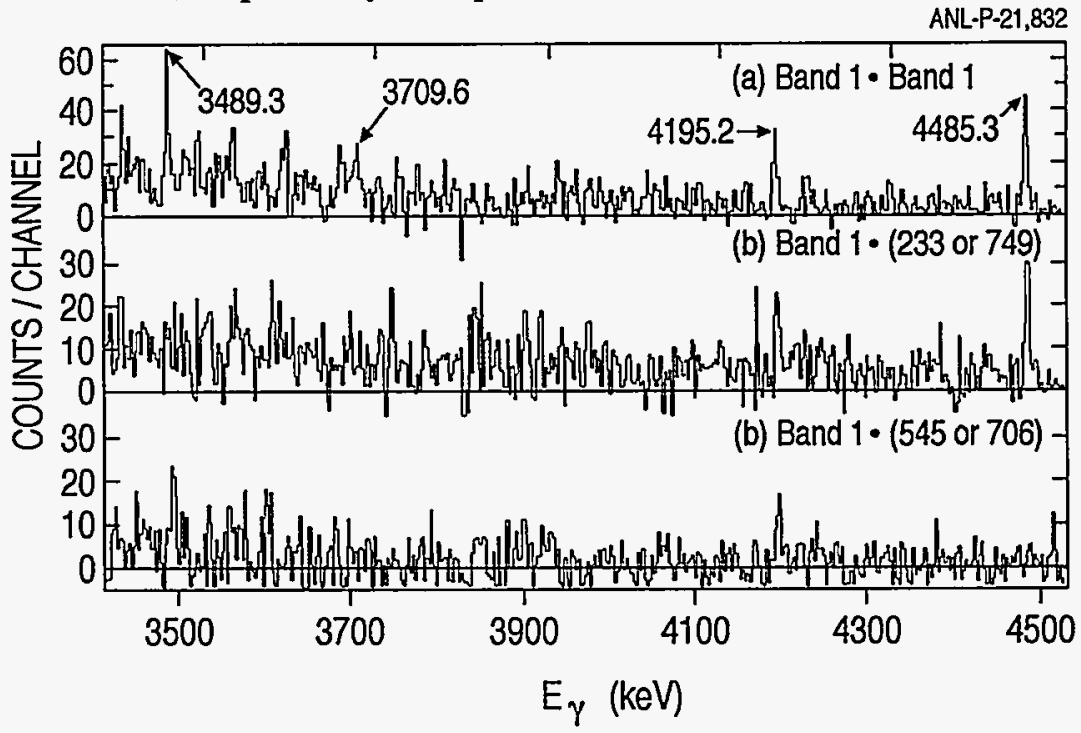

Fig. I-10. Spectra from (a) pairwise coincidences of transitions in SD band 1 and (b), (c) pairwise coincidences with a band $1 \gamma$ ray and a transition deexciting a ND negative parity level. SD band 1 coincidence gates have energies of 255 to $492 \mathrm{keV}$. Transitions directly connecting SD and ND yrast levels are labeled in (a): other weaker lines probably feed excited ND levels. The absence of the 4485-keV line in (c) demonstrates that it feeds the normaldeformed 9- level. The 3489-keV line is weak in (b) due to statistical fluctuations.

*North Carolina State University and Triangle Universities Nuclear Laboratory, + Centre de Spectrometrie Nucleaire et de Spectrometrie de Masse, Orsay, France, §Niels Bohr Institute, Copenhagen, Denmark, qLawrence Berkeley National Laboratory, IILawrence Livermore National Laboratory, **Institut Physique Nucleaire, Orsay, France 
ANL-P-21,823

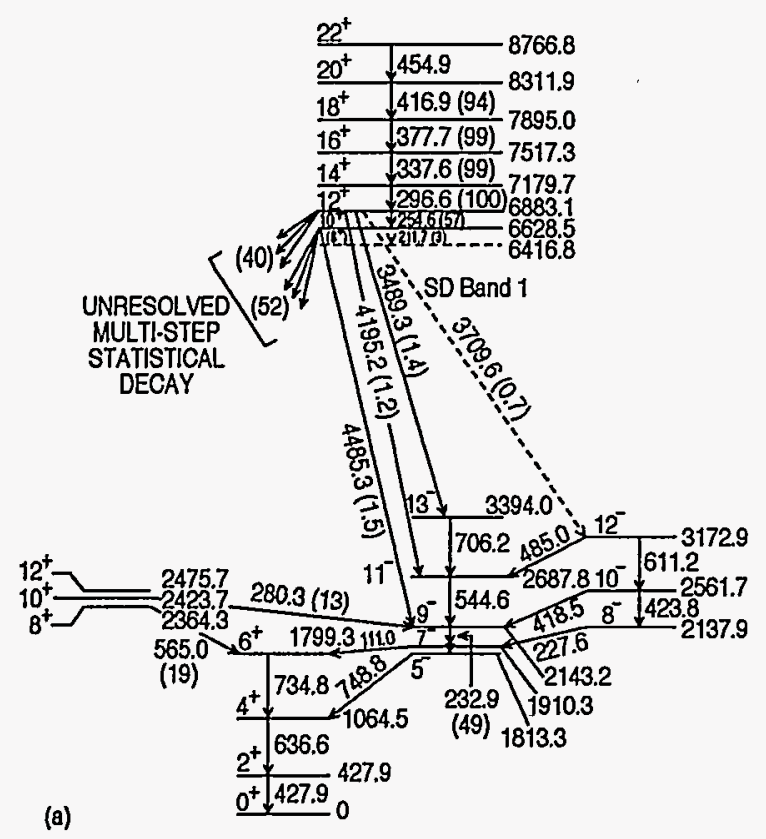

(b)

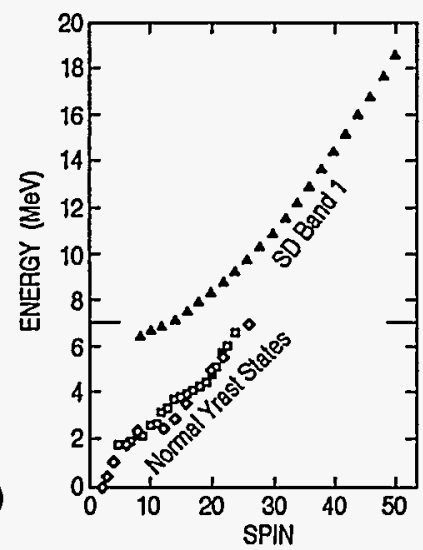

Fig. I-1I. (a) Decay scheme of SD band 1 in ${ }^{194} \mathrm{Hg}$. The excitation energies and spin assignments are firm, while the parity is very probable. The relative intensities of transitions are given in parentheses. Dashed lines indicate tentative assignments. (b) Energy vs spin diagram for $S D$ and ND states in $194 \mathrm{Hg}$. extracted the total decay spectrum of gamma rays connecting SD and ND states, and calculated this spectrum. In addition, we determined the experimental and theoretical gamma spectra following thermal neutron capture, which represents an analogous decay from narrow highly-excited states. These studies led us to propose that the best way to accurately locate a SD band is to find the highest energy primary gamma rays, i. e. the direct one-step transitions to the ND yrast states. We discovered the discrete gamma rays directly connecting states of a superdeformed (SD) band in ${ }^{194} \mathrm{Hg}$ to the yrast states from a Gammasphere experiment (see Fig. I-10). The exact connections with the yrast transitions were defined by coincidence relationships (see Fig. I-11). Thus, the excitation energies and spins of all members of the lowest SD band are established for the first time, together with their likely parity. The SD band decays from its $10^{+}$ and $12^{+}$states, which lie 4204.8 and $4407.4 \mathrm{keV}$ above the normal-deformed yrast states of the same spins. The decays from these levels are mostly through unresolved transitions, with the 1-step gamma rays carrying only a small fraction of the decay intensity: $3.3 \%$ and $1.5 \%$ from the $12^{+} \mathrm{SD}$ and $10^{+} \mathrm{SD}$ levels, respectively. This is consistent with our theoretical estimate of $5 \%$. However, this agreement may be fortuitous. Since the decay out of SD states occurs through a small admixture of the complex compound ND states in which it is embedded, the transition strengths of the 1step gamma rays are expected to exhibit fluctuations.

This first determination of the spins and of the most probable parity of the SD levels is significant. By using our proposed technique and an approximately tenfold increase in statistics expected with the full Gammasphere and Eurogam arrays, it should be possible to find the one-step decays of many more SD bands and to characterize their quantum numbers. In a forthcoming Gammasphere experiment we expect to be able to determine the spins of two SD bands in $192,194 \mathrm{Hg}$ which have identical energies. A paper reporting our results was accepted for publication. 


\section{a.2. Search for One-Step Decay Gamma Rays Connecting Superdeformed and} Normal States in ${ }^{192} \mathrm{Hg}$ (D. Ackermann, T. L. Khoo, M. P. Carpenter, T. Lauritsen, I. Ahmad, D. J. Blumenthal, S. M. Fischer, R. V. F. Janssens,

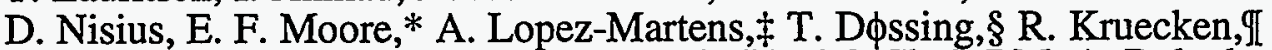
S. J. Asztalos,II J. A. Becker,\| L. Bernstein, Il R. M. Clark, II M. A. Deleplanque,I R. M. Diamond, I P. Fallon, II L. P. Farris, Il F. Hannachi, $\ddagger$ E. A. Henry, ll A. Korichi,** I. Y. Lee,II A. O. Macchiavelli,I and F. S. Stephens\$D

We have discovered the gamma rays directly connecting a super- and normal- deformed band in ${ }^{194} \mathrm{Hg}$ (see B.a.1). A similar search was also undertaken for the 1step decay gamma rays from the lowest superdeformed band in ${ }^{192} \mathrm{Hg}$, for which we have Gammasphere data of comparable statistics as for ${ }^{194} \mathrm{Hg}$. The highest energy transition is a line at $3116 \mathrm{keV}$. No transitions above $3.2 \mathrm{MeV}$ were found above a 3 sigma level. The difference in the 1-step decays in the two cases is probably due to fluctuations in the decay strengths (of Porter-Thomas type) expected in the decay of complex normal states. It is through the admixtures of such complex states that SD levels decay. Another possible interpretation is that the SD band lies only $3.1 \mathrm{MeV}$ above the normal yrast line, more than $1 \mathrm{MeV}$ lower than in ${ }^{194} \mathrm{Hg}$. However, the total quasicontinuum spectrum suggests an excitation energy in ${ }^{192} \mathrm{Hg}$ of 4.3 $\pm 0.9 \mathrm{MeV}$.

We shall shortly perform another Gammasphere experiment to search for the 1-step decay transitions from the SD band in ${ }^{192} \mathrm{Hg}$. With an 6-fold increase in statistics from the 86 detectors currently in operation, we are optimistic about finding these transitions. The transition energies for the ${ }^{192} \mathrm{Hg}$ SD band are almost identical to those of an excited band in ${ }^{194} \mathrm{Hg}$. Thus, we shall soon be able to ascertain if the identical transitions emanate from states of the same spin. This is a critical question for understanding the reason for identical bands.

*North Carolina State University and Triangle Universities Nuclear Laboratory, $\ddagger$ Centre de Spectrometrie Nucleaire et de Spectrometrie de Masse, Orsay, France, §Niels Bohr Institute, Copenhagen, Denmark, qLawrence Berkeley National Laboratory, IILawrence Livermore National Laboratory, **Institut de Physique Nucleaire, Orsay, France

\section{a.3. The ${ }^{194} \mathrm{~Pb}$ Decay-Out Quasi-Continuum of Gamma Rays} (T. Lauritsen, I. Ahmad, M. P. Carpenter, R. V. F. Janssens, T. L. Khoo, D. Nisius, M. J. Brinkman, * A. E. Henry, $\dagger$ and J. A. Becker $\dagger$ )

The excitation energy and spin of superdeformed (SD) bands were recently found in ${ }^{194} \mathrm{Hg}$ in a direct decay to the normal deformed band. Such direct one-step decays were hitherto difficult to observe and most of the measurements of the excitation energy and spin were made from the analysis of the quasi-continuum of gamma-rays (QC) in coincidence with the SD bands. An early analysis of the QC in ${ }^{194} \mathrm{~Pb}$ stipulated that the $\mathrm{QC}$ in this nucleus had less strength than that seen in $191-194 \mathrm{Hg}$ - perhaps as a result of the nucleus' almost spherical shell, favoring direct, discrete decays and a different feeding pattern.
We have undertaken an independent re-analysis of the data from this controversial experiment. Our analysis shows that the QC largely looks like the QC found in the $\mathrm{Hg}$ nuclei - in disagreement with the previous analysis. Two new experiments on ${ }^{194} \mathrm{~Pb}$ are planned, one at Eurogam and one at Gammasphere. The former was just completed and the latter will commence shortly. We hope to be able to find the direct one-step decay links and analyze the $\mathrm{QC}$ in coincidence with the SD bands. We will have orders of magnitude more statistics in these experiments because many more detectors were added since the first experiment was done with Gammasphere in its early implementation phase.

*Oak Ridge National Laboratory, $\nmid$ Lawrence Livermore National Laboratory 


\section{a.4. 2nd and 3rd Moments of Low Statistics (High-Energy) $\gamma$-ray Spectra (D. Ackermann, T. L. Khoo, M. P. Carpenter, T. Lauritsen, I. Ahmad, D. J. Blumenthal, S. M. Fischer, G. Hackman, R. V. F. Janssens, D. Nisius, and J. Young)}

The primary $\gamma$-rays connecting the states of highly deformed bands to the yrast states are often hidden in the fluctuations of the high-energy part of $\gamma$-spectra. The goal was to determine the presence of peaks in a way that does not depend on the individual judgment of the scientist inspecting the spectrum. The 2 nd and 3 rd moments, the variance and skewness of a spectrum were used to locate the position and significance of such transitions in an effective and objective way. The method was applied to spectra in coincidence with superdeformed bànds in ${ }^{192,194} \mathrm{Hg}$, where candidates for primary transitions were found previously $\left({ }^{194} \mathrm{Hg}\right)$ or are expected $\left({ }^{192} \mathrm{Hg}\right)$. In the first case the observed transitions could be confirmed whereas in the second case no clear candidate could be found. On the basis of the observed skewnesses and variances for peaks with relatively low statistical significance the method will be extended to extract the total transition strength in a wider region of the spectrum.

\section{a.5. A Technique for the Measurement of the Spin of Superdeformed Bands in ${ }^{194} \mathrm{Hg}$ (D. J. Blumenthal, T. L. Khoo, M. P. Carpenter, T. Lauritsen, D. Ackermann, I. Ahmad, S. M. Fischer, G. Hackman, R. V. F. Janssens, D. Nisius, R. Polender, * E. F. Moore,* T. D $\phi s s i n g \dagger$ )}

Despite the abundance of superdeformed (SD) bands which were discovered in nuclei, very little is known about their properties such as spin and excitation above the normally deformed (ND) states. This is due to the inability to unambiguously observe (until recently) the discrete single-step gamma-ray transitions linking the SD and ND states. A technique was developed to extract the spin of SD bands without the need for such direct observation. The predominant mode of SD decay involves the mixing of these discrete states with the continuum and the emission of statistical gamma-rays followed by the decay between discrete ND states to the ground state. The spin at which the SD band decays out into the continuum is merely the sum of the spin carried away by the statistical gamma rays and the average spin at which the ND states are populated. Model calculations provide the spin carried away by statistical gamma rays. Careful measurement of the intensities of the ND gamma-ray transitions following SD decay gives the average ND spin populated. This technique was applied to three superdeformed bands observed in ${ }^{194} \mathrm{Hg}$. An experiment was performed using the Gammasphere array and the ${ }^{150} \mathrm{Nd}\left({ }^{48} \mathrm{Ca}, 4 \mathrm{n}\right){ }^{194} \mathrm{Hg}$ reaction at $205 \mathrm{MeV}$. The tentative spin extracted for the strongest SD band agrees with a direct measurement of one-step discrete decays to the yrast line. Future experiments are planned which will determine the accuracy of the above method. If it proves to be successful, it will remain useful in those cases where the discrete transitions are not observed.

*North Carolina State University and Triangle Universities Nuclear Laboratory, †Niels Bohr Institute, Copenhagen, Denmark 


\section{a.6. Spin Distributions and Entry Points of Super- and Normal-Deformed}

States in ${ }^{192} \mathrm{Hg}$ (T. L. Khoo, T. Lauritsen, I. Ahmad, M. P. Carpenter, P. Fernandez, R. V. F. Janssens, E. F. Moore,II S. Ridley, J. Winn, F. L. H. Wolfs, Ph. Benet,* K. B. Beard,§ P. J. Daly,* M. W. Drigert,§ U. Garg,§ Z. Grabowski,* and D. Ye*)

The population of discrete superdeformed (SD) levels at high spin is much larger than might be expected from an extrapolation of the intensities of normal-deformed (ND) states. Hence, it is interesting to understand the mechanism whereby the $\gamma$ decay cascade ends up trapped in the SD minimum. The spin distributions and entry points leading to feeding of SD and ND levels provide information on the feeding mechanism. We measured these quantities at several beam energies for ${ }^{192} \mathrm{Hg}$ as populated in the ${ }^{160} \mathrm{Gd}\left({ }^{36} \mathrm{~S}, 4 \mathrm{n}\right)$ reaction. The results show that the SD states are fed from the higher angular momentum portion of the spin distribution for the groundstate of the nucleus (see Fig. I-12). Increasing beam energy results in larger initial partial waves but there is a saturation. This saturation is due to the depletion of the highest partial waves by fission. A paper is being written on this work.

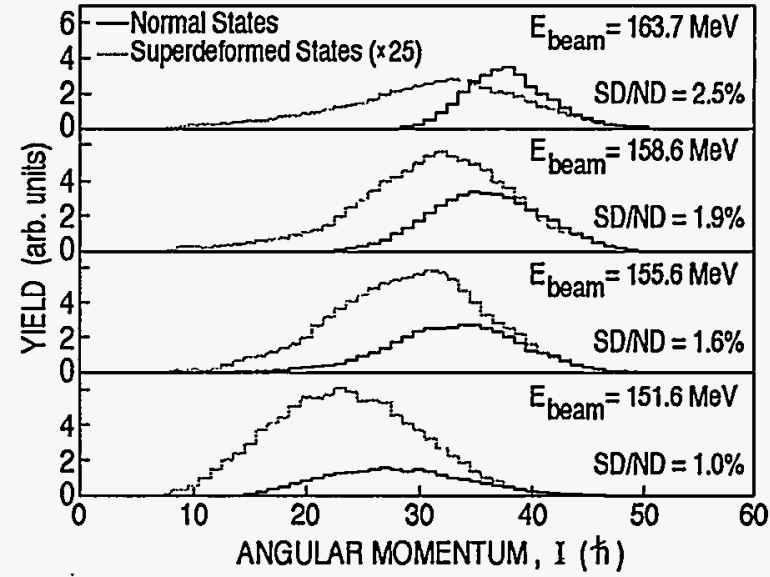

Fig. I-12. Spin distributions for $\gamma$ rays leading to normal deformed and superdeformed states in $192 \mathrm{Hg}$ as obtained at four beam energies with the $\left({ }^{36} S, 4 n\right)$ reaction.

*Purdue University, §University of Notre Dame, $\mathbb{I N o r t h}$ Carolina State University and Triangle Universities Nuclear Laboratory

\section{a.7. Calculation of the Spectrum of $\gamma$ Rays Connecting Superdeformed and} Normally Deformed Nuclear States (T. D $\phi s s i n g, *$ T. L. Khoo, T. Lauritsen, I. Ahmad, D. Blumenthal, M. P. Carpenter, B. Crowell, D. Gassmann, R. G. Henry, R. V. F. Janssens, and D. Nisius)

The decay out of superdeformed states occurs by a coupling to compound nuclear states of normal deformation. The coupling is very weak, resulting in mixing of the SD state with one or two normal compound states. With a high energy available for decay, a statistical spectrum ensues. The shape of this statistical spectrum contains information on the level densities of the excited states below the SD level. The level densities are sensitively affected by the pair correlations. Thus decay-out of a SD state (which presents us with a means to study a statistical cascade from a highly-excited sharp state) provides a method for investigating the reduction of pairing with increasing thermal excitation energy.

*Niels Bohr Institute, Copenhagen, Denmark

${ }^{1}$ T. D $\phi s s i n g$ et al., Phys. Rev. Lett. $\underline{75}, 1276$ (1995)
The energies of $0,2,4 \ldots$ quasiparticle states are calculated using a BCS approximation with a selfconsistent pair field, followed by particle-number projection and diagonalization. A step-wise reduction of the pair correlation energy with increasing quasiparticle number is obtained. The resultant levels are used to calculate the statistical spectrum from an initial state $4.3 \mathrm{MeV}$ above the yrast line. Spectra for even-even, odd-even and odd-odd nuclei were calculated (see Fig. I-13), and characteristic features for each type are observed. Qualitative agreement with the decay-out spectra from SD bands in ${ }^{192} \mathrm{Hg}$ (even-even) and ${ }^{191} \mathrm{Hg}$ (odd-even) are observed, indicating that structures in the spectra are due to pairing effects. 


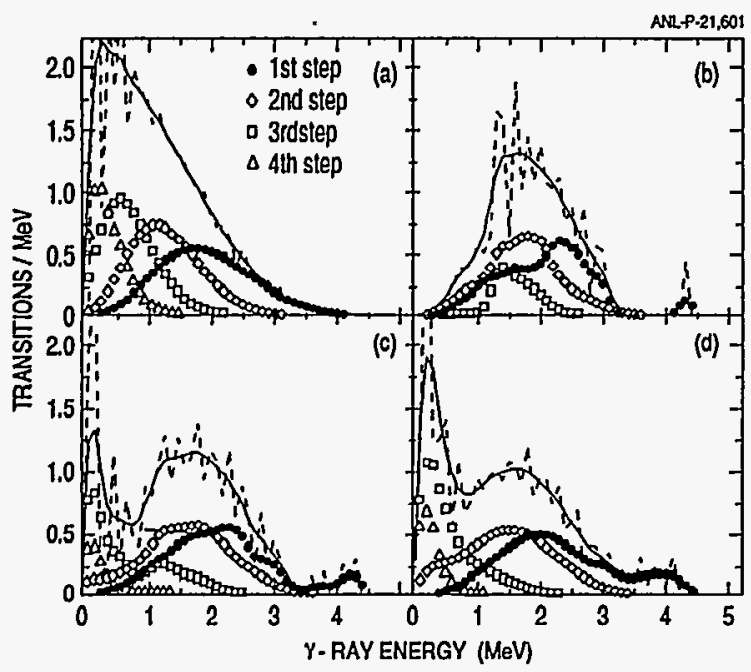

Fig. 1-13. Statistical decay spectra, with 100-keV bins, for initial energy $U=4.3 \mathrm{MeV}$ (dashed lines) calculated with (a) the unpaired even-even level density, and $(b)-(d)$ with the level densities obtained in the diagonalization procedure with $(b)$ even-even, $(c)$ odd-even, and (d) odd-odd particles numbers. The solid lines in the panels are obtained by folding the spectra by a Gaussian function of FWHM $=3 \mathrm{MeV}$, chosen to be equal to the distance between doubly degenerate neutron single-particle levels. The contributions to the full spectrum from individual cascade steps, denoted by the symbols in panel (a), are also folded by the Gaussian function.
The calculated decay spectrum also exhibits a peak at $4.3 \mathrm{MeV}$, corresponding to 1-step decay directly to the yrast line, which has a branching ratio of 3-5\%. This calculated peak provided one of the stimulations for us to search for 1-step decays in our data. This turned out to be successful in ${ }^{194} \mathrm{Hg}$. This work was published. ${ }^{1}$ The calculations were extended to compute the statistical $\gamma$ spectrum following thermal neutron capture, where the similar physics applies. Comparison with the measured spectrum from the ${ }^{167} \mathrm{Er}(\mathrm{n}, \gamma)^{168} \mathrm{Er}$ reaction (see B.b.3.) gives fair agreement for the spectral shape and multiplicity. However, differences in details are present, mainly because the calculations do not include vibrational and rotational levels, which have the effect of decreasing the pair gap. Future work will concentrate on using realistic single-particle energies from the cranked shell-model (instead of the schematic equi-spaced single-particle levels used so far), and on allowing for removal of angular momentum in the statistical decay. The calculated decay-out multiplicity is $\sim 25 \%$ lower than the experimental one; the cause for this will be sought.

a.8. Search for Hyperdeformed Structures in 144,145 Gd (S. M. Fischer, R. V. F. Janssens, M. P. Carpenter, T. Lauritsen, I. Ahmad, D. J. Blumenthal, G. Hackman, T. L. Khoo, D. Nisius, P. Fallon,* S. Asztalos,* R. M. Clark,* M.-A. Delaplanque-Stephens, * R. M. Diamond,* I.-Y. Lee, * A. O. Macchiavelli,* F. S. Stephens, $*$ D. G. Sarantites, $\dagger$ M. Devlin, $\dagger$ D. R. LaFosse, $\dagger$ and F. Lerma $\dagger$ )

The ${ }^{98} \mathrm{Mo}\left({ }^{51} \mathrm{~V}, \mathrm{pxn}\right)$ reaction at a beam energy of 230 $\mathrm{MeV}$ was studied at Gammasphere in order to search for possible hyperdeformed (HD) structures in $144,145 \mathrm{Gd}$. If found, these HD bands indicate the existence of extremely deformed nuclear shapes with $\sim$ 3:1 axis ratios. Theoretically, these HD structures were predicted to be yrast above spin $\sim 70 \hbar$. Earlier works from Chalk River, GASP and Gammasphere showed some experimental evidence for $\mathrm{HD}$ bands in ${ }^{152} \mathrm{Dy}$ and $147_{\mathrm{Gd}}$.
The present experiment was performed in conjunction with the MicroBall particle detector array, which was used to provide charged-particle selection for the different reaction channels. The data were analyzed and they display no strong evidence for HD structures. However, they do.contain a wealth of information on superdeformed structures in several neighboring nuclei. The results are being written up for publication.

*Lawrence Berkeley National Laboratory, $\uparrow$ Washington University 
a.9. Superdeformation Studies in ${ }^{150} \mathrm{~Tb}$ (D. Nisius, R. V. F. Janssens, B. Crowell, I. Ahmad, M. P. Carpenter, R. G. Henry, T. L. Khoo, T. Lauritsen, S. Asztalos,* P. Fallon,* B. Cederwall,* M. A. Deleplanque,* R. M. Diamond,* I. Y. Lee, * A. O. Machiavelli, * F. S. Stephens, * P. J. Twin, $\dagger$ C. W. Beausang, $\dagger$ M. Bergstrom, $\dagger$ and S. Clarke $\dagger$ )

There are now over 40 superdeformed (SD) bands known in the $\mathrm{A} \sim 150$ region and in most cases the properties of these bands are understood in terms of single-particle excitations in the absence of pairing. By continuing the search for new SD bands we hope to gain insight into (i) the ordering of the proton and neutron orbitals near the Fermi surface in the SD well, (ii) the effects that the alignment of those orbitals has on the moments of inertia, and (iii) the collective excitations in the SD well. For ${ }^{150} \mathrm{~Tb}$, which is one proton and one neutron away from the SD doubly-magic nucleus ${ }^{152} \mathrm{Dy}$, it should be possible to study SD bands based on both proton and neutron hole excitations.
Two new SD bands were observed in ${ }^{150} \mathrm{~Tb}$ using the reaction ${ }^{124} \mathrm{Sn}\left({ }^{31} \mathrm{P}, 5 \mathrm{n}\right)$ at $167 \mathrm{MeV}$ with the Gammasphere detector array. This brings the total number of SD bands in this nucleus to three. These new bands can be interpreted as excited SD configurations involving a proton and a neutron particle-hole excitation, respectively. The data provide information on (i) the contribution to the dynamic moments of inertia due to the occupation of specific orbitals, (ii) the deformation driving effects of the particle-hole states, and (iii) the consequences of these effects on pairing and band crossings. A paper presenting these results was published. ${ }^{1}$

*Lawrence Berkeley National Laboratory, $\nmid$ Liverpool University, United Kingdom

1P. Fallon, Phys. Rev. C 52.93 (1995)

a.10. Superdeformation Studies in ${ }^{151}$ Dy (D. Nisius, R. V. F. Janssens, I. Ahmad, B. Crowell, M. P. Carpenter, D. Blumenthal, D. Gassmann, S. M. Fischer, T. L. Khoo, T. Lauritsen, E. F. Moore, * P. Fallon, $\uparrow$ S. Asztalos, $\uparrow$ B. Cederwall, $\uparrow$ R. Clark, $\uparrow$ M. A. Deleplanque-Stephens, $\uparrow$ R. M. Diamond, $\uparrow$ I. Y. Lee $\nmid$ A. O. Macchiavelli, $\uparrow$ F. S. Stephens, $\uparrow$ P. J. Daly $₫$ Z. W. Grabowski, $\ddagger$ R. H. Mayer, $\S$ and P. ChowdhuryII)

Following the first reports of "identical" superdeformed (SD) bands in the pairs $\left(151 \mathrm{~Tb}^{*}, 152 \mathrm{Dy}\right)$, $\left({ }^{150} \mathrm{Gd}^{*},{ }^{151} \mathrm{~Tb}\right)$ and $\left({ }^{153} \mathrm{Dy} * 1{ }^{152} \mathrm{Dy}\right)$ (where * denotes an excited SD band), Nazarewicz et al., 1 proposed that these observations could be understood in the strong coupling limit of a pseudo SU(3) symmetry scheme. In this scenario a valence particle or hole is coupled to the core SD band for which there exists three limiting values of the decoupling parameter, $a= \pm 1$ and $a=0$. The first two cases, where the identical bands have nearly identical transition energies, corresponds to $a=$ +1 . Signature partner excited SD bands in 153 Dy lie at the $3 / 4$ and $1 / 4$ point energies of the ${ }^{152}$ Dy core SD band and correspond to an $a=0$ case. Previously, no band based on a decoupling parameter of $a=-1$ was seen. However, such a case was predicted to occur for an excited SD band in ${ }^{151} \mathrm{Dy}$. The configuration of this band would be a pseudo [ $\tilde{3} \tilde{0} \tilde{1}] 1 / 2$ neutron hole coupled to the ${ }^{152}$ Dy core and it would have transition energies that lie midway to those of the core SD band. In an early implementation experiment at Gammasphere, this band (band 4) was identified as well as 3 other new excited SD bands in the ${ }^{151}$ Dy nucleus. These results were published. ${ }^{2}$

Inherent in the-pseudo-spin picture of identical bands is the assumption that the moments of inertia be identical as well. The expected $A^{5 / 3}$ scaling of the moment of inertia contradicts this assumption. However, if the occupation of one of these pseudo-spin orbitals leads to a change in deformation between the "identical" bands, the mass dependence of the moment of inertia could be offset. Thus, lifetime measurements of the identical bands are necessary to establish their deformations.

\footnotetext{
*North Carolina State University and Triangle Universities Nuclear Laboratory, †Lawrence Berkeley National Laboratory, $\ddagger$ Purdue University, §Rutgers University, IUUniversity of Massachusetts

$1_{V}$. Nazarewicz, Phys. Rev. Lett. 64,1654 (1995)

2D. Nisius, Phys. Lett. B346, 15 (1995).
} 


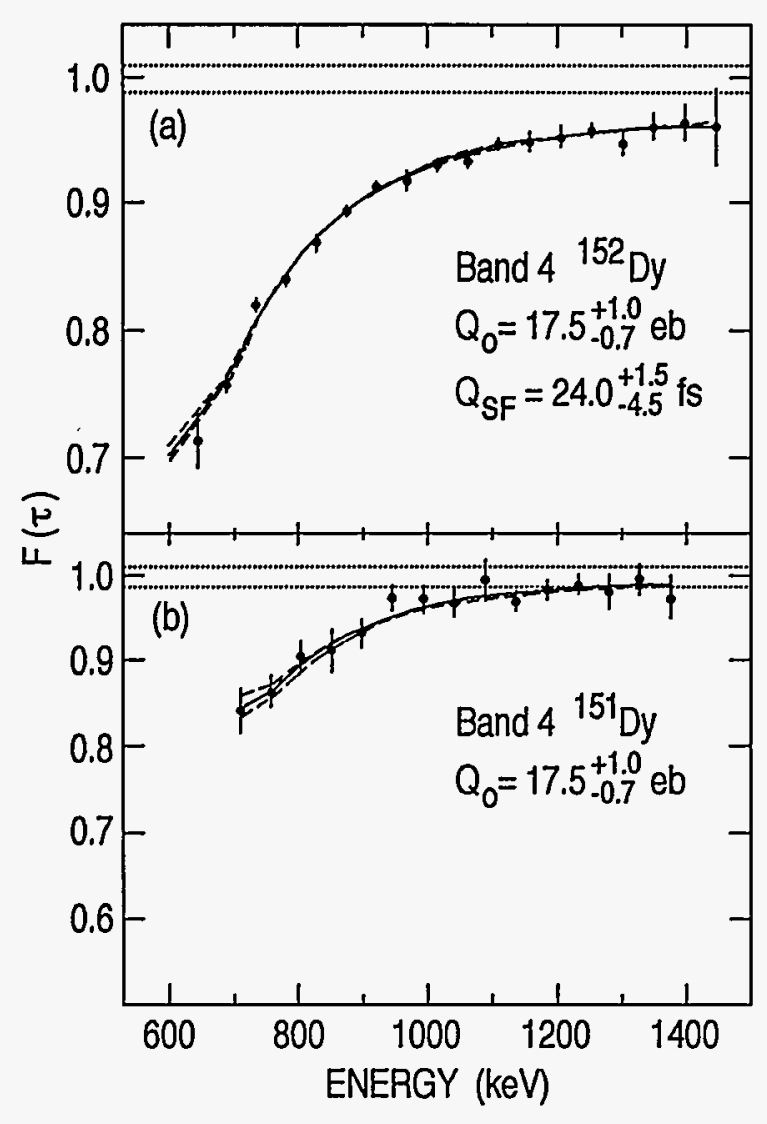

Fig. I-14. Top: Quadrupole moment (Q0) fit to the $F(\tau)$ data for band $I$ in $152 D y$. Note the long feeding delay into the band of $24 \mathrm{fs}$. Bottom: Qo fit for band 4 in 151 Dy (i.e. the identical bend to ${ }^{152} \mathrm{Dy}$ band 1). It has an identical Qo as well.

A Doppler Shift Attenuation Method (DSAM) measurement was performed to extract the lifetimes of superdeformed states in 151,152 Dy nuclei populated in the ${ }^{122} \mathrm{Sn}\left({ }^{34} \mathrm{~S}, 5 \& 4 \mathrm{n}\right)$ reaction at a beam energy of 175
$\mathrm{MeV}$. The Phase I implementation of Gammasphere, which consisted of 56 detectors at the time, was employed for the measurement and a total of 1.5 billion triple and higher fold coincidences were recorded. In addition to the five known SD bands in ${ }^{151} \mathrm{Dy},{ }^{2}$ two more SD bands were tentatively assigned to that nucleus. Since SD bands from $151,152 \mathrm{Dy}$ and ${ }^{151} \mathrm{~Tb}$ are populated in this reaction, a differential lifetime measurement can be made. Only with the increased detection sensitivity of the new large arrays is such a precise measurement possible because the reaction conditions are not optimum for the ${ }^{152} \mathrm{Dy}$ and ${ }^{151} \mathrm{~Tb}$ bands.

The results of the data analysis of this DSAM measurement are in a preliminary stage, but already several interesting features can be discussed. Fractions of full Doppler shift (from centroid measurements of gamma-ray energies from angle-sorted spectra) were extracted. It appears that the identical bands (band 4 in ${ }^{151}$ Dy and the yrast SD band in 152 Dy) have identical quadrupole moments within experimental errors (see Fig. I-14). There are substantial differences $(\sim 20 \%)$ in intrinsic quadrupole moments for the SD bands in $151 \mathrm{Dy}$ indicating a configuration dependence on deformation. A surprising result is that the ${ }^{152} \mathrm{Dy} S \mathrm{SD}$ band has a considerable feeding delay compared to all the other SD bands populated in this reaction. Possible explanations such as changes in the SD production cross section across the target were investigated, but cannot explain such a large delay. We are currently in the process of developing a more realistic model of the sidefeeding structure in an attempt to quantify the differences in feeding times.

\section{a.11. A Superdeformed Band in ${ }^{154}$ Dy (D. Nisius, R. V. F. Janssens,}

I. Ahmad, B. Crowell, M. P. Carpenter, D. Blumenthal, D. Gassmann, T. L. Khoo, T. Lauritsen, W. C. Ma, J. H. Hamilton, $\uparrow$ A. V. Ramayya, $\uparrow$ P. Bhattacharyya, $\ddagger$ L. Ciszewski, $\ddagger$ P. J. Daly $\ddagger$ Z. W. Grabowski $\ddagger$ R. H. Mayer $\ddagger$ and C. T. Zhang $\ddagger)$

The island of superdeformation around the doubly-magic superdeformed (SD) nucleus ${ }^{152} \mathrm{Dy}$ is thought to be well understood in the framework of cranked mean field calculations. In particular, the calculations ${ }^{1}$ suggest that there should be no SD minimum in the ${ }^{154} \mathrm{Dy}$ nucleus in the 40-60 $\hbar$ spin range, where SD bands in this mass region are thought to be populated. In an attempt to probe this limit, the reaction ${ }^{122} \mathrm{Sn}\left({ }^{36} \mathrm{~S}, 4 \mathrm{n}\right)$ at a beam energy of $165 \mathrm{MeV}$ was employed to

*Mississippi State University, $†$ Vanderbilt University, $\ddagger$ Purdue University,

${ }^{1}$ For example, J. Dudek, Phys. Rev. C 31, 298 (1985)

2D. Nisius, Phys. Rev. C 51, R1061 (1995) 
populate high-spin states in the ${ }^{154} \mathrm{Dy}$ nucleus and decay gamma rays were detected with the Gammasphere spectrometer in its early implementation phase. One new SD band was identified, which is iso-spectral to an excited band in ${ }^{153} \mathrm{Dy}$, and was assigned to ${ }^{154} \mathrm{Dy}$ on the basis of coincidence relationships with the gamma rays de-exciting the yrast and near yrast states of that nucleus.

The ${ }^{154}$ Dy SD band has a nearly identical $\mathfrak{I}^{(2)}$ moment of inertia to the yrast SD band in ${ }^{152} \mathrm{Dy}$ (see Fig. I-15), which suggests both bands have the same high-N intruder content (i.e. two neutrons in $\mathrm{j} 15 / 2, \mathrm{~N}=7$ orbitals). In contrast, the yrast SD band in ${ }^{153} \mathrm{Dy}$ is believed to have an additional neutron in a $\mathrm{N}=7$ orbital, which results in its higher $\mathfrak{S}^{(2)}$ moment of inertia. Cranked mean field calculations indicated that a slight decrease in deformation would shift the energy spacings of the Nilsson orbitals such that the third $\mathrm{N}=$ 7 intruder would remain vacant in the yrast SD band of ${ }^{154} \mathrm{Dy}$. Indeed, guided by the $\mathfrak{S}^{(2)}$ information and the results of these calculations a configuration based on the occupancy of two [514]9/2 neutron orbitals coupled to the ${ }^{152}$ Dy SD core was proposed for the ${ }^{154} \mathrm{Dy}$ band. The results were reported. 2 The ${ }^{154}$ Dy SD band was also populated in a DSAM experiment that focussed on

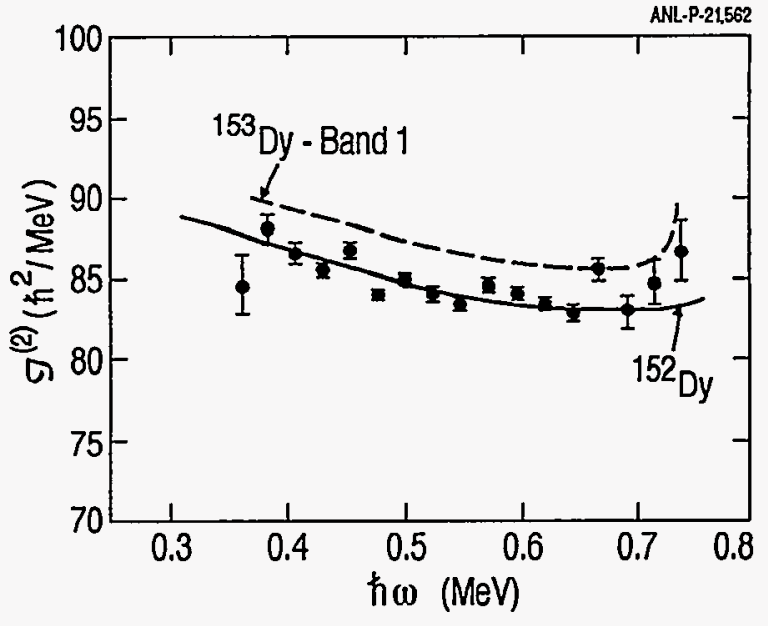

Fig. 1-15. The $\mathfrak{I}^{(2)}$ moment of inertia for the new SD band in ${ }^{154}$ Dy as a function of rotational frequency. The solid curve corresponds to the $\mathfrak{I}^{(2)}$ of the yrast $S D$ band in $152 D y$, while the dashed curve is the $\mathfrak{I}^{(2)}$ of the ${ }^{153}$ Dy yrast $S D$ band.

lifetime measurements of the many normally deformed bands in ${ }^{155} \mathrm{Dy}$. The intrinsic quadrupole moment of the ${ }^{154} \mathrm{Dy}$ band will be extracted from this data set and the deformation determined.

\section{a.12. Superdeformation, New Shape Minima and Band Termination} Spectroscopy in ${ }^{155}$ Dy (S. M. Fischer, R. V. F. Janssens, D. Nisius, I. Ahmad, D. J. Blumenthal, M. P. Carpenter, G. Hackman, T. L. Khoo, T. Lauritsen, M. A. Riley,* T. B. Brown,* D. J. Hartley,* W. C. Ma, † and J. Simpson $\ddagger)$

A search for superdeformed (SD) bands in the nucleus ${ }^{155} \mathrm{Dy}$ was performed via the ${ }^{124} \mathrm{Sn}\left({ }^{32} \mathrm{~S}, 5 \mathrm{n}\right)$ reaction at $175 \mathrm{MeV}$. Five-fold $\gamma$ ray coincidences were detected by $68 \mathrm{HPGe}$ detectors in the Gammasphere array. Recent results also from Gammasphere extended the frontiers of the $A \sim 150 \mathrm{SD}$ region to the $\mathrm{N}=88, \mathrm{Z}=$ $66{ }^{154}$ Dy nucleus. This is of particular interest since cranked mean field calculations show no indication for a pronounced SD minimum which would become yrast in the spin range where known SD bands of this mass region are believed to be populated.

Preliminary results from the analysis of the present data set indicate the presence of two SD bands. The previously observed SD band in ${ }^{154} \mathrm{Dy}$ is confirmed, along with a new band tentatively assigned to ${ }^{155} \mathrm{Dy}$. These SD bands will also be analyzed to extract lifetime information, as the data were obtained from a thick target measurement. A search for rotational structures corresponding to even larger deformations will also be performed in order to test recent calculations by $\mathrm{R}$. Chasman described elsewhere in this report. The normal deformed states in ${ }^{155}$ Dy and 154 Dy are also being analyzed. In particular, 155 Dy offers an opportunity to study the effects of band termination, as the high spin structures in this nucleus correspond to the highest angular momentum states observed to date in a normal deformed odd-A nucleus. The data are currently being analyzed.

*Florida State University, $\uparrow$ Mississippi State University, $\$$ SERC Daresbury Laboratory, United Kingdom 


\section{a.13. Experimental Search for Very Extended Shapes in the A $\sim 180$ Region}

(M. P. Carpenter, R. R. Chasman, R. V. F. Janssens, I. Ahmad, H. Amro,

D. J. Blumenthal, B. Crowell, T. L. Khoo, T. Lauritsen, C. J. Lister, D. Nisius, and P. Chowdhury*)

We performed an experiment at Gammasphere to search for elongated shapes in the nucleus ${ }^{180}$ Os. Recent calculations by $R$. R. Chasman showed that this nucleus is one of most promising for finding structures with major-to-minor axis ratios of 2.2:1 or greater. These calculations include a necking degree of freedom which is thought to be an improvement over past cranked Strutinsky calculations where predictions for extended shapes in $\mathrm{Yb}$ and $\mathrm{Er}$ nuclei were made.

In order to populate ${ }^{180} \mathrm{Os}$ at spins $>60 \hbar$, we utilized the ${ }^{130} \mathrm{Te}\left({ }^{54} \mathrm{Cr}, 4 \mathrm{n}\right)$ reaction at $240 \mathrm{MeV}$. The beam was supplied by the 88-inch cyclotron at LBNL. A single target was used and the ${ }^{130} \mathrm{Te}$ had a thickness of $0.5 \mathrm{mg} / \mathrm{cm}^{2}$. The run lasted four days and $\sim 1 \times 10^{9}$ unfolded triple events were histogrammed into a 3dimensional cube for analysis. At this beam energy, the dominant channel is ${ }^{179}$ Os (5n) and the $180 \mathrm{Os}$ residues are populated at about $25 \%$ of the ${ }^{179}$ Os crosssection. The quality of the data is excellent and we were able to extend the known level schemes in both nuclei. However, no evidence for elongated shapes was found. Analysis of the data is still underway.

*University of Massachusetts at Lowell

\section{a.14. Evidence for Octupole Vibration in the Superdeformed Well of} ${ }_{190} \mathrm{Hg}$ from Eurogam (B. Crowell, M. P. Carpenter, R. V. F. Janssens, D. J. Blumenthal, I. Ahmad, T. Lauritsen, T. L. Khoo, D. Nisius, J. Timar,*

A. N. Wilson,* J. F. Sharpey-Schafer,* T. Nakatsukasa, A. Astier, § F. Azaiez,II

L. du Croux,§ B. J. P. Gall, II F. Hannachi, ** A. Korichi, $\{$ A. Lopez-Martens,**

M. Meyer,§ E. S. Paul,* M. G. Porquet,** and N. Redon§)

Gammasphere experiments in 1993-94 brought to light the existence of an excited superdeformed (SD) band in $190 \mathrm{Hg}$ with the unusual property of decaying entirely to the lowest (yrast) SD band over 3-4 transitions, rather than to the normally deformed states as is usually the case in the $\mathrm{A} \sim 150$ and $\mathrm{A} \sim 190$ regions of superdeformation. Although M1 transitions between signature-partner SD bands were previously observed in ${ }^{193} \mathrm{Hg}$ and other SD nuclei of the region, no such mechanism was available to explain the situation in the even-even nucleus $190 \mathrm{Hg}$, whose yrast SD band has no signature partner. The best explanation appears to lie in long-standing theoretical predictions that the SD minimum in the potential energy surface would be quite soft with respect to octupole vibrations. This would lead to enhanced E1 transitions connecting the one-phonon and zerophonon states. The data and this interpretation were published. A shortcoming of the Gammasphere experiments was that they did not allow the definitive measurement of the energies of the gamma-ray transitions connecting the two bands, due to the very weak population of the excited band $(\sim 0.05 \%$ of the ${ }^{190} \mathrm{Hg}$ channel) and also partly, we believed, to the angular distributions of the transitions, which were peaked near 90 degrees, where Gammasphere had few detectors.

An experiment was therefore performed using the Eurogam Phase II spectrometer, which was more efficient than the partially completed Gammasphere array, and which has detectors at side angles. This experiment confirmed the existence of the excited SD band. Furthermore, the energies of the interband transitions were firmly established, and Directional Correlation ratios (DCO) were extracted, indicating that the lines are of dipole character. This constitutes a measurement of the relative spins and excitation energies of the two SD bands. RPA calculations performed by Nakatsukasa are in excellent agreement with the data, supporting the octupole-vibrational interpretation. A paper describing the Eurogam results and the RPA calculations was published. ${ }^{1}$

\footnotetext{
*University of Liverpool, United Kingdom, $\ddagger$ Chalk River Laboratories, Ontario, Canada, §Institut de Physique Nucléaire, Lyon, France, IIIPN, Orsay, France, IICentre de Recherches Nucleaires, Strasbourg, France, **CSNSM, Orsay, France

${ }^{1}$ B. Crowell et al., Phys. Rev. C 51, R1599 (1995).
} 
Further analysis of the same data has also provided evidence for two additional SD bands in $190 \mathrm{Hg}$. One of these bands also appears to decay towards the yrast SD band in $190_{\mathrm{Hg}}$ and can be understood in the RPA calculations discussed above as resulting from an octupole excitation while the other is understood as a two-quasiparticle excitation. An extended report is being prepared for publication. An experiment to measure the lifetimes of the SD states in all the bands is scheduled to run at Gammasphere in the Spring of 1996.

a.15. Superdeformation Studies in ${ }^{191} \mathrm{Hg}$ (M. P. Carpenter, R. V. F. Janssens, B. Crowell, I. Ahmad, D. Gassmann, R. G. Henry, T. L. Khoo, T. Lauritsen, D. Nisius, B. Cederwall,* J. A. Becker, $\ddagger$ M. J. Brinkman $\ddagger$ M. A. Deleplanque,* R. M. Diamond,* P. Fallon,* L. P. Farris, $\ddagger$ U. Garg, $\S$ E. A. Henry, $\ddagger$ J. R. Hughes, I. Y. Lee,* A. O. Machiavelli,* E. F. Moore, II and F. S. Stephens*)

From an initial measurement at Gammasphere on ${ }^{191} \mathrm{Hg}$, the three SD bands identified previously were extended, and their feeding into the yrast states was delineated. In addition, a new SD band was observed whose properties indicate that it is built on the unfavored signature of the $\mathrm{j} 15 / 2$ intruder configuration. A new measurement to study superdeformed (SD) structures in ${ }^{191} \mathrm{Hg}(\mathrm{N}=111)$ has now been carried out at Gammasphere with the ${ }^{160} \mathrm{Gd}\left({ }^{36} \mathrm{~S}, 5 \mathrm{n}\right)$ reaction at $172 \mathrm{MeV}$. The goal of the experiment was to identify new SD bands in the data in order to obtain valuable information on the relative placement of single-particle orbitals near the $\mathrm{N}=112 \mathrm{SD}$ shell gap.

This recent data set was combined with data from the previous Gammasphere experiment on ${ }^{191} \mathrm{Hg}$, and an analysis of the data allowed for the identification of three new SD bands in ${ }^{191} \mathrm{Hg}$, bringing the total number of bands to seven. Two of the new bands behave as signature partners, with one of the bands having transition energies nearly identical to the yrast $\mathrm{SD}$ band of ${ }^{192} \mathrm{Hg}$. These two new bands, coupled with the four previously identified bands, show striking similarities to the six SD bands identified in ${ }^{193} \mathrm{~Pb}(\mathrm{~N}$ =111). A comparison with cranked shell-model calculations allowed us to propose quasiparticle assignments for these bands in ${ }^{191} \mathrm{Hg}$. The final SD band (band 7) identified in the data has a moment of inertia which is not similar to any other band known in the $A=190 \mathrm{SD}$ region, and the underlying structure is uncertain at the present time.

*Lawrence Berkeley National Laboratory, $\ddagger$ Lawrence Livermore National Laboratory, §University of Notre Dame, INorth Carolina State University and Triangle Universities Nuclear Laboratory

\section{a.16. Verification of the $\Delta \mathrm{I}=4$ Oscillations in ${ }^{194} \mathrm{Hg}$ Superdeformed Bands}

(G. Hackman, R. V. F. Janssens, I. Ahmad, D. J. Blumenthal, M. P. Carpenter, S. M. Fischer, T. L. Khoo, T. Lauritsen, D. T. Nisius, R. Kruecken,* S. Astaloz,* R. M. Clark,* M. A. Deleplanque,* R. M. Diamond,* P. Fallon,* I-Y. Lee,* A. O. Machiavelli, ${ }^{*}$ F. S. Stephens, ${ }^{*}$ and B. Herskind $\dagger$ )

A Gammasphere experiment to study the nucleus ${ }^{194} \mathrm{Hg}$ was undertaken with two main goals: (1) measure, to the highest precision possible, the energies of $\gamma$ rays in the known SD bands to establish the $\Delta \mathrm{I}=$ 4 staggering phenomenon; (2) search for new superdeformed bands. The reaction ${ }^{150} \mathrm{Nd}\left({ }^{48} \mathrm{Ca}, \mathrm{mn}\right){ }^{198-x} \mathrm{Hg}$ at a beam energy of $201 \mathrm{MeV}$ was selected for optimal population of the ${ }^{194} \mathrm{Hg} \mathrm{SD}$ bands via the $4 \mathrm{n}$ channel.
The $\Delta \mathrm{I}=4$ staggering phenomenon has solicited a flurry of theoretical interest, mostly in terms of the breaking of a yet-unknown symmetry in the nuclear Hamiltonian. It was also proposed that it is a consequence of less exotic phenomena such as band interactions. The experimental data to date have largely been inadequate for detailed, unambiguous comparison to the predictions of any model.

*Lawrence Berkeley National Laboratory, $\uparrow$ Niels Bohr Institute, Copenhagen, Denmark 
From the data taken during this experiment, triple- and quadruple-coincidence data were rigorously investigated. Gated spectra were extracted, and in these spectra, the centroids of the peaks in the ${ }^{194} \mathrm{Hg}$ strongest SD band were fit with uncertainties as low as $\sim 5 \%$ of an ADC channel, corresponding to $\sim 6 \mathrm{eV}$ at a nominal gain of $0.125 \mathrm{keV} / \mathrm{channel}$. This is a purely statistical uncertainty and may not completely account for the effects of interfering (non-SD) lines. The current status is that the staggering effects are confirmed for bands 2 and 3 and are consistent with earlier published results. For band 1 the staggering is found not to be significant within the statistical accuracy. Ongoing work includes formulation of a method for extracting the very fine deviations in $\gamma$-ray energy from a smooth rotational behavior which will not introduce artificial oscillations.

\section{a.17. Differential Quadrupole Moments of Identical Superdeformed Bands in} ${ }^{192,194}$ Hg (T. L. Khoo, M. P. Carpenter, T. Lauritsen, D. Ackermann, I. Ahmad, D. J. Blumenthal, S. M. Fischer, R. V. F. Janssens, D. Nisius, E. F. Moore,* A. Lopez-Martens, ‡ S. J. Asztalos,§ J. A. Becker,IL L. Bernstein,\{ R. M. Clark,§ M. A. Deleplanque, $\S$ R. M. Diamond,§ P. Fallon,§ L. P. Farris, IL F. Hannachi, ; E. A. Henry, II A. Korichi,ll I. Y. Lee,§ A. O. Macchiavelli,§ and F. S. Stephens§)

One of the two most critical questions for understanding the origin of identical bands is whether identical bands have the same deformation. An answer to this question will help reveal whether the cause is due to some unidentified symmetry or to accidental cancellations. We are in the process of analyzing data from a Gammasphere experiment, with the goal of measuring the differential quadrupole moments of SD bands in $192,194 \mathrm{Hg}$, which have identical transition energies. Differential quadrupole moments can, in principle, be measured with sufficient accuracy to provide an answer, even though sufficiently precise absolute measurements are not yet feasible. Preliminary indications are that the identical bands have the same quadrupole moments.

We shall also perform Gammasphere experiments to answer the other critical question regarding identical bands, namely do transitions of identical energies emanate from states of the same spin? With this twopronged approach, we shall provide data which should lead to a significant advance in understanding the origin of identical bands.

*North Carolina State University and Triangle Universities Nuclear Laboratory, $\ddagger$ Centre de Spectrometrie Nucleaire et de Spectrometrie de Masse, Orsay, France, §Lawrence Berkeley National Laboratory, ILawrence Livermore National Laboratory, IIInstitut de Physique Nucleaire, Orsay, France

\section{a.18. Alignment Additivity in the Two-Quasiparticle Superdeformed Bands of ${ }_{192}$ Tl (S. M. Fischer, M. P. Carpenter, R. V. F. Janssens, B. Crowell, I. Ahmad, D. J. Blumenthal, T. L. Khoo, T. Lauritsen, D. Nisius, W. Reviol,* W. F. Mueller,* L. L. Riedinger, ${ }^{*}$ B. H. Smith, ${ }^{*}$ and B. Cederwall $\dagger$ )}

The superdeformed (SD) structure of the odd-odd nucleus ${ }^{192} \mathrm{Tl}$ was studied via the ${ }^{160} \mathrm{Gd}\left({ }^{37} \mathrm{Cl}, 5 \mathrm{n}\right)$ reaction at $178 \mathrm{MeV}$. Recently, several detailed studies of odd-A nuclei in the mass 190 region were quite successful in presenting a coherent picture of the various quasiparticle excitations occurring in the SD well. By studying SD states in odd-odd nuclei, we are able to test the extent to which ideas such as the additivity of quasiparticle alignments in the cranking model remain valid at these very large deformations. Specifically, it is possible to test whether the alignments observed in a twoquasiparticle SD band can be accounted for by the addition of the alignments measured in the onequasiparticle bands of the neighboring odd-A nuclei.

The experiment was performed using the early implementation phase of Gammasphere in which threefold $\gamma$-ray coincidences were detected by $36 \mathrm{HPGe}$ detectors. Four SD bands were confirmed to belong to ${ }_{192} \mathrm{Tl}$ (see Figs I-16 and I-17). Two of these bands exhibit nearly constant dynamic moments of inertia, $\mathfrak{S}^{(2)}$, as a function of frequency. This supports the proposal that these "flat" bands correspond to quasiparticle configurations involving the occupation

*University of Tennessee, †Lawrence Berkeley National Laboratory 
Fig. I-16. (a): Double gated coincidence spectra for $S D$ bands $A$ and $B$. Yrast transitions in 192 Tl are labeled by the spins of the initial and final levels. The transition labeled " $C$ " is a known contaminant. (b): Spectra obtained from sums of all double coincidence gates in band $A$ for $E_{\gamma} \geq 437 \mathrm{keV}$ or in band $B$ for $E_{\gamma}$ $\geq 451 \mathrm{keV}$ showing the weakcross-talk between the bands. The lines at 344.9 and $387.3 \mathrm{keV}$ are yrast transitions which are coincident with $\gamma$ rays of energies nearly identical to those of members of band $A$.

of the $\pi i_{13 / 2}$ and $v j_{15 / 2}$ intruder orbitals which block both proton and neutron alignments. The other observed SD bands display increasing $\mathfrak{S}^{(2)_{\text {s }} \text { as a }}$ function of frequency, typical of other SD bands in this region. From comparisons with the odd-A neighbors, it is found that the alignments of these bands relative to a ${ }^{192} \mathrm{Hg}$ core can be accounted for from the additive contributions of the assigned quasiproton and quasineutron orbitals. This method will likely be applied in the interpretation of the SD structure of other odd-odd nuclei.

The results of this work were submitted for publication.

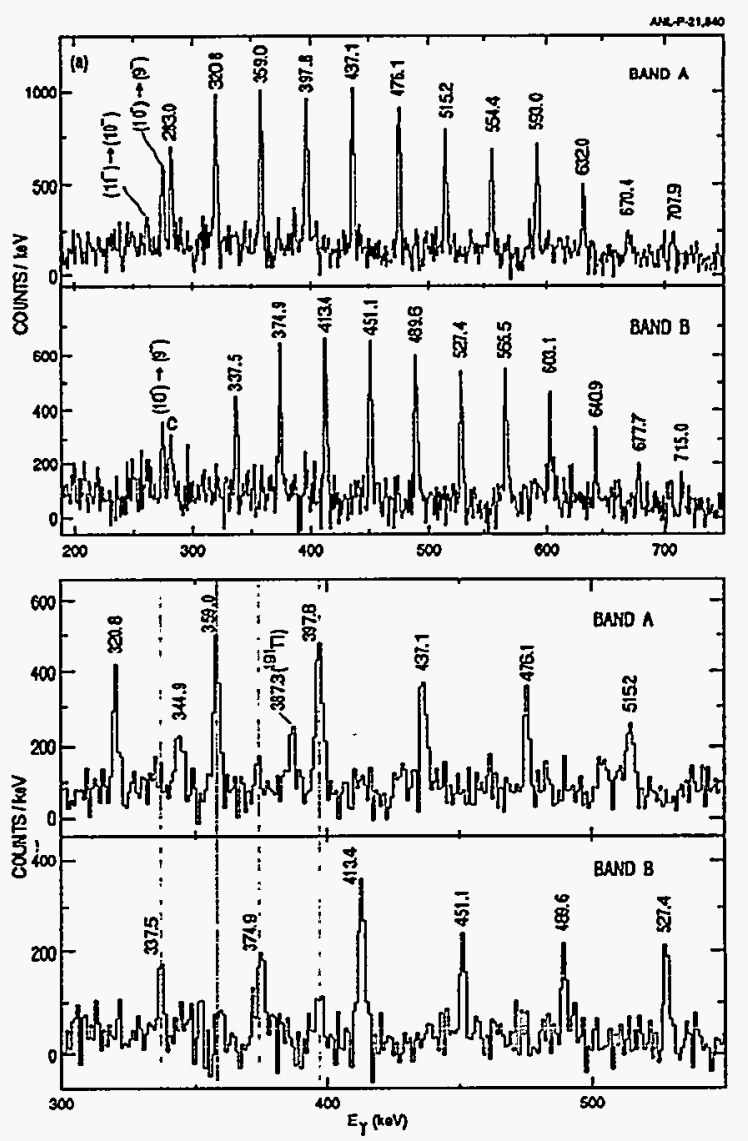

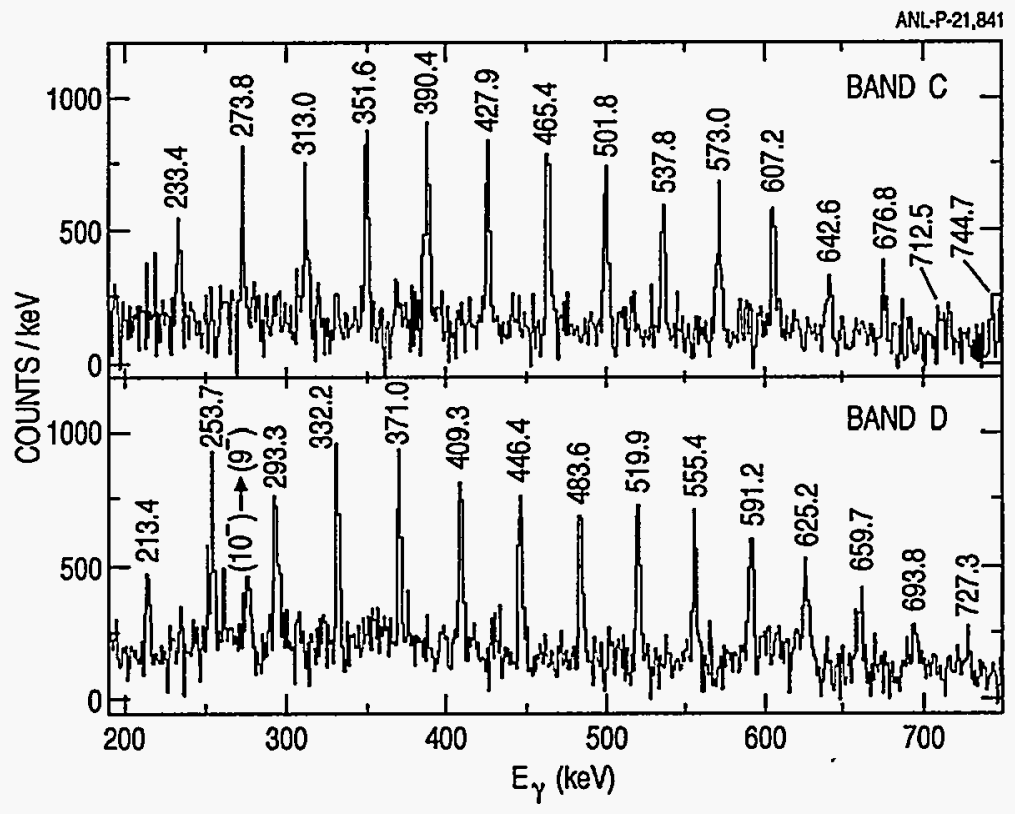

Fig. I-17. Double gated coincidence spectra for SD bands C and D in ${ }^{192} \mathrm{Tl}$. An yrast transition in ${ }^{192} \mathrm{Tl}$ is labeled by the spins of the initial and final levels. 
a.19. Gammasphere Study of Superdeformation in ${ }^{195} \mathrm{Hg}$ (G. Hackman, R. V. F. Janssens, I. Ahmad, D. J. Blumenthal, M. P. Carpenter, S. M. Fischer, T. L. Khoo, T. Lauritsen, D. T. Nisius, R. Kruecken, ${ }^{*}$ S. Astaloz, ${ }^{*}$ R. M. Clark, ${ }^{*}$ M. A. Deleplanque,* R. M. Diamond,* P. Fallon, ${ }^{*}$ I-Y. Lee, * A. O. Machiavelli, * F. S. Stephens, ${ }^{*}$ and B. Herskind $\dagger$ )

A Gammasphere experiment was undertaken with two main goals: (1) measure the energies of $\gamma$ rays in the known ${ }^{194} \mathrm{Hg}$ SD bands to high precision; (2) search for new excited superdeformed bands, including a predicted $\mathrm{K}=7$ two-quasineutron band in ${ }^{194} \mathrm{Hg}$. The reaction ${ }^{150} \mathrm{Nd}\left({ }^{48} \mathrm{Ca}, x \mathrm{n}\right){ }^{198-x} \mathrm{Hg}$ at a beam energy of $201 \mathrm{MeV}$ was selected for optimal population of the ${ }^{194} \mathrm{Hg}$ SD bands via the $4 \mathrm{n}$ channel.

A well-established search algorithm produced positive results for the three known bands in ${ }^{194} \mathrm{Hg}$ and the five known bands in ${ }^{193} \mathrm{Hg}$, which are populated via the $5 \mathrm{n}$ channel. The search algorithm also uncovered three candidates for new superdeformed bands, but the coincidence data suggest that they are not in ${ }^{194} \mathrm{Hg}$, but rather in $195 \mathrm{Hg}$. The three new bands have a slightly higher dynamic moment of inertia $\mathfrak{S}(2)$ than the bands of ${ }^{194} \mathrm{Hg}$, and their $\gamma$-ray energies are not trivially related to the ${ }^{194} \mathrm{Hg}$ SD bands. This would suggest either that (a) the ${ }^{195} \mathrm{Hg}$ SD bands are better represented as quasiparticle states in a yet unknown ${ }^{196} \mathrm{Hg}$ core, rather than the ${ }^{194} \mathrm{Hg}$ core, or (b) the special symmetries which lead to identical bands are broken with increasing neutron number. The current effort is directed towards understanding the microscopic structure of these new bands.

This result provides a strong incentive to look for SD bands in $196 \mathrm{Hg}$, although a standard neutronevaporation reaction will not be appropriate. Evaluation of charged-particle reactions for such an experiment is ongoing, and if a suitable reaction is found, the experiment will be proposed to the next Gammasphere Program Advisory Committee.

*Lawrence Berkeley National Laboratory, †Niels Bohr Institute, Copenhagen, Denmark,

\section{B.b. Other Nuclear Structure Studies}

Study of the evolution of the nuclear shape as a function of proton and neutron number as well as spin and excitation energy was an important component of the research program at Argonne for many years. At this particular time, a substantial fraction of the data for these studies comes from experiments performed mainly to investigate properties associated with superdeformation. The data are of such quality that their use for other purposes like the one described above is obvious. Over the last year, the studies have concentrated on (1) the competition between collective and single-particle degrees of freedom along and above the yrast line in ${ }^{154} \mathrm{Dy}$, (2) the degree in which $\mathrm{K}$ is a good quantum number at moderate and high spins in rare-earth nuclei, (3) the yrast and near-yrast spectroscopy of $\mathrm{Hg}$ and $\mathrm{Pb}$ nuclei where superdeformation occurs, and (4) the structure of neutron-rich nuclei from the prompt radiation of fission fragments. Other aspects of the research program include (1) the search for 2-octupole phonon excitations in ${ }^{208} \mathrm{~Pb},(2)$ the search for collectivity in nuclei just above the $N=50$ shell gap and (3) the spectroscopy of neutron-rich nuclei around ${ }^{132} \mathrm{Sn}$. Many aspects of the program involve major efforts by collaborators from outside institutions. 


\section{b.1. "Anatomy" of a Phase Transition Along the Yrast Line at ${ }^{154} \mathrm{Dy}$}

(T. L. Khoo, R. V. F. Janssens, T. Lauritsen, D. Nisius, I. Ahmad, D. Blumenthal, M. P. Carpenter, B. Crowell, D. Gassmann, W. C. Ma, * P. G. Varmette, ${ }^{*}$

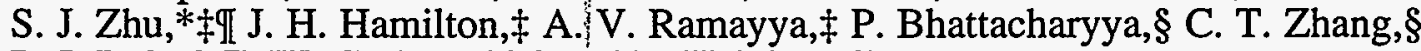
P. J. Daly,§ Z. W. Grabowski,§ and R. H. Mayer§)

The transitional nucleus ${ }^{154} \mathrm{Dy}$, with 88 neutrons, straddles the well-deformed nuclei (which rotate collectively) and oblate nuclei (with spin generated by particle alignment). The yrast structure of ${ }^{154} \mathrm{Dy}$ changes rapidly with spin, with an abrupt phase transition from collective to aligned-particle character along the yrast line. Thus, the fluctuations inherent in the mesoscopic nucleus do not wash out the signatures of the phase transition, which is predicted by mean-field theory.

We used data from an early-implementation phase at Gammasphere to probe the "anatomy" of this phase transition. The ground-state band and the positiveparity s-band in ${ }^{154} \mathrm{Dy}$ were both extended from spin $32^{+}$to $44^{+}$. A negative parity rotational band was found to extend from $27^{-}$to $45^{-}$. These three bands are rather weak, with intensities of one to two percent of the groundstate transition at spins $32^{+}$and $33^{-}$. They are not seen in a separate experiment at Gammasphere using a thick target (with a gold backing), based on a preliminary analysis. That means that they have shorter lifetimes than the terminating states, indicating a collective rotational behavior with, presumably, prolate

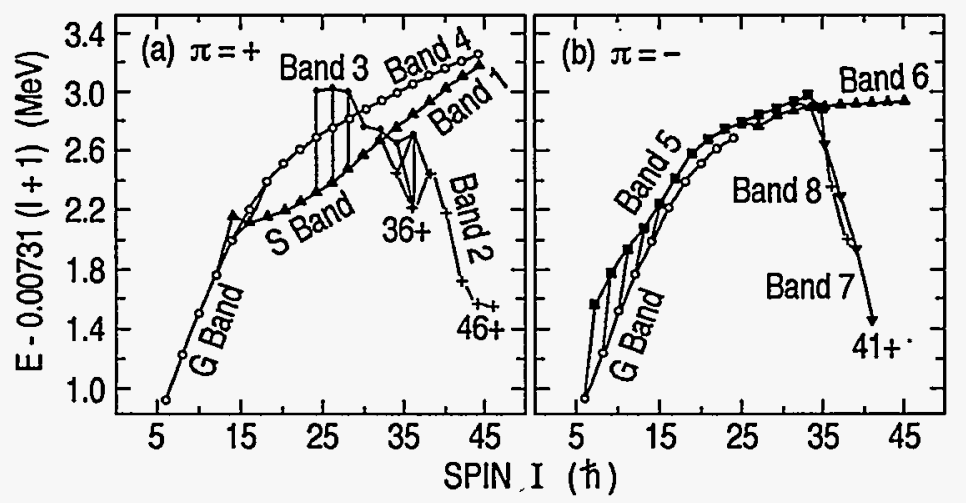

Fig. 1-18. Level energy (after subtraction of a rotational term) vs spin in ${ }^{154} \mathrm{Dy}$.

deformation. The discovery and extension of these three bands illustrates beautifully the coexisistence of terminating states with the prolate collective rotational bands, with the latter lying at higher escitation energies. This coexistence is illustrated in Fig. I-18.

An interesting feature is that the two positive-parity collective rotational bands have similar energies at the highest spins and approach each other. The establishment of a near-yrast decay sequence, together with several other near-yrast states, illustrates how aligned-particle states dip towards yrast line, and finally cross it to become yrast. We shall collaborate with theorists from Lund to understand the microscopic configurations of the near-yrast bands. Emphasis will be given on identifying the cause for a re-emergence of collective states at the highest spins.

*Mississippi State University, $¥$ Vanderbilt University, §Purdue University, ITsinghua University, Beijing, China 1 


\section{b.2. Probing the Phase Transition Boundary of Hot States in ${ }^{154} \mathrm{Dy}$} (T. L. Khoo, R. V. F. Janssens, T. Lauritsen, D. Nisius, I. Ahmad, D. Blumenthal, M. P. Carpenter, B. Crowell, D. Gassmann, W. C. Ma, * P. G. Varmette,*

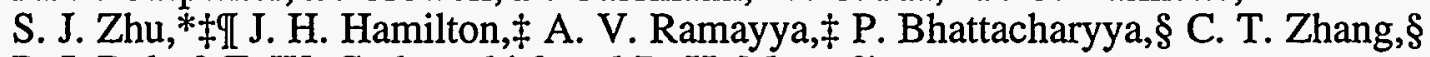
P. J. Daly,§ Z. W. Grabowski,§ and R. H. Mayer§)

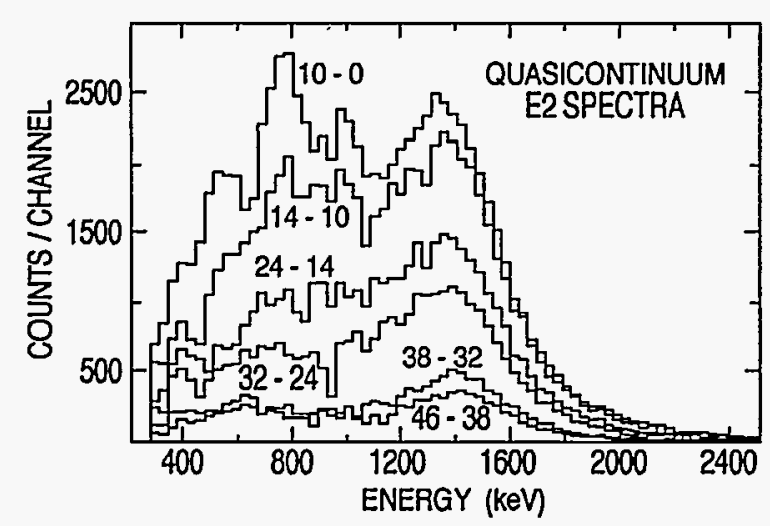

Fig. I-19. Quasicontinuum E2 spectra gated on different spin regions.

Mean-field theories predict phase transitions in nuclei, such as a transition from collective to oblate shapes. However, fluctuations in the finite nucleus smear out the transition, and it is an interesting problem in mesoscopic physics to search for a remnant signature of the phase transition. Temperature-dependent HartreeFock theory predicts that the collective-to-oblate phase transition boundaries occur in a domain that can be favorably probed in experiments in $152,154,156 \mathrm{Dy}$.
We measured and calculated the quasicontinuous spectra feeding yrast states of specified spin intervals in ${ }^{154}$ Dy. By selecting definite spin intervals of the yrast line, it is possible to select for the first time definite regions of the energy-spin plane. Data were obtained from an experiment with Gammasphere, when 36 detectors were available. The quasicontinuum $\gamma$ spectrum feeding all states in ${ }^{154}$ Dy exhibits a unique two-bump feature for the E2 transitions. However, as the feeding spin into the yrast line increases, the lower energy bump decreases in intensity (see Fig. I-19).

We calculated the theoretical $\gamma$ spectra corresponding to the experimental gates. The overall features and trends (with spin) of the calculated spectra are in qualitative agreement with the experimental ones. The calculations are based on the results of temperature-dependent Hartree-Fock theory. The experimental and theoretical spectra together provide strong confirmation of our previous interpretation that the two bumps arise from $\gamma$ transitions which lie on either side of the phase boundary. Hence, the signature for a phase transition in the nuclear mesoscopic system persists.

Future work will examine the E $\gamma$-E $\gamma$ correlation spectra to obtain more detailed information on the character of the highly-excited states above the yrast line, and to provide further tests of the validity of temperaturedependent Hartree-Fock theory.

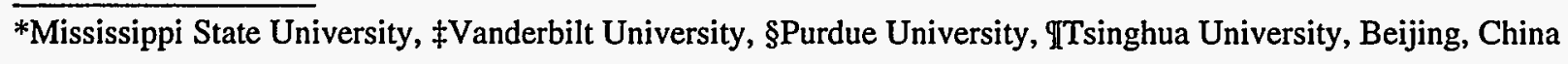




\section{b.3. Gamma Spectrum Following Neutron Capture in ${ }^{167} \mathrm{Er}$}

(T. L. Khoo, D. Visser, F. Soramel,I C. J. Lister, D. Blumenthal, R. G. Henry, T. Lauritsen, I. Ahmad, M. P. Carpenter, B. Crowell, R. V. F. Janssens, D. Nisius, J. L. Durell,* W. R. Phillips, * J. A. Shannon,* B. J. Varley,* R. Casten, $\ddagger$ R. Gill, and T. D $\phi s s i n g l l)$

Statistical decay from a highly excited state samples all the lower-lying states and, hence, provides a sensitive measure of the level density. Pairing has a major impact on the level density, e.g. it creates a pair gap between the 0 - and 2-quasiparticle configurations. Hence, the shape of the statistical spectrum contains information on pairing, and can be used to provide information on the reduction of pairing with thermal excitation energy. For this reason, we measured the complete spectrum of $\gamma$ rays following thermal neutron capture in ${ }^{167} \mathrm{Er}$. The experiment was performed at the Brookhaven reactor using Comptonsuppressed $\mathrm{Ge}$ detectors from TESSA. The spectrum, which was corrected for detector response and efficiency, reveals primary (first-step, high-energy) transitions up to nearly $8 \mathrm{MeV}$, secondary (last-step, lower-energy) transitions, as well as a continuous statistical component. (see Fig. I-20). A major effort was expanded to identify all lines from contaminant sources and an upper limit of 5\% was set for their contributions. The spectral shape of the statistical spectrum is compared with the theoretical spectrum obtained from a calculation of pairing which accounts for a stepwise reduction of the pair correlations as the number of quasiparticles increases (see B.a.7.). The calculated spectrum reproduces the general features and

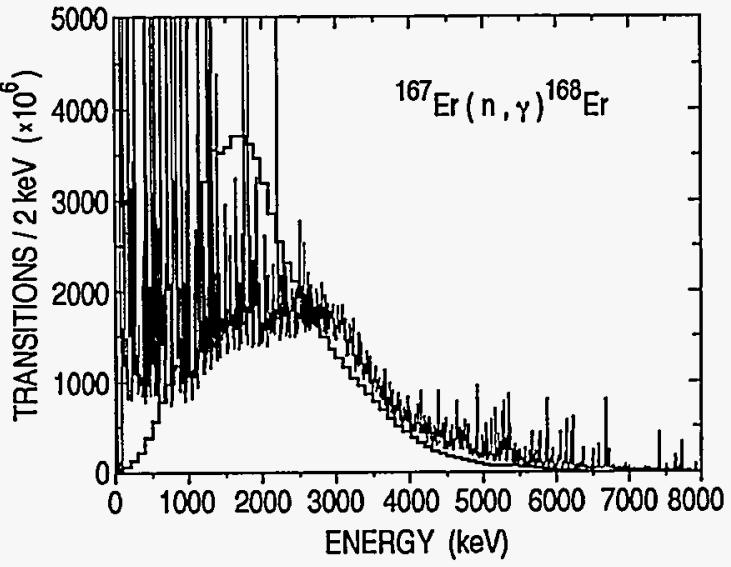

Fig. I-20. Gamma spectrum following thermal

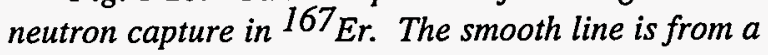
calculation.

the multiplicity of the measured spectrum. However, there are discrepancies in detail, which can be traced to the fact that vibrational and rotational states are not specifically included in the calculation. Fluctuation properties of the spectra will be examined to deduce information on the level density of excited states.

*University of Manchester, United Kingdom, $¥$ Brookhaven National Laboratory, IUUniversity of Udine, Italy, INiels Bohr Institute, Copenhagen, Denmark

\section{b.4. High-K Isomers in ${ }^{176} \mathrm{~W}$ and Mechanisms of $\mathrm{K}$-Violation}

(B. Crowell, R. V. F. Janssens, D. J. Blumenthal, C. J. Lister, T. L. Khoo, I. Ahmad, M. P. Carpenter, D. Gassmann, R. G. Henry, T. Lauritsen, Y. Liang, D. Nisius, F. Soramel, I. G. Bearden, P. Chowdhury, ${ }^{*}$ and S. J. Freeman†)

$\mathrm{K}$-isomers are states in deformed nuclei whose $\gamma$ decay is hindered by selection rules involving $\mathrm{K}$, the projection of the angular momentum along the axis of symmetry of the nucleus. Previous work with the Argonne-Notre Dame BGO gamma-ray facility delineated the existence of two K-isomers in ${ }^{176} \mathrm{~W}$, one of which had a very unusual pattern of decay. A short description of this work was published as a letter, ${ }^{1}$ and a more complete account has recently been accepted for publication. These results provided evidence that quantum-mechanical fluctuations in the nuclear shape may be responsible for some of the observed $\mathrm{K}$-violating transitions.

*University of Massachusetts, $\uparrow$ Schuster Laboratory, Manchester, United Kingdom

${ }^{1}$ B. Crowell et al., Phys. Rev. Lett. 72 , 1164 (1994). 
The existence of a new isomer of higher spin was confirmed in an experiment performed at ATLAS and a tentative level scheme associated with the decay was constructed. The feeding of the isomer is only about $0.02 \%$ of the $176 \mathrm{~W}$ reaction channel and final conclusions regarding the properties of the isomer could not be drawn. As a result, another experiment was subsequently performed at Gammasphere. The analysis of the latter is in progress. Preliminary results indicate that the decay of the new isomer is now delineated completely. Furthermore, spectroscopic information was obtained on at least 16 rotational bands in the ${ }^{176} \mathrm{~W}$ nucleus. The analysis continues.

\section{b.5. Yrast and Near-Yrast Structure of ${ }^{191} \mathrm{Hg}$ (M. P. Carpenter, T. Ishii,*}

R. V. F. Janssens, B. Crowell, I. Ahmad, D. Gassmann, R. G. Henry, T. L. Khoo, T. Lauritsen, D. Nisius, B. Cederwall, $\ddagger$ J. A. Becker,§ R. Clark,

M. A. Deleplanque, $\ddagger$ R. M. Diamond $\ddagger$ P. Fallon, $\ddagger$ L. P. Farris,$\S U$. Garg,,$I$

E. A. Henry,§ I. Y. Lee, A. O. Machiavelli,ł E. F. Moore,ll and F. S. Stephens $\ddagger)$

Recently, we have performed several measurements at Gammasphere on the ${ }^{191} \mathrm{Hg}$ nucleus. ${ }^{1}$ The main purpose of these investigations is to identify and study states in the superdeformed well. High-spin states in ${ }^{191} \mathrm{Hg}$ were populated with the $160 \mathrm{Gd}\left({ }^{36} \mathrm{~S}, 5 \mathrm{n}\right)$ and ${ }^{174} \mathrm{Yb}\left({ }^{22} \mathrm{Ne}, 5 \mathrm{n}\right)$ reactions at 172 and $120 \mathrm{MeV}$, respectively. As a result of these studies, four new superdeformed bands were identified in ${ }^{191} \mathrm{Hg}$, bringing the total number of SD bands in this nucleus to seven.

In addition to studying superdeformed structures, we have also examined the yrast and near-yrast spectroscopy of the normal states. From our previous work at ATLAS, ${ }^{2}$ the level structure of ${ }^{191} \mathrm{Hg}$ was extended and both collective and non-collective excitations observed. Indeed most of the high-spin negative parity levels were associated with a non-collective prolate shape. In the current work, the level structure of ${ }^{191} \mathrm{Hg}$ was examined using triple-coincidence data and the program LEVIT8R. While the analysis allowed for the extension of the known bands by only one or two transitions, a number of new rotational bands were identified. From our previous study, the negative parity states seemed dominated at high-spin by non-collective degrees of freedom, however, the present work shows that the negative-parity collective structures compete with the non-collective states up to the highest observed spins. The analysis of the data continues.

*JAERI, Ibaraki, Japan, łLawrence Berkeley National Laboratory, §Lawrence Livermore National Laboratory, TUniversity of Notre Dame, INNorth Carolina State University and Triangle Universities Nuclear Laboratory

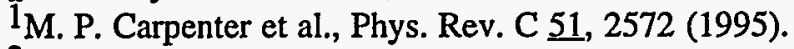

2D. Ye et al., Nucl. Phys. A537, 207 (1992).

b.6. g-Factor Measurement of a High-Spin $\mathrm{M1}$ Band in ${ }^{197} \mathrm{~Pb}$ (M. P. Carpenter, I. Ahmad, D. Blumenthal, B. Crowell, R. V. F. Janssens, D. Nisius, J. Cizewski,* N. Benczer-Köller,* G. Baldsiefen,* M. Satteson,* R. Mayer,* W. Younes,* N. Matt,* and D. McNabb*)

Recent measurements of lifetimes of M1 oblate bands in $196-198 \mathrm{~Pb}$ indicate enhancements in the $\mathrm{M1}$ transition rates. These enhanced $M 1$ transitions are consistent with configurations in which two protons are excited into high-j, high- $\Omega$ orbitals and are coupled to low $\Omega$ neutron orbitals. But the lifetimes are not sensitive enough to establish the microscopic structure of these states. The single-particle orbitals involved in these states can be probed by the measurement of the $g$ factors. Measurements of g-factors of short-lived states require large magnetic fields to precess the angular distribution of gamma rays by an observable amount. Previous experiments have demonstrated that g-factors of such short-lived states can be measured by the transient field technique. In this technique, the reaction product recoils into a ferromagnet with high recoil velocity. We tested the feasibility of measuring the $\mathrm{g}$ factors in ${ }^{197} \mathrm{~Pb}$ by using the ${ }^{124} \mathrm{Sn}\left({ }^{76} \mathrm{Ge}, 3 \mathrm{n}\right){ }^{197} \mathrm{~Pb}$ reaction. Using a high multiplicity cut, we obtained a quite clean spectrum of ${ }^{197} \mathrm{~Pb}$ gamma rays. The moderate yield of the gamma rays connecting members of the M1 band and the good peak-to background

*Rutgers University 
ratio indicate that the g-factors in ${ }^{197} \mathrm{~Pb}$ can be measured by the transient field technique. An experiment is planned where the ${ }^{197} \mathrm{~Pb}$ recoils formed by the bombardment of a ${ }^{124} \mathrm{Sn}$ target by a $305-\mathrm{MeV}$ ${ }^{76} \mathrm{Ge}$ beam will recoil in a Gd foil. Gamma- and x-ray spectra will be measured by the Argonne-Notre Dame BGO gamma-ray facility.

\section{b.7. Gamma Rays from Fission Fragments (I. Ahmad and W. R. Phillips*)}

A review article on "Gamma Rays from Fission Fragments" was published. 1 The article summarizes the current experimental knowledge about the nuclear structure of very neutron-rich fragments produced in low-energy fission. Fission is one of the best ways to produce neutron-rich nuclei. The availability of large Ge detector arrays provided high sensitivity to study the structure of fission products. Since fission is a binary process, levels in a pair of nuclei with very low yield can be studied by three- and higher-fold coincidence measurements. Many aspects of nuclear shapes and structures were studied by this technique. Level schemes of more than a dozen nuclei about which nothing was known previously were determined. The article concludes that studies of fission gamma rays with large Ge arrays and particle detectors will in the future provide more details about shapes and structures of neutron-rich nuclei and they may also provide information on the fission mechanism itself.

*University of Manchester, United Kingdom

${ }^{1}$ I. Ahmad and W. R. Phillips, Rep. Prog. Phys. 58, 1415 (1995).

\section{b.8. Harmonic Two-phonon Gamma Vibrational State in Neutron-Rich ${ }^{106} \mathrm{Mo}$ (I. Ahmad, L. R. Morss, * A. Guessous, $\uparrow$ N. Schulz, $\uparrow$ M. Bentaleb,$\uparrow$ E. Lubkiewicz, $\uparrow$ W. R. Phillips $\ddagger$ J. L. Durell $\ddagger$ M. A. Jones,$\ddagger$ M. Leddy $\ddagger$ A. G. Smith $\ddagger$ W. Urban,$\ddagger$ B. J. Varley, $\ddagger$ and R. Piepenbring§)}

Vibrational states are known in many nuclei, but so far experimental evidence for a two-phonon vibration (which is the characteristic signature of a harmonic vibrator) was reported in a few nuclei only. If vibrations in nuclei are harmonic the energy of the twophonon vibration should be exactly twice the energy of the one-phonon state. Such a two-phonon vibrational state was observed in $106 \mathrm{Mo}$. The $\mathrm{K} \pi=4^{+}$twophonon $\gamma$ vibrational state in $106 \mathrm{Mo}$ was observed at $1435 \mathrm{keV}$ which is very close to twice the energy of the one-phonon $\gamma$ vibrational state at $710 \mathrm{keV}$. The twophonon band was identified in triple coincidence gamma-ray spectra of $248 \mathrm{Cm}$ fission fragments measured with the Eurogam2 array. The Eurogam2 array for this experiment was located at Strasbourg and consisted of $124 \mathrm{Ge}$ detectors. For the detection of lowenergy gamma rays (below $300 \mathrm{keV}$ ) with high resolution, four low-energy planar spectrometers (LEPS) were also added to the array. Several rotational bands were identified in ${ }^{106} \mathrm{Mo}$. The spin-parity assignments to the members of the two-phonon band are based on the level energies, moment of inertia and the $\mathrm{E} 2$ branching ratios. The level scheme of $106_{\mathrm{Mo}}$, shown in Fig. I-21, provides one of the best examples of harmonic two-phonon excitations. The results of this investigation were published. ${ }^{1}$

${ }^{*}$ Chemistry Division, ANL, †CRN, Strasbourg, France, $\ddagger$ University of Manchester, United Kingdom, §ISN, Grenoble, France

${ }^{1}$ A. Guessous et al., Phys. Rev. Lett. 75, 2280 (1995) 


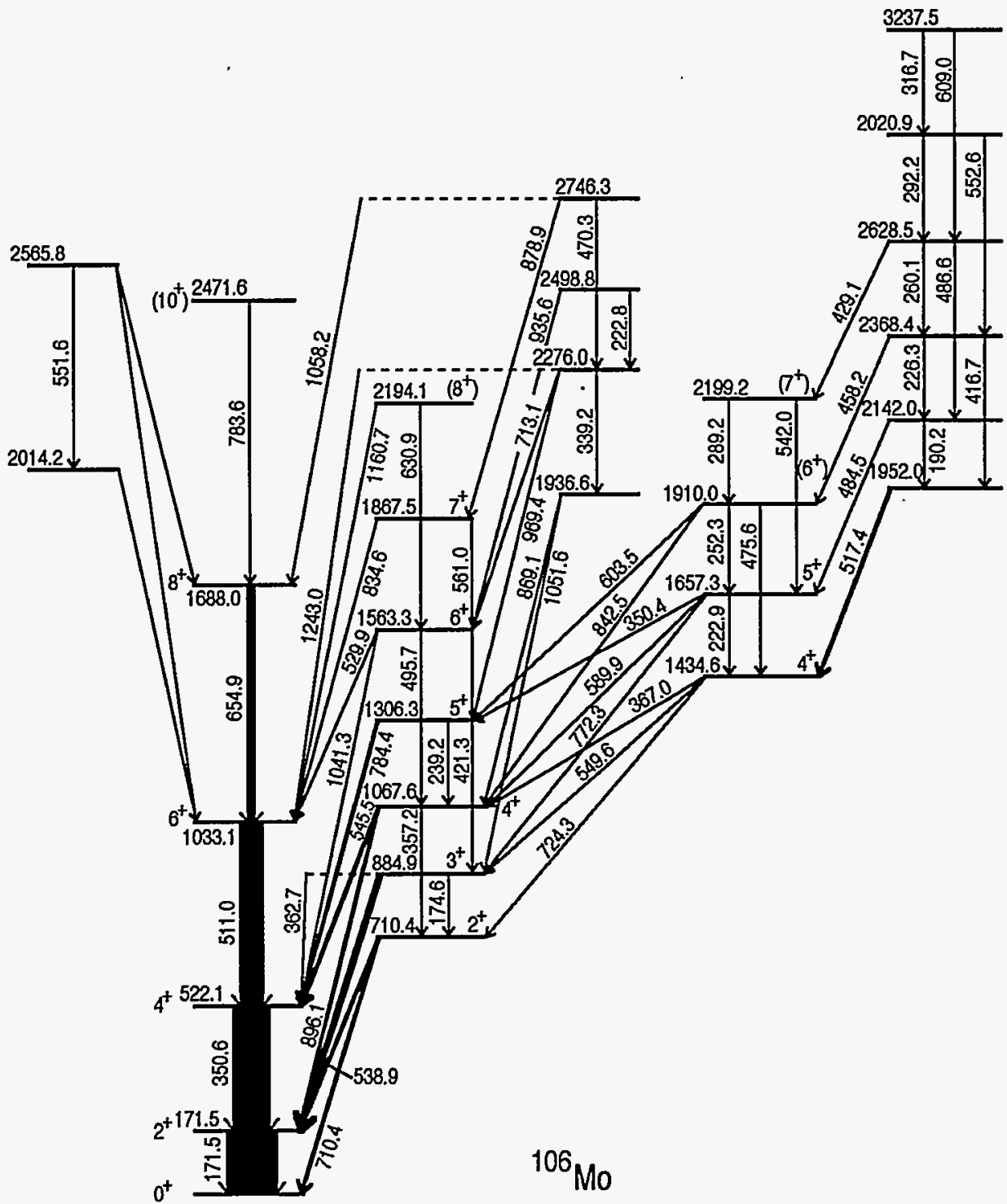

Fig. I-21. Partial decay scheme for ${ }^{106}$ Mo. The widths of the arrows are proportional to the $\gamma$-ray intensities.

b.9. Pairing Strength in Neutron-Rich Isotopes of $\mathbf{Z r}$ (I. Ahmad, C. J. Lister, L. R. Morss, ${ }^{*}$ K. L. Nash,* C. W. Clayton, ${ }^{*}$ J. L. Durell, $\dagger$ W. R. Phillips, $\dagger$

C. J. Pearson, $\uparrow$ J. A. Shanon, $\uparrow$ W. Urban, $\uparrow$ B. J. Varley, $\uparrow$ N. Schulz, $\ddagger$

E. Lubkiewicz, $¥$ M. Bentaleb, $\ddagger$ K. Jain,§ and N. Rowley†§)

Although the pairing strength is quite well known in stable nuclei, very little data exist for neutron-rich nuclei. Also, how the pairing strength changes when the level density is low is not clear. One of the ways to deduce the pairing strength is to measure the energies of high-K two-quasiparticle bands and to compare them with calculations within the BCS theory. We identified rotational bands built on two-quasiparticle states in ${ }^{100} \mathrm{Zr}$ and ${ }^{102} \mathrm{Zr}$. The level energies and gamma-ray branching ratios were determined from triple-gamma coincidence data obtained with the Eurogam 1 array and a ${ }^{248} \mathrm{Cm}$ source. The Eurogam1 array consisted of 45 Compton-suppressed $\mathrm{Ge}$ detectors and 5 LEPS spectrometers. The configurations of the $\mathrm{K} \pi=6^{+}$band in ${ }^{100} \mathrm{Zr}$ and the $\mathrm{K} \pi=4^{-}$band in ${ }^{102} \mathrm{Zr}$ were

*Chemistry Division, ANL, †University of Manchester, United Kingdom, $¥ C R N$, Strasbourg, France, §University of Surrey, United Kingdom 
deduced. The energies of the two-quasiparticle bandheads were used within the BCS model of pairing to deduce the strength of pairing interaction, $G$. Values of $G$ were deduced to be 0.21 and $0.19 \mathrm{MeV}$ for ${ }^{100} \mathrm{Zr}$ and ${ }^{102} \mathrm{Zr}$, respectively (see Fig. I-22). These are somewhat smaller than the value of 23/A used to describe even-even nuclei near stability in this region. Results of this study were published. ${ }^{1}$

Fig. I-22. (a) The pairing strength $G$ determined as a function of the quadrupole deformation parameter $\varepsilon_{2}$ by fitting the bandhead in $100_{\mathrm{Zr}}$; (b) similar curves for ${ }_{102} \mathrm{Zr}$. Solid squares show the results for $\& 4$ equal to zero, with no spin-spin shift; triangles show results for $\varepsilon_{4}$ equal to zero with the spin-spin shift taken into account; circles show results for $G$ including the spinspin shift and the effect of the theoretically predicted values of $\varepsilon 4$.

1J. L. Durrell et al., Phys Rev. C $\underline{52}$, R2306 (1995)

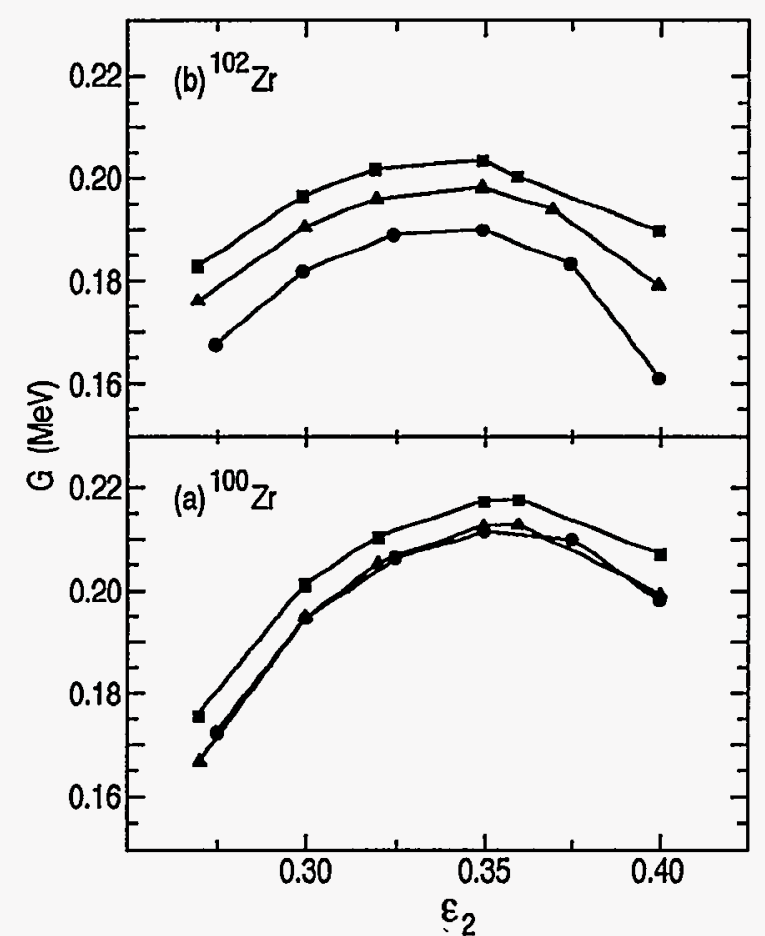

\section{b.10. Search for Octupole Deformation in Neutron-Rich Cs Nuclei} (I. Ahmad, C. J. Lister, L. R. Morss, ${ }^{*}$ K. L. Nash, ${ }^{*}$ C. W. Williams, ${ }^{*}$ T. Rzaca-Urban, $\uparrow$ W. R. Phillips, $\dagger$ J. L. Durell, $\uparrow$ W. Urban, $\dagger$ B. J. Varley, $\dagger$ C. J. Pearson, $\uparrow$ J. A. Shannon, $\uparrow$ M. Bentaleb, $\ddagger$ E. Lubkiewicz, $\ddagger$ and N. Schulz $\ddagger)$

Previous experiments showed that neutron-rich eveneven $\mathrm{Ba}$ and $\mathrm{Ce}$ nuclei have large octupole correlations. Theory predicts even larger octupole correlations in the neighboring odd-mass Cs isotopes. For this reason level schemes of the very neutron-rich nuclei $141,143,145 \mathrm{Cs}$ were studied from triple gamma coincidence measurements of ${ }^{248} \mathrm{Cm}$ fission fragments. The experiment was performed with the Eurogam 1 array which, for this experiment, consisted of 45 Comptonsuppressed Ge detectors and 5 LEPS spectrometers. Excited states in ${ }^{143} \mathrm{Cs}$ and ${ }^{145} \mathrm{Cs}$ were observed for the first time (see Fig. I-23). No parity doublets, the characteristic signature of octupole deformation, were observed suggesting lack of large octupole correlations in these nuclei. The results of this investigation were published. 1

*Chemistry Division, ANL, †University of Manchester, United Kingdom, $\ddagger$ CRN, Strasbourg, France

1T. Rzaca-Urban et al., Phys. Lett. B348, 336 (1995)

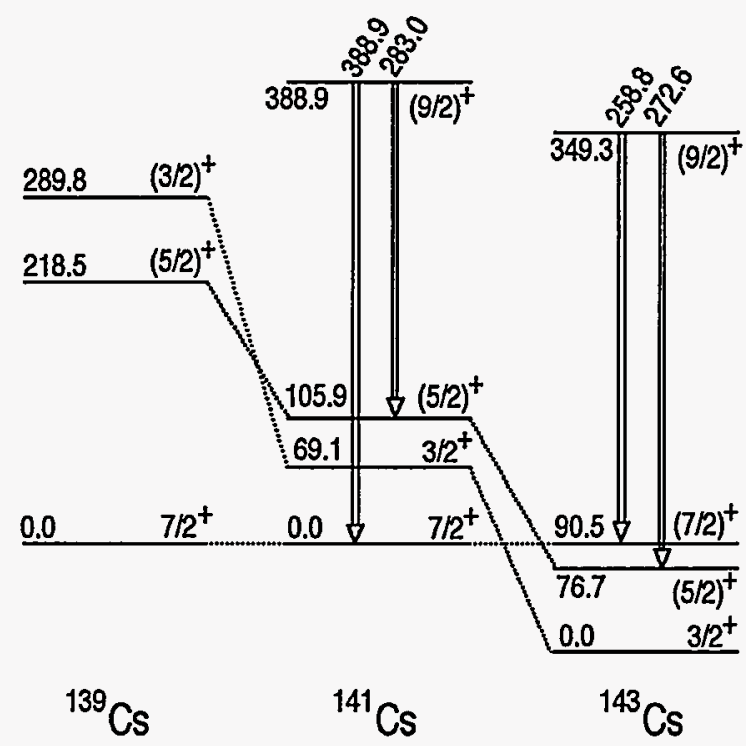

Fig. I-23. Systematics of the low-lying excitations in $\mathrm{Cs}$ isotopes. 


\section{b.11. Search for the Two-Phonon Octupole Vibrational States in ${ }^{208} \mathbf{P b}$ (H. Amro, R. V. F. Janssens, E. F. Moore,* W. Henning, T. L. Khoo, I. Ahmad, M. P. Carpenter, T. Lauritsen, C. J. Lister, D. J. Blumenthal, G. Hackman, S. Fischer, S. J. Sanders, $\dagger$ and S. Yates $\ddagger$ )}

We performed an experiment to search for the twophonon octupole vibrational states in ${ }^{208} \mathrm{~Pb}$. Thick targets of ${ }^{208} \mathrm{~Pb}$ and ${ }^{209} \mathrm{Bi}$ were bombarded with 1305 $\mathrm{MeV}$ beam of ${ }^{208} \mathrm{~Pb}$ supplied by ATLAS. Gamma rays were detected using the Argonne-Notre Dame BGO gamma-ray facility, consisting of 12 Comptonsuppressed germanium detectors surrounding an array of 50 BGO scintillators. We identified all known gamma rays from ${ }^{208} \mathrm{~Pb}$ in the spectra gated by the $5^{-} \rightarrow 3^{-}$and $3^{-} \rightarrow 0^{+}$transitions in ${ }^{208} \mathrm{~Pb}$ and constructed the level scheme for this nucleus. In addition, after unfolding these spectra for the Compton response, we observed weak broad candidates in the energy region expected for the 2-phonon states. When gating on those candidates, the coincidence spectra suggest the assignment of those weak candidates to the transition, from either the $4^{+}$or $6^{+}$members of the 2-phonon states to the $5^{-}$level. An additional experiment is being proposed at Gammasphere to collect much higher statistics for a ${ }^{132} \mathrm{Xe}$ beam on a thick ${ }^{208} \mathrm{~Pb}$ target since our present data were not sufficient to confirm the assignment of those weak candidates.

We also studied the transfer reactions in our data and calculated the relative probability of one or more nucleon transfer relative to Coulomb excitation.

*North Carolina State University and Triangle Universities Nuclear Laboratory, †University of Kansas, łUniversity of Kentucky

\section{b.12. Level Structures at High Spins in Nuclei Near and at $\mathbf{N}=\mathbf{5 2}$}

M. P. Carpenter, B. Crowell, R. V. F. Janssens, T. L. Khoo, T. Lauritsen, S. S. Ghugre, * U. Garg,* B. Kharraja, * G. Smith,* W. Mueller, $\dagger$ W. Reviol, $\dagger$ L. L. Riedinger, $\dagger$ and R. Kaczarowski $\ddagger$ )

The level sequences in the $\mathrm{N}=50$ isotones of $\mathrm{Mo}, \mathrm{Tc}, \mathrm{Ru}$ and $\mathrm{Rh}$ nuclei exhibit single-particle characteristics even at high spins $(I \sim 20 \hbar)$ and excitation energies $\left(E_{X} \sim 12 \mathrm{MeV}\right)$. On the other hand, rotational excitations are observed in nuclei with $\mathrm{N}>$ 54.2 Thus, the study of high spin states of the $\mathrm{N}=$ $52,53,54$ isotones of $\mathrm{Mo}, \mathrm{Tc}, \mathrm{Ru}$ and $\mathrm{Rh}$ would greatly help in understanding the mechanism for the generation of the higher angular momentum states in this region, and to observe the transition from the spherical singleparticle mode to the collective degrees of freedom. High-spin states in $94,95 \mathrm{Mo}, 94,95,96 \mathrm{Tc}, 96,97,98_{\mathrm{Ru}}$ and $97,98 \mathrm{Rh}$ were populated using a $142-\mathrm{MeV}{ }^{36} \mathrm{~S}$ beam on a ${ }^{65} \mathrm{Cu}$ target. Triple- $\gamma$ coincidences were measured using the early implementation phase of Gammasphere.

Several new transitions were identified in these nuclei, and the level sequences were substantially extended (up to a spin of $\mathrm{I} \sim 20 \hbar$ and an excitation energy of $\mathrm{E}_{\mathrm{X}} \sim$ $12-14 \mathrm{MeV}){ }^{1}$ Shell-model calculations, using different model spaces and effective interactions, were carried out for several of these nuclei. The $N=51,52$ isotones exhibit single-particle nature and their higher angular momentum states are dominated by the excitation of a single g9/2 neutron across the $\mathrm{N}=50$ magic shell. The "breaking" of the $N=50$ core is evidenced by a number of transitions of $\sim 2 \mathrm{MeV}$ followed by a fragmentation of intensity into several parallel cascades. The level sequences of the $\mathrm{N}=53,54$ isotones, on the other hand, begin to indicate an onset of collectivity. Weakcoupling model calculations have been quite successful in interpreting the level sequences of these nuclei. Vibrational model calculations as well as lifetime measurements would greatly facilitate a more complete understanding of the level sequences of these nuclei.

*University of Notre Dame, $\nmid$ University of Tennessee, $\ddagger$ Soltan Institute for Nuclear Studies, Swierk, Poland

${ }_{1}^{1}$ S. S. Chugre et al., Phys. Rev. C 51, 2809

2V. Ravikumar et al., J. Phys. G 20, 441 (1994) 


\section{b.13. Search of Collectivity at $N>52$ via Lifetime Measurements in $96-98 R u$ (M. P. Carpenter, I. Ahmad, D. Blumenthal, B. Crowell, R. V. F. Janssens, T. L. Khoo, T. Lauritsen, D. Nisius, B. Kharraja,* A. Frohlich,* U. Garg,* S.S.Ghugre,* W. Reviol, $\neq$ W. Mueller, $\neq$ I. M.Govil, $\neq$ W. C. Ma,§ R. Kaczarowski,II and E. RuchowskaID}

Level structures of nuclei with $\mathrm{N} \sim 50$ and $\mathrm{Z} \sim 40$ exhibit an interesting interplay between single-particle and collective degrees of freedom. The nuclei ${ }^{96-98} \mathrm{Ru}$ were recently studied in some detail at ATLAS and at Gammasphere, and their level sequences extended up to high spin and excitation energies $\left(\mathrm{I} \sim 34 \hbar, \mathrm{E}_{\mathrm{X}} \sim 20\right.$ $\mathrm{MeV}){ }^{1}$ Most of the observed levels can be explained within the spherical shell-model framework, even at high spins. However, a series of consecutive E2 transitions with increasing energies was also observed in these nuclei. These E2 transitions provided tantalizing hints of the onset of collectivity as low as $\mathrm{N}$ $=52$ in this region. It transpires that both the shellmodel and collective models can explain these E2 sequences with similar success and, hence, lifetime measurements are crucial to ascertain the true intrinsic structure of these "band-like" cascades.

This motivated us to undertake RDM lifetime measurements in $96-98 \mathrm{Ru}$. These nuclei were populated via the ${ }^{65} \mathrm{Cu}\left({ }^{36} \mathrm{Si}\right.$,pyn) reaction at a beam energy of $142 \mathrm{MeV}$, and the Argonne-Notre Dame gamma-ray facility was employed in conjunction with the Notre Dame plunger. Because of the fragmentation of the total reaction strength among a number of competing reaction channels and the concomitant complexity of the observed spectra, data were collected in the coincidence mode at 12 distances from $10 \mu \mathrm{m}$ to $1000 \mu \mathrm{m}$, giving us an effective lifetime range of $1 \mathrm{ps}$ 400 ps. Detailed analysis of the data, sorted into angledependent matrices, is in progress.

*University of Notre Dame, †Panjab University, Pakistan, †University of Tennessee, §University of Mississippi, ISoltan Institute for Nuclear Studies, Swierk, Poland,

${ }^{1} \mathrm{U}$. Garg et al., Proceedings of the Workshop on Gammasphere Physics, Lawrence Berkeley National Laboratory, Berkeley, CA, Dec. 1-2, 1995, in press.

\section{b.14. Lifetime Measurements in 180,182,184Pt Beyond the Backbend}

(M. P. Carpenter, I. Ahmad, B. Crowell, R. V. F. Janssens, T. L. Khoo, T. Lauritsen, S. S. Ghugre,* U. Garg,* B. Kharraja,*, B. Prause,* G. Smith,* E. F. Moore, $\uparrow$ W. Mueller $\ddagger$ W. Reviol, $\ddagger$ L. L. Riedinger,$\ddagger$ R. Kaczarowski, $\S$ and I. M. Govil|D

In ${ }^{180-186} \mathrm{Pt}$ nuclei, a sharp increase in the transition quadrupole moment, $\mathrm{Q}_{t}$, was observed at low frequencies followed by a rapid and significant decline in the backbending region. ${ }^{1}$ It was suggested that this decline is attributable to a transition to triaxial shapes. The next question, then, is what happens beyond the backbending region? This interesting aspect of nuclear structure was explored in $180-184 \mathrm{Pt}$ nuclei, populated via the ${ }^{120-124} \mathrm{Sn}\left({ }^{64} \mathrm{Ni}, 4 \mathrm{n}\right) 180,182,184$ Pt reactions. DSAM lifetime measurements were carried out at Gammasphere. The targets were isotopically enriched foils $\left(\sim 1 \mathrm{mg} / \mathrm{cm}^{2}\right.$ thick), on a $\mathrm{Pb}$ backing ( 75 $\mathrm{mg} / \mathrm{cm}^{2}$ thick).
During the analysis, the $\gamma-\gamma-\gamma$ coincidence events were sorted into angle-dependent gated matrices. The computer code LILIFI was used to extract lifetimes from the Doppler-broadened lines. Lineshapes were calculated using the slowing down histories, nuclear decay schemes, detector geometries, and sidefeeding (to each state, approximated by a single rotational cascade of 5 transitions). The data analysis is in progress. It is hoped that the measured lifetimes will help us understand any shape changes caused by the alignment of specific orbitals and the deformation-driving capabilities of these orbitals.

\footnotetext{
*University of Notre Dame, $\uparrow$ North Carolina State University and Triangle Universities Nuclear Laboratory,. University of Tennessee, §Soltan Institute for Nuclear Studies, Swierk, Poland, IPanjab University, Pakistan

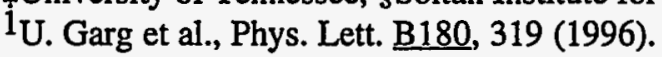


b.15. Yrast Spectroscopy Around ${ }^{132}$ Sn (I. Ahmad, M. P. Carpenter, T. Ishii,ll L. R. Morss, ${ }^{*}$ D. Nisius, C. T. Zhang, $\ddagger$ P. Bhattacharyya $\ddagger$ R. Broda, $\ddagger$

Z. W. Grabowski,ł P. J. Daly, W. R. Phillips,§ J. L. Durell,§ M.A. Jones,§

M. Leddy,§ A. G. Smith,§ W. Urban,§ B. J. Varley,§ N. Schulz,ף

E. Lubkiewicz, I and M. BentalebID)

One of the interesting problems in nuclear physics is to measure and understand the single-particle potential in very neutron-rich closed-shell nuclei and see how it differs from the structure of stable spherical nuclei. One of the sources of neutron-rich nuclei is low-energy fission. Nuclei around ${ }^{132} \mathrm{Sn}$ are produced with reasonable yields in the fission of $248 \mathrm{Cm}$. For this reason we embarked on an analysis of fission data obtained with the Eurogam 2 array and a ${ }^{248} \mathrm{Cm}$ source to determine the structures of spherical and nearspherical nuclei with $\mathrm{N}=80-84$ and $\mathrm{Z}=50-54$. The Eurogam2 array for this measurement consisted of 124 $\mathrm{Ge}$ detectors and 4 LEPS spectrometers. At this early stage of the analysis, yrast spectra of several $\mathrm{N}=83$ and 84 nuclei were extended to higher spins and higher excitation energies than previously known and we expect to learn a great deal more about nuclear structure in the vicinity of ${ }^{132} \mathrm{Sn}$ in the coming months.

*Chemistry Division, ANL, ¥Purdue University, §University of Manchester, United Kingdom, đCRN, Strasbourg, France, IVisiting Scientist from JAERI, Ibaraki, Japan

b.16. Single-Particle States in Heavy Nuclei (I. Ahmad, B. B. Back, T. Ishii,* R. R. Chasman, J. P. Greene, L. R. Morss, $\uparrow$ G. P. A. Berg, $\ddagger$ A. Bacher,$\ddagger$ C. C. Foster $\ddagger$ W. R. Lozowski, $\ddagger$ W. Schmitt, $\ddagger$ and E. J. Stephenson $\ddagger$ )

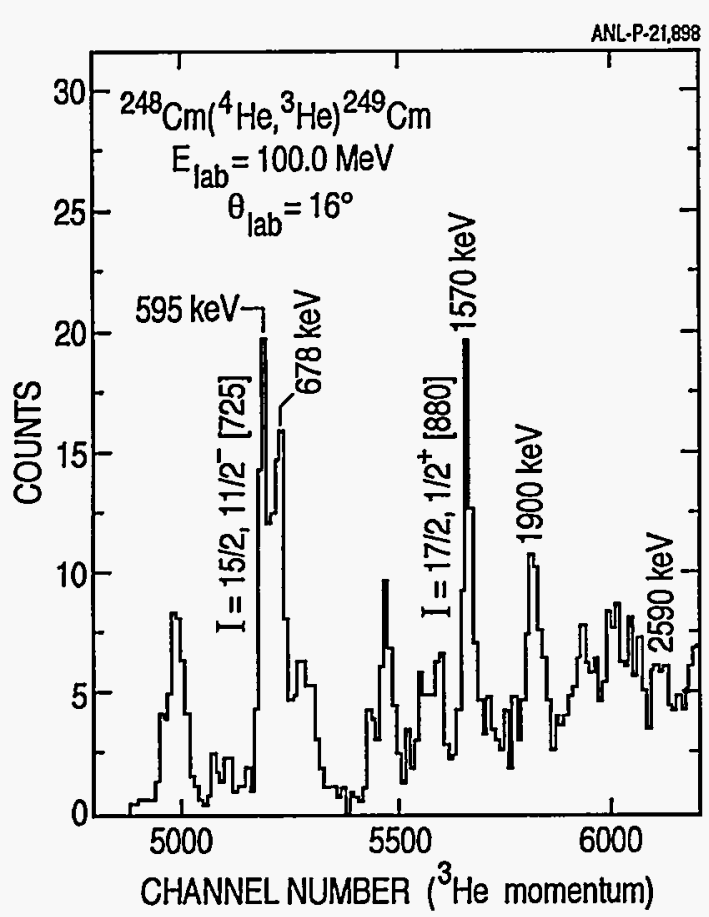

Fig. I-24. Spectrum of ${ }^{3}$ He ions from the ${ }^{248} \mathrm{Cm}\left({ }^{4} \mathrm{He},{ }^{3} \mathrm{He}\right)$ reaction and measured with the K600 magnetic spectrometer.

The half-lives of the superheavy elements depend critically on the shell correction, which in turn depends on the location of the single-particle states near the Fermi surface. Most of the single-particle states (neutron orbitals between $\mathrm{N}=152$ and 162 and proton states near $Z=100$ ) were identified in previous studies. One of the states which has a crucial influence on the shell correction is the $k_{17 / 2}$ single-particle state. This high-j state is only very weakly populated in $(d, p)$ reactions and, hence, has so far not been identified. This state is expected to be strongly populated in $\left({ }^{4} \mathrm{He},{ }^{3} \mathrm{He}\right)$ reactions. We therefore performed the $\left({ }^{4} \mathrm{He},{ }^{3} \mathrm{He}\right)$ reaction on a ${ }^{248} \mathrm{Cm}$ target. The experiment was performed at the Indiana University Cyclotron Facility at a ${ }^{4} \mathrm{He}$ energy of $100 \mathrm{MeV}$. The emerging ${ }^{3} \mathrm{He}$ ions were momentum analyzed with the $\mathrm{K} 600$ magnetic spectrometer. Our test experiment with a ${ }^{232}$ Th target shows that the high-j value states are clearly the most strongly populated levels. The ${ }^{3} \mathrm{He}$ spectra from the ${ }^{248} \mathrm{Cm}\left({ }^{4} \mathrm{He},{ }^{3} \mathrm{He}\right)$ reaction were measured at several angles in order to distinguish peaks in ${ }^{249} \mathrm{Cm}$ from those in light elements. In the ${ }^{248} \mathrm{Cm}\left({ }^{4} \mathrm{He},{ }^{3} \mathrm{He}\right){ }^{249} \mathrm{Cm}$ spectrum (see Fig. I-24), the two strongest peaks are observed at $600 \mathrm{keV}$ and 1.56 $\mathrm{MeV}$. The $600-\mathrm{keV}$ state is assigned to the $15 / 2$ member of the 11/2-[725] band which is known in the isotone ${ }^{251} \mathrm{Cf}$ to be located at $569 \mathrm{keV}$. The strong peak at $1.56 \mathrm{MeV}$ is assigned to the $\mathrm{I}=17 / 2$ member of the $1 / 2+[880]$ band. The data are still being analyzed.

*JAERI, Ibaraki, Japan, †Chemistry Division, ANL, ‡Indiana University 


\section{REACTION STUDIES}

Reaction studies at ATLAS cover a broad range of topics including processes in the vicinity of the Coulomb Barrier, fusion and fission reactions at higher energies and the production and decay of exotic nuclear cluster states in s-d shell nuclei. These studies take advantage of the characteristics of the ATLAS accelerator such as excellent beam quality, outstanding timing properties and easy energy variability. This research utilizes a variety of detection systems such as the magnetic spectrographs, the fragment mass analyzer, and an array of double-sided silicon strip-detectors with the accompanying modular electronics developed for this specific usage.

Some of the achievements over the past year are:

- the successful application of the particle-particle angular correlation technique to spin assignments of resonances in the ${ }^{12} \mathrm{C}+{ }^{12} \mathrm{C}$ system.

- the demonstration (with two complementary experimental techniques) of the presence of fission hindrance in hot nuclei by measurements of enhanced probabilities for survival of the composite system as compared to predictions of statistical models.

- the measurement of the time scale associated with quasi-fission reactions by making use of the gamma decay of the Giant Dipole resonance as a clock.

- the extraction of barrier distributions from fusion excitation functions at energies near or below the coulomb barrier in cases involving transitional (i.e. "soft") nuclei.

a.1. Angular Correlation Measurements for ${ }^{12} \mathrm{C}\left({ }^{12} \mathrm{C},{ }^{12} \mathrm{C}\right){ }^{12} \mathrm{C}\left(3^{-}\right)$Scattering (A. H. Wuosmaa, B. B. Back, R. R. Betts, D. J. Blumenthal, S. Fischer, B. G. Glagola, D. J. Henderson, D. Hofman, R. V. F. Janssens, C. J. Lister, V. Nanal, D. Nisius, M. D. Rhein, P. R. Wilt, and M. Freer*)

Many previous studies of inelastic scattering in the ${ }^{12} \mathrm{C}$ $+{ }^{12} \mathrm{C}$ system revealed strong resonance-like behavior. Several theoretical pictures emerged which attempt to describe this behavior, either as superdeformed cluster states in the compound system ${ }^{24} \mathrm{Mg}$, or as potential scattering resonances whose strength is enhanced due to favorable angular momentum coupling conditions at particular energies. These models make predictions about the angular momenta expected to dominate the scattering process at resonance energies. Many of the reaction channels in which the strongest resonances are observed have non-zero channel spin, making the extraction of a resonance angular momentum difficult, if not impossible, using the traditional technique of angular distribution measurements. If, however, the radiation from the decay of the level in the inelastically excited nucleus can be detected, the angular correlations between that radiation, and the scattered nucleus becomes sensitive to the total angular momentum of the system and hence the resonance spin.

We applied the technique of particle-particle angular correlations to the problem of making spin assignments for resonances observed in the $3^{-}+$g.s. excitation in ${ }^{12} \mathrm{C}+{ }^{12} \mathrm{C}$ scattering, ${ }^{1}$ where previous experiments identified a number of strong resonances. Data from two experiments, conducted with arrays of four, and six double-sided strip detectors (DSSDs) were obtained at several energies in the range of $E(c . m)=$.26 to 40 $\mathrm{MeV}$. The alpha particles from the decay of the $3^{-}$

\footnotetext{
*University of Birmingham, United Kingdom

${ }^{1}$ W. Reilly et al., Nuovo Cimento 13A, 913 (1973); B. R. Fulton et al., Phys. Rev. C 21 , 198 (1980)

2S. Marsh and W. D. M. Rae, Phys. Lett. B153, 21 (1985)

${ }^{3}$ Y. Kondo, Y. Abe, and T. Matsuse, Phys. Rev. C 19, 1356 (1980)
} 

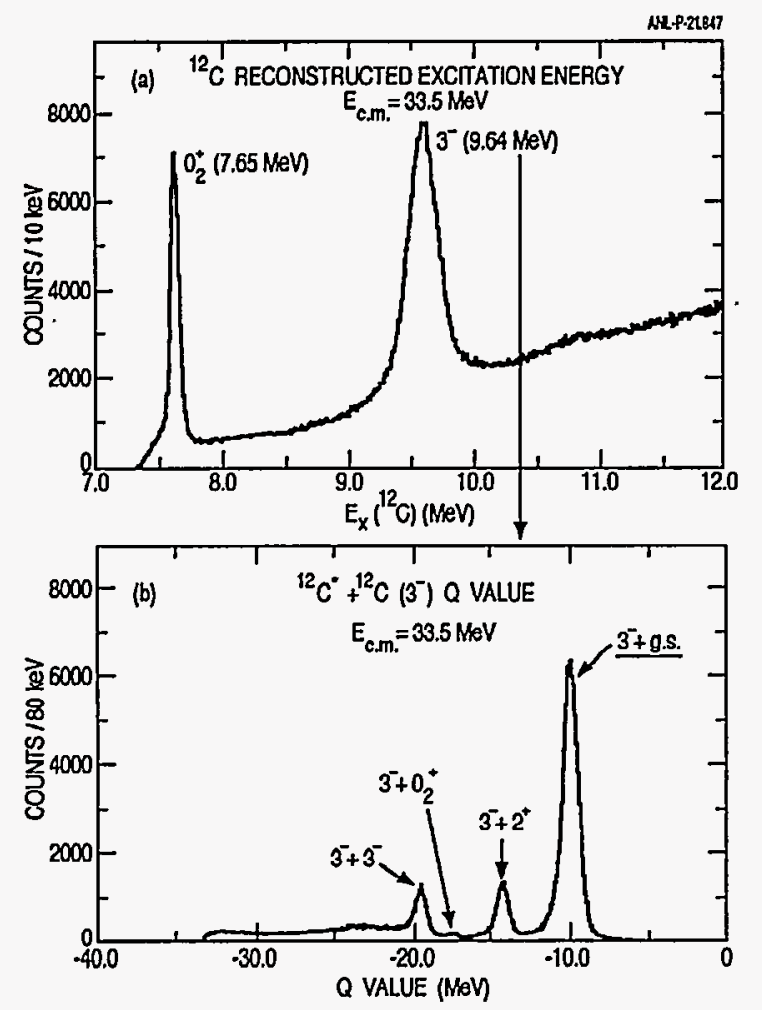

Fig. I-25. (a) Reconstructed ${ }^{12}$ C excitation energy spectrum from three-alpha particle coincidences, with the requirement that two of the three alpha particles emerge from the decay of a ${ }^{8} \mathrm{Be} .(\mathrm{b}){ }^{12} \mathrm{C}+{ }^{12} \mathrm{CQ}$ value spectrum at $E$ (c.m.) $=32.5 \mathrm{MeV}$, for events where one ${ }^{12} \mathrm{C}$ is in its 3- $(9.64 \mathrm{MeV})$ level.

(9.64 MeV) state in ${ }^{12} \mathrm{C}$ were detected in the DSSD arrays, and the energy and momentum correlations between them were used to identify the reaction channel of interest. as shown in Fig. I-25.

Figure I-26(a) shows a matrix of the angular correlation data obtained at an energy of $32.5 \mathrm{MeV}$, the peak of a resonance in the $3^{-}+$g.s. channel. Here the abscissa corresponds to the decay angle of the alpha particle in the ${ }^{12} \mathrm{C}$ center-of-mass frame, measured relative to the beam axis, and the ordinate the center-of-mass scattering angle of the ${ }^{12} \mathrm{C}$. The ridge structure in the correlation matrix contains information about the coupling of the spin of the decaying ${ }^{12} \mathrm{C}$, and the orbital and total angular momentum of the ${ }^{12} \mathrm{C}+{ }^{12} \mathrm{C}$ system. ${ }^{2}$ In a simple picture, the slope of the parallel ridges are equal to the ratio of the ${ }^{12} \mathrm{C}$ spin (3) to the orbital angular momentum, here unambiguously identified as 15 . The kinematics of the reaction favor the assumption of an aligned configuration, where the $12 \mathrm{C}$ spin and the orbital angular momentum are parallel, and assumption supported by the alpha-particle angular dependence of
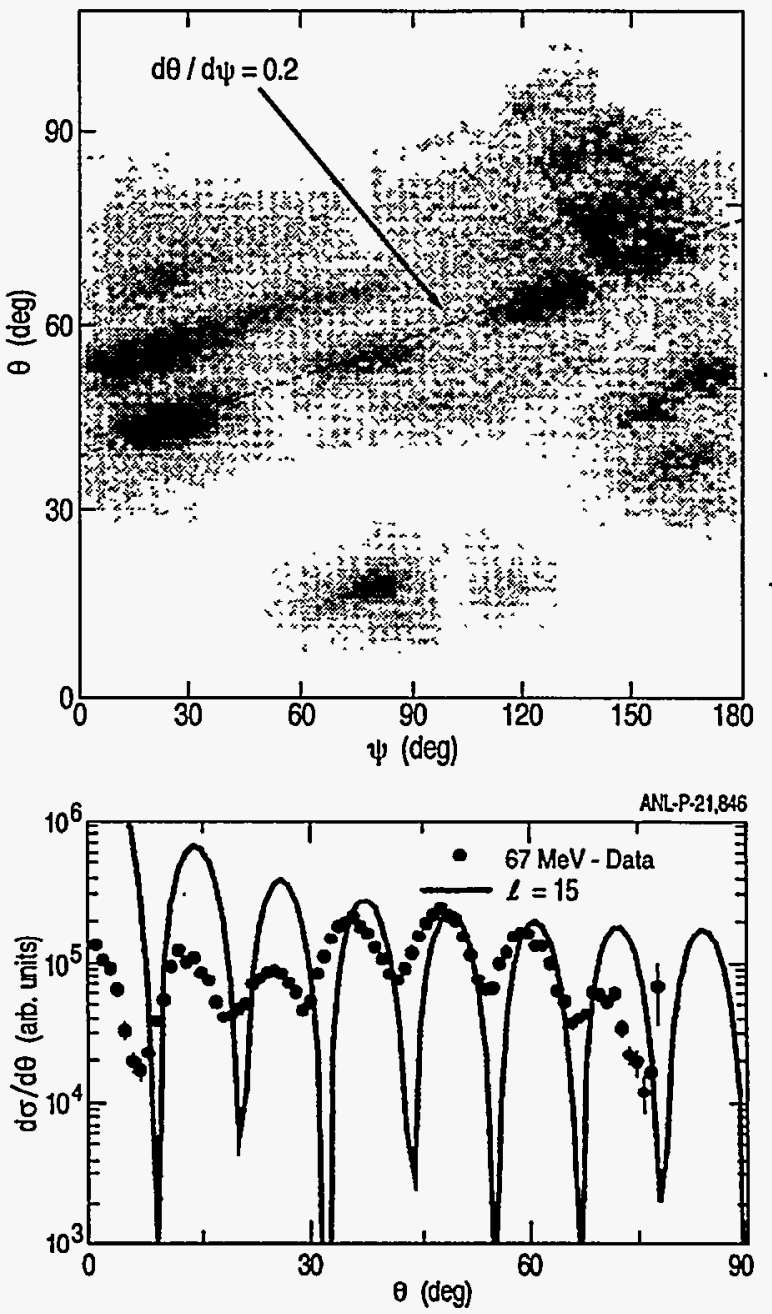

Fig. I-26. (a) ${ }^{12} C+{ }^{12} C\left(3^{-}\right)$angular correlation matrix at $E($ c.m. $)=33.5 \mathrm{MeV}$. (b) Projection of (a) along the correlation ridges, on the $\theta$ axis, with $\left|P_{15}(\cos \theta)\right|^{2}$ (solid curve).

the correlation matrix. Under such an assumption, we can assign a spin value of 18 to the resonance at 32.5 $\mathrm{MeV}$. This value is surprising, as it is in disagreement with various reaction model calculations for resonances in this channel. 3

The data from a more recent measurement carried out with six DSSDs are at an early stage of analysis, but the preliminary results already confirm the assignment of $18^{+}$for the resonance at $\mathrm{E}(\mathrm{c} . \mathrm{m})=.33.5 \mathrm{MeV}$, and suggest an assignment of $16^{+}$for two resonances at lower energies $[\mathrm{E}$ (c.m.) $=27$ and $28.5 \mathrm{MeV}$ ]. This analysis is continuing, and a manuscript is in preparation describing the results of the first measurements. 
a.2. Study of the ${ }^{12} \mathrm{C}\left({ }^{20} \mathrm{Ne},{ }^{20} \mathrm{Ne}\right){ }^{12} \mathrm{C} *$ Reaction (A. H. Wuosmaa, B. B. Back, R. R. Betts, D. J. Blumenthal, S. Fischer, B. G. Glagola, D. J. Henderson, D. Hofman, R. V. F. *† B. R. Fulton,* and W. D. M. Rae $\dagger$ )

Recent cluster model calculations suggested that extremely deformed shapes might exist at high excitation energy in the nucleus ${ }^{32} \mathrm{~S}$; and that these structures could have large decay branches to final states involving $20 \mathrm{Ne}$ and excited, deformed states in ${ }^{12} \mathrm{C}$, such as the first excited $0^{+}$level at $7.65 \mathrm{MeV}$. Observation of non-statistical features in the excitation function for inelastic scattering in the ${ }^{12} \mathrm{C}\left({ }^{20} \mathrm{Ne},{ }^{20} \mathrm{Ne}\right){ }^{12} \mathrm{C} *$ reaction represents a possible signature for this behavior. To examine this possibility, we obtained excitation function data for the ${ }^{20} \mathrm{Ne}+{ }^{12} \mathrm{C}$ system, for final states in which either the ${ }^{12} \mathrm{C}$, or the ${ }^{20} \mathrm{Ne}$, or both are excited above their particle decay thresholds. Data were obtained in 1-MeV steps, at laboratory energies from 80 to $100 \mathrm{MeV}$. The decay products from the scattered nuclei were detected in an array of six double-sided strip detectors. Preliminary results show that the reaction channel of interest, the ${ }^{20} \mathrm{Ne}+{ }^{12} \mathrm{C}\left(0^{+} 2\right)$ final state, is populated and the analysis of the energy dependence of the yield for this channel is continuing.

*University of Birmingham, United Kingdom, †University of Oxford, United Kingdom

a.3. Studies of Fission Hindrance in Hot Nuclei (D. Ackermann, B. B. Back, D. J. Blumenthal, C. N. Davids, G. S. Hackman, D. J. Henderson, W. F. Henning, D. J. Hofman, C. L. Jiang, P. Paul, * V. Nanal, and A. H. Wuosmaa)

Experimental studies of the time-scale of fission of hot nuclei have been carried out recently using the emission rates of neutrons, gamma rays, and charged particles as "clocks" for the fission process. These experiments showed that the fission process is strongly retarded (or hindered) relative to expectation (based on the statistical model description of the process) well beyond any uncertainties in the model parameters. It therefore appears that a dynamical description of the fission process at these energies is more relevant and that the experimental data are able to shed light on the dissipation in the shape degree of freedom. However, these experiments are not very sensitive to whether the emission occurs mainly before or after the traversal of the saddle point as the system proceeds toward scission. Various dissipation models are, however, strongly dependent on the deformation and shape symmetry of the system. As an alternative to these methods we therefore measure the evaporation probability for hot nuclei formed in heavy-ion fusion reactions. As the hot system cools down by the emission of neutrons and charged particles there is a finite chance to undergo fission after each evaporation step. If the fission branch is suppressed due to dissipation there is therefore a strongly enhanced probability for survival of the composite system as compared with the prediction of pure statistical models. Two types of experiments are presently being pursued to utilize this sensitivity to study fission hindrance in excited systems. One is the measurement of evaporation residue cross sections as a function of excitation energy, in which three systems are studied, namely, ${ }^{32} \mathrm{~S}+{ }^{182} \mathrm{~W},{ }^{64} \mathrm{Ni}+{ }^{154} \mathrm{Sm}$, and $100 \mathrm{Mo}+{ }^{116} \mathrm{Cd}$. The second is the direct measurement of the survival probability of target-like deeply inelastic products in kinematic coincidence with binary reactions partners. Both methods have strengths and weaknesses in terms of the interpretation of the data, but since these are in different areas they complement each other to hopefully derive more rigorous information about the fission hindrance and dissipation of hot nuclei.

*SUNY at Stony Brook 


\section{a.4. Evaporation Residue Cross Sections for ${ }^{32} \mathrm{~S}+{ }^{184} \mathrm{~W}$ (B. B. Back, D. J. Blumenthal, C. N. Davids, D. J . Henderson, R. Hermann, D. J. Hofman, C. L. Jiang, P. Paul, * H. T. Penttila, and A. H. Wuosmaa)}

Measurements of the evaporation residue cross sections for the ${ }^{32} \mathrm{~S}+{ }^{184} \mathrm{~W}$ system were carried out over a wide range of beam energies using the Argonne Fragment Mass Analyzer (FMA). Absolute cross sections were obtained on the basis of the recent determination of the transmission probability through the FMA of heavy, slow-moving reaction products.

The analysis of the data is complete and the results are presently being prepared for publication.

*SUNY at Stony Brook

\section{a.5. Evaporation Residue Cross Sections for the ${ }^{100} \mathrm{Mo}+{ }^{116} \mathrm{Cd}$ Reaction (B. B. Back, D. J. Blumenthal, C. N. Davids, R. H. Hermann, D. J. Henderson, W. F. Henning, D. J. Hofman, P. Paul,* H. T. Penttilä, and A. H. Wuosmaa)}

Evaporation residue cross sections for the $100 \mathrm{Mo}+$ $116 \mathrm{Cd}$ reaction were measured with the FMA at beam energies of $E_{\text {beam }}=460,490$, and $521 \mathrm{MeV}$, which correspond to excitation energies of $E_{\text {exc }}=62,78$, and $95 \mathrm{MeV}$, respectively.

*SUNY at Stony Brook
By comparing to results for the ${ }^{32} \mathrm{~S}+{ }^{184} \mathrm{~W}$ reactions, we expect to demonstrate a strong entrance channel effect related to the hindrance of complete fusion in near-symmetric heavy systems (a fusion hindrance factor of the order 7-10 is expected on the basis of the ExtraPush Model).

The data are presently being analyzed.

\section{a.6. Time-Scale in Quasi-Fission Reactions (B. B. Back, P. Paul,* J. Nestler,*} K. S. Drese,* D. J. Hofman, S. Schadmand,* and R. Varma*)

The quasifission process arises from the hindrance of the complete fusion process when heavy-ion beams are used. The strong dissipation in the system tends to prevent fusion and leads the system towards reseparation into two final products of similar mass reminiscent of a fission process. This dissipation slows down the mass transfer and shape transformation and allows for the emission of high-energy $\gamma$ rays during the process, albeit with a low probability. Giant Dipole $\gamma$ rays emitted during this time have a characteristic spectral shape and may thus be discerned in the presence of a background of $\gamma$ rays emitted from the final fission-like fragments. Since the rate of GDR $\gamma$ emission is very well established, the strength of this component may therefore be used to measure the timescale of the quasifission process. In this experiment we studied the reaction between $368-\mathrm{MeV} 58 \mathrm{Ni}$ and a ${ }^{165} \mathrm{Ho}$ target, where deep inelastic scattering and quasifission

*SUNY at Stony Brook

1B. B. Back et al., Phys. Rev. C 51, 2218 (1995). processes are dominant. Coincidences between fission fragments (detected in four position-sensitive avalanche detectors) and high energy $\gamma$ rays (measured in a $10^{\prime \prime} \times$ 10 " actively shielded NaI detector) were registered. Beams were provided by the Stony Brook Superconducting Linac.

The $\gamma$-ray spectrum associated with deep inelastic scattering events is well reproduced by statistical cooling of projectile and target-like fragments with close to equal initial excitation energy sharing (see Fig. I-27). The $\gamma$ spectrum associated with quasifission events is well described by statistical emission from the fission fragments alone, with only weak evidence for GDR emission from the mono-nucleus. A $1 \sigma$ limit of $t_{\text {mono }}<11 \times 10^{-21} \mathrm{~s}$ is obtained for the mono-nucleus lifetime, which is consistent with the lifetime obtained from quasifission fragment angular distributions. This work is completed and was published. ${ }^{1}$ 


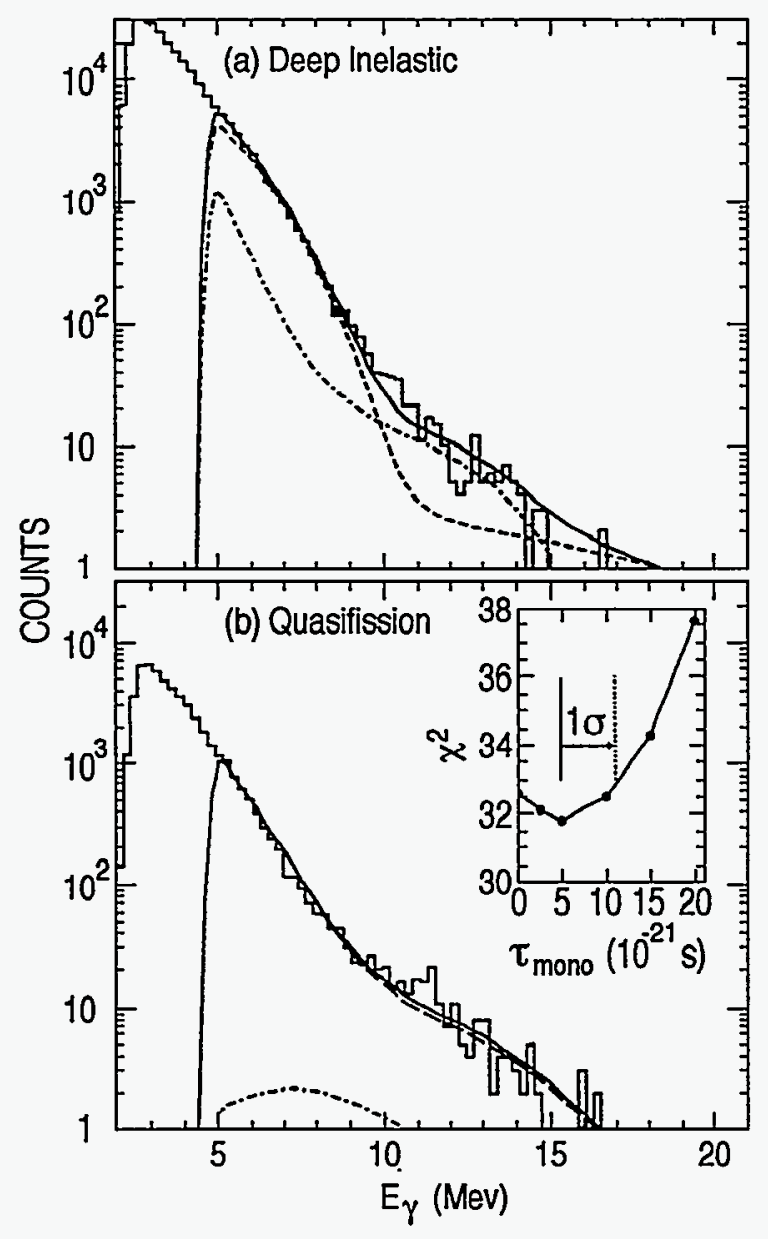

Fig. I-27. (a) Top: experimental $\gamma$-ray energy spectrum (histogram) observed in coincidence with deep inelastic scattering events. The curves are fits assuming statistical emission of GDR yrays from the PLF's (dashed line) and TLF's (dot-dashed line) and their sum (solid curve) assuming almost equal energy sharing. (b) Bottom: experimental y ray spectrum (histogram) observed in coincidence with quasifission events. The curves are fits assuming statistical emission from the fission fragments only (dashed line) and the sum (solid line) including remission from the intermediate system (dot-dashed line) calculated for t mono $<5 \times 10^{-21} \mathrm{~s}$.

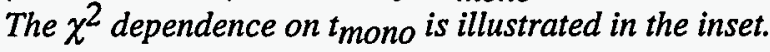

\section{a.7. Evaporation Residue Cross Sections for the ${ }^{64} \mathrm{Ni}+144,154 \mathrm{Sm}$ Reaction (B. B. Back, D. Ackermann, D. J. Blumenthal, C. N. Davids, G. Hackman, D. J. Henderson, W. F. Henning, D. J. Hofman, and V. Nanal)}

The fission hindrance of hot nuclei was deduced recently from an enhanced emission of GDR gamma rays, neutrons and charged particles prior to scission of heavy nuclei. Recent measurements of the pre-scission gamma rays and evaporation residues from the ${ }^{32} \mathrm{~S}+$ ${ }^{184} \mathrm{~W}$ reaction, found that a rather sharp transition from negligible to full one-body dissipation occurs over the excitation energy region $E_{\text {exc }}=60-100 \mathrm{MeV}$. However, the evaporation residue cross section does not appear to level out or start to decline again at the upper end of the energy range as expected in this interpretation. The extension of the excitation energy range of these types of measurements is therefore required to look for such an effect in order to either corroborate or refute this interpretation.

The aim of this work is two-fold: 1) to study the fission hindrance in hot nuclei as a result of an increase in the dissipation strength with excitation energy and 2) to study the increased fusion hindrance expected for more mass symmetric entrance channel systems by comparing the measured evaporation residue cross section to those obtained earlier using the ${ }^{32} S+{ }^{182} W$ reaction.

Measurements of the evaporation residue cross section for the ${ }^{64} \mathrm{Ni}+{ }^{154} \mathrm{Sm}$ reaction were carried out and the data are presently being analyzed. 


\section{a.8. Survival Probability of Th-like Nuclei as a Function of Excitation Energy} (B. B. Back, D. Ackermann, D. Henderson, D. J. Hofman, V. Nanal, and A. Wuosmaa)

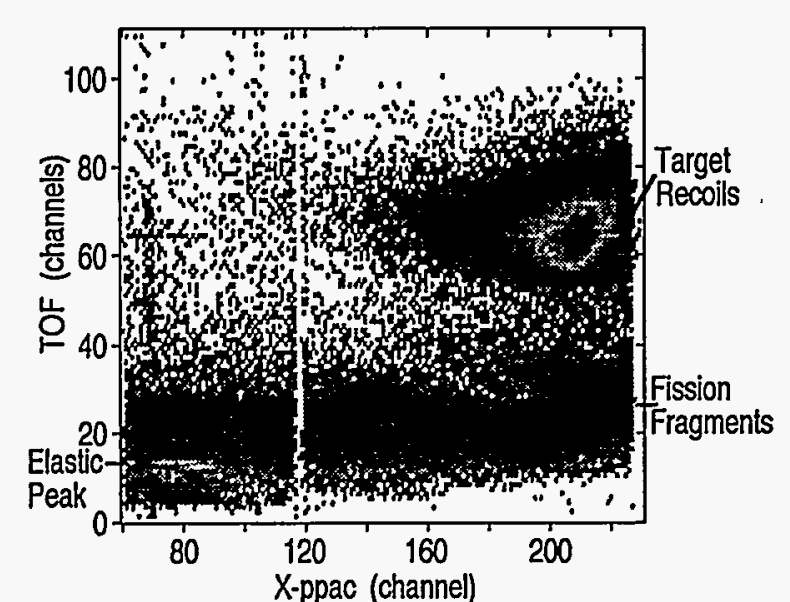

We measured the survival probability of Th-like nuclei as a function of excitation energy using the deepinelastic reaction ${ }^{40} \mathrm{Ar}+{ }^{232} \mathrm{Th}$ in order to search for possible effects of nuclear dissipation on the fission process. This experiment represents a new type of measurement which can provide insight into the many open questions surrounding the observed excesses of pre-fission particle, gamma-ray, and evaporation residue yields at high excitation energies.

The measurement was carried out in Argonne's large scattering chamber (ATSCAT) using a $400-\mathrm{MeV} 40 \mathrm{Ar}$ beam from the ATLAS superconducting linac. A $\triangle E-E$ silicon telescope was used to measure the charge and energy of the Ar projectile-like-fragment (PLF), and a large multi-wire gas-filled proportional counter measured the time-of-flight and position of the surviving target-like-fragment (TLF) (see Fig. I-28). In this manner the survival probability of the TLF could be derived as a function of total available excitation energy. Five silicon-CsI $(\Delta E-E)$ telescopes were developed and used for this experiment to measure the light-charged-particle emission in coincidence with the Ar-PLF in order to obtain experimental limits on the excitation energy division between the PLF and TLF.

The experimental method was found to work very well, with the surviving Th-TLF's being cleanly separable from the large fission background (see Fig. I-28). Preliminary analysis of the data indicates that the hot Th-like fragments have a larger survival probability than predicted by standard statistical model calculations by an order of magnitude at the highest excitation energy (see Fig. I-28).

The success of this experiment has motivated us to continue this work for other heavy nuclei, and a proposal for measuring the survival probability of $\mathrm{Pb}$ and Au-like nuclei was allocated beam time at ATLAS. temperature dependent dissipaton strength (solid curve) which was found to describe fusion-fission GDR $\gamma$-ray results for $16 \mathrm{O}+{ }^{208} \mathrm{~Pb} \rightarrow{ }^{224} \mathrm{Th} . \mathrm{I}^{\mathrm{I}}$

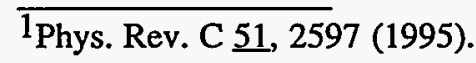




\section{a.9. Fusion Excitation Functions Involving Transitional Nuclei (K. E. Rehm, C. L. Jiang, H. Esbensen, B. Crowell, J. Gehring, B. Glagola, M. Rhein, and A. H. Wuosmaa)}

Measurements of fusion excitation functions involving transitional nuclei, e.g. ${ }^{78} \mathrm{Kr},{ }^{76} \mathrm{Ge},{ }^{100} \mathrm{Mo}$ and ${ }^{104} \mathrm{Ru}$ showed a different behavior at low energies, if compared to similar experiments with "stiff" closed-shell nuclei, e.g. ${ }^{86} \mathrm{Kr}$ or ${ }^{92} \mathrm{Mo}$. The main difference is the much shallower falloff of the excitation functions at subbarrier energies which points to a larger subbarrier fusion enhancement in systems involving transitional nuclei. In order to study possible correlations with the underlying nuclear structure of these nuclei we are presently extracting the barrier distributions from the first and second derivatives of the measured cross sections.

\section{a.10. Multi-Neutron Transfer Reaction in the System ${ }^{58} \mathrm{Ni}+{ }^{124} \mathrm{Sn}$ at Subbarrier Energies (C. L. Jiang, K. E. Rehm, D. Blumenthal, B. Crowell, J. Gehring, B. Glagola, J. P. Schiffer, and A. H. Wuosmaa)}

It was suggested that in grazing collisions between two heavy nuclei with large pair correlations (e.g. the even Sn-isotopes) an enhancement of multi-pair transfer reactions might occur. In order to investigate these predictions we studied multi-neutron transfer reactions in the system ${ }^{58} \mathrm{Ni}+{ }^{124} \mathrm{Sn}$ at $\mathrm{cm}$ energies between 150-160 MeV. The Coulomb barrier calculated with a radius parameter $r_{0}=1.44 \mathrm{fm}$ is $V_{B}=158 \mathrm{MeV}$. The experiments were performed in inverse kinematics with $470-500-\mathrm{MeV}{ }^{124} \mathrm{Sn}$ beams bombarding a $58 \mathrm{Ni}$ target. The scattered particles were analyzed according to their magnetic rigidity in the split-pole spectrograph and detected in a position-sensitive ionization chamber in the focal plane. Mass and $\mathrm{Z}$ resolutions of $\mathrm{A} / \Delta \mathrm{A}=150$ and $Z / \Delta Z=70$ were achieved. The transfer reactions are all backward peaked and are dominated by neutron transfers. At all bombarding energies the transfer of up to six neutrons was observed. At $\mathrm{E}_{\mathrm{cm}}=153 \mathrm{MeV}$ the transfer of seven neutrons from ${ }^{124} \mathrm{Sn}$ to ${ }^{58} \mathrm{Ni}$ was observed. Fig. I-29 shows a mass spectrum measured at $\theta_{\mathrm{cm}}=140^{\circ}$. The energy - and angle-integrated cross sections for the one- to six-neutron transfers show an exponential decrease with a reduction in cross sections per transferred neutron by factors which change from 4.0 (at $160 \mathrm{MeV}$ ) to 5.3 (at $150 \mathrm{MeV}$ ). No enhancement of the energy-integrated cross sections for $2 n, 4 n$ and $6 n$ transfer with respect to the $1 n, 3 n$ or $5 n$ yields is observed.

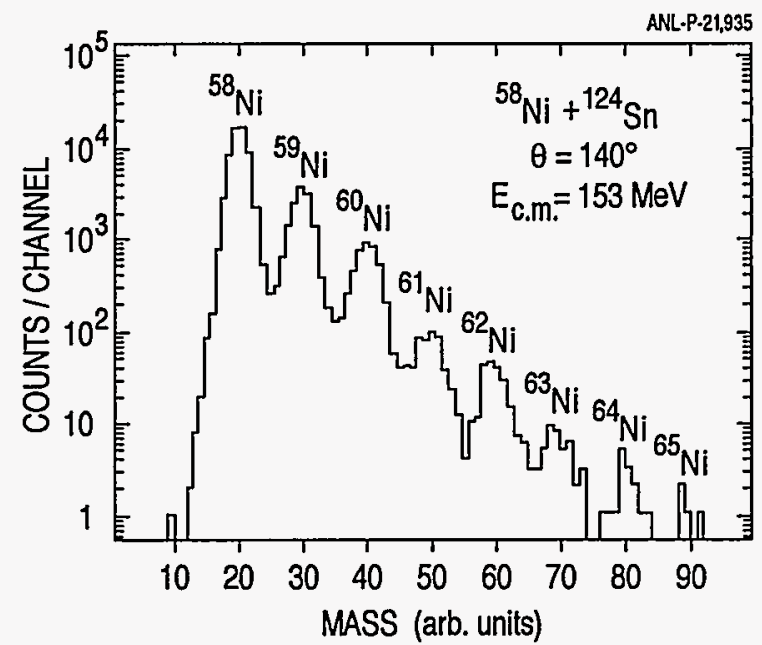

Fig. 1-29. Mass spectrum measured at $E_{c m}=153$ $\mathrm{MeV}$ and $\theta_{\mathrm{cm}}=140^{\circ}$ in the system ${ }^{58} \mathrm{Ni}+124 \mathrm{Sn}$ with the focal plane detector and the split-pole spectrograph. 
a.11. Deep-Inelastic Scattering near the Coulomb Barrier (J. Gehring, B. Back, K. Chan,* M. Freer, $\dagger$ D. Henderson, C. L. Jiang, K. E. Rehm, J. P. Schiffer, M. Wolanski, and A. Wuosmaa)

Deep-inelastic scattering is a process that has traditionally been explained in terms of frictional forces which operate in a nuclear collision. These frictional forces become negligible at bombarding energies below the Coulomb Barrier due to a decrease in the product of the nuclear overlap and the relative velocity between the two nuclei. Hence, the observation of deep-inelastic scattering at energies near and below the Coulomb barrier requires a qualitatively different explanation.

We made measurements of the angular distributions, mass distributions, and total cross-sections for deepinelastic scattering in two $\mathrm{Xe}+\mathrm{Ni}$ systems $\left({ }^{124} \mathrm{Xe}+\right.$ ${ }^{58} \mathrm{Ni}$ and $\left.{ }^{136} \mathrm{Xe}+{ }^{64} \mathrm{Ni}\right)$ at five energies near and below the barrier. The experiments were performed with inverse kinematics using large-area $x-y$ positionsensitive parallel-grid avalanche detectors in a kinematic coincidence setup. A mass resolution $\Delta \mathrm{m}=1 \mathrm{u}$ and a Q-value resolution of $\sim 5 \mathrm{MeV}$ was achieved. Fig. I-30 shows $\mathrm{Q}$-value vs mass spectra measured in the systems ${ }^{124} \mathrm{Xe}+{ }^{58} \mathrm{Ni}$ and ${ }^{136} \mathrm{Xe}+{ }^{64} \mathrm{Ni}$, respectively. The various components originate from projectile- and target-like reaction products $(A=130$ and $A=60)$ and from fission fragments $(A=100)$. Because of the use of inverse kinematics, the present measurements allowed a more detailed study of the angular and mass distributions for deep-inelastic scattering. These results and the total cross-sections are presently being compared with new theoretical calculations. These data will be a part of the thesis of J. Gehring.

*Yale University, †University of Birmingham, United Kingdom
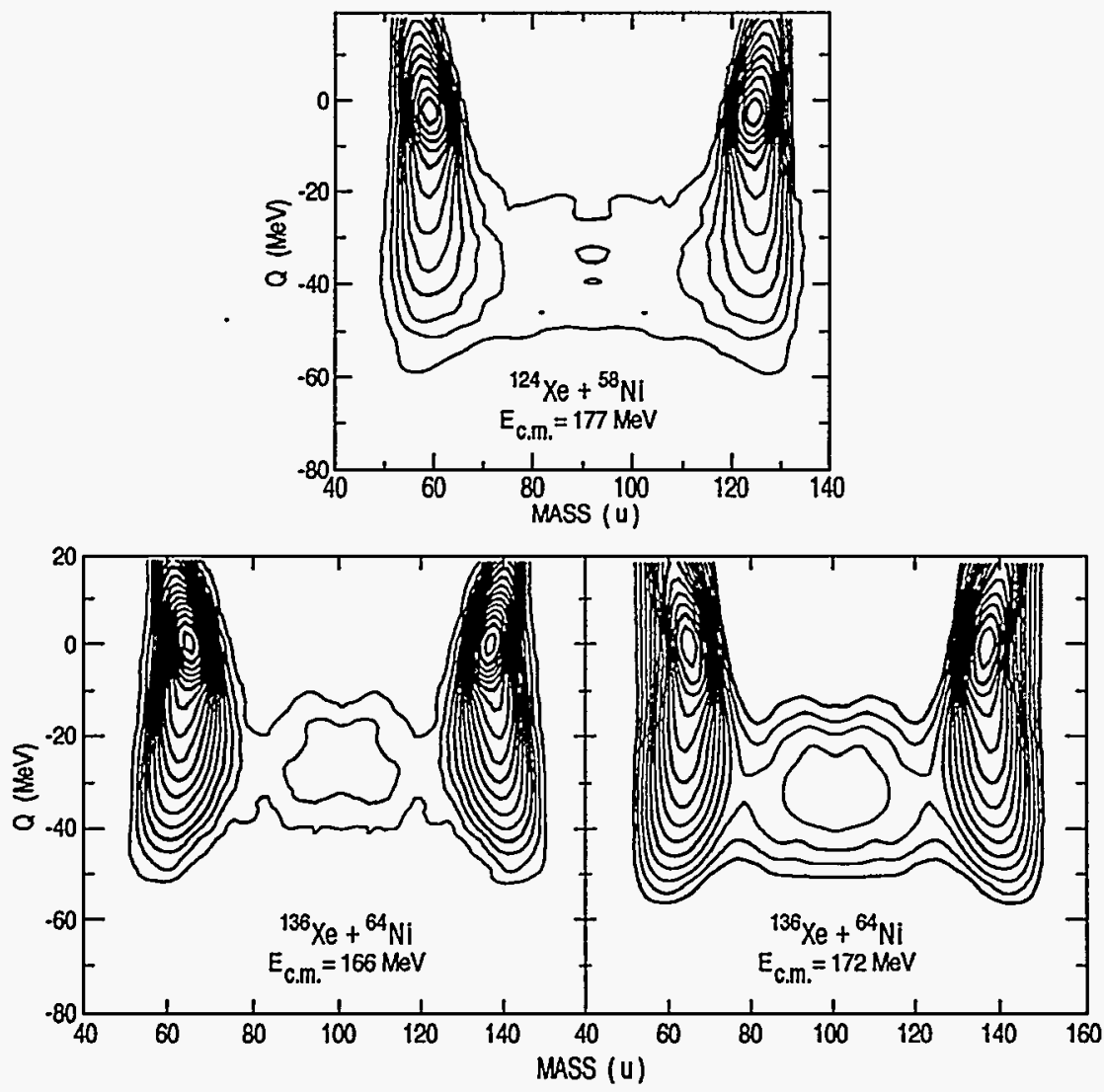

Fig. 1-30. Two-dimensional histograms of counts vs mass and $Q$-value for ${ }^{136} \mathrm{Xe}+{ }^{64} \mathrm{Ni}$ at CM energies of $166 \mathrm{MeV}$ and $172 \mathrm{MeV}$ and for ${ }^{124} \mathrm{Xe}+58 \mathrm{Ni}$ at $176 \mathrm{MeV}$. Two components are visible in mass, one peaked around the target and projectile masses and a broad distribution around half the mass of the composite system. 


\section{a.12. Production of Nuclei in the $\mathbf{N}<126$ and $Z>82$ Region (D. J. Blumenthal, D. Seweryniak, C. N. Davids, C. J. Lister, K. E. Rehm, S. J. Freeman,* J. L. Durrell,* M. J. Leddy,* A. G. Smith,* and R. B. E. Taylor*)}

In pursuit of deformation effects in light pre-actinide systems, the production of isotopes heavier than radon is needed. The evaporation residue cross sections in such systems are severely limited by rapidly increasing fission competition. The fusion of two heavy ions is known to depend on several competing factors and it is useful to draw on the experience built up over many years in the manufacture of isotopes with the very highest atomic numbers. Several influences were identified arising from the production reaction mechanisms and energetics, dissipative processes and the characteristics of the evaporation process following fusion. These indicate that so-called cold fusion reactions facilitate the production of heavy elements. The application of such reactions is therefore sensible to the problem of production of isotopes away from stability in the light preactinide region. The strategy for production appears to be to minimize the excitation energy of the system to reduce fission competition and enhance shell stabilization, while choosing a reaction where extra-push effects are small and the nuclear structure of the reacting partners produces large subbarrier enhancement. Such enhancement facilitates the use of even lower beam energies reducing the excitation energy further.

A reasonable choice of reaction would be the nearsymmetric reactions of the $\mathrm{Ru}$ isotopes in order to produce very neutron-deficient radium compound systems. These reactions are expected to exhibit strong sub-barrier effects via the coupling to low-lying phonon states with large vibrational B(E2) strengths. Although still small in comparison to heavy-ion fusion cross sections above the Coulomb barrier, the excitation energies of the system at sub-barrier energies should be low enough that fragmentation between many evaporation channels is not expected. In fact, measurements of similar systems observed residue production cross sections of the order of a millibarn. It is expected that such systems might experience extrapush effects, however, the results of previous measurements indicate that despite these effects there is still cross section at sub-barrier energies which might be employed in the production of cold residues.

An experiment was performed using ${ }^{104} \mathrm{Ru}$ beams to bombard targets of $104,102 \mathrm{Ru}$ and $102 \mathrm{Mo}$. Recoiling residues were mass analyzed by the FMA and transported to the focal plane where they were implanted in a double-sided silicon strip detector. Particular nuclides were identified by their alpha-decay characteristics. Although some experimental difficulties associated with preparation of ruthenium targets were encountered, several data sets were collected at energies below and at the Bass barrier. Analysis is underway to assess the yield and production cross sections for residues of interest. However, a preliminary study indicates a substantial amount of scattered beam was detected at the focal plane which lowers the sensitivity to fusion products produced at low cross section. The scattered beam could be substantially suppressed by measurement of the time-of-flight of residues in transit through the spectrometer, and an improved experiment is being designed.

*University of Manchester, United Kingdom

\section{a.13. Study of the ${ }^{9}$ Li(d,p) Reaction (A. H. Wuosmaa, J. J. Kolata, ${ }^{*}$ M. Belbot, $\dagger$ K. Lamkin, $\dagger$ P. Santi,* J. Schwarzenberg,* and B. Sherrill $\dagger$ )}

The behavior of the so-called "halo" nucleus ${ }^{11} \mathrm{Li}$ has attracted a great deal of attention recently. This nucleus is of particular interest due to the extended two-neutron halo that arises from the small binding energy of the two neutrons outside the ${ }^{9} \mathrm{Li}$ core. The nucleus becomes bound with respect to neutron emission only due to the interaction of the two neutrons; in fact the nucleus ${ }^{10} \mathrm{Li}$ is unbound to neutron decay. One of the ingredients needed in a theoretical description of the structure of ${ }^{11} \mathrm{Li}$ is the level structure of the unbound ${ }^{10} \mathrm{Li}$ near threshold, which can be used to described the $\mathrm{n}+{ }^{9} \mathrm{Li}$ interaction. An $\mathrm{n}+{ }^{9} \mathrm{Li}$ resonance is expected, however, its energy and spin are somewhat uncertain. One experimental method for examining this resonance is to populate ${ }^{10} \mathrm{Li}$ via the ${ }^{9} \mathrm{Li}(\mathrm{d}, \mathrm{p}){ }^{10} \mathrm{Li}$ reaction. $\mathrm{A}$ test measurement to study this reaction was carried out at the NSCL at Michigan State University, in inverse kinematics using a ${ }^{9} \mathrm{Li}$ beam and a $\mathrm{CD} 2$ target, at a 
beam energy of $72 \mathrm{MeV}$. The ${ }^{9} \mathrm{Li}$ nuclei from the decaying ${ }^{10} \mathrm{Li}$ populated in the reaction, as well as those from elastic scattering, are detected in large annular silicon strip detectors at forward angles. The recoil protons, whose energies are used to separate elastic and inelastic scattering from the transfer reaction

*Univ. of Notre Dame, †Michigan State University are detected at backward angles. The test run demonstrated the feasibility of transmitting a low energy ${ }^{9} \mathrm{Li}$ beam from the $\mathrm{A} 1200$ separator at NSCL.

Another experiment is planned using a more efficient recoil detector arrangement, as well as tracking detectors to isolate the trajectories of the incident ${ }^{9} \mathrm{Li}$ ions. It is hoped that this experiment will be able to identify the near-threshold resonance in ${ }^{10} \mathrm{Li}$, and be able to yield some information on its energy, width, and angular momentum properties.

\section{SUPERCRITICAL FIELDS AND OTHER TOPICS}

A major effort was mounted in the area of the physics associated with the strong fields produced in near-barrier collisions of very heavy ions such as $U+T h$. A new experiment (APEX), led by the Argonne group, was designed and constructed to study the phenomena hinted at by the results of experiments carried out over the past decade at GSI. Coupled with the completion of the ATLAS U-upgrade, APEX moved from the construction and testing phase to data production about 2 years ago. Several highly successful runs were carried out, resulting in data sets for positron-electron pairs far greater than any of the published results. Many aspects of these data were analyzed and the first results were published. For the $U+T h$ and $U+T a$ systems, no sharp $\mathrm{e}^{+}-\mathrm{e}^{-}$sum-energy lines were found, in contradiction with the earlier GSI results. Upper limits on production cross sections for these type of events are 10 to 100 times smaller than the values that can be deduced from the earlier reports.

In addition to the studies of strong fields and research programs outlined in previous sections, some effort devoted to other topics is also contained in this section. One of these efforts relates to the behavior of cooled beams of charged particles confined in storage rings and ion traps. This effort takes place both at the theoretical and experimental levels. In particular, measurements are performed at the ASTRID storage ring.

Our measurements of the half-life of ${ }^{44} \mathrm{Ti}$ are continuing with the goal of achieving a precision of $\sim 5 \%$ for this isotope of importance in astrophysics. This section also describes efforts within the PHOBOS Collaboration.

\section{a.1. Studies of Positron Electron Pair Production in ${ }^{238} \mathrm{U}+{ }^{181} \mathrm{Ta}$}

(I. Ahmad, B. B. Back, R. R. Betts, R. W. Dunford, W. Kutschera, C. J. Lister, M. D. Rhein, J. P. Schiffer, P. Wilt, M. Wolanski, A. H. Wuosmaa, S. M. Austin, * F. P. Calaprice, $\nmid$ K. C. Chan,§ A. Chishti,§ P. Chowdhury,§ C. Conner, J. D. Fox, II S. J. Freedman,ll M. Freer,** S. Gazes, ††

J. S. Greenberg,§ A. L. Hallin, \$ T. Happ,§§ N. Kaloskamis,§ E. Kashy,* M. Liu, \$屯 M. R. Maier,II A. Perera,III E. Roa,II T. Trainor,IIII J. S. Winfield,* F. L. H. Wolfs, IIT G. Xu,§ A. Young, $†$ and J. E. Yurkon*)

The analysis of the positron-electron coincidence data from two experiments carried out at APEX, studying the ${ }^{238} \mathrm{U}+{ }^{181} \mathrm{Ta}$ system, is essentially complete.
These experiments, conducted with approximately 1 $\mathrm{mg} / \mathrm{cm}^{2}$ targets, at beam energies of $5.95,6.1$ and 6.3 $\mathrm{MeV} /$ nucleon provided data in which the previously

\footnotetext{
*Michigan State University, $\uparrow$ Princeton University, §Yale University, qFlorida State University, IIUniversity of California, Berkeley, ${ }^{* *}$ University of Birmingham, United Kingdom, ††The University of Chicago, $\ddagger$ Queen's University, Kingston, Ontario, §§GSI, Darmstadt, Germany, đTIUniversity of Rochester, IlllUniversity of Washington
} 


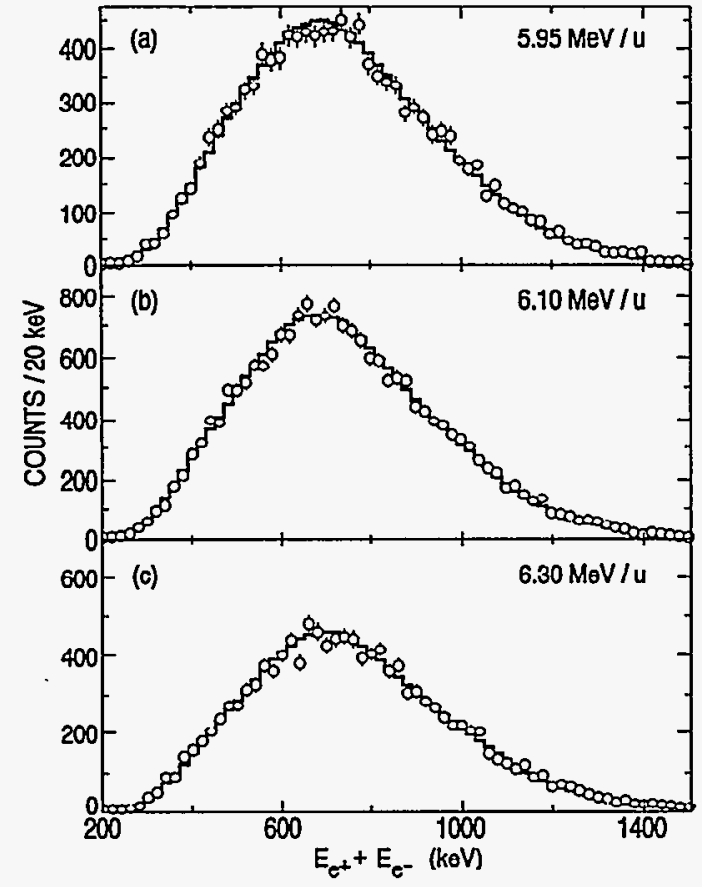

Fig. I-31. Sum-energy spectra for ${ }^{238} U+{ }^{181} T a$ at (a) 5.95, (b) 6.10 , and (c) $6.30 \mathrm{MeV} / u$, analyzed according to the selection on lepton energies for the $748-k e V$ line previously reported. The superimposed histograms represent spectra obtained by event mixing. reported sharp sum-energy lines should appear. Data from earlier experiments show evidence for sharp sumenergy lines at 625,748 and $805 \mathrm{keV}$, observed at bombarding energies from 5.9 to $6.3 \mathrm{MeV} / \mathrm{u}$. The 625- and 809-keV lires display the characteristics of equal-energy back-to-back emission whereas the 748$\mathrm{keV}$ line shows a rather different behavior. In our measurements, average beam currents of 2-3 pnA from the ATLAS accelerator were used to bombard 1$\mathrm{mg} / \mathrm{cm}^{2}$ rolled ${ }^{181} \mathrm{Ta}$ targets, the energy loss in which corresponds to the ranges of bombarding energies over which the sharp sum-energy lines were reported previously.

These data were analyzed extensively, under a variety of conditions which enhance the sensitivity to sharp lines produced under different scenarios. No evidence is found for the sharp sum-energy lines reported previously (see Fig. I-31) and, depending on the scenario assumed for the production mechanism and kinematics of the pairs, upper limits on cross sections at the $99 \%$ confidence limit range from 10100 times smaller than the values that can be deduced from the earlier reports. Furthermore, in all cases, the shape of the sum energy spectrum for the positron-electron pairs may be well reproduced by an event-mixing calculation of the uncorrelated positronelectron background. These results were described in a recent publication.

\section{a.2. Studies of Positron Electron Pair Production in ${ }^{238} \mathrm{U}+{ }^{232} \mathrm{Th}$}

(I. Ahmad, B. B. Back, R. R. Betts, R. W. Dunford, W. Kutschera, C. J. Lister, M. D. Rhein, J. P. Schiffer, P. Wilt, M. Wolanski, A. H. Wuosmaa, S. M. Austin,* F. P. Calaprice, $\dagger$ K. C. Chan, $\S$ A. Chishti, $\S$ P. Chowdhury, $\S$ C. Conner,*** J. D. Fox, II S. J. Freedman,ll M. Freer,** S. Gazes, ††

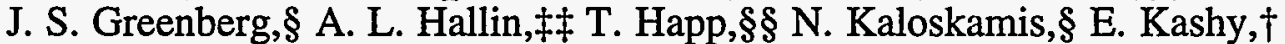
M. Liu, + M. R. Maier,ll A. Perera,ITI E. Roa,II T. Trainor,IIII J. S. Winfield, $\uparrow$ F. L. H. Wolfs, $₫ \mathbb{I}$ G. Xu, $\S$ A. Young, $\dagger$ and J. E. Yurkon $\dagger$ )

Following the non-observation of sharp sum-energy lines in our earlier ${ }^{238} U+{ }^{181}$ Ta measurements, it was decided to pursue measurements of the $238 \mathrm{U}+$ ${ }^{232} \mathrm{Th}$ system which, in the previously published work, showed the most striking evidence for nearequal-energy back-to-back pairs leading to sharp sumenergy lines. Following the refurbishing of the
APEX silicon arrays and extensive tests of the rotating target wheel assembly, a major positron run took place in November 1994. Rolled $1-\mathrm{mg} / \mathrm{cm}^{2}$ $232 \mathrm{Th}$ targets were bombarded with $5.95-\mathrm{MeV} / \mathrm{u}$ ${ }^{238} \mathrm{U}$. The target rotation allowed up to $2 \mathrm{pnA}$ of beam to be used without serious deterioration of the targets.

*Michigan State University, †Princeton University, §Yale University, IFlorida State University, IIUniversity of California, Berkeley, ${ }^{* *}$ University of Birmingham, United Kingdom, $\dagger \dagger$ The University of Chicago, $\ddagger$ Queen's University, Kingston, Ontario, §§GSI, Darmstadt, Germany, ITIUniversity of Rochester, IIIIUniversity of Washington, ${ }^{* * *}$ University of Illinois at Chicago 
Over $300,000 \mathrm{e}^{+}+\mathrm{e}^{-}$pairs were accumulated, representing an order-of-magnitude improvement in statistics over the previously published results.

The initial analysis of these coincidence data is now effectively complete. No evidence was found for positron-electron sum energy lines with characteristics similar to those described in earlier work, nor were sharp lines observed under any of a large number of analysis conditions. The coincidence spectra are entirely consistent with completely uncorrelated positron-electron pairs, and are well described by an event-mixing calculation of the uncorrelated positronelectron sum-energy spectrum. Two analyses designed to study the suggested scenario of a decaying light neutral particle were quantitatively examined. Using an analysis described in the literature, as well as one applying more stringent cuts on the data, specifically designed to be sensitive to positronelectron coincidences with the kinematics expected for particle decay, we established production cross section limits that are on the order of one to two orders of magnitude smaller than those previously reported as shown in Fig. I-32. These results were described in a recent publication.

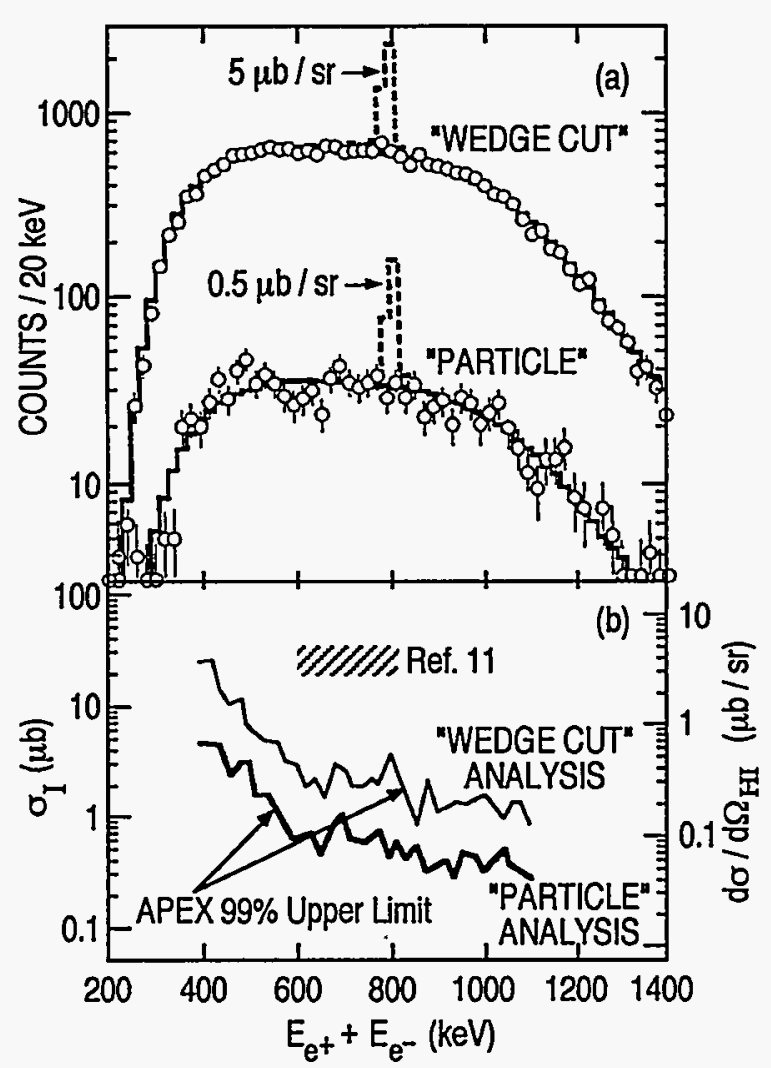

Fig. I-32. (a) Sum-energy spectra for ${ }^{238} U+{ }^{232} \mathrm{Th}$ at $5.95 \mathrm{MeV} / \mathrm{u}$ analyzed according to the expectations for the isotropic decay of a particle produced at rest in the center of mass $(\beta=0.06)$, selected on positron and electron energies (wedge cut), and further selected on correlated energies and azimuthal angles (particle). The superimposed curves correspond to event-mixed spectra. The expected additional yields for a $1.8 \mathrm{MeV} / \mathrm{c}^{2}$ particle, produced with cross sections of 5 and $0.5 \mu \mathrm{b} / \mathrm{sr}$, respectively, are shown dashed. (b) Upper limits (99\% C.L.) for the cross section derived from the wedge cut and particle analyses as a function of sum energy. The shaded area indicates the energy range of peaks and the level of cross section from previous work.

\section{a.3. Dynamic Positron Production in ${ }^{238} \mathrm{U}+{ }^{181} \mathrm{Ta}$ and ${ }^{238} \mathrm{U}+{ }^{232} \mathrm{Th}$}

Collisions (I. Ahmad, B. B. Back, R. R. Betts, R. W. Dunford, W. Kutschera, C. J. Lister, M. D. Rhein, J. P. Schiffer, P. Wilt, M. Wolanski, A. H. Wuosmaa, S. M. Austin,* F. P. Calaprice, $\dagger$ K. C. Chan, $\S$ A. Chishti, $\S$ P. Chowdhury, $\S$ C. Conner,*** J. D. Fox, II S. J. Freedman,ll M. Freer,** S. Gazes, $\uparrow \dagger$ J. S. Greenberg,§ A. L. Hallin, $\ddagger$ T. Happ, $\S$ N. Kaloskamis,§ E. Kashy,* M. Liu, + M. R. Maier,ll A. Perera,III E. Roa,II T. Trainor,Illl J. S. Winfield,* F. L. H. Wolfs, III G. Xu,§ A. Young, † and J. E. Yurkon*)

There exist two dominant mechanisms by which positrons can be produced in the collisions of heavy ions with high charge. The first, Internal Pair
Conversion (IPC) of high-energy gamma-ray transitions in either of the two colliding nuclei, is a conventional and well understood process. The second, so-called "dynamic" production mechanism,

*Michigan State University, †Princeton University, §Yale University, qFlorida State University, IIUniversity of California, Berkeley, **University of Birmingham, United Kingdom, $\dagger \dagger$ The University of Chicago, $\ddagger$ Queen's University, Kingston, Ontario, §§GSI, Darmstadt, Germany, TIUniversity of Rochester, IllIUniversity of Washington, ***University of Illinois at Chicago 
arises from the strong, time-varying Coulomb fields which exist for short times during these collisions. In this process, the virtual photons can excite positron-electron pairs directly from the vacuum. The probability for the production of a positron in a given heavy-ion collision depends on the combined charge $Z$ (tot) of the system, the energies of emitted positrons, and the properties of the nuclear reaction such as the distance of closest approach for the two ions, as well as the effective duration of the collision. In previous experiments for different systems, there has been a wide range of level of agreement between experiment and theory, ranging from nearly perfect agreement, to experiments exceeding theory by more than a factor of two.

Analysis of positron singles data from ${ }^{238} \mathrm{U}+{ }^{181} \mathrm{Ta}$ and ${ }^{238} \mathrm{U}+{ }^{232} \mathrm{Th}$ collisions is currently underway, in combination with the gamma-ray spectra obtained during these experiments with high purity $\mathrm{Ge}$ detectors. The extracted dynamic spectra are then compared to the predictions of a coupled-channels model of the reaction. Preliminary results are that the theory and experimental data for the ${ }^{238} \mathrm{U}+{ }^{181} \mathrm{Ta}$ system agree on approximately the $10 \%$ level. Efforts to refine these results are in progress.

a.4. Monte Carlo Studies of APEX (I. Ahmad, B. B. Back, R. R. Betts, R. W. Dunford, W. Kutschera, C. J. Lister, M. D. Rhein, J. P. Schiffer, P. Wilt, M. Wolanski, A. H. Wuosmaa, S. M. Austin,* F. P. Calaprice, $\dagger$ K. C. Chan, $\S$ A. Chishti,§ P. Chowdhury, § C. Conner,*** J. D. Fox,II S. J. Freedman,II M. Freer, ${ }^{* *}$ S. Gazes, $\uparrow \dagger$ J. S. Greenberg, $\S$ A. L. Hallin, $¥ \div$ T. Happ, $\S \S$

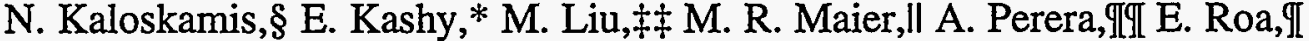
T. Trainor,IIII J. S. Winfield,* F. L. H. Wolfs, ItI G. Xu,§ A. Young, † and J. E. Yurkon*)

One of the most important elements in the evaluation of the data obtained from the various APEX measurements is the understanding of the acceptance and response of the apparatus. This understanding requires detailed simulation of the response of the spectrometer to various sources, as well as a simulation of predicted signals expected in in-beam data for specific, well-defined production scenarios. These simulations were carried out using the codes EGS and GEANT. In each case, as many important components of the apparatus and detector system as possible were considered. The results of these simulations were compared to data obtained with conversion electron sources, positron emitting sources (e.g. ${ }^{68} \mathrm{Ge}$ ), and to positron-electron pair emitting sources (e.g. ${ }^{90} \mathrm{Y}$ ). In each case, the agreement between simulation and data in absolute efficiency as well as in the shapes of the energy and angle acceptance, is excellent. Another important comparison is the simulation of electrons and positrons from an internal pair converting transition in $206 \mathrm{~Pb}$, populated in Coulomb excitation (see D.6). Here again, the agreement is excellent.

The confidence gained from these simulations allows us to establish, from our in-beam positron-electron coincidence data, production limits for electronpositron coincidence lines arising from different, welldefined scenarios. In the specific case of ${ }^{238} \mathrm{U}+$ $232 \mathrm{Th}$, where it was suggested previously that the observed lines corresponded to the decay of a light neutral particle with mass $1.5-2.0 \mathrm{MeV} / \mathrm{c}^{2}$, the limits we obtain are one to two orders of magnitude below the production cross sections reported in the literature.

\footnotetext{
*Michigan State University, †Princeton University, §Yale University, IFFlorida State University, IIUniversity of California, Berkeley, **University of Birmingham, United Kingdom, ††The University of Chicago, $\ddagger \ddagger Q u e e n ' s$ University, Kingston, Ontario, §§GSI, Darmstadt, Germany, GIIUniversity of Rochester, IIIIUniversity of Washington, ***University of Illinois at Chicago
} 
a.5. Silicon Detector Timing Measurements at APEX (I. Ahmad, B. B. Back,

R. R. Betts, R. W. Dunford, W. Kutschera, C. J. Lister, M. D. Rhein, J. P. Schiffer,

P. Wilt, M. Wolanski, A. H. Wuosmaa, S. M. Austin,* F. P. Calaprice, + K. C. Chan,

A. Chishti, $\ddagger$ P. Chowdhury, $\ddagger$ C. Conner, $* * *$ J. D. Fox,II S. J. Freedman,ll M. Freer,**

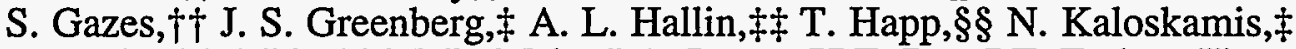

E. Kashy,* M. Liu, $\neq$ M. R. Maier,\|l A. Perera,III E. Roa, TI T. Trainor,IIII

J. S. Winfield, * F. L. H. Wolfs, III G. Xu, A. Young,† and J. E. Yurkon*)

One of the very few remaining unresolved questions regarding the performance of the APEX spectrometer is the intrinsic timing resolution that can be obtained using the current silicon detector arrays and associated electronics. Previous estimates were obtained from indirect measurements, based upon comparisons to Monte Carlo simulations. Previously, a timing source was used which signaled the time of emission of a ${ }^{137} \mathrm{Cs}$ beta-decay electron and provided a time reference with which to compare the timing response of the silicon detectors. The chief difficulty in this measurement lies in the fact that for a broad range of lepton energies and angles, many trajectories with different flight times will reach the silicon detector arrays. Recently the situation was improved with the implementation of a timing source utilizing an avalanche photo-diode (APD). The APD detects the
Auger electrons produced in coincidence with conversion electrons from a ${ }^{113} \mathrm{Sn}$ source. The conversion electrons are detected in the silicon arrays. The intrinsic timing resolution of the APD is less than $1 \mathrm{~ns}$ for the $\sim 50 \mathrm{keV}$ Auger electrons. In addition, the source is fitted with a collimating mask which passes electrons only at specific angles $(\sim 50$ degrees relative to the solenoid axis), so that the electron trajectories are well defined and the timing response is single valued for a given element on the silicon array. These measurements showed that the resolution attainable, previously thought to be on the order of 4 to $6 \mathrm{~ns}$, is actually substantially better, on the order of 2 to $3 \mathrm{~ns}$. This result has important implications for the angle reconstruction procedure for APEX lepton data, in particular for Doppler reconstruction of known internal pair converting transitions (see D.6.).

*Michigan State University, †Princeton University, $¥$ Yale University, IFlorida State University, IIUniversity of California, Berkeley, **University of Birmingham, United Kingdom, $\dagger \dagger$ The University of Chicago, $\ddagger$ Queen's University, Kingston, Ontario, §§GSI, Darmstadt, Germany, ITIUniversity of Rochester, IIIIUniversity of Washington, ${ }^{* * *}$ University of Illinois at Chicago

a.6. Measurements of Internal Pairs from 206Pb (I. Ahmad, B. B. Back, R. R. Betts, R. W. Dunford, W. Kutschera, C. J. Lister, M. D. Rhein, J. P. Schiffer, P. Wilt, M. Wolanski, A. H. Wuosmaa, S. M. Austin,* F. P. Calaprice, † K. C. Chan, $\ddagger$ A. Chishti, $\ddagger$ P. Chowdhury, $\ddagger$ C. Conner,*** J. D. Fox, $\$$ S. J. Freedman,I M. Freer, $* *$

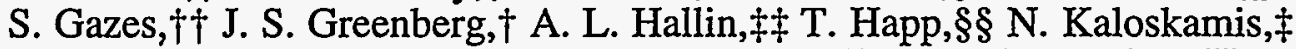
E. Kashy,* M. Liu, + M. R. Maier, T A. Perera, III E. Roa,II T. Trainor, IIII J. S. Winfield,* F. L. H. Wolfs, III G. Xu, $\ddagger$ A. Young, $\ddagger$ and J. E. Yurkon*)

The failure to observe sharp sum-energy lines in measurements of ${ }^{238} \mathrm{U}+{ }^{181} \mathrm{Ta}$ and ${ }^{238} \mathrm{U}+{ }^{232} \mathrm{Th}$ raises the issue of the correct functioning of APEX under in-beam conditions. Extensive measurements with electron, and positron-electron pair emitting sources were used to demonstrate the resolution and acceptance of APEX, but the possibility remains that some unforeseen background or other effects might compromise the ability to see peaks in-beam. In order to test the functioning of APEX under the most stringent conditions, we also performed a measurement of internal pairs produced in the decay of the $2.648-\mathrm{MeV} \mathrm{3}^{-}$state in ${ }^{206} \mathrm{~Pb}$ to the $2^{+}$state at $0.803 \mathrm{MeV}$. The $3^{-}$state was excited in the

\footnotetext{
*Michigan State University, †Princeton University, $¥$ Yale University, §Florida State University, IUUniversity of California, Berkeley, **University of Birmingham, United Kingdom, $\dagger \dagger$ The University of Chicago, $\ddagger \ddagger Q u e e n ' s$ University, Kingston, Ontario, §§GSI, Darmstadt, Germany, IIIUniversity of Rochester, IlllUniversity of Washington, ***University of Illinois at Chicago
} 


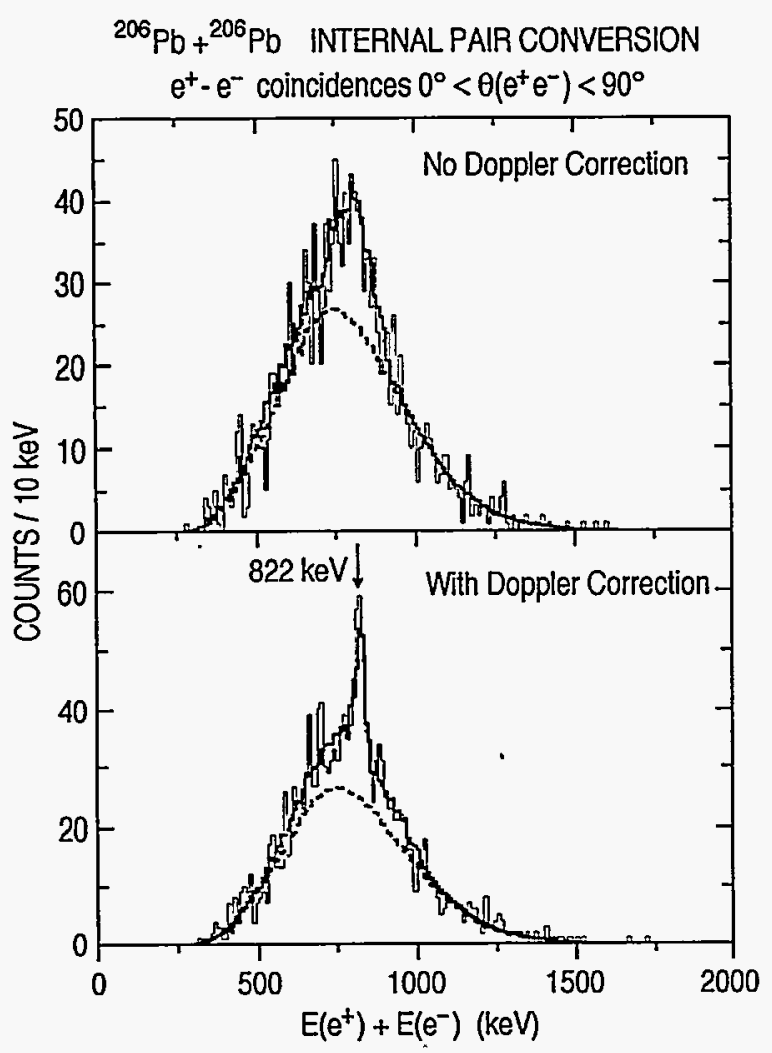

Fig. 1-33. (a) Measured $e^{+}-e^{-}$sum energy spectra with no Doppler correction applied (light histogram). The dark histogram is the sum of a simulated IPC transition added to a model of the uncorrelated $e^{+}-e^{-}$ background (dashed histogram). (b) The same as (a), with Doppler corrections applied to the energies of the positron and electron, clearly showing the 822$\mathrm{keV}$ peak from the pair conversion of the $1844-\mathrm{keV}$ El transition in $206_{P b}$.
${ }^{206} \mathrm{~Pb}+206 \mathrm{~Pb}$ reaction at $5.9 \mathrm{MeV} / \mathrm{u}$ with a cross section of roughly $40 \mathrm{mb}$, resulting in an expected pair cross section of approximately 16 microbarns. It should be emphasized that this measurement represents a much stricter test of the functioning of the apparatus than the observation of sum-energy lines would represent, as the internal pair measurement requires a Doppler-shift correction of the lepton energies before the peak corresponding to the pair transition can be observed.

These data have been fully analyzed and the expected peak corresponding to the $822-\mathrm{keV}$ pair transition is clearly observed (see Fig. I-33). Using the GEANT Monte-Carlo codes adapted for APEX, this transition was simulated. In these calculations, the realistic silicon-detector time resolution determined from recent measurements (see D.5) has been included. The calculated shapes of the positron-electron pair spectra are in excellent agreement with their measured counterparts, for both the situations in which the lepton energies were, or were not corrected for Doppler shift. The strength of the calculated pair transition was adjusted to match the yield expected from the calculated Coulomb excitation cross section for the $3^{-}$state in $206 \mathrm{~Pb}$, combined with the theoretical pair conversion coefficient, and was also in excellent agreement with the data. These results convincingly demonstrate the proper functioning of APEX in an in-beam environment, as well as the ability to calculate the absolute value of the pair detection efficiency for a well-known pair-production scenario.

\section{a.7. Spectroscopy of High-Lying States in Actinide Nuclei (I. Ahmad,}

B. B. Back, R. R. Betts, M. P. Carpenter, B. Crowell, R. W. Dunford,

R. V. F. Janssens, T. L. Khoo, W. Kutschera, T. Lauritsen, C. J. Lister, M. D. Rhein, J. P. Schiffer, P. Wilt, M. Wolanski, A. H. Wuosmaa, A. Aprahamian,*

S. M. Austin, $\uparrow$ F. P. Calaprice $\neq$ K. C. Chan,$\S$ A. Chishti, $\S$ P. Chowdhury, $\S$

C. Conner, $\dagger \dagger \dagger$ J. D. Fox, II S. J. Freedman,II M. Freer, $\dagger \dagger$ U. Garg, * S. Gazes, $\ddagger$

J. S. Greenberg,§ A. L. Hallin, §§ T. Happ, $\mathbb{I I I}$ N. Kaloskamis, § E. Kashy, $\dagger$

M. Liu,§§ M. R. Maier,Il E. F. Moore,IIII A. Perera,** E. Roa,II T. Trainor,***

J. S. Winfield, $\uparrow$ F. L. H. Wolfs, ${ }^{* *}$ G. Xu, $\S$ A. Young, $\ddagger$ and J. E. Yurkon $\dagger$ )

In the course of studying positron-electron production during the collisions of very heavy ions, gamma ray spectra were accumulated, with the intent of monitoring the target condition, the efficiency of the heavy-ion counters, and to measure the total gammaray flux. However, during these measurements a series of new high-energy transitions were

*University of Notre Dame, †Michigan State University, $\ddagger$ Princeton University, §Yale University, qFlorida State University, IIUniversity of California, Berkeley, ${ }^{* *}$ University of Rochester, $\dagger+$ University of Birmingham, United Kingdom, The University of Chicago, §Queen's University, Kingston, Ontario, TIIGSI, Darmstadt, Germany, IIIINorth Carolina State University, $* * *$ University of Washington, $+\dagger \dagger$ University of Illinois at Chicago 
located in uranium, tantalum and thorium. To confirm the origin of these states and understand their nuclear structure, a separate series of experiments was conducted.

The studies used large $(>55 \%)$ Ge detectors mounted in the APEX chamber. States in ${ }^{238} \mathrm{U}$ and ${ }^{232} \mathrm{Th}$ were Coulomb excited using a ${ }^{208} \mathrm{~Pb}$ beam of 5.8 $\mathrm{MeV} / \mathrm{u}$. Heavy ions were detected in the large-area APEX multiwire proportional counters. The extensive beam monitoring of the APEX setup allowed precise normalization and accurate crosssection determinations. The precision of these absolute measurements was checked in a determination of the population of states in $206 \mathrm{~Pb}$, for which experiment and calculation (using known matrix elements) agreed at better than the $10 \%$ level. The Doppler shifts from upstream and downstream detectors permitted a precise confirmation of the incident beam energy to less than $0.05 \mathrm{MeV} / \mathrm{u}$.

A strong transition was found in ${ }^{238} \mathrm{U}$ at $1782 \mathrm{keV}$. This is surprising, as most discrete transitions lie below' $1200 \mathrm{keV}$ and at higher energies an exponentially falling continuum of transitions is observed. The impact parameter dependence of the $1782-\mathrm{keV}$ line precluded its origin arising from a transfer reaction. The width of the peak was larger than expected from Doppler reconstruction, indicating that it could be a composite of several transitions. No evidence was found to support the hypothesis that the state from which this gamma ray originates is of two-phonon origin, as no decay branches to the known one-phonon vibrational states could be found.

In ${ }^{232} \mathrm{Th}$ a rather different structure was observed, with many new weak transitions up to $1900 \mathrm{keV}$ being found. Preliminary analysis indicates that the integrated strength of the lines in the $1750-1900$ $\mathrm{keV}$ region in both ${ }^{238} \mathrm{U}$ and ${ }^{232} \mathrm{Th}$ are about $24 \mathrm{mb}$.

A further experiment was performed at Florida State University. ${ }^{238} \mathrm{U}$ was Coulomb excited with a ${ }^{12} \mathrm{C}$ beam to further elucidate the structure of the state from which the $1782-\mathrm{keV}$ transition originates. These data were just collected, but the $1782-\mathrm{keV}$ transition is strong, and the comparison of ${ }^{238} \mathrm{U}$ excitation with photons, ${ }^{4} \mathrm{He},{ }^{12} \mathrm{C}$, and ${ }^{208} \mathrm{~Pb}$ should clarify this interesting nuclear structure problem.

A letter on the IPC decay of ${ }^{206} \mathrm{~Pb}$ and the IPC branches from high-lying states in actinide nuclei is being finalized. In short, it appears that the IPC branches from the new states found in ${ }^{232} \mathrm{Th}$ and ${ }^{238} \mathrm{U}$ cannot ever produce large peaks in summed, Doppler-corrected, electron-positron spectra from our spectrometer. Under the optimal conditions for enhancing the IPC signal relative to background, the IPC peaks lie at the one sigma level.

A further publication of the gamma-ray spectroscopy of high lying states should be completed during the next year, and this will form part of the thesis of one of the APEX students (E. Roa).

\section{a.8. Nature of Ordering in Confined Crystalline Ionic Systems (J. P. Schiffer)}

The configurations of confined cold ions were simulated under a variety of confining geometries from the three-dimensionally isotropic (spherical) -- to the two-dimensional beam-like ones. For all of these, when the number of ions exceeds a certain limit, layered shell structures are seen that follow equipotential lines. Recent experimental work at the NIST Laboratories in Boulder found that this shell structure does not continue indefinitely for very large systems but that in large ion clouds (larger than 50,000 ions) the interior of the cloud seems to contain crystallites that are body-centered cubic (bcc) in character. Such ordering is the lowest state for an infinite Coulombic system. In the past year, a simulation run was started on the ER Cray2 to attempt to reproduce this transition. In the simulations the shells appear gradually from the outer surface inward -- the structure seems to have stabilized in the last several months with a significant 17th shell, but with the 18th shell not clearly discernible. The simulation is continuing and the cooling is at the point where cubic ordering may start to emerge in the center of the cloud within the next few months. 


\section{a.9. The Influence of Repeating Cells on Simulations of Crystallized Beams (J. P. Schiffer)}

Simulations of very cold ion beams were studied by a number of investigators, attempting to include the dynamic conditions in the accelerator lattice. Such simulations, of necessity, must be restricted to a small slice of a beam, where the particles on one end of the slice match into those at the other end. One of the questions is whether this "repeating boundary condition", particularly with small unit cells, can influence the conclusions of the simulations. A systematic study was tried. Figure I-34 shows the effect. The two top boxes show, for a fixed number of particles per unit length, the pattern obtained in a simulation for 15 and for 16 particles in the repeating cell, viewed from the direction of the beam. The plots on the bottom show the arrangement along the beam with the angle the particles make around the beam plotted on the vertical scale (the particles are at the same radius). The bottom left is the same data as the top left for 15 particles; the bottom right shows the differences between 49,50 , and 51 particles in the cell. Such differences are seen in the simulations up to several hundred particles-- for 1000 or more particles per unit cell these differences seem to be lost in thermal and computer noise. Clearly these differences can be crucially important in conclusions reached from silulations regarding the stability of the solutions under dynamic conditions of focusing and bending.
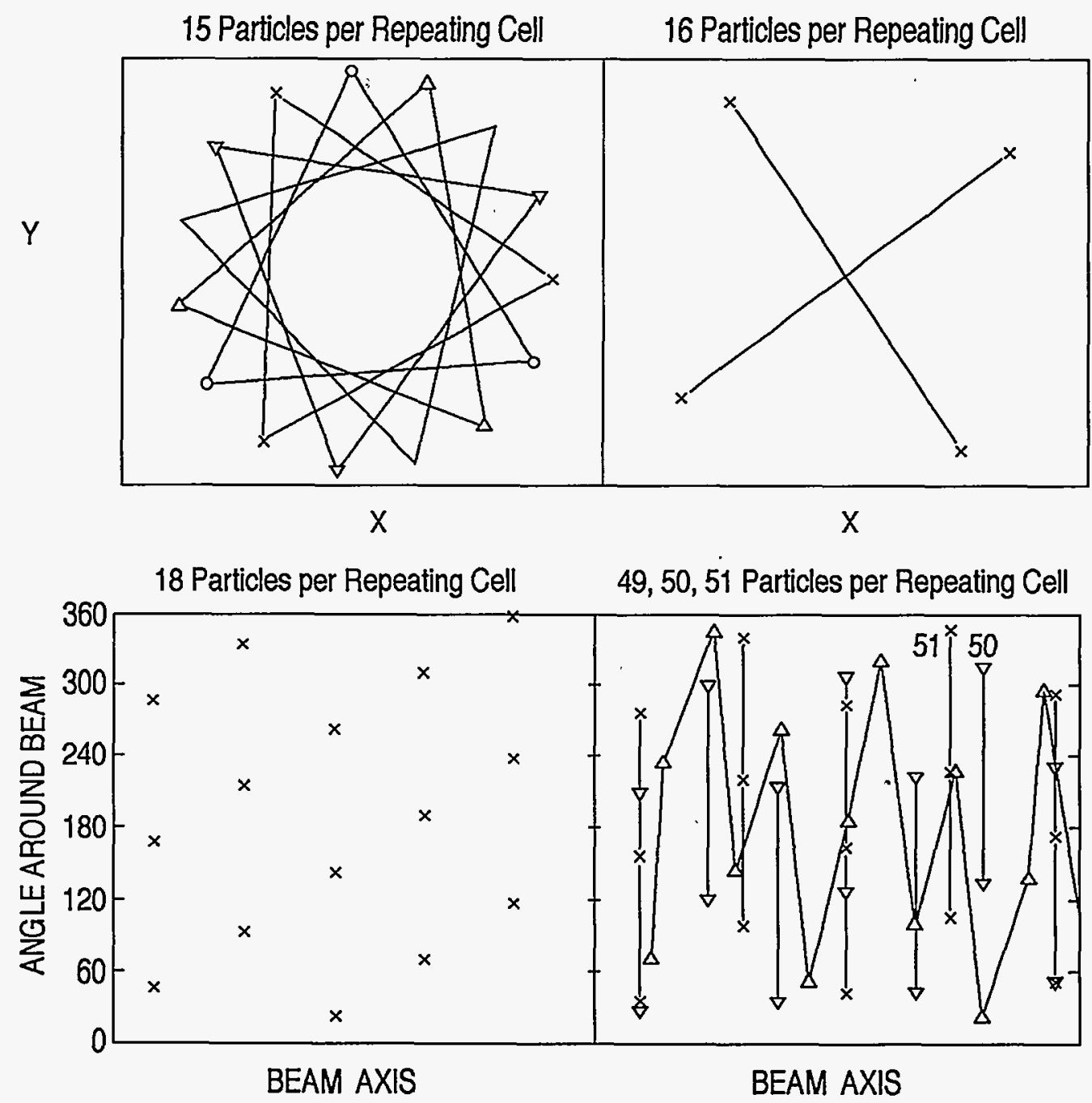

Fig. I-34. Influence on choice of particle number for repeating cells in simulations of crystalline ion beams. 


\section{a.10. Apparent Temperatures of "Cold" Beams (J. P. Schiffer)}

A "cold" beam is normally considered as one where the ions in the beam are all traveling with the same velocity, while transversely the focusing forces maintain a balance against the internal space-charge repulsion within the beam and the particles have zero velocity in the directions perpendicular to the travel. Real focusing forces are periodic in time and thus the beam will undergo coherent transverse oscillations that need not be considered as a random 'temperature', yet any measurement of transverse velocities would see these as an apparent temperature given by

$$
\mathrm{T}_{\text {apparent }} \approx\left(\mathrm{r}_{\text {beam }} / \mathrm{R}_{\text {ring }}\right)^{2} \mathrm{E}_{\mathrm{kin}}
$$

where rbeam $_{\text {is }}$ the radius of the beam (set by space charge in the cold limit) $R_{\text {ring }}$ is the radius of the storage ring. In the "cold" limit this will be on the order of the energy of the beam. Similarly, a 'cold' beam with finite radius (imposed by the space-charge repulsion) in a storage ring must be bent in a closed orbit by magnets. This implies that the lowest state of the beam should be one where the linear velocity of particles has a gradient so that all particles complete an orbit in the same time. Cooling techniques to achieve such a gradient do not yet exist. The coherent velocity spread of the beam would appear as an energy spread or temperature:

$$
\left.\mathrm{T}_{\text {apparent }} \approx 2 v^{4} \beta / \mathrm{N}^{2} \text { period. (rbeam } / \mathrm{R}_{\text {ring }}\right)^{2} \mathrm{E}_{\text {kin }}
$$

where $v \beta$ is the betatron tune (the number of oscillations in the focusing lattice per turn) of the storage ring, and $\mathrm{N}_{\text {period }}$ is the number of focusing cells around the ring. This is of the same order as the above estimate. These coherent kinetic energies with an imperfect beam can be a source of 'heating' (mixing into more random velocities). More quantitative expressions were derived for the kinetic energies contained in these coherent aspects of a stored ion beam.

\section{a.11. Laser-Cooled Ion Beams (J. P. Schiffer, J. S. Hangst,* A. Labrador,* V. Lebedev,* N. Madsen,* J. S. Nielsen,* O. Poulsen,* and P. Shi*)}

The collaboration with the group in Åarhus, Denmark to study the preparation of very cold laser-cooled ion beams with their storage ring ASTRID, continued. During the past year work focused on a better understanding of the transverse properties of a cooled ${ }^{24} \mathrm{Mg}$ beam using the more powerful diagnostics capabilities of a recently purchased CCD camera. Data obtained recently, that are not yet fully analyzed, show the power of this technique in yielding accurate information of the transverse beam size -- and thus of the transverse temperature of the beam. Experimental time has unfortunately been rather limited because of conflicting demands on beam time -- plans are under way to build an additional facility at Åarhus in order to make it possible to extend this class of investigations with very cold ion beams.

*Åarhus University, Denmark 


\section{a.12. Half-Life of ${ }^{44}$ Ti (I. Ahmad, W. Kutschera, ${ }^{*}$ G. Castagnoli, $\dagger$ and M. Paul )}

An accurate value of the ${ }^{44} \mathrm{Ti}$ half-life is needed by cosmologists to determine the production of heavy elements in supernovae. The half-life of ${ }^{44} \mathrm{Ti}$ was measured previously by following its decay rate and by accelerator mass spectrometry. There is a large variation among the published values. For this reason we undertook the measurement of the ${ }^{44} \mathrm{Ti}$ half-life by carefully following the decay of the ${ }^{44} \mathrm{Ti}$ activity. Three sets of samples - a pure $200-\mathrm{nCi}{ }^{44} \mathrm{Ti}$ sample, a $300-\mathrm{nCi}{ }^{60} \mathrm{Co}$ source and a ${ }^{44} \mathrm{Ti}-{ }^{60} \mathrm{Co}$ mixed source of similar strength - were prepared and their spectra are being measured with $\mathrm{Ge}$ spectrometers at Argonne, Torino and Jerusalem. Each sample is measured for a period of 2 days at approximately 4 month intervals. The room background is also measured for the same length of time. Two sets of spectra are being measured at two different source-to-detector distances in order to understand summing and pile-up effects. We have sufficient points to determine the half-life and are currently analyzing the data.

*University of Vienna, Austria, †Instituto di Cosmogeofisica, CNR Torino, Italy, $\ddagger$ Hebrew University, Jerusalem, Israel

\section{a.13. Lifetimes of the Isomeric States in ${ }^{57} \mathrm{Fe}$ and ${ }^{83} \mathrm{Kr}$ (I. Ahmad, K. E. Rehm,} E. P. Kanter, W. Kutschera, W. R. Phillips, * and A. R. Barnett*)

Precise lifetimes of isomeric states are required to refine nuclear model calculations. Isomeric states have usually low-excitation energies and, hence, large internal conversion coefficients. Such isomeric states were used in experiments to measure changes in conversion coefficients with ionic charge $q$. For this reason the half-lives of the $14.4-\mathrm{keV}$ level in ${ }^{57} \mathrm{Fe}$ and the $9.4-\mathrm{keV}$ state in ${ }^{83} \mathrm{Kr}$ were measured by the delayed coincidence technique. In both cases, the high-energy gamma ray was detected with a $25 \% \mathrm{Ge}$ detector and the low-energy gamma ray with a planar Ge detector (LEPS). Our measurements gave a halflife of $99.2(4) \mathrm{ns}$ for the $14.4 \mathrm{keV}$-level in ${ }^{57} \mathrm{Fe}$ and $155.1(12) \mathrm{ns}$ for the $9.4-\mathrm{keV}$ state in ${ }^{83} \mathrm{Kr}$. Results of these measurements were published.

*University of Manchester, United Kingdom

\section{a.14. The PHOBOS Experiment (R. R. Betts, PHOBOS Collaboration*)}

PHOBOS is an experiment designed to study $\mathrm{Au}+$ $\mathrm{Au}$ collisions at RHIC which will produce unprecedented energy densities leading, it is hoped, to new physics of deconfined quarks and gluons. The apparatus consists of a $4 \pi$ multiplicity array and two spectrometer arms and is also equipped with a vertex finding detector and various trigger detectors (as shown in Fig. I-35). The multiplicity detector will measure the rapidity and azimuthal dependence of the number of charged particles on an event-by-event basis allowing the selection of interesting events for further study in the spectrometer arms. The spectrometer arms are optimized for the measurement of low $\mathrm{p}_{\mathrm{t}}$ particles produced near mid-rapidity which are expected to show the greatest sensitivity to collective effects which may characterize new physics. PHOBOS proceeded from the Letter of Intent through
Proposal and Conceptual Design Review to full construction approval. It is expected that PHOBOS will be ready to take data when the first beams from RHIC are available in 1999.

The ANL and UIC groups have overall responsibility for the design and construction of the multiplicity and vertex detectors as well as responsibilities for off-line software.

The PHOBOS multiplicity detector consists of an octagonal array of silicon detectors surrounding the beam pipe between $\mathrm{z}= \pm 52 \mathrm{~cm}$ from the nominal collision vertex. It is comprised of approximately 100 silicon wafers each divided into 64 individual elements and read out with a custom designed preamplifier and ADC. Higher segmentation detectors placed in the immediate vicinity of the collision vertex are used to determine precisely the 


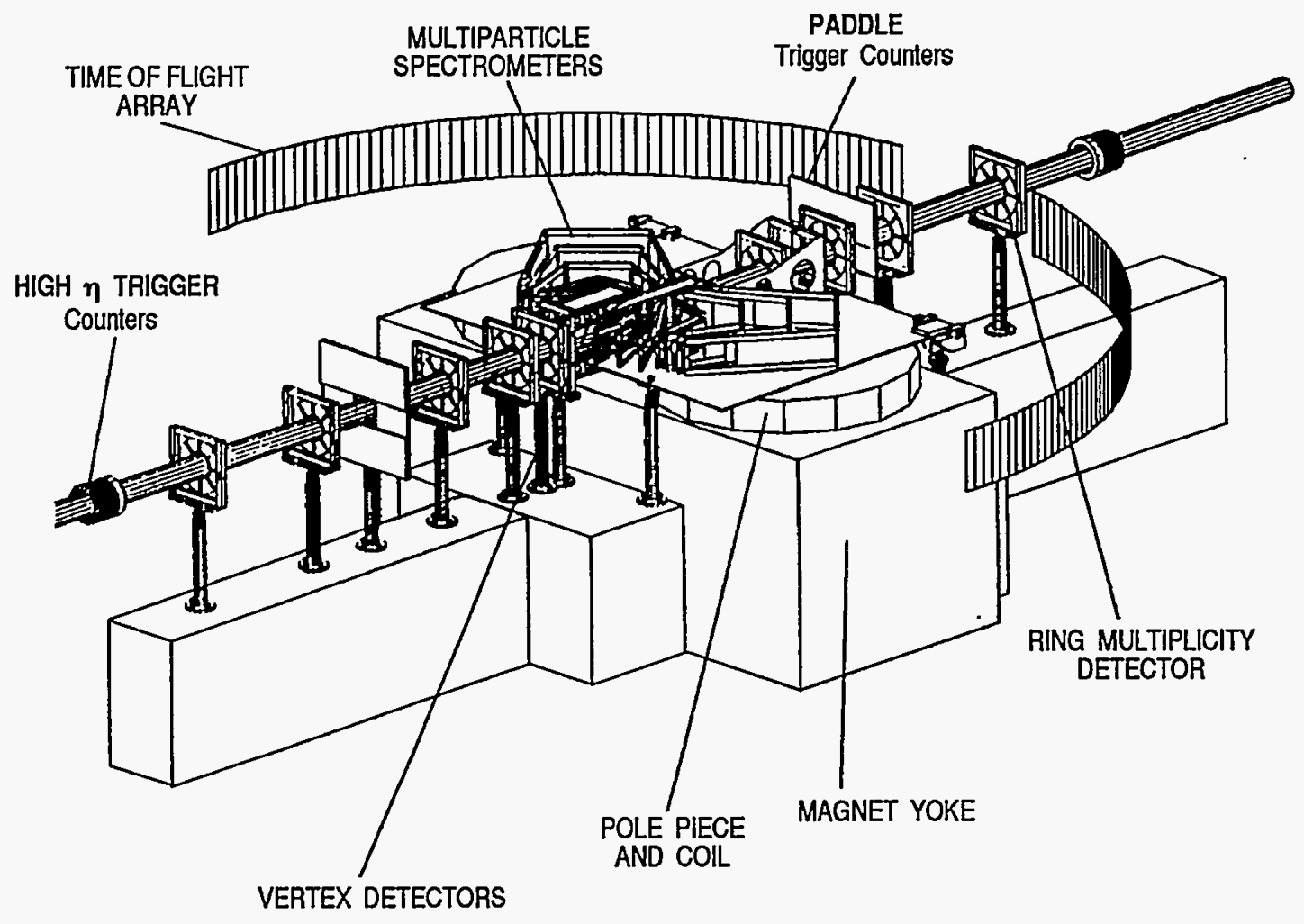

Fig. I-35. Overall view of the PHOBOS Experiment -- the top half of the magnet has been removed.

collision point for each event. Rings of silicon detectors placed at distances of $\pm 107, \pm 223$ and \pm $479 \mathrm{~cm}$ from the nominal collision vertex complete the coverage of the multiplicity array.

Much of the past year was spent modifying the design subject to constraints imposed by the beam pipe, spectrometer arm magnets etc., and we are now close to finalizing this and proceeding to detailed design of the mechanical supports and cooling systems. This will be done in coordination with the engineering group at Krakow. Together with this, the overall design of the silicon detector elements was fixed. Considerable effort went into the general specification of the PHOBOS silicon detectors through internal discussions within the collaboration and in consultation with commercial vendors. The basic concept of reading out each sub-element through strip lines running on top of a second dielectric layer was verified using prototypes supplied by NCU-Taiwan and the final, pre-production, prototypes of some of the PHOBOS detectors are now ordered. We expect to obtain prototypes of the octagon multiplicity elements to test the soundness of the scheme of placing the readout electronics on top of the active face of the detector. All other detector types are similar enough to the successfully produced prototypes that we expect to go straight to production.

We are carrying out tests to explore the possibility of generating a "multiplicity trigger" by reading out the back face of each silicon wafer thus giving a signal proportional to the total multiplicity in that wafer. In this way, from the segmentation given by the wafers themselves, a crude picture of $\partial^{2} \mathrm{M} / \partial \eta \partial \phi$ can be obtained and can be used to generate triggers selected on multiplicity or other features of the event topology such as fluctuations.

*Brookhaven National Laboratory, Institute of Nuclear Physics, Krakow, Poland, Jagiellonian University, Krakow, Poland, Massachusetts Institute of Technology, National Central University, Taiwan, Oak Ridge National Laboratory, University of Illinois at Chicago, University of Maryland, University of Rochester, Yale University 


\section{E. EQUIPMENT DEVELOPMENT AT THE ATLAS FACILITY}

During the past year the development of equipment around the Fragment Mass Analyzer continued at a brisk pace. This is understandable as in excess of $50 \%$ of the experiments run at ATLAS use this instrument. Several new developments range from the development of a very small Faraday cup to intercept the beam at the entrance of the FMA to the development of new detectors for the focal plane of the device such as the Silicon box or an apparatus for the measurement of Coulomb excitation cross sections.

The most important development at the FMA was the installation of a large array of gamma-ray detectors at the target location. AYE-Ball (the Argonne-Yale-European Ball) is an array using as many as twenty gamma-ray spectrometers assembled in a spherical geometry around the FMA target. Up to nine Germanium detectors were of large volume (i.e. 70\% efficient). They were inserted in BGO Compton-suppression shields on loan from the Eurogam collaboration. This collaboration also provided 7 of the large Ge detectors, the two others coming from Yale University and ANL. Nine Compton-suppressed spectrometers with Germanium counters of smaller volume on loan from the TESSA collaboration and from Yale University were also installed, together with two X-ray detectors. A series of thirteen experiments was performed between August and December, at which time the array was dismantled.

In the Gammasphere project, the Argonne Physics Division took responsibility for procuring and testing the BGO Compton suppressor shields, for the design and fabrication of the target chamber, and for part of the software development. Most of these tasks have now come to an end. The last BGO shields were delivered to the Lawrence Berkeley National Laboratory, work on the target chamber only involved minor modifications, and the software effort is essentially complete. The Division is now actively preparing for a move of Gammasphere to ATLAS. A proposal was written and reviewed by an advisory committee appointed by the DOE. The decision was made to move Gammasphere to Argonne beginning in September of 1997 for a minimum of one year of operation at ATLAS.

Development of the LEPPEX detector has begun. This detector consists of an array of gas counters mounted in a barrel assembly around the target, surrounded by $\mathrm{BaF}_{2}$ detectors. The system will investigate dynamics aspects of fusion and fission reactions. Extensive tests of the $\mathrm{BaF}_{2}$ response under various conditions were performed and design studies are under way.

This section also describes other efforts under way in the Division such as those related to radioactive beam experiments at the spectrographs, software and other computing developments in the area of data acquisition and data analysis, and the activities related to target preparation.

\section{a.1. The AYE-Ball (Argonne-Yale-European Gamma-Ray Spectrometer)}

(D. J. Blumenthal, M. P. Carpenter, C. N. Davids, R. V. F. Janssens, C. J. Lister, D. Nisius, D. Seweryniak, B. Field, *S. J. Freeman, $\uparrow$ W. Gelletly, $\ddagger$ P. Regan, $\ddagger$ J. Schwartz, J. Simpson,§ D. D. Warner,§ and P. J. WoodsI)

The AYE-Ball gamma-ray spectrometer was designed for operation with the Fragment Mass Analyzer (FMA) in order to increase the efficiency for massspectrometer-gated prompt spectroscopy by a significant factor. In practice, this factor was about an order-of-magnitude larger than initial experiments in the U.K. a decade ago, but about an order-ofmagnitude less than the efficiency which may be hoped for when operating the largest present array (Gammasphere) with the FMA.

*Oberlin College, †University of Manchester, United Kingdom, łUniversity of Surrey, United Kingdom, $\S$ Daresbury Laboratory, United Kingdom, qUniversity of Edinburgh, United Kingdom 
The concept for such a device was developed in collaboration with a group of scientists from the U.K., who were interested in this area of physics and had access to some of the necessary equipment. The U.K. groups designed the support frame and provided large volume gamma-ray detectors, while the ANL group developed the target chamber and the detector cooling system, designed the electronics and dataacquisition systems and coordinated the overall assembly and operation of the array.

The AYE-Ball spectrometer consisted of up to 9 highpurity intrinsic germanium ( $\mathrm{HpGe}$ ) detectors, each of greater than $70 \%$ relative efficiency, and up to 9 more conventional $25 \% \mathrm{HpGe}$ detectors, all mounted in Compton-suppression shields. Most of the large Ge detectors were on loan from the Eurogam collaboration with others being provided by Yale University and ANL. They were all placed in Eurogam suppression shields. The $25 \%$ detectors and their BGO shields came in part from the old TESSA array (Daresbury) and from Yale University. Arrays

\section{a.2. Fragment Mass Analyzer Project D. J. Henderson, and D. Seweryniak)}

The FMA was heavily used in the past year. The beam time was split equally between target and focalplane experiments.

A gamma detector array consisting of 18 Comptonsuppressed Ge detectors (AYE-ball array) was mounted around the target position for a period of 4 months. About 13 experiments were run, including several that used a trigger from alpha and proton decays of residues in a double-sided silicon strip detector (DSSD) behind the focal plane. This of X-ray detectors and neutron detectors were installed and operated in coincidence with the main array for experiments which needed them. All the detectors surrounded the normal Fragment Mass Analyzer (FMA) target position in order to view prompt radiation and particles. The array was measured to have an absolute photopeak efficiency of $1.2 \%$ for the $1.33-\mathrm{MeV}$ radiation from ${ }^{60} \mathrm{Co}$, placing it at about twice the efficiency of the TESSA arrays at Daresbury. Thus, when operated with the FMA, the combined device had previously unsurpassed efficiency for studying nuclei far from stability.

The array was almost always triggered by detectors at the focal plane of the FMA. A variety of devices were used to select the physics process of interest. These included triggering with the focal-plane parallel-gridded avalanche counter, with an ion chamber mounted behind the focal plane, and with silicon double-sided strip detectors (DSSDs) of a variety of sizes. method, termed recoil-decay tagging, enabled in-beam gamma-ray spectroscopy measurements to be done on nuclei near and beyond the proton drip line.

A new ion-optical mode of focusing was developed which provides for a parallel beam of small crosssection at the FMA focal plane. This was tested with ${ }^{79} \mathrm{Rb}$ ions from the ${ }^{58} \mathrm{Ni}+{ }^{24} \mathrm{Mg}$ reaction, with the result that over $80 \%$ of the ions passing through the focal plane were detected in a $400-\mathrm{mm}^{2}$ silicon detector placed $60 \mathrm{~cm}$ behind the focal plane.

\section{a.3. Development of an Ion Chamber for Use with the FMA (C. J. Lister, C. N. Davids, and D. Henderson)}

A highly segmented ion chamber for use with the FMA was commissioned. Its physical size matches the current FMA focal-plane detectors, $15 \mathrm{~cm} \times 5 \mathrm{~cm}$. It has two energy-loss measuring stages and a stopping section, of thicknesses 5-, 5-, and 10-cm deep. The anode is also segmented into eight separate horizontal sectors, which can be ganged together by jumpers to form one large detector, or, if count-rates are very high, can be operated as eight separate chambers side-by-side in a common gas volume. It has a left-right position sense wire plane. The normal pressure window is of $220-\mu \mathrm{g} / \mathrm{cm}^{2}$ mylar. It is normally filled with isobutane gas and operated at pressures from 3 to 45 torr. The energy resolution was measured to be $2.5 \%$ with alpha particles and the energy-loss signals to be about $7.5 \%$. 
The ion chamber was operated in a variety of projects and its performance is being improved with time and operating experience. In most studies to date, it was operated behind the focal plane PGAC, which causes sufficient energy loss and straggling of incident ions that the ion chamber performance is severely degraded. To remedy this, a focal-plane channel plate detector is being developed, which should have an order-ofmagnitude less energy loss and scattering. In radiative capture studies on light nuclei, where the ions under study have low energies, the ion chamber was operated successfully at low pressure in a common volume with PGAC.

\section{a.4. A Position-Sensitive Channel Plate Detector System for the FMA (C. J. Lister, C. N. Davids, D. Henderson, B. J. Varley, * S. J. Freeman,* and J. Durell*)}

The evolution of the FMA research program is based on detecting ions arriving at the focal plane with a parallel gridded avalanche counter (PGAC). This detector has been a workhorse, with very robust performance, and is widely used. However, as the many FMA research projects evolve, the PGAC is being pushed to its limits and new detector designs are being considered. The main shortcoming of the PGAC is that it is physically thick, with more than $250 \mu \mathrm{g} / \mathrm{cm}^{2}$ of material in the beamline to degrade ions crossing the focal plane. The detector we use also appears to have count-rate limitations: it starts to spark at high rates.

To overcome these limitations a foil-and-channelplate design is being considered. Initially, an existing 8-cm diameter detector, with $\mathrm{x}, \mathrm{y}$ position sensitivity of 1.3-mm FWHM, is being loaned by the University of Manchester, U.K. to appraise the advantages of such a system. A test experiment is planned to study position resolution, count-rate capability, and time resolution. In the light of these tests, a dedicated system will be built, probably of asymmetric shape to match the $5 \times 15-\mathrm{cm}$ FMA focal plane. The successful completion of a reliable detector has many interesting upgrade paths in addition to the improvement of most "post-focal-plane" detector systems. With two channel-plate detectors, ray tracing of ions crossing the focal plane will become possible. This will allow a new method of rejecting scattered beam particles, and allow correction of angular aberrations of the FMA. This may allow new ion-optic solutions for the device which permit operation with a greater solid angle (and thus higher efficiency) without degrading reconstructed mass resolution. Three-channel-plate operation will allow atomic number $(\mathrm{Z})$ determination through differential time of flight with a passive absorber, a method which should have potentially extremely high countrate capability, and possibly better Z-measurement than conventional ion-chambers.

*University of Manchester, United Kingdom

\section{a.5. High-Efficiency Silicon Box Detector Array for the FMA Focal Plane (C. N. Davids, B. B. Back, D. J. Blumenthal, L. T. Brown, L. F. Conticchio, T. Davinson, * R. J. Irvine, * D. Seweryniak, W. B. Walters, $†$ and P. J. Woods*)}

For a forthcoming series of experiments searching for new heavy proton radioactivities and alpha decay of elements with $Z>100$, it is planned to use a large 40-mm $\times$ 40-mm double-sided silicon strip detector (DSSD) placed behind the FMA focal plane. The average implantation depth in the DSSD is about 10 microns, while the average proton range is 12 microns and the average alpha-particle range is 30 microns. This means that some fraction of the energy of the protons and alphas will be lost when they escape from the detector into the backward hemisphere, causing the identification of particles in a decay chain to be incomplete. Capturing the energy of escaping particles is crucial for these experiments. This can be accomplished by surrounding the backward hemisphere with a "silicon box", made up of 4 detectors each of area $60 \mathrm{~mm} \times 40 \mathrm{~mm}$. This array will cover approximately $80 \%$ of the backward hemisphere, and allow the energy measured there to be added back into events in the implantation detector. The system is designed and built, and will be tested in the coming year.

\footnotetext{
*University of Edinburgh, United Kingdom, †University of Maryland
} 


\section{a.6. Determination of a Mass Spectrum with the FMA from Time-of-Flight Measurements (C.-L. Jiang, M. P. Carpenter, C. J. Lister, G. Hackman, D. J. Blumenthal, C. N. Davids, W. F. Henning, R. V. F. Janssens, D. Ackermann, S. M. Fischer, T. L. Khoo, T. Lauritsen, V. Nanal, D. Nisius, D. Seweryniak, H. Amro, and J. Schwartz)}

The Fragment Mass Analyzer (FMA) is an 8.2-meterlong recoil mass spectrometer which separates reaction products produced in a heavy-ion fusion reaction and disperses them by mass/charge $(\mathrm{M} / \mathrm{Q})$ at the focal plane. As a result, unequal masses having different charge states can appear at the same focalplane position resulting in $M / Q$ ambiguities. However, by measuring both the time-of-flight of the ions through the FMA and the energies of the residues, it should be possible to determine the mass of the detected residues, and thus resolve these M/Q ambiguities.

In our "double" reaction studies, we have shown that this is possible. In these studies, the recoil energies from residues produced in the $58 \mathrm{Ni}+{ }^{40} \mathrm{Ca}$ reaction at $310 \mathrm{MeV}$ were measured in the Daresbury ionization chamber placed at the back of the FMA, and the timeof-flight for the ions was determined by measuring their arrival at the focal plane PPAC detector against the RF beam structure. The measured M/Q spectrum is very complicated and contains $M / Q$ ambiguities. When the calculated mass spectrum is plotted against the M/Q spectrum, individual masses can be separated even though there is a large spread in the calculated mass spectrum. For example, $A=93$ ions with $Q=$ 26 are degenerate with $A=85$ ions with $Q=24$ in the $M / Q$ spectrum. However, these two masses are completely resolvable from each other when $M / Q$ is plotted against calculated mass (from time of flight).

The large spread in the mass spectrum results from the fact that ions passing through the PPAC focalplane detector experience a substantial amount of energy loss. For our recoils, this is $>20 \%$. By replacing the PPAC detector with a combination thin foil and position-sensitive channel-plate detector, the energy loss and the energy dispersion will be greatly limited. Thus, the residue energies measured in the ion-chamber should have less energy dispersion and result in a better mass determination. A channel-plate detector is currently in house and will be tested at the FMA focal plane in the near future.

\section{a.7. Using the FMA for Radiative Capture Cross-Section Measurements of Interest to Astrophysics (C. N. Davids, B. B. Back, D. J. Blumenthal, L. T. Brown,* B. Busse, $†$ M. Gai, $\ddagger$ A. Garcia,§ J. Görres,§ D. J. Henderson, J. E. McDonald, $\ddagger$ S. Vouzoukas,§ E. L. Wilds, $\ddagger$ and P. J. WoodsID}

After the successful test of the FMA as a tool to study radiative capture reactions of astrophysical interest using inverse kinematics, we modified the focal-plane detection system in order to reduce the number of windows through which the reaction recoils pass. A new parallel-grid avalanche detector was fabricated. It differs from the original detector by the fact that the through pumping port can be open or closed using a cover plate. For regular experiments the port will be opened, and for radiative capture reaction measurements the port will be closed. This allows the rear gas window to be removed while not permitting counter gas to enter the beamline. Behind this detector will be mounted the large ionization detector, with its gas window also removed. A 5-cm $\times 5-\mathrm{cm}$ silicon detector was placed at the back of the ionization chamber. The two gas detectors are run together as a common gas volume. Energy loss signals will be obtained from the ionization chamber anode, and the remaining energy of the ion will be measured with the silicon detector. This system will be tested in the coming year.

*Vanderbilt University, †Oregon State University, $\ddagger$ University of Connecticut, §University of Notre Dame, qUniversity of Edinburgh, United Kingdom 


\section{a.8. First In-Beam Test of the Vanderbilt-ANL-LSU Array of Neutron Detectors (D. Seweryniak)}

Since the FMA disperses recoiling nuclei according to their mass-to-charge state ratio, additional detectors are necessary to obtain complete reaction channel selection. One possibility is to use an ion chamber placed behind the focal plane of the FMA. The energy losses in the ion chamber depend on the atomic number of the incident particles and enable separation of different elements within each mass. This method works well when incident particles have large velocity. For heavy-ion fusion-evaporation reactions leading to heavier compound systems, however, the $\mathrm{Z}$ resolution becomes limited and other methods have to be used. Detection of evaporated neutrons is a possible alternative. It proved to be a very powerful tool in the search for neutron deficient nuclei far from the line of stability.

An array of up to 16 neutron liquid scintillator detectors was placed between the AYEBALL array and the FMA to detect evaporated neutrons. The detectors were built by Vanderbilt University, ANL and LSU and were not used in-beam before. The detectors are filled with a BC501 scintillator. Light produced in the scintillator has two components which have different decay times (a few ns and about $300 \mathrm{~ns}$ ). Relative intensities of these two components can be used to discriminate between gamma rays and neutrons bombarding the detectors. To exploit this phenomenon an electronic circuit was set up to integrate the fast and slow light components separately. In addition, neutron time of flight was measured to improve neutron-gamma discrimination. A layer of $\mathrm{Pb}, 1-\mathrm{cm}$ thick, was also placed in front of the detectors to decrease the gamma-ray background.

The detectors performed well during several in-beam experiments with the AYEBALL array aimed at neutron-deficient nuclei far from stability. Less than $1 \%$ of the detected gamma rays were misassigned as neutrons. Neutron detection efficiency between 5 and $8 \%$ was obtained depending on the reaction used. The efficiency could be greatly improved by moving the detectors closer to the target. This was impossible due to mechanical restrictions. The results obtained with the neutron detectors are satisfactory and are promising for future use of the neutron detectors in combination with the FMA.

\section{a.9. Development of a Radioactive Beam Coulomb Excitation Chamber for the FMA (C. N. Davids, S. Fischer, D. Henderson, C. J. Lister, M. P. Carpenter, R. V. F. Janssens, T. L. Khoo, D. Nisius, J. Schwartz, S. J. Freeman, * and B. J. Varley*)}

Consideration of useful experiments with lowintensity radioactive beams reveals Coulomb excitation to be an even more powerful technique than when used near stability. Far from stability, the radioactive beams are poorly bound, so the relatively gentle electromagnetic probe becomes an essential tool. Further, the relatively large cross sections and well-developed understanding of the reaction mechanism make the translation from raw data to useful observables rather clear-cut.

In order to develop the necessary experimental methodology, we used the Fragment Mass Analyzer (FMA) to produce a parallel radioactive beam of $10^{5}$ particles/second. The primary reaction used was ${ }^{58} \mathrm{Ni}$ on ${ }^{24} \mathrm{Mg}$ at $210-240 \mathrm{MeV}$, which allowed a mass- selected $\mathrm{A}=79$ beam of mean energy $135 \mathrm{MeV}$ to be isolated. The beam had a diameter of approximately 1-cm FWHM. Coulomb excitation of the beam on lead and nickel foils was studied. The problems associated with the prolific background of radioactivity from the beam stopping in the target vicinity were investigated.

Several technical improvements are being made to the experiment before our next run. The FMA focalplane PGAC is to be replaced by a channel-plate detector. This will improve the radioactive beam quality, both as the beam energy will not be degraded in energy in the gas counter (which led to a loss of Coulomb excitation yield of a factor of two), will not be so badly broadened by multiple scattering, nor have count rate limitations of less than 30,000

*University of Manchester, United Kingdom 
counts/second. Further, a four-quadrant PGAC is being developed for operation after the target position to detect ions which scatter through angles greater than $15^{\circ}$. These ions are the ones most likely to be Coulomb excited and detection of scattered-ion gamma-ray coincidence events should suppress the intense background from decaying beam particles, should allow full Doppler correction of gamma-ray transitions emitted from moving ions, and allow the impact parameter dependence of gamma rays to be established.

\section{a.10. Measurements of the Transport Efficiency of the Fragment \\ Mass Analyzer (B. B. Back, D. J. Blumenthal, C. N. Davids, D. J. Henderson, R. Hermann, D. J. Hofman, C. L. Jiang, H. T. Penttila, and A. H. Wuosmaa)}

Absolute transmission efficiencies for the Fragment Mass Analyzer (FMA) were measured for ions of ${ }^{184} \mathrm{~W},{ }^{208} \mathrm{~Pb}$, and ${ }^{232} \mathrm{Th}$ with energies in the range $\mathrm{E}=30-45 \mathrm{MeV}$. These were obtained by Rutherford scattering of ${ }^{32} \mathrm{~S}$ beams of 50,80 , and $110 \mathrm{MeV}$ into backward angles. Measurements were performed for a range of settings of the FMA in order to determine the acceptance of the instrument as a function of energy and angle of the incident ion. The transport efficiency across the focal plane was also measured. The results are compared with predictions of the transport code GIOS, which agree well with the data (see Fig. I-36). With these data we conclude that the transport efficiency of the FMA is sufficiently well known to support measurements of absolute cross sections with an accuracy of $\pm 10 \%$.

A manuscript describing these results was submitted for publication.
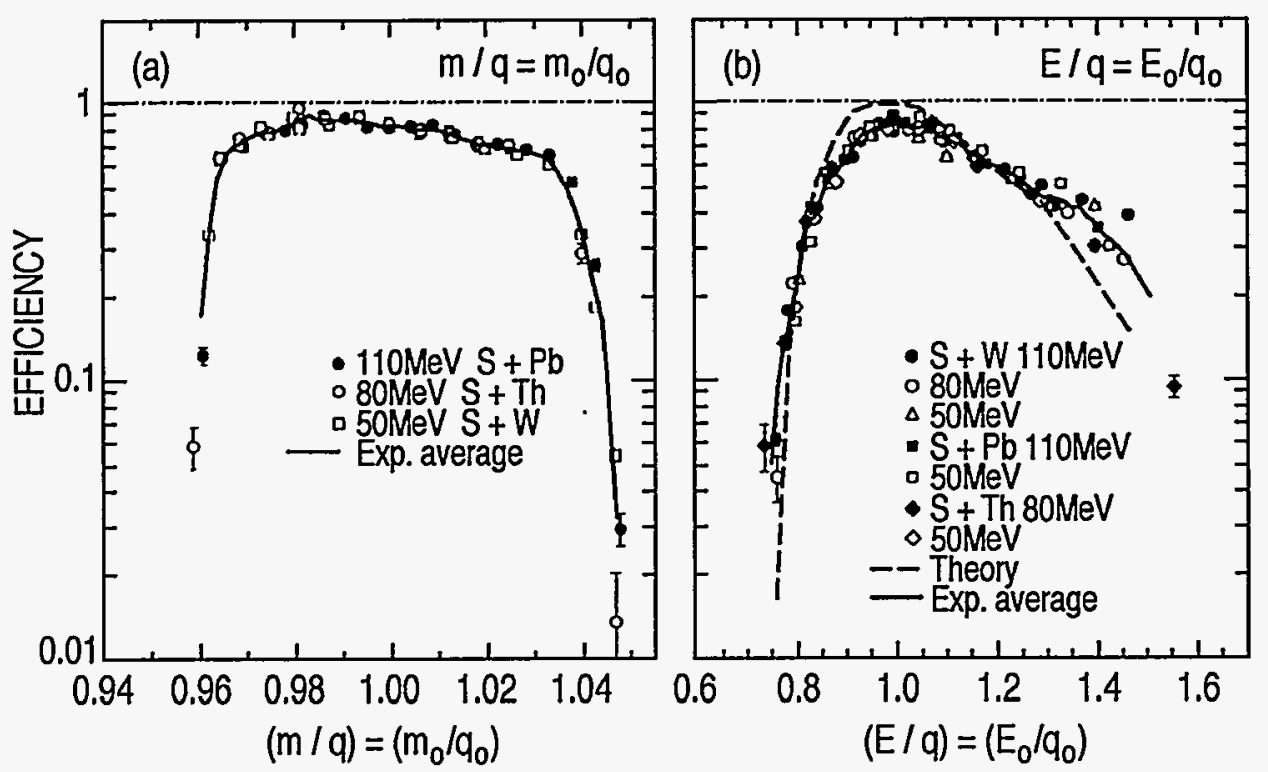

Fig. I-36. Measured absolute transport efficiencies for mono-energetic recoil ions (points labeled by the sulfur bombarding energies and targets) as a function of deviation from the central $\mathrm{m} / \mathrm{q}$ setting, (left) and the $E / q$ setting, $E_{0} / q 0$ (right) of the FMA with $a 4.5^{\circ} \times 4.5^{\circ}$ aperture. A transport efficiency of $I$ would mean that all the ions of a given charge state, passing the $4.5^{\circ} \times 4.5^{\circ}$ aperture, were detected. The calculated value of the efficiency as a function of the charge-state setting of the instrument using the code GIOS is shown on the right as a dashed curve. 


\section{a.11. Development of a $0^{\circ}$ Faraday Cup for the FMA (B. B. Back, R. Kickert, and J. Sendera)}

The main source of background in the FMA when operated at $0^{\circ}$ arises from the forward scattering of beam particles hitting the anode of the first electrostatic dipole. In order to reduce this background, a small Faraday cup was developed which stops the beam at the entrance to the FMA. This cup, which covers angles up to $0.7^{\circ}$, is mounted on one of the four insertable entrance apertures, and can thus easily be inserted or removed without breaking the vacuum. The alignment of the $0^{\circ}$ cup is critical to the success of this scheme, but is complicated by the fact that one cannot mount a telescope to establish the beamline. Instead a telescope line was established at $90^{\circ}$ to the beamline. By placing a pentaprism at the target position this was used for the alignment of the $0^{\circ}$ Faraday cup, as well as detectors in the scattering chamber. This Faraday cup was used successfully in one experiment, and it is planned to use it whenever the small loss in solid angle (about $5 \%$ ) is acceptable and a reduction in beam background is imperative.

\section{a.12. $\mathrm{BaF}_{2}$ High-Energy Gamma Array - LEPPEX (B. B. Back, D. Ackermann, G. Hackman, D. J. Hofman, D. Henderson, V. Nanal, P. Paul, * and P. Wilt)}

The LEPPEX project involves the construction of two high-efficiency 30 -element $\mathrm{BaF}_{2}$ detector arrays and a high-efficiency fission fragment detection system consisting of eight position-sensitive parallel grid avalanche detectors arranged in a barrel around the beam axis.

This setup is designed for optimal efficiency for fission-gamma coincidence measurements to study the pre-scission gamma-emission. Such studies were shown to provide unique information about the timescale in fission and other strongly damped reactions, but the systematic study using this method was hampered by small detection efficiencies in available experiments.

*SUNY at Stony Brook
The geometrical design of the $\mathrm{BaF}_{2}$ arrays which each consist of $305 \times 5 \times 25$-cm crystals surrounded by an anti-scattering shield of either plastic or liquid scintillator, was optimized for energy resolution, pileup reduction and efficiency. Further enhancement of the energy resolution will be obtained by cooling the crystals to about $0^{\circ} \mathrm{F}$.

The first set of $16 \mathrm{BaF}_{2}$ crystals were ordered in FY 1995 and will be assembled into a sub-assembly for on-line testing and first physics experiments in FY 1996.

\section{a.13. Temperature Dependence of $\mathrm{BaF}_{2}$ Scintillation (B. B. Back, D. J. Hofman, and V. Nanal)}

We studied the scintillation characteristics of $\mathrm{BaF}_{2}$ as a function of temperature in the range $-50^{\circ} \mathrm{C}$ to $27^{\circ} \mathrm{C}$ using an ${ }^{88} \mathrm{Y}$ gamma-ray source.

$\mathrm{BaF}_{2}$ crystals of different thicknesses 0.5 "-2.5" (about 1 " diameter) were cooled by using dry ice. The detailed line shape of the scintillator pulse was measured at various temperatures in the above mentioned range using the Bollinger-Thomas method. The light output of the $\mathrm{BaF}_{2}$ crystal was also measured as a function of temperature. The stability using both a blue and green Light Emitting Diode (LED).

The scintillation pulse of $\mathrm{BaF}_{2}$ is known to have a very fast component (decay time $\sim 600 \mathrm{ps}$ ) followed by a slow component (decay time $\sim 600 \mathrm{~ns}$ ) at room temperature. We observed that both the decay time and the intensity of the fast component are unaffected by cooling. The decay time of the slow component increases with decreasing temperature reaching a value of $4 \mu$ s at $-45^{\circ} \mathrm{C}$ (see Fig. I-37). A third component with decay time $0.5 \mu \mathrm{s}$ is clearly observed at 


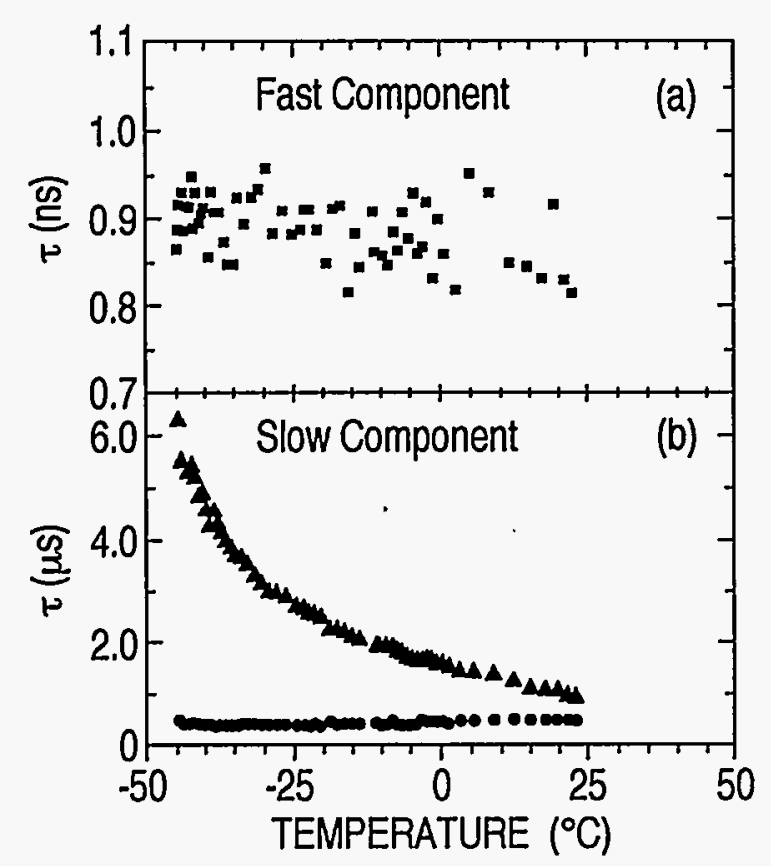

temperatures lower than $-30^{\circ} \mathrm{C}$. The intensity of the slow component initially increases with decreasing temperature down to $-20^{\circ} \mathrm{C}$ and is reduced with further cooling. At $-20^{\circ} \mathrm{C}$ the intensity of the slow component is increased by about $100 \%$ over its room temperature value. This is different from the observations reported in the literature. ${ }^{1}$

The total energy resolution also shows improvement at this temperature which is consistent with the increased light output.

A manuscript describing these results was submitted for publication.

Fig. 1-37. The variation of the fast (a) and slow (b) scintillation decay times for $\mathrm{BaF}_{2}$ crystal as a function of temperature as determined using the Bollinger-Thomas method.

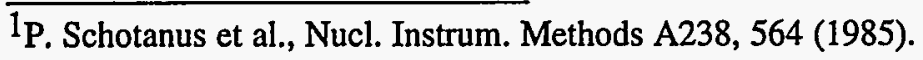

\section{a.14. Development of Si-CsI $\Delta \mathrm{E}$-E Light Charged Particle Detectors (B. B. Back, D. J. Hofman, and V. Nanal)}

We have constructed five silicon-CsI $(\Delta \mathrm{E}-\mathrm{E})$ detectors for use in experiments measuring energetic light charged particles. A $400-\mathrm{mm}^{2}$ silicon detector measures the time and $\Delta \mathrm{E}$ signal, and a $\mathrm{CsI}(\mathrm{Tl})$ crystal coupled to a photo-diode provides a measure of the total energy.

The response of the CsI detectors to protons, deuterons and alpha particles was determined using the 9-MV tandem at the University of Notre Dame. The energy response to protons and deuterons was measured up to $15 \mathrm{MeV}$ and was found to be linear.
The energy response to alpha particles is slightly non-linear below $10 \mathrm{MeV}$ and was measured over a larger energy range of $2.0-22.5 \mathrm{MeV}$. The detectors were designed within the framework of the detector mounting system established for Argonne's large scattering chamber (ATSCAT).

These detectors were first used in a successful experiment at ATLAS measuring the survival probability of Th-like nuclei. An energy resolution of $4.5 \%$ for $8.8-\mathrm{MeV}$ alpha particles was obtained for the CsI detectors during this experiment.

\section{a.15. Use of the Gas-Filled-Magnet Technique for Particle Identification at Low Energies (K. E. Rehm, C. L. Jiang, and M. Paul*)}

Reaction studies of interest to astrophysics with radioactive ion beams will be performed mainly in inverse reaction kinematics, i.e., heavy particles bombarding a hydrogen target. The low energy of the outgoing heavy reaction products makes particle identification with respect to mass and nuclear charge a major challenge. For the ${ }^{18} \mathrm{~F}(\mathrm{p}, \alpha)$ experiment, five

*Hebrew University, Jerusalem, Israel different types of particles in the outgoing channels are observed: ${ }^{18} \mathrm{~F}$ and ${ }^{18} \mathrm{O}$ (from elastic scattering of ${ }^{18} \mathrm{~F}$ and ${ }^{18} \mathrm{O}$ on ${ }^{12} \mathrm{C}$ ), ${ }^{15} \mathrm{O}$ and ${ }^{15} \mathrm{~N}$ [from the ${ }^{18} \mathrm{~F}$ and $18 \mathrm{O}$ induced $(p, \alpha)$ reactions] and ${ }^{12} \mathrm{C}$ recoils from the polypropylene target. While mass determination can be achieved easily by time-of-flight (TOF) measurements, a determination of the nuclear charge presents a challenge, especially if the energy of the particles is below $500 \mathrm{keV} / \mathrm{u}$. We studied the gas- 
filled magnet technique for Z-identification of light ions between $Z=6-9$. In a gas-filled magnet the particles move with an average charge state $\bar{q}$ which in one parameterization is given by $\overline{\mathrm{q}}=\mathrm{Z}$ $\ln \left(a v Z^{\alpha}\right) / \ln \left(b Z^{\beta}\right)$ where $Z$ is the nuclear charge of the ions and $v$ their velocity. Introducing $\bar{q}$ into the expression for the magnetic rigidity $B \rho=m v / \bar{q}$ results in a $Z$ dependence of $B \rho$ which is valid to very low velocities. As a magnet we used the Enge splitpole spectrograph which was filled with nitrogen gas at a pressure of 0.5 Torr. The particles were detected in the focal plane with a $50 \times 10-\mathrm{cm}^{2}$ parallel-gridavalanche counter which measured TOF and magnetic rigidity. The mass and $\mathrm{Z}$ separation was tested with
${ }^{13} \mathrm{C}$ and ${ }^{18} \mathrm{O}$ beams at energies of about $600 \mathrm{keV} / \mathrm{u}$ and recoil particles ranging from ${ }^{12} \mathrm{C}$ to ${ }^{19} \mathrm{~F}$.

From these measurements a parameterization of the average charge state $\bar{q}$ was obtained which, together with the RAYTRACE program, allows a simulation of the trajectories through the split-pole spectrograph including the charge-changing collisions between ions and gas-molecules. The $Z$ separation in these experiments was $Z / \Delta Z=30$ (FWHM). This resolution together with the time-of-flight measurement is sufficient to separate the $A=18$ isobars ${ }^{18} \mathrm{~F}$ and ${ }^{18} \mathrm{O}$ with intensity ratios of about $10^{5}$. A paper with these results was published.

\section{a.16. Production of 18F Beams from a SNICS Ion Source} (K. E. Rehm, M. Paul, * A. Roberts, $\dagger$ and J. Nickles $\dagger$ )

In continuation of our earlier experiments studying the optimum conditions for producing a usable ${ }^{18} \mathrm{~F}$ beam at the tandem accelerator, we made several improvements in the production technique. Aqueous $18 \mathrm{~F}^{-}$ions are produced at the University of Wisconsin cyclotron via the ${ }^{18} \mathrm{O}(\mathrm{p}, \mathrm{n})^{18} \mathrm{~F}$ reaction with $11-\mathrm{MeV}$ protons bombarding an enriched $\left[{ }^{18} \mathrm{O}\right]$ water target. In the first experiment electrodeposition was used to deposit the active ${ }^{18} \mathrm{~F}$ ions onto the SNICS cathode inserts. Since this resulted in sparking inside the ion source requiring a one-hour conditioning procedure, we changed the deposition process by directly placing small drops of the $\mathrm{H}_{2} \mathrm{O}+{ }^{18} \mathrm{~F}$ solution on a heated $\mathrm{Al}$ + Ag cathode insert. With these samples no arcing in the source was observed. By reducing the Cs sputter rate, a considerable increase in the ion source extraction efficiency was observed. In the latest experiments up to $1 \%$ of the ${ }^{18} \mathrm{~F}$ atoms in the sample could be converted to ${ }^{18} \mathrm{~F}$ - ions and extracted from the source during a three-hour run. On the other hand, test measurements using gaseous $\mathrm{CH}_{3} \mathrm{~F}$ material resulted in efficiencies which were lower by factors of 10-30. A publication describing the production technique was published.

*Hebrew University, Jerusalem, Israel, †University of Wisconsin, Madison

\section{a.17. Test of the Beam Transport System at Low Velocities (R. C. Pardo and K. E. Rehm)}

All experiments with low-intensity radioactive ion beams require the optimum transmission from the ion source to the target. Earlier test measurements with $18 \mathrm{O}$ beams showed that about $10 \%$ of the ions injected into the tandem accelerator could be transported to the target located in the scattering chamber of the spectrograph in area II. After the replacement of the corona tubes in the tandem accelerator in 1995, this transmission decreased by a factor of 2-3. Ion-optical transport calculations for the ion-source-tandem area were performed, showing that a reduction of the ion source pre-acceleration voltage should result in an improved transmission through the tandem accelerator. First test measurements with $18 \mathrm{O}$ beams confirmed this increase and improvements in the tandem transmission by about $50 \%$ were observed. More tests are planned for early 1996 . For the ${ }^{18} \mathrm{~F}(\mathrm{p}, \gamma){ }^{19} \mathrm{Ne}$ experiment the transmission through the beam transport system to the Fragment Mass Analyzer has been optimized using ${ }^{18} \mathrm{O}^{4+}$ ions. It was found that $90 \%$ of the beam coming out of the tandem accelerator could be transported to the FMA target position. 


\section{a.18. Status of the Argonne-Notre Dame BGO Gamma-Ray Facility at ATLAS \\ (R. V. F. Janssens, D. T. Nisius, H. Amro, D. J. Blumenthal, M. P. Carpenter, B. F. Crowell, J. W. Falout, S. M. Fischer, J. M. Joswick, T. L. Khoo, G. S. Hackman, T. Lauritsen, J. P. Timm, and P. R. Wilt)}

The gamma-ray facility at ATLAS consists of (a) a $4 \pi$ gamma-sum/multiplicity spectrometer with 50 BGO hexagonal elements (inner array) and (b) 12 Compton-suppressed germanium detectors (CSG) external to the inner array. The facility was heavily used until July 15, 1995. At that point it was turned off for the remainder of the year so that a good fraction of the dedicated electronics could be used with the AYE-Ball array described elsewhere in this report. During the past year the effort related to this facility continued on several fronts.

-- Because of neutron damage, annealing was performed on all Ge detectors. Three of these were annealed twice. The performance of the detectors was recovered in all but two cases. In the latter, the detectors were returned to the manufacturer for repair.

-- Maintenance and repairs had to be performed on several electronics modules and, in particular, on some of the CAMAC units. None of these problems affected an experiment for more than a couple of hours.

-- Preventive maintenance was performed on the $\mathrm{LN}_{2}$ filling system (inspection of all filling lines and check of the various functions of the control modules).

-- New discriminator modules to handle time signals from the inner BGO array detectors were designed and construction is progressing.

\section{a.19. Completion of Testing and Procurement of BGO Compton-Suppression} Detectors for Gammasphere (M. P. Carpenter, I. Ahmad, R. V. F. Janssens, and T. L. Khoo)

Gammasphere, the national $\gamma$-ray facility, when completed will consist of 110 Compton-suppressed Ge detectors. The bismuth germanate (BGO) Comptonsuppression detector system for each $\mathrm{Ge}$ detector consists of one tapered hexagonal BGO side shield and one slotted BGO back plug. Due to the geometry of the array, three types of annular shields are required. These types are referred to as B, C and D, and the array consists of 60,30 and 20 of these units, respectively. Shield types B, C and D have a hexagonal geometry. They are divided into six optically separate sections, each with its own pair of photomultiplier tubes.

Argonne assumed responsibility for the procurement and testing of the BGO Compton-suppression units. All detectors were accepted and are now in Berkeley bringing to a close ANL's responsibility for this task. Over the last several years, all backplugs and eightyeight side shields were tested at ANL, and twenty-four side shields were evaluated by Prof. D. Fossan's group at Stony Brook. During this past year, thirteen detectors were evaluated by our group and delivered to LBL for use in Gammasphere.

\section{a.20. Software Developments for Gammasphere ( $T$. Lauritsen)}

Gammasphere in its final implementation makes use of a series of calibration and monitoring tasks developed at ANL specifically for this array. Gammasphere employs a slow control system, EPICS, which is well suited for complex computer control. Basic calibration tasks were completed and thoroughly debugged and tested under running conditions during the last year. Graphical User Interfaces (GUIs) for the numerous calibration and monitoring tasks were improved and made more user friendly, and the online and WEB-served documentation 
was expanded significantly. Many new utilities were added as we found a need for them in order to operate the array more efficiently. Well designed GUIs allowed outside users, with a minimum knowledge of the array control system, to perform almost all calibration and monitoring tasks with minimum guidance by the Gammasphere staff.

In addition, a more complex calibration and monitoring task was started for the BGO detectors.
There are a maximum of 1540 tubes in the array, each having its own adjustable high voltage. This task involves: (i) taking data over the Gammasphere LAN, (ii) analyzing $2 \mathrm{D}$ data and (iii) the recovery, recalculation and deposit of new voltages via EPICS to electronics modules in VXI crates. A working prototype of this task was just tested successfully and the first on-line BGO calibration will be tested shortly.

\section{a.21. High-Spin Gamma-Ray Spectroscopy Software Developments (T. Lauritsen)}

With the new powerful gamma-ray arrays getting near completion, the data from a typical experiment now comprise well in excess of one billion events with very high multiplicity. In addition, the number of different polar angles of the detectors has gone from a few to more than ten $(17 \max )$. For the analysis of the quasi-continuum of gamma rays and peak shape analysis for lifetime determination (DSAM) it is necessary to sort the data into angles and take into account peaks (on which we set gates) that are moving.

Our old software did not allow us to do this efficiently and the task of sorting a particular band typically took a week because the tapes had to be spun once for every angle. We developed a new sorting package that will angle sort data from Gammasphere and Eurogam in one pass. This cuts the sorting time down by an order of magnitude. The new sorting software also makes very efficient use of the memory; only the necessary spectra and 2D matrices are kept - allowing the sort to be done in memory rather than on disk. The program makes use of a background subtraction method previously developed at ANL, is written in C and takes advantage of some of the dynamic memory allocation features found in this programming language.

\section{a.22. Towards a One-Cube DCO Method with Gammasphere (G. Hackman)}

To date, the very powerful and well-established directional correlation from oriented states method (DCO) has not been applied to high-spin experiments with Gammasphere in a way which best exploits the sensitivity near the optimal coincidence fold. DCO and angular distribution measurements are two means of determining the multipolarity and spin change of a given $\gamma$-ray transition between nuclear states. Current results relied on the latter method, specifically for measuring the angular distributions of one or some small set of $\gamma$ rays in coincidence with a superdeformed band. These measurements were adequate for these purposes, but cannot be applied easily to more general problems such as the deduction of a complex level scheme.

A code to calculate triple-gamma angular distributions is currently being developed. The code was designed to be as flexible as possible, to permit any possible detector arrangement and initial state distributions. Currently the code is being employed to determine the best angles for a "one-cube" DCO method. This would be a logical extension of the well-known "one-matrix" method, whose main advantage is that all efficiency factors cancel, reducing the problem of determining transition multipolarity to simply measuring peak areas. 


\section{a.23. Triple Angular Correlations of $\boldsymbol{\gamma}$ Rays Following Spontaneous Fission (I. Ahmad, L. R. Morss, * W. Urban, $\uparrow$ M. A. Jones, $\uparrow$ C. J. Pearson, $\uparrow$ J. L. Durell, $\uparrow$ W. R. Phillips, $\dagger$ M. J. Leddy, $\dagger$ B. J. Varley, $\dagger$ M. Bentaleb, $\ddagger$ E. Lubkiewicz, $\ddagger$ and N. Schulz $\ddagger$ )}

Triple angular correlations between gamma rays emitted by fission fragments of ${ }^{248} \mathrm{Cm}$ were studied with the Eurogam2 array. The measured values of Directional Correlation from Oriented Nuclei (DCO ratios) for even-even nuclei are found to be in good agreement with the predictions of angular correlation theory. These studies provide a technique to determine the multipolarities of strong transitions in the neutronrich nuclei produced in fission. The DCO ratios of even-even $\mathrm{Ba}$ and $\mathrm{Ce}$ nuclei are consistent with the previously assigned spins and parities. The technique is currently being applied to establish spins and parities of levels in other fission fragments. The results of this investigation were published.

*Chemistry Division, ANL, †University of Manchester, United Kingdom, $¥$ CRN, Strasbourg, France

\section{a.24. Neutron Detectors for Operation with Gammasphere (C. J. Lister, J. Schwartz, D. Balamuth,* P. Hausladen,* K. Pohl,* M. Leddy, $\uparrow$ S. J. Freeman, $\uparrow$ and B. J.Varley†)}

In order to enhance the channel selection available for Gammasphere at LBNL, several triggering systems were developed. To date, the CsI array "microball" is the most used. It is a large solid-angle array for selecting light charged particles. To complement this, an array of neutron detectors is being developed. Neutron selection is important for isolating nuclei near the proton dripline. Fifteen detectors will be mounted in the Gammasphere frame, replacing the most downstream rings of Germanium counters. Each is approximately $5^{\prime \prime} \times 6^{\prime \prime}$ of NE213 liquid scintillator. The detectors are presently at ANL for testing and development of a readout system which is compatible with the Gammasphere data stream. Three experiments are scheduled for the Spring of 1996.

*University of Pennsylvania, $\uparrow$ University of Manchester, United Kingdom

\section{a.25. Development of a Plan to Move Gammasphere to ANL in 1997 \\ (M. P. Carpenter, C. N. Davids, R. F. V. Janssens, T. L. Khoo, C. J. Lister, J. P. Schiffer, and W. Henning)}

From its original concept, the national gamma-ray facility Gammasphere was designed as a detector which could be moved. Many laboratories, including Argonne, contributed resources to its construction with the understanding that after completion the physics case for moving the device to another laboratory would be reviewed. The completion of the detector and its dedication were reached at the end of 1995. Consequently, at ANL, consideration was given to the case for moving the detector to ATLAS for a period of operation, and a document "The Science and Operation of Gammasphere at ATLAS" was prepared. A DOE review of the document and its underlying Physics case was held at ANL in January 1996.
The physics case for moving the detector centers around the interest in marginally bound nuclei near the proton dripline. This interest is both theoretical and experimental and was the driving force behind the development of the concepts of a national radioactive beam facility. To reach many proton dripline nuclei, there are two experimental strategies which may be employed: developing accelerated radioactive beams and forming nuclei with good cross section, or alternatively using stable beams and developing hypersensitive experiments to isolate nuclei of interest which are produced with low cross sections. These techniques are very complementary. One hypersensitive channel selection method was already developed. Through the observation and study of groundstate proton decay using the Fragment Mass 
Analyzer (FMA) and silicon strip detector technology, it was demonstrated that nuclei at and beyond the proton dripline can cleanly be isolated. The potential for using these decays as a trigger for prompt gammaray and particle spectroscopy beyond the dripline was demonstrated, both using Eurogam1 at Daresbury and using the AYE-Ball at ANL. However, the efficiency and granularity of Gammasphere are needed to turn this potential into a powerful tool.

Thus, the proposal is to move Gammasphere to the target position of the FMA and use beams from the ATLAS accelerator to form nuclei far from stability. This arrangement opens up a wide variety of other interesting and novel projects, including spectroscopy of light nuclei, studies of the shell structure stabilizing the heaviest nuclei, and several reaction mechanism investigations including sub-barrier fusion and the possibility of exploring "double" nuclear reactions to produce rare isotopes. In addition, the availability of very heavy beams, like lead and uranium, at the Coulomb barrier with good intensity will allow excellent Coulomb excitation research. The excellent timing of the ATLAS beam structure will allow unique experiments on isomers. Finally, the entire range of beams presently available to Gammasphere will still be offered for important high-angular momentum projects.

Many technical details remain to be worked out. A suitable management plan is being devised and the mechanical options and costs involved are being explored in detail.

\section{a.26. Nuclear Target Development (J. P. Greene and G. E. Thomas)}

The Physics Division operates a target development laboratory that produces the thin foil targets needed for experiments performed at the ATLAS and Dynamitron accelerators. Targets are not only produced for the Physics Division but also for other divisions and occasionally for other laboratories and universities.

In the past year, numerous targets were fabricated either as self-supporting foils on various substrates or as sandwich targets. Targets produced included acrylic, $\mathrm{Au}, 10,1^{1} \mathrm{~B}, \mathrm{Bi},{ }^{12} \mathrm{C},{ }^{40} \mathrm{Ca},{ }^{176} \mathrm{HfO}_{2}$, In, ${ }^{24} \mathrm{Mg}$, ${ }^{92} \mathrm{Mo}, 148,150 \mathrm{Nd}$, Ni, p-terphenyl, $206,208 \mathrm{~Pb}, \mathrm{Pr}$, ${ }^{104} \mathrm{Ru}, 144,150,154_{\mathrm{Sm}}, 112,122,124_{\mathrm{Sn}},{ }^{124,130} \mathrm{Te}$ and $168,170,172 \mathrm{Yb}$. The first experiments using radioactive beams of ${ }^{18} \mathrm{~F}$ employed hydrogen targets consisting of kapton, polyethylene and stretched polypropylene plastic foils. The first experiments using the FMA rotating target wheel have begun and employed targets of ${ }^{208} \mathrm{~Pb}$ on carbon backings. Ten of these sector targets are needed for each complete wheel.

An increased output of foils fabricated using our small rolling mill included targets of $\mathrm{Au}, \mathrm{Bi}, \mathrm{Ca},{ }^{106} \mathrm{Cd}$, ${ }^{50} \mathrm{Cr},{ }^{65} \mathrm{Cu},{ }^{160} \mathrm{Dy}$, Havar, $\mathrm{Mg}, 92,94,95_{\mathrm{Mo}},{ }^{58} \mathrm{Ni}$, ${ }^{208} \mathrm{~Pb},{ }^{103} \mathrm{Rh},{ }^{144} \mathrm{Sm},{ }^{232} \mathrm{Th}$ and Ti. Rolling has become the method of choice for most targets used in FMA experiments where the required areal densities range from $0.7 \mathrm{mg} / \mathrm{cm}^{2}$ up to $1 \mathrm{mg} / \mathrm{cm}^{2}$. Similarly for gamma-ray experiments using the AYE-Ball array at the FMA, the areal densities needed decrease to 0.3 $\mathrm{mg} / \mathrm{cm}^{2}$ and either rolling or evaporation are the methods of choice.

With Gammasphere now operational, there is an increase in target requests for these experiments. Targets of $160 \mathrm{Gd},{ }^{122} \mathrm{Sn}$ and ${ }^{130} \mathrm{Te}$ foils were produced for Argonne runs at Berkeley. Sandwiched targets of $\mathrm{Au}-40^{\mathrm{Ca}-\mathrm{Au}}$ and $\mathrm{Au}-148,150 \mathrm{Nd}-\mathrm{Au}$, both very reactive elements, were prepared successfully and transported under vacuum or argon to Berkeley for Gammasphere experiments. Targets supplied for other institutions included ${ }^{10} \mathrm{~B},{ }^{65} \mathrm{Cu}, 58 \mathrm{Ni}$, Ta and ${ }^{208} \mathrm{~Pb}$.

In all, approximately 785 targets were prepared for these various experiments during the past year. As part of ATLAS support, carbon stripper foils of 2 $\mu \mathrm{g} / \mathrm{cm}^{2}$ for use in the Tandem as well as other thicknesses for additional stripping are being routinely produced by the target lab. A total of 981 carbon stripper and gold foils of various types were prepared in calendar year 1995.

The target development laboratory includes state-ofthe-art equipment used for thin-film deposition. The available techniques consist of multiple resistive heating, focused ion beam sputtering, electron beam and electron bombardment evaporation. The evaporators are maintained under high vacuum and each vessel contains a quartz-crystal film-thickness monitor with deposition rate indicators. Also included are movable shutters, quartz-lamp substrate heaters and 
thermocouple temperature sensors. This allows for complete process monitoring during target deposition.

A wide-beam Saddle Field Ion Source, purchased for it's larger beam spot size has enabled the development of new ion milling applications. An exhaustive study was undertaken, for the most part by co-op student Stacey Schiel, involving the deposition rates obtained with this source for many common target materials. In conjunction with this effort was the use of TRIM calculations to predict the observed sputter yields. This source will also provide increased sputtering rates during target depositions.

Auxiliary equipment used for target development includes a glow discharge apparatus for plasma deposition, mainly of carbon foils. Reactivation of this system was recently realized using a diffusion pump to produce diamond-like carbon foils to be used as hydrogen containing targets. This technique was used successfully at TUNL to prepare deuterium targets.

Also present are a small rolling mill, mentioned previously, an alpha-particle thickness gauge (see below), inert atmosphere glove box, laminar flow clean bench, a reduction furnace, and a variety of precision balances.

The laboratory's IBM computer saw increased use for a number of purposes, particularly for INTERNET access and the wealth of information available on the
WORLD WIDE WEB. File archives maintained on this system include all targets produced, dating back to 1978. Computer listings can be generated for inventories of all stable isotopes and chemicals maintained by the target lab. An ADC board and associated software allows for acquisition and analysis of alpha particle energy loss measurements needed for film thickness determinations.

A target storage facility is in operation for maintaining, under high vacuum, those targets which can readily oxidize in air. This system utilizes a turbo pump and employs computer-controlled circuitry to prevent targets from exposure to atmosphere during power interruptions. This equipment is currently installed in ATLAS Area II for the storage of those reactive targets which were activated by the beam and therefore kept within the controlled Area of ATLAS. A second, turbo-pumped high vacuum chamber is in routine use for target storage within the target laboratory. This system uses electronically controlled valves for preserving the targets under high vacuum.

There also exists a bank of vacuum desiccators connected to a mechanically-pumped manifold for use by individual experimenters. The mechanical pumping system includes an emergency shut-off valve which prevents venting of the desiccators to atmosphere during power interruptions. A second, similar system has been constructed at ATLAS for storage of targets which were activated by the beam during experiments.

\section{a.27. Physics Computing Facilities (D. R. Cyborski, T. Lauritsen, and K. Teh)}

The Physics Division maintains several computer systems for data analysis, computation, and general computing. These systems are conveniently grouped into various clusters and are described briefly below. This past year saw significant improvements to the computing facilities and resources in the division. More noteworthy is the fact that these improvements were achieved at minimal cost through a mix of efforts by the Computer Support Group; by taking advantage of vendor trade-in offerings, decommissioning older computers and discontinuing their maintenance contracts, redeploying existing computers to meet immediate needs, adopting more "off-the-shelf" hardware and greater reliance on free public domain software.

The Division's Unix cluster consists of five Sparcstations and four DEC 3000/300LXs Alphas.
One of the Sparcstations was obtained from the disposition of SSC equipment. Two DEC 3000/300LX Alphas which were previously part of the Unix cluster were redeployed as VMS machines; one was added to the data-acquisition cluster, and the other to the analysis cluster. The cluster also hosts eight Xterminals, providing for a total of seventeen graphics seats. Its primary function is data analysis and some general computing. While the Unix Cluster suffered a net loss of one machine, it was subsequently enhanced with the addition of eight PCs running the Linux Operating System. The adoption of the Linux PC workstation was motivated by the need to provide the postdoctoral researchers greater access to graphics capabilities and computing resources on the primary clusters. The choice of a PC running Linux was based on the following factors: Linux is a free public domain Unix clone, fully compatible with the existing 
Unix cluster. Software tools such as the CERN analysis packages and the $\mathrm{TeX}$ document preparation tools, both widely used in the physics community, run on it. Investigations also indicate that the Linux workstation can be used as a primary computing resource. Indeed, the Computer Support Group measured its performance and found it to compare favorably with commercial workstations costing five times as much. Finally, the low cost of the PC hardware platform and the free software tools combine to make the Linux workstation a persuasive option.

The Analysis Cluster is a cluster of five DEC Alpha machines running the OpenVMS operating system. It is hosted by a DEC Alphaserver 2000 4/233, obtained at reduced cost by trading in a decommissioned computer, and consists of three DEC 3000/300LXs and an Alphastation 200. Its primary function is data analysis. The choice of a cluster based on the DEC Alpha CPU running OpenVMS is motivated by the following reasons: the primary analysis package is DAPHNE, a VMS-based analysis software package, developed here in the Division. In addition to offline analysis, DAPHNE is also used for online monitoring during data acquisition. The DEC Alpha CPU is the highest performance CPU chip on the market. Coupled with the fact that the CPU supports multiple operating systems, it was the logical choice for the division to quickly provide additional analysis resources while maintaining a long-term flexibility to adopt alternative analysis packages running on alternative operating systems.

With the bulk of data analysis now being done on the Unix and Analysis clusters, the goal of the Computer
Support Group is to provide support to fully utilize these resources. One option already under study is to share storage resources available on the primary clusters with the Linux workstations. Another is to provide software support to utilize the Linux workstations as true computing machines. These two options should enable greater utilization of existing resources. Because of its older technology, the original VAX Cluster is no longer used for data analysis. This cluster which consists of two VAX 3300 servers, configured as a dual-host system, and six satellite VAX workstations continue to provide general computing services to the division. In particular, it provides users of PCs and Macintosh computers in the division, access to additional disk storage, printing, and mail services. There will be no more improvements to this cluster. Time permitting, the Computer Support Group hopes to take preliminary steps toward finding a more cost-effective alternative to providing general computing resources.

In addition to the main divisional clusters, the division also operates three additional clusters. The Theory Group has two IBM RS/6000 workstations which serve several $\mathrm{X}$-window terminals. The group also utilizes the Argonne IBM SP2, a massively parallel computing machine, for major numerical computations. The Medium Energy Group has a small cluster of two Silicon Graphics workstations, and a larger cluster of DEC machines running OpenVMS. The larger cluster is a mixed architecture cluster, consisting of a DEC Alpha 3000/300X, a VAXstation 4000, and other smaller VAXstations.

\section{a.28. Data-Acquisition Systems (D. R. Cyborski, T. Lauritsen, and K. Teh)}

The original DAPHNE data-acquisition system was decommissioned last year. In its place, the Division now operates three MSU/DAPHNE systems for data acquisition. The MSU/DAPHNE system is a hybrid data-acquisition system which combines the front end of the Michigan State University (MSU) DA system with the traditional DAPHNE back end. The first such system was obtained for the APEX experiment and was used there successfully. A second system, purchased as a backup for the APEX front end, was installed the previous year with the procurement of the DEC 3000 Alpha host computer. During the past year, additional hardware was procured for a third MSU front end. The necessary network infrastructure was installed and a DEC 3000 Alpha, originally part of the Division's Unix Cluster, was redeployed as a VMS machine, and is now used as the host for the third MSU front-end. Memory was upgraded to allow larger online monitoring setups and the two DEC 3000 hosts were clustered to form the data-acquisition cluster. Two 8-mm tape drives, also redeployed from the offline computer clusters, were added, making available a total of four 8-mm tape drives for data taking and tape copying. We are pleased to report that the system was used extensively with great success for the past year.

Two important goals for this year include the procurement of larger color monitors for the dataacquisition cluster to replace the aging Tektronix graphics monitor, and to develop an alternative online monitoring package. 


\section{F. ASSISTANCE TO OUTSIDE USERS OF ATLAS}

\section{B. G. Glagola}

There is continuing strong interest by outside users in doing research at ATLAS, with outside users involved in about $90 \%$ of all experiments performed in FY 1995. The user assistance program is essential in facilitating the effective performance of research by outside scientists.

The outside user involvement continued to increase in FY 1995. The user liaison physicist continues to play a key role in channeling assistance to outside users. Major components of his responsibility are: (1) provide users with technical information about ATLAS and its experimental systems, and to provide instruction in its use; (2) assist outside users in all aspects of initiating and planning an experiment; (3) provide the needed information and organizational assistance to committees, workshops, and other meetings involving outside users; (4) instruct the users in the safety procedures to be followed when using the ATLAS facility; (5) the extent that is appropriate and feasible, to assist users in the actual performance of experiments; (6) provide instruction and help with the use of computer hardware and software; (7) assist in coordinating the operation of the technical support group; and (8) provide an interface between the user and the technical support and ATLAS operation groups.

Two PAC meetings were held during FY 1995. The Program Advisory Committee (PAC) for ATLAS (nominally consisting of six members from other institutions and two from Argonne) continues to meet regularly during the year. PAC meetings were held on December 2-3, 1994 and May 26-27, 1995 to recommend experiments for running time at ATLAS. In FY 1995 the PAC members were David Church (Texas A\&M University), Cary Davids (ANL), Teng Lek Khoo (ANL), Paul Kienle (GSI), Christopher J. Lister (Yale University), Mark A. Riley (Florida State University), Michael C. Wiescher (University of Notre Dame), and Frank L. H. Wolfs (University of Rochester). The Committee is chaired by John Schiffer (ANL). The PAC reviewed 32 proposals for 172 days of running time and 28 proposals for 158 days of running time at the meetings, respectively. The demand for running time at ATLAS continues to be about twice the time available on the accelerator.

The ATLAS User Executive Committee organized a User Group meeting during the October 1995 Division of Nuclear Physics APS meeting held in Bloomington, Indiana. The meeting was attended by approximately 42 scientists. The main topics of discussion were a presentation of the Users' perspective of ATLAS, institutional issues concerning an exotic beam facility and the cycling of Gammasphere to ATLAS, the status of ATLAS, accelerator R\&D, a review of the AYEBall runs at the FMA, and a discussion of PAC matters. A workshop on radioactive beams was held at ANL in January 1995. In FY 1995 the ATLAS Executive Committee consisted of Partha Chowdhury (University of Massachusetts, Lowell), John Fox (Florida State University), Lee Riedinger (University of Tennessee), and Frank Wolfs (University of Rochester), as Chairperson.

Outside users are heavily involved in the Fragment Mass Analyzer project with a rather large number participating in the setup and use of the AYEBall at the FMA in the last quarter of FY 1995 and first quarter of FY 1996. For more details see Section E.a.1.

The magnitude of the outside use of the accelerator during the past year was substantial, evidenced by the following two lists giving (A) the experiments performed by outside users and (B) the institutions represented. As may be seen from the names associated with each experiment, university groups are playing a major role in an important fraction of the experiments and a dominant role in some. 


\section{a. Experiments Involving Outside Users}

All experiments in which outside users participated during FY 1995 are listed below. The spokesperson for each experiment is given in square brackets after the title. The names in parentheses are Argonne collaborators.

Onset of Deformation in Light Polonium Nuclei: Further Study of ${ }^{194}$ Po [Cizewski]

J. Cizewski, W. Younes, R. Mayer, G. Baldsiefen, D. McNabb, Rutgers University; M. Drigert, Idaho National Engineering Laboratory; D. Nisius, Purdue University; H. Amro, North Carolina State University; (T. Khoo, S. Fischer, M. Carpenter,

B. Crowell, T. Lauritsen, D. Blumenthal, R. Janssens)

(2) Further Investigation of Possible Emergence of Collectivity at $\mathrm{N} \geq 52$ via Lifetime

Measurements in $96-98 \mathrm{Ru}$ [Garg]

U. Garg, S. Arnold, A. Frohlich, S. Ghugre, B. Kharraja, University of Notre Dame; R. Kaczarowski,

E. Ruchowska, SINS, Swierk; W. Reviol, University of Tennessee; I. Govil, Panjab University;

D. Nisius, Purdue University;

(M. Carpenter, T. Lauritsen, R. Janssens)

Study of the Two-Phonon Octupole Vibrational State in ${ }^{208} \mathrm{~Pb}$ [Henning]

E.F. Moore, North Carolina State University; S. Sanders, University of Kansas; D. Nisius, Purdue University; H. Amro, North Carolina State University; (W. Henning, T. Khoo, R. Janssens,

S. Fischer, M. Carpenter, B. Crowell, T. Lauritsen, D. Blumenthal, I. Ahmad)

Search for Shape-Coexisting Structures in $188,190 \mathrm{~Pb}$ via Fine Structure in the Alpha

Decay of ${ }^{192,194} \mathrm{Po}$ : Request for Additional Time [Zimmerman]

B. Zimmerman, C. Bingham, W. Reviol, X.-S. Chen, University of Tennessee; W. Walters,

L. Conticchio, University of Maryland; M. Huyse, N. Bijnens, J. Wauters, University of Leuven;

J. Batchelder, E. Zganjar, Louisiana State University; H. Penttilä, University of Jyväskylä; (C. Davids, R. Janssens, I. Ahmad, D. Blumenthal)

${ }^{68} \mathrm{Se}$ and the Use of the FMA for Highly Inverse Reactions [Lister]

D. Warner, J. Simpson, Daresbury Laboratory; J. Schwartz, Yale University; (C. Davids,

D. Blumenthal, D. Seweryniak, D. Ackermann, C. Lister)

Atomic Spectroscopy and Lifetime Measurements in Copper-Like Lead and Uranium

[Cheng]

S. Cheng, L. Curtis, R. Hellborg, University of Toledo; A. E. Livingston, H. G. Berry, University of Notre Dame; (R. Dunford)

APEX - Positron Production in Heavy-Ion Collisions [APEX]

S. Austin, E. Kashy, J. Winfield, J. Yurkon, Michigan State University; A. Perrera, F. Wolfs, University of Rochester; S. Gazes, M. Wolanski, University of Chicago; J. Greenberg, K. Chan, N. Kaloskamis, A. Chishti, Yale University; A. Hallin, M. Liu, Queens University; T. Trainor, University of Washington; J. Fox, E. Roa, Florida State University; T. Happ, GSI; M. Freer, University of Birmingham; C. Conner, University of Illinois, Chicago; D. Bazin, GANIL; S. Freedman, M. Maier, Lawrence Berkeley Laboratory; F. Calaprice, A. Young, Princeton University; (R. Betts, A. Wuosmaa, W. Kutschera, B. Back, J. Schiffer, I. Ahmad, D. Henderson, C. Lister, R. Dunford, M. Rhein, P. Wilt)

Production of Actinide Nuclei by Multi-Nucleon Transfer [Butler] G. Jones, J. Cocks, P. Butler, University of Liverpool; D. Nisius, Purdue University; D. Gassmann, Technical University of Munich; (R. Janssens, B. Crowell, M. Carpenter, T. Khoo, )

Spectroscopy of ${ }^{187} \mathrm{~Pb}$ using the Fragment Mass Analyzer and the Ge Detector Array [Baxter]

A. Baxter, G. Dracoulis, A. Byrne, Australian National University; D. Nisius, Purdue University; T. Brown, Vanderbilt University; L. Conticchio, University of Maryland; D. Gassmann, Technical University of Munich; (T. Khoo, R. Janssens, M. Carpenter, T. Lauritsen, C. Davids, C. Lister, D. Blumenthal) 
(10) Angular Correlation Measurements for $4 \alpha$-Decaying States in ${ }^{16}$ O Populated in ${ }^{12} \mathrm{C}+{ }^{12} \mathrm{C}$ Scattering [Wuosmaa]

D. Nisius, Purdue University; (A. Wuosmaa, B. Back, R. Betts, D. Blumenthal, D. Henderson, R. Janssens, B. Glagola, M. Rhein, P. Wilt, C. Lister)

(11) Heavy-Element Production via Asymmetric Entrance Channel Systems with ${ }^{34,36} \mathrm{~S}$ and 44,48 Ca Beams [Henning]

B. Busse, Oregon State University; T. Brown, Vanderbilt University; L. Conticchio, University of Maryland; (I. Ahmad, B. Back, C. Davids, D. Blumenthal, S. Fischer, W. Henning, D. Hofman, R. Janssens, C. Jiang, D. Seweryniak, A. Wuosmaa)

Study of the ${ }^{18} \mathrm{~F}(\mathrm{p}, \alpha)$ and ${ }^{18} \mathrm{~F}(\mathrm{p}, \gamma)$ Reactions Using a Radioactive ${ }^{18} \mathrm{~F}$ Beam - II [Rehm] M. Paul, Hebrew University; J. Gehring, University of Chicago; R. Segel, Northwestern University; A. Roberts, J. Nickles, University of Wisconsin; (K. E. Rehm, C. Jiang, D. Blumenthal, S. Fischer, J. Nolen, R. Pardo, D. Henderson, J. Schiffer)

Study of the ${ }^{18} \mathrm{~F}(\mathrm{p}, \alpha)$ and ${ }^{18} \mathrm{~F}(\mathrm{p}, \gamma)$ Reactions Using a Radioactive ${ }^{18} \mathrm{~F}$ Beam - III [Rehm] M. Paul, Hebrew University; J. Gehring, University of Chicago; R. Segel, Northwestern University; A. Roberts, J. Nickles, University of Wisconsin; J. Schwartz, Yale University; (K. E. Rehm, C. Jiang, D. Blumenthal, S. Fischer, J. Nolen, R. Pardo, D. Henderson, J. Schiffer)

Gamma-Ray Studies of Neutron-Rich Products of Deep-Inelastic ${ }^{124} \mathrm{Sn}+{ }^{208} \mathrm{~Pb}$

Collisions [Daly]

P. Daly, P. Bhattacharyya, C.-T. Zhang, L. Richter, Z. Grabowski, D. Nisius, Purdue University; R. Mayer, Rutgers University; (R. Janssens, I. Ahmad, T. Khoo, T. Lauritsen)

(15) First Study of ${ }^{179} \mathrm{Au}$ [Riedinger]

L. Riedinger, W. Reviol, W. Mueller, B. Smith, C. Bingham, B. Zimmerman, J. Richards, University of Tennessee; H. Jin, Oak Ridge National Laboratory; W. Ma, Mississippi State University; (M. Carpenter, R. Janssens, C. Davids, D. Blumenthal, T. Khoo, B. Crowell, T. Lauritsen, I. Ahmad, D. Henderson)

Isomeric and Ground-State Decays of ${ }^{183} \mathrm{Hg}$. Search for E0 Transitions in ${ }^{183} \mathrm{Au}$ [Ramayya]

A. Ramayya, B. Balabhadrapatruni, T. Brown, T. Ginter, Vanderbilt University; W. Ma, Mississippi State University; E. Zganjar, Louisiana State University; L. Conticchio, University of Maryland; B. Busse, Oregon State University; (C. Davids, R. Janssens)

(17) Proton Radioactivity in Light Bismuth Isotopes [Davids]

T. Brown, Vanderbilt University; B. Busse, Oregon State University; L. Conticchio, W. Walters, University of Maryland; K. Toth, Oak Ridge National Laboratory; J. Batchelder, Louisiana State University; B. Zimmerman, C. Bingham, X.-S. Chen, University of Tennessee; P. Woods, T. Davinson, R. Irvine, Edinburgh University; H. Penttilä, University of Jyväskylä; (C. Davids, D. Blumenthal, D. Seweryniak)

(18) Feasibility Study for Magnetic-Moment Measurements of M1 Bands in ${ }^{197} \mathrm{~Pb}$ [Cizewski] J. Cizewski, G. Baldsiefen, M. Satteson, R. Mayer, W. Younes, N. Matt, D. McNabb, Rutgers University; L. Bernstein, Lawrence Livermore National Laboratory; D. Nisius, Purdue University; (R. Janssens, M. Carpenter, D. Blumenthal, B. Crowell)

Coulomb Excitation of Radioactive ${ }^{79} \mathrm{Rb}$ [Lister]

R. Gill, Brookhaven National Laboratory; J. Schwartz, Yale University; (C. Lister, D. Blumenthal, C. Davids, S. Fischer, M. Carpenter, R. Janssens)

(20) Search for the Ternary Fission of ${ }^{48} \mathrm{Cr}$ [Sanders] S. Sanders, F. Prosser, K. Farrar, A. Dummer, University of Kansas; (A. Wuosmaa, R. Betts) 
(21) Wavelengths and Lifetimes of $\Delta \mathrm{n}=0, \mathrm{n}=2$ Transitions in Highly-Ionized Krypton [Berry]

A. E. Livingston, H. G. Berry, K. Kukla, C. Vogel-Vogt, University of Notre Dame; L. Curtis, University of Toledo; (R. Dunford)

(22) Search for Collective Bands in ${ }^{181} \mathrm{Hg}$ [Ma]

W. Ma, P. Varmette, J. Winger, W. L. Croft, Mississippi State University; A. Ramayya, B. Balabhadrapatruni, T. Brown, J. Kormicki, S. Zhu, Vanderbilt University; D. Nisius, Purdue University; (R. Janssens, D. Blumenthal, B. Crowell)

(23) Study of Proton Intruder Bands in the ${ }^{183}$ Ir Nucleus [Kaczarowski]

R. Kaczarowski, Soltan Institute for Nuclear Studies; U. Garg, S. Ghugre, S. Naguleswaran, University of Notre Dame; D. Nisius, Purdue University; H. Amro, North Carolina State University; (R. Janssens, T. Lauritsen, D. Blumenthal, B. Crowell, T. Khoo, M. Carpenter)

Spectral Distribution of the Two-Photon Decay of $\mathrm{Kr}^{34+}$ [Ali]

R. Ali, University of Nevada; P. Mokler, GSI; A. E. Livingston, University of Notre Dame; S. Cheng, L. Curtis, University of Toledo; (R. Dunford, E. Kanter, C. Kurtz, B. Zabransky)

Study of Octupole Deformation in ${ }^{220} \mathrm{Ac}$ and ${ }^{221} \mathrm{Ac}$ [Ahmad]

L. Conticchio, University of Maryland; T. Brown, Vanderbilt University; D. Nisius, Purdue University; (B. Back, C. Davids, S. Fischer, B. Crowell, R. Janssens, T. Khoo, T. Lauritsen, M. Carpenter, I. Ahmad)

Proton Radioactivity of ${ }^{167}$ Ir and ${ }^{166}$ Ir [Davids]

P. Woods, R. Irvine, Edinburgh University; C. Bingham, B. Zimmerman, J. Das, University of Tennessee; K. Toth, Oak Ridge National Laboratory; J. Batchelder, Louisiana State University; L. Conticchio, University of Maryland; B. Busse, Oregon State University; T. Brown, Vanderbilt University; (C. Davids, D. Seweryniak, D. Blumenthal)

Studies of the $\mathrm{N}=\mathrm{Z}{ }^{88} \mathrm{Ru}$ and ${ }^{92} \mathrm{Pd}$ with the FMA [Gelletly]

W. Gelletly, P. Regan, S. Vincent, C. Pearson, S. Mohammadi, University of Surrey; J. Simpson, Daresbury Laboratory; D. Nisius, Purdue University; J. Schwartz, Yale University; (C. Lister, D. Blumenthal, R. Janssens, M. Carpenter, D. Henderson, C. Davids)

(28) Fission Fragment Mass Distributions as a Probe to the Pre-Saddle Time Scale [Charity] R. Charity, L. Sobotka, J. Dempsey, Washington University

Ion Irradiations of Highly Anisotropic High-Tc Superconductors: Probing Dynamics of Magnetic Vortices [Hettinger]

J. Hettinger, B. Washburn, D. Miller, D. Steel, ANL, Material Science Division; D. Kim, Yeungnam University

Study of the Fusion Reactions between "Stiff" and "Soft" Nuclei [Rehm]

J. Gehring, University of Chicago; (K.E. Rehm, B. Crowell, B. Glagola, M. Rhein, A. Wuosmaa, C. Jiang)

Search for Shape Eigenstate in ${ }^{32} \mathrm{~S}$

M. Freer, B. Fulton, W. Rae, N. Clarke, S. Hoad, P. Fry, University of Birmingham; (A. Wuosmaa, R. Betts)

Study of Multi-Neutron Transfer Reactions in the System ${ }^{58} \mathrm{Ni}+{ }^{124} \mathrm{Sn}$ at Sub-Barrier Energies [Rehm]

J. Gehring, University of Chicago; (K. E. Rehm, C. Jiang, D. Blumenthal, B. Crowell, B. Glagola, A. Wuosmaa, J. P. Schiffer)

(33) Study of Nuclide Production Near ${ }^{100}$ Sn Using a Radioactive Beam [Carpenter]

D. Nisius, Purdue University; J. Schwartz, Yale University; H. Amro, North Carolina State University;

(M. Carpenter, C. Lister, R. Janssens, D. Blumenthal, D. Seweryniak, C. Jiang, W. Henning,

C. Davids, T. Khoo) 
Identification of $\gamma$-Ray Transitions in the Neutron-Deficient ${ }_{51}^{110} \mathrm{Sb},{ }_{53}^{110} \mathrm{I}$, and ${ }_{54}^{110} \mathrm{Xe}$ Isobars [Paul]

E. Paul, University of Liverpool; I. Thorslund, G. Lane, SUNY at Stony Brook; W. Gelletly, S. Vincent, University of Surrey; J. Simpson, Daresbury Laboratory; (C. Lister, R. Janssens, T. Khoo, D. Seweryniak)

In-Beam Spectroscopy of ${ }^{103}$ Sn [Seweryniak]

L. Conticchio, W. Walters, University of Maryland; T. Brown, Vanderbilt University; D. Nisius, Purdue University; B. Busse, Oregon State University; J. Schwartz, Yale University; (C. Davids, D. Seweryniak, C. Lister, S. Fischer, M. Carpenter, D. Blumenthal, R. Janssens, T. Khoo, D. Ackermann)

(36) Angular Correlation Measurements for ${ }^{12} \mathrm{C}\left({ }^{12} \mathrm{C},{ }^{12} \mathrm{C}\right){ }^{12} \mathrm{C}\left(3^{-}\right)$Inelastic Scattering [Wuosmaa]

D. Nisius, Purdue University; (A. Wuosmaa, B. Back, R. Betts, S. Fischer, B. Glagola,

D. Hofman, D. Blumenthal, C. Lister, R. Janssens)

Heavy Ion Irradiation of High-Temperature Superconducting Single Crystals [Fendrich] J. Fendrich, W. Kwok, G. Crabtree, Materials Science Division, ANL

${ }^{40}$ Ca Beam Test [Pardo]

D. Nisius, Purdue University; (R. Pardo, R. Harkewicz, P. Billquist, G. Zinkann, R. Janssens, M. Carpenter)

Study of the Doubly-Odd $\mathrm{T}=0$ Nucleus ${ }^{6}{ }_{\mathrm{Ga}}$ [Warner]

D. Warner, J. Simpson, Daresbury Laboratory; P. Regan, S. Vincent, University of Surrey; D. Nisius, Purdue University; (C. Davids, D. Blumenthal, C. Lister, D. Seweryniak, R. Janssens)

Neutron-Rich Isotope Production via Protons on Uranium [Nolen]

R. Ronningen, Michigan State University; (I. Ahmad, B. Back, J. Schiffer, J. Nolen, C. Davids)

Radiative Capture via Inverse Kinematics [Davids]

M. Gai, J. McDonald, University of Connecticut; J. Goerres, A. Garcia, M. Wiescher, University of Notre Dame; P. Woods, Edinburgh University; L. Conticchio, University of Maryland; T. Brown, Vanderbilt University; H.Penttilä, University of Jyväskylä; (C. Davids, D.Henderson, D. Blumenthal)

\section{b. Outside Users of ATLAS and of ATLAS Technology -- 10/1/94 - 9/30/95}

This list includes only those who were present at ATLAS for an experiment or other related research. An asterisk denotes students.

(1)

$$
\begin{aligned}
& \text { University of Kansas } \\
& \text { * A. Dummer } \\
& \text { K. Farrar } \\
& \text { F. Prosser } \\
& \text { S. Sanders }
\end{aligned}
$$

$\begin{array}{ll}\text { Purdue University } \\ \text { * } & \text { P. Bhattacharyya } \\ & \text { P. Daly } \\ & \text { Z. Grabowski } \\ * & \text { D. Nisius } \\ * & \text { L. Richter } \\ & \text { C. } \text { Zhang }\end{array}$

$$
\begin{aligned}
& \text { Washington University } \\
& \text { * } \quad \text { R. Charity } \\
& \text { J. Dempsey } \\
& \text { L. Sobotka }
\end{aligned}
$$

\begin{tabular}{ll}
\multicolumn{2}{c}{ Vanderbilt University } \\
& B. Balabhadrapatruni \\
$*$ & T. Brown \\
$*$ & T. Ginter \\
& J. Kormicki \\
& A. Ramayya \\
$*$ & D. Shi \\
& S. Zhu
\end{tabular}


(5) University of Notre Dame

$\begin{array}{ll}* & \text { S. Arnold } \\ * & \text { A. Frohlich } \\ & \text { H. G. Berry } \\ & \text { A. Garcia } \\ & \text { U. Garg } \\ & \text { S. Ghugre } \\ & \text { J. Goerres } \\ & \text { B. Kharraja } \\ & \text { K. Kukla } \\ & \text { A. E. Livingston } \\ & \text { S. Naguleswaran } \\ * & \text { C. Vogel-Vogt } \\ & \text { M. Wiescher }\end{array}$

(6) Rutgers University

$$
\begin{array}{ll} 
& \text { G. Baldsiefen } \\
& \text { J. Cizewski } \\
* & \text { N. Matt } \\
& \text { R. Mayer } \\
* & \text { D. McNabb } \\
* & \text { W. Younes }
\end{array}
$$

(7) Lawrence Livermore National Lab

$$
\text { L. Bernstein }
$$

(8) University of Rochester

$$
\begin{aligned}
& \text { * A. Perrera } \\
& \text { F. Wolfs }
\end{aligned}
$$

(9) University of Connecticut

$$
\text { * } \quad \text { M. Gai }
$$

(10) University of Chicago

$$
\begin{array}{ll} 
& \text { S. Gazes } \\
* & \text { J. Gehring } \\
* & \text { M. Wolànski }
\end{array}
$$

(11) University of Nevada

$$
\text { R. Ali }
$$

Idaho National Engineering Lab M. Drigert

University of Toledo

S. Cheng

L. Curtis

* R. Hellborg

(14) NSC, New Delhi
G. Mehta
R. Mehta
P. Potukuchi
A. Roy

(15) Technical University, Munich

D. Gassmann
(17)

$\begin{array}{ll}\text { University of Tennessee } \\ \text { C. Bingham } \\ \text { * } & \text { X.-S. Chen } \\ & \text { J. Das } \\ * & \text { W. Mueller } \\ & \text { L. Riedinger } \\ & \text { W. Reviol } \\ * & \text { J. Richards } \\ * & \text { B. Smith } \\ & \text { B. Zimmerman }\end{array}$
R. Segel

University of Wisconsin J. Nickles

A. Roberts
A. Baxter
A. Byrne
G. Dracoulis

University of Maryland

L. Conticchio

W. Walters

Yale University

$\begin{array}{ll}* & \text { K. Chan } \\ & \text { A. Chishti } \\ & \text { J. Greenberg } \\ * & \text { N. Kaloskamis } \\ * & \text { J. Schwartz }\end{array}$

Florida State University

J. Fox

* E. Roa
G. Crabtree
J. Fendrich
K. Gray
J. Hettinger
W. Kwok
D. Miller

* B. Washburn

Hebrew University M. Paul

University of Tennessee

University of Sao Paulo N. Added
Northwestern University

Australian National University

Materials Science Division, ANL
A. Szanto de Toledo

Michigan State University
S. Austin
R. Fox
E. Kashy
R. Ronningen
J. Winfield
J. Yurkon

J. Batchelder

E. Zganjar 
(28) Princeton University

F. Calaprice

A. Young

(29) Daresbury Laboratory

R. Cunningham

J. Simpson

D. Warner

(30) Mississippi State University

L. Croft

W.-C. Ma

* P. Varmette

J. Winger

(31) Oak Ridge National Laboratory

* H. Jin

K. Toth

(32) Brookhaven National Laboratory

R. Gill

(33) Lawrence Berkeley Laboratory

S. Freedman

M. Maier

(34) University of Birmingham

N. Clarke

M. Freer

* P. Fry

B. Fulton

* S. Hoad

W. Rae

University of Leuven

* N. Bijnens

M. Huyse

J. Wauters

(36) GSI, Darmstadt

T. Happ

P. Mokler

(37) Queens University, Ontario

A. Hallin

M. Liu
(45)

(38)

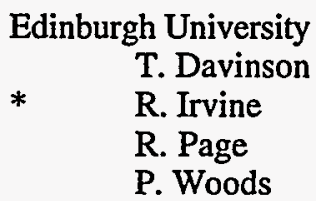

(39)

University of Illinois, Chicago

* C. Conner

(40) GANIL

North Carolina State University

E. F. Moore

* H. Amro

University of Manchester

J. Durell

S. Freeman

W. Phillips

University of Washington T. Trainor

University of Surrey

W. Gelletly

* S. Mohammadi

C. Pearson

P. Regan

* S. Vincent

SINS, Swierk

R. Kaczarowski

E. Ruchowska

Oregon State University

* B. Busse

(47) University of Liverpool

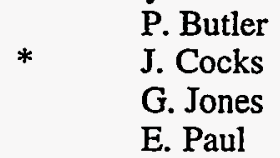

SUNY, Stony Brook

I. Thorslund

G. Lane

(49) Panjab University

I. Govil

(50) University of Jyväskylä

H. Penttilä 


\section{c. Summaries of the Continuing User Programs for FY 1995}

\section{c.a. The University of Notre Dame}

c.a.1. Nuclear Physics (U. Garg, A. Frohlich, S. Ghugre, B. Kharraja, S. Naguleswaran, G. Smith, J. Walpe, W. Reviol, L. Riedinger (University of Tennessee), R. Kaczarowski, E. Ruchowska, (SINS, Swierk, Poland), I. Govil (Panjab University, Chadigarh, India)

Several groups from the University of Notre Dame are playing an important role in developing the research program at ATLAS. One of their main interests is the study, in collaboration with ANL staff members, of the behavior of nuclei at high spin in the transitional region near $A=180$ (i.e. the $\mathrm{Hg}-\mathrm{Pt}-\mathrm{Os}$ nuclei), and $A=100$ (i.e. the $R u-T c$ nuclei), with emphasis on shape coexistence and configuration mixing. The $\gamma$-ray groups at ANL and Notre Dame also had collaborative experiments at Gammasphere. The Notre Dame group built, tested, and used extensively a state-of-the-art plunger device for lifetime measurements in conjunction with the ATLAS $\gamma$-ray facility. An adapted version of this device is now under construction at Notre Dame, under contract with ANL, for use at the Gammasphere facility.

\section{c.a.2. Atomic Physics (A. E. Livingston, H. G. Berry, K. W. Kukla, C. Vogel Vogt)}

In a collaboration with the Atomic Physics groups at Argonne and the University of Toledo, the Atomic Physics group at the University of Notre Dame is measuring the energy structures and lifetimes of excited fine structure in highly-charged few-electron ions. Extreme ultraviolet spectroscopy is applied with the beam-foil excitation technique and positionsensitive photon detection. A precise measurement of the $2{ }^{3} \mathrm{~S}_{1}-2{ }^{3} \mathrm{P}_{0,2}$ transition energies in heliumlike $\mathrm{Ar}^{16+}$ was published which provides sensitivity to uncalculated higher-order screening corrections in the QED contributions. Further measurements of the $n$ $=2$ state atomic structures are planned for the higher- $Z$ heliumlike ions $\mathrm{Ni}^{26+}$ and $\mathrm{Kr}^{34+}$. Measurements of excited-state spectra and lifetimes of $\mathrm{n}=2$ states in lithiumlike $\mathrm{Kr}^{33+}$ and berylliumlike $\mathrm{Kr}^{32+}$ were initiated, and extension of these measurements to higher beam energies is planned. Wavelength and lifetime measurements in copperlike $\mathrm{U}^{63+}$ were also undertaken, and data analysis is in progress. In a collaboration with P. Mokler of GSI-Darmstadt, measurements were made of the spectral distribution of the $2 \mathrm{E} 1$ decay continuum in heliumlike $\mathrm{Kr}^{34+}$, and a measurement comparing the $2 \mathrm{E} 1$ continua in heliumlike $\mathrm{Ni}^{26+}$ and hydrogenlike $\mathrm{Ni}^{27+}$ is planned.

\section{c.b. Purdue University (P. Daly, Z. Grabowski, D. Nisius, L. Richter, P. Bhattacharyya, C. T. Zhang)}

The Purdue University group, which includes several thesis students, is working on a measurement of highspin nuclear states at ATLAS. They use in-beam gamma-ray techniques to investigate several aspects of nuclear structure at high spin, testing the validity of shell-model calculations for high-spin yrast states. The nuclei are produced via deep inelastic reactions, rather than with the more conventional fusion reactions. This technique allows the study of neutron-rich nuclei that cannot be studied by other means. In the past year this study was extended to the ${ }^{238} \mathrm{U}+{ }^{124} \mathrm{Sn}$ system. The group is studying proton-rich nuclei with $\mathrm{N} \sim 82$ using the FMA and an electron spectrometer to investigate the ${ }^{58} \mathrm{Ni}+{ }^{92} \mathrm{Mo}$ reaction. Furthermore, D. Nisius is a Ph.D. student, resident at ANL, performing his thesis work under the supervision of R. V. F. Janssens. 


\section{c.c. University of Kansas (S. Sanders, F. W. Prosser, and A. Dummer)}

During the past year, three projects started in previous years were largely brought to conclusion. The groups' study of the ${ }^{136} \mathrm{Xe}+{ }^{64} \mathrm{Ni}$ reaction started out as a search for gamma-ray transitions resulting from a predicted superdeformed configuration in $66 \mathrm{Ni}$. Although no evidence for such a configuration was observed, the extensive data that were accumulated proved valuable in a study of single- and multi-nucleon transfer reactions in the ${ }^{136} \mathrm{Xe}+{ }^{64} \mathrm{Ni}$ system and the role of cluster transfer in these reactions. For the past several years the group explored the fusion-fission mechanism in light nuclei. During the past year, data for three separate entrance channels reaching the ${ }^{48} \mathrm{Cr}$ compound system were used to establish the competition between the fission and heavy-ion resonance mechanisms in this system. The third project that was completed last year was the construction of a

c.d. Vanderbilt University (A.V. Ramayya, J. H. Hamilton, J. Kormicki, L. T. Brown, B. R. S. Balabhadrapatruni, D. T. Shi, and W.-C. Ma [Mississippi State University] )

An experiment identifying gamma-ray transitions in ${ }^{183} \mathrm{Hg}$ using $\gamma-\gamma$ and recoil- $\gamma$ coincidences was performed at the FMA and published. Five bands were observed, of which two are associated with the [624]9/2 ${ }^{+}$orbital and exhibit signature splitting. Two other bands which do not show signature splitting were associated with the [514]7/2- orbital and exhibit transition energies almost identical to bands with the same configuration in $185 \mathrm{Hg}$. New experiments on identification of bands in ${ }^{181} \mathrm{Hg}$ and

\section{c.e. Idaho National Engineering Laboratory}

This activity is associated with many research programs done with the Argonne-Notre Dame BGO $\gamma$ ray facility and is heavily involved with the superdeformation studies in the $\mathrm{Dy}$ and $\mathrm{Pb}$ mass regions. There is also participation in the study of octupole stability in the light actinide mass region. hybrid MWPC-Si strip detector. This compact detector, which was developed to allow good position, time, and energy measurement in a close geometry, was used successfully in a new experiment at ATLAS searching for the ternary breakup of ${ }^{48} \mathrm{Cr}$.

The measurement of the ternary breakup of ${ }^{48} \mathrm{Cr}$ into three ${ }^{16} \mathrm{O}$ fragments, using the ${ }^{36} \mathrm{Ar}+{ }^{12} \mathrm{C}$ reaction, was motivated by the renewed interest in the role of clustering in light nuclei and specific predictions of a three-16 0 cluster-like arrangement in ${ }^{48} \mathrm{Cr}$. Although the data analysis is incomplete, preliminary results suggest a significant population of the ${ }^{16} \mathrm{O}-16_{0}-16_{0}$ final state, possibly indicating an ${ }^{16} \mathrm{O}$ cluster configuration in ${ }^{48} \mathrm{Cr}$. Work started on developing a new experiment based on these results, which is expected to become A. Dummer's thesis project. an in-beam study of conversion electrons from ${ }^{183} \mathrm{Hg}$ were performed in 1995 at the focal plane of the FMA using the tape transport, PIN detectors and cooled $\mathrm{Si}(\mathrm{Li})$ detectors, and a $\mathrm{Ge}$ detector to investigate the isomeric and ground-state decays of $183 \mathrm{Hg}$. In particular, a search for E0 transitions in ${ }^{183} \mathrm{Au}$ was undertaken to indicate the presence of shape coexistence. One member of the collaboration, L. Brown, who is a Ph.D. student, is working full-time under the direction of Cary Davids.

\section{c.f. University of Tennessee, University of Maryland, and Oak Ridge National Laboratory (C. R. Bingham, J. D. Richards, B. Zimmerman, W. B. Walters, L. F. Conticchio, and K. S. Toth)}

This collaboration was involved in the study of alphadecaying nuclei in the $\mathrm{A}>180$ region using the double-sided silicon strip detector implantation facility at the FMA. They are also active participants in the proton radioactivity experiments (see Sec. I.A.a.13). 


\section{c.g. University of Toledo (L. J. Curtis, S. Cheng)}

A program of fast ion beam studies of the structure of highly-ionized atoms is being carried out in collaboration with the Atomic Physics groups at Argonne and the University of Notre Dame. Transition energies and lifetimes are measured using time resolved methods which employ positionsensitive multiplexed detection of wavelengths and coincidence and energy summing discrimination. Particular emphasis is given to examining isoelectronic trends which can be exploited for interpolative and extrapolative predictions. Current projects include studies of lifetimes for two-photon decays in Heliumlike $\mathrm{Ni}, \mathrm{Ar}$, and $\mathrm{Kr}$; lifetimes of highly ionized members of $\mathrm{Li}, \mathrm{Be}$, and $\mathrm{B}$ isoelectronic sequences; energies and lifetimes of Copperlike and Zinclike Uranium ions; Z-dependence of the energy distribution shapes for two-photon decays; ultra-short lifetime measurement by the two-foil technique; and the study of giant $\mathrm{C} 60$ molecules by atomic collisions.

\section{d. ATLAS - Technology Transfer}

In addition to outside users of the ATLAS beam, we also provide assistance in the use of ATLAS technology at other laboratories.

\section{d.a. Florida State University and Kansas State University}

Argonne fabricated the niobium resonators and some auxiliary devices for the superconducting linac energy booster built at Florida State University and the accel/decel linac now in operation at Kansas State University. There was substantial interaction with personnel from both FSU and KSU in the later
1980 s and early 1990s during the construction phases when several of their staff spent extended periods of time at ANL and during the commissioning and early operations phases of both accelerators. Recently there is very little interaction with these facilities.

\section{d.b. University of Sao Paulo (N. Added and A. Szanto de Toledo)}

Argonne is assisting the University of Sao Paulo (USP) in the construction of a small superconducting heavy-ion linac to serve as an energy booster for projectiles from their 8-MV tandem. This booster will be similar in many respects to the ANL booster linac built in the late 1970s. The ANL contribution to this project will be (1) to build (at USP expense) 14 splitring niobium resonators and some of the associated if electronics, (2) to provide technical information, and (3) to train USP staff members in several phases of superconducting-linac technology. Two Brazilian engineers worked at Argonne for one year, gaining experience in cryogenics and in superconductingresonator technology. Another engineer worked on the new control system at ATLAS for two years, the first year supported by Sao Paulo and the second with direct ANL support. Sao Paulo personnel returned to ANL in 1993 for assembly and testing of the first batch of completed resonators. The fabrication of the resonators was completed in 1995 when the Sao Paulo personnel came for final assembly and testing. Eleven resonators were shipped to Sao Paulo and three remain at ANL for final testing. Fabrication of electronics modules at ANL is still in progress.

\section{d.c. Nuclear Science Centre, New Delhi (G. Mehta, P. Potukuchi, A. Roy, R. Mehta)}

Argonne is collaborating with the Nuclear Science Centre (NSC), New Delhi, to develop a new type of superconducting accelerating structure for low-velocity heavy ions. A copper model were evaluated and tests on the niobium prototype are currently in progress. Some technical details of this project are described in the Superconducting Linac Development section of this FWP. The NSC stationed staff members at ATLAS for extended time periods during the past three years to gain experience and to work on this project. Two NSC staff members are scheduled to spend several months at ANL during 1996 to finalize development of the prototype resonators and to initiate fabrication of the production models for their linac project. 


\section{OPERATION AND DEVELOPMENT OF ATLAS}

\section{OVERVIEW}

This section reports on the operation of the Argonne Tandem Linear Accelerator System (ATLAS) as a national user facility. ATLAS is used for basic research in nuclear physics and atomic physics, as well as occasionally for other areas of research, such as materials science. Over half of the beam time is allocated to outside users.

Currently, the facility is on a seven-day week operating schedule. An increase in funding as part of the DOE Scientific Facilities Initiative began in 1996, to bring the staff level and $M \& S$ budget up to that required for steady-state seven-day operation. The present budget permits the delivery of 5600 hours per year of beam on target for research. This enhances our ability to serve the high level of demand for beam time from the ATLAS user community.

An upgrade of the three ATLAS liquid helium refrigerators to reduce electrical power usage, funded by the DOE in-house energy management program, was completed in the summer of 1995 . The project is paying off in three ways: the cryogenics efficiency is about $30 \%$ better at normal refrigeration loads, the maximum refrigerator capacity is somewhat increased at the original electrical input when needed for the heaviest beams, and the wet expansion engines proved to provide much smoother flow-rate control than the original Joule-Thompson expansion valves.

A second, and more advanced Electron Cyclotron Resonance (ECR) ion source system, funded as an Accelerator Improvement Project, is under construction, and is scheduled for completion in 1996. AIP funding in 1997 will provide the beam line for coupling the new source into the linacs. Beam on target for research will be available from the new ion source System by mid-1997. Improved beam monitoring and faster linac tuning will be possible with a new type of nonintercepting beam intensity and phase pickup system. A prototype is working well at the entrance of the PII linac, and units are currently being commissioned at the Booster and ATLAS linac entrances. 


\section{ATLAS Beams for FY 1995}

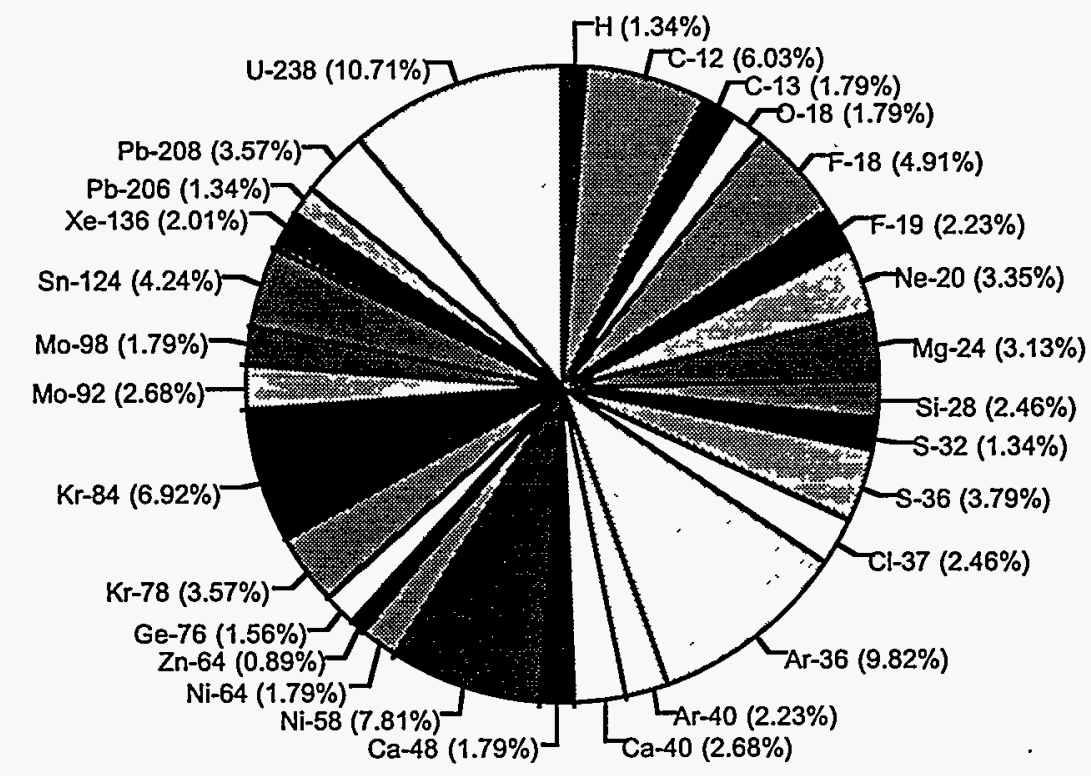

Fig. II-1. Chart of the isotopes delivered by ATLAS for research during FY 1995. Relative usage is indicted.

TABLE I. Statustics related to ATLAS Operations and Users.

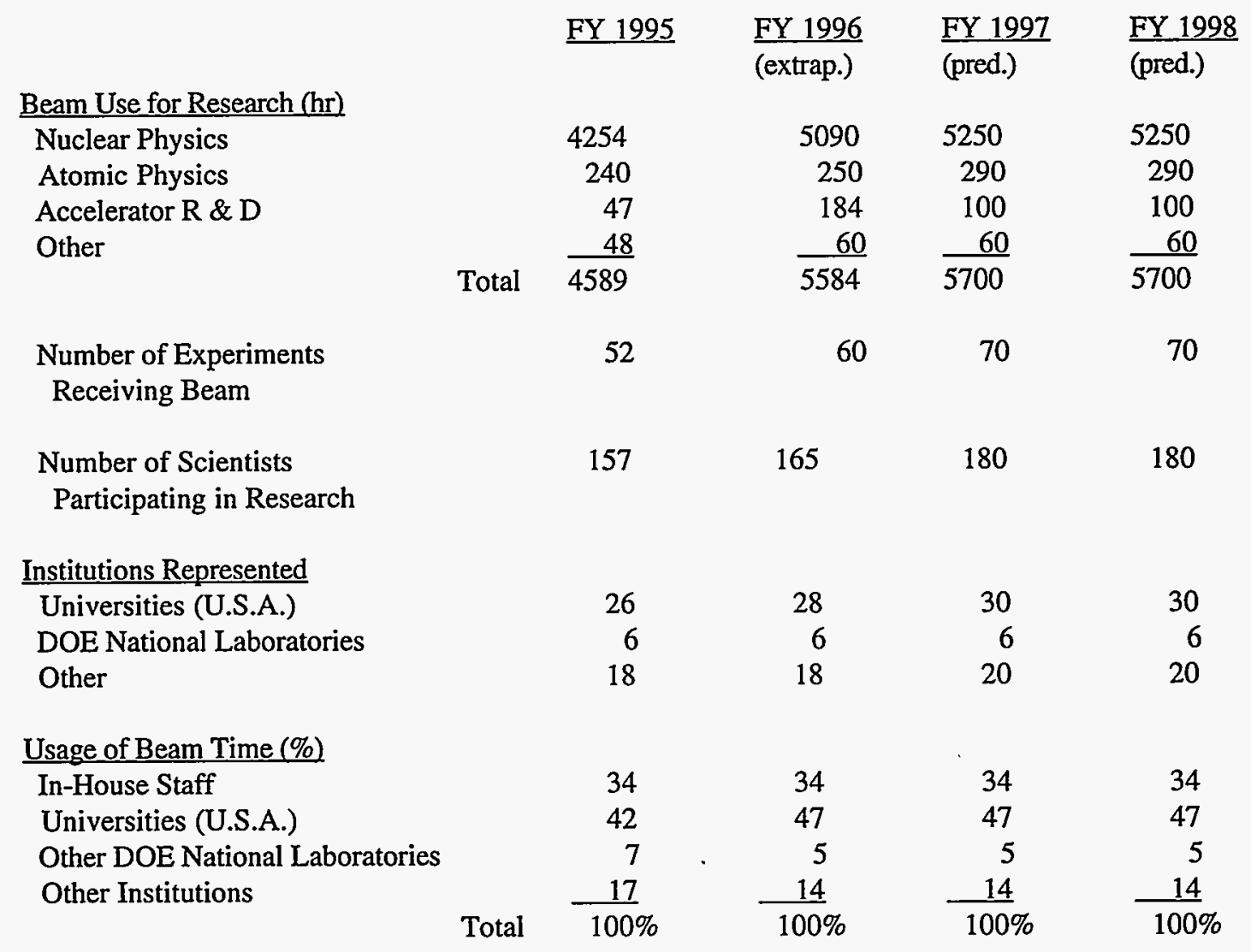




\section{A. OPERATION OF THE ACCELERATOR}

(R. C. Pardo, B. Batzka, P.J. Billquist, J. Bogaty, B. E. Clifft, S. L. Craig, R. E. Harden, B. Millar, F. H. Munson, Jr., K. Nakagawa, D. R. Phillips, C. Roehrig, A. Ruthenberg, M. Schlapp, J. R. Specht, P. Strickhorn, A. Sutherland, B. Tieman, I. R. Tilbrook, R. Vondrasek, and G. P. Zinkann)

During FY 1995 ATLAS delivered 4589 hours of research time. A two-month shutdown of the facility for the installation of two additional 'wet engines' on the cryogenic refrigerators reduced the research hours delivered this year compared to FY 1994, the first year of seven-day operation. A total of 51 experiments were performed at the facility. The reliability factor for the fiscal year was a good $89.5 \%$ which included some lost time as part of the restart of the facility with the new 'wet engines'. Statistics related to hours of opertion and the distributon of research time and origin or users are given in Table I.

Seven-day operation was made possible two years ago with the addition of two additional operators, the bare minimum needed to initiate seven-day operation. With some increased funds in FY 1996 due to the Scientific Facilities Initiative, we have begun the process of adding two additional persons to the staff. The new staff members will provide additional staff to our electronics repair and development group and will give some increased depth in the operator pool by adding one person to the group who will serve as a roaming operator assigned to fill-in as much as possible during periods of illness and vacation. This person will also have $30-50 \%$ of their time assigned to the vacuum and mechanical maintenance group to provide added manpower in that area. Other staff rearrangements allowed us to assign a second person to ECR ion source operations and to provide a cryogenics person during eight hours of each weekend day. We believe these improvements in staff levels will significantly improve our ability to respond to weekend problems and make all employee's task assignments more manageable. Due to the nine month training time for new operators, the improved staff arrangements will not be fully functional until early in FY 1997.

ATLAS provided a total of 29 different isotopes for the research program in FY1995 with the relative usage shown in Figure II-1. New beams of zinc and ruthenium were developed from the ECR source this year. This is a new record number for any fiscal year even though the operating year was shortened by two months for the cryogenic system upgrade. Uranium and lead isotopes were used for $13.4 \%$ of the beam time and $22 \%$ of the beam time utilized isotopes with
$A>100$. The Positive-Ion Injector provided beams $68 \%$ of the research time during the year, with the tandem being used as the injector for the rest. The tandem was used to provide the radioactive beam ${ }^{18} \mathrm{~F}$ for a series of nuclear astrophysics measurements during FY 1995. The use of ATLAS for research with radioactive beams is increasing in FY 1996.

The facility was down ten weeks for a major cryogenics upgrade from April 14 to June 27. The dominant task was the installation of a new 'wet expander' engine on each of the two ATLAS 2800 refrigerators. In FY 1994 the first 'wet expander' engine was installed on the smaller 1630 refrigerator. The project was made possible by an 'energy efficiency' fund from the DOE in-house energy management program. The results of the program are improved energy efficiency in the operation of the cryogenic system during typical accelerator operation and increased maximum capacity for high-energy runs.

While work proceeded on the cryogenics system, other significant maintenance and improvement projects were undertaken by the rest of the ATLAS crew. Resonator maintenance and cryostat repairs on five cryostats were undertaken, the last two ' $\mathrm{V}$-series' (matched beta of 0.15 or about $12 \mathrm{MeV}$ per nucleon) were replaced with ' $\mathrm{H}$ series' resonators (matched beta of 0.10 or about $5 \mathrm{MeV}$ per nucleon). The new resonator configuration is better matched for heavy beams such as lead and uranium in the 5 to $8-\mathrm{MeV}$ per nucleon range. The $40^{\circ}$ bend region was also completely rebuilt, improving the beam transmission in the region significantly, and a new beam stripper assembly was installed at the booster exit.

Over the past two years ATLAS experienced problems with the reliable delivery of primary electrical power to the facility. In the past 18 months, the Laboratory has undertaken a major upgrade and replacement of the primary power feed system to the ATLAS facility and the entire Argonne 200 Area. In FY 1995 we began to see the results of these improvements when the facility had only 68 hours of lost time due to building utility problems. The upgrade of the primary power delivery system is complete for the ATLAS facility and Building 203 and we now expect to have very little lost time due to power failures. 


\section{Recent Improvements at ATLAS}

\section{a Upgrade of the Linac Control System (F. Munson, B. Tieman, S. Kramer)}

The new ATLAS control system, based on the commercial "VISTA" software package, continues to evolve. Control of all the system hardware is available within the new control system and a number of 'highlevel' functions are now available, including scaling saved tunes, and routine logging of system parameters. Programs for changing the linac energy and setting up the initial linac configuration are under active development with initial testing of these expected in the spring and summer of 1996. The last 'high-level' program which will be ported to the new system will be the resonator 'auto-scan' program which empirically determines the beam-resonator phase for a new, unscaled linac configuration.

Control of the new ECR source will also be incorporated into the new control system rather than using a separate stand-alone control system as for the existing source.

\section{b. Linac Beam-phase Monitoring at the Entrance to Each Section (J. Bogaty, B. E. Clifft)}

In last year's Annual Report, the planned use and status of capacitive pickups to measure the arrival time of the beam at the entrance to each of the major sections of the linac (PII, Booster, ATLAS) were discussed. This system is expected to allow the accurate setting of the beam arrival time at each of the major linac sections so that scaled tune configurations may be reliably used and a much more flexible scaling algorithm can be implemented.

The system located at the entrance of the PII linac was fully implemented in FY 1995 and is being used routinely. In the past four months, pickups at the entrance to the Booster and ATLAS were installed and the associated electronics are now also complete.

The PII system already demonstrated its utility in permitting rapid setting of the arrival time at the entrance to the linac which greatly facilitates the configuration of the PII linac. The time required for linac tuning as a percentage of available research hours was $13 \%$ for FY 1995. That value has continued to decrease from 15\% in FY 1994 and 22\% in FY 1993. With the operation of the Booster and ATLAS pickups, a further reduction of beam tuning time is expected to be realized.

\section{c. Helium Refrigerator System (J. R. Specht, B. Millar, A. Sutherland)}

An energy conservation upgrade was completed in 1995. This project included adding a wet expansion engine to each of the three existing ATLAS liquid helium refrigerators thus replacing the Joule-Thompson valves. Also, a variable-speed motor drive was added to one compressor on each refrigerator. These improvements resulted in approximately a $30 \%$ reduction in energy use for the refrigerators and allow smoother control of the system. The actual cost savings of these measures depend on the actual load on the refrigerators, being less for higher loads.

The installation of uninterruptable power supplies in the refrigerators' and compressor control systems is also complete. This improvement allows automatic restart of the refrigeration system in case of short (10-15 seconds) interuptions of electrical power. This upgrade functioned last summer restarting the systems after a ten-second power failure. A new programmable-logic, PC-based cryogenic alarm system was also brought fully on line this year. This fail-safe system allows for additional alarm circuits and is readable from the ATLAS control room as well as at each refrigerator. The charcoal filters in the $2800 \mathrm{~W}$ refrigerator were nearly plugged and un-cleanable for several years. A new set of filters was purchased, built into a new valve box, and mounted externally to the cold box. The operation of the refrigerator is much improved with these new filters. The first step in a project to improve the temperature profile of the nitrogen heat shield on the PII linac cryostats is complete. The goal of the project is to reduce the liquid nitrogen consumption and the quiescent cryostat heat load to the helium refrigeration system. By adding an additional heat shield component in one PII cryostat a significant improvement in the quiescent helium system heat load of approximately 10 watts was observed and some improvement in liquid nitrogen consumption was also achieved. We plan to extend these improvements to the remaining two cryostats as time permits. 


\section{Current Projects and Future Plans}

ATLAS is expected to operate for research a total of 5700 hours in FY 1997. This level of productivity is made possible by the two new positions funded by the DOE Scientific Facilities Initiative in FY 1996.

The project to construct a second ECR ion source and associated high-voltage platform has progressed well this past year. Components of the $14 \mathrm{GHz}$ source are shown in Fig. II-2. The new source will be commissioned in FY 1996. Funds for the beamline connecting the new ECR ion source to the PII injector will be provided via an Accelerator Improvement Project in FY 1997. First beams from the source for research should be available in Summer 1997. This source will make possible uranium, and other high mass beams, at Coulomb barrier energies without any stripping stage. The benefits will be higher intensity, better beam quality, and more reliable operation for the very heavy beams from ATLAS. Beams of rare isotopes from natural feed materials will then be possible for interesting ( $\approx \mathrm{pnA})$ beam currents due to the higher intensity beams which will be provided by the advanced ECR ion source.

System reliability, stability, and efficiency of the superconducting linacs will be improved by the fabrication and installation of upgraded resonator if control modules. The 20,000 gallon $\mathrm{LN}_{2}$ storage tank, obtained as surplus equipment from the SSC and shown in Fig. II-3, will also be installed this year.

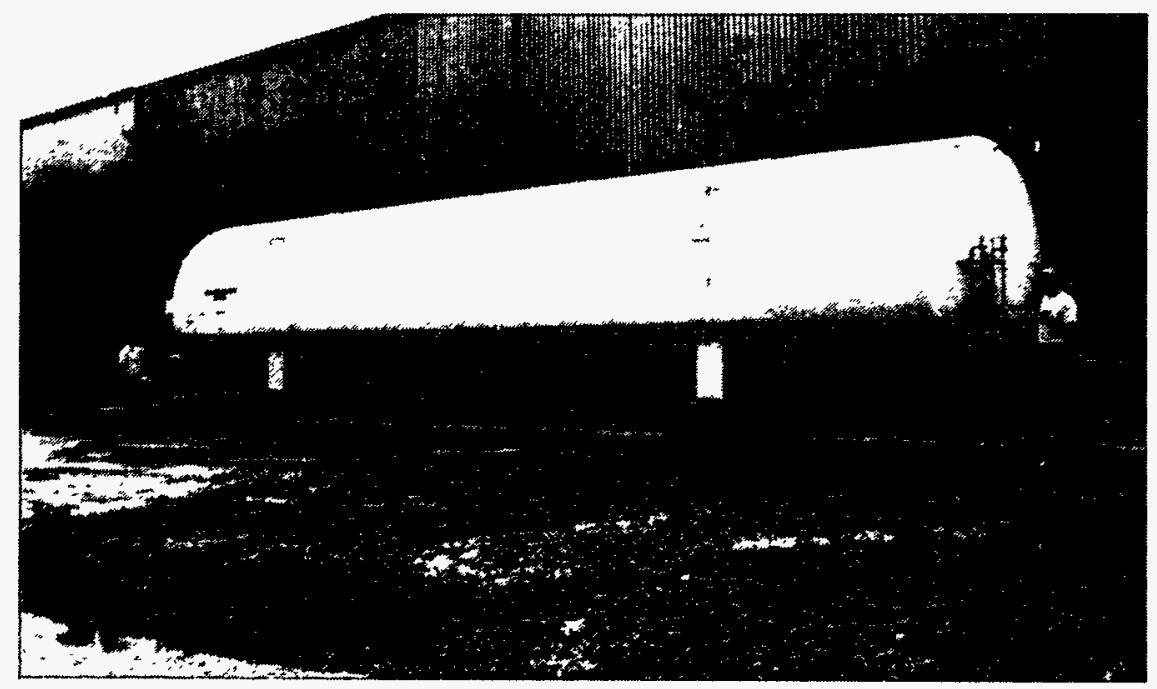

Fig. II-3. The 20,000 gallon liquid nitrogen storage tank, obtained as surplus equipment from the SSC project,

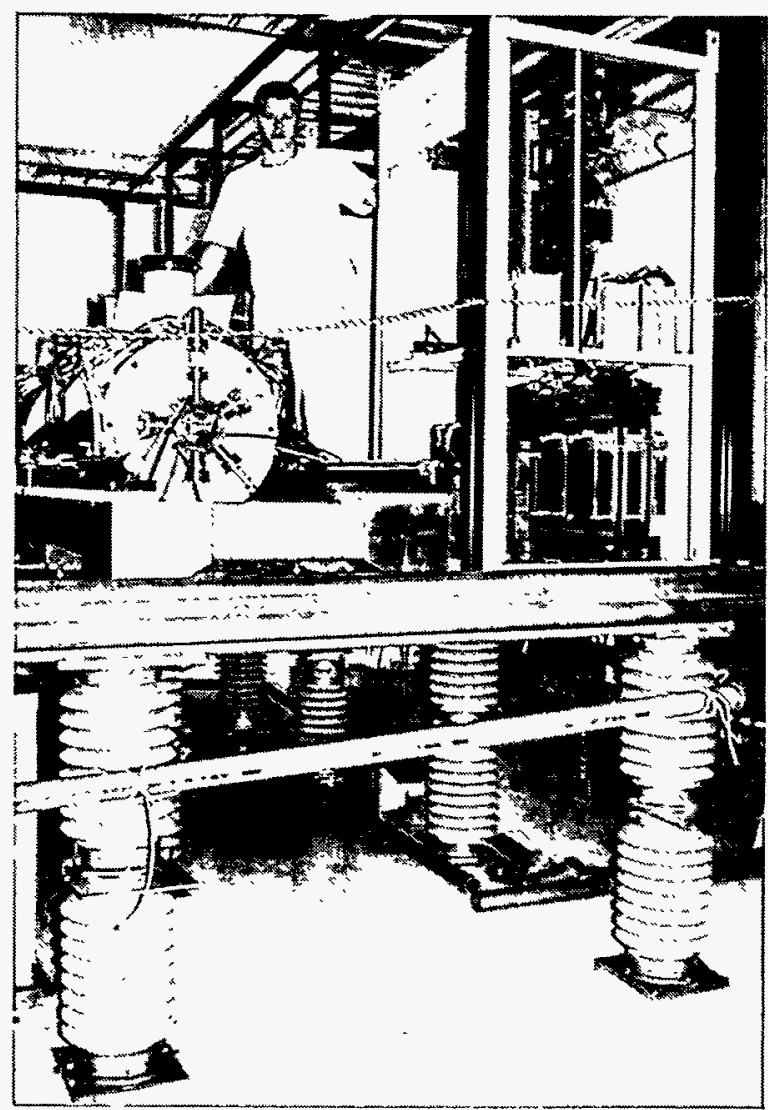

Fig. II-2. A view of the $14 \mathrm{GHz} E C R$ ion source and its high-voltage platform under construction in the recently completed ATLAS building addition.

\footnotetext{
arrived at Argonne recently and is currently being installed at ATLAS.
} 
All of these upgrades will be completed in time to be of benefit for operation of ATLAS with Gammasphere beginning in early FY 1998.

The present plan is for Gammasphere to be moved to ATLAS early in FY 1998 and for research with the Gammasphere/FMA system to use a large fraction of the beamtime for at least 12 months. Our intent is to plan major maintenance periods to coordinate with the Gammasphere schedule so as to provide 6000 hours of beam time for research during that 12 month period. The second ECR ion source will be fully commissioned during FY 1997 so as to be available with its increased capabilities for expanded operations during FY 1998.
Scheduling flexibility will be enhanced by the availability of three injectors, the two ECR ion source systems, and the tandem.

The Accelerator Improvement projects for FY 1998 will not interfere with beam time that year. It is proposed to install the large helium compressor obtained from the SSC to reduce the electrical cost of running ATLAS. It is also proposed to construct three new L-type superconducting resonators to replace two of the existing $\mathrm{H}$-type resonators in the booster, in order to augment the energies of the heaviest beams, such as lead and uranium, from the new ECR ion source.

\title{
B. ACCELERATOR PHYSICS AND LINAC DEVELOPMENT
}

\author{
J. A. Nolen, R. C. Pardo, K. W. Shepard, R. Harkewicz, J.-W. Kim, J. Bogaty, \\ B. Clifft, G. Zinkann, M. Kedzie, T. Barlow, and K. Beyer
}

This section describes the accelerator-physics program that initially developed the underlying methods which made the ATLAS facility possible. This is now an ongoing program with several related goals: one is to maintain the forefront expertise of the small group who developed the technology of $\mathrm{Nb}$ superconducting structures for low-beta ion accelerators. The collective know-how of the Argonne team in the design, fabrication, and use of this technology is unique in the world. Another goal is to continue development as necessary in superconducting-linac-related specialties to keep ATLAS modern and competitive as a National User Facility. These related technologies include highcharge-state ion sources, beam diagnostics, acceleratorcontrol systems, fast-timing techniques, cryogenics, and linac-beam-dynamics studies. Much of the technology of ATLAS is of increasing interest for a possible future radioactive-beam facility and some aspects of it are also potentially of industrial significance. Research related to acceleration of radioactive beams is discussed in Section $\mathrm{C}$ below. Many of these accelerator-physics developments are fundamental and involve both undergraduate and Ph.D. students, as well as postdoctoral appointees and the permanent ATLAS scientific and technical staff.

Recent efforts at ATLAS were concentrated on finishing and commissioning the new positive-ion injector (PII). This involved the highly successful development of a new class of 4-gap niobium accelerating structures (in four variations) useful over the ion-velocity range from $0.008 \mathrm{c}$ to $0.05 \mathrm{c}$ for ions with $\mathrm{q} / \mathrm{m}$ as low as 0.1 . The superconducting injector linac of PII is fed with ions from an ECR source mounted on a deck which is floated at potentials up to $350 \mathrm{kV}$. The overall system works extremely well, and together with ATLAS, now routinely produces $\mathrm{CW}$ beams of lead and uranium in the 6- to $6.8 \mathrm{MeV}$ per-nucleon energy range at intensities on target of up to $5 \mathrm{pnA}$. Lower mass ions are available at higher energies per nucleon and higher intensities.

Other developments include the design, construction, testing, and commissioning of an advanced ECR ion source for ATLAS. Implementation of nonintercepting beam phase and intensity monitors along the ATLAS beam lines is also in the final stages of completion. 


\section{a. High-Charge-State Ion Sources (P. J. Billquist, R. Vondrasek, R. Harkewicz,} R. C. Pardo, M. Schlapp, Z. Q. Xie, ${ }^{*}$, C. Lyneis*)

The feasibility of using a 30-watt pulsed NdYAG laser to ablate or evaporate material directly into the ECR had some initial exploratory runs and produced two distinctly interesting results. The first being detailed information about the time evolution of the chargestate distribution in an ECR ion source, and the second being the discovery of the biased-sample sputtering method of feeding solid materials into the plasma.

The first tests of the laser ablation idea were carried out using a bismuth sample. The inherent pulsed nature of the technique allowed us to immediately study the time evolution of charge states in the ECR plasma. The results are directly comparable to model calculations and are completely consistent with the sequential stepwise stripping process which

*LBNL, Berkeley was assumed to dominate the high charge state production process. A paper describing our results was presented at the 1995 International Ion Source Conference.

The sputtering technique for introducing solid materials into the ECR plasma and producing beams from certain materials is now in routine use at ATLAS and at several other heavy ion facilities around the world. Beams of calcium, nickel, germanium, tellurium, gold, lead, and uranium, for example, are produced in this manner.

A $14 \mathrm{GHz}$ ECR ion source is currently under construction at ATLAS; the project status is discussed in the Operations Section above. The design of this source is an evolution of the LBNL "AECR" in collaboration with the Berkeley group.

\section{b. Superconducting Accelerating Structures for Low-Beta Heavy-Ions (K. W. Shepard, M. Kedzie)}

One aspect of this work is a collaborative project with the Nuclear Science Centre, New Delhi, India to develop new superconducting ion accelerating structures. The development of a prototype quarter-wave resonator with a new fabrication technique is in progress. The new method eliminates the need for bonded copper-niobium material, and hence, should be more cost effective. A method of optically observing bubbles in liquid helium to locate regions of excessive rf loss in superconducting cavities was developed and has been used to diagnose defective welds during the prototype resonator development.
The laboratory used for electrochemically processing, cleaning, and assembling superconducting cavities as substantially upgraded during FY 1995 and early 1996, to modernize the laboratory, to improve methods of handling large resonators and quantities of acid, and to provide an improved class 100 clean area facility for rinsing and assembling niobium rf cavities.

Maintaining and even increasing the operating resonator accelerating fields will be a major focus of our activities in the coming years. Continuing to research the causes of field limitations in on-line performance is crucial to the viability and continued improvement of ATLAS and will be emphasized during this period.

\section{ACCELERATOR PHYSICS RELATED TO THE ACCELERATION OF RADIOACTIVE BEAMS}

During FY 1995 the Physics Division developed a concept for an advanced facility for nuclear physics reasearch with accelerated radioactive beams. Preliminary work on conceptual design and some prototype hardware in support of a proposal in this area has been done with ANL discretionary funding during FY 1994-96. Programmatic support of generic R\&D for radioactive beams began in FY 1996.

A design is being developed for a low-charge-state linac suitable for injecting ATLAS with radioactive beams. Initial work indicates that the existing ATLAS interdigital superconducting accelerating structures, together with superconducting quadrupole transverse focussing elements, provide a basis for a high-performance low-charge-state linac. The initial 2 or $3 \mathrm{MV}$ of such a linac could be based on a normallyconducting, low-frequency RFQ. Preliminary beam dynamics studies of the whole low-charge-state postaccelerator section were carried out in early FY 1995. 


\section{a. Acceleration of Very Low Velocity and $q / m$ Radioactive Ions} (K. W. Shepard, W. Sellyey, M. Kedzie, B. Clifft, J. Nolen)

A half-scale model RFQ section was designed, constructed, and tested (without beam) in order to determine the voltage limits on $\mathrm{CW}$ operation of an RFQ with frequency and aperture suitable for a lowcharge-state, high-quality beam. A photograph of the model is shown in Fig. II-4. Electrodynamic measurements on the model indicate that sufficiently high shunt impedance has been obtained to permit operation of a 2 meter injector section with less than $40 \mathrm{kw}$ of RF input. In (pulsed) high-voltage tests, the $1 / 2$ scale model sustained electric fields of at least 1.25 times the Kilpatrick limit (which is $11 \mathrm{MV} / \mathrm{m}$ for the model structure), a value higher than is required for operation of the injector RFQ. The model was taken to the NSCL at Michigan State University for these pulsed high-voltage tests. These results indicate that a low-frequency $(12 \mathrm{MHz}) \mathrm{RFQ}$, operated on a highvoltage platform, and injected with a pre-bunched beam, can provide ATLAS quality beams of ions of charge-to-mass ratio less than $1 / 132$.

The details of the vane shape at the entrance of the RFQ for proper matching of the prebunched beam, both transversely and longitudinally are currently being calculated. The use of a drift tube to provide additional energy gain at the entrance and exit of the RFQ is being pursued.

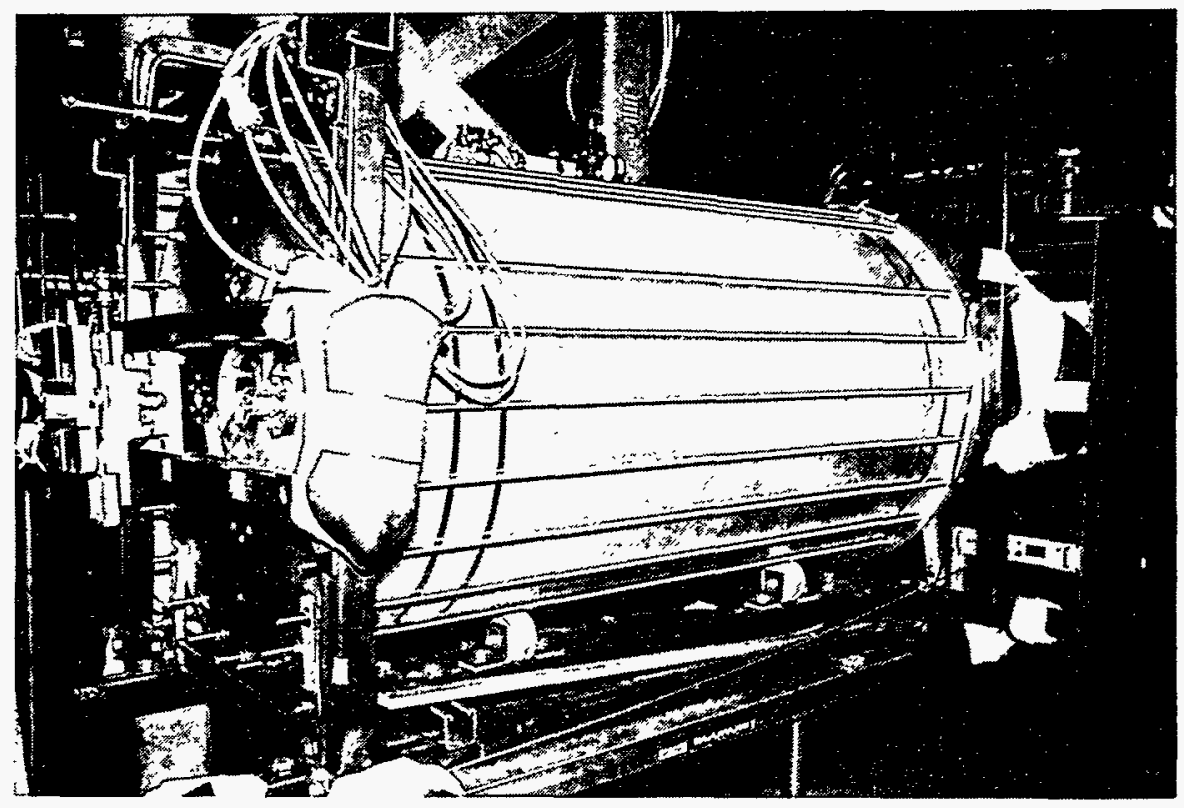

Fig. II-4. The half-scale model of a $12 \mathrm{MHz} R F Q$. Tests and field measurements were performed on this model during 1996.

\section{b. Superconducting Quadrupoles for Focussing in Low-beta, Low-q/m Linacs (J. W. Kim, K. W. Shepard, M. Kedzie, J. Nolen)}

A design was developed for a $350 \mathrm{~T} / \mathrm{m}, 2.6-\mathrm{cm}$ clear aperture superconducting quadrupole focussing element for use in the very low $\mathrm{q} / \mathrm{m}$ superconducting linac. The quadrupole incorporates holmium pole tips, and a rectangular-section winding using standard commercially-available $\mathrm{Nb}-\mathrm{Ti}$ wire. The magnet was modeled numerically using both $2 \mathrm{D}$ and $3 \mathrm{D}$ codes, as a basis for numerical ray tracing using the quadrupole as a linac element. Two prototype coils (single pole tips) for a singlet were procured during FY 1995. Tests of the prototype coils at the vendor's plant quenched at fields about $20 \%$ below design values. During FY 1996 tests of improved coil clamping proceedures will be carried out to see if the gradients can be increased. Once the individual coils are optimized the singlet will be completed and tested. The resonator spacing in the superconducting radioactive beam injector linac will be determined by the fields obtainable in the prototype triplet. 


\section{c. Ion Sources and Targetry for Use in an Advanced Radioactive Beam Facility (J. Nolen, P. DeCrock, T. Mullen, A. Ruthenberg, T. Barlow, K. Beyer, M. Portillo, J. Berger)}

A high-intensity ISOL-type radioactive beam facility depends critically on the performance of the target/ion source system. We developed a concept for producing high-intensity secondary beams of fission fragments, such as ${ }^{132} \mathrm{Sn}$, using a two-part target and ion source combination. The idea involves stopping a $100-\mathrm{kW}$ beam of $200-\mathrm{MeV}$ deuterons in a target of $\mathrm{Be}$ or $\mathrm{U}$ to produce a secondary beam of neutrons. Just behind the neutron production target is a second target, typically a porous form of UC, coupled to an ISOL-type ion source. In December 1994, we tested this concept with $200-\mathrm{MeV}$ deuterons at low intensity in an experiment at the NSCL. The yields of characteristic gamma rays were measured and confirmed our predictions.

To continue developing expertise with targets and ion sources required for an advanced ISOL-type radioactive beam facility we obtained a source from the TRISTAN group at Brookhaven. This source was installed and operated at the ATLAS ion source test beam line. The test stand is being upgraded to measure the transverse emittance and ionization efficiency of this source in various operating modes. After this initial experience the source will be moved to the Physics Division Dynamitron accelerator to produce low-intensity radioactive beams via neutron-induced fission. The Dynamitron is currently being upgraded to operate as a neutron generator using the $\mathrm{d}+\mathrm{Be}$ reaction with 100 microamperes of deuterons. The neutron generator/ion source system will be mounted in a small cave which has a neutron attenuation factor of $10^{4}$. The cave is currently under construction as shown in Fig. II-5.

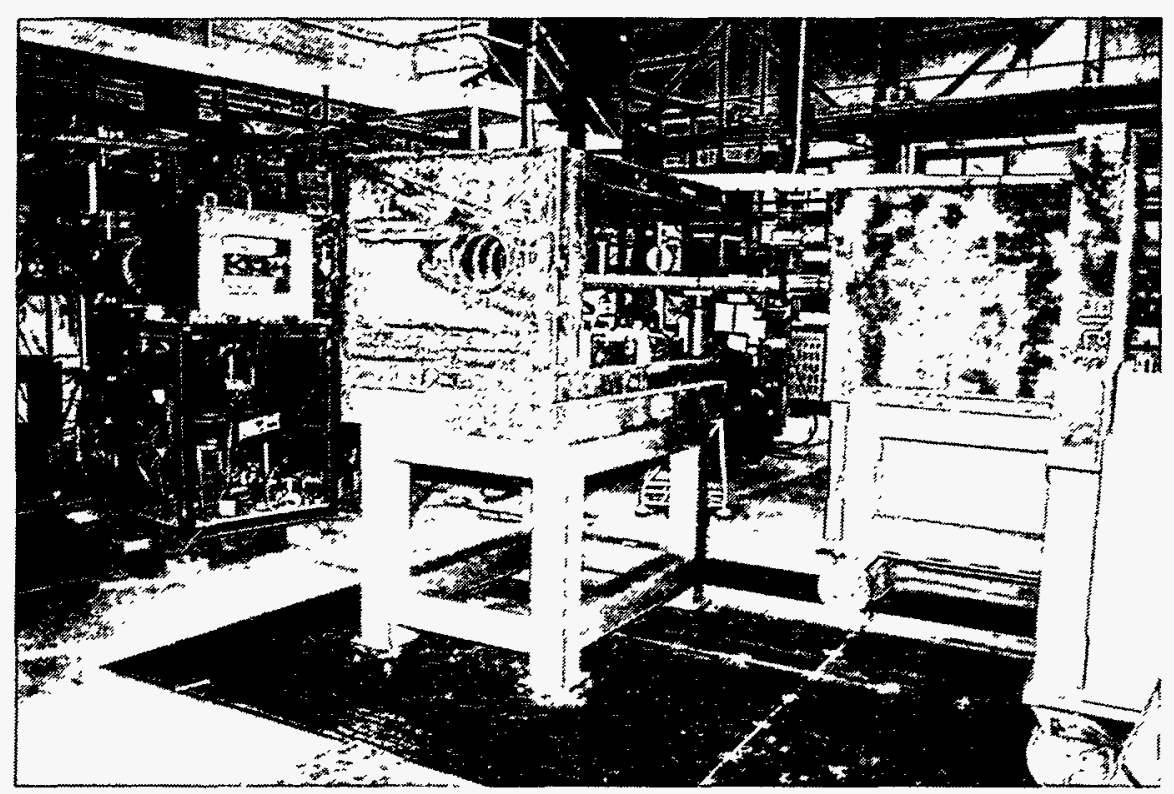

Fig. II-5. Upper: Mechanical drawing of the ISOL-type ion source, neutron generator, and shielding to be installed at the Dynamitron facility. Lower:

Photograph of the shielding under construction. The neutron generator and ion source mount in the central part, while the section on the right is on wheels to permit access.
Experience at the ISOLDE facility at CERN showed that liquid targets are very useful for coupling to ion sources for radioactive beam production. Liquid targets of lanthanum, gold, and lead, for example, were quite successful. However, so far a reliable liquid target of uranium was not developed. Such a target would be very useful, especially for fission products $32^{\text {vith }}$ lifetimes on the order of $30 \mathrm{sec}$ or more, e.g. ${ }^{132} \mathrm{Sn}$. After initial ion source tests with standard UC samples, further measurements will be done to evaluate some alloys for liquid uranium containment. 


\section{d. Charge-State Enhancement Concepts for Radioactive Beam Accelerators (J. Dooling, J. Nolen, P. Decrock)}

A critical question for an ISOL-type radioactive-beam facility, such as that being discussed by the North American Isospin Laboratory Committee, is the efficiency and $q / m$ of the ion source for the radioactive species. ISOLDE at CERN demonstrated that high efficiency is obtained for a wide variety of species in the $1+$ charge state. These ion sources also generally have excellent transverse emittances and low energy spreads. One possibility is to use this proven technology plus an ionizer stage to increase the output of such sources to 2,3 , or $4+$ with high efficiency. We are currently investigating technical options for such charge-state enhancement. There is a proposal by a Heidelberg/ISOLDE collaboration to build a "chargestate breeder" as part of an experiment called REXISOLDE. This concept would deliver batches of radioactive ions with low duty cycle, optimized for relatiyely low-intensity secondary beams, on the order of $10 \%$ sec. We are independently doing simulations of an alternative approach, called the Electron-Beam Charge-State Amplifier (EBQA), which would yield DC beams and would not have the intensity limitation. The cost and efficiency of the EBQA will have to be compared with those of a normally-conducting $\mathrm{CW}$ RFQ followed by ion stripping, as alternatives for the first stage of a secondary ion accelerator.

To be quantitative in the comparison of stripping efficiencies with various other methods of obtaining higher charge states, measurements are being carried out at the Dynamitron with $1+$ xenon beams in the energy range appropriate for the ANL radioactive beam proposal. This work began in early FY 1996 and will continue into FY 1997.

Alternative technologies, which we will be following closely as they develop, include the ECR "plasma catcher" being studied at Grenoble, direct production of higher charge states in ECR ion sources, and the possible use of multicusp ion sources for charge states above one.

\section{e. Concepts for Remote Handling and Shielding at an Advanced Radioactive Facility (J. Wendlandt, J. Nolen)}

An advanced radioactive beam facility will produce intense prompt and residual radiation in the vicinity of the production target. The radiological problems are generally not as severe as those currently encountered at meson factories and in hot cells associated with reactor component handling. Preliminary designs of production-target-area components which build on the experiences of such facilities are being developed. The incorporation of modern robotics devices and techniques will be considered as appropriate. In some cases the application of "virtual reality" sensor and visualization technology to real-time remote handling and mechanical manipulation of radioactive components such as ion sources may be beneficial.

As discussed above, a shielded cave is being constructed at the Physcis Division Dynamitron facility for operation of an ISOL-type ion source adjacent to a neutron-production target. The construction and operation of this facility is giving us experience relevant to the design of an advanced facility with much higher radiological intensities. 


\section{MEDIUM-ENERGY NUCLEAR PHYSICS RESEARCH}

\section{OVERVIEW}

In order to understand how to incorporate the quark-gluon structure of the nucleon into a fundamental description of nuclear forces, the medium-energy research program in the Argonne Physics Division emphasizes the study of processes in nuclei in which interactions with the constituents of the nucleon describe the basic physics. Specific research topics include short-range properties of nuclear forces, nuclear pion fields, and quark degrees of freedom in the nuclear medium. Because energetic leptons provide an accurate well-understood probe of these phenomena, primary emphasis is placed on experiments involving electron and deep-inelastic muon scattering.

The Argonne medium-energy physics program has a major presence in the research program at CEBAF. Staff members are actively involved in construction of the experimental facilities, and are spokesmen for four approved experiments. In 1995, the group completed construction of a broad-purpose short-orbit spectrometer (SOS) which forms half of the coincidence spectrometer pair that is the primary base experimental equipment in Hall C. Argonne led the first experiment to be carried out at CEBAF, a study of the energy dependence of proton propagation in nuclei in quasifree electron scattering reactions which built on previous work by this group at MIT and SLAC.

The most direct way to probe the quark structure of nuclei is to use a high-energy probe which interacts with a single quark at a time. In FNAL experiment E665, the deep inelastic scattering of $490-\mathrm{GeV}$ muons at Fermi National Accelerator Laboratory measures the changes of the quark distributions in the nucleus compared to the nucleon, and the interactions of high-energy quarks and hadrons with nuclear material. New results identified diffractive scattering for the first time in deep inelastic scattering from nuclei. Inelastic lepton scattering makes it possible to control separately the transverse and longitudinal energy scales of exclusive reactions. In exclusive $\rho$-meson production, a signal for color transparency is observed in the increase of the relative cross section on lead compared to that on deuterium as a function of momentum transfer squared. This complements and extends our search for color transparency in exclusive proton knock-out reactions at SLAC and CEBAF. New data on the ratio of deep inelastic scattering from hydrogen and deuterium show that nuclear effects are important in the deuteron but also confirm previous results which suggest that the $\overline{\mathrm{u}}$ distributions and the $\overline{\mathrm{d}}$ distributions are quite different. A new FNAL experiment, E866, will measure directly this difference using the Drell-Yan process in the next FY1996-97 fixedtarget cycle. 
HERMES, a broadly-based North American-European collaboration, is studying the spin structure of the nucleon using internal polarized targets in the HERA storage ring at DESY. The first production run measured polarized positron scattering on polarized helium-3 nuclei. Argonne contributed the Cerenkov counter which will provide the primary hadron identification for the experiment. Argonne scientists have also extended the physics program to begin the first studies of vector meson production in polarized-beam polarized-target experiments. These measurements will also provide insight into color transparency in an important new kinematic range.

Considerable technical resources of the medium-energy program were devoted to developing a new technology for producing polarized hydrogen and deuterium targets for storage rings experiments. Significant progress was made in establishing stable operation of laser-driven spin-exchange targets and measuring the nuclear polarization. A collaboration between Argonne and a Russian group at Novosibirsk is constructing a laser-driven source to measure tensor polarization in elastic electron-deuteron scattering, a reaction which is very sensitive to subnucleonic effects. Argonne also continues to develop the laser-pumped technology for hydrogen and deuterium targets for HERMES. A new effort is underway to produce a polarized tritium target for external beam experiments at CEBAF. This is a substantial extension of the laser-pumped spin-exchange technology to sealed target cells.

Activities involving the NPAS program at the Stanford Linear Accelerator Center are essentially completed. Several of these physics programs will continue using the more powerful capabilities at CEBAF. 


\section{A. SUBNUCLEONIC EFFECTS IN NUCLEI}

a. Deep-Inelastic Muon Scattering from Nuclei with Hadron Detection (D. Geesaman, H. Jackson, S. Kaufman, E. Kinney, V. Papavassiliou, D. Potterveld, A. Zghiche, T. Kirk,* H. J. Trost,* R. Kennedy, $\dagger$ H. Kobrak, $\uparrow$ P. Madden, $\dagger$ A. Salvarani, $\uparrow$ Robert A. Swanson,$\uparrow$ A. Eskreys $₫$ P. Malecki $\ddagger$ K. Eskreys,$\ddagger$ K. Olkiewicz $\ddagger$ B. Pawlik, $\ddagger$ B. Baller,$\S$ G. B. Coutrakon,$\S$ J. Hanlon, $\S$ H. Melanson, $\S$ H. E. Montgomery,§ J. G. Morfin,§ C. Salgado,§ S. Wolbers, § T. Dreyer,, $\mathbb{T}$ M. Erdmann, IT J. Haas, II W. Mohr,II H. Stier, I M. Wilhelm,II J. M. Conrad,II G. Fang,II A. Kotwal,\| D. G. Michael,\| R. B. Nickerson,\| F. M. Pipkin,\| M. Schmitt,\| Richard Wilson,ll M. R. Adams, ** D. A. Averill,** T. Carroll,** R. Guo,** C. Halliwell,** S. Magill, ** D. McLeod,** S. Aid, $\uparrow \uparrow$ S. Kunori, $\uparrow \dagger$ S. O'Day, $\dagger$

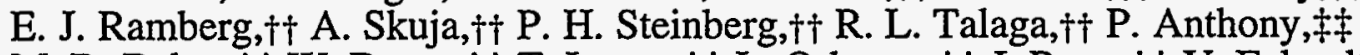

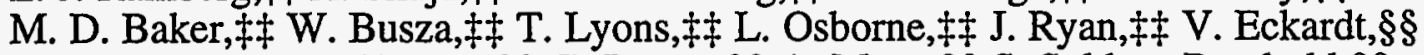

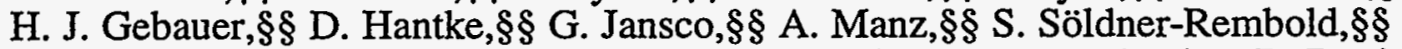

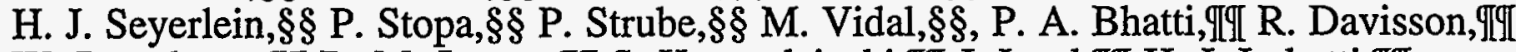
W. Dougherty, TIT D. M. Jansen, TII S. Krzywdzinski, TIT J. Lord,TIT H. J. Lubatti, TII

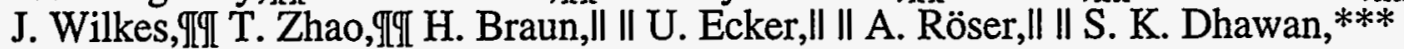
V. W. Hughes, $* * *$ K. P. Schüler, $* * *$, H. Venkataramania, $* * *$ F. Dietrich, $+\dagger \dagger$

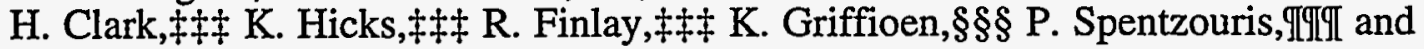
H. SchellmanIIII)

Deep-inelastic lepton scattering from nuclei provides a direct look at the quark structure of nuclear matter. These reactions revealed the first convincing evidence that the structure of nucleons is modified in the nuclear medium and had profound implications on the understanding of nuclear dynamics. FNAL experiment E665, using the $490-\mathrm{GeV}$ muon beams at Fermi National Accelerator Laboratory, provides new information on the nuclear effects on nucleon properties by studying deep-inelastic muon scattering with coincident hadron detection. The high beam energy makes the experiment particularly suited to the study of the region of $x<0.1$ (where $x$ is the fraction of the momentum of the nucleon carried by the struck quark in the infinite momentum frame), and total center-ofmass hadronic energy $>25 \mathrm{GeV}$, where hard QCD processes are expected to become evident and there are little data from earlier deep-inelastic measurements.

The data-acquisition phase of E665 was completed in January 1992 following the third block of beam time. During the 1987-1988 run, data were accumulated at $490 \mathrm{GeV}$ on targets of liquid deuterium (luminosities of $2 \times 10^{36}$ muon-nucleon $/ \mathrm{cm}^{2}$ ) liquid-hydrogen $\left(7 \times 10^{35}\right.$ muon-nucleon $\left./ \mathrm{cm}^{2}\right)$ and gaseous xenon $(7 \times$ $10^{35}$ muon-nucleon $/ \mathrm{cm}^{2}$ ). In this period the target was surrounded by a streamer chamber to provide essentially $4 \pi$ acceptance. During the 1990 run, luminosities of $4 \times 10^{35}$ were collected on targets of hydrogen, deuterium, carbon, calcium, and lead. The targets were changed every 1-3 minutes to reduce systematic uncertainties in the target dependence. In the final 1991 running period, luminosities of $4 \times 10^{36}$ were collected on hydrogen and deuterium.

Final results are now available in eighteen publications. Twenty eight students completed Ph.D. theses on this experiment. The most important new results obtained in 1995 were absolute cross section measurements on hydrogen and deuterium. These cover an extremely wide kinematic range from $8 \times 10^{-4}<\mathrm{x}<0.6$ and $0.2<\mathrm{Q}^{2}$ $<75(\mathrm{GeV} / \mathrm{c})^{2}$. In regions of overlap, E665 results agree well with measurements from other experiments. At low $x$ they extrapolate smoothly to the high $Q^{2}$ data from HERA. The combination of the E665 and HERA data (see Fig. III-1) cover four orders of magnitude in $\mathrm{Q}^{2}$ and measure the transition in the nature of the photon from a hadronic-likeobject at low $\mathrm{Q}^{2}$ to a pointlike object at high $\mathrm{Q}^{2}$.

\footnotetext{
*High Energy Physics Division, ANL, †University of California at San Diego, $\$$ Institute of Nuclear Physics, Cracow, Poland, §Fermi National Accelerator Laboratory, TUniversity of Freiburg, Germany, ॥Harvard University, **University of Illinois at Chicago, $\dagger \dagger$ University of Maryland, $\ddagger$ Massachusetts Institute of Technology, §§Max-Planck-Institute, Heidelberg, Germany, 㧃University of Washington, || IIUniversity of Wuppertal, Germany, ***Yale University, $\dagger \dagger \dagger$ Lawrence Livermore National Laboratory, $\ddagger$ University, §§§University of Pennsylvania đIIINorthwestern University.
} 


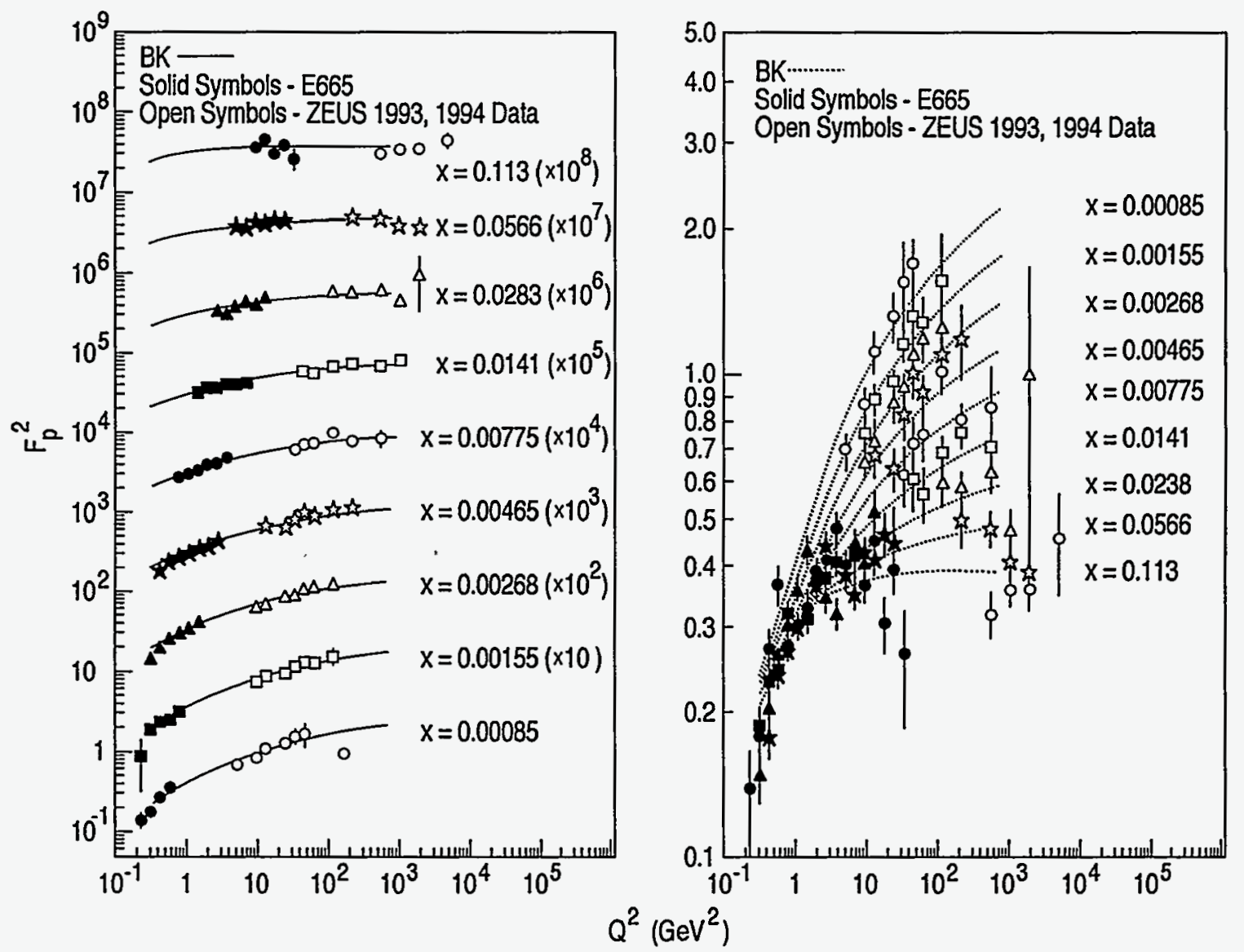

Fig. III-1. Proton $F_{2}$ vs $Q^{2}\left(\mathrm{GeV}^{2}\right)$ in $x_{B j}$ bins, from E665 and ZEUS. ${ }^{1}$ The Badelek-Kwiecinski $(B K)^{2}$ model is also shown. (Left) the data points and the model curves have been multiplied by the factors indicated in parentheses for clarity. (Right) the points and curves are plotted with no scale factors, to show the trends.

Studies continue on the role of diffractive scattering in deep inelastic scattering on nuclear targets. E665 was the first experiment to show that diffractive events can account for up to $20 \%$ of the nuclear deep inelastic cross section at low $x$ on heavy targets. In a related diffractive process, exclusive $\rho$ meson production in nuclei reveals some evidence of color transparency. Further work continues to extract the $\rho$ meson polarization from the decay angular distributions. Important extensions of this line of research are being carried out by ANL staff in the HERMES experiment.

1M. Derrick et al., Z. Phys. $\underline{\mathrm{C} 69}, 607$ (1995)

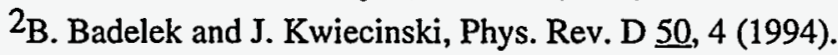


b. Electron-Deuteron Scattering With a Polarized Deuterium Gas Target in the VEPP-3 Electron Storage Ring (C. E. Jones, J. A. Fedchak, R. S. Kowalczyk, D. H. Potterveld, L. Young, B. Zeidman, R. J. Holt, * K. P. Coulter $\dagger$ R. Gilman, $\ddagger$ E. R. Kinney,§ S. I. Mishnev, I D. M. Nikolenko,II S. G. Popov,II I. A. Rachek, II A. B. Temnyhk, II D. K. Toporkov, II E. P. Tsentalovich, II D. K. Vesnovsky, $\mathbb{I}$ B. B. Wojtsekhowski, II K. de Jager,ll H. de Vries,ll and G. Retzlaffll)

The collaborative effort between Argonne and the Budker Institute for Nuclear Physics in Novosibirsk to measure the tensor analyzing power of the deuteron at high momentum transfer continues. This measurement allows the experimental separation of the deuteron charge and quadrupole form factors, which cannot be obtained from unpolarized scattering alone. In preparation for Phase 3 running of the experiment, tests were completed of a new set of quadrupoles used to reduce the beam size at the internal target region; the beam size was verified to be sufficiently small for the planned storage cell. A small experimental area where tests can be done on the laser-driven source (LDS) coupled to the storage cell was set up. This area has the full internal target setup, with the storage cell connected to the Rabi polarimeter and all differential pumping stations. A room to house the lasers was constructed outside the VEPP-3 storage ring so that the lasers can be easily adjusted and maintained while beam is in the experimental area. The lasers are operational, with less than 3 Watts output power at the operational frequency of the LDS. Although this is less power than obtained at Argonne, it is sufficient for optically pumping the LDS at the flow rates required for the VEPP-3 storage ring. Dmitri Toporkov of the Budker Institute visited Argonne for three months to work with the operational laser-driven source here and to gain experience in coating the source and target.

*University of Illinois, $\uparrow$ University of Michigan, $\ddagger$ Rutgers University, §University of Colorado, TBudker Institute for Nuclear Physics, Novosibirsk, Russia, IINIKHEF, Amsterdam, The Netherlands.

\section{c. Laser-Driven Polarized Hydrogen and Deuterium Internal Targets (C. E. Jones, W. J. Cummings, J. A. Fedchak, R. S. Kowalczyk, and D. K. Toporkov*)}

This past year we worked to get stable, reproducible operation of the laser-driven source. We tested a new drifilm coating which permits the source to operate over time spans of months in the presence of potassium without noticeable degradation of the atomic fraction. We continued measurements of the atomic fraction and deuterium atomic polarization of the flow from the source alone and the source coupled to a storage cell in an internal target configuration. We are currently testing a diode probe laser setup which will allow us to measure the potassium polarization and density in the source simultaneously with measurements of the deuterium polarization and atomic fraction. In parallel with the lab work, we began to develop a realistic computer model of the source that takes into account $K$ optical pumping, K-D and D-D spin exchange, and wall relaxation in a flow-through system with an optical pumping cell and a transport tube. We hope to use our experimental data to verify that the simulation provides a reasonable model of the LDS, and to then optimize the geometry and operating $\mathrm{K}$ and $\mathrm{D}$ densities for the proposed HERMES laser-driven H/D internal target using the model.

*Budker Institute for Nuclear Physics, Novosibirsk, Russia 


\section{d. Nuclear and $\mathbf{Q}^{2}$ Dependence of Quasielastic (e,e'p) Scattering at Large}

Momentum Transfer (T. G. O'Neill, N. C. Makins, D. F. Geesaman, H. E. Jackson, D. Potterveld, B. Zeidman, R. J. Holt,* K. Coulter, $\dagger$ E.Kinney, $\ddagger$ E. Beise, $\S$ B. Filippone, $\S$

W. Lorenzon,§ R. Arnold,II S. Rock, Il Z. Szalata,IL M. Epstein,Il J. J. Napolitano,ll

R. C. Minehart,** R. Ent, $\uparrow \dagger$ R. Milner, $\dagger \dagger$ D. Beck,

An experiment was completed at the Stanford Linear Accelerator Center in which measurements of the (e,e'p) coincidence quasielastic cross section in nuclei were extended to the largest possible $\mathrm{Q}^{2}$ attainable with the Nuclear Physics Injector and the End Station A spectrometers. Coincidence measurements of the quasielastic (e,e'p) cross section were made on nuclei from carbon to gold in the $\mathrm{Q}^{2}$ range of $1-7(\mathrm{GeV} / \mathrm{c})^{2}$. The analysis of the data was finished in 1995, and several papers describing the results were published. In summary, the cross section for quasielastic ${ }^{12} \mathrm{C}(\mathrm{e}, \mathrm{e} \cdot \mathrm{p})$ scattering was measured at momentum transfer $Q^{2}=1$, 3,5 , and $6.8(\mathrm{GeV} / \mathrm{c})^{2}$. The results are consistent with scattering from a single nucleon as the dominant process. The nuclear transparency $T\left(A, Q^{2}\right)$, a measure of the average probability that the struck proton escapes from the nucleus A without interaction, was extracted. The nuclear transparency was compared with theoretical calculations that incorporate color transparency effects.
Measurements of the quasielastic $\mathrm{A}\left(\mathrm{e}, \mathrm{e}^{\prime} \mathrm{p}\right)$ reaction on the nuclei ${ }^{2} \mathrm{H}, \mathrm{C}, \mathrm{Fe}$, and $\mathrm{Au}$ allowed determination of the simultaneous $Q^{2}$ and A-dependence of the nuclear transparency $T\left(A, Q^{2}\right)$. Several calculations including color transparency predict a significant rise in the nuclear transparency with momentum transfer. No significant rise within errors is seen for any of the nuclei studied. In addition the A-dependence is seen to be independent of $Q^{2}$ for $Q^{2} \geq 3 \mathrm{GeV}^{2}$, also indicating a lack of color transparency effects. Cross sections were reported for the reaction ${ }^{2} \mathrm{H}(\mathrm{e}, \mathrm{e} \mathrm{p}) \mathrm{n}$ for momentum transfers in the range $1.2 \leq \mathrm{Q}^{2} \leq 6.8(\mathrm{GeV} / \mathrm{c})^{2}$ and for missing momenta from 0 to $250 \mathrm{MeV} / \mathrm{c}$. The longitudinal-transverse interference structure function was separated at $\mathrm{Q}^{2}=1.5(\mathrm{GeV} / \mathrm{c})^{2}$. The observables were compared to calculations performed in nonrelativistic and relativistic frameworks. The data are best described by a fully relativistic calculation.

*Univ.of Illinois, $\nmid$ Univ. of Michigan, $\ddagger$ Univ. of Colorado, §Calitech, $\mathbb{I A m e r i c a n ~ U n i v , ~} \ddagger$ California State Univ., IIRensselaer Polytechnic Institute, **Univ. of Virginia, ††MIT, $\ddagger$ Univ. of Illinois, §§Univ.of Wisconsin.

\section{e. Polarized Tritium Target Development (W. Cummings, J. A. Fedchak, C. E. Jones, and R. S. Kowalczyk)}

The first step toward demonstrating the feasibility of a sealed polarized tritium target was completed. This past year we were able to establish and maintain a discharge in the dissociator region of a simple sealed two-cell glass system containing purified $\mathrm{H}_{2}$ gas and prevent the discharge from extending into the target cell by applying a transverse magnetic field in the region between the two cells. Separating the discharge from the target cell is vital to the polarized target operation because bombardment by electrons and ions will cause irreversible degradation of the coated surface of the target.

Studies began of various organosilicon surface coatings to find a coating with very good outgassing properties that is resistant to alkalis and which does not depolarize or recombine the hydrogen in wall collisions. We used an atomic force microscope (AFM) to scan the coated surfaces to study the small-scale structure of liquiddeposited drifilm, vapor-deposited drifilm (SC-77) and liquid-deposited octadecyltrichlorosilane (OTS). These scans revealed that the SC-77 showed significant large-scale structures while the OTS coating was

essentially flat. Because both this group and researchers outside of Argonne have noted long-term degradation of SC-77 when exposed to alkalis, we speculated that OTS would be a better coating than SC-77, which potentially has more cracks through which the alkali can directly attack the bonds between the drifilm and the glass. We then did tests of the viability of OTS under long-term exposure to alkali and found that OTS could not withstand alkali exposure at the operating temperatures needed for the laser-driven source/target. However, the information from the AFM scans suggested that a smoother version of the SC-77 surface might be excellent, so we tried a standard SC-77 coating followed with an afterwash of a second silane compound that should, in principle, bond to any remaining exposed $\mathrm{OH}$ sites. The resultant coating is as smooth as OTS and more resistant to alkalis than OTS. Initial tests of the laser-driven source with this coating show that it does not degrade after months of standard operation, a significant improvement over previous coatings that were used. It appears that this new coating is very stable under long term operation and an excellent candidate for coating the sealed polarized target. 
f. Electroproduction of Kaons and Light Hypernuclei (D. F. Geesaman, J.-O. Hansen, H. E. Jackson, C. E. Jones, D. H. Potterveld, S. B. Kaufman, T. G. O'Neill, J. Reinhold, J. P. Schiffer, B. Zeidman, R. J. Holt, * R. E. Chrien, $\dagger$ S. Bart, †R. Sawafta, † R. J. Sutter, $\uparrow$ B. W. Filippone, $\ddagger$ W. Lorenzon,$\ddagger$ J. Napolitano, $\S$ R. Carlini,TI R. Ent,TI D. Mack,T S. A. Wood,T E. R. Kinney,II O. K. Baker,** W. W. Buck, ** J.-S. Cha, ** L. Tang,** E. V. Hungerford, $\uparrow \dagger$ K. Lan, $\dagger \dagger$

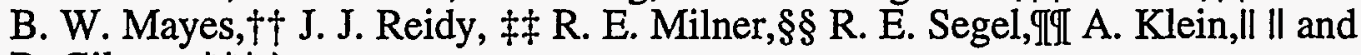
R. Gilman,***)

A detailed investigation of the basic hyperon-nucleon interactions in nuclei is one of the aims of Experiment 91-016, approved with high priority at CEBAF, to study the electroproduction of kaons on targets of deuterium, ${ }^{3} \mathrm{He}$, and ${ }^{4} \mathrm{He}$. Inasmuch as both the electron and $\mathrm{K}^{+}$are particles that interact relatively weakly with nucleons, electroproduction of light hypernuclei provides a low-distortion method for investigating the fundamental interactions between nucleons, $\Lambda$ 's, and $\Sigma$ 's in few-body systems. In particular, the $\left(e, \mathrm{e}^{\prime} \mathrm{K}^{+}\right)$reactions on cryogenic targets of $\mathrm{D},{ }^{3} \mathrm{He}$, and ${ }^{4} \mathrm{He}$ will be studied at incident electron energies near $3 \mathrm{GeV}$ with coincident detection of the emergent $\mathrm{e}$ and $\mathrm{K}^{+}$in the HMS and SOS magnetic spectrometers in Hall C. The first data runs for E91016 on $\mathrm{H}$ and D, are expected to begin in August 1996 and will be the basis for a doctoral thesis at Hampton University. The He target, operating at $\sim 10 \mathrm{~atm}$, will be used in the run cycles planned for the fall of 1996. In addition to providing new information on the phases of hyperon-nucleon interactions, measurements of cross sections for hypernuclear formation, and interference phenomena, the data may provide evidence for the presence of bound $\Sigma$ 's and strange dibaryonic states that are the subject of considerable theoretical discussion.

*Univ. of Illinois, †Brookhaven National Laboratory, ‡California Inst. of Technology, §Rensselaer Polytechnic Inst., ICEBAF, IIUniv. of Colorado, **Hampton Univ., ††Univ. of Houston, 择Univ. of Mississippi, $\S \S M a s s a c h u s e t t s$ Inst. of Technology, TITNorthwestern Univ., II IIOld Dominion Univ., ***Rutgers Univ.

\section{g. Two-Body Photodisintegration of the Deuteron at Forward Angles and} Photon Energies Between 1.5 and 4.0 GeV (W. J. Cummings, J. Fedchak, D. F. Geesaman, J.-O. Hansen, H. E. Jackson, C. Jones, S. Kaufman, T. G. O'Neill, D. Potterveld, J. Reinhold, B. Zeidman, J. Arrington,* B. Bray,* A. Dvoredsky,* B. W. Filippone,* W. Korsch,* A. Lung,* R. D. McKeown,* M. Pitt,* E. Belz, $†$ E. Kinney, $\uparrow$ D. van Westrum, $\dagger$ D. Abbott $\ddagger$ L. S. Cardman,$\ddagger$ R. Carlini, $\ddagger$ J. Dunne, $\ddagger$

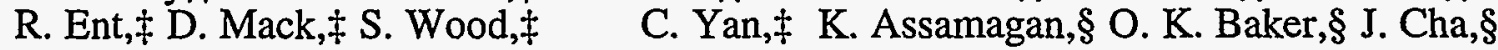

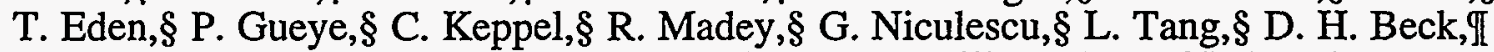
C. Bochna, II T. Forest, II H. Gao,II R. J. Holt, $\mathbb{I}$ M. A. Miller, $\mathbb{I}$ A. M. Nathan, II N. Simicevic, $\mathbb{I}$ B. Terburg,, E. Beise,\| H. Breuer,\| N. Chant,\|l F. Duncan,\|l J. J. Kelly,\| P. Roos,ll D. DeSchepper,** R. Miller,** S. Pate, $\dagger \dagger$ M. Khandaker, $\neq$ K. McFarlane,

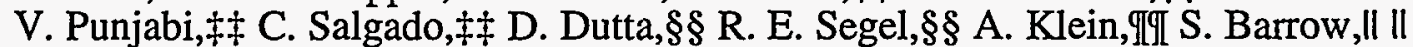
D. Beatty, || || H. T. Fortune, || || D. Koltenuk, || || W. Lorenzon, || || J. Yu, || || V. Frolov, *** J. Napolitano, $* * *$ N. Nozar, *** J. Price, ${ }^{* * *}$ P. Stoler,*** M. T. Witkowski, ***

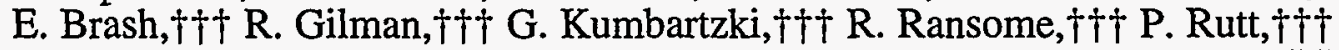
P. Bosted, H. Mkrtchyan, I| I| II and V. Tadevosyan, II II II

It was discovered in ANL experiments NE8 and NE17 at SLAC that the differential cross section near $\theta_{\mathrm{cm}}=$ $90^{\circ}$ for the $\gamma \mathrm{d} \rightarrow \mathrm{pn}$ reaction at the highest measured photon energies $\left(\mathrm{E}_{\gamma}=1.3-2.8 \mathrm{GeV}\right)$ has an energy dependence consistent with the constituent counting rules. Experiment NE17 at SLAC indicates the sdependence of the cross section is not consistent with constituent counting at forward angles.

*California Inst. of Technology, $\nmid$ Univ. of Colorado, $\ddagger$ CEBAF, $\S$ Hampton Univ., qUniv. of Illinois at ChampaignUrbana, IIUniv. of Maryland, **MIT, $\dagger \dagger$ New Mexico State Univ., $\ddagger$ Norfolk State Univ., §§Northwestern Univ., IITOld Dominion Univ., II IUUniv. of Pennsylvania, ***Rensselaer Polytechnic Inst., †††Rutgers Univ.,

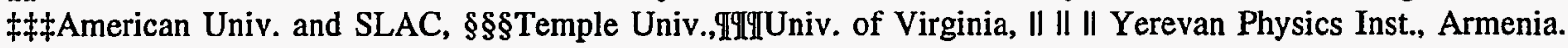


The second experiment scheduled to run at CEBAF is a continuation of these measurements for two of the simplest exclusive binary reactions involving a deuteron in the initial or final state: 1) $\gamma \mathrm{d} \rightarrow$ pn between $E_{\gamma}=$ 1.5 and $4.0 \mathrm{GeV}$, and 2) $\gamma \mathrm{d} \rightarrow \pi^{0} \mathrm{~d}$ between $\mathrm{E} \gamma=1.0$ and $3.0 \mathrm{GeV}$. This experiment was given high scientific priority by the PAC and the Hall $C$ collaboration chose this as a commissioning experiment.

The constituent counting rules predict an energy dependence of $\mathrm{s}^{-11}$ and $\mathrm{s}^{-13}$ for the $\gamma \mathrm{d} \rightarrow \mathrm{pn}$ and $\gamma \mathrm{d} \rightarrow$ $\pi^{0} \mathrm{~d}$ cross sections, respectively, where $s$ is the square of the energy in the center of mass. Since more complex nuclei involve more constituents and consequently a more rapid falloff of the cross section as a function of $\mathrm{s}$, these two cases may represent the only practical nuclear reactions that can be studied at large values of $\mathrm{s}$ where asymptotic scaling is most likely to be observed. These experiments are practical at CEBAF with the large beam current $(\sim 30 \mu \mathrm{A})$ and the large solid-angle spectrometer. The experiment is scheduled to run in early 1996.

\section{h. A Study of Longitudinal Charged-Pion Electroproduction in $\mathbf{D},{ }^{3} \mathrm{He}$, and ${ }^{4} \mathrm{He}$ (H. E. Jackson, W. Cummings, D. F. Geesaman, J.-O. Hansen, C. E. Jones, S. Kaufman, D. Potterveld, T. G. O'Neill, B. Zeidman, D. Mack, * J. Mougey, † B. Saghai, † C. Armstrong, $\ddagger$ R. E. Segel,§ R. Gilman, II R. J. Holt,ll E. Kinney,** S. Barrow, $\dagger \dagger$ P. Beatty, $\dagger \dagger$ H. T. Fortune, $\uparrow \dagger$ D. Koltenuk, $\dagger \dagger$ W. Lorenzon, $\uparrow \dagger$ T. Amatuni, $\neq \ddagger$ H. Mkrtchyan, $\neq \neq$ and V. Tadevosyan $+\ddagger)$}

Studies of pion electroproduction on the deuteron carried out by the ANL group at ALS, Saclay, in the 1980's show that even in the weakly-bound deuteron, multinucleon processes alter the electro-production amplitudes in the forward direction. The data provide the first experimental indications for a significant change in the effective pion-nucleon coupling for nucleons bound in nuclei. It is clear that forward-angle electroproduction may be a sensitive probe of the properties of the pion coupling in the nuclear medium. At CEBAF, we will study longitudinal charged-pion electroproduction (in the excitation region below the delta isobar) along the direction of the momentum transfer where the charge scattering process dominates. Direct comparison of the cross section per nucleon in deuterium and the helium isotopes with the experimental value for the free nucleon will provide estimates of the strength of the nuclear pion field. A Rosenbluth separation of the longitudinal and transverse cross sections will be performed for four-momentum transfers of 2.5 and $10 \mathrm{fm}^{-2}$. Measurements for a number of light nuclei will provide useful data on the sensitivity of longitudinal electroproduction to nuclear binding effects. If current conceptions of pion-exchange currents in nuclei are correct, longitudinal electroproduction will be suppressed at the lower momentum transfer and enhanced at the higher momentum transfer by multinucleon processes. If on the other hand, as suggested by recent data from DrellYan studies of antiquark structure functions, there is no such enhancement, a reformulation of pion exchange models of the medium- and short-range properties of nuclear forces will be required. The experiment is scheduled to run in Fall 1996 at CEBAF. This is one of the experiments that highlighted the need for a relatively short spectrometer for unstable particles at CEBAF. The ANL Medium-Energy group responded to this need by constructing the short-orbit spectrometer (SOS) as the hadron arm in Hall C.

*CEBAF, †CEN Saclay, France, $\Varangle$ College of William and Mary, §Northwestern University, TRutgers University, IUniversity of Illinois at Champaign-Urbana, **University of Colorado, ††University of Pennsylvania, $\$$ Yerevan Physics Institute, Armenia 
i. The Energy Dependence of Nucleon Propagation in Nuclei as Measured in the (e,e'p) Reaction (D. F. Geesaman, W. J. Cummings, J. Fedchak, J.-O. Hansen, H. E. Jackson, C. E. Jones, S. Kaufman, T. G. O'Neill, D. Potterveld, J. P. Schiffer, B. Zeidman, J. Arrington, * B. W. Filippone,* A. Lung,* R. D. McKeown,* D. Abbott, $\dagger$ R. Carlini, $\uparrow$ R. Ent,$\uparrow$ D. Mack,$\uparrow$ D. Meekins,$\uparrow$ J. Mitchell, $\uparrow$ S. Wood,$\uparrow$ C. Yan, $\uparrow$ Jae-Choon Yang,‡ E. Belz,§ E. Kinney,§ D. van Westrum,§ P. Markowitz,II O. K. Baker,॥ J. Cha,\| T. Eden,\| P. Gueye,\| C. Keppel,\| R. Madey,\|l G. Niculescu,\|l L. Tang,\| Wooyong Kim,** C. Bochna, $\uparrow \dagger$ H. Gao, $\uparrow \dagger$ R. Holt, $\uparrow \dagger$ M. Miller, $\uparrow \dagger$ B. Terburg, $\dagger \dagger$ R. Milner, M. Khandaker,,III K. McFarlane, III C. Salgado, đIT S. Beedoe, II II S. Dangoulian, II II C. Jackson, II II D. Dutta,*** R. E. Segel,*** Pat Welch, $\uparrow \dagger \dagger$ A. Klein,

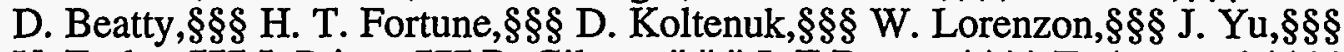

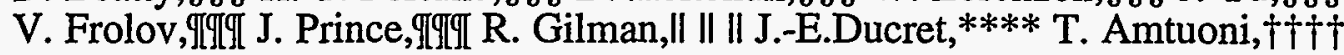

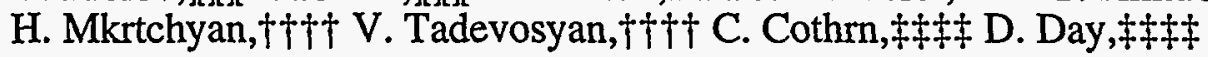

ANL led the first experiment to be carried out at CEBAF in November-December 1995. This experiment continued the ANL work at MIT and SLAC using the $\left(e, e^{\prime} p\right)$ reaction to study the propagation of 0.35 to $1.80-\mathrm{GeV}$ protons through nuclear material. The Hall $\mathrm{C}$ collaboration selected this experiment as one of the two commissioning experiment. Electrons were detected in the HMS $6-\mathrm{GeV}$ spectrometer and protons were detected in the SOS $1.8-\mathrm{GeV}$ spectrometer. The experiment utilized CEBAF beams of 0.845 , $1.645,2.445$ and $3.245 \mathrm{GeV}$ with up to $25 \mu \mathrm{A}$ intensity on targets of $\mathrm{C}, \mathrm{Fe}$ and Au. Full commissioning studies of each spectrometer were performed to calibrate this experiment and to serve as a baseline for future experiments.

In this first run over 4 million quasifree electron-proton coincidence events were accumulated along with 16 million singles events for normalization. The $\mathrm{Q}^{2}$ points of $0.64,1.3$ and $1.8(\mathrm{GeV} / \mathrm{c})^{2}\left(\mathrm{~T}_{\mathrm{p}}=0.350\right.$, 0.700 and $0.970 \mathrm{GeV}$ ) span the threshold for pion production in p-p collisions where the nature of the p-p cross section changes from dominantly elastic to dominantly inelastic. Preliminary missing energy spectra on each target at $\mathrm{Q}^{2}=1.8(\mathrm{GeV} / \mathrm{c})^{2}$ for parallel kinematics are shown in Fig. III-2. At the lowest $Q^{2}$, data were taken at two values of the virtual photon polarization to examine the separate contributions of longitudinal and transverse photon exchange. This $L / T$ separation will address the role of two-body and manybody photon absorption mechanisms in the quasifree region. In all aspects the experimental equipment performed as expected. The statistics, kinematic coverage and experimental resolution significantly exceed those of previous results. This data set should provide precise measurements of nuclear transparency as

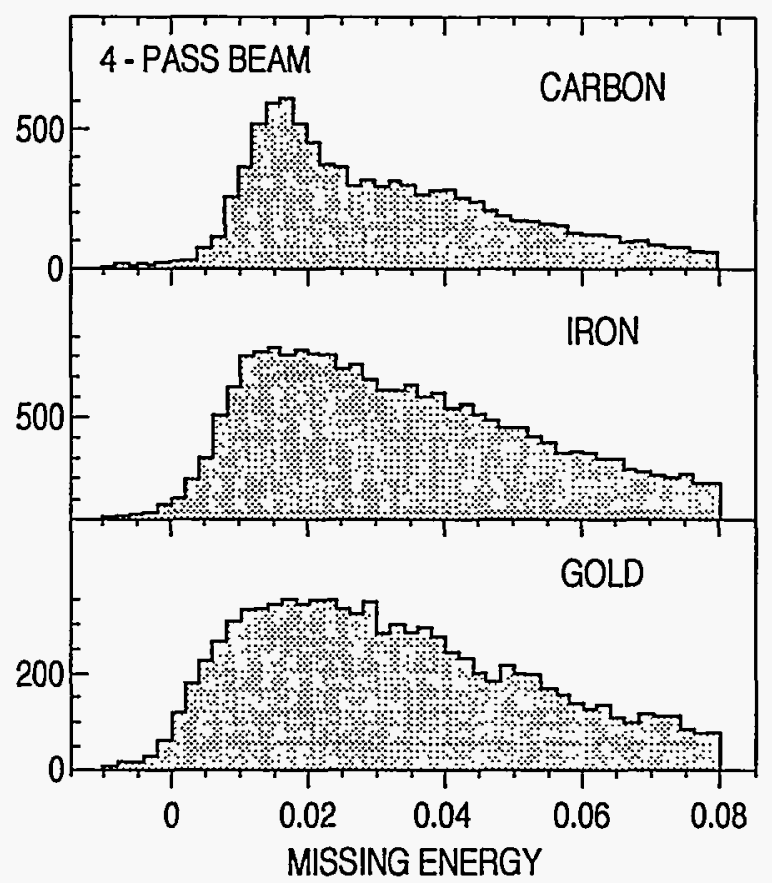

Fig. III-2. Preliminary missing energy spectra for quasifree $\left(e, e^{\prime} p\right)$ scattering in parallel kinematics. The electron beam energy is $3.245 \mathrm{GeV}$ and average momentum transfer is $1.8(\mathrm{GeV} / \mathrm{c})^{2}$.

well as a broad survey of nuclear spectral functions from recoil momentum of 0 to $300 \mathrm{MeV} / \mathrm{c}$ and missing energy of 0 to $150 \mathrm{MeV}$.

In spring 1996, the last phase of the experiment will take backward angle data at $T_{p}$ of $0.970 \mathrm{GeV}$ and a higher $Q^{2}$ point at $T_{p}$ of $1.80 \mathrm{GeV}$. A companion CEBAF experiment will extend these data to $T_{p}$ of 3.00 $\mathrm{GeV}$.

*California Inst. of Tech., †CEBAF, $\ddagger$ Chungnam National Univ., Taejon, Korea, §Univ.of Colorado, IFlorida International Univ., llHampton Univ., **Hoseo Univ., Chungnam, S. Korea, ††Univ. of Illinois at UrbanaChampaign, $\ddagger$ MIT, §§Univ. of Maryland, IINorfolk State Univ., II IINorth Carolina A\&T Univ., ***Northwestern

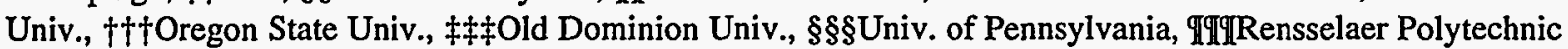
Inst., I| I| IIRutgers Univ., ****CE Saclay, France, $\dagger \dagger \dagger \dagger$ Yerevan Physics Inst., Armenia, $\div+\div$ Univ. of Virginia 
j. Measurement of Proton Polarization in the $d(\gamma, p) n$ Reaction (D. F. Geesaman, H. E. Jackson, C. E. Jones, D. H. Potterveld, B. Zeidman, K. P. Coulter,*

E. R. Kinney, $\nmid$ E. Beise,$\ddagger$ B. Filippone,$\ddagger$ R. D. McKeown, $\ddagger$ J. Napolitano, $\S$ R. Milner, $\mathbb{I}$ R. E. Segel,ll D. Beatty,** R. Gilman,** C. Glashausser,** G. Kumbartzki,**

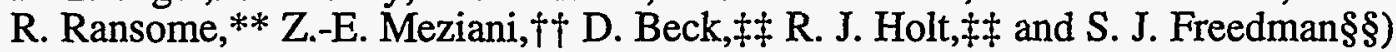

A proposal was approved by CEBAF PAC7 to measure angular distributions of the proton polarization for the $\mathrm{d}(\gamma, \overrightarrow{\mathrm{p}}) \mathrm{n}$ reaction in the $\mathrm{GeV}$ region. This proposed measurement will test the validity of extensions of conventional nuclear-physics theories to the higher energy regime. The results of the experiment will further constrain the evidence from SLAC experiments NE8 and NE17 that asymptotic scaling was observed above a photon energy of $1.3 \mathrm{GeV}$. Photoproton polarization measurements at lower energy indicate that the magnitude of the polarization increases with energy.
This is consistent with the observation that polarizations are large in high-energy processes, e.g. $\mathrm{A}_{\text {inn }}$ in $\mathrm{pp} \rightarrow \mathrm{pp}$ scattering or $\mathrm{A}_{\mathrm{y}}$ in $\mathrm{pp} \rightarrow \pi^{0} \mathrm{X}$. However, the polarizations in hadron-hadron scattering are believed to arise from Landshoff mechanisms. The higher energy photoproton experiment will permit the first measurements of polarization for a reaction, $\gamma \mathrm{d} \rightarrow \overrightarrow{\mathrm{p} n}$, where there are no Landshoff terms. The experiment would make use of a polarimeter installed in either the High Resolution Spectrometer in Hall A or the Short Orbit Spectrometer in Hall C at CEBAF.

*University of Michigan, †University of Colorado, ¥California Institute of Technology, §Rensselaer Polytechnic Institute, TMassachusetts Institute of Téchnology, INorthwestern University, **Rutgers University, $\uparrow+$ Stanford University, $\ddagger \ddagger$ University of Illinois, §§University of California at Berkeley

\section{k. Short-Orbit Spectrometer for Hall C at CEBAF (H. E. Jackson, D. H. Potterveld, and B. Zeidman)}

The Short Orbit Spectrometer (SOS) which the Argonne Medium Energy Physics Group built under contract to CEBAF passed its initial commissioning tests and is now being used in the first production experiments to be carried out at CEBAF. The SOS is a large-acceptance, low-loss, general-purpose magnetic particle spectrometer which will be used as the second coincident arm in electron scattering experiments planned in Hall C. It will be a resource of the CEBAF facility available to all users. The optical design is point-to-point in both the dispersive (vertical) and the transverse (scattering) planes. For a 1-mm target spot, the first-order resolving power is approximately 2200 , while the angular resolution is $<5 \mathrm{mr}$. Because of the reverse bend in the second dipole, there is a relatively small net deflection of the beam through the spectrometer, a property particularly useful for polarization measurements. Because of the strong edge focusing, the optical length of the spectrometer is only $\sim 7.4$ meters. Relatively short spectrometer drift lengths are required in experiments involving detection of pions or kaons in order to minimize decay losses.
The rigid structural design, coupled with a compact focal-plane detector package, yields a device that is readily adapted to out-of-plane measurements. Operation of SOS in conjunction with the High Momentum Spectrometer in Hall $\mathrm{C}$ will provide a coincidence capability and will serve as a generalpurpose second arm in a wide variety of experiments planned at CEBAF. A unique feature of the SOS, operation on a spherical bearing at the pivot point with a hydraulic lifting system, will allow measurements of scattered hadrons at angles up to 20 degrees out of the horizontal scattering plane. Substantial cost savings in its construction resulted from the participation of LANL engineers who had constructed a similar instrument at LAMPF. Initial tests using $800-\mathrm{MeV}$ electron beams from the CEBAF accelerator indicate performance as designed. The current schedule of experiments in the Hall call for its use in a broad spectrum of studies ranging from the photodisintegration of the deuteron and quasifree electron scattering to electroproduction of mesons and hypernuclei.

\section{1. ${ }^{3} \mathrm{He}$ Target for Hall C at CEBAF (B. Zeidman and A. Zeuli)}

A major fraction of the physics program for Hall $\mathrm{C}$ involves scattering from cryogenic targets of the lightest nuclei, i.e. H, D, and $3,4 \mathrm{He}$. Argonne is constructing the $\mathrm{He}$ target that will consist of a $4-\mathrm{cm}$ cylinder, operating at a pressure of 10 atmospheres and a temperature of $\sim 5.2$ degrees Kelvin. CEBAF is currently constructing a cryo-target system that includes liquid $\mathrm{H}$ and $\mathrm{D}$ cells and, in addition, the cooled, 
pressurized helium target. The He target system includes cell loop, the He supply systems, and the additional equipment needed to ensure minimum loss of ${ }^{3} \mathrm{He}$ in the event of target rupture. The major components have been completed, and are ready for installation. In a test of the He cell, the wall ruptured at 27 atmospheres, well above the 5-10 atm operating pressure.

m. HERMES, Measurements of Spin-Structure Functions and Semi-Inclusive Asymmetries for the Proton and Neutron at HERA (H. E. Jackson, J.-O. Hansen, C. E. Jones, N.C.R. Makins, T. G. O'Neill, D. H. Potterveld, and collaborators at 29 other institutions)

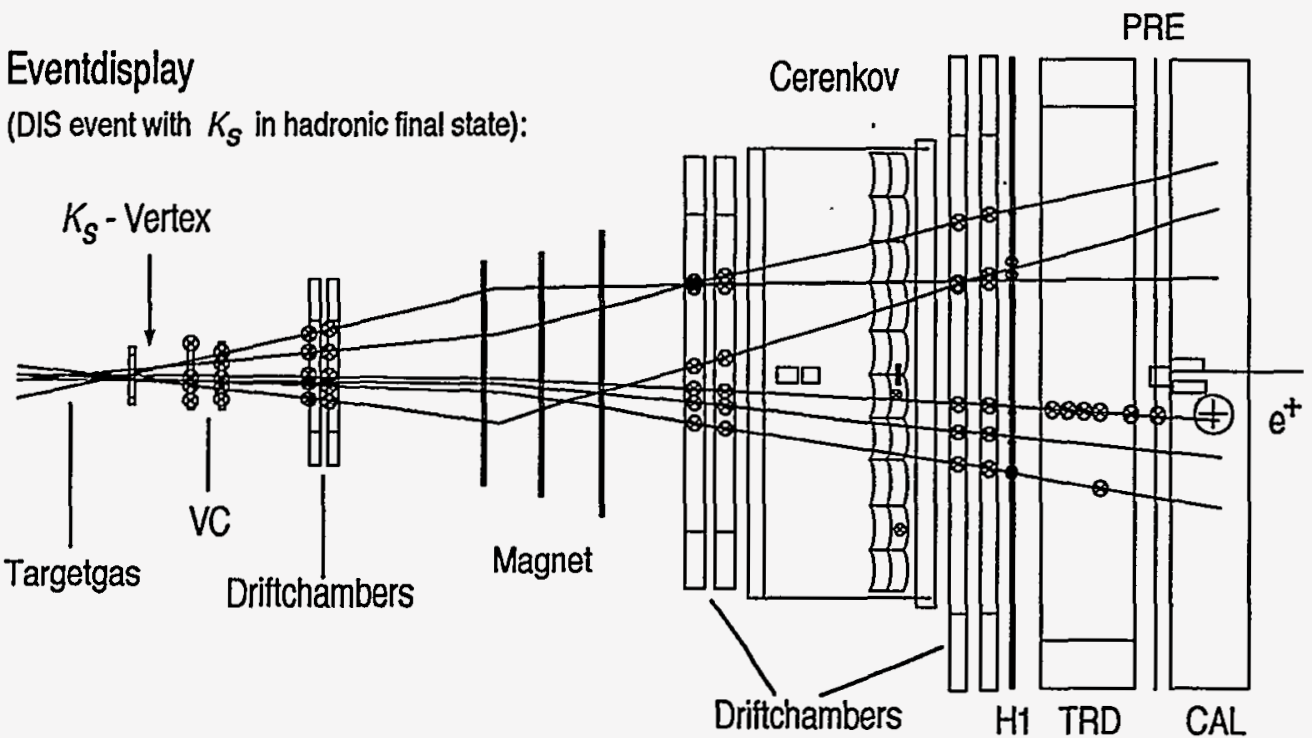

Transverse vertex distribution:

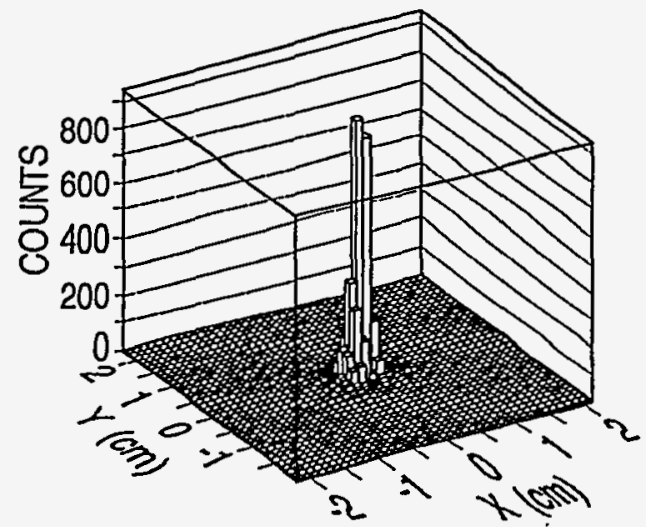

No background from wall scattering
$K_{S}$ mass peak:

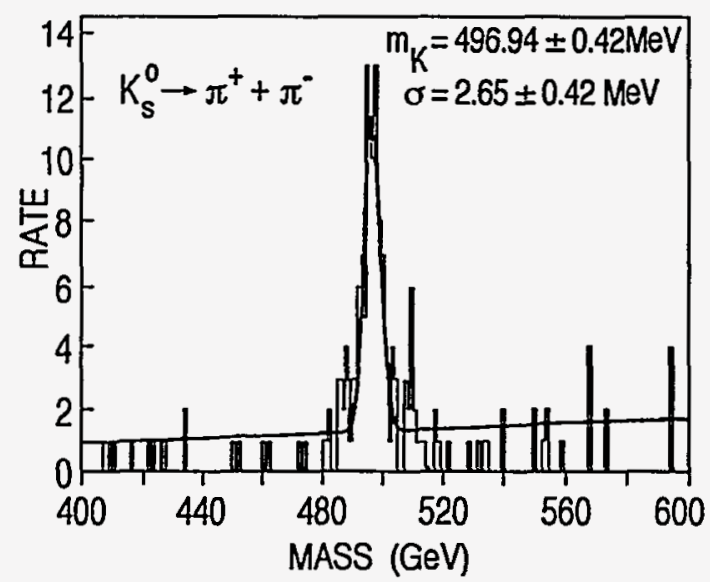

Fig. III-3. The upper section shows the HERMES event display for a typical HERMES deep-inelastic scattering event, in this case containing a neutral kaon and its decay. The lower left section contains a plot of the reconstructed vertex distribution for deep-inelastic scattering events. The lower right section is the invariant mass distribution for reconstructed neutral kaons.

HERMES is an international collaboration that is carrying out a program of studies of the spin structure of the nucleon at the DESY Laboratory in Hamburg Germany, using internal polarized gas targets in the stored electron beam of the HERA accelerator. The collaboration currently includes approximately 30 institutions from North America and Europe including several institutions from CIS. The full experiment 
with the complete spectrometer was commissioned during the first few months of the 1995 operating cycle at DESY. By September 1, the experimental systems were in routine operation (see Fig. III-3). Subsequent efforts were devoted to accumulating data on the spindependent structure function, $g_{1}(x)$, for the neutron using a polarized ${ }^{3} \mathrm{He}$ target. A limited amount of data was also taken with unpolarized hydrogen and deuterium in order to calibrate the spectrometer and to begin a measurement of the flavor asymmetry of the quark sea in the nucleon. Over the course of the experiment, expected to be 3-4 years, a complete series of measurements of spin-dependent properties of the nucleon and few-body nuclei will be made; the spin structure function $g_{1}(x)$ of the proton and neutron will be measured to test the Bjorken sum rule and quarkgluon correlations, and the structure functions $b_{1}(x)$ and $\Delta(\mathrm{x})$, sensitive to nuclear binding will be measured. Using the particle identification capability of the HERMES spectrometer, pions will be detected in coincidence with the scattered electrons. The coincident hadron measurements represent the most important extension that can be made to the existing measurements on the quark spin distribution in the nucleon. Argonne provided the Cerenkov counter used for particle identification and developed the drifilm coating technique for the ultrathin target cell required for this experiment. The HERMES collaboration intends to use polarized targets with the highest available figures of merit and the Argonne laser-driven source offers the most promise for a significant advance in present-day targets.

Preliminary analyses of the data taken during 1995 on a longitudinally polarized ${ }^{3} \mathrm{He}$ target (shown in Fig. III-4) indicate that the final results for $\mathrm{g}_{1}^{\mathrm{n}}(\mathrm{x})$ will be competitive with those published by other groups.

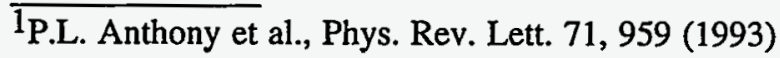

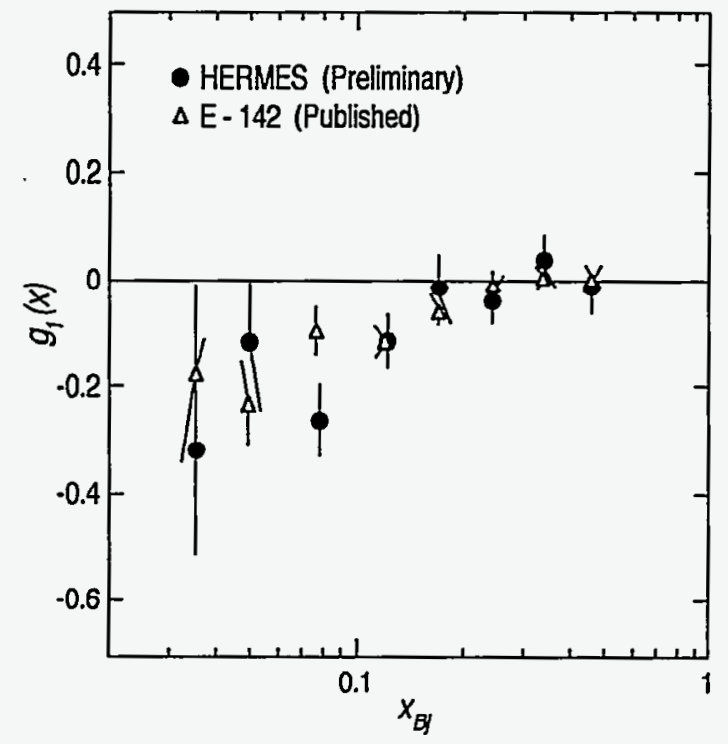

Fig. III-4. Results for the spin structure function $g_{1}(x)$ of the neutron from a preliminary analysis of data taken in 1995. Results are compared with measurements made in an experiment $(E-142)^{l}$ at the Stanford Linear Accelerator.

Data on semi-inclusive pion asymmetries already give the first direct measurements of the polarization of sea quarks in the nucleon. One objective of the unpolarized $\mathrm{H} / \mathrm{D}$ measurements is the determination of the charged pion multiplicities which will then be used to make a direct determination of the flavor asymmetry in the quark sea in the nucleon. Analysis of a portion of the data is completed. Although a definitive experiment will require additional running in 1996, the data obtained this year demonstrate the feasibility of the technique. Analysis of the complete data set from the 1995 run may already provide a statistically significant result, but it is too early to be certain. 


\section{n. Nuclear Transparency and Longitudinal Asymmetry in $A\left(e, e^{\prime} \rho^{0}\right)$ at High}

Energies T. G. O'Neill, H. E. Jackson, J.-O. Hansen, N. C. Makins, D. H. Potterveld, C. E. Jones, and collaborators at 29 other institutions.

Data on $A\left(e, e^{\prime} \rho^{0}\right)$, the exclusive electroproduction of the neutral $\rho$ meson, was isolated from the inclusive $A\left(e, e^{\prime}\right)$ data used for the HERMES measurement of the spindependent structure functions. Data were taken on ${ }^{1} \mathrm{H}$, ${ }^{2} \mathrm{H}$, and ${ }^{3} \mathrm{He}$ targets. The $\rho^{0}$ meson, which decays into 2 pions, manifests itself in a peak at $\mathrm{M} \rho=0.77 \mathrm{GeV}$ in the invariant mass of the 2 pion system. The $\rho^{0}$ mass peak is shown in Fig. III-5 for exclusive $A\left(e, e^{\prime} \rho^{0}\right)$ events, in which there are no additional articles in the final state. The preliminary analysis of the data has yielded results for the nuclear transparency of ${ }^{3} \mathrm{He}$ and also for the reaction asymmetry in the case where the ${ }^{3} \mathrm{He}$ target and the electron beam were both polarized longitudinally. The longitudinal asymmetry can be used to help understand the reaction mechanism. The nuclear transparency data exhibit a strong statistically significant increase with the momentum transfer $Q^{2}$, which can be interpreted as evidence of the color transparency effect and of variations in the hadronic structure of the virtual photon mediating the reaction. These data can help illuminate the photon structure and the interaction of the outgoing $\rho$ meson with the nuclear medium. In addition they could represent the culmination of the decade-long search for definitive evidence of the color transparency phenomenon.

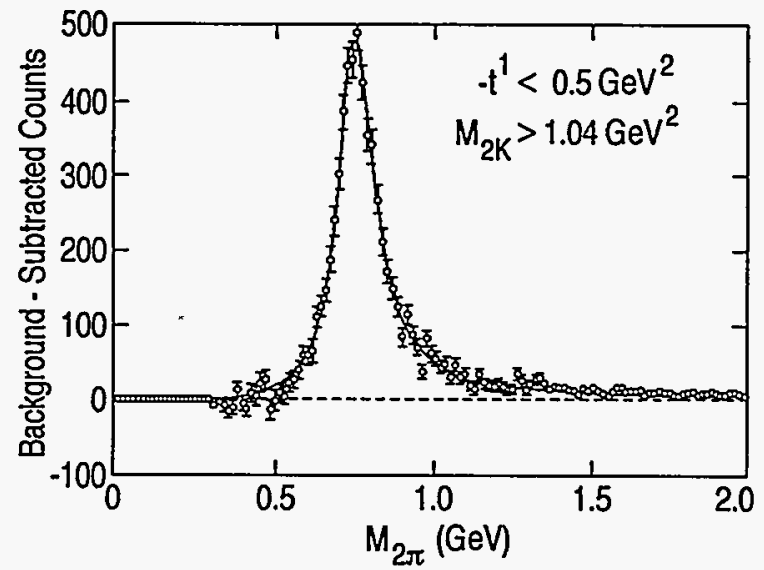

Fig. III-5. Histogram of the invariant mass of the 2-pion final state in exclusive $A\left(e, e^{\prime} \pi^{ \pm} \pi^{-}\right)$events, exhibiting a peak at $M_{\rho}=0.77 \mathrm{GeV}$ due to exclusive $A\left(e, e^{\prime} \rho^{0}\right)$. The background contribution from nonexclusive events has been subtracted. The curve is a fit to a relativistic $p$-wave Breit-Wigner with a mass skewing factor.

o. Measurement of $\overline{\mathrm{d}} / \overline{\mathrm{u}}$ in the Nucleon (D. F. Geesaman, H. E. Jackson, C. E. Jones, S. Kaufman, N. C. Makins, D. Potterveld, B. Zeidman, D. Isenhower,* M. Sadler,* Y. C. Chen, $\dagger$ G. C. Kiang, $†$ P. K. Teng, $\dagger$ M. J. Wang, $\dagger$ B. Filippone, $\ddagger$ R. McKeown, $\ddagger$ C. N. Brown, $\S$ W. E. Cooper, $\S$ C. S. Mishra, $\S$ T. A. Carey, ,

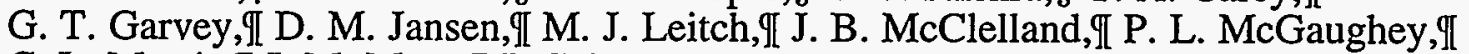
C. L. Morris,I J. M. Moss,I J. C. Peng,II D. M. Kaplan,II T. C. Awes, **

F. E. Obenshain,** G. R. Young, ** F. Z. Plasil, ** Hee Kim,** S. Saini, ** P. Stankus, ** S. P. Sorenson, $\dagger \dagger$ C. A Gagliardi, $\neq \ddagger$ and R. E. Tribble $\ddagger$ )

Recent experimental results on the deep inelastic structure functions on hydrogen and deuterium, combined in a sum rule analysis, suggest that there is a substantial difference between $\overline{\mathrm{u}}$ and $\overline{\mathrm{d}}$ sea in the proton. The Drell-Yan process, where a quark from a projectile annihilates with an anti-quark in the target to form a timelike-virtual-photon, can provide a direct measurement of the $x$ dependence of the anti-quark distributions. A first examination of this process in
CERN experiment NA-51 confirmed that the $\overline{\mathrm{u}}$ and $\overline{\mathrm{d}}$ distributions are indeed different near $x$ of 0.15 .

A new experiment, E866, was approved for the next fixed target cycle at FNAL to measure accurately the Drell-Yan production of di-muon pairs using an 800$\mathrm{GeV}$ proton beam on liquid hydrogen and deuterium targets, with relative systematic errors of $\sim 1 \%$. This will measure $\overline{\mathrm{d}} / \overline{\mathrm{u}}$ to $1 \%$ accuracy for $0.05<\mathrm{x}<0.15$ and with lesser statistical accuracy out to $x \sim 0.3$,

*Abilene Christian University, $\uparrow$ Academia Sinica, Taiwan, ROC, $\ddagger$ California Institute of Technology, §Fermi National Accelerator Laboratory, ILos Alamos National Laboratory, INNorthern Illinois University, **Oak Ridge National Laboratory, $\dagger$ †University of Tennessee, $\ddagger$ Texas A\&M University 
with one to two orders of magnitude higher statistics than NA-51. High statistics $\mathrm{J} / \Psi$, and $\psi^{\prime}$ and $\Upsilon$ production data will also be obtained.

Minimizing the systematic errors is an essential factor in these measurements. ANL is concentrating on two aspects of this. The magnetic fields in the analysis magnets will be carefully mapped in a region where the strengths of two magnets are coupled. A mapping apparatus was constructed and the mapping will occur in winter 1996. Also to address the experiment's known sensitivity to the microscopic beam structure, a Cerenkov counter and flash ADC readout system will provide a microsecond history of the $53-\mathrm{MHz}$ beam structure.

The experiment will begin taking data in summer 1996 and continue in 1997.

p. First Measurement of the Triton Asymmetry in Muon Capture by Polarized Muonic ${ }^{3}$ He (W. J. Cummings, J. Behr,* A. Gorelov,* O. Hausser, ${ }^{*}$ M. D. Hasinoff, $\dagger$ J. C. Huang, $\dagger$ E. Saettler, $\uparrow$ P. Michaux, $\ddagger$ P. Bogorad,§ G. D. Cates, $\S$ K. Kumar, $\S$ H. Middleton,§ D. Siegel,§ A. Young,§ R. Holmes, II J. McCracken, $\mathbb{1}$ P. A. Souder, $\mathbb{I}$ X. Wang,II D. Swenson,II D. Tupa,II K. Hicks, ** B. Larson, ** W. Lorenzon, $† \dagger$ E. J. Brash $\ddagger$ )

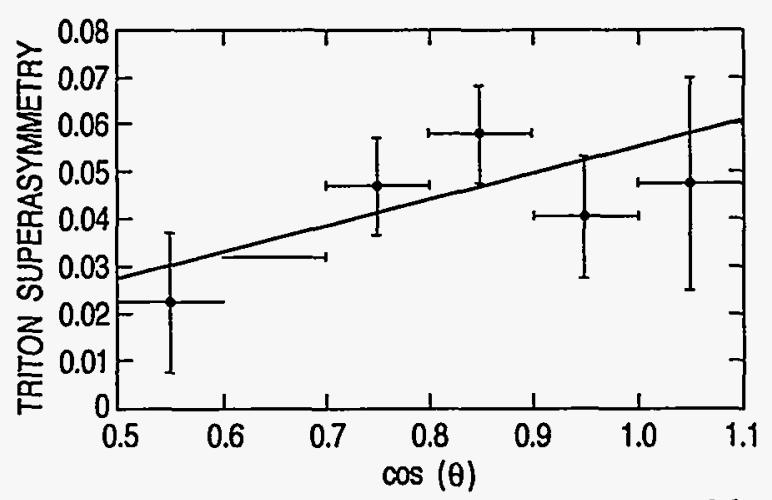

Fig. III-6 Triton asymmetry as measured by TRIUMF E683 shown as a function of $\cos (\theta)$, where $\theta$ is the angle between the muon spin and triton recoil directions. The solid line is a fit to the theoretically expected angular dependence.

Of particular interest in studying semi-leptonic weak interactions is the value of the induced pseudoscalar coupling $\mathrm{g}_{\mathrm{P}}$. A comparison of $\mathrm{g}_{\mathrm{P}}$ from muon capture in nuclei with $g_{p}$ from muon capture in hydrogen is very sensitive to the inclusion of meson exchange currents (MEC). Of all nuclear targets, ${ }^{3} \mathrm{He}$ is the most interesting for muon capture studies. Muon capture on $3 \mathrm{He}$ has the advantage that the ${ }^{3} \mathrm{He} \rightarrow{ }^{3} \mathrm{H}$ transition has the same spin, isospin and parity as the $p \rightarrow n$ transition. The wave functions of the $A=3$ isodoublet can be calculated with little uncertainty from Faddeev methods. Tritons emitted following muon capture by polarized muonic $3 \mathrm{He}$ exhibit a characteristic asymmetry which allows a nearly model independent extraction of $g_{P}$ with enhanced sensitivity over capture rate measurements. TRIUMF Experiment 683 recently completed the first measurement of this asymmetry.

The experiment requires that the muon beam is stopped, the muonic ${ }^{3} \mathrm{He}$ is polarized, and the triton is detected in the same gas volume. The experiment was performed at TRIUMF using very low momentum (21-MeV/c) muons from the M9B superconducting muon channel. These muons stop in an ionization chamber containing high pressure ${ }^{3} \mathrm{He}$ gas and form muonic $\mathrm{He}$ atoms. The muonic ${ }^{3} \mathrm{He}$ atoms are polarized by spin exchange collisions with the optically pumped $\mathrm{Rb}$ vapor.

The results of this first experiment, which are shown in Fig. III-6, give a value for $g_{p}$ which is consistent with previous total capture rate measurements and slightly less accurate. However, the main limitation on these results was the low degree of polarization of the muonic atoms. Recent advances in diode laser technology provide the opportunity to overcome this limitation. The collaboration plans a new measurement with these more powerful laser beams and an improved ionization chamber. The ultimate goal is a measurement of $g_{P}$ of $10 \%$ accuracy.

*Simon Fraser University, Burnaby, Canada, †University of British Columbia, Vancouver, Canada, $¥$ TRIUMF, Vancouver, Canada, §Princeton University, ISSyracuse University, IILANL, **Ohio University, ††University of Pennsylvania, $\ddagger \ddagger$ Rutgers University 


\title{
IV. THEORETICAL PHYSICS
}

\section{OVERVIEW}

\begin{abstract}
Theoretical nuclear research at Argonne addresses a broad range of problems involving the structure and dynamics of hadrons and nuclei. There is a strong emphasis on comparison to data provided by experimental groups at Argonne and at other facilities around the world. The principal areas of research include nuclear dynamics with sub-nucleonic degrees of freedom, nuclear forces and nuclear systems, and heavy-ion reactions and nuclear structure studies. Our work includes the modeling of quantum chromodynamics in meson and baryon structure, developing reaction theories for medium-energy nucleon-nucleon interactions and meson production, and studying electron scattering within the framework of relativistic Hamiltonian particle dynamics. We also construct realistic nucleonnucleon potentials that give very accurate fits to elastic scattering data, and then use these in detailed many-body calculations of the properties of few-body nuclei, light closed-shell nuclei, hypernuclei, nuclear matter and neutron stars. Heavy-ion research includes the structure and reactions of neutron-rich nuclei produced at radioactive beam facilities and coupled-channels calculations of reactions near the Coulomb barrier, while our nuclear structure research concentrates on effective two-body interaction studies of deformed and superdeformed nuclei observed at ATLAS. Several of these projects require major numerical simulations using stateof-the-art computers, including Argonne's massively parallel IBM SP. Much of our work is done in collaboration with other researchers at domestic and foreign universities and other national laboratories.
\end{abstract}

\section{A. NUCLEAR DYNAMICS WITH SUBNUCLEONIC DEGREES OF FREEDOM}

The objective of this research program is to investigate the role of mesons, nucleon resonances, and quark-gluon degrees of freedom in nuclear dynamics.

We are continuing our effort to address theoretical questions concerning intermediate-energy reactions. By employing a unitary transformation method, we developed an energy-independent effective Hamiltonian for a successful description of both the pion photo-production on the nucleon and the pion threshold production in NN scattering. A Pomeron-exchange model for exclusive electroproduction of $\rho$-mesons was developed in conjunction with the recent HERMES experiments at HERA. To facilitate relativistic heavy-ion calculations, many experimentally inaccessible cross sections of binary collisions with the $\Delta$ resonance were predicted within the unitary $\pi \mathrm{NN}$ model. A meson-exchange model of $\pi \mathrm{N}$ and $\gamma \mathrm{N}$ reactions was developed to interpret the $N^{*}$ excitations within the chiral constituent-quark model.

The Dyson-Schwinger equation (DSE) approach is both an alternative and complement to numerical simulations of the lattice-QCD Lagrangian. We made extensive progress in employing the DSEs as a semi-phenomenological tool for nonperturbative studies of QCD. In the course of 
this research we have undertaken nonperturbative studies of QED, which provide us with important qualitative input for our QCD studies and contribute to a deeper understanding of the application of this approach to the solution of gauge field theories. Important recent successes are the calculations of the electromagnetic pion form factor and the $\gamma^{*} \pi^{0} \rightarrow \gamma$ transition form factor, which manifestly demonstrate the unique ability of this approach to unify the perturbative and nonperturbative domains of $Q C D$, where confinement and dynamical chiral symmetry breaking are crucial. We are currently applying this framework to the study of baryon spectroscopy and electromagnetic properties.

We are continuing our investigation of electron scattering of few-nucleon systems, and constituentquark methods of hadrons, in the framework of relativistic Hamiltonian few-body dynamics. Euclidean space-time formulations of Lagrangian quantum field theories provide the mathematical basis for non-perturbative approximations, and for precisely defined models of finite systems. We are exploring the implications of this approach, in particular the requirements quantum theory imposes on model Schwinger functions. Independently we found that the well-known Lorentz covariant representations of current kernels for spin $1 / 2$ and spin 1 can be generalized to arbitrary integral and half-odd integral spins by emphasizing the $O(1,2)$ subgroup that leaves the fourmomentum transfer invariant. This representation may provide a basis for the construction of dynamically determined interaction currents in the framework of Hamiltonian light-front dynamics.

\section{a. Production of Mesons and Nucleon Resonances at GeV Energies} (T.-S. H. Lee and T. Sato*)

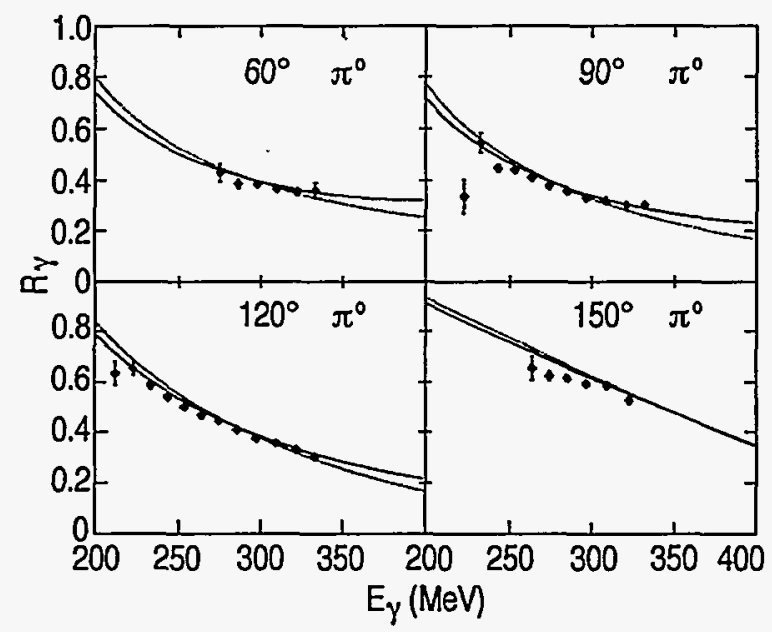

Fig. IV-1. The ratio $d \sigma_{||} / d \sigma_{\perp}$ of the $\gamma p \rightarrow \pi^{o} p$ reaction are compared with the LEGS data. The solid and dotted curves are respectively for $\left(G_{M}, J_{W N N}\right)=$ (1.85, 105), (1.95.7).

The objective of this work is to develop a theoretical model for investigating the nucleon resonances $\left(\mathrm{N}^{*}\right)$ at energies accessible to CEBAF and relativistic heavy-ion facilities. Motivated by the existing QCD-based hadron

\footnotetext{
*Osaka University, Japan
}

models, we assume that the basic resonant interaction mechanisms of the model Hamiltonian are the absorption and emission of photons and mesons by a bare quark core. The matrix elements of nonresonant interactions are deduced from an effective Lagrangian with chiral symmetry by using a method of unitary transformation. The standard projection operator technique was applied to obtain a set of unitary scattering equations for describing $\pi \mathrm{N}$ and $\gamma \mathrm{N}$ reactions up to the GeV energy region. In FY 1995, a mesonexchange Hamiltonian was obtained for a consistent description of $\pi \pi, \pi N$ and $\gamma N \rightarrow \pi N$ reactions. The predicted cross sections are in excellent agreement with very extensive data as well as the new polarization data from LEGS. We explored in detail the dependence of the predicted $\gamma \mathrm{N} \rightarrow \pi \mathrm{N}$ observables on the deformation of the $\Delta$ resonance. The extracted E2/M1 ratio for $\Delta \rightarrow \gamma \mathrm{N}$ transition is $-0.015 \pm 0.02$. Our results for the ratio LEGS $d \sigma_{\|} / \mathrm{d} \sigma_{\perp}$ are compared in Fig. IV-1 with the data. The uncertainties are mainly due to the lack of accurate values of the coupling constants, $\gamma_{\gamma \rho \pi}$, $\gamma \gamma \omega \pi, \gamma_{\rho N} N$ and $\gamma_{\omega N N}$ of the nonresonant amplitudes. A paper describing our results is being prepared publication. Our current focus is to extend the meson-exchange Hamiltonian to include highermass nucleon resonances. 


\section{b. Pomeron-Exchange and Exclusive Electroproduction of $\rho$-Mesons (T.-S. H. Lee and M. A. Pichowsky)}

The exclusive electroproduction of vector mesons is considered to be very effective in probing the structure of the Pomeron using data from HERMES. To facilitate such an investigation, a Pomeron-exchange model of exclusive electroproduction of $\rho$-mesons is being examined using a dressed-quark propagator. We showed that by representing the photon- $\rho$-mesonPomeron coupling by a nonperturbative-quark look, one can obtain predictions for $\rho$-meson electroproduction that are in good agreement with the existing data as well as the new data from the ZEUS collaboration. In contrast to the earlier work by Donnachie and Lanshoff, a quark-Pomeron form factor is unnecessary. Our results are displayed in Fig. IV-2. A paper describing our results was submitted for publication. We are extending the model to also describe the vector meson electroproduction at CEBAF energies.

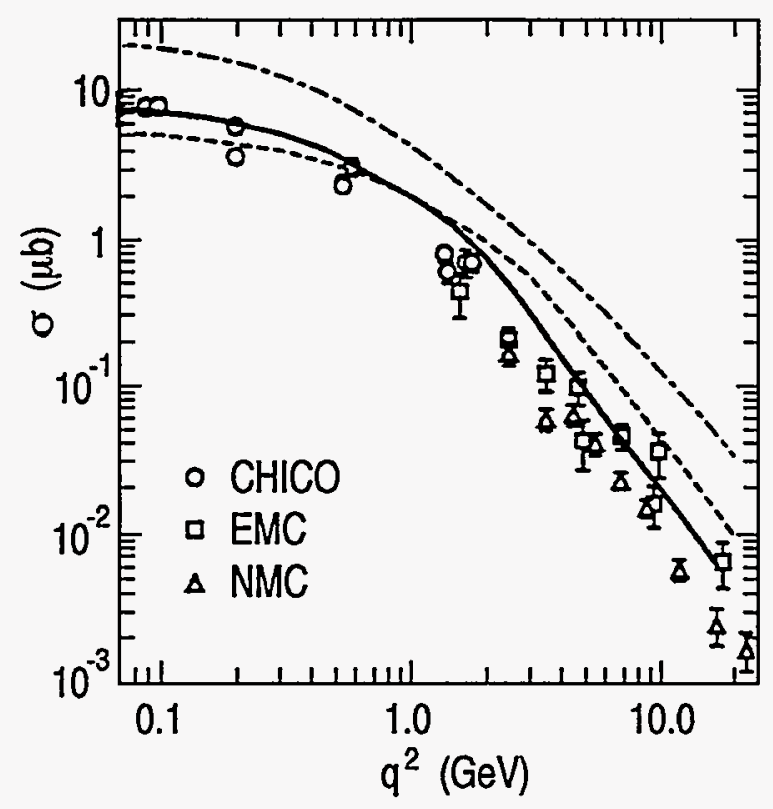

Fig. IV-2. The total $\rho-m e s o n$ electroproduction cross section. The solid line is our model, the dash-dotted line is obtained using the "onshell approximation", and the dashed line is our model with a simplified dressed-quark propagator.

\section{c. Calculation of E2/M1 Ratio of the $\Delta \rightarrow \gamma \mathrm{N}$ Within the Chiral Constituent Quark Model (T.-S. H. Lee, Y.-B. Dong,* P. Shen,* Y. Yu,* and Z.-Y. Zhang*)}

The E2/M1 ratio of the $\gamma \mathrm{N} \rightarrow \pi \mathrm{N}$ reaction measures the deformation of the $\Delta$ resonance. This quantity is crucial for testing various QCD-based hadron models. The objective of this work is to examine whether the $\Delta$ deformation predicted by the chiral quark model is consistent with the data of pion photo- and electroproduction. The calculation was carried out including $\mathrm{N}=0,2,4$ excitations in the oscillator basis. The predicted E2/M1 ratio at the $\mathrm{q}^{2}=0$ photon point is

*Academia Sinica, Beijing, PRC consistent with the value determined recently by Lee and Sato ( see IV.A.a). At high $\mathrm{q}^{2}=3.2(\mathrm{GeV} / \mathrm{c})^{2}$ where $\mathrm{p}\left(\mathrm{e}, \mathrm{e}^{\prime} \pi^{0}\right)$ data are available, our prediction is $\mathrm{E} 2 / \mathrm{M} 1=$ -0.06 which is much larger than the value of about -0.02 predicted by the constituent quark model of Capstick. The difference is due to the presence of the pion coupling in our model. To further test the model, we made predictions for future experiments at Mainz and CEBAF. A paper describing our results is being prepared for publication. 


\section{d. Phenomenological Coupled-Channel Model for $\pi \mathbf{N}$ and $\gamma \mathbf{N}$ Reactions With $\mathbf{N}^{*}$ Excitations (T.-S. H. Lee, T. Vrana,* and S. Dytman*)}

Under CEBAF's $\mathrm{N}^{*}$ program, extensive $\gamma \mathrm{N} \rightarrow \pi \mathrm{N}, \pi \pi \mathrm{N}$ data with unprecedented accuracy will be available. One of the main tasks is to extract from the data the multipole amplitudes which can then be used to test various hadron models. Because of multi-channel complications, the available methods based on dispersion relations and $\mathrm{K}$-matrix methods are not directly applicable at CEBAF energies. We adapted the coupled-channel method developed by Cutkosky and his collaborators in analyzing the $\mathrm{N}^{*}$ in $\pi \mathrm{N}$ reactions. The channels included in our analysis are $\pi N, \pi \Delta, \rho N$, and $\varepsilon N$. The parameters for all $\mathrm{N}^{*}$ listed in the Particle Data Tables are then determined by fitting Manley's representation of $\pi N \rightarrow \pi N, \pi \pi N$ data. A paper describing our results is being prepared for publication. The model is being extended to include the $\gamma \mathrm{N}$ channel in order to analyze the forthcoming data from CEBAF. The constructed model was also used to generate cross sections for many experimentally inaccessible processes, such as $\rho \mathrm{N} \rightarrow \pi \mathrm{N}$ and $\pi \Delta \rightarrow \pi \Delta$, which are needed in relativistic heavy-ion calculations. A paper on this subject is also being prepared for publication.

*University of Pittsburgh

\section{e. Electroproduction of Pions on the Three-Nucleon Systems (T.-S. H. Lee, C. Chmielewski, * and P. U. Sauer*)}

The electroproduction of pions on ${ }^{3} \mathrm{He}$ is being studied with the assumption that the basic pion production mechanisms can be described by the $N\left(e, e^{\prime} \pi\right)$ and $\Delta\left(e, e^{\prime} \pi\right)$ mechanisms. In the impulse approximation, the ${ }^{3} \mathrm{He}(\mathrm{e}, \mathrm{e} \cdot \pi)$ cross section is then determined from the $\mathrm{N}$ and $\Delta$ spectral functions generated from Hannover's three-body calculation including the $\Delta$. The

*University of Hannover, Germany objective is to investigate the effects due to the $\Delta$ components in ${ }^{3} \mathrm{He}$, as suggested by Lipkin and Lee. In FY 1995, the computer program for calculating the ${ }^{3} \mathrm{He}\left(\mathrm{e}, \mathrm{e}^{\prime} \pi^{+}\right)$and ${ }^{3} \mathrm{He}\left(\mathrm{e}, \mathrm{e}^{\prime} \pi^{-}\right)$cross sections was developed. The non-resonant $\Delta$ knockout mechanism was found to be important, and was included in the calculation. A paper describing our results is being prepared. Predictions for future experimental efforts at Mainz and CEBAF will be made.

\section{f. Threshold Pion Production from Nucleon-Nucleon Collisions (T.-S. H. Lee)}

The threshold production of $\pi^{0} \mathrm{pp}, \pi^{+} \mathrm{np}$, and $\pi^{+} \mathrm{d}$ from proton-proton collisions are investigated using a model consisting of pion s-wave rescattering and $\mathrm{NN}$ pairterms of heavy-meson exchanges. In a calculation using the Paris potential, we find that the data can be best reproduced by using a soft $\pi N N$ form factor with $\Lambda=650 \mathrm{MeV}$ for a monopole form. This is consistent with our earlier studies of pion production in the $\Delta$-excitation region. The dependence of our results on the NN wave functions and the $\pi N$ off-shell dynamics was explored in detail. Attempts are being made to relate the model to a more fundamental approach using the method of unitary transformation and chiral perturbation theory.

\section{g. Production of $\eta$ Meson in Photo-Nucleon and Nucleon-Nucleon Reactions (T.-S. H. Lee, A. Svarc,* M. Batnic,* and C. Fasano $\dagger$ )}

It is generally believed that $\eta$ production is predominantly due to excitation of the $\mathrm{N}^{*}(1550)$. But a recent analysis of the $\pi \mathrm{N} \rightarrow \eta \mathrm{N}$ reaction by Batnic and Svarc has indicated that the effects due to the other $\mathrm{N}^{*}$ resonances are significant. This raises an important

*Rudjer Boskovic Institute, Zagreb, Croatia

†Francis Marion University question concerning the dynamical content of the $\eta$ meson which was traditionally classified within the SU(6) symmetry. To clarify the situation, we are exploring the consequences of the $\pi N \rightarrow \eta N$ amplitudes determined by Batnic and Svarc in predicting $\eta$ production in photo-nucleon and nucleon-nucleon reactions. The predicted $\mathrm{pp} \rightarrow \mathrm{pp \eta}$ cross sections are found to be lower than the recent data by a factor similar 
to what was found in the threshold $\mathrm{pp} \rightarrow \mathrm{pp} \pi^{0}$ reaction. We are exploring whether this process can be described by including the short-range dynamics developed by Lee and Riska. The calculation of $\eta$ photoproduction has revealed the sensitivity to the $\eta N N$ coupling and the associated non-resonant mechanisms. Predictions will be made for determining the $\eta N N$ coupling constant and $\mathrm{N}^{*}$ excitation parameters using the data from Mainz and future CEBAF experiments.

\section{h. Medium Effects on Binary Collisions With the $\Delta$ Resonance (T.-S. H. Lee)}

To facilitate the theoretical calculations of relativistic heavy-ion collisions, the binary collisions involving a $\Delta$ resonance in either the entrance channel or the exit channel are investigated within a Hamiltonian formulation of $\pi \mathrm{NN}$ interactions. An averaging procedure is developed to define a quasi-particle $\Delta^{*}$ and to express the experimentally measured $\mathrm{NN} \rightarrow \pi \mathrm{NN}$ cross section in terms of an effective $\mathrm{NN} \rightarrow \mathrm{N} \Delta^{*}$ cross section. In contrast to previous works, the main feature of the present approach is that the mass and the momentum of the produced $\Delta^{*}$ at each collision energy are calculated dynamically from the bare $\Delta \rightarrow \pi \mathrm{N}$ vertex interaction of the model Hamiltonian and are constrained by the unitarity condition. The procedure is then extended to define the effective cross sections for the experimentally inaccessible $\mathrm{N} \Delta^{*} \rightarrow \mathrm{NN}$ and $\mathrm{N} \Delta^{*} \rightarrow \mathrm{N} \Delta^{*}$ reactions. By including the medium effects on the $\Delta$ propagation, the dependence of the effective cross sections of the $N N \rightarrow N \Delta^{*}, N \Delta^{*} \rightarrow N N$ and $\mathrm{N} \Delta^{*} \rightarrow \mathrm{N} \Delta^{*}$ processes on the nuclear density are examined. In Fig. IV-3 we show that the predicted $\mathrm{N} \Delta^{*} \rightarrow \mathrm{NN}$ and $\mathrm{N} \Delta^{*} \rightarrow \mathrm{N} \Delta^{*}$ cross sections depend strongly on the density and momentum in the region near pion production threshold. Our predictions can be used in relativistic heavy-ion calculations based on transport 'equations. A paper describing results is being prepared for publication.

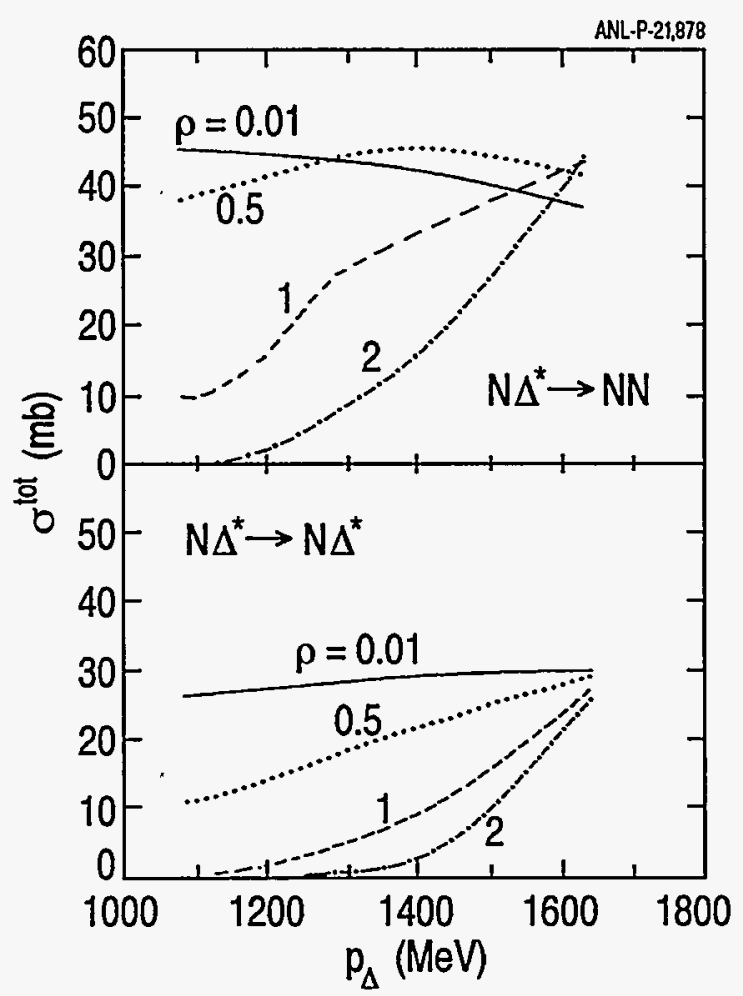

Fig. IV-3. The density dependence and momentum dependence of the predicted $N \Delta^{*} \rightarrow N N$ and $N \Delta^{*} \rightarrow N \Delta^{*}$ reactions.

\section{i. The Effect of the $\Delta$ Excitation on Proton-Proton Bremsstrahlung (T.-S. H. Lee, F. de Jong,* and K. Nakayama*)}

The proton-proton bremsstrahlung is investigated with a coupled-channel model with $\pi$ and $\Delta$ degrees of freedom. The model is consistent with the NN scattering up to $1 \mathrm{GeV}$ and the $\gamma \mathrm{N} \Delta$ vertex determined in the study of pion photoproduction on the nucleon. We find that the $\Delta$ excitation can significantly improve

*University of Georgia, and Institute of Theoretical Physics, Jülich, Germany the agreement with the $\mathrm{pp} \rightarrow \mathrm{pp} \gamma$ data at $\mathrm{E}_{\mathrm{L}}=280 \mathrm{MeV}$. The $\mathrm{N} \Delta$ rescattering plays an important role in determining the angular distribution and analyzing powers. Predictions at $\mathrm{E}_{\mathrm{L}}=550$ and $800 \mathrm{MeV}$ were made for the forthcoming experimental tests at COSY of Jülich. A paper describing our results was published. 


\section{j. The Effect of the $\Delta$ Three-Body Force on Effective Nucleon-Nucleon Interactions of the Nuclear Shell-Model (T.-S. H. Lee, T. T. S. Kuo, and $\mathrm{Y}$. Tzeng $\dagger$ )}

The effect of the $\Delta$ three-nucleon force on the shellmodel effective interaction is investigated by evaluating the $\Delta$ particle-nucleon hole core polarization diagrams $\mathrm{G}_{\mathrm{pp} \Delta \mathrm{h}}$ within the folded-diagram formulation. The calculation was performed using the $\mathrm{NN} \leftrightarrow \mathrm{N} \Delta$ transition G-matrix generated from a coupled-channel

*State University of New York at Stony Brook, $\dagger$ Institute of Physics, Academia Sinica, Taipei, Taiwan, ROC $\pi \mathrm{NN}$ model which is constrained by the NN data up to $1 \mathrm{GeV}$ and is based on a $\Delta$-subtracted Paris potential. Satisfactory convergence of the calculation is reached by including the $\Delta$ excitations up to 20 oscillator shells. The $\Delta$-hole core-polarization diagrams $G_{p p} \Delta h$ are found to be very small for the sd-shell valence nucleons. A paper describing our results was published.

\section{k. Excitation of $\mathbf{N}^{*}$ Resonances (D. Kurath)}

Coupled-channel calculations of the excitation of nucleon and delta resonances are underway by T.-S. H. Lee and his collaborators. Necessary ingredients are matrix elements for either $\gamma$ or $\pi$ excitation.
Previously constructed programs to calculate these matrix elements with the harmonic oscillator quark model were extended to include resonances of the $\Delta$, and the desired matrix elements were calculated.

\section{Pion Loop Contribution to the Electromagnetic Pion Charge Radius} (C. D. Roberts, A. Bender, and R. Alkofer*)

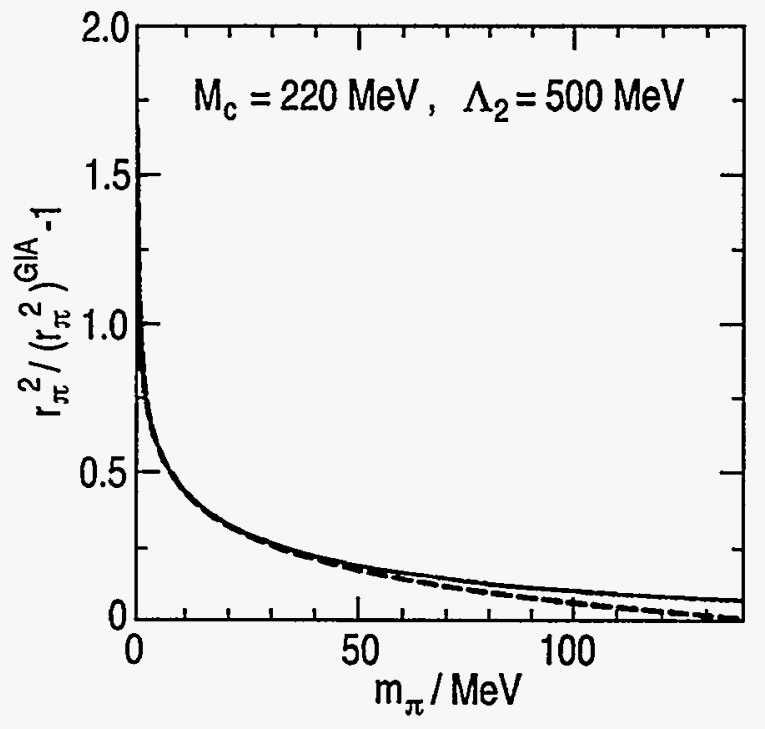

Fig. IV-4. Here the relative contribution of the pion loop to $\left\langle\mathrm{r}_{\pi}^{2}\right\rangle$ is shown as a function of the pion mass $m_{x}$, in $\mathrm{MeV}$. The solid line is the result of our calculation; the dashed line is a fit of the form $a+b \ln m_{x}$. The figure illustrates the onset of the pion-loop induced ln $m_{x}$ divergence of $\left\langle\mathrm{r}_{\pi}^{2}\right\rangle$. It is clear that even for $m_{x}=50 \mathrm{MeV}$ the pion-loop contributes less than $25 \%$ of the charge radius.
There is a widely held misconception that the electromagnetic structure of the pion is dominated by the pion's own pion-cloud. To clarify this the Global Color-symmetry Model (GCM) was used to calculate the electromagnetic charge radius of the pion. In this calculation the contributions from the quark core and pion loop were identified and compared. It was shown explicitly that the divergence of the charge radius in the chiral limit is due solely to the pion loop and that, at the physical value of the pion mass, this loop contributes less than $15 \%$ to $\left\langle\mathrm{r}_{\pi}^{2}\right\rangle$ as illustrated in Fig. IV-4; i.e. the quark core is the dominant determining characteristic for the pion. This suggests that quark-based models that fail to reproduce the $\mathrm{m}_{\pi}$ divergence of $\left\langle\mathrm{r}_{\pi}^{2}\right\rangle$ may nevertheless incorporate the dominant characteristic of the pion: its quark core. The results of this study lend further support to the contention that, away from resonances, the dominant determining characteristic of kinematic and dynamical properties of hadrons is their quark core. A paper describing this work was published.

\footnotetext{
*University of Tübingen, Germany
} 


\section{m. Analytic Structure of the Quark Propagator in a Model With an Infrared- Vanishing Gluon Propagator (A. Bender and R. Alkofer*)}

A model Dyson-Schwinger equation for the quark selfenergy in rainbow approximation was studied using an Ansatz for the gluon propagator that vanishes for zero four-momentum and that introduces an infrared mass scale $b$. The aim was to determine whether such a form of the gluon propagator, which is suggested by a number of studies including recent lattice simulations of QCD, can support dynamical chiral symmetry breaking and ensure quark confinement. The form of the gluon propagator at small space-like momenta is crucial to the nature of hadron phenomena but is

*University of Tübingen, Germany

\section{n. Electromagnetic Pion Form Factor}

The electromagnetic pion form factor, $F_{\pi}\left(q^{2}\right)$, is calculated in impulse approximation for space-like $q^{2}$ on the domain $[0,20] \mathrm{GeV}^{2}$ using a confining quark propagator and a dressed quark-photon vertex obtained from realistic, non-perturbative Dyson-Schwinger equation studies. Good agreement with the available data is obtained for $F_{\pi}\left(q^{2}\right)$ and other pion observables, including the decay $\pi^{0} \rightarrow \gamma \gamma$. It is found that, at presently accessible momentum-transfer, nonperturbative, bound-state effects dominate the form presently unknown. Information gathered in studies such as this is invaluable in verifying or invalidating given hypotheses. It was found that there is a bdependent critical coupling, $\mathrm{g}_{\mathrm{c}}^{2}(\mathrm{~b})$, with chiral symmetry dynamically broken for $\mathrm{g}^{2}(\mathrm{~b})>\mathrm{g}_{\mathrm{c}}^{2}(\mathrm{~b})$. For large $b, g_{c}^{2}(b) \rightarrow \infty$ and chiral symmetry is not broken. For small b, the critical coupling and scale of dynamical chiral symmetry breaking are independent of $b$. In addition, it was shown that at time-like momenta the quark propagator possesses a pole and hence quarks are not confined by this model gluon propagator. These features indicate that this model gluon propagator is unable to yield the observed spectrum of QCD and hence is phenomenologically untenable. A paper describing this work was published.

\section{(C. D. Roberts)}

factor. This application is an illustration of the efficacy of the phenomenological Dyson-Schwinger equation approach, whereby a large range of observables can be correlated via a few parameters that characterize the long-range piece of the quark-quark interaction, and which it may be possible to relate to the underlying parameters in QCD. This work was described at international conferences and summary papers appeared in the proceedings.

\section{o. The Off-Shell Axial Anomaly via the $\gamma * \pi^{0} \rightarrow \gamma$ Transition (C. D. Roberts,} M. R. Frank, * K. L. Mitchell, $\dagger$ and P. C. Tandy $\ddagger$ )

The $\gamma * \pi^{0} \rightarrow \gamma$ form factor, $\mathrm{F}^{0} \gamma^{0}(\mathrm{~s})$, including the extension off the pion mass-shell, is calculated in impulse approximation within the Dyson-Schwinger Equation framework used to provide an excellent description of the pion charge form factor, described above. This anomalous process is a fundamentally important characteristic of the quantum field theoretical structure of QCD because it signals the breaking of the $\mathrm{U}_{\mathrm{A}}(1)$ symmetry by quantization. This form factor was measured by the CELLO collaboration at the PETRA storage ring using the process $\mathrm{e}^{+} \mathrm{e}^{-} \rightarrow \mathrm{e}^{+} \mathrm{e}^{-} \pi^{0}$. There is a letter-of-intent at CEBAF to re-measure this form factor in virtual Compton scattering from a proton target. In this case a (virtual) pion is supplied by the

*University of Washington, †TRIUMF, Canada, $\ddagger$ Kent State University target and a final real photon selected through the excellent missing mass spectrometry available at CEBAF. An extrapolation to the pion mass shell will be needed to deduce the physical transition form factor. Our calculation shows that the dependence on the virtual-pion momentum is smooth and well described by a simple suppression factor, which is qualitatively independent of the details of the pion interpolating field. The correct mass-shell value of this form factor is naturally generated in our approach and the $q^{2}$. dependence is in accord with the available CELLO data. No parameters are adjusted to achieve this; they are fixed at the values derived in the study of $F_{\pi}\left(q^{2}\right)$. A significant result of our study is that for this anomalous process, soft nonperturbative effects remain significant in the domain of experimentally accessible momentumtransfer. A paper describing this work was published. 


\section{p. Model Gluon Propagator and Pion and $\rho$-Meson Observables (C. D. Roberts and M. R. Frank*)}

The Dyson-Schwinger equation for the quark propagator and Bethe-Salpeter equations for the $\pi$ and $\rho$-mesons are solved in the rainbow/ladder approximation using a oneparameter model confined-gluon propagator. The results are used to calculate a range of $\pi$ and $p$-meson properties: masses, decay widths and scattering lengths, and good agreement with the data is obtained. The quark propagator obtained as a solution has no pole on the real-time-like axis and hence may be interpreted as representing a confined particle. It agrees well in form

*University of Washington and magnitude with the parametrization employed in studies of the pion and kaon form factors. In this calculation a large range of observables is correlated via a single parameter, which marks the transition between perturbative and nonperturbative domains. This emphasizes the phenomen-ological efficacy of this approach. A mass formula for the pion, involving only the vacuum, dressed-quark propagator, is presented and shown to provide an accurate estimate of the mass obtained via a direct solution of the Bethe-Salpeter equation. An article describing this work was published.

\section{q. Study of the Anomalous Process $\gamma \pi \rightarrow \pi \pi \quad$ (C. D. Roberts and R. Alkofer*)}

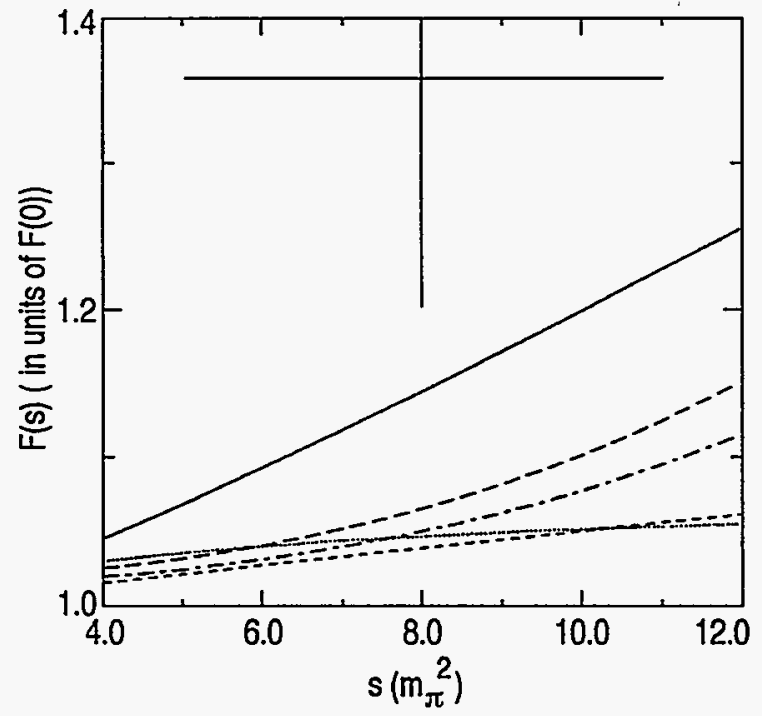

Fig. IV-5. The $\gamma \pi^{*} \pi \pi$ amplitude $F^{3 \pi}(s, t, u)$, normalized to $I$ at $s=t=u=0$, as function of the Mandelstam variable s for $u=\left(-1.5-4.28 x+0.12 x^{2}\right)$, $x=(s /[4 m 2 \pi]-1)$ and $t=-m 2 \pi$. Our result: solid line; our result with $m_{x} \rightarrow m_{\pi} / 2:$ short-dash line; vector meson dominance: long-dash and dash-dot lines; chiral expansion plus vector meson saturation Ansatz: dotted line. The single data point was obtained at Serpukhov via the Primakov reaction $\pi^{-} A \rightarrow \pi^{\prime} \pi^{0} A^{\prime}$.
The anomalous form factor for the $\gamma \pi^{*} \rightarrow \pi \pi$ transition, denoted by $\mathrm{F}^{3 \pi}(\mathrm{s}, \mathrm{t}, \mathrm{u})$, was measured at Serpukhov in the Primakov reaction $\pi^{-} \mathrm{A} \rightarrow \pi^{-1} \pi^{0} \mathrm{~A}^{\prime}$. In this experiment the considerable uncertainty in both the kinematic range and result make it difficult to draw a conclusion regarding the accuracy of the theoretical prediction for the chiral limit value of $F^{3 \pi}(0,0,0)$. New experiments are planned at CEBAF: $\gamma \pi \rightarrow \pi^{+} \pi^{0} n$, in the range $s / \mathrm{m}_{\pi}^{2}=4-15$ and at Fermilab via the Primakov reaction using a $600-\mathrm{GeV}$ pion beam, in the range $s / m_{\pi}^{2}=4-6$. Such processes are of particular interest because they are intimately connected to the anomaly structure of QCD, which is tied to the quantum field-theoretic character of the theory.

We calculated this transition form factor as a phenomenological application of the QCD DysonSchwinger equations. The chiral-limit value dictated by the electro-magnetic, anomalous chiral Ward identity, is reproduced, independent of the details of the modeling of the gluon and quark 2-point Schwinger functions. Using a paramet-erization of the dressed u-d quark 2point Schwinger function that provides a good description of pion observables, $\mathrm{F}^{3 \pi}(\mathrm{s}, \mathrm{t}, \mathrm{u})$ is calculated on a kinematic range that proposed experiments plan to explore. Our result confirms the general trend of othercalculations; i.e., a monotonic increase with sat fixed $t$ and $u$, but is uniformly larger and exhibits a more rapid rise with $s$ as illustratred in Fig. IV-5. An article describing this work has been accepted for publication.

*University of Tûbingen, Germany 


\section{r. Electromagnetic Charged and Neutral Kaon Form Factors (C. D. Roberts, C. J. Burden,* and M. J. Thomson $\dagger$ )}

The electromagnetic form factors of the charged and neutral kaon are calculated using the approach applied in the successful study of the pion form factor, described above. The charged-kaon form factor will be measured in forthcoming experiments at CEBAF. Our calculation involves the dressed strange-quark propagator, to which $\mathrm{F}_{\pi}\left(\mathrm{q}^{2}\right)$ is not sensitive, and hence it provides us with constraints on the strange-quark sector of QCD. This calculation is the first to yield a value of $\mathrm{f}_{\mathrm{K}} / \mathrm{f}_{\pi}$ that is in good agreement with experiment and also yields $\mathrm{rK}_{\mathrm{K}}+\mathrm{r}_{\pi}$ in good agreement with experiment. The results are sensitive to the difference between the kaon and pion Bethe-Salpeter amplitude and the u- and s-quark propagation characteristics, demonstrating that such exclusive processes are a probe of nonperturbative, quark-gluon bound-state dynamics. We find that on the range of $Q^{2}$ currently accessible to experiment $F_{K} \pm\left(Q^{2}\right)>F_{\pi}\left(Q^{2}\right)$. Further, $\mathrm{Q}^{2} \mathrm{~F}_{\mathrm{K}}\left(\mathrm{Q}^{2}\right)$ has a peak, at approximately the same position as $Q^{2} F_{\pi}\left(Q^{2}\right)$; this is a signal of quarkantiquark recombination tnto the final-state meson in exclusive elastic scattering processes. We find for the neutral Kaon that $\mathrm{r}_{\mathrm{K}^{0}}^{2}<0$.

*Australian National University, Canberra, Australia $\dagger$ University of Melbourne, Australia
These features are illustrated in Fig. IV-6. A paper describing this work was accepted for publication.

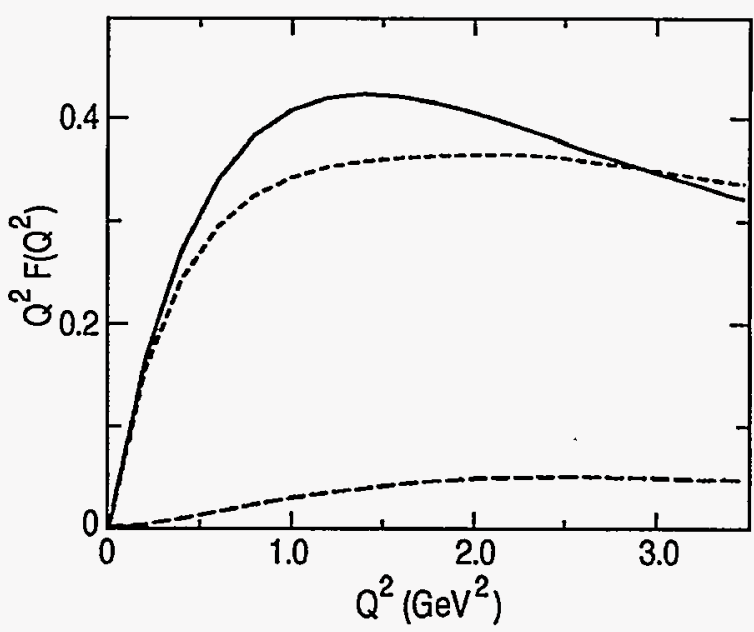

Fig. IV-6. Calculated form factors: $Q^{2} F_{K} \pm$ solid line; $Q^{2} F_{\pi \pm}$ - short-dashed line; $Q^{2} F_{K} 0$ long dashed line. The difference between $F_{K} \pm$ and $F_{\pi \pm \text { for }} Q^{2}>3 \mathrm{Gev}^{2} 2$ is small but is amplified in this figure because of the multioplication by $Q^{2}$.

\section{s . Bethe-Salpeter Equation Studies of $\mathrm{SU}_{\mathrm{f}}(3)$ Mesons (C. D. Roberts, C. J. Burden, * Lu Qian, $\dagger$ P. C. Tandy, $\dagger$ and M. J. Thomson $\doteqdot$ )}

The spectroscopy and weak decays of mesons composed of $u, d$ and $s$ quarks is studied using a separable ansatz for the quark-quark scattering kernel, which is obtained by inverting the Dyson-Schwinger equation for the quark propagator. The quark propagator used is the phenomen-ologically-efficacious, confining model form derived in the study of the pion and kaon form factors, described above. One obtains an excellent description of the spectrum and decays, which indicates that pion and kaon observables can be used to constrain and quark-quark interaction in QCD. The simplicity of the approach allows one to study the influence of subleading Dirac-components in the meson Bethe-Salpeter amplitude; for example, the effect of pseudoscalarpseudovector mixing, with the interesting result that, in this framework at least, these may be significant in the pion channel. This work was described at international conferences and summaries of these presentations will appear in the proceedings. A detailed article is being prepared for submission.

*Australian National University, Canberra, Australia $\dagger$ Kent State University, $\ddagger$ University of Melbourne, Australia 


\section{t. Electromagnetic Nucleon Form Factors (A. Bender, C. D. Roberts, and M. R. Frank*)}

The Dyson-Schwinger equation framework is employed to obtain expressions for the electromagnetic nucleon form factor. In impulse approximation the form factor depends on the dressed quark propagator, the dressed quark-photon vertex, which is crucial to ensuring current conservation, and the nucleon Faddeev amplitude. The approach manifestly incorporates the large spacelike $\mathrm{q}^{2}$ renormalization group properties of QCD and allows a realistic extrapolation to small spacelike $\mathrm{q}^{2}$. This extrapolation allows one to relate experimental data to the form of the quark-quark

*University of Washington interaction at small spacelike $q^{2}$, which is presently unknown. The approach provides a means of unifying, within a single framework, the treatment of the perturbative and nonperturbative regimes of $\mathrm{QCD}$. The wealth of experimental nucleon form factor data, over a large range of $q^{2}$, entails that this application will provide an excellent environment to test, improve and extend our approach, which was very successful in its application to meson observables. This is particularly true since the nucleon is a system in which explicit three-gluon vertices can play a qualitatively new role, attaching to each of the three dressed-quarks in the bound state, a process which one cannot model as a two-body force.

\section{u. Quark and Diquark Confinement Beyond Rainbow/Ladder Approximation} (A. Bender, C. D. Roberts, and L. von Smekal)

Confinement of color is an important feature of the strong interaction spectrum at accessible energy scales; this means not only that isolated quarks and gluons have not been observed but also that the only bound states that have been observed are color neutral. In quantum field theory two-body bound states are studied via the inhomogeneous Bethe-Salpeter equation for the T-matrix. A pole in $\mathrm{T}$, at a given total momentum, corresponds to a bound state and enables one to extract the bound-state Bethe-Salpeter amplitude. This approach allows one to study quark-antiquark (meson) bound states and also quark-quark (diquark) systems, which can occur in two color-charge states denoted by $\overline{3}$ and 6 . The simplest approximation to the coupled Dyson-Schwinger/Bethe-Salpeter equation system, which is used to study bound states of strongly-dressed quarks and anti-quarks, is the rainbow/ladder approximation and may be described mnemonically as $O\left(g^{2}\right)$. This provides the basis for an excellent description of meson spectroscopy and dynamics but admits $\overline{3}$ diquark bound states of mass $\geq 700 \mathrm{MeV}$, which are not observed. In the first study of its type, we employed a simple model gluon propagator in studies of this coupled system of equations at the next level of approximation, which may be denoted as $\mathrm{O}\left(\mathrm{g}^{4}\right)$.
The $\mathrm{O}\left(\mathrm{g}^{2}\right)$ studies have the feature that one has a manifest realization of Goldstone's theorem; i.e., the pseudoscalar bound state is massless if chiral symmetry is dynamically broken. We found that the $\mathrm{O}\left(\mathrm{g}^{4}\right)$ approximation preserves this property. We also found that, in meson systems, these higher-order corrections shift the meson mass by less than $10 \%$, which is an indication that the $\mathrm{O}\left(\mathrm{g}^{2}\right)$ (rainbow/ladder) approximation in this channel is qualitatively and quantitatively reliable. However, the $\mathrm{O}\left(\mathrm{g}^{4}\right)$ corrections have a dramatic effect in the diquark channel: they ensure that there is no pole at real time-like $\mathrm{P}^{2}$ and hence that there are no stable diquark excitations, an observational fact that previous studies were not able to reproduce. In this simple model the quark and gluon excitations are confined; i.e., their propagators are such that there is no asymptotic state associated with them, and this calculation provides an illustration of how an interaction with this feature can also entail the absence of colored bound states from the particle spectrum. In this study one obtains confinement without explicit three-gluon vertices. Their contribution is nevertheless crucial since they are responsible for the enhancement of the gluon (and hence quark) propagators in the infrared. 


\section{v. Dynamical Chiral-Symmetry Breaking and Confinement at Finite Temperature (A. Bender, C. D. Roberts, D. Blaschke,* and Yu. Kalinovsky*)}

The Dyson-Schwinger equation for the quark propagator is being studied at finite temperature using the oneparameter, model gluon propagator used successfully in the studies of $\pi$ and $\rho$-meson observables described above. This study can provide insight into the existence and nature of chiral-symmetry restoration and deconfinement in QCD, which is a significant part of the physics program at RHIC. With the one parameter in the gluon propagator fixed in calculations of observables at $\mathrm{T}=0$, the study will allow a parameterfree determination of the chiral-symmetry restoration temperature, $\mathrm{T}_{c}^{\chi}$, and the deconfinement temperature, $\mathrm{T}_{\mathrm{c}}^{\mathrm{D}}$, which may differ. This is the first

*University of Rostock, Germany

\section{w . Nucleon Faddeev Equation Studies}

The bound-state studies described above, although concentrated on two-body systems, provide experience and insights that are useful in studying the baryon as a three-body bound state of strongly-dressed quarks; $u, d$ and $\mathrm{s}$. The nucleon form factor studies described above provide useful information about the importance of diquark correlations in the baryon. As emphasized by the two-body Bethe-Salpeter equation studies, there are no diquark bound states and diquarks are merely loose correlations that can be used as an artifice in the solution of the three-body Faddeev equation. We are studying the nucleon Faddeev equation, which will study to take into account the momentum dependence of the two-quark wave-function renormalization at finite temperature, which can play a significant role, especially in ensuring or eliminating confinement. This is the first phase of the study. The second phase will be a study of how observables, such as the $\pi$ - and $\rho$-meson masses and $\pi-\pi$ scattering lengths, vary with temperature. This study is significant because it is one of the first to study the response of the nonperturbative, internal quark-gluon structure of meson bound states to changes in temperature, and can provide a means of relating the response in observables to the underlying parameters in QCD.

\section{x . QCD Greens Functions in the Infrared (L. von Smekal, R. Alkofer,* and A. Hauck*)}

The behavior of QCD Schwinger functions in the infrared has important consequences for the spectroscopy and interactions of hadronic bound states. This region is inaccessible in perturbation theory. Of fundamental importance is the behavior of the twopoint gluon Schwinger function, which is a measure of the quark-quark interaction and is believed to be responsible for confinement and dynamical chiral symmetry breaking. There have been a number of studies of the infrared behavior of the gluon two-point function, some using lattice techniques and others using the Dyson-Schwinger equations. At present there is no consensus: some studies suggest a suppression in the infrared and others a strong enhancement with respect to perturbative behavior. Most studies of the DysonSchwinger equation for this two-point function have

*University Tübingen, Germany neglected the contribution made by ghost fields, which arise because of gauge-fixing in QCD and are necessary to preserve unitarity of the S-matrix. We are studying the coupled system of gluon and ghost DysonSchwinger equations in such a manner as to preserve the Slavnov-Taylor identities, which are entailed by gauge invariance and are the QCD analogues of the Ward-Takahashi identities in QED. This study will allow us to explore the importance of ghost fields in connection with confinement and dynamical chiral symmetry breaking. It will provide information relevant to the dispute over the infrared behavior of the gluon two-point function, indicating whether ghost fields can qualitatively or quantitatively modify this behavior. As a byproduct we will be able to assess, for the first time, the claims made by other authors that ghost fields only provide small quantitative contributions to hadronic observables. 


\section{y $\quad$ Relativistic Approaches to Hadrons and Nuclei at Medium Energies (F. Coester, V. A. Karmanov,* F. M. Lev, $\dagger$ R. Schiavilla, $\ddagger$ A. Stadler, $\S$ and J. A. TjongD}

A Round Table Discussion was held during the International Conference on Intermediate Energy Nuclear Physics, ICTP, Trieste, Italy, May 8-12, 1995. The description of hadrons and nuclei at medium

*Lebedev Physical Institute, Moscow, Russia, $\dagger$ †oint Inst. for Nuclear Research, Dubna, Russia, ¥Old Dominion University and CEBAF, §College of William and Mary and CEBAF, IRijksuniversiteit, Utrecht, The Netherlands energies requires nonperturbative relativistic dynamics.. The purpose of these discussions was to illuminate a variety of different approaches and their interrelations. The discussions covered: a) Quantum Field Theory and Covariant Wave Functions;b) Fock-space Hamiltonian Dynamics; c) Relativistic Hamiltonian Few-Body dynamics; d) Nonrelativistic Quantum Mechanics with corrections of order $(v / c)^{2}$. These discussions will appear in the proceedings of the conference.

\section{z. Electroweak Transitions in Quark Model Baryons (F. Coester and D. O. Riska*)}

The recently developed oscillator model of Riska and Glozman has natural applications to a fully relativistic

*University of Helsinki, Finland treatment of electroweak transitions. We are exploring the theoretical problems and the quantitative consequences of such a treatment.

\section{aa. Current Operators in Relativistic Few-Body Systems (F. Coester, W. H. Klink, * and W. N. Polyzou*)}

An important area of medium-energy hadronic physics is the study of the structure of few-body compounds with electro-weak probes. The observables are mass spectra and cross sections related to matrix elements of current density operators. The relevant group is the Poincaré group and its subgroups. Single-particle states are defined by irreducible representations. The Hilbert space of states is a tensor product of singleparticle Hilbert spaces or a finite direct sum of such spaces. The structure of the state space is independent of the dynamics. Maximal subgroups are called kinematic system is specified by generators not in the kinematic Lie algebra. subgroups. The dynamics of an interacting. The choice of the kinematic subgroup determines the "form of dynamics". Explicit constructions start with a mass and spin operator determining a representation of the Little Group, and

*University of Iowa proceed to an induced representation of the full group. Current densities $\mathrm{I} \mu(\mathrm{x})$ are covariant, operator-valued tempered-distributions with Fourier transforms $\tilde{I}^{\mu}(Q)$. They are well defined for single particles. In an interacting system single-particle currents are covariant only under the kinematic subgroup. We investigated the problem of determining dynamically required interaction currents in the context of null-plane dynamics where the kinematic subgroup is the stability group of a null plane. The key to this construction is found in the spinor representations of the subgroup $\mathrm{O}(1,2)$ that leaves the spacelike four-momentum transfer invariant. This representation of the current kernel is related to the light-front spin representation by representations of light-front boosts in which the role of the dynamics can be isolated. An invited talk on this subject will be given at a meeting of the American Mathematical Society in March 1996. A paper is being prepared for publication. 


\section{bb. Theory of Hadronic Nonperturbative Models (F. Coester and W. N. Polyzou*)}

As more data probing hadron structure becomes available, hadron models based on nonperturbative relativistic dynamics will be increasingly important for their interpretation. Relativistic Hamiltonian dynamics of few-body systems (constituent-quark models) and many-body systems (parton models) provides a precisely defined approach and a useful phenomenology. However such models lack a quantitative foundation in quantum field theory. The specification of a quantum field theory by a Euclidean action provides a basis for the construction of non-perturbative models designed to maintain essential features of the field theory. For

*University of Iowa finite systems it is possible to satisfy axioms which guarantee the existence of a Hilbert space with a unitary representation of the Poincare group and the spectral condition which ensures that the spectrum of the fourmomentum operator is in the forward light cone. The separate axiom which guarantees locality of the field operators can be weakened for the construction for fewbody models. In this context we are investigating algebraic and analytic properties of model Schwinger functions. This approach promises insight into the relations between hadronic models based on relativistic Hamiltonian dynamics on one hand and Bethe-Salpeter Green-function equations on the other.

\section{cc. Osterwalder-Schrader Positivity of Euclidean Quark Propagators (F. Coester and W. N. Polyzou*)}

OS (Osterwalder-Schrader) positivity is the key property of Schwinger functions that permits the construction of a Hilbert space with a unitary representation of the Poincaré group. Successful nonperturbative hadron

*University of Iowa models were based on solutions of truncated DysonSchwinger equations which realize conditions for confinement and asymptotic freedom. We are exploring sufficient conditions for OS positivity of the quark propagators of such models.

\section{B. NUCLEAR FORCES AND NUCLEAR SYSTEMS}

The goal of this program is to achieve a description of nuclear systems ranging in size from the deuteron to nuclear matter and neutron stars using a single parametrization of the nuclear forces. Aspects of our program include both the construction of two- and three-nucleon potentials and the development of many-body techniques for computing nuclear properties with these interactions. Detailed quantitative, computationally-intensive studies are essential parts of this program.

A new nucleon-nucleon potential, Argonne $v_{18}$, was completed last year. It includes a dominant charge-independent piece plus additional charge-dependent and charge-asymmetric terms. It fits $4301 \mathrm{pp}$ and $\mathrm{np}$ elastic scattering data with a $\chi^{2}$ of 1.09 /degree of freedom, as well as low-energy $\mathrm{nn}$ scattering parameters and deuteron properties. We are also studying three-nucleon potentials to be used in conjunction with the $v_{18}$ model in microscopic many-body calculations. In addition, Argonne $v_{18}$ is being used as input to folded-diagram calculations of effective interactions for use in the nuclear shell model.

The many-body calculations at Argonne are based primarily on the variational method with correlated-operator trial functions. Parameters in the trial functions are varied to minimize the energy, and these optimized variational wave functions are then used to study other nuclear properties. The calculations can be separated into three groups, according to the size of the system: (1) direct Monte Carlo integration for few-body $(A \leq 8)$ nuclei, $(2)$ a cluster expansion with Monte Carlo integrations for larger $(8 \leq \mathrm{A} \leq 40)$ nuclei, and (3) a diagrammatic cluster expansion with integral-equation summation methods for nuclear and neutron matter. Although the methods vary, the calculations are all linked by the same Hamiltonian and very similar trial functions. 
Because minimization of the ground-state energy is the key to determining the wave function, much work has been devoted to evaluating binding energies and searching for accurate variational wave functions. Energies in ${ }^{3} \mathrm{H}$ and ${ }^{4} \mathrm{He}$ that are $\sim 2 \%$ above the values from available exact methods have now been achieved. A very significant development the last two years was the conversion of our few-body and light-nuclei programs to run on Argonne's new 128-processor IBM SP at speeds up to 7.5 GFLOPS. This made possible extensive calculations of the $A=6$ and 7 nuclei, five-body cluster contributions to $16 \mathrm{O}$, and the first extensive studies of ${ }^{40} \mathrm{Ca}$.

The few-body trial functions, were also used as input to six- and seven-body Green's function Monte Carlo (GFMC) calculations in collaboration with researchers at Urbana and Los Alamos. These are the first exact calculations of such nuclei with realistic interactions. In addition to ground-state calculations, we made extensive studies of the excitation spectrum of ${ }^{6} \mathrm{He}$, ${ }^{6} \mathrm{Li}$, and ${ }_{\mathrm{Li}}$, using both variational and GFMC methods. A study of elastic and transition form factors in ${ }^{6} \mathrm{Li}$ was begun, including two-body charge and current operators constructed to be consistent with the Hamiltonian.

The charge-independence-breaking terms of the new Argonne $v_{18}$ interaction are being used to study the energy differences of mirror nuclei, i.e., the Nolen-Schiffer anomaly. We are computing the properties of neutron drops with the goal of providing additional constraints for the construction of Skyrme interactions for modeling neutron-rich nuclei in the crusts of neutron stars. We also continue to work on improving our nuclear and neutron matter calculations and to study the effects of our new interactions on the properties of dense matter.

Studies of hypernuclei are continuing on several fronts. We are examining $\Lambda$ single-particle energies and the effect of $\Lambda$-induced distortion of nuclear cores in light hypernuclei such as ${ }_{\Lambda} \mathrm{He}$ and ${ }^{17} \mathrm{O}$. We also continue to use our studies of hypernuclei to deduce features of hyperonnucleon interactions, such as space-exchange forces, charge-symmetry breaking, and $\Lambda N-\Sigma N$ coupling.

\section{a. Three-Body Correlations in Few-Body Nuclei (R. B. Wiringa, A. Arriaga,* and V.R. Pandharipande $\dagger$ )}

A detailed comparison of Faddeev and variational wave functions for ${ }^{3} \mathrm{H}$, calculated with realistic nuclear forces, was made to study the form of three-body correlations in few-body nuclei. Three new three-body correlations for use in variational wave functions were identified, which substantially reduce the difference with the Faddeev wave function (supplied by the Los Alamos-Iowa

*University of Lisbon, Portugal

tUniversity of Illinois at Urbana-Champaign group). The difference between the variational upper bound and the Faddeev binding energy is reduced by half, to typically $<2 \%$. These three-body correlations also produce a significant lowering of the variational binding energy for ${ }^{4} \mathrm{He}$ and larger nuclei. This work was recently published in Phys. Rev. C $\underline{52}, 2362$ (1995). We hope to use similar comparisons with GFMC calculations in six-body nuclei (Sec. B.b) to find improvements for the variational ansatz in p-shell nuclei.

\section{b. Variational and Green's Function Monte Carlo Calculations of Light p-Shell Nuclei (R. B. Wiringa, B. S. Pudliner, * V. R. Pandharipande, ${ }^{*}$ and J. Carlson $\dagger$ )}

An extensive series of variational Monte Carlo (VMC) and Green's Function Monte Carlo (GFMC) calculations for few-body nuclei using a Hamiltonian, $\mathrm{H}$, containing the new Argonne $\mathrm{v}_{18} \mathrm{NN}$ interaction

\footnotetext{
* University of Illinois at Urbana-Champaign $\nmid$ Los Alamos National Laboratory
}

supplemented by the Urbana model IX three-nucleon (3N) potential were performed. These calculations include the ground-state binding energy and low-lying excited states for nuclei up to $A=7$. The variational wave functions, $\Psi_{V}(\mathbf{R})$, include central, spin, isospin, tensor, and spin-orbit two- and three-body correlations. calculations in ${ }^{3} \mathrm{H}$ and ${ }^{4} \mathrm{He}$. 


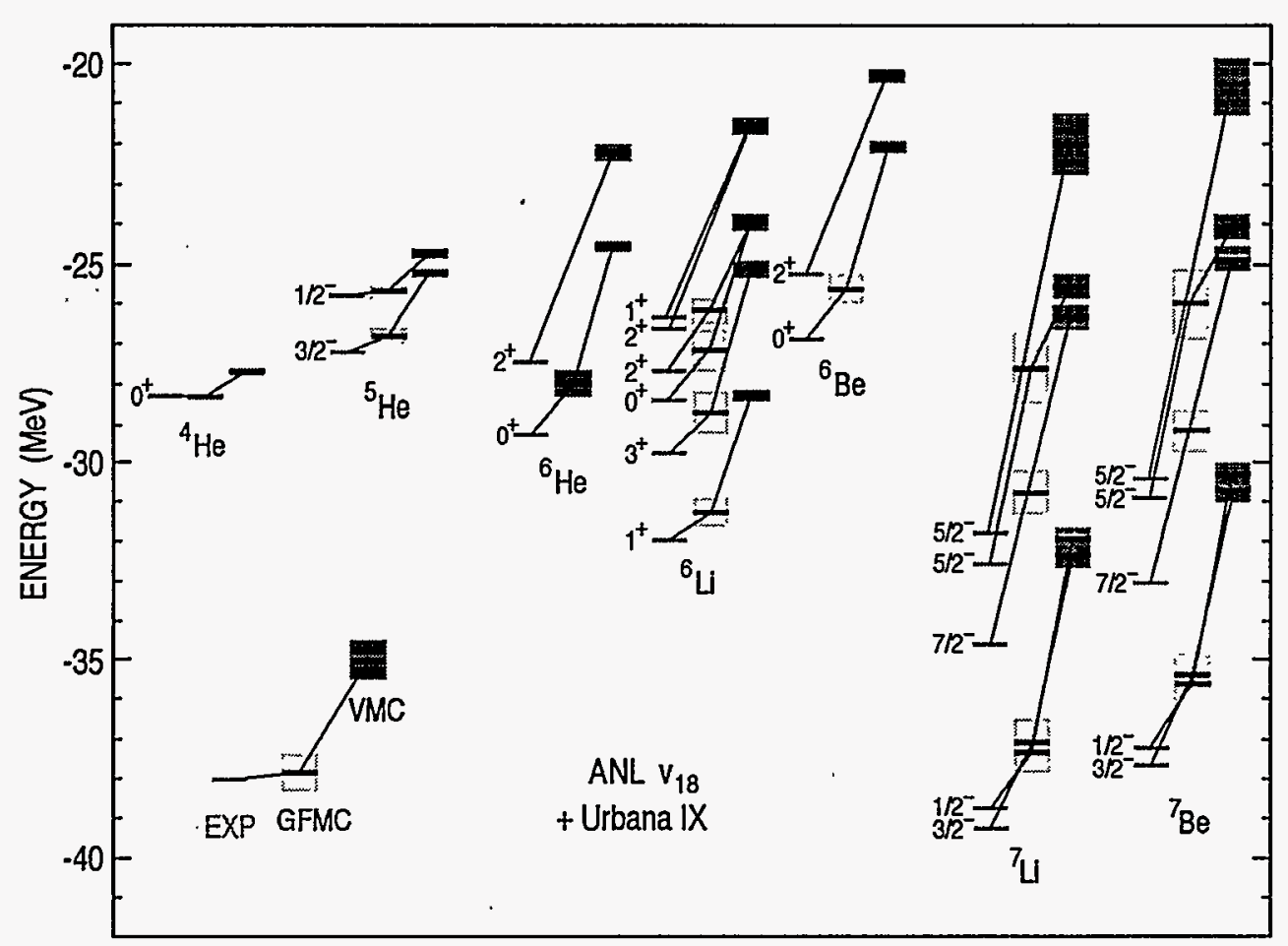

Fig. IV-7 VMC and GFMC spectral calculations compared to experimnent. Lines connect the same states (some states have not been computed with GFMC yet). Shaded regions denote Monte Carlo error bars.

These trial functions give upper bounds to the groundstate binding energy $\sim 2 \%$ above exact GFMC calculations in ${ }^{3} \mathrm{He}$ and ${ }^{4} \mathrm{He}$.

The $\Psi_{\mathrm{V}}(\mathbf{R})$ serve as input to the GFMC calculations, which are made for a somewhat simplified version of the Hamiltonian, $\mathrm{H}^{\prime}$, which omits potential terms quadratic in $\mathrm{L}$ and charge-independence-breaking (CIB) terms. The GFMC algorithm produces values of $\Psi(\tau, \mathbf{R})=\exp \left(-\mathrm{H}^{\prime} \tau\right) \Psi_{\mathrm{V}}(\mathbf{R})$ at configurations $\mathbf{R}(\tau)$ distributed with probability $\left|\Psi_{\mathrm{V}} \dagger(\mathbf{R}) \Psi(\tau, \mathbf{R})\right|$. The $\exp \left(-\mathrm{H}^{\prime} \tau\right)$ is considered as a product of many small imaginary time steps $\exp \left(-\mathrm{H}^{\prime} \Delta \tau\right)$. The present calculations have typically propagated 10,000 configurations to $\tau=0.06 \mathrm{MeV}^{-1}$. The transient estimate for the energy, $E(\tau)$, is well converged for $A=3,4$, but needs some extrapolation for $A=6$ and 7 nuclei. The correction $\mathrm{H}^{-\mathrm{H}^{\prime}}$ is computed by perturbation and is relatively small.

For the full $\mathrm{H}$, in which the $3 \mathrm{~N}$ potential was adjusted to give the correct $A=3,4$ ground state energies, we find a GFMC upper bound for the ground state of ${ }^{6} \mathrm{Li}$ of $-31.3 \pm 0.4 \mathrm{MeV}$, which is stable against $\alpha-\mathrm{d}$ breakup. We estimate the converged result to be $-31.8 \mathrm{MeV}$, in reasonable agreement with the experimental value of
$-32.0 \mathrm{MeV}$. We find the correct order for excited states in ${ }^{6} \mathrm{Li}:\left(\mathrm{J}^{\pi} ; \mathrm{T}\right)=\left(3^{+} ; 0\right),\left(0^{+} ; 1\right),\left(2^{+} ; 0\right)$, and $\left(2^{+} ; 1\right)$, and $\left(1^{+} ; 0\right)$.but the excitation energies are a little too large on average. A first letter on the results through $A=6$ was published in Phys. Rev. Lett. 74, 4396 (1995).

Our first results for ${ }^{7} \mathrm{Li}$ place the $\left(3 / 2^{-}\right)$ground state binding energy at $-37.1 \pm 0.6 \mathrm{MeV}$, compared to the experimental value of $-39.2 \mathrm{MeV}$. We are also calculating the lowest-lying $\left(1 / 2^{-}\right),\left(7 / 2^{-}\right)$, and $\left(5 / 2^{-}\right)$ states in the spectrum, as well as ${ }^{7} \mathrm{Be}$. The current status of our VMC and GFMC spectral calculations is shown in Fig. IV-7. In general the VMC calculation gives the correct ordering of states, but insufficient binding. The GFMC results are significantly closer to experiment. We are now beginning preparatory work for studies of ${ }^{8} \mathrm{He},{ }^{8} \mathrm{Li},{ }^{8} \mathrm{Be}$, and ${ }^{8} \mathrm{~B}$.

The VMC and $A=6$ GFMC calculations were made possible by the 128-processor IBM SP parallel supercomputer in Argonne's MCS Division, on which we achieved speeds of up to 6 GFLOPS. The $A=7$ GFMC calculations are being made on a larger IBM SP2 at the Cornell Theory Center, where a large block of time was awarded to our collaboration. 


\section{c. Ground States of Larger Nuclei (S. C. Pieper, R. B. Wiringa, and V. R. Pandharipande*)}

The methods (Sec. IV.B.b.) used for the few-body nuclei require operations on the complete spin-isospin vector; the size of this vector makes such methods impractical for nuclei with $A>8$. During the last few years we developed cluster expansion methods that do not require operations on the complete vector. We use the same Hamiltonians as for the few-body nuclei and variational wave functions of a form similar to the fewbody wave functions. The cluster expansions are made for the noncentral parts of the wave functions and for the operators whose expectation values are being evaluated. The central pair correlations in the wave functions are treated exactly and this requires the evaluation of 3A-dimensional integrals which are done with Monte Carlo techniques. Most of our effort was on ${ }^{16} \mathrm{O}$, other p-shell nuclei, and ${ }^{40} \mathrm{Ca}$.

In 1993 the Mathematics and Computer Science Division acquired a 128-processor IBM SP which has a theoretical peak speed of 16 Gigaflops (GFLOPS) and we converted our program to run on this machine.

* University of Illinois at Urbana-Champaign
The program has achieved 60 MFLOPS on a single processor or 7.5 GFLOPS on the whole machine, which we believe to be the best speed measured on the Argonne SP. All of our production runs are made on the SP.

The SP made possible complete five-body cluster calculations of 160 for the first time; previously we could only do four-body cluster calculations. In addition, the L-dependent correlation and potential terms are now being done in all orders of the cluster expansion; previously they were done only at the twobody level. At the two-body level, these L-dependent terms make a small $(\sim-0.5 \mathrm{MeV} / \mathrm{A})$ contribution and we had felt justified in not programming them in the higher orders. However, we find a large, repulsive, contribution from the three- and four-body clusters due to these terms. This results in ${ }^{16} \mathrm{O}$ being significantly underbound in our calculations. These results are now being prepared for publication and we are starting to investigate possible improvements to the variational wave functions.

d. Short-Range Structure of the Deuteron and Other Nuclei (S. C. Pieper, R. B. Wiringa, J. L. Forest, * V. R. Pandharipande,* R. Schiavilla, $\dagger$ and A. Arriaga $\left.{ }^{\ddagger}\right)$

The most important correlations among the nucleons in nuclei are induced by the tensor and repulsive-core parts of the two-nucleon interaction. These correlations are strongest in the isospin $\mathrm{T}=0$, spin $\mathrm{S}=1$ two nucleon states. We find that these correlations result in tensorpolarized deuterons with $M_{S}= \pm 1$ having a dumbbell shape oriented along the $\hat{\mathrm{z}}$ axis, while equidensity surfaces for $M_{S}=0$ are tori. This torus shape, resulting from conventional nucleon-nucleon potentials, does not seem to have been remarked on previously, although Skyrme models do predict such a shape. We can relate the sizes of these geometric shapes to the location of minima and maxima in various deuteron form factors. We also used our variational wave functions for nuclei from ${ }^{3} \mathrm{He}$ to ${ }^{16} \mathrm{O}$ to compute the density distributions of pairs of nucleons in various $\mathrm{T}, \mathrm{S}, \mathrm{M}_{\mathrm{T}}, \mathrm{M}_{\mathrm{S}}$ states.

\footnotetext{
*University of Illinois at Urbana-Champaign †CEBAF and Old Dominion University $\doteqdot$ University of Lisbon, Portugal
}

We find that the distributions for $T=0, S=1$ are very similar in shape to those of the deuteron. Furthermore, the ratio of the peak value of these densities to the peak value of the corresponding deuteron density provides an estimate for the number of "quasi-deuterons" in the nuclei. This estimate gives a good prediction of the observed photo- and pion-absorption cross sections. A similar situation is found for the other channel $(T=1$, $S=0$ ) that has nucleon pairs in $L=0$ partial waves. In this case, the ${ }^{1} S_{0}$ virtual bound-state wave function provides a good prediction of the $T=1, S=0$ pair distributions computed for the nuclei.

Also of interest are two-cluster distributions within nuclei, such as d-p in ${ }^{3} \mathrm{He}$, d-d in ${ }^{4} \mathrm{He}$, and $\alpha$-d in ${ }^{6} \mathrm{Li}$. These distributions all have $S$ - and $D$-wave components, which may be detectable in (e,e' $\vec{p}$ ) and (e,e'd) experiments. Their spatial structure can be directly related to the deuteron dumbbell and torus shapes. These results have been submitted for publication. 
e. Charge-Independence Breaking in Light Nuclei (R. B. Wiringa, S. C. Pieper, and B. S. Pudliner*)

We are using Green's function Monte Carlo (GFMC) and cluster variational Monte Carlo (CVMC) calculations to study energy differences in mirror nuclei with $A=3,7,15,17,39$, and 41 , and in the $A=6$ isotriplet. We use a realistic Hamiltonian containing the Argonne v18 two-nucleon potential and the Urbana IX three-nucleon potential. This Hamiltonian includes strong interaction charge-dependent (CD) and chargesymmetry-breaking (CSB) terms as well as a complete electromagnetic potential, and gives an excellent fit to $\mathrm{pp}, \mathrm{np}$, and $\mathrm{nn}$ data.

The exact GFMC calculations (Sec. IV.B.b) yleld energy differences of $0.757 \pm 0.001 \mathrm{MeV}$ for ${ }^{3} \mathrm{H}-{ }^{3} \mathrm{He}$, and $1.66 \pm 0.03 \mathrm{MeV}$ : for ${ }^{7} \mathrm{Li}-{ }^{7} \mathrm{Be}$, compared to experimental differences of 0.764 and $1.644 \mathrm{MeV}$, respectively. The CVMC calculations (Sec. IV.B.c) are made with wave functions adjusted to give the experimentally observed charge radii. The differences obtained are $3.56 \pm 0.01$ for ${ }^{15} \mathrm{~N}-15 \mathrm{O}$ and $3.48 \pm 0.01$ for ${ }^{17} \mathrm{O}-17 \mathrm{~F}$, compared to experimental values of

*University of Illinois at Urbana-Champaign
3.537 and $3.544 \mathrm{MeV}$, respectively. In each case, the Coulomb contribution dominates, but is insufficient to explain the full energy difference. The strong interaction CSB terms contribute $66,95,118$, and 90 $\mathrm{keV}$ in these four cases, while the additional electromagnetic interactions add $30,49,53$, and 35 $\mathrm{keV}$, respectively. We also compute the kinetic energy contribution due to the difference in proton and neutron masses.

The CVMC calculations of $\mathrm{A}=39$ and 41 nuclei are more problematic due to uncertainties in the variational wave function and the dominance of the Coulomb interaction. However the CSB contributions continue to be of order 120 to $140 \mathrm{keV}$ in these nuclei. GFMC calculations of the $\dot{A}=6$ isotriplet yield $0.55 \pm 0.05$ $\mathrm{MeV}$ for the isotensor difference $1 / 2\left({ }^{6} \mathrm{He}+{ }^{6} \mathrm{Be}\right)-{ }^{6} \mathrm{Li}$ compared to an experimental value of $0.334 \mathrm{MeV}$. This poses an interesting problem, since the strong interaction $\mathrm{CD}$ terms are more constrained by the twonucleon data than the CSB ones. We also looked at three-nucleon $\mathrm{CD}$ contributions and find them to be small.

\section{f. Microscopic Calculation of Elastic and Transition Form Factors of Lithium} (R. B. Wiringa, R. Schiavilla,* and B. S. Pudliner†)

Variational and Green's function Monte Carlo calculations are being made for the elastic and inelastic form factors of ${ }^{6} \mathrm{Li}$, The six-body wave functions are computed for a realistic Hamiltonian containing the Argonne $v_{18}$ two-nucleon potential and the Urbana IX three-nucleon potential. Consistent charge and current operators including one- and two-body terms are evaluated. Elastic results include the longitudinal and transverse form factors and tensor polarization. Transition form factors are calculated for the $\left(\mathrm{J}^{\pi} ; \mathrm{T}\right)=$ $\left(3^{+} ; 0\right),\left(0^{+} ; 1\right) ;\left(2^{+} ; 0\right)$, and $\left.2^{+} ; 1\right)$ states.

The first round of varioational calculations have been completed as shown in Fig. IV-8. We find that the elastic longitudinal form factor $F_{L}{ }^{2}(q)$ is generally well reproduced in impulse approximation (IA) and that

* CEBAF and Old Dominion University †University of Illinois at Urbana-Champaign meson-exchange current (MEC) contributions are small but do improve the agreement with data. The location of the first minimum and height of the second maximum are in good agreement with data. The elastic transverse form factor $\mathrm{FT}^{2}(\mathrm{q})$ is good to the first minimum, but the height of the second maximum is twice as large as experiment in IA, and the MEC increase the discrepancy. However the magnetic moment is reproduced within $5 \%$.

The longitudinal transition form factor to the $\left(3^{+} ; 0\right)$ excited state is again in good agreement with data at the IA level, and addition of the small MEC corrections improves the agreement. The calculated B(E2) is 16.8 compared to the quoted experimental value $21.8 \pm 4.8$ $\mathrm{e}^{2}-\mathrm{fm}^{4}$. The transverse transition form factor to the $\left(0^{+} ; 1\right)$ state is bracketed by the IA and MEC calculations. These calculations will be repeated with the GFMC wave functions in the near future. 

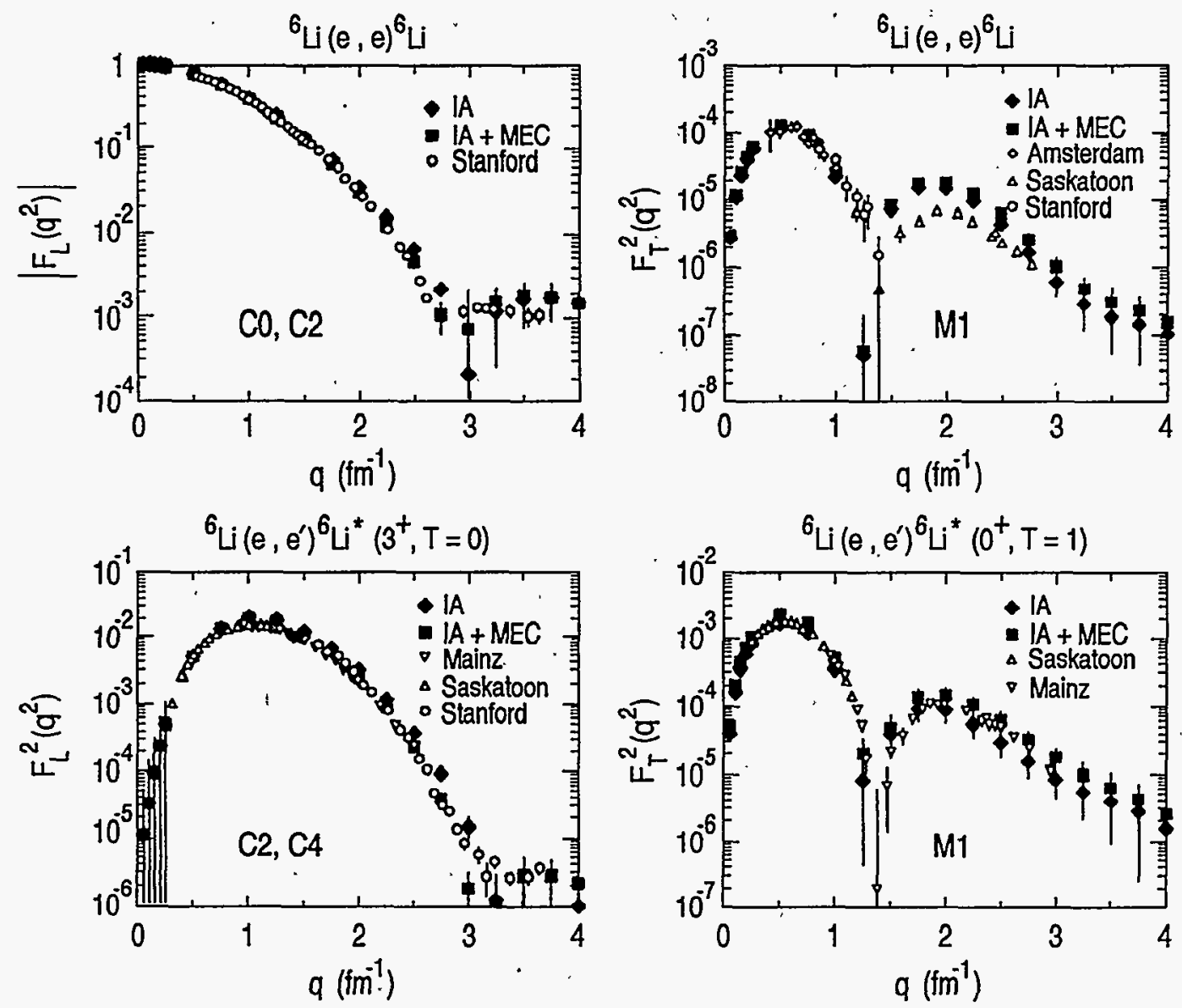

Fig. IV-8. Elastic and transition form factors in ${ }^{6} \mathrm{Li}$ calculated in impulse approximation (IA) and with meson-exchange currents added (IA+MEC) compared to various experimental data.

\section{g. Nuclear and Neutron Matter Studies V.R. Pandharipande*)}

We are studying nuclear and neutron matter with the new Argonne $v_{18}$ two-nucleon and Urbana threenucleon potentials. We use variational wave functions and a diagrammatic cluster expansion with Fermi hypernetted and single-operator chain (FHNC/SOC) integral equations to evaluate the energy expectation value. Initial results show some interesting differences with our previous calculations with the older Argonne $\mathrm{v}_{14}$ potential. In particular, there are a number of

*University of Mllinois at Urbana-Champaign diagrams involving $\mathbf{L} \cdot \mathbf{S}$ and $\mathrm{L}^{2}$ terms which were small with the older model and were rather crudely estimated or even neglected. It appéars that these terms are more important with the new potential and will have to be evaluated more accurately. Work on this subject is in progress. A simple line of attack is to just add additional diagrams at the three-body cluster level. A longer-term approach may be to adapt some of the methods for evaluating nucleon clusters used in the few-body and closed-shell nuclei described above (Secs. B.b, B.c).

\section{h. Elementary Diagrams in Nuclear and Neutron Matter (R. B. Wiringa)}

Variational calculations of nuclear and neutron matter are currently performed using a diagrammatic cluster expansion with the aid of nonlinear integral equations for evaluating expectation values. These are the Fermi hypernetted chain (FHNC) and single-operator chain
(SOC) equations, which are a way of doing partial diagram summations to infinite order. A more complete summation can be made by adding elementary diagrams to the procedure. The simplest elementary diagrams appear at the four-body cluster level; there is 
one such $\mathrm{E}_{4}$ diagram in Bose systems, but 35 diagrams in Fermi systems, which gives a level of approximation called FHNC/4. We developed a novel technique for evaluating these diagrams, by computing and storing 6 three-point functions, $S_{x y z}\left(r_{12}, r_{13}\right.$, r23), where $x y z=c c d, c c e$, ddd, dde, dee, or eee denotes the exchange character at the vertices 1,2 , and 3 . All 35 Fermi $E_{4}$ diagrams can be constructed from these 6 functions and other two-point functions that are already calculated.

The elementary diagrams are known to be important in some systems like liquid ${ }^{3} \mathrm{He}$. We expect them to be small in nuclear matter at normal density, but they might become significant at higher densities appropriate for neutron-star calculations. This year we programmed

\section{i. Spin-Orbit Splitting in Neutron Drops D. G. Ravenhall,* and A. Smerzi*)}

Hartree-Fock calculations of very neutron-rich nuclei are an essential source of input for calculations of the properties of neutron-star crusts. The Hartree-Fock calculations often use Skyrme models whose parameters are determined by fits to known (hence not neutron-rich) nuclei and extrapolations to the $\mathrm{N} \gg \mathrm{Z}$ case. The Vautherin and Brink (VB) prescription for the isospin dependence of the spin-orbit potential, $V_{\text {so }}$, is usually used; this is based on the assumption that most of $V_{\text {So }}$ comes from a short-range $L \cdot S$ nucleon-nucleon interaction.

In 1993 we showed that more than half of the spin-orbit splitting in ${ }^{15} \mathrm{~N}$ comes from long-range three-nucleon potentials and correlations, which violate the VB assumption. To investigate the isospin dependence of the spin-orbit splitting and other terms in the Skyrme model, we made VMC calculations of the type described in Sec. B.c. for systems of 7 and 8 (p-shell) and 19 and 20 (s-d shell) neutrons. The neutrons were confined in external potentials that were adjusted to give physically reasonable densities. GFMC calculations of the type described in Sec. B.b. were made for the 7- and 8neutron drops. The GFMC results indicate that our VMC results for neutron drops are significantly more accurate than is the case for comparable-sized nuclei --

*University of Illinois at Urbana-Champaign the FHNC/4 contributions to the energy and tested them in a number of simple model cases, including liquid ${ }^{3} \mathrm{He}$ and Bethe's homework problem. We get reasonable, but not exact agreement with earlier published work. In nuclear and neutron matter with the Argonne $v_{14}$ interaction these contributions are indeed small corrections at normal density and grow to only 5$10 \mathrm{MeV} /$ nucleon at 5 times normal density.

The same method can also be used to construct some elementary single-operator chain diagrams, at the SOC/4 level. These corrections are probably also small, but might make some contribution in highdensity matter. Work on these terms is now in progress.

(S. C. Pieper, V. R. Pandharipande,

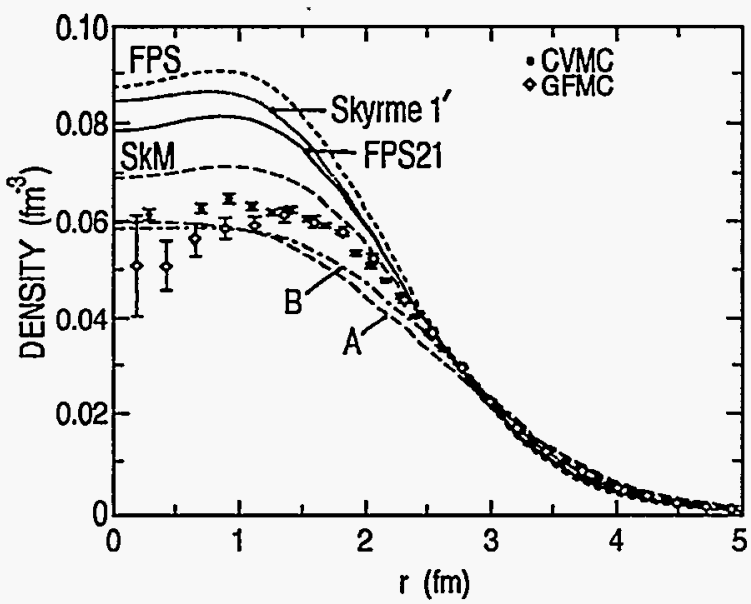

Fig. IV-9. The points and error bars show the VMC and GFMC computed density profiles for an eight-neutron drop. The upper four curves show calculations of the same system with four conventional Skyrme potentials; the lower two result from adding a quadratic gradient term to FPS21.

presumably due to the reduced strength of tensor correlations. We find that the spin-orbit splitting of these drops is less than half the ${ }^{15_{N}}$ value. These values can be used to determine an isospin dependence of $V_{\text {so }}$ that is very different from that of VB. In addition, as shown in Fig. IV-9, the surface of the drops is significantly more diffuse than is predicted by typical Skyrme models. These results have been published in Phys. Rev. Lett. 76, 2416 (1996). 


\section{j. Calculation of Effective Interactions of the Nuclear Shell Model Using the Argonne v18 Potential (T.-S. H. Lee, R. B. Wiringa, and T. T. S. Kuo*)}

The derivation of the effective interactions of the nuclear shell-model from a realistic nucleon-nucleon potential has long been a challenge to theoretical physics. In previous work, the employed $\mathrm{NN}$ potentials, such as the Paris and Bonn potentials, do not describe accurately the NN data in both the total isospin $\mathrm{T}=0$ and $\mathrm{T}=1$ channels. The resulting

*SUNY, Stony Brook, and Academia Sinica, Taiwan, ROC effective interactions only reproduce the general trend of the empirical values. With the newly developed Argonne v18 potential, we applied the folded-diagram approach to calculate the effective interactions in sdshells. The predicted spectra for the $A=18$ systems are in much better agreement with the data than in previous work. A paper describing our results is being prepared for publication.

\section{k. $\quad \Lambda$ Single-Particle Energies (A. R. Bodmer, and Q. N. Usmani*)}

We are continuing our work on the $\Lambda$-particle singleparticle energies and their interpretation in terms of the basic $\Lambda$-nuclear interactions taking account of recent developments in the variational calculation of $5 \mathrm{He}$ and also of ${ }_{\Lambda}^{17} \mathrm{O}$. We are also modifying and extending

*Jamia Millia Islamia, New Delhi, India our treatment of the fringing field (that part of the $\Lambda$ nucleus potential which extends beyond the nuclear density distribution because of the finite range of the $\Lambda$ nuclear interactions). We are also considering a complementary, more phenomenological approach to the single-particle energies.

\section{Core-Nucleus Distortion in Hypernuclei (A. R. Bodmer and Q. N. Usmani*)}

We have completed a study of the effects of the spherical distortion of the core nucleus by the $\Lambda$ in a hypernucleus. The response of the core was determined by an appropriately chosen energy-density functional which depends, in particular, on the nuclear compressibility. The forcing action of the $\Lambda$ is determined by the nuclear density dependence of the $\Lambda$ binding in nuclear matter which is obtained from our work on the $\Lambda$ single-particle energies. Because of the strongly repulsive $\Lambda \mathrm{NN}$ forces, this $\Lambda$ binding saturates at a density close to the central density of nuclei, and

*Jamia Millia Islamia, New Delhi, India results in a reduced core-nucleus distortion much less than would otherwise be obtained. The effects of the core distortion then turns out to be very small even for quite light hypernuclei. We also considered two toy models which display explicitly the role of the forcing action of the $\Lambda$ as well as that of the core response.

Our results justify the usual assumption that core distortion effects of spherical core nuclei are quite small and can be neglected. This work was done almost entirely at Jamia Millia Islamia while ARB was a visitor there. A report on this work was published in a conference proceedings. A more complete version was submitted for publication.

\section{m. Space-Exchange Effects in Light Hypernuclei (A. R. Bodmer, M. Shoeb,* M. Murali, $\dagger$ and Q. N. Usmani†)}

As a result of comments on center-of-mass problems of our treatment of space exchange, we concluded that for few- ( $>$ 2) body systems our rather literal implementation of the space-exchange operator was invalid. We are now implementing this operator withan $\ell^{2}$-dependent operator $(\ell$ is the relative angular

*Muslim University, Aligarh, India

$\dagger$ Jamia Millia Islamia, New Delhi, India momentum between the $\Lambda$ and a nucleon). Preliminary calculations (by Murali and Usmani) for $5 \mathrm{He}$ indicate that such a nonlocal exchange operator can give surprisingly large effects, especially if used together with realistic nucleon-nucleon potentials (such as Argonne v18) which involve momentum-dependent terms. 


\section{n. Charge-Symmetry Breaking $\Lambda$-Nucleon Interaction (A. R. Bodmer, M. Murali,* and Q. N. Usmani*)}

Some time ago we showed that the charge-symmetrybreaking interaction, as obtained from the mass-four hypernuclei $\left({ }_{\Lambda}^{4} \mathrm{H}, 4 \mathrm{He}\right)$, was spin-independent; a result which cannot be understood with the

*Jamia Millia Islamia, New Delhi, India conventional meson-exchange models. The calculations of $\left({ }_{\Lambda}^{4} \mathrm{H},{ }_{\Lambda}^{4} \mathrm{He}\right)$ are currently being extended to include realistic nucleon-nucleon forces, in particular ones which include noncentral and momentum-dependent terms, which could possibly modify this result.

\section{o. Suppression of the $\Lambda-\Sigma$ Coupling in Nuclear Matter (A. R. Bodmer and Q. N. Usmani*)}

We initiated a study of the modification of the coupling of the $\Lambda \mathrm{N}$ to the $\Sigma \mathrm{N}$ channel in nuclear matter with the Fermi hypernetted-chain variational approach. This modification of the $\Lambda \mathrm{N}-\Sigma \mathrm{N}$ coupling is a central problem in hypernuclear physics and is related closely to the strongly repulsive three-body forces which are needed to account for hypernuclear binding energies.
All earlier calculations only considered this problem in the so-called G-matrix approximation which neglects important higher-order effects. An important result of this work will be a better understanding of the density dependence of $\Lambda$ binding in nuclear matter, which can then be tested in the calculation of the $\Lambda$ single-particle energies.

*Jamia Millia Islamia, New Delhi, India

\section{p. Nuclear Core Dynamics and Rearrangement Energy for Hypernuclei} (A. R. Bodmer, S. Murali, * and Q. N. Usmani*)

The effect of the nuclear core dynamics on the binding energies of $\Lambda$ hypernuclei was studied in the framework of variational correlated wave functions. In addition to the core energy and to a $\Lambda$ single-particle contribution $\varepsilon_{\Lambda}$, we exhibit a rearrangement energy $E_{R}$ which in particular depends on the $\Lambda \mathrm{N}$ correlations $\mathrm{F}$ and on the difference $\delta \Phi_{N}$ between the core wave function and its exact ground state value. $E_{R}$ is non-zero only if there are both $\Lambda N$ correlations $(F \neq 1)$ and if $\delta \Phi_{N} \neq 0$. The latter can result from core polarization by the $\Lambda$ or from errors in the ground-state variational wave function of

*Jamia Millia Islamia, New Delhi, India the core. $E_{R}$ varies linearly with $\delta \Phi_{N}$ for small $\delta \Phi_{N}$. Detailed numerical results for $5 \mathrm{He}$ using central $\Lambda \mathrm{N}$ and $\mathrm{NN}$ potentials with repulsive cores confirm these general features. However, the effect of $E_{R}$ leads to an expansion of the core by the $\Lambda$ rather than a contraction obtained with only $\varepsilon_{\Lambda}$. The core polarization energy is quite small $-0.4 \mathrm{MeV}$. We studied the effects of errors in the variational core wave function by detuning this as a function of one of the variational parameters. This work was submitted for publication. It is being continued by Murali and Usmani for more realistic NN interactions.

\section{NUCLEAR STRUCTURE AND HEAVY-ION REACTIONS}

This research focuses on nuclear structure in unusual regimes: neutron-rich nuclei far from stability, and superdeformed nuclei at high spin. We also study heavy-ion reactions near the Coulomb barrier and some traditional shell-model problems. Much of this work is closely tied to experiments performed at ATLAS and at radioactive-beam facilities.

Our studies of drip-line nuclei are closely related to experimental programs at radioactive beam facilities. We focused on breakup reactions induced by the Coulomb and nuclear fields from a target nucleus, and analyzed decay energy spectra and momentum distributions of the emitted 
fragments. A critical issue is how reliably one can extract the low-lying dipole response of weakly bound nuclei and we have, in particular, investigated the Coulomb dissociation of ${ }^{8} \mathrm{~B}$.

The studies of heavy-ion reactions at energies close to the Coulomb barrier are based on applications of the coupled-channels technique. The objective is to obtain a consistent, unified, quantum-mechanical explanation of fusion reactions, compound-nucleus spin distributions, elastic and inelastic scattering, and transfer reactions. The calculations are constrained by the nuclear structure of the interacting nuclei.

Our studies of superdeformed nuclei, at both low and high spins, address new regions of superdeformation, rotational moments of inertia, and the transition from superdeformed to normal states. Other areas of interest are the density-dependence of residual interactions and the structure of the heaviest elements. We try to understand these phenomena on the basis of correlations induced by the effective two-body interaction. The techniques used include a deformed one-body potential for surveying nuclear structure over a large regime, self-consistent mean-field calculations for more detailed studies of particular nuclides and many-body wave functions when residual interactions are weak and a mean-field approach is inadequate.

Much of our work is computer intensive and we adapted our codes to exploit the massively parallel IBM SP supercomputer at Argonne. This allowed us to calculate energy surfaces using the Strutinsky method in a four-dimensional shape space that consists of quadrupole, octupole, hexadecapole and necking degrees of freedom. We completed studies of the A 180 and the A 150 mass regions. These studies are being complemented with Hartree-Fock studies of selected nuclides in these regions. There seems to be a better-than-semi-quantitative agreement between the two approaches at even the highest spins and the largest deformations. We are extending these studies to the $A \sim 130$ mass region. We are looking at improved treatments of pairing in superdeformed rotational bands. We are involved in experimental searches for new regions of superdeformation, and the experimental study of single-particle states in the heaviest elements.

\section{a. Coulomb Dissociation of ${ }^{8}$ B (H. Esbensen, G. F. Bertsch,* S. M. Austin, $\dagger$ J. H. Kelley, $\dagger$ and others $\dagger$ )}

We have studied the ${ }^{8} \mathrm{~B} \rightarrow{ }^{7} \mathrm{Be}+\mathrm{p}$ breakup, induced by the Coulomb field from a heavy target. The breakup is dominated by $\mathrm{E} 1$ transitions and attempts were made to extract the low-lying dipole strength from a recent measurement. The extracted dipole strength can be used to infer the radiative capture rate of protons on $7 \mathrm{Be}$, which is relevant to solar neutrino physics. We have focused on the influence of E2 transitions and of higherorder processes in our study of the Coulomb dissociation. These processes may play a significant role and thereby affect the interpretation of the data.

We developed a single-particle model for the weaklybound valence proton in ${ }^{8} \mathrm{~B}$ and used it to calculate the Coulomb dissociation. Our calculations predict a significant contribution from first-order E2 transitions. Our calculations also predict a large asymmetry in the longitudinal momentum distribution of ${ }^{7} \mathrm{Be}$ fragments,

\footnotetext{
*Institute for Nuclear Theory, University of
} Washington, †Michigan State Univèrsity due to the interference between the E1 and E2 amplitudes. By measuring the asymmetry it may be possible to extract information about the magnitude of the E2 strength. This work was published in Phys. Lett. B359, 13 (1995)..

In order to study the influence of higher-order processes, we solve numerically the time evolution of the wave function for the two-body, Coulomb-induced breakup, ${ }^{8} \mathrm{~B} \rightarrow{ }^{7} \mathrm{Be}+\mathrm{p}$. Our calculations show that higher-order processes can reduce the asymmetry in the longitudinal momentum distribution of ${ }^{7} \mathrm{Be}$ fragments, compared to the first-order prediction. However, their influence can be diminished, for example, by choosing a lighter target.

Higher-order processes have a peculiar effect on the decay energy spectrum: the effects of the E1 and E2 fields are not additive as they are in first-order perturbation theory. In a calculation that simulates a recent measurement, we find that the peak height obtained in the full calculation, based on the E1 and E2 
Coulomb fields from the target, is smaller than the result obtained in first-order perturbation theory. However, it is close to the prediction based on firstorder E1 transitions alone. This result explains and partly resolves a recent controversy about the magnitude of the E2 strength as illustrated in Fig. IV-10. There may be an appreciable E2 strength but its effect on the peak height of the decay. energy spectrum is apparently quenched by higher-order processes. This work was accepted for publication in Nucl. Phys. A.

A recent measurement of the Coulomb dissociation of ${ }^{8} \mathrm{~B}$ seems to indicate an asymmetry in the longitudinal momentum distribution of ${ }^{7} \mathrm{Be}$ fragments. However, the experimental uncertainty is too large to make an accurate assessment of the magnitude of the E2 strength. An analysis of the data is being prepared for publication, and more precise measurements are planned.

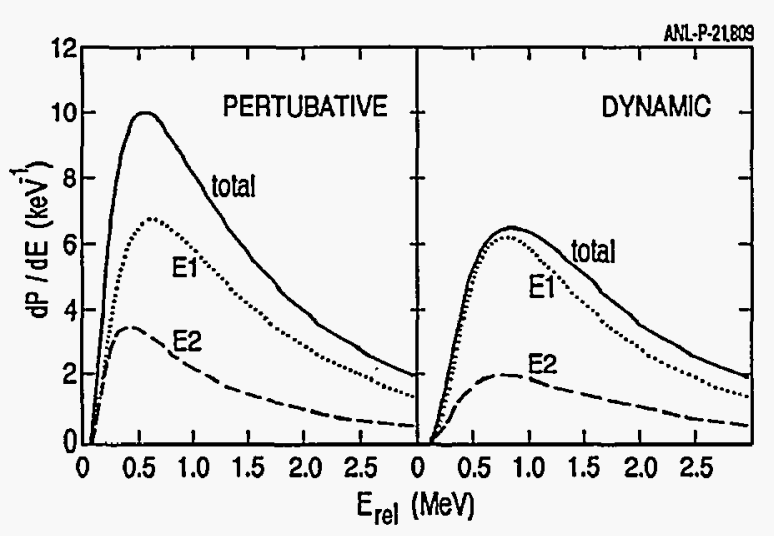

Fig. IV-10. Decay energy spectra from the Coulomb dissociation of ${ }^{8}$ Be at an impact parameter of $20 \mathrm{fm}$ with respect to a lead nucleus and a beam energy of $46.5 \mathrm{MeV} / \mathrm{u}$. The results of first-order perturbation theory and higher-order dynamical calculations are shown separately. The solid curves show the combined effect of the EI and E2 Coulomb fields from the target. The dotted and dashed curves are the results obtained from the EI and E2 fields separately.

\section{b. Momentum Distributions in Nuclear-induced Breakup Reactions (H. Esbensen, G. F. Bertsch,* and K. Hencken*)}

We started a program to study the momentum distributions of fragments which are produced in the nuclear-induced breakup of halo nuclei on light targets. We focus on the ${ }^{11} \mathrm{Be} \rightarrow{ }^{10} \mathrm{Be}+\mathrm{n}$ and ${ }^{8} \mathrm{~B} \rightarrow{ }^{7} \mathrm{Be}+\mathrm{p}$ breakup reactions, and use a single-particle description to model the wave function of the valence nucleon. Measurements of the longitudinal momentum distribution (LMD) of projectile-like fragments are commonly analyzed in the transparent limit of the Serber model. The basic breakup mechanism in this model is stripping, and in the transparent limit one ignores the effect of absorption of the projectile-like fragment. This implies that the width of the observed LMD is identical to the width of the ground-state momentum distribution of the removed valence nucleon.

We calculated the LMDs in the more realistic, strongabsorption limit of the Serber model and include the absorption of the projectile-like fragment. We find that the width of the LMD of $10_{\mathrm{Be}}$ fragments, produced in the breakup of ${ }^{11} \mathrm{Be}$, is only slightly reduced compared to the result obtained in the transparent limit.

\footnotetext{
*Institute for Nuclear Theory, University of Washington
}

However, the width of the LMD of ${ }^{7} \mathrm{Be}$ fragments, produced in the breakup of ${ }^{8} \mathrm{~B}$, is reduced by about a factor of two. The LMDs obtained in the strongabsorption limit are both in good agreement with recent measurements.

The reason why the transparent limit fails so dramatically for the ${ }^{8} \mathrm{~B}$ breakup is that the valence proton is bound in a p-orbit. In the strong-absorption limit one therefore has to consider the dependence of the breakup on the orientation of the proton orbit. Thus one finds that the production of $7 \mathrm{Be}$ fragments is strongly suppressed when the proton orbit is aligned with the beam direction. If one eliminates this component of the LMD, one obtains indeed a much smaller width in the transparent limit. The valence neutron in ${ }^{11} \mathrm{Be}$, on the other hand, is mainly bound in an s-orbit. The feature that the breakup probes mainly the transverse tail of the single-particle wave function has only a minor effect for a weakly-bound system when the wave function is an s-wave. This work was submitted for publication.

We are presently investigating the breakup contribution from nuclear-induced diffraction. This contribution is small compared to stripping at high beam energies but it becomes more significant at lower energies. 


\section{c. Pairing Gap in the Inner Crust of Neutron Stars (H. Esbensen, R. A. Broglia,* E. Vigezzi, * and F. Barranco $\dagger$ )}

The pairing gap in a neutron star can be affected by the presence of heavy nuclei. The effect is commonly estimated in a semiclassical description, using the localdensity approximation. Modifications of the pairing gap will also affect the nuclear specific heat, and it has been shown that it can become comparable to the electronic specific heat at certain densities and temperatures which may exist in the inner crust of a neutron star.

$\mathrm{n}$ order to investigate the validity of the semiclassical approach, we solve the quantal BCS equations for neutrons that are confined to the Wigner-Seitz cell, which surrounds a heavy nucleus. We performed calculations with the Gogny pairing force. We find that the presence of a heavy nucleus does enhance the nuclear specific heat but the enhancement is much smaller than found in the semiclassical approach. The discrepancy is attributed to the fact that the spatial extension of a Cooper pair is much larger than the size of a heavy nucleus. In fact, by smearing the pairing gap obtained in the local density approximation with the density of a typical Cooper pair, we obtain a pairing gap and a specific heat that is in reasonable agreement with the result of the quantal BCS calculation. This work is still in progress.

\footnotetext{
*Istituto Nazionale de Fisica Nucleare, Milano, Italy †University of Sevilla, Spain
}

\section{d. Pair Correlations in Heavy, Neutron-rich Nuclei (H. Esbensen)}

We started a program to study the properties of neutronrich nuclei using the Hartree-Fock-Bogolyubov (HFB) approximation. This appears to be the most realistic approach one has at present for heavy nuclei that contain many loosely-bound valence neutrons. The two-neutron density can be decomposed into two components, associated with the mean-field and the pairfield, respectively. The latter has a structure which is quite similar to the pair-density one obtains' by diagonalizing the Hamiltonian for a two-neutron halo. Thus one can compare the HFB solutions against numerically exact solutions. Preliminary results indicate that the two approaches give almost identical binding energies for a two-neutron halo.

The HFB method is applied to calculate the neutron separation energy for a series of isotopes and to predict the location of the neutron drip-line. The successful outcome depends crucially upon the two-body interaction that is used. This is parameterized as an effective, density-dependent interaction which approaches a realistic free interaction at low density. This work is in progress.

\section{e. Nuclear Energy Surfaces at High-Spin in the A 180 Mass Region (R. R. Chasman, J. L. Egido,* and L. M. Robledo*)}

We are studying nuclear energy surfaces at high spin, with an emphasis on very deformed shapes using two complementary methods: 1) the Strutinsky method for making surveys of mass regions and; 2) Hartree-Fock calculations using a Gogny interaction to study specific nuclei that appear to be particularly interesting from the Strutinsky method calculations. The great advantage of the Strutinsky method is that one can study the energy surfaces of many nuclides ( 300$)$ with a single set of calculations. Although the Hartree-Fock calculations are quite time consuming relative to the Strutinsky calculations, they determine the shape at a minimum without being limited to a few deformation modes. We

*Universidad Autonoma de Madrid, Spain have completed a study of $182 \mathrm{Os}$ using both approaches. In our cranked-Strutinsky calculations, that incorporate a necking mode deformation in addition to quadrupole and hexadecapole deformations, we found three well-separated, deep, strongly deformed minima. The first is characterized by nuclear shapes with axis ratios of 1.5:1; the second by axis ratios of 2.2:1 and the third by axis ratios of $2.9: 1$.

We also studied this nuclide with the density-dependent Gogny interaction at $I=60$ using the Hartree-Fock method and found minima characterized by shapes with axis ratios of 1.5:1 and 2.2:1. A comparison of the shapes at these minima, generated in the two calculations, shows that the necking mode of 
deformation is extremely useful for generating nuclear shapes at large deformation that minimize the energy. The Hartree-Fock calculations are being extended to larger deformations in order to further explore the energy surface in the region of the 2.9:1 minimum.

Using the Argonne IBM SP and NERSC resources, we are carrying out Strutinsky calculations on a large fourdimensional grid in a deformation space that includes octupole deformation in addition to quadrupole, hexadecapole and necking deformations. We are carrying out this study of the $A \sim 180$ region concentrating on the questions: 1) how do the inclusion of necking and reflection asymmetric degrees of freedom modify nuclear energy surfaces, and 2) how soft are the many known very-deformed nuclear shapes in this region to octupole deformation.

We completed the first phase of these studies, using the Strutinsky method to calculate nuclear energy surfaces in the four-dimensional space discussed above. Comparing the results obtained with and without. octupole deformation, we found major modifications of the energy surface in many nuclei in the region around ${ }^{176} \mathrm{~W}$. These effects are strong at all values of the angular momentum. There are reductions of the total energy of $\sim 7 \mathrm{MeV}$ for the necked-in shapes at the largest deformations. This feature can be understood in terms of incipient fission fragments. The reflection asymmetric shape is necked-in in such a way as to exploit the large shell corrections associated with a strongly-deformed fragment in the vicinity of ${ }^{100} \mathrm{Zr}$ and a spherical fragment in the vicinity of ${ }^{80} \mathrm{Zr}$. The symmetric necked-in shape at these large deformations corresponds to two strongly-deformed incipient fission fragments near $\mathrm{A}=90$ and is not favored. The effects are huge here, compared to typical octupole deformation energy gains of less than $1 \mathrm{MeV}$. These results have been published in Phys. Lett. B351, 18 (1995).

As in the case for Strutinsky calculations, the introduction of reflection asymmetry doubles the basis space that must be used in Hartree-Fock calculations. We extended the Hartree-Fock codes to allow us to calculate the properties of very-deformed reflectionasymmetric shapes. We finished a calculation of the energy surface of ${ }^{176} \mathrm{~W}$, using the Gogny interaction. We find that the inclusion of reflection asymmetry in the Hartree-Fock calculations causes a significant lowering of the energy surface at the largest deformations. In fact, the magnitude of the effects appears to be slightly larger than we found in the Strutinsky calculations. We are now making a survey of other regions of nuclei, and hope to find other cases where the octupole effects are large.

\section{f. Very Extended Shapes in the A $\sim 150$ Mass Region (R. R. Chasman)}

In the past few years, large computer resources have become available on the massively parallel processor IBM SP system at Argonne, in addition to the resources provided by NERSC. We parallelized our codes to exploit the SP system and devoted a large part of our efforts in the past year to calculating energy surface in a four-dimensional shape space that includes reflectionasymmetric shapes. We study the nuclear energy surfaces as a function of mass, charge, shape and angular momentum, using the Strutinsky method. In this approach, one makes quantum corrections to a smooth liquid drop behavior using the calculated meanfield energy levels. In earlier studies, we found that it is often not sufficient to use only quadrupole and hexadecapole deformations to describe very extended reflection-symmetric nuclear shapes. When we added a necking degree of freedom, we found previously unknown minima. These minima are characterized by very extended capsule-shaped nuclei with axis ratios of 2.2:1 in the $A=180$ mass region. We have now added octupole deformation to this shape space. The inclusion of these two degrees of freedom to our shape space substantially increases our ability to describe nuclear shapes compared to a typical shape space consisting only of quadrupole and hexadecapole deformations. As parity is no longer a good quantum number when octupole deformation is included, the size of the matrices we diagonalize is doubled. In a typical calculation, we diagonalize matrices that are $600 \times 600$. Several thousand such diagonalizations are needed to determine energy surfaces.

There was a report of a rotational band in ${ }^{152} \mathrm{Dy}$ or ${ }^{153}$ Dy that is characterized by a dynamic moment of inertia of $130 \hbar^{2} \mathrm{MeV}^{-1}$. More recently, there was a report of rotational bands associated with hyperdeformed shapes in ${ }^{147} \mathrm{Gd}$ with a dynamic moment of inertia of $\sim 140 \hbar^{2} \mathrm{MeV}^{-1}$. For purposes of orientation, it should be noted that the well-known superdeformed bands in this region are characterized by moments of inertia of only $\sim 90 \hbar^{2} \mathrm{MeV}^{-1}$. Some calculations were carried out in two- and three-dimensional shape spaces, in order to understand this experimental observation. Some of these calculations suggest that the hyperdeformed bands are associated with a reflection-asymmetric minimum, that becomes yrast near $\mathrm{I}=90 \hbar$. We extended our fourdimensional Strutinsky calculations to a study of this mass region, with the hope of getting some 


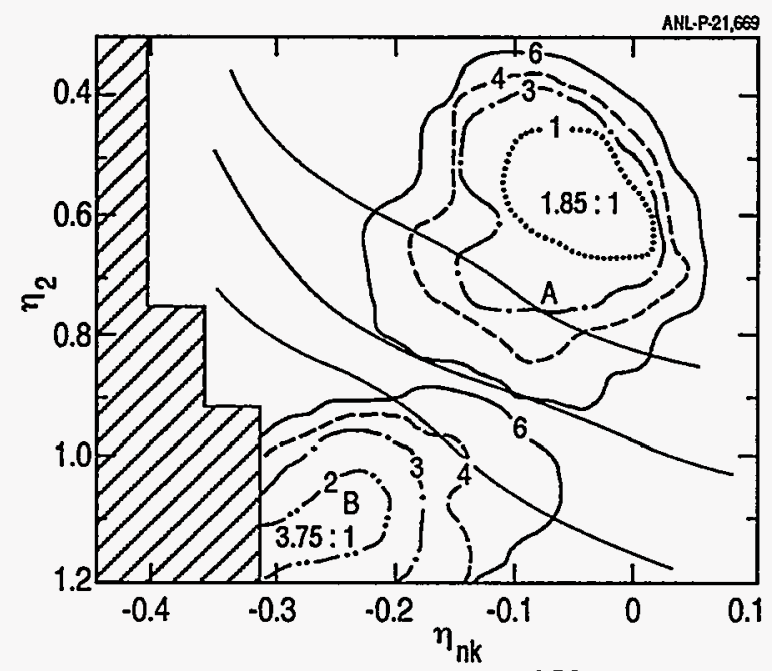

Fig. IV-11. Energy surface of ${ }^{152}$ Dy at $I=80$ : octupole deformation effects are included in the energy surfaces. The vertical axis gives elongation and the horizontal axis is the amount of necking relative to an ellipsoidal shape. The numbers on the contour lines are energy in $\mathrm{MeV}$. The lines denoted as 110 , 130 , and 150 are lines of constant moment of inertia. The ratios in the minima are the axis ratios of superdeformed shapes in these minima. The shaded areas are regions where the surface area is greather than 1.27.

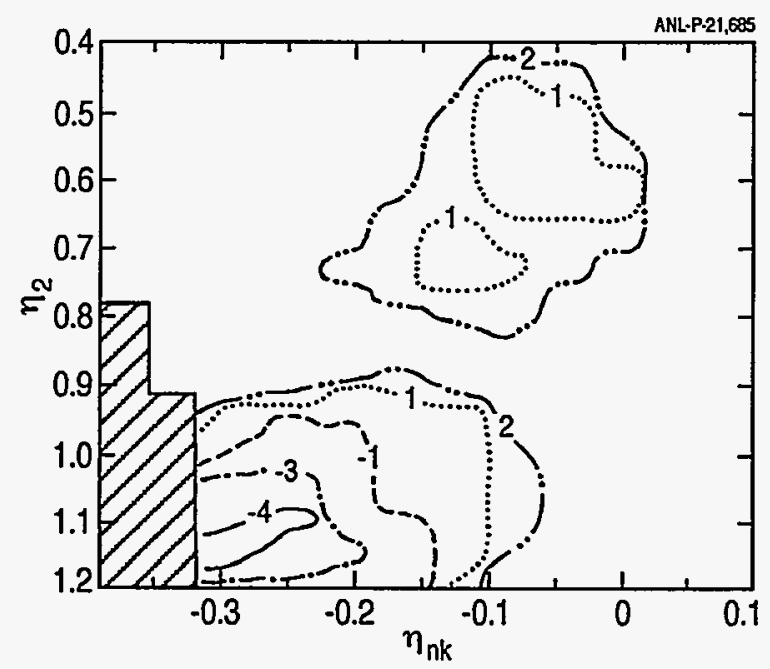

Fig. IV-12. Energy surface of ${ }^{152}$ Dy at $I=90$. See caption for Fig. IV-11. Note that the minimum leading to fission (in the lower left) is roughly 5 $\mathrm{MeV}$ below the superdeformed minima.

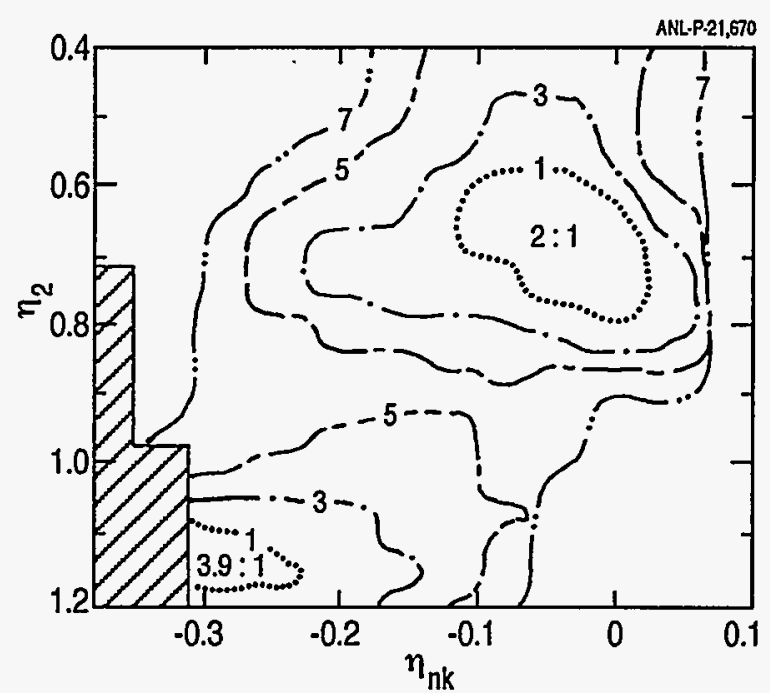

Fig. IV-13. Energy surface of 158 Dr at $I=180$. See caption of Fig. IV-II. Note the increase in elongation and in axis ratios relative to the superdeformed minimum of ${ }^{152} \mathrm{Dy}$.

understanding of these hyperdeformed bands. We did not find hyperdeformed minima in this mass region that appear to be stable to fission. Our calculations suggest that the explanation for these bands is something other than hyperdeformation. In Figs. IV-11 and IV-12 we show the energy surface of ${ }^{152}$ Dy at $I=80 \hbar$ and $\mathrm{I}=90 \hbar$. Since the completion of these calculations, and the submission of our results for publication in Phys. Lett. B364, 137 (1995), the claims of experimental observation of hyperdeformed bands in ${ }^{152} \mathrm{Dy}$ and in ${ }^{147} \mathrm{Gd}$ were withdrawn.

We did, however, find several nuclei with superdeformed minima that are somewhat more extended than any observed in the A 150 mass region. Specifically, these are nuclides near ${ }^{158} \mathrm{Er}$ in mass and are characterized by axis ratios as large as $2: 1$ in contrast to the $1.85: 1$ axis ratio of the super-deformed shapes in ${ }^{152}$ Dy (see Fig. IV-13) The single-particle level structure associated with the very superdeformed shapes of the $A=156$ region is particularly fascinating. The single particle orbitals that appear at the Fermi levelat $\mathrm{I}=80 \hbar$ in these minima are orbitals that are characteristic of undeformed superheavy elements $(\mathrm{Z}=$ $114, N=184$ ) at $I=0 \hbar$. It would be one of the more exciting findings of the heavy-ion research program to identify these orbitals in the light rare earths.

\section{g. Rotational Spacings in Superdeformed Bands of Nuclei (R. R. Chasman)}

An unexpected result of the experimental investigation of superdeformed rotational bands is the observation of nearly identical dynamic moments of inertia in different nuclei. This phenomenon was noted in normally deformed rotational bands. A priori, the BCS method is suspect at $I=0$ for the treatment of superdeformed 
nuclear shapes because the single-particle level density near the nuclear surface is small. If it were large, there would be no superdeformed minimum. At high spin, pairing correlations are further weakened, and the BCS method becomes even worse.

In our studies of nuclear structure of the actinides, we found that a conventional pairing force with constant matrix elements does not do a good job of explaining low-energy spectra. A density-dependent pairing force does a considerably better job of describing these spectra. In a superdeformed nucleus, the ratio of surface to volume is much larger than it is in the case of the normally deformed actinides, and we expect densitydependent effects to play an even more important role.The above considerations motivated us to undertake a many-body calculation of moments of inertia, going beyond the usual BCS treatment of pairing correlations in two ways: 1) we use wave functions with good particle number and; 2) we do a configuration interaction calculation. In the configuration interaction calculations, we use the proton and neutron pairing interaction strengths and the cranking frequency to generate configurations for the interaction calculation. Our many-body wave functions are products of sums of terms. Each such wave function has $\sim 10^{12}$ amplitudes, and contains $\sim 2000$ variational parameters. We minimize the variational parameters after projecting states of good particle number, good parity and good signature.

As a first step, we carried out a calculation of rotational spacings in the superdeformed band of ${ }^{192} \mathrm{Hg}$, using constant pairing matrix elements, to test the adequacy of conventional calculations of rotational spacings. This study shows that it is extremely important to take configuration interaction effects into account. Above I $=30$, the energy can be lowered by $>300 \mathrm{keV}$ by including configuration interaction effects with the wave functions generated by varying the pairing interaction strengths. We get additional lowering of $\sim 200 \mathrm{keV}$ when states generated from all cranking frequencies are also included in the configuration interaction calculation. These shifts are relative to a wave function with good particle number. The shifts are even larger relative to wave functions calculated in the BCS approximation.

These results suggest that all calculations of dynamic moments of inertia using the cranked quasiparticle approximation are not adequate for treating superdeformed bands. It is extremely difficult to understand the success of such methods in describing superdeformed bands.

We carried out a similar calculation using a densitydependent interaction. On the whole, our results with the density-dependent interaction are similar to those that we obtain with constant-pairing-force matrix elements. The overall strengths of the two interactions must be the same, and it appears that it is this overall strength that is the crucial ingredient. Because the level densities are low in superdeformed minima, the gap parameters are small. This in turn reduces pairinginduced shifts in single-particle energies, as a function of particle number. The shifts we calculate in this region are smaller by a factor of two than those we obtained in the actinides.

\section{h. Experimental Search for Very Extended Shapes in the A $\sim 180$ Region (R. R. Chasman, M. P. Carpenter, R.V.F. Janssens, I. Ahmad, B. Crowell, R. G. Henry, T. L. Khoo, T. Lauritsen, and D. Nisius)}

A very important question in nuclear structure studies is the adequacy of the Strutinsky method for states at very large deformation. According to our calculations, nuclides in the vicinity of ${ }^{182} \mathrm{Os}$ are expected to have an accessible minimum that is more deformed than any minimum that has so far been populated at high spin in heavy-ion reactions. The shapes of nuclei associated with states in this extended minimum are quite different from those of the conventional superdeformed nuclide that bulge out like a football at the center. These shapes are flat and one needs a necking degree of freedom in the deformation space to calculate this minimum. Studies of this region will provide useful tests of the Strutinsky approach, and perhaps allow us to fine tune some of the ingredients of these calculations dealing with extended shapes.
An attempt was made to populate this minimum in $182 \mathrm{Os}$, by bombarding ${ }^{138} \mathrm{Ba}$ with a $220-\mathrm{MeV}{ }^{48} \mathrm{Ca}$ beam at the Gammasphere facility. This produces states in ${ }^{182} \mathrm{Os}$ with angular momenta of $\mathrm{I} \sim 70$. Our calculations predict that the very extended minimum (2.2:1) is yrast at this angular momentum. This search was not successful because of oxygen contamination in the target giving rise to many unwanted $\gamma$-rays.

According to our calculations, it should be almost as easy to make states in the $2.2: 1$ minimum of $180 \mathrm{Os}$ as it is in ${ }^{182} \mathrm{Os}$. To make ${ }^{180} \mathrm{Os}$, a target of ${ }^{130} \mathrm{Te}$ was bombarded with a $240-\mathrm{MeV}{ }^{54} \mathrm{Cr}$ beam, at the Gammasphere facility. The gamma-ray coincidence data from that experiment are being analyzed. 


\section{i. Single-particle States in the Heaviest Elements (I. Ahmad and R. R. Chasman )}

The search for superheavy elements has been a major theme of nuclear structure research for the past twenty years. Theoretical predictions of the stability of superheavy elements depend crucially on the singleparticle energy level spacings in the vicinity of 114 protons and 184 neutrons. The approach we are taking is to learn as much as possible about these levels from spectroscopic studies of nuclides in the $A=250$ region. This is possible because there are members of the relevant spherical multiplets that drop rapidly in energy with increasing deformation, and are fairly close to ground in the strongly deformed nuclides near $\mathrm{A}=250$. The orbitals that are important for fixing the shell corrections near $\mathrm{N}=184$ are the $\mathrm{h}_{11 / 2}, \mathrm{j}_{13 / 2}$ and $\mathrm{k}_{17 / 2}$ spherical states. For each of these spherical orbitals, there is a corresponding deformed orbital whose energy in the $A=250$ region is quite sensitive to one of

\section{j. $\quad$ Many-Body Wave Functions}

In the past few years, we have developed many-body variational wave functions that allow one to treat pairing and particle-hole two-body interactions on an equal footing. The complexity of these wave functions depends on the number of levels included in the valence space, but does not depend on the number of nucleons in the system. By using residual interaction strengths (e.g. the quadrupole-interaction strength or pairinginteraction strength) as generator coordinates, one gets many different wave functions, each having a different expectation value for the relevant interaction mode. These wave functions are particularly useful when one is dealing with a situation in which the mean-field approximation is inadequate. Because the same basis these spherical states, e.g. the $1 / 2-[761]$ orbital that has already been identified in ${ }^{251} \mathrm{Cf}$ is quite sensitive to the spherical $\mathrm{j} 13 / 2$ orbital. The position of the $1 / 2+[880]$ deformed orbital is very sensitive to the $\mathrm{k}_{17 / 2}$ spherical state. According to our calculations, this state should be found at $\sim 1500 \mathrm{keV}$ in ${ }^{251} \mathrm{Cf}$ and should be populated in a one-nucleon transfer reaction using an $\left(\alpha,{ }^{3} \mathrm{He}\right)$ reaction. We calculated signatures for the lowlying states in $251 \mathrm{Cf}$ and the calculated energies and signatures are in good agreement with the experimentally observed $(d, p)$ spectrum. We expect to see the high-J states in a ( $\left.\alpha,{ }^{3} \mathrm{He}\right)$ study. Our analysis of low-lying states in ${ }^{251} \mathrm{Cf}$ was published.

The $\left(\alpha,{ }^{3} \mathrm{He}\right)$ experiment was carried out and several high-J peaks were identified at excitation energies above $1500 \mathrm{keV}$. We are in the process of analyzing these data.

\section{R. R. Chasman)}

states are used in the construction of the many-body wave functions, it is possible to calculate overlaps and interaction matrix elements for the many-body wave functions (which are not in general orthogonal) easily. The valence space can contain a large number of singleparticle basis states, when there are constants of motion that can be used to break the levels up into groups. We added a cranking term to the many-body Hamiltonian and modified the projection procedure to get states of good signature before variation.

In the past year, we extended this code to the calculation of spectroscopic factors for proton decay. With minor modifications, this should also allow us to calculate spectroscopic factors for one-nucleon transfer reactions.

\section{k. Nuclear Structure Near the Drip Line (R. R. Chasman)}

Very recently, proton emission was observed in the light $\mathrm{Pb}$ nuclei. It seems one might hope to use this phenomenon to extract some interesting spectroscopic data on nuclear shapes and orbitals in much the same way one gets spectroscopic information through singlenucleon transfer reactions such as $(d, p)$ and $(d, t)$ reactions. In order to explore this possibility, we developed a treatment of proton emission spectroscopic factors using many-body wave functions. In the case we studied ${ }^{185} \mathrm{Bi} \rightarrow{ }^{184} \mathrm{~Pb}$, the energetics of the reaction is such that only the ground state is populated. However, we calculate a very small spectroscopic factor for the proton decay to the ground state $(0.1 \%)$ in lowest order. To understand the observed spectroscopic factor $(\sim 3 \%)$, it is necessary to carry out configuration mixing of the many-body wave functions. We are carrying out such calculations and extending them to neighboring nuclides. 


\section{Survey of Nuclear Energy Surfaces in the A 120 Region (R. R. Chasman)}

We have extended the calculation of nuclear energy surfaces in a four-dimensional deformation space to the A $\sim 120$ region. Our goal is to find new regions of nuclei in which very extended shapes are experimentally accessible. The results of these calculations are being analyzed.

\section{m. Monopole Transition in ${ }^{10 B}$ (D. Kurath and D. J. Millener*)}

Before recent inelastic electron scattering experiments were carried out on ${ }^{10} \mathrm{~B}$, shell-model calculations could account for most observations. However, the longitudinal excitation of the $\mathrm{J}, \mathrm{T}=3,0$ state at 4.77 $\mathrm{MeV}$ has a magnitude much larger than is consistent with the gamma decay to the 3,0 ground state.

The width of the form factor is also much narrower than that of a C2 excitation. We investigated the possibility

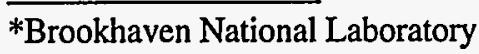

that this is a monopole $\mathrm{C} 0$ excitation by comparing the observed form factor with that obtained for GMR, the giant monopole resonance. The shapes as functions of momentum transfer are very similar, but the magnitude observed is only a few percent of the GMR case. This is consistent with what is seen in ${ }^{13} \mathrm{C}$ where a $\mathrm{J}=1 / 2$ to $1 / 2$ - transition can only be a pure monopole transition. The extreme weakness of the $\mathrm{C} 2$ transition is the reason the monopole dominates in the ${ }^{10} \mathrm{~B}$ case. Our conclusion was incorporated into the experimental paper on ${ }^{10} \mathrm{~B}$.

\section{n. Ptolemy on New Computers (S. C. Pieper)}

The program Ptolemy was written by $\mathrm{M}$. $\mathrm{H}$. Macfarlane, M. Rhoades-Brown and me in the late 70's and early 80 's to do calculations of heavy-ion reactions (optical-model fits, DWBA for transfer and coupledchannels for inelastic scattering). Since then it has been in continual use by Argonne experimentalists and at a number of other sites. However, the large size of the program inhibited moving it to new computers. I have modified the non-portable sections of the program using mostly Fortran 77 constructs (which were not widely available at the time Ptolemy was written) and Fortran 90 for the dynamic memory allocation. The new version has been extensively tested on IBM RS6000 (Unix) and DEC-alpha VMS workstations. The DEC version is now the production version at Argonne.

\section{FUNDAMENTAL QUANTUM MECHANICS}

\section{a. Aharonov-Bohm Effects in Normal Metal Shields (M. Peshkin)}

In the Aharonov-Bohm (AB) effect, the motion of a charged beam particle is influenced by a magnetic flux through a region which the particle cannot enter. This occurs only when the domain of the particle is multiply connected and the flux threads the hole in that domain. This phenomenon has attracted considerable interest because of its fundamental significance in quantum mechanics and because of applications to studying the dynamics of mesoscopic electrical circuits. There is a long-standing question as to whether a conducting shield around the magnetic field can influence the $\mathrm{AB}$ effect on the beam particles. The conduction electrons in the shield provide moving image charges and other currents induced by the magnetic fields of which the beam particle is the source. Those conduction electrons may have their own $A B$ effect and that may affect the quantum mechanical phases that are responsible for the observed $A B$ effect. In all real experiments, the magnetic field is surrounded by a conductor and no such influence is seen, but the beams in those experiments have speeds around $10^{9} \mathrm{~cm} / \mathrm{sec}$ and they go rapidly past conductors whose widths are typically not more than $100 \mathrm{~nm}$, and it may be that experiments with slower beams could reveal such an $\mathrm{AB}$ effect in the conductor if it exists.

For superconducting shields there is no problem. Flux quantization protects the conduction electrons from changes in the external magnetic flux. However for normal metals, the question has not previously been 
answered definitively. We now show that there can be no such effect in a normal metal conductor, assuming only that a normal metal is one that lacks off-diagonal long-range order (ODLRO). The central point of the proof is that for the limited Hilbert space of density matrices lacking ODLRO, it is possible to define a gauge transformation that removes the interaction of the conduction electrons with the magnetic flux from the Hamiltonian. (In the general case of electrons moving in a multiply-connected domain, no such gauge transformation exists except for quantized values of the flux.) This proof applies to macroscopic shields only. For mesoscopic shields on the nanometer scale, the range of the order has only to exceed the dimensions of the shield to evade the conditions of the proof. It may be that $A B$ effect experiments on mesoscopic circuits can provide information about the range of off-diagonal order in those circuits. This work was published in Phys. Rev. Lett. 76, 2207 (1996).

\section{b. The Electron (M. Peshkin and Yosef Imry*)}

The year 1997 marks the one hundredth anniversary of the discovery of the electron by J. J. Thompson. In honor of that event, a book entitled "The Electron" will be published by Cambridge University Press. The book, intended to be accessible to advanced undergraduate physics students, will be edited by

*Weizmann Institute of Science, Rehovot, Israel
Michael Springford (Bristol University). We are preparing a chapter entitled "The Coherent Electron". The principal emphasis of that chapter will be on coherence length in electron beams and in conductors, electron interferometry and holography, and on the physics learned from Aharonov-Bohm effect on electron beams and on mesoscopic normal circuits.

\section{c. Quantum Ballistic Evolution: Application to Quantum Computation (Paul Benioff)}

The techniques of quantum ballistic evolution, used in quantum computation, have been expanded to any process to which a step operator can be associated. Quantum ballistic evolution describes motion along a collection of distinct (nonintersecting) paths in any basis. Examples of step operators as sums over elementary steps can be obtained by translation of computer programs, as sums of elementary steps, into sums of products of projection operators, arbitrary unitary bit transformations, and local particle shifts on space lattices. If a bounded linear operator $T$ is a step operator for a process, then powers of $\mathrm{T}$ or its adjoint describe the successive steps of the process.

For paths defined as sequences of states in a basis, a theorem was proved which gives necessary and sufficient conditions that any bounded linear operator $T$ must satisfy such that there exists a Hamiltonian description of quantum ballistic evolution for any process described by iterations of powers of $T$ or its adjoint. It was also shown that in the case of deterministic quantum Turing machine models (ones where the only bit transformations allowed are $\mid 0>$ to $|1\rangle$ and $\mid 1>$ to $\mid 0>$ ) there is an effective decision procedure for determining if the associated step operator satisfies the conditions of the theorem. For nondeterministic models, (ones where any bit transformation in $U(2)$ is allowed), it is an open question if an effective decision procedure exists.

It is hoped to expand this work in the future by generalization of the definition of quantum ballistic evolution to more general definitions of paths and to include state-dependent potentials along the paths. It is also hoped to apply this work to fundamental questions on the relationship between the foundations of mathematics and physics.

\section{E. ATOMIC THEORY}

\section{a. Interactions of High-Energy Photons with Matter (M. Inokuti)}

In support of experimental work in atomic physics with the use of synchrotron radiation, theoretical studies are conducted in the two following areas, focusing on topics selected in view of basic importance, timeliness, and potentials in applications.
Physics of the Compton scattering by bound electrons. When the photon energy greatly exceeds the binding energy of an electron, the effect of electron binding to an atom is well understood in the framework of an impulse approximation, which accounts for the momentum of an electron at the instant of the 
Compton scattering. However, the physics remains to be explored for a more general case, where full details of atomic structure, described for instance in terms of the Green's function, need to be considered. For a hydrogen-like atom, or within a single-electron approximation, full analysis of the binding effect is theoretically feasible. Efforts will be made to elucidate basic points of the physics involved and to suggest items for experimental exploration.

Dispersion relations and sum rules for photoabsorption cross sections and related data. Within the dipole approximation, these data are subject to numerous theoretical constraints, which are closely related to the electronic structure of atoms, and more generally to their aggregates. Comprehensive analysis of the data for a specific material proved valuable for many purposes, as exemplified in earlier work on many atoms, several molecules, and metallic aluminum. Similar analysis of data for basic materials such as carbon and silicon will be carried out. Results will be useful in the design of many experiments in which these materials are used for optical devices.

\section{b. Interactions of Fast-Charged Particles with Matter (M. Inokuti)}

Stopping power, the total yield of ionization, its fluctuation, ionization cross sections, and other quantities related to the energy loss of fast-charged particles penetrating through matter are important in many applications such as the detection of particles and the analysis of their charges and kinetic energies. Despite its long history of study, the subject remains to be further studied in several respects, including the differences between particles of the same speed with

\section{c. Beam Polarization and Non-Dipole Electron Ejection (M. Peshkin)}

When X-rays strike unpolarized atoms or molecules, the angular distribution of anything that emerges depends trivially upon the polarization state of the Xrays, as long as one can make use of the dipole approximation. To obtain dynamical information from non-dipolar angular distributions, typically measured at only limited angles, one must unfold purely kinematic angular-momentum coefficients. Those coefficients depend non-trivially upon the polarization of the X-ray beam, which may be imperfectly known. All that has been known for many years, as has the complete angular momentum analysis required for extracting the dynamical coefficients. However, the results are not always transparent and the calculations needed are not always simple. We developed a simple treatment of the polarization dependence of angular distributions of scattered X-rays and other ejecta from unpolarized targets that appears to be useful both for the analysis of angular distribution data and for the planning of angular-distribution experiments. We represent the polarization state of the beam in the photon helicity representation. When the missing longitudinally polarized state is eliminated, the remaining two-by-two density matrix for the photon spin has the form $\rho=(1 / 2)(1+\sigma \cdot P)$, where the components of $\sigma$ are the usual Pauli matrices but not the generators of different charges and the differences of the stopping power of the same material, depending on the phase or the chemical state. Charged-particle collisions at high energies, and especially at low-momentum transfers, are intimately connected with photon interactions. Therefore, a study on such collisions also contribute to the full understanding of photon-interaction physics. Efforts are being devoted to the clarification of these and related current issues.

\section{Effects in X-Ray Scattering and}

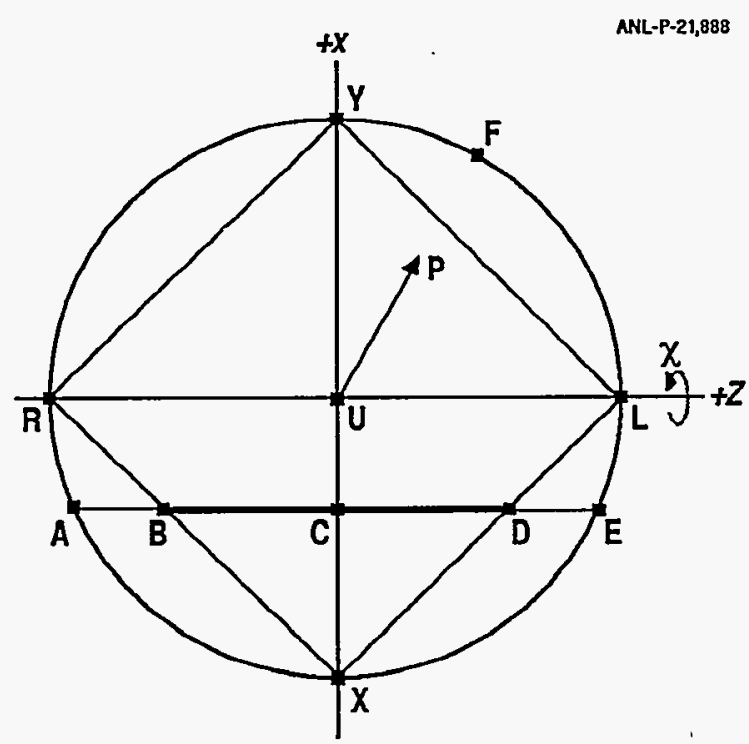

Fig. IV-14. The polarization sphere. U respresents unpolarized light. On the unit circle, $L$ and $R$ are leftand right-curcular polarizaton states, $X$ and $Y$ are $X$ plane and Y-plane. All other points are elliptically polarized pure states. Points within the unit circle are statistical mixtures of pure polarizations states. The sphere is completed by rotations of the polarization vector $\boldsymbol{P}$ around the $z$ axis, the angle of rotation of $\boldsymbol{P}$ being twice the rotation angle in real space. States on any one line parallel to the $z$ axis, such as the line $A E$, lead to the same angular distributions. 
rotations in real space, and $P$ is a vector characterizing the polarization state of the beam. ( $P$ is related to but significantly reprdifferent from Poincare's polarization vector.) This representation (Fig. IV-14) appears, incredibly, to be new and has the advantage that it enables a simple geometric representation of polarization in which the relations of different polarization states are transparent and the consequences of polarization for angular distributions are very simple. Some results that may be useful are the following: 1) If the intensity of an outgoing X-ray or electron is measured at angles chosen so that the intensity at $(\vartheta, \varphi)$ can be averaged with the intensity at $\left(\vartheta, \varphi+90^{\circ}\right)$, where $(\vartheta, \varphi)$ are the polar angles with respect to the beam axis, then the angular distribution is independent of the beam polarization, but no dynamical information is lost except possibly for a factor $\sqrt{2}$ in some statistical errors. 2) To get total cross sections and total forward-backward asymmetries, it is enough to measure at four "doubly magic" angles whose direction cosines with respect to three axes all have magnitude $\sqrt{2 / 3}$. One axis should be the beam axis, but the other two axes can be arbitrary, regardless of the polarization. 3) If the absorption of the incident X-ray is dominated by the electric dipole interaction so that other multipoles appear in the, angular distribution only linearly, i.e. through their interference with electric dipole, then every coefficient in the angular distribution is a product of a dynamical factor and a geometrical factor, the former independent of the polarization and the latter a universal function of the polarization alone.

All these results apply to the angular distribution of any vector in the final state. Examples are the momentum of an X-ray of a selected energy, the momentum of an Auger electron, and the sum of the momenta of all electrons. This work was submitted for publication.

\section{F. OTHER ACTIVITIES}

\section{a. Theory Workshop on Pairing Forces (R. R. Chasman and H. Esbensen)}

The Theory Group hosted a workshop on Pairing Forces in Nuclei, June 26-30, 1995. About 25 theorists from the US and Europe were brought together to discuss recent developments in the description of phenomena that are sensitive to pairing forces. The topics included the nuclear structure of superdeformed states and nuclei far from stability, isotope shifts, pairtransfer at high spins and the properties of neutron stars. While the conventional approach in nuclear physics has been to use a simplified model of the pairing force, namely one with constant pairing matrix element, the focus of the workshop was on more realistic forces, such as the Gogny interaction and density-dependent delta interactions. Many of the results presented at the workshop showed that such interactions provide an improved description of nuclear properties. This conclusion will be further tested in the next few years, under the extreme conditions that can be observed with large arrays of gamma-detectors and produced with radioactive beams. The workshop was funded by the Associate Laboratory Director for Physical Research.

\section{b. Eighth Annual Midwest Nuclear Théory Get-Together (R. B. Wiringa)}

The Theory Group hosted the Eighth Annual Midwest Nuclear Theory Get-Together on September 29 and 30, 1995. Nuclear theorists from a number of midwest universities get together every fall to find out what different people and groups in the area are working on. The organizational duties rotate among the participants, but Argonne has become the regular meeting place by virtue of its facilities and central location. The organizer for 1995 was John Clark of Washington University in St. Louis. The meeting provides a good chance for students to broaden their horizons and get some practical speaking experience in a friendly atmosphere. The format is very informal, with an agenda of talks being volunteered at the beginning of the meeting. This year 36 faculty, postdocs and graduate students from ten different institutions in Illinois, Indiana, Iowa, Kentucky, Michigan, Missouri, Ohio and Wisconsin, along with the Argonne staff, gathered to discuss their current work in nuclear physics. Some 26 presentations were made over Friday afternoon and Saturday morning. Topics included QCD, Skyrme models, charge-symmetry breaking and parity nonconservation, the NN interaction, halo nuclei, the shell model, chaos, relativistic heavy-ion collisions, and neutrino astrophysics. A good time was had by all. 


\section{ATOMIC AND MOLECULAR PHYSICS RESEARCH}

\section{OVERVIEW}

Atomic physics research is focused principally on measurements of atomic structure and dynamics. Within that domain, precision measurements useful in testing many-body relativistic atomic calculations are emphasized. The program addresses current problems in atomic physics using the unique facilities available at Argonne, namely, ATLAS and the Advanced Photon Source (APS). In addition, scientific interests led to collaborative experiments at the heavy-ion storage ring in Darmstadt, Germany (GSI) and the synchrotron facilities, ESRF in Grenoble, ALS in Berkeley, and NSLS at Brookhaven.

In 1995, the ATLAS-based heavy-ion program to measure the spectral distribution of the two-photon decay in heliumlike ions was continued. The $2{ }^{1} \mathrm{~S}_{0}$ state in heliumlike ions is forbidden to decay to the ground state by the emission of a single photon, so the dominant decay mode is emission of two E1 photons. The energies of the individual photons sum to the transition energy and the shape of the spectrum is broad, with a peak at half the transition energy. The spectral shape provides a sensitive probe of the calculation of the transition probability for this decay. In particular, this class of measurements tests our understanding of the entire structure of an ion since a sum over the complete set of intermediate states is required and both energy levels and wavefunctions must be understood.

In 1995, the two-foil technique to measure ultrashort lifetimes in the 100 fs to 10 ps regime for highly-charged ions was assessed. The idea behind the method was to use the first foil as an "exciter" and the second as a "probe" of excited states. Atoms in excited states are preferentially stripped and thus a change in the chargestate distribution and/or X-ray yield as a function of foil separation is manifested. The specific case used to demonstrate the feasibility of the technique was the decay of the $2{ }^{3} \mathrm{P}_{2}$ level in heliumlike $\mathrm{Kr}$, with a calculated lifetime of 9.5 ps. A measurement of this lifetime would contribute to the understanding of atomic structure when both electron correlations and relativistic effects are simultaneously important. An earlier measurement observing X-ray emission from $n=2$, gave the lifetime of the ${ }^{3} \mathrm{P}_{2}$ level in heliumlike $\mathrm{Kr}$ to an accuracy of $10 \%$. The method was extended by observing charge-state fractions as a function of foil separation. The charge-state observation method led to decays that were consistent with a powerlaw dependence of Rydberg-fed atomic transitions, but the utility of the technique to isolate the lifetime of a single level with high accuracy was found to be limited.

The ATLAS-based VUV spectroscopy program shifted emphasis from 2-electron systems to more complex systems where substantial theoretical progress has been made with little corresponding experimental work. Measurements were made on multielectron $\mathrm{Kr}$ in the far ultraviolet region. In addition, a program to study copperlike uranium was initiated. These data are being analyzed. 
Collision studies of fast highly-charged ions with $\mathrm{C}_{60}$ were analyzed. These measurements constitute the highest energy collisional system studied for fullerenes and were extended to detect the weak photon emission branch of the collisionally-excited system.

Experimental work at BLASE on precision hyperfine structure and lifetime measurements was put on hold as preparations for an experimental program at the APS took priority. Efforts at Argonne were made to isolate a systematic problem in the time-correlated single-photon counting measurements of the excited-state lifetime of a single trapped $\mathrm{Ba}^{+}$ion. The time-correlated single-photon counting method applied to a single trapped ion could yield a lifetime measurement of unprecedented accuracy, $<0.1 \%$. The enhanced precision is of interest as a test of $a b$ initio theories of atomic structure which are needed to interpret atomic parity nonconservation measurements. Efforts in this area are continuing in collaboration with R. G. DeVoe at IBM Almaden.

The synchrotron-based atomic physics program continued with joint support from both DOE and Argonne Laboratory Director's Discretionary Funding, as part of a lab-wide initiative in support of the 7-GeV Advanced Photon Source (APS). During this year, the program shifted its emphasis from conducting experiments at the National Synchrotron Light Source (NSLS) at Brookhaven National Laboratory to preparing for experiments at the APS. The general scientific theme of this program is to increase the understanding of photon-atom interactions at high photon energies. Specifically, the intense X-ray probe will enable detailed studies of structure and dynamics of atoms and molecules in a regime where relativistic and quantum electrodynamic effects play a major role. These studies also have relevance in other fields since understanding of the X-ray - atom interaction in free atoms forms the basis for interpretation of many solid-state results.

In 1995, a program to measure non-dipolar contributions to photoionization was initiated using an X-ray beamline at Brookhaven's NSLS. Current understanding of photoionization is based largely on the dipole approximation, however, the contribution of non-dipolar terms is expected to become increasingly important at higher photon energies (as will be available at the APS). These non-dipolar interactions (e.g. electric quadrupole and magnetic dipole) were predicted to strongly modify the angular distribution of photoelectrons. A new instrument capable of measuring photoelectron angular distributions was designed, constructed, and implemented in the measurement of the angular distribution of photoelectrons ejected from the $\mathrm{Ar} 1 \mathrm{~s}, \mathrm{Kr} 2 \mathrm{~s}$ and $2 \mathrm{p}$ shells using $2-5-\mathrm{keV}$ polarized X-rays. In these cases, the measured asymmetries were in good agreement with a non-relativistic, independent particle model. However, deviations from these simple models are expected at higher energies in heavier atoms where relativistic effects are expected to play a significant role.

In 1995 , the atomic physics group joined an existing collaboration with the Frankfurt, GSI and Kansas State University groups to use the COLTRIMS (coldtarget recoil-ion momentum spectroscopy) technique to make kinematically complete measurements of photon-atom interaction in He over a wide range of energies ( $85 \mathrm{eV}$ to $60 \mathrm{keV}$ ). These experiments were conducted at the Advanced Light Source in Berkeley and the ESRF in Grenoble, France. The experiments measure the cross sections for single-and double-photo or Compton ionization of He. The ratio $R=\sigma^{++} / \sigma^{+}$is sensitive to approximations and assumptions about electron correlations in the bound initial state and in the final state of the two escaping electrons and thus has been the subject of much theoretical and experimental investigation recently. The COLTRIMS technique is able to cleanly 
separate the contributions of the photo- and Compton-ionization channels, unlike simple time-of-flight techniques. The low-energy study, photon energy range of $85-400 \mathrm{eV}$, obtained values for $\mathrm{R}$ that were roughly $25 \%$ lower than previous measurements and resolved a discrepancy between earlier measurements and the value expected from numerous charged-particle impact experiments. The highenergy study, $60 \mathrm{keV}$, determined a value for $\mathrm{R}$ with sufficient precision to discriminate between various theories.

During this year, the development of Raman spectroscopy as a structural tool continued. Specifically, systematic measurements of the $X$-ray scattering from the L-shell of Xe as a function of excitation energy through the threshold region confirmed current theoretical understanding of these resonance processes. However, these studies were performed with experimental resolution comparable to the natural width of the core-excited state and thus some finer details of the scattering process (e.g. interference effects) were obscured. Efforts to develop optics and spectrometers with enhanced resolution are currently underway.

The development of photon-electron coincidence techniques to enable the detailed understanding of complex vacancy decay mechanisms continued. In particular, the complex decay of the 1 s hole state in Ar created by X-ray absorption was studied. The initial demonstration showed that by recording the $\mathrm{Ar} 1 \mathrm{~s}$ photoelectron spectrum in coincidence with X-ray fluorescence one could eliminate the line-broadening and shifting effects associated with post-collision interaction with $\mathrm{K}$-Auger electrons. The technique was also applied to the problem of understanding the L-Auger electron spectra produced by vacancy cascades. An additional photon-detection channel was added at $90^{\circ}$ to explore angular correlations between the electrons and photons.

\section{A. ACCELERATOR-BASED ATOMIC PHYSICS}

The narrow energy spread of beams from ATLAS together with the continuous energy variability and capability of operation in a deceleration mode make it an ideal machine for the study of the atomic physics of highly-ionized atoms. The opportunities offered by ATLAS attract a number of outside groups. Atomic structure studies are done by a group from the University of Notre Dame led by A. E. Livingston and H. G. Berry, and groups from the University of Toledo (L. Curtis and S. Cheng), University of Nevada (R. Ali), and GSI in Germany (led by P. Mokler) pursue atomic physics experiments at ATLAS. Also, the ATLAS atomic physics group has participated in experiments in other labs including the atomic collisions laboratory at Kansas State University and the GSI laboratory in Germany. Atomic physics experiments at ATLAS produced some of the most precise determinations of lifetimes of few-electron ions in high-Z systems. Such measurements are sensitive to higher-order relativistic corrections to the calculations which depend strongly on Z. Other spectroscopy programs at ATLAS are aimed at studying the atomic structure of highly-charged few-electron ions. Precise measurements of transition energies test relativistic and radiative corrections to the energy-level calculations as well as correlation effects in the simplest systems where such effects are present. Ultraviolet spectroscopy is used to study transitions within the $n=2$ shell of two- and three-electron ions.

The BLASE (beam-laser) facility provides ion beams for precision measurements of structure parameters (hyperfine structure and lifetimes) of atoms in low ionization states. The hyperfine structure measurements have stimulated and continue to guide development of $a b$ initio theoretical techniques for transition metal ions with highly interleaved configurations. The lifetime measurements provide benchmark data with which to test many-body perturbation theory calculations in simple systems with a single valence electron and have bearing on the interpretation of atomic parity nonconservation experiments. 


\section{a. Measurement of Short Lifetimes in Highly-Charged Ions using a Two-Foil Target (R. Ali, * R. W. Dunford, D. S. Gemmell, M Jung, E. P. Kanter, C. Kurtz, T. LeBrun, K. E. Rehm, L. Young, B. J. Zabransky, and S. Cheng†ं)}

One of the frontiers in the study of the atomic physics of highly-charged ions is the measurement of lifetimes in the $100 \mathrm{fs}$ to $10 \mathrm{ps}$ regime. The standard technique for measuring lifetimes of states in highly-charged ions is the beam-foil time-of-flight method in which the intensity of an emission line is monitored as a function of the separation between the exciting foil and the portion of the beam being viewed by the detector. This method becomes increasingly difficult as the decay lengths of the states of interest become shorter. At a typical beam velocity of $10 \%$ of the speed of light, the beam travels 30 microns in a picosecond. The standard beam-foil time-of-flight method necessitates observation of the decay radiation within one or two decay lengths from the foil while preventing the detectors from observing the beam spot at the foil. For short-lived states this requires tight collimation of the detector with a resulting loss in solid angle. In an attempt to circumvent these difficulties, we have begun exploring a new method for measuring ultrashort atomic lifetimes utilizing a twofoil target.

As a specific case to demonstrate the feasibility of our method, we began studying the decay of the $2{ }^{3} \mathrm{P}_{2}$ level in He-like $\mathrm{Kr}^{34+}$. This level has a calculated lifetime of 9.5 ps which corresponds to a decay length of $380 \mu \mathrm{m}$. For krypton, theory predicts that $90 \%$ of the $2{ }^{3} \mathrm{P}_{2}$ states decay via $\mathrm{M} 2$ radiation to the ground state. A measurement of the lifetime of this state would contribute to an important current problem which concerns the understanding of atomic structure when both electron correlations and relativistic effects are simultaneously important.

Our target consists of two foils arranged parallel to each other and perpendicular to the beam velocity. The separation between the foils can be varied accurately from a few microns to several $\mathrm{mm}$. In the experiment $700-\mathrm{MeV} \mathrm{Kr}$ ions are stripped to the He-like charge state after ATLAS and directed to the spectrographbeam line (Area III) where they enter a target chamber containing the two-foil target.

\footnotetext{
*University of Nevada, Reno, †University of Toledo
}

In the first foil, some of the ions are excited to the Helike ${ }^{3}{ }^{3}{ }_{2}$ state (as well as other long-lived excited states). After drifting in the vacuum between the foils, the ions pass through the second foil where ions which remain in excited states are preferentially stripped because of the enhanced loss cross section for $n \geq 2$ electrons. In contrast, those ions which decay to the He-like ground state between foils preferentially remain in that state after passage through the second foil. As the distance between the foils is increased, the fraction of ions in the $\mathrm{H}$-like charge state decreases because more of the ions have decayed before reaching the second foil. Thus, by monitoring the fraction of the beam in the H-like charge state as a function of the foil separation, one can, in principle, determine the decay length. The charge states emerging from the second foil are monitored using the Area III spectrograph.

Our initial runs utilizing the two-foil target provided encouraging results. By observing $n=2 X$-ray emission downstream of the second target, we were able to use the foil separation to provide a $10 \%$ measurement of the lifetime of the He-like $2{ }^{3} \mathrm{P}_{2}$ level. The expected dependence of the $35^{+}$charge-state fraction on the foil separation was confirmed. Surprisingly though, we also saw significant changes in the intensities of all the other charge states emerging from the second foil.

We extended those measurements of charge-state fractions as a function of foil separation to demonstrate that the principal source of that dependence originates from the decay of $n>2$ excited states between the foils. In contrast to the previous X-ray measurements, the less selective charge-state measurements probe all excited states simultaneously. The results are found to be consistent with the well-known power-law dependence of Rydberg-fed atomic transitions observed in beam-foil studies. However, these measurements probe such cascades on a much shorter time scale than has hitherto been accessible. Furthermore, this work represents the first clear demonstration of the influence of Rydberg populations on the charge-state evolution of such highly-charged foil-excited ions. 


\section{b. Spectral Distribution of the Two-Photon Decay of He-Like Krypton

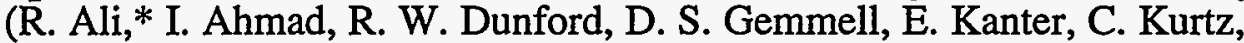 B. J. Zabransky, S. Cheng, $\uparrow$ L. Curtis, $\uparrow$ A. E. Livingston, $\ddagger$ and P. H. Mokler§)}

The $2{ }^{1} \mathrm{~S}_{0}$ level in He-like ions cannot decay to the ${ }^{1}{ }^{1} S_{0}$ ground state by emission of a single photon because of the requirement to conserve angular momentum in the decay. So this state decays to the ground state by emission of two photons. The singles spectra from these decays are continua which have a maximum at half the transition energy. The sum of the energies of the two photons equals the transition energy. Two-photon decay is a second-order process involving virtual intermediate states, so the two photons are emitted in coincidence. The sum over intermediate states involves all discrete states plus the positive- and negative-energy continuum states.

We measured the shape of the continuum radiation from decay of the $2{ }^{1} \mathrm{~S}_{0}$ level in He-like krypton (Fig. V-1). These data provide information on the details of the two-photon transition probability, beyond existing lifetime measurements; much as measurements of differential cross sections give more information than total cross-section measurements. Also, the measurements explicitly address the question of the handling of the negative-energy states in He-like ions, and this is an issue of current theoretical interest. Two-photon decay measurements test our understanding of the entire structure of an ion since a sum over the complete set of intermediate states is required and both energy levels and wavefunctions must be understood.

In the experiment, a beam of ${ }^{84} \mathrm{Kr}$ ions provided by ATLAS was stripped in a $200-\mu \mathrm{g} / \mathrm{cm}^{2}$ carbon foil and the $34^{+}$charge state was magnetically selected and directed to our target chamber. The ions were excited by a $10-\mu \mathrm{g} / \mathrm{cm}^{2}$ carbon foil. The region of the beam downstream of the foil was viewed by an array of four $\mathrm{X}$-ray detectors. Two-photon decays were identified as coincidences for which the energies of the two X-rays added up to the transition energy.

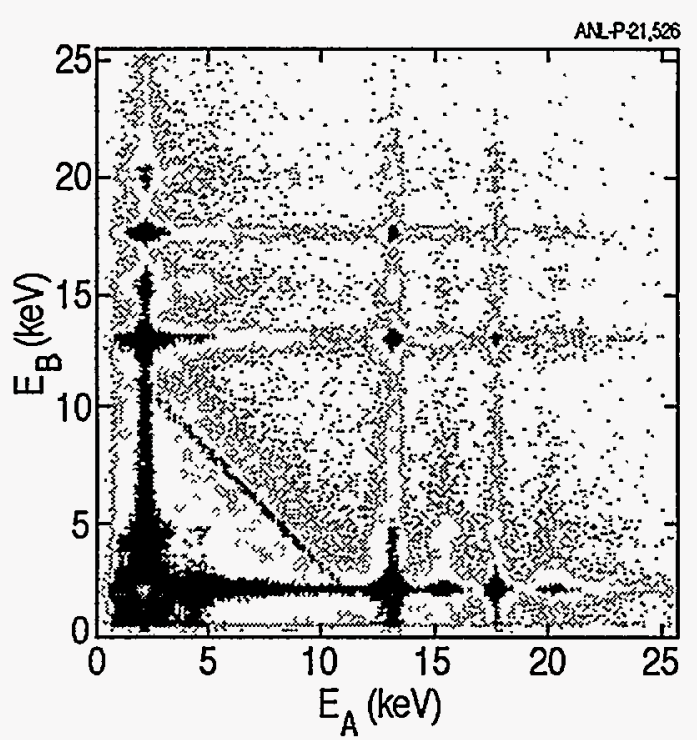

Fig. V-1. Two-dimensional scatter plot for coincidence events in detectors $A$ and $B$. The diagonal line represents the two-photon decays from the $2{ }^{1} S_{0}$ level of He-like $\mathrm{Kr}$.

The interpretation of our data requires that we determine the coincidence efficiency of our detection system. Several considerations are involved in this. We must characterize both the intrinsic detector efficiency and the efficiency of the acquisition electronics as a function of photon energy. In addition, we must take into account such details as the beam velocity, the decay distribution of beam ions, the angular distribution of decay radiation and the detailed geometry of our detectors. In order to properly account for all of these factors, we developed a Monte-Carlo simulation program to model our experiment. To provide precise information for inclusion in the code, careful measurements were made of the geometry of the foil holders, collimators, and detector crystals. The intrinsic efficiencies of the detectors were determined using a set of radioactive sources whose absolute intensities were measured.

\footnotetext{
*University of Nevada, Reno, $\nmid$ University of Toledo, $\ddagger$ University of Notre Dame, §GSI, Darmstadt, Germany
} 


\section{c. Wavelength and Lifetime Measurements in Highly-Ionized Atoms}

(H. G. Berry,* S. Cheng, $\uparrow$ L. Curtis, $\uparrow$ R. W. Dunford, D. S. Gemmell, E. P. Kanter, K. Kukla* A. E. Livingston,* F. G. Serpa,* and C. Vogel-Vogt*)

In the past year, final data analysis was completed for measurements of the $1 \mathrm{~s} 2 \mathrm{~s}{ }^{3} \mathrm{~S}-1 \mathrm{~s} 2 \mathrm{p}{ }^{3} \mathrm{P}$ transition wavelengths in $\mathrm{He}-\mathrm{like} \mathrm{Ar}^{16+}$. The experiments were done with the 1-m normal-incidence monochromator on the atomic physics beamline at ATLAS. The experiment utilized beam-foil spectroscopy with position-sensitive photon detection. The wavelength results are 559.944(16) $\AA$ for the $\mathrm{J}=1-2$ transition and relativistic corrections to the $n=2$ state fine structures

*University of Notre Dame, †University of Toledo measurements confirm the significance of $(\mathrm{Z} \alpha)^{4}$ $661.533(18) \AA$ for the $\mathrm{J}=1-0$ transition. The experiments are sensitive to the relativistic contributions in the transition energies to $<500 \mathrm{ppm}$ and to the total QED correction to $0.5 \%$. The suggested by recent calculations. The small remaining discrepancies between these measurements and the accurate theoretical values suggest the magnitude of uncalculated higher-order QED corrections is about $0.15(Z \alpha)^{4}$ a.u.

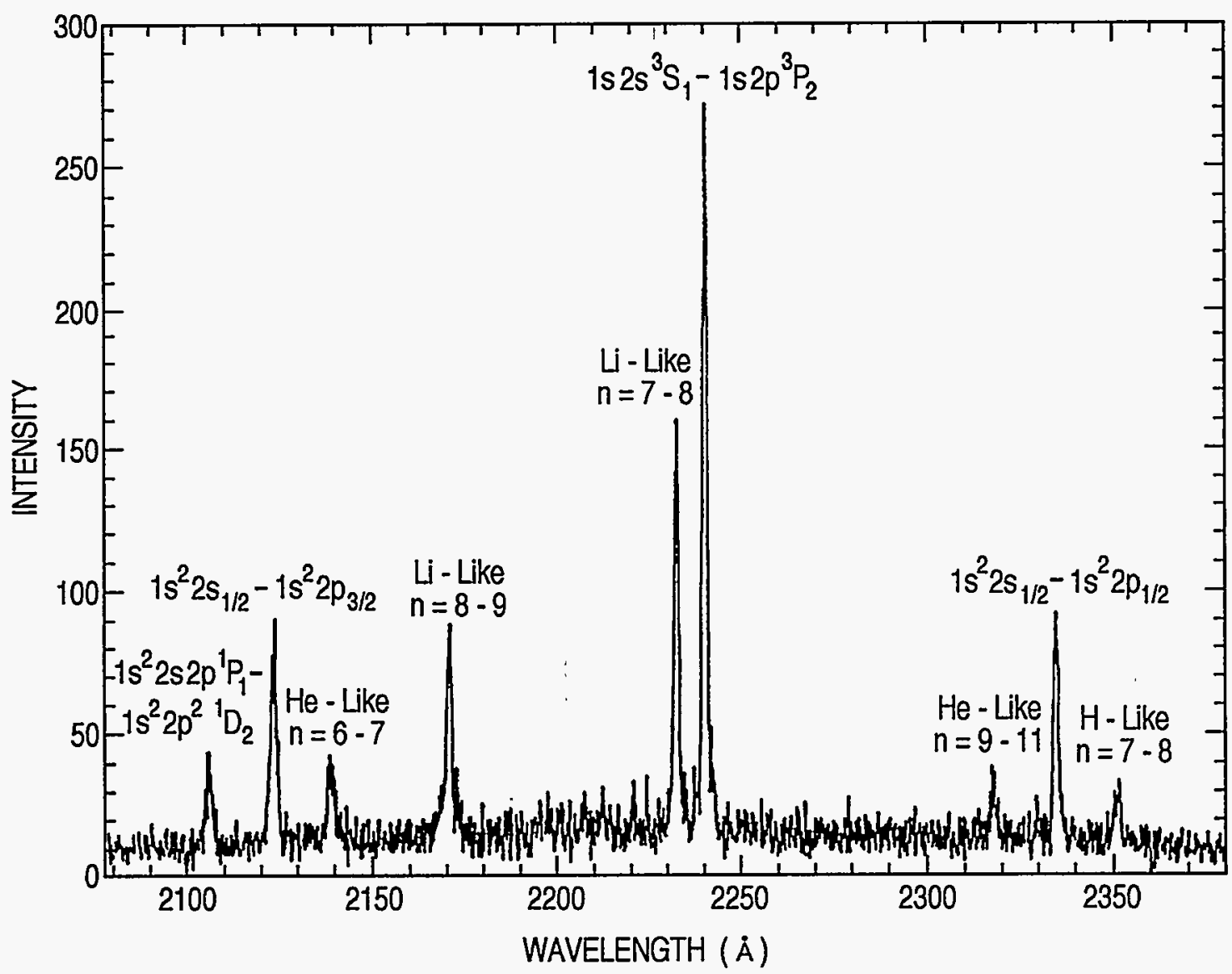

Fig. V-2. Prompt spectrum of highly ionized argon showing the $1 s 2 s^{3} \mathrm{Si}_{1--1 s 2 p}{ }^{3} \mathrm{P}_{2}$ transition in the fourth order of diffraction. (Intensity is in arbitrary units.) 
We have also begun a program to test relativistic Hartree-Fock calculations in 3-, 4- and 5-electron systems by making precision wavelength and lifetime measurements. We made accurate wavelength and lifetime measurements of the spectra of multielectron krypton in the far ultraviolet region, at wavelengths of 50 to $400 \AA$ (Fig. V-2). Data analyses are still in progress. Our spectra are taken using a beam-foil chamber coupled to a $2.2-\mathrm{m} \mathrm{McPherson}$ grazingincidence monochromator. This system provides efficient light collection and takes advantage of the time structure of ATLAS. The exit slits of the monochromator were replaced by a position-sensitive channel plate with high spatial and time resolution. The channel plate is mounted on a movable chariot on the Rowland circle of the monochromator. The chariot can be translated along the circle, and it can be rotated about a tangent point of the circle. This latter movement allows us to optimize resolution and efficiency depending on the needs of the experiment.
Backgrounds due to electrons, neutrons, gamma rays, and dark count from the detector are greatly reduced using a time window (1-2 ns) triggered from the ATLAS beam-pulse structure ( 82 ns pulse separation).

Another program recently begun at ATLAS, is the spectroscopy of copperlike uranium. Although some information is known about the transition wavelengths in this species, there are no lifetime data for well-resolved $\mathrm{Cu}$-like spectra for $\mathrm{Z}>55$. Our experiments test the relativistic calculations for complex, many-electron atomic systems. The beamfoil spectroscopy station on the ATLAS atomic physics beamline was used with the grazing-incidence monochromator mounted. Transition energies in the $23 \AA$ to $120 \AA \AA$ regime were measured. The foil holder was mounted on a translation mechanism driven by a stepper motor to allow lifetimes to be measured by the beam-foil, time-of-flight technique. Preliminary data were obtained and analysis is in progress.

\section{d. Positron Production in Heavy-Ion Collisions (R. W. Dunford and the APEX collaboration)}

The ATLAS Positron Experiment, APEX, was built to study positron emission in collisions between very heavy ions. Narrow peaks were observed in such collisions at GSI Darmstadt in the spectra of positrons and in the sum-energy spectra of electron-positron coincidences. APEX is a second-generation experiment which was specifically designed to look for

\section{e. Spectroscopy of H-Like Uranium GSI, Darmstadt, Germany)}

Precision data were taken of the ground-state transition energies in H-like uranium at the ESR storage ring at GSI in Darmstadt, Germany. The experiment is sensitive to quantum electrodynamics (QED) in strong Coulomb fields. Bare uranium ions at $360 \mathrm{MeV} / \mathrm{u}$ produced by stripping a beam from the heavy-ion synchrotron SIS were injected into the ESR storage ring. Up to $10^{8}$ stored ions were accumulated and cooled by an electron cooler. A gas-jet target in the ESR with thickness of about $10^{12}$ particles $/ \mathrm{cm}^{2}$ was used to produce excited H-like uranium ions via electron capture. The $\mathrm{X}$-rays emitted following capture the coincidence events and measure the opening angle between electrons and positrons. The initial results from APEX indicate no evidence for the sharp sumenergy lines. Data were collected for ${ }^{238} \mathrm{U}+{ }^{181} \mathrm{Ta}$ at $5.95,6.10$ and $6.3 \mathrm{MeV} / \mathrm{u}$ and ${ }^{238} \mathrm{U}+{ }^{232} \mathrm{Th}$ at 5.95 $\mathrm{MeV} / \mathrm{u}$. The data were analyzed to place limits on the possibility of the existence of a neutral particle of mass $1.4-2.1 \mathrm{MeV} / \mathrm{c}^{2}$.

were measured with an array of solid-state detectors. Data were taken at the incident energy of $360 \mathrm{MeV} / \mathrm{u}$ and at energies of $220 \mathrm{MeV} / \mathrm{u}, 68 \mathrm{MeV} / \mathrm{u}$, and 49 $\mathrm{MeV} / \mathrm{u}$. The latter three energies were obtained using the deceleration capability of the ESR. This was the first dedicated spectroscopy experiment at the ESR performed with decelerated heavy ions. The resulting high-quality spectra show the Lyman, Balmer, and Paschen series of $\mathrm{H}$-like $\mathrm{U}^{91+}$. These data are being analyzed to obtain a precision measurement of the ground-state Lamb shift in H-like uranium 


\section{f. Interactions of High-Energy, Highly-Charged Xe Ions with $\mathrm{C}_{60}$}

(R. Ali, * H. G. Berry, $\dagger$ S. Cheng, $\ddagger$ R. W. Dunford, H. Esbensen, D. S. Gemmell,

E. P. Kanter, T. LeBrun, L. Young, and W. Bauer§)

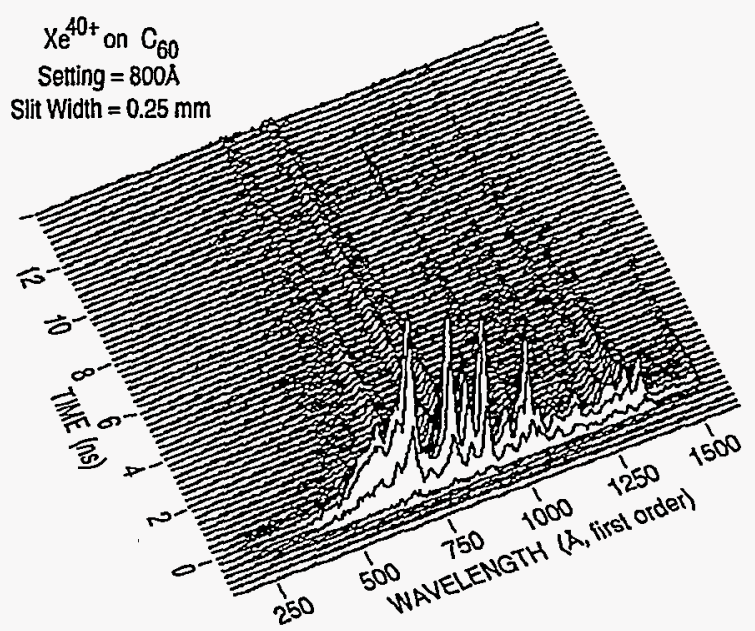

Fig. V-3. Spectrum of wavelength vs. time relative to the pulse structure of the ion beam. Zero time corresponds to the center of the prompt peak in the spectrum. These data were taken with a $625 \mathrm{MeV}$ $\mathrm{Xe}^{40+}$ beam incident on the $C_{60}$ jet target. The spectrometer dial was set to $800 \AA$ (first order).
$\mathrm{C}_{60}$ molecules in the vapor phase were bombarded by $\mathrm{Xe}^{35+}$ and $\mathrm{Xe}^{18+}$ ions with energies in the range 420 - $625 \mathrm{MeV}$ and the ionization and fragmentation measured by time-of flight techniques. The bombarding energies exceeded those used in previous studies by several orders of magnitude. The mass distribution of the resulting positively-charged fragments was determined. We developed a theoretical model indicating that the total interaction cross section contains roughly equal contributions from a) excitation of the giant plasmon resonance, and b) large-energytransfer processes that lead to multiple fragmentation of the molecule. Additional spectroscopic and lifetime measurements were made on the VUV emissions from the fragments generated in these interactions (Fig. V-3).

*University of Nevada, Reno, tUniversity of Notre Dame, $\ddagger$ University of Toledo, §NSCL, Michigan State Univ.

\section{g. Precision Lifetime Measurements by Single-Photon Counting (L. Young, C. A. Kurtz, K. Lykke,* R. G. DeVoe†)}

There is active interest in the accurate measurement of lifetimes of excited states in alkalis in order to test $a b$ initio theories of atomic structure. The general interest is heightened because dipole matrix elements (which can be directly extracted from lifetime measurements) are required for the interpretation of atomic parity nonconservation (PNC) measurements which now approach accuracy sufficient to probe physics beyond the standard model. There are currently several methods accurate at the sub- $1 \%$ level employed for determination of dipole matrix elements, including the fast-beam laser method, photoassociative spectroscopy, and time-correlated single-photon counting. While the fast-beam laser method yielded the most precise published lifetimes to date, measurements using this method consistently yielded lifetimes that are long $(\approx 5 \sigma)$ relative to theory in light alkalis. Earlier, we demonstrated that an alternative method, time-correlated single-photon counting, is capable of measuring lifetimes with comparable accuracy. Current efforts focus on achieving an extreme level of accuracy in lifetime measurements, i.e. $<0.1 \%$, using the single-photon counting technique on a single trapped $\mathrm{Ba}^{+}$ion. The $\mathrm{Ba}^{+}$ion is isoelectronic to $\mathrm{Cs}$ and thus the accurate $a b$ initio calculations performed for $\mathrm{Cs}$ are directly transportable.
This year at Argonne, we isolated an important systematic problem in the detection channel for $\mathrm{Ba}^{+}$. A chamber was constructed for measuring the instrument function (response to a delta function light pulse) of the photomultiplier tube used for the $\mathrm{Ba}^{+}$ experiments as a function of position on the photocathode. The delta function light pulse at $\approx 400$ $\mathrm{nm}$ was produced by frequency doubling the output of an ultrafast Ti-sapphire laser ( $\approx 200 \mathrm{fs}$ pulsewidth) and Rayleigh scattering the pulse into the photomultiplier. The photomultiplier response was measured using time-correlated single-photon counting. Variation of the position illuminated on the photocathode drastically altered the pulse shape. More quantitatively, the pulse width varied by a factor of $\approx 2$ with a change in position of $0.18 "$. Since the image of the trapped ion moves around on the photocathode during a run, these position-sensitive effects could lead to variations in the mean lifetime of $\approx 40 \mathrm{ps}(\approx 0.5 \%)$. This illuminates the need for extra stability in the detection optics chain, which has a magnification of $\mathrm{x} 1200$.

\footnotetext{
*Chemistry Division, ANL, †IBM Almaden Research Laboratory
} 
h. Hyperfine Structure Studies of Transition Metals (L. Young, C. Kurtz, S. Hasegawa,* D. R. Beck, $\dagger$ and D. Datta $\dagger$ )

Experimental work on hyperfine structure in transition metal ions is motivated by the inability of $a b$ initio theories to predict magnetic dipole and electric quadrupole interaction constants with a reasonable degree of accuracy for systems with more than a single-valence electron. These constants measure $\left(1 / \mathrm{r}^{3}\right)$ matrix elements of specific electronic states, and thus probe our knowledge of the wavefunction near the nucleus unlike the lifetime studies described above. Our early work on a two-valence electron ion, Sc II, demonstrated the inability of the multiconfiguration Dirac-Fock method to predict even the correct sign of the magnetic dipole interaction constant, A.

*University of Tokyo, Japan

†Michigan Technological University
This in turn stimulated theoretical work using the relativistic configuration interaction method, which was markedly more successful. Our experimental work systematically investigated two-, three- and fourvalence electron-transition metals using the laser-rf double resonance method. During this past year, our combined experimental and theoretical work on $(5 \mathrm{~s}$ 4d) ${ }^{4}$ manifold of $\mathrm{Nb} I I$ was published. Experimental studies of the four-valence electron system $\mathrm{V}^{+}$in the $(4 \mathrm{~s}+3 \mathrm{~d})^{4}$ manifold are complete. A manuscript is in preparation. The theoretical difficulties for the $3 d$ transition metals, noted earlier for the three-valence electron systems, relative to the $4 \mathrm{~d}$ transition metals, appear to be repeated for the four-valence electron systems $\left(\mathrm{Nb}^{+}\right.$and $\left.\mathrm{V}^{+}\right)$.

\section{B. ATOMIC PHYSICS AT SYNCHROTRON LIGHT SOURCES}

The research program in atomic, molecular, and optical physics with X-rays complements the program of accelerator-based atomic physics research within the Division. Both programs involve basic research focused principally on atomic structure. The synchrotron radiation work also enables the study of photon interactions with matter at extremely high energy. The selective nature of the photon excitation provides unique advantages in the detailed study of inner-shell dynamical processes. We are members of the Basic Energy Sciences Synchrotron Radiation Facility (BESSRC) which will eventually operate two sectors (four beamlines) at the Argonne Advanced Photon Source (APS). Initially, three beamlines will be instrumented with an undulator, elliptical multipole wiggler, and bending magnet. As members we will have access to all three beamlines on a timeshare basis with the other members of BESSRC, namely, materials science, chemistry, geosciences and Northern Illinois University.

The atomic physics group operated Brookhaven's X24A NSLS beamline jointly with the Quantum Metrology Division of NIST through September 1995. This agreement provided the group with priority access to this state-of-the-art facility for research in AMO physics with synchrotron radiation. The experimental techniques developed at the NSLS are in large measure transferable to the APS. These techniques include electron-photon coincidence studies, X-ray resonant Raman spectroscopy, Auger resonant Raman spectroscopy, and X-ray absorption spectroscopy.

As the experimental program for AMO physics at the Argonne APS is being readied, we have actively sought collaborations with groups performing experiments at other facilities that possess technical expertise that will be useful in the future. In particular, the COLTRIMS technique (coldtarget recoil-ion momentum spectroscopy), developed by the Frankfurt and GSI groups and further utilized by the Kansas State University group, provides kinematically complete information on single and double photo- and Compton-ionization events in light gases and the great advantage of $4 \pi$ solid-angle detection. Already two experiments were performed at other synchrotron facilities (ALS in Berkeley and ESRF in Grenoble) in collaboration with these outside groups. We anticipate ongoing and strengthened collaborations in the future. 
a Non-Dipolar Asymmetries of Photoelectron Angular Distributions

(D. S. Gemmell, E. P. Kanter, B. Krässig, M. Jung, T. LeBrun, S. H. Southworth, and L. Young)

Current understanding of atomic photoionization phenomena is largely based on the dipole approximation of the photon/atom interaction. However, non-dipolar interactions were predicted to strongly modify photoelectron angular distributions due to interference between dipolar and non-dipolar amplitudes. These effects were generally ignored in experimental studies. We measured the non-dipolar contributions to the $\mathrm{Ar} 1 \mathrm{~s}$, $\mathrm{Kr} 2 \mathrm{~s}$, and $\mathrm{Kr} 2 \mathrm{p}$ photoelectron angular distributions using $2-5 \mathrm{keV}$ polarized $\mathrm{X}$-rays. The non-dipolar interaction results in a forward/backward asymmetry with respect to the photon beam which was measured by rotating an electron spectrometer about the polarization vector with a fixed polar angle of $54.7^{\circ}$, the "magic angle" which eliminates the angular dependence on the dipolar asymmetry parameter $\beta$ (Fig. V-4). The non-dipolar asymmetries for different atomic subshells vary with energy in different ways; at certain energies the photoelectron intensity is enhanced in the backward direction, while at other energies the asymmetry vanishes or is enhanced in the forward direction. In all

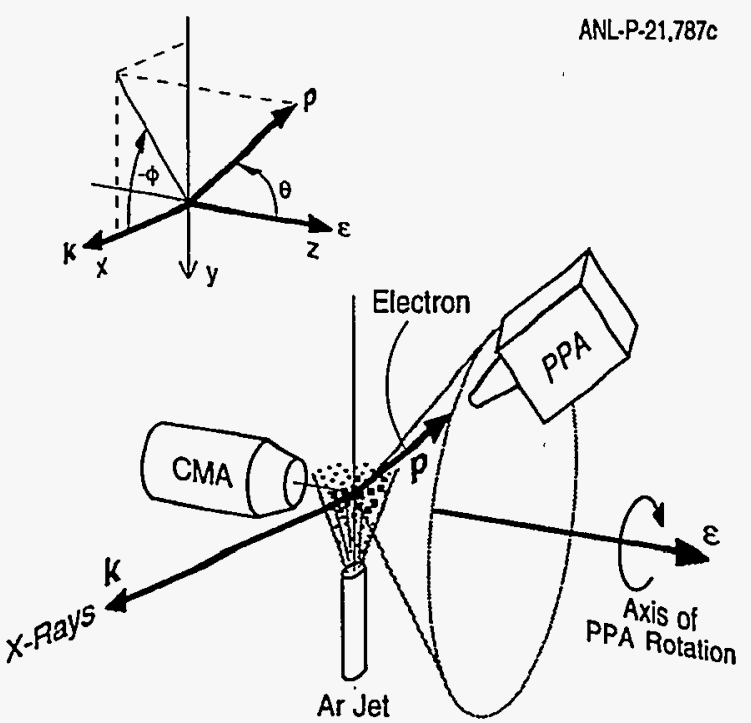

Fig. V-4. Schematic of the experimental setup used and its orientation with respect to the coordinate frame. The vectors $p, k$, and $\varepsilon$ indicate the directions of photoelectron momentum, photon propagation and linear polarization, respectively.
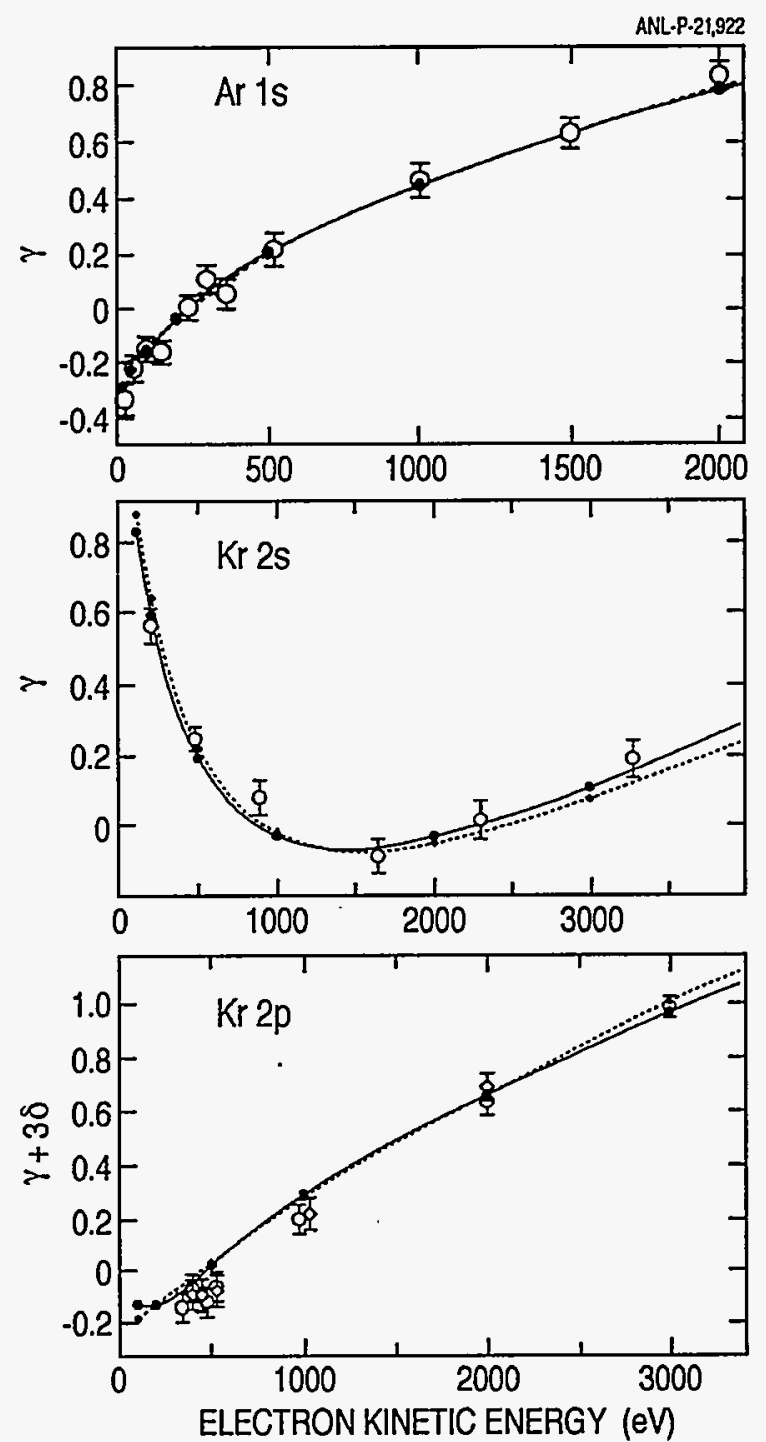

Fig. V-5 Energy dependence of the non-dipolar asymmetry parameters for $\operatorname{Ar}(1 s), \operatorname{Kr}(2 s)$ and $\operatorname{Kr}(2 p)$ photoelectrons.

cases, the measured asymmetries are in good agreementwith recent non-relativistic calculations which include interference between the electric-dipole and electric-quadrupole photoionization amplitudes (Fig. V-5). Future experiments at APS beamlines will investigate photoelectron angular distrịbutions at higher energies in heavier atoms where relativistic effects are expected to play a significant role. 


\section{b. Double and Single Photoionization of Helium by $60-\mathrm{keV}$ X-Rays (D. S. Gemmell, B. Krässig, L. Spielberger,* O. Jagutzki,* U. Meyer,* K. Khayyat,* R. Dörner,* and H. Schmidt-Böcking*)}

Understanding the role of electron-electron interactions in helium, which is the simplest neutral atomic system exhibiting such correlation effects, is basic to an understanding of the more general cases of manyelectron atoms and molecules. To this end there have been many experimental and theoretical studies of ionization of helium by photons. As a measure of the relative importance of electron-correlation effects, the determination of the ratio, $R$, of cross sections for double and single photoionization $\left(R=\sigma^{++} / \sigma^{+}\right)$as a function of photon energy is of particular interest. Calculations of this quantity are highly sensitive to approximations and assumptions about electron correlations both in the bound initial state and in the final state of the two escaping electrons. Electron correlations govern the energy dependence of the ratio, $R$, from threshold to high energies: in the threshold region the delicate correlated motion of the two slow electrons gives rise to a photon-energy dependence of the cross section described by the Wannier threshold law. With increasing excess energies the correlation effects enhance the cross section for double ionization and the ratio, $R$, rises until at about $175-\mathrm{eV}$ photon energy a broad maximum, $R_{\max }$, is reached. For higher photon energies $R$ decreases and approaches an asymptotic value. Whereas at low energies, including the region of the maximum, the ionization cross sections are dominated by photoabsorption, at higher photon energies (above about $6 \mathrm{keV}$ ) the major contributing process comes from Compton scattering, and the question of an asymptotic value of $\mathrm{R}$ at photon high energies becomes contingent on which of the two mechanisms is considered. 'At these high energies, the Compton scattering cross section is roughly constant at $\sim 1$ barn, while the photoabsorption cross section decreases rapidly with photon energy: $\sigma \approx 480 \mathrm{E}^{-7 / 2}$ barns, where $\mathrm{E}$ is in $\mathrm{keV}$.

*Institute for Nuclear Physics, Universität Frankfurt, Germany

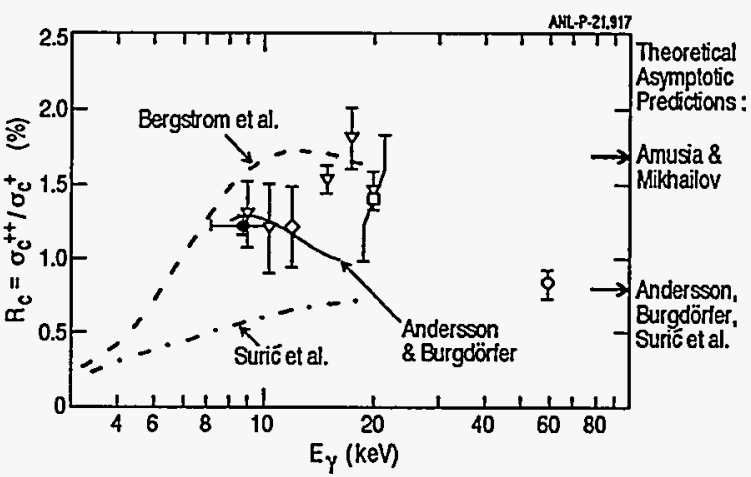

Fig. V-6. Ratios of double to single ionization, $R_{c}$, after Compton scattering. $O$ timework. Previous experimental data, and various theoretical predictions are also shown.

Our experiment was motivated by the desirability of making a measurement of $R$ at a photon energy significantly higher than those explored previously. For this we chose the $60-\mathrm{keV}$ X-ray beam at the ID15, beamline 25 of the European Synchrotron Radiation Facility (ESRF) in Grenoble, France. This beam arises from a wiggler insertion device and a Bragg-type monochromator employing a single bent $\mathrm{Si}(311)$ crystal. To minimize background problems, we employed the COLTRIMS (Cold-Target Recoil-Ion Momentum Spectroscopy) technique and a supersonic cold jet of helium gas. Using a position-sensitive channel-plate detector and time-of-flight measurement, we were able to cleanly identify the $\mathrm{He}^{++}$ions, which were produced at the rate of one every few minutes. We were able to determine a value of $R=0.84$ $(+.08 \%,-.11 \%)$ and this serves to discriminate between several theoretical calculations of the asymptotic limit (Fig. V-6). 


\section{c. Inclusive Recoil Momentum Spectroscopy of Single and Double Ionization of He by 85-400 eV Photons (M. Jung, E. P. Kanter, R. Dörner, * S. Kravis,* C. L. Cocke, ${ }^{*}$ T. Vogt, $\uparrow$ V. Mergel,$\uparrow$ M. Unverzagt, $\uparrow$ L. Spielberger,$\uparrow$ M. Damrau, $\uparrow$ O. Jagutzki, $\uparrow$ I. Ali, $\uparrow$, B. Weaver,$\uparrow K$. Ullmann, $\uparrow$ H. Schmidt-Böcking, $\uparrow$ H. Khemliche, $\ddagger$ M. H. Prior, $\ddagger$ T. Warwick, $\ddagger$ J. Ullrich,§ C. C. Hsu,II B. Sonntag,** E. Rotenberg, $\dagger \dagger$ J. Denlinger, $\ddagger \ddagger$ S. T. Manson, $\S \S$ and J. FeaginđID}

Double ionization of He by a single photon is perhaps the most fundamental many-electron process. Strictly forbidden in an independent electron approximation, this process only occurs because of electron-electron correlations in the ground state of helium and in the final continuum states. Theoretical descriptions of double ionization are therefore extremely sensitive to the approximations employed to describe these correlated wave functions and hence provide a challenging test case for the desciption of electronelectron correlations. In recent years, several groups have attempted to provide such a test by measuring the asymptotic limit of the ratio ( $R$ ) between double and single ionization (see Section 1) at high energies where Compton scattering dominates. Equally challenging, however, is the low-energy regime where photabsorption dominates. In an effort to explore the region from threshold to beyond the maximum in $R$, we used cold-target recoil-ion momentum spectroscopy (COLTRIMS) to measure the ratio between double and single ionization of $\mathrm{He}$ by photons between $85 \mathrm{eV}$ and $400 \mathrm{eV}$. Because this technique is kinematically complete, we were able to eliminate many sources of systematic errors which plagued previous attempts to measure $\mathrm{R}$. The experiment was performed at the LBL Advanced Light Source. The values of $\mathrm{R}$ obtained are generally about $25 \%$ below those previously reported, but they are in good agreement with several recent theoretical calculations.

We also began examining the angular correlations between the two electrons in the case of double ionization and extracted triply-differential cross sections starting about $1 \mathrm{eV}$ above threshold.

\section{*Kansas State University, $\nmid$ Institute for Nuclear Physics, Universität Frankfurt, Germany, $\neq$ Lawrence Berkeley National Laboratory, §GSI, Darmstadt, Germany, \INational Tsing Hua University, Taiwan, **Universität

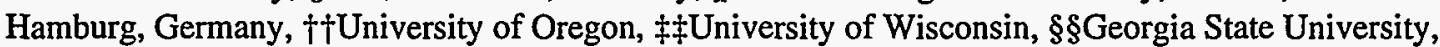 ФTCalifornia State University, Fullerton}

\section{d. Auger Resonant Raman Spectroscopy (T. LeBrun, S. H. Southworth, M. A. MacDonald, * and J. C. Levin $\dagger$ )}

Auger resonant Raman spectroscopy is analogous to inelastic X-ray scattering in which the outgoing photon is replaced by an ejected electron. As such it presents many of the same advantages as X-ray resonant Raman scattering, notably the potential for very high-resolution studies of deep inner shells. Indeed, because the final state consists of a singlycharged ion, the effective resolution below threshold is enhanced by the increased spacing of the Rydberg levels.

The ionic character of the final state also leads to other significant differences with respect to $\mathrm{X}$-ray scattering. For example, near threshold the photoelectron has little kinetic energy and can easily be overtaken and affected by the subsequently emitted Auger electron, a phenomenon known as post-collision interaction (PCI).

We recorded Auger resonant Raman spectra at both the $\mathrm{Ar} \mathrm{K}$ and $\mathrm{Xe}_{\mathrm{L}}$ edges. The Ar KLL Auger resonant
Raman spectrum, for example, clearly reveals resonant peaks with positive-linear dispersion and line shapes which correspond to resonant Auger Raman scattering. In addition, the continuum peak displays negative dispersion and asymmetric broadening characteristic of post-collision interaction with the photoelectron. Such features, resulting from bound and continuum excitations of the $K$ shell, can only be inferred from the X-ray absorption spectrum.

While some of the X-ray optics under development for the X-ray Raman measurements will also be used for Auger Raman studies, these measurements will also require high-resolution electron spectrometers suitable for electrons with kinetic energies up to 50 or even $100 \mathrm{keV}$ in order to take advantage of the promise of the APS. This is a significant challenge, but the small source sizes available at the APS coupled with recent advances in precision numerical machining techniques are making promising new options possible.

*Daresbury Laboratory, UK, †University of Tennessee 


\section{e. X-Ray Scattering and Fluorescence Studies (S. H. Southworth, K. E. Miyano,* Y. Ma, † and U. Arp $\ddagger)$}

$\mathrm{X}$-ray scattering and fluorescence studies were performed on atomic Ar, molecular OCS, and crystalline $\mathrm{KCl}$ using a bent-crystal spectrometer and tunable synchrotron radiation over the $2-5-\mathrm{keV}$ energy range. There is much current interest in understanding the basic physics of atomic excitation and ionization processes by $\mathrm{X}$-ray absorption and scattering. These processes were studied in atomic Ar by recording $\mathrm{X}$-ray fluorescence and elastic- and inelastic-scattering spectra as the excitation energy was tuned above and below the K-edge. Comparison will be made with calculated X-ray scattering spectra which include atomic binding effects.

Molecular X-ray absorption spectra typically display strong, subthreshold resonances due to excitation of an inner-shell electron to unoccupied valence molecular orbitals of specific symmetry. Excitation of these resonant states with a polarized $\mathrm{X}$-ray beam produces an aligned (spatially anisotropic) ensemble of excited molecules. Consequently, the X-ray fluorescence from resonantly-excited molecules can be strongly polarized and anisotropic. This phenomenon was observed in OCS molecules resonantly excited at the S K-edge. The observed resonant energy shifts and polarizations are in rough qualitative agreement with calculated resonant $\mathrm{X}$ ray spectra.

The states produced by resonant excitation of an innershell electron to a bound or near-threshold continuum level are highly nonstationary and participate as intermediate states in the excitation and decay process known as resonant Raman scattering. We have studied $\mathrm{X}$-ray resonant Raman scattering at both the $\mathrm{Cl}$ and $\mathrm{K}$ (potassium) K-edges of crystalline $\mathrm{KCl}$. An approximate model of the resonant Raman process was used to relate variations observed in the scattered X-ray spectra to resonant features in the X-ray absorption spectra.

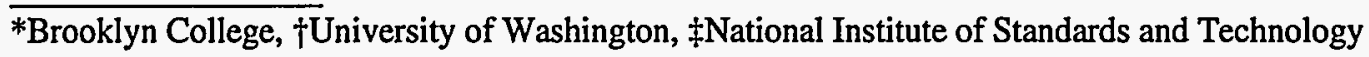

\section{f. X-Ray Resonance Raman Scattering (T. LeBrun, S. H. Southworth, M. A. MacDonald,* and R. D. Deslattes, $\uparrow$ )}

X-ray resonant Raman spectroscopy (resonant inelastic $\mathrm{X}$-ray scattering) recently emerged as an important spectroscopic tool for studying the electronic structure of the deepest inner shells of atoms, molecules, and solids. The great potential of the technique results from the ability to avoid the lifetime broadening associated with short-lived core-excited states and therefore resolve highly-excited states on a much finer scale. Resonant Raman scattering also presents other advantages that were barely tapped: measurements of polarization and angular anisotropies give information on the symmetries of the electronic states of atoms and molecules, including final states normally forbidden by dipole selection rules.

We performed measurements of the scattering from the $\mathrm{L}$ shell of Xe which confirm several aspects of the current theoretical understanding of these processes: e.g. the narrowing of lines on resonance and the behavior of Raman scattering near absorption edges. Our results, taken in the regime where the experimental resolution is similar to the natural width

*Daresbury Laboratory, UK, †National Institute of Standards and Technólogy of the core-excited state, are well reproduced by calculations based on the widely used model by Tulkki and Åberg. But such models neglect interference between the many possible intermediate states effects that are expected to be large when the experimental resolution is substantially less than the natural width of the intermediate states. The manner in which these interference effects will be manifested is still a subject of debate, and is a point of some importance because it is central to the issue of resolving electronic structure at a level finer than the natural width.

To address these important questions we have begun developing optics and spectrometers at the Advanced Photon Source to allow measurement of these processes in a regime where the experimental resolution is up to two orders of magnitude finer than the lifetime broadening. Measurements performed with this apparatus should not only reveal much about Raman scattering itself, but also allow the inner shells of atoms and molecules to be studied with a new level of sensitivity and precision. 


\section{g. Electron/X-Ray Coincidence Studies of Inner-Shell Processes (T. LeBrun, M. Jung, S. H. Southworth, and U. Arp,* J. W. Cooper, † and M. A. MacDonald $\ddagger$ )}

A new coincidence technique was developed to disentangle the complex processes involved in the decay of the $1 \mathrm{~s}$ vacancy in Ar following X-ray absorption (Fig. V-7). After excitation of the $1 \mathrm{~s}$

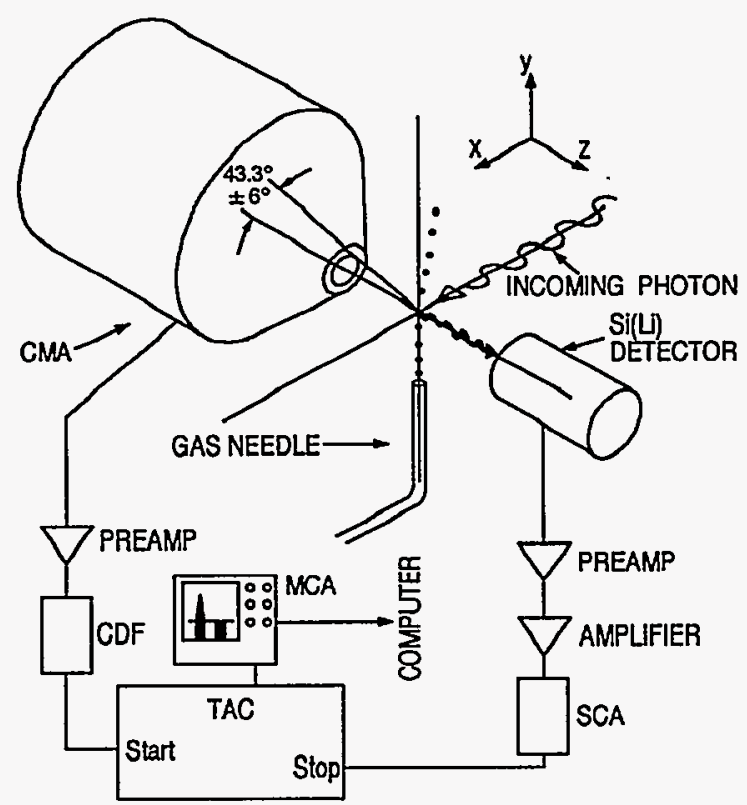

Fig. V-7. Diagram of instrumentation used to record electron spectra in coincidence with $x$-ray fluorescence following excitation by synchrotron radiation.

electron to a bound or continuum state the atom relaxes rapidly by Auger-electron emission or X-ray fluorescence. Most of these decay processes lead to unstable states which decay further, creating a cascade of decay processes and an array of final charge states, $\mathrm{Ar}^{1+}$ to $\mathrm{Ar}^{8+}$. The new technique uses coincident detection of X-rays and electrons to select decay pathways that involve emission of both an X-ray photon and electrons. The kinetic energy spectra of

*Natl. Inst. of Standards and Technology, †Inst. Sci and Tech., University of Maryland, $\ddagger$ EPSEC, Daresbury Lab., U.K. photoelectrons and Auger electrons produced in coincidence with fluorescent $\mathrm{X}$-rays are selectively recorded.

In the first demonstration of this technique, the $\mathrm{Ar} 1 \mathrm{~s}$ photoelectron spectrum was recorded in coincidence with X-ray fluorescence to eliminate the asymmetric broadening and shifting of the energy distribution which result due to post-collision interaction (PCI) with K-Auger electrons (Fig. V-8). These measurements allowed us to isolate PCI between different relaxation steps, yielding information about PCI effects due to secondary relaxation steps which were inaccessible previously.

We also applied this technique to the complex problem of understanding the L-Auger electron spectra produced by vacancy cascades. The Auger-cascade spectra recorded in coincidence with $\mathrm{X}$-ray fluorescence are relatively simple to interpret and give information on inner-shell processes which were not previously observed. These effects include the observation of resonant L-Auger spectra produced via initial resonant excitation of the K-shell and PCI between $1 \mathrm{~s}$ photoelectrons and L-Auger vacancy-cascade electrons.

Most recently we began to explore the angular correlations between the electrons and photons in these experiments by adding photon detectors at multiple positions. Fixing the angle between the ejected electron and photon also limits the kinematics of the processes one can observe. By selectively studying a subset of PCI processes in this way, we are able to observe unusual events in which the energy sharing between the photoelectron and Auger electron is opposite to that normally encountered.

At the APS these experiments will benefit from the increased intensity and higher photon energy which generates more complex relaxation processes while enabling new and more powerful detection strategies. The experience acquired by initiating these experiments at existing sources should allow us to extend the technique to yield a much more complete understanding of the decay of highly-excited states of atoms and ions. 


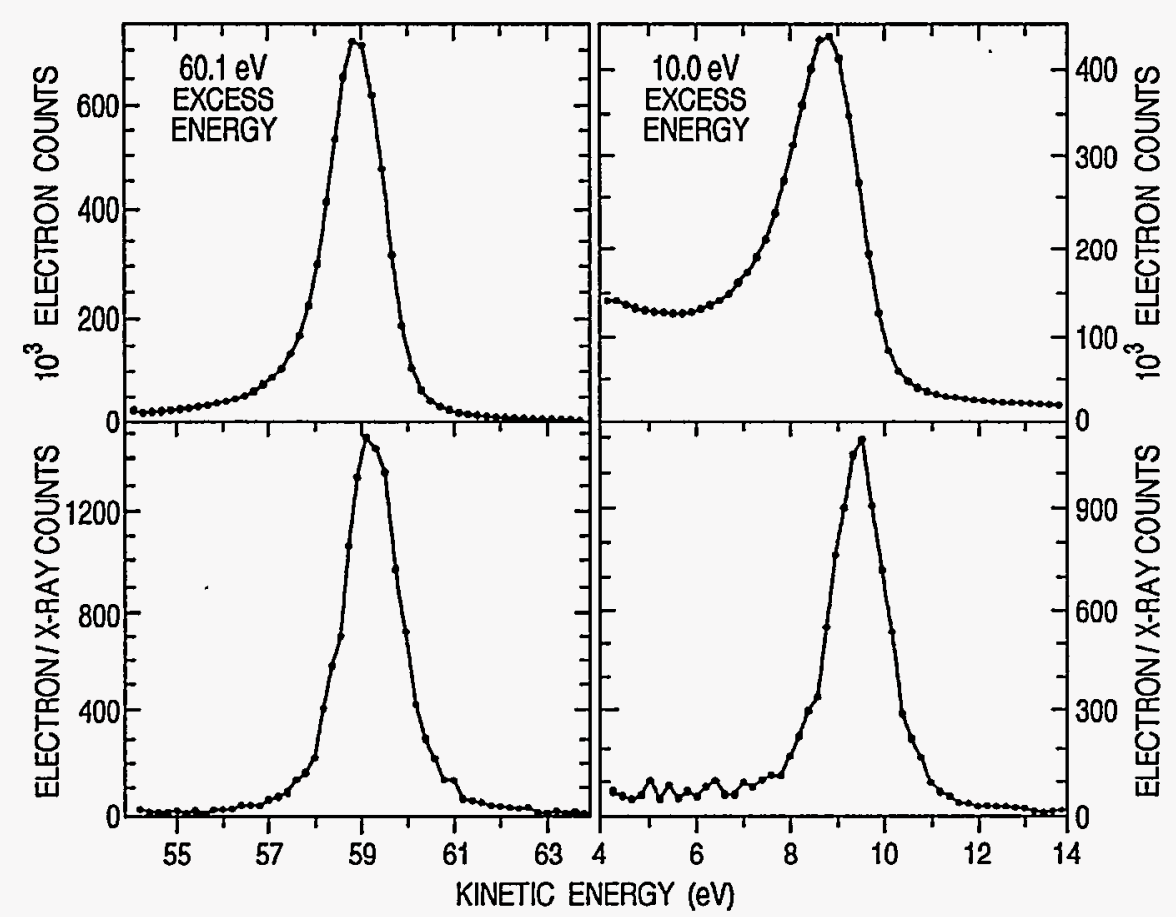

Fig. V-8. Argon $1 s$ photoelectron spectra (upper panels) and coincidence spectra between argon $1 s$ photoelectrons and $k$-shell $x$-ray fluorescence (lower panels). Results are shown for 10.0 and $60.1 \mathrm{eV}$ excess energies.

h. Preparations for Atomic Physics at the Advanced Photon Source (D. S. Gemmell, M. Jung, E. P. Kanter, B. Krässig, C. A. Kurtz, T. LeBrun, S. H. Southworth, L. Young, and B. J. Zabransky)

The Advanced Photon Source (APS) at Argonne produced the first $\mathrm{X}$-ray beam from a bending magnet source on March 26, 1995 and from an undulator source on August 9, 1995. In January 1996, the APS design goal for a stored beam of $100 \mathrm{~mA}$ at $7 \mathrm{GeV}$ was achieved with storage times of several hours. Data on the properties of the X-ray beams have been acquired and the process of commissioning user beamlines has begun. Construction of the lab/office modules surrounding the experimental hall is well under way, as is the construction of the central lab/office building, the APS conference center, and the user residence facility.

The Basic Energy Sciences Synchrotron Research Center (BESSRC) was allocated two out of the 34 sectors available for research at the APS. Each sector contains a bending-magnet source and an insertion device source. Initially three beamlines will be constructed: a bending magnet line (expected to be ready for use in January 1996), an undulator line (March 1996), and an elliptical multipole wiggler line (June 1996). Much of the atomic physics research conducted at the APS will use these beamlines. Indeed, a large hutch designed primarily for atomic physics and capable of containing sizable items such as a small accelerator and/or various lasers, is part of the planned installation at the undulator beamline. Our group has undertaken responsibilities in such areas as hutch design, interfacing and equipment, sagittal focussing in the cryogenically-cooled monochromator systems, user policy, evaluation of undulator performance, etc. Over the coming months we will be heavily involved with beamline construction and testing.

The initial experimental program, being prepared in collaboration with atomic physicists from the University of Western Michigan, the University of Tennessee, and the University of Notre Dame, is beginning to take shape. Even before the BESSRC beamlines are completed, we hope to be able to exploit the high X-ray energies and intensities to measure photoattenuation and photoionization cross sections in the rare gases in the $1-100-\mathrm{keV}$ energy range. Then, as the beamlines and associated apparatus come into operation, we expect to refine these measurements and to conduct a broad research program aimed at the basic physics of photon/atom interactions in the hard X-ray regime where currently very few data exist and where atomic physics theory remains largely untested. 


\section{OTHER EDUCATIONAL ACTIVITIES IN THE PHYSICS DIVISION}

\section{a. Enhancement of Minority Involvement in DOE Nuclear Physics Programs (B. Zeidman)}

As a result of continuing efforts to interact with a large number of minority students, the Minority Program in the ANL Physics Division succeeded in attracting many highly qualified students to apply for participation in the programs of the Physics Division and other ANL divisions. The program is directed toward the identification of institutions with relatively strong physics programs and with faculty interested in stimulating their students to pursue research activities, particularly summer programs. During visits to colleges, lectures are presented and are followed by discussion of activities in physics, at Argonne and other national laboratories, and the possibilities for graduate study, employment, etc. Additional activities included attending meetings of the Society for the Advancement of Chicanos and Native Americans in Science and of the
Society of Black Physics Students. As a result of these efforts, 37 applications were received for the summer program in 1995. A total of 19 offers were made for the Summer Research Participation program in conjunction with Argonne's Department of Educational Programs. A number of former participants are currently enrolled in graduate programs in physics; one student is nearing completion of his Ph.D. thesis in Nuclear Physics in the Physics Division. Various institutions will be visited during this year and several meetings of minority groups will be attended. These ongoing interactions are generating institutional relationships that will enrich the physics programs in minority institutions and substantially enhance minority involvement not only in nuclear physics, but in other branches of physics and science.

\section{b. Nuclear Physics Award for Faculty in Undergraduate Institutions (B. Zeidman)}

The goal of the "Faculty Program" is to enhance undergraduate science education through faculty awards for minority and historically black colleges and universities (HBCU) faculty that will allow them to participate directly in the ANL Physics Division research program and increase the number of undergraduates involved in research. The program began during the summer of 1994 with the appointment of a Hispanic theorist. Undergraduate students are already working with the faculty member who submitted an independent funding proposal for a continuing research collaboration. Other minority faculty members and their students were involved in research collaborations in the Physics Division during the summer of 1995. They are in the process of preparing funding proposals. In order to extend the limited resources of this program, participants are placed through existing educational programs whenever possible, thereby obtaining supplemental support. 


\section{c. Scientific Support of SciTech Museum Exhibits and Outreach Programs (M. Peshkin)}

SciTech (Science and Technology Interactive Center) is a hands-on science museum located in Aurora, Illinois, near Argonne. It serves communities from prosperous suburbs to disadvantaged minority groups in a wide area that includes Chicago, providing both hands-on scientific experiences on the museum floor and outreach programs in schools. With the assistance of scientists and engineers from Argonne, Fermilab, and several technological companies, SciTech has become a recognized leader in developing exhibits that bring modern science to the public.

Argonne's Director, Alan Schriesheim, is a member of SciTech's Board of Directors. Murray Peshkin and Dale Henderson work part-time as SciTech's Senior Scientist and as an exhibit developer, respectively. In addition, several members of the Physics Division voluntarily assist with exhibit development from time to time, and the Division makes facilities available for that effort.

\section{d. The "Museum in a School" Program (M. Peshkin and D. Henderson)}

For most of FY 1995, Peshkin has primarily served by teaching science to Scitech's "explainers" for the "Museum in a School" outreach program. In that program, groups of exhibits, each group focused on a single subject such as light, heat, or astronomy, are taken to a school for a week. Early in that week, all students in grades 4 and 5 and sometimes 6 have an opportunity to experiment with the exhibits for an hour. Later in the week, a SciTech explainer discusses the exhibits with the children. SciTech provides written materials for teachers and for children. The explainers are typically capable people with teaching backgrounds but not necessarily strong in science. Peshkin assists them by conducting discussions of the exhibits and of the science they illustrate. This activity is expected to continue in FY 1996 and probably will be supplemented by some upgrading of the exhibits on the museum floor.

\section{e. The $\mathbf{E}=\mathbf{m} \mathbf{c}^{\mathbf{2}}$ Exhibition (D. Henderson and R.V.F. Janssens)}

The goal of this DOE-supported exhibition is to demystify Einstein's formula $\mathrm{E}=\mathrm{mc}^{2}$ by illustrating the interchangeability of matter $(m)$ and energy $(E), c^{2}$ being the exchange rate. The exhibition has two major parts, "matter into energy" and "energy into matter", plus a video to connect them. "Matter into energy" has now been on the museum floor for over a year and has been evaluated for durability and effectiveness as an exhibit and now awaits relocation to an area which will contain other nuclear exhibits. Positrons from a ${ }^{22} \mathrm{Na}$ source are annihilated to produce gamma rays that are caught in NaI detectors. The viewer can alter the alignment of the detectors and observe the consequences for the rates of single and coincident counts. The viewer can also observe the effects of placing absorbers in front of the counters. Prototype explanatory graphics were placed around the exhibit and those will probably be changed after we have some experience with their effectiveness. The connecting video is in the process of being produced in collaboration with Fermilab. The electronic circuitry was made by L. Bagby (Fermilab). A cloud chamber for the demonstration of "energy into matter" purchased from a scientific instrument company was tested with a Th source for pair production. The low yield of pairs produced combined with the large number of events produced by other reactions limited the success of this project. In order to overcome this difficulty, a video camera combined with a computer is being programmed to allow the viewer to review events that the viewer finds interesting either in a freeze-frame mode or in slow motion.

This and the two preceding activities are largely voluntary or paid out of non-programmatic funds. 


\section{STAFF MEMBERS OF THE PHYSICS DIVISION •}

Listed below are the staff of the Physics Division for the year ending March 31, 1996.

The program headings indicate only the individual's current primary activity.

\section{SCIENTIFIC STAFF \\ EXPERIMENTAL NUCLEAR PHYSICS STAFF}

\section{Regular Staff}

Irshad Ahmad, Ph.D., University of California, 1966

Birger B. Back, Ph.D., University of Copenhagen, 1974

* R. Russell Betts, Ph.D., University of Pennsylvania, 1972

Michael P. Carpenter, Ph.D., University of Tennessee, 1987

$\dagger$ Cary N. Davids, Ph.D., California Institute of Technology, 1967

¥ Stuart J. Freedman, Ph.D., University of California, 1972

Donald F. Geesaman, Ph.D., State University of N.Y., Stony Brook, 1976

Bruce G. Glagola, Ph.D., University of Maryland, 1978

$\S$ Walter F. Henning, Ph.D., Technical University of Munich, 1968

If Harold E. Jackson, Jr., Ph.D., Cornell University, 1959

ii Robert V.F. Janssens, Ph.D. Univ. Catholique de Louvain, Belgium, 1978

Cheng-lie Jiang, Ph.D. China Institute of Atomic Energy, 1960

Cathleen Jones, Ph.D., California Institute of Technology, 1991 (until January 1996)

Teng Lek Khoo, Ph.D., McMaster University, 1972

** Walter Kutschera, Ph.D., University of Graz, Austria, 1965

Torben Lauritsen, Ph.D., State University of New York, 1990

Christopher J. Lister, Ph.D., University of Liverpool, 1977

†† Jerry A. Nolen, Jr., Ph.D., Princeton University, 1965

Thomas P. O'Neill, Ph.D., California Institute of Technology, 1994

Richard C. Pardo, Ph.D., University of Texas, 1976

David H. Potterveld, Ph.D., Caltech, 1988

Karl Ernst Rehm, Ph.D., Technical University, Munich, 1973

III John P. Schiffer, Ph.D., Yale University, 1954

Kenneth W. Shepard, Ph.D., Stanford University, 1970

Kenneth Teh, Ph.D., Vanderbilt University, 1988

Alan H. Wuosmaa, Ph.D., University of Pennsylvania, 1989

II II Benjamin Zeidman, Ph.D., Washington University, 1957

* Joint appointment with the University of Illiinois-Chicago.

$\dagger$ Adjunct Professor, Vanderbilt University.

\$ On leave of absence at the University of California, Berkeley.

$\S$ Director of the Physics Division.

II Associate Director of the Physics Division.

II Adjunct Professor, North Carolina State University.

** On leave of absence at the University of Vienna.

t† Director of the ATLAS Facility. Adjunct Professor, Michigan State University.

IIT Associate Director of the Physics Division. Joint appointment with the University of Chicago.

II II Adjunct Professor, Hampton University. 


\section{Special Appointments}

Lowell M. Bollinger, Ph.D., Cornell University, 1951

Melvin S. Freedman, Ph.D., University of Chicago, 1942

Sheldon B. Kaufman, Ph.D., University of Chicago, 1953

Alexander Langsdorf, Jr., Ph.D., Massachusetts Inst. of Technology, 1937

* Michael Paul, Ph.D., Hebrew University of Jerusalem, 1973

G. Roy Ringo, Ph.D., University of Chicago, 1940

Ralph E. Segel, Ph.D., Johns Hopkins University, 1955

George E. Thomas, B.A., Illinois Wesleyan, 1943

Jan L. Yntema, Ph.D., Free University of Amsterdam, 1952

\section{Term Appointments}

William Cummings, Ph.D., Stanford University, 1992

Richard Harkewicz, Ph.D. Michigan State University, 1992 (until May 1995)

William Sellyey, Ph.D. Bryn Mawr College, 1974

\section{THEORETICAL NUCLEAR PHYSICS STAFF}

\section{Regular Staff}

Richard R. Chasman, Ph.D., University of California, 1959

Fritz Coester, Ph.D., University of Zurich, 1944

Henning Esbensen, Ph.D., University of Aarhus, 1977

Stephen Landowne, Ph.D., Carnegie-Mellon University, 1970 (until January 1996)

$\dagger$ Tsung-Shung Harry Lee, Ph.D., University of Pittsburgh, 1973

Steven C. Pieper, Ph.D., University of Illinois, 1970

Craig T. Roberts, Ph.D., Flinders University of South Australia, 1989

Robert B. Wiringa, Ph.D., University of Illinois, 1978

\section{Special Appointments}

Paul Benioff, Ph.D., University of California, 1959

Arnold R. Bodmer, Ph.D., Manchester University, 1953

Mitio Inokuti, Ph.D., University of Tokyo, 1962

Dieter Kurath, Ph.D., University of Chicago, 1951

Harry J. Lipkin, Ph.D., Princeton University, 1950

$\ddagger$ Vijay Pandharipande, Ph.D., University of Bombay, 1969

Murray Peshkin, Ph.D., Cornell University, 1951

* Special Term Appointee from the Hebrew University of Jerusalem, Israel.

$\dagger$ Adjunct Professor, University of Pittsburgh.

$¥$ Special Term Appointee from the University of Illinois, Urbana. 


\section{ATOMIC AND MOLECULAR PHYSICS STAFF}

\section{Regular Staff}

Robert Dunford, Ph.D., University of Michigan, 1978

Donald S. Gemmell, Ph.D., Australian National University, 1960

Elliot P. Kanter, Ph.D., Rutgers University, 1977

Stephen H. Southworth, Ph.D., University of California, Berkeley, 1982

* Zeev Vager, Ph.D. Weizmann Institute of Science, 1962

Linda Young, Ph.D., University of California, Berkeley, 1981

\section{Special Appointments}

William J. Childs, Ph.D., University of Michigan, 1956

F. Paul Mooring, Ph.D., University of Wisconsin, 1951

Gilbert J. Perlow, Ph.D., University of Chicago, 1940

\section{Term Appointment}

Thomas LeBrun, Ph.D. University of Paris, 1991

\section{TEMPORARY APPOINTMENTS}

\section{Postdoctoral Appointees}

Dieter Ackermann (from Laboratorie Nazionale di Legnaro, Italy):

Heavy-ion research at ATLAS.

(June 1995--

Axel Bender (University of Tubingen, Germany):

Nuclear theory studies.

(January 1995--

)

Daniel Blumenthal (University of Pennsylvania, Philadelphia, Pennsylvania):

Heavy-ion research at ATLAS.

(January 1994-- )

Benjamin F. Crowell (from Yale University, New Haven, Connecticut):

Heavy-ion research at ATLAS.

(June 1993--August 1995)

Patrick Decrock (from K. U., Leuven, Belgium):

Accelerator development.

(January 1996--

James Fedchak (from College of William and Mary, Williamsburg, Virginia): Medium-energy physics.

(October 1994--

* Joint Appointment with Weizmann Institute of Science, Rehovot, Israel. 
Susan Fischer (from University of Notre Dame, Notre Dame, Indiana):

Heavy-ion research at ATLAS.

(January 1995-- )

Gregory Hackman (from McMaster University, Hamilton, Ontaro, Canada):

Heavy-ion research at ATLAS.

(September 1995-- )

Jens-Ole Hansen (from Massachusetts Inst. of Technology, Cambridge, Massachusetts):

(Medium-energy studies.

(October 1994--

David J. Hofman (from SUNY, Stony Brook, New York):

Heavy-ion research at ATLAS.

(August 1994-- )

Martin Jung (from GSI, Darmstadt, Germany):

Atomic physics research.

(December 1994-- )

Jong-won Kim (from Michigan State University, E. Lansing, Michigan):

ATLAS development.

(June 1994--October 1995)

Bertold Kraessig (from University of Freiburg, Germany):

Atomic physics with synchrotron light sources.

(March 1995--

)

Naomi Makins (from Massachusetts Inst. of Technology, Cambridge, Massachusetts):

Medium energy physics.

(July 1994--

Vandana Nanal (from Tata Institute, Bombay, India):

Heavy-ion research at ATLAS.

(August 1995-- )

* Thomas P. O'Neill (from California Inst. of Technology, Pasadena, California):

Medium-energy physics.

(March 1994--September 1995)

$\dagger$ Jorg Reinhold (from Technical University of Munich, Germany):

Medium-energy research.

(September 1995-- )

Michael Schlapp (from University of Giessen, Germany):

Accelerator development.

(October 1995--

$\ddagger$ Dariusz Seweryniak (from University of Uppsala, Sweden):

Heavy-ion research at ATLAS.

(January 1995-- )

Lorenz von Smekal (from University of Tubingen, Germany):

Theoretical studies.

(November 1995-- )

*Joined the regular staff of the Physics Division in September 1995.

$\dagger$ Feodor-Lynen Postdoctoral Fellowship from Alexander von Humboldt Foundation.

$\ddagger$ Joint appointment with the University of Maryland. 


\section{TECHNICAL AND ENGINEERING STAFF (and areas of activity)}

Kevin G. Bailey (B.S. University of Nebraska, 1989). SOS construction for CEBAF.

Brian T. Batzka (B.S. University of Houston, 1992). ATLAS operator.

Peter J. Billquist ECR heavy-ion source, ATLAS operation.

John M. Bogaty (A.A.S. DeVry, 1961).

Electrical systems, ATLAS operation and development.

Benny G. Clifft (A.S.E.E., DeVry, 1959).

Electrical systems, ATLAS operation and development.

Joseph Falout (B.S.M.E. University of Illinois; 1970).

Experimental equipment design.

John P. Greene (M.S. DePaul University, 1982). Target preparation.

Ray E. Harden (A.A.S. Milwaukee School of Engineering, 1957). ATLAS operator

* Dale J. Henderson (B.S. Elmhurst College, 1951).

Detector development, technical assistance, heavy-ion physics.

James M. Joswick (A.A.S. Milwaukee School of Engineering, 1964).

ATLAS experimental equipment maintenance, technical assistance, heavy-ion physics.

* Raymond B. Kickert

ATLAS experimental equipment maintenance, technical assistance, heavy-ion physics.

Robert Kowalczyk (M.S. Northeastern Illinois University, 1983).

Technical assistance, medium-energy physics.

Charles A. Kurtz (M.S. University of Arkansas, 1984).

Technical assistance, atomic physics.

* Paul Markovich (B.S. Purdue University, 1972).

Surface chemistry, ATLAS development and operation.

Thomas P. Mullen (B.S. Marquette University, 1966).

Division ESH/QA engineer.

Floyd Munson, Jr. (A.A.S. DeVry, 1966, B.S. Lewis University, 1993). Control system for ATLAS.

Kirt Nakagawa (B.S. University of Illinois, 1988). ATLAS operator.

Bruce G. Nardi (A.A.S. Morton Jr. College, 1967; A.A.S. DeVry, 1969). Electronics design and maintenance.

*Special Term Appointee. 
James R. Specht (A.A.S. DeVry, 1964).

Cryogenics engineer. ATLAS development and operation.

Philip Strickhorn (B.S. DeVry, 1990).

Electrical and technical assistance with ATLAS operations.

Anne Sutherland (B.S. University of Rochester, 1993).

Operate, upgrade and maintain ATLAS cryogenic system.

Brian J. Tieman (B. A. North Central College, 1992).

ATLAS operator.

Ian R. Tilbrook (B.S. Pennsylvania State University, 1987; MBA, Keller Graduate School of Management, 1994).

ATLAS operator.

Richard Vondrasek (B.S. University of Illinois, 1990). ATLAS operator.

Philip R. Wilt (Johnstown Technical School 1973).

Electronics design and maintenance.

* Bruce J. Zabransky (M.S. University of Illinois, Chicago, 1973).

Dynamitron operation.

Anthony R. Zeuli (B.A. Hamline University, 1990).

Technical assistance. Medium-energy physics.

Gary P. Zinkann (B.S. DeVry, 1975).

ATLAS operations supervisor.

\section{ADMINISTRATIVE STAFF}

$\dagger$ Allan Bernstein, M.B.A., Rosary College, 1986

$\dagger$ James E. Nelson, B.A., University of Illinois, 1975

$\ddagger$ Karen J. Thayer

\section{VISITORS AND STUDENTS}

Long-Term Visitors (at Argonne more than 4 months)

Jeffrey Hangst (University of Aarhus, Denmark):

Heavy-ion research at ATLAS. -

(April 1995--

$\S$ Donald McLeod (University of Illinois, Chicago, Illinois):

Heavy-ion research at ATLAS.

(June 1994--

$\S$ Nora Mansour (Western Michigan University, Kalamazoo, Michigan):

Atomic physics research.

(October 1991--

* In charge of Dynamitron operations.

$\dagger$ Assistant Director of the Physics Division.

Staff Assistant.

$\S$ Guest Faculty Research Participant. 
Rajeev Mehta (Nuclear Science Centre, New Delhi, India):

ATLAS development.

(February 1995--May 1996)

Amit Roy (Nuclear Science Center, New Delhi, India):

ATLAS development.

(February-August 1995)

Norbert Schmidt (Fachhochschule, Munich, Germany):

Heavy-ion research at ATLAS.

(March 1994--August 1994)

* Carol Tanner (University of Notre Dame, Indiana):

Atomic physics at BLASE.

(March 1992--

* Guangsheng Xu (Yale University, New Haven, Connecticut):

Heavy-ion research at ATLAS.

(January 1994--

Short-Term Visitors (at ANL less than 4 months)

$\dagger$ Richardo Cossyleon (Benito Juarez High School, Cicero, Illinois):

Medium-energy physics.

(July-August 1995)

Christopher Fasano (Francis Marion University, Florence, South Carolina):

Theoretical studies.

(May-June 1995)

Igor Gontchar (Omsk State Railway Engineering Academy, Omsk, Russia):

Heavy-ion research.

(July-August 1995)

Edward Hohman (York Township High School, Lyons, Illinois):

Summer student coordinator.

(June-August 1995)

Wen-chao Ma (Mississippi State University, Mississippi State, Missisppi):

Heavy-ion research at ATLAS.

(May-July 1995)

Miloslave M. Michailova (Bulgarian Academy of Sciences, Sofia, Bulgaria):

Theoretical physics studies.

(December 1995--March 1996)

$\ddagger$ Robert Perry (Talladega College, Talladega, Alabama)

Atomic physics studies.

(June-August 1995)

* Guest Faculty Research Participant.

$\dagger 1995$ DOE Trac Teacher.

$\$$ Faculty Research Participant. 
* Akunuri Ramayya (Vanderbilt University, Nashville, Tennessee):

Heavy-ion research at ATLAS.

(June--July 1994)

Toru Sato (Osaka University, Osaka, Japan):

Theoretical physics studies.

(July-August 1995)

Ryochi Seki (California State University, Northridge, California):

Theoretical physics studies.

(June-August 1995)

Dmitri Toporkov (Institute for Nuclear Physics, Novosibirsk, Russia):

Medium-energy physics.

(August-November 1995)

\section{Resident Graduate Students}

Hanan Amro (North Carolina State University, Raleigh, North Carolina):

Heavy-ion Research at ATLAS.

(January 1995-- )

Kevin Beyer (Michigan State University, E. Lansing, Michigan):

Heavy-ion research at ATLAS.

(August 1992--October 1995)

Larry Todd Brown (Vanderbilt University, Nashville, Tennessee):

FMA development at ATLAS

(January 1994-- )

Brian Busse (Oregon State University, Corvallis, Oregon):

FMA development at ATLAS.

(June 1994--December 1995)

Isaac Chappell (Massachusetts Institute of Technology, Cambridge, Massachusetts):

Theoretical physics studies.

(June-August 1995)

Christopher Conner (University of Illinois-Chicago):

Heavy-ion research at ATLAS.

(June 1994-- )

Louis Conticchio (University of Maryland, College Park, Maryland):

Heavy-ion research at ATLAS.

(October 1994-- )

John C. Gehring (University of Chicago, Chicago, Illiniois):

Heavy-ion research at ATLAS.

(June 1990--

David Nisius (Purdue Univ ersity, W. Lafayette, Indiana):

Heavy-ion research at ATLAS.

(June 1993 --

* Faculty Research Participant. 
Aloy Perera (University of Rochester, Rochester, New York): APEX experiment at ATLAS.

(August 1993 -- August 1995)

Michael Pichowsky (University of Pittsburgh, Pennsylvania): Theoretical physics studies.

(September 1993--November 1996)

Mauricio Portillo (Michigan State University, E. Lansing, Michigan): Accelerator development. (September 1995-- )

Dante Roa (Florida State University): Research with APEX at ATLAS.

(May 1992--

Michael Rogers (University of Iowa, Ames, Iowa):

Nuclear theory studies.

(March 1994--June 1995)

Jazmin Schwartz (Yale University, New Haven, Connecticut):

Heavy-ion research at ATLAS.

(May 1995--

Mark Wolanski (University of Chicago, Chicago, Illinois):

Weak interaction studies.

(July 1991--August 1995)

\section{Guest Graduate Students}

Konstantin Akimov (University of Illinois, Chicago, Illinois): Heavy-ion research at ATLÁS.

(May 1994--

Kin-chi Chan (University of Chicago):

APEX experiment.

(March-August 1995)

Dipangkar Dutta (Northwestern University, Evanston, Illinois): Medium-energy studies.

(June 1994--

Niels Madsen (University of Aarhus, Aarhus, Denmark):

Heavy-ion research.

(July-August 1995)

Robert Rafac (University of Notre Dame, Notre Dame, Indiana):

Atomic physics research.

(March 1992--March 1996) 


\section{Undergraduate Students}

Gordon Annan (Dillard University)

Teresa Barlow (Illinois Benedictine College)

Kevin Beyer (Michigan State University)

Samir Bhargava (California State University-Long Beach)

Daniel Brathwaite (University of Chicago)

Peter Cabauy (Florida International University)

Jesse Canterbury (Florida State University)

George Chancellor (North Central College)

Shannon Cyborski (Southern Illinois University)

Brendan Field (Oberlin College)

Steven Kramer (Lewis University)

Daniel McKinsey (University of Michigan)

Jennifer Nemanich (North Central College)

Juan Sebastian Oddone (Florida International University)

Richard Pelaia (Florida Institute of Technology)

Kara Perry (College of St. Francis)

Robert Polender (Wheaton College)

Mauricio Portillo (University of Texas, El Paso)

Christian Roehrig (North Central College)

Stacey Schiel (North Central College)

Max Schoenberg (University of Illinois-Urbana)

Teresa Soledad (University of Illinois - Urbana)

Jerette Spann (Francis Marion University)

John Szezech (University of Illinois-Chicago)

Jared Torres (Loyola University)

Erik Tryggestad (Wittenberg University)

Patricia Vahle (New Mexico State University)

Jeff Wendlandt (University of California-Berkeley)

James Young (Florida State University)

Pre-College Program (Just Graduated from High School)

(June--August 1995)

Robert Berles (Maine East High School)

Daniel Braithwaite (Oak Park/River Forest High School)

John Frederiksen (Maine Township High School)

James Moawad (Hinsdale South High School)

Elaine Pugeda (Benet Academy) 


\section{PUBLICATIONS FROM APRIL 1, 1995 THROUGH MARCH 31, 1996}

(The arrangement follows approximately the Table of Contents)

\section{HEAVY-ION RESEARCH}

Energy Dependence of Collective Flow of Neutrons and Protons in ${ }^{197} \mathrm{Au}+{ }^{197} \mathrm{Au}$ Collisions

D. Lambrecht, et al.

Zeit. fuer Physik $\underline{\text { A350, }} 115$ (1994)

Collective Motion in Selected Central Collisions of $\mathrm{Au}$ on $\mathrm{Au}$ at $150 \mathrm{AMeV}$

S. C. Jeong, et al.

Phys. Rev. Lett. $\underline{72}, 3468$ (1994)

Recent Advances in the Study of Nuclear Clusters

A. H. Wuosmaa, R. R. Betts, M. Freer, and B. R. Fulton

Ann. Rev. Nucl. and Part. Sci. 45, 89-131 (1995)

Investigation of High-Spin States in the Neutron-Rich ${ }^{106}$ Mo Nucleus

A. Guessous, N. Schulz, M. Bentaleb, I. Ahmad, J. L. Durell, S. Khazrouni, F. Liden,

C. J. Lister, E. Lubkiewicz, L. R. Morss, K. L. Nash, C. J. Pearson, W. R. Phillips,

J. Shannon, B. J. Varley, and C. W. Williams

Appl. Radiat. Isot. 46, 549-550 (1995)

Targets for the APEX Experiment at ATLAS

J. P. Greene, G. E. Thomas, and R. H. Leonard

Nucl. Instrum. Methods A $1362,81-89$ (1995)

Description of the Argonne National Laboratory Target Making Facility

G. E. Thomas and J. P. Greene

Nucl. Instrum. Methods A362, 201-204 (1995)

Triple Angular Correlations of $\gamma$ Rays Following Spontaneous Fission

W. Urban, M. A. Jones, C. J. Pearson, J. L. Durell, M. Leddy, W. R. Phillips, A. G. Smith,

B. J. Varley, I. Ahmad, L. R. Morss, E. Lubkiewicz, and N. Schulz

Nucl. Instrum. Methods A365, 596-599 (1995)

Search for the Two-phonon Octupole Vibrational State in ${ }^{208} \mathrm{~Pb}$

E. F. Moore, W. Henning, R. V. F. Janssens, T. L. Khoo, S. J. Sanders, I. Ahmad,

H. Amro, D. Blumenthal, M. P. Carpenter, B. Crowell, M. W. Drigert, D. Gassmann,

R. G. Henry, T. Lauritsen, C. J. Lister, and D. Nisius

Nucl. Instrum. Methods B99, 308-311 (1995)

Cooling and Diffusion in the $\gamma$ Decay of Rotating Nuclei

$\mathrm{T}$. D $\phi s s i n g$ and $\mathrm{E}$. Vigezzi

Nucl. Phys. A587, 13-35 (1995)

Relationship Between the Deformed Harmonic Oscillator and Clustering in Light Nuclei

M. Freer, R. R. Betts, and A. H. Wuosmaa

Nucl. Phys. A587, 36-54 (1995) 
Observation and Lifetime of the First Excited Superdeformed Band in ${ }^{192} \mathrm{Hg}$

A. Korichi, F. Hannachi, I. Deloncle, W. Korten, I. Ahmad, F. Azaiez, G. Bastin,

C. W. Beausang, C. Bourgeois, M. P. Carpenter, R. M. Clark, R. Duffait, J. Duprat,

B. J. P. Gall, R. H. Henry, H. Hubel, R. V. F. Janssens, M. J. Joyce, M. Kaci, T. L. Khoo,

T. Lauritsen, Y. LeCoz, M. Meyer, E. S. Paul, N. Perrin, N. Poffe, M. G. Porquet,

N. Redon, C. Schuck. H. Sergolle, J. F. Sharpey-Schafer, J. Simpson, A. G. Smith,

R. Wadsworth, and P. Willsau

Phys. Lett. B345, 403-407.(1995)

"Identical" Superdeformed Band in 151Dy: Further Evidence for the Pseudospin Coupling Scheme

D. Nisius, R. V. F. Janssens, P. Fallon, B. Crowell, I. Ahmad, C. W. Beausang,

M. P. Carpenter, B. Cederwall, P. J. Daly, M. A. Deleplanque, R. M. Diamond,

D. Gassmann, Z. W. Grabowski, R. G. Henry, T. L. Khoo, T. Lauritsen, I. Y. Lee,

A. O. Macchiavelli, R. H. Mayer, F. S. Stephens, and P. J. Twin

Phys. Lett. B346, 15-20 (1995)

Search for Octupole Deformation in Neutron-rich Cs Nuclei

T. Rzaca-Urban, W. R. Phillips, J. L. Durell, W. Urban, B. J. Varley, C. J. Pearson,

J. A. Shannon, I. Ahmad, C. J. Lister, L. R. Morss, K. L. Nash, C. W. Williams,

M. Bentaleb, E. Lubkiewicz, and N. Schulz

Phys. Lett. B348, 336-342 (1995)

Search for Entrance Channel Dependence in the Population of Superdeformed Bands in ${ }^{191} \mathrm{Hg}$

F. Soramel, T. L. Khoo, Ph. Benet, K. B. Beard, R. V. F. Janssens, I. Ahmad, I. Bearden,

M. P. Carpenter, P. J. Daly, M. W. Drigert, B. Fornal, U. Garg, Z. Grabowski, T. Lauritsen,

Y. Liang, R. Mayer, E. F. Moore, W. Reviol, and D. Ye

Phys. Lett. B350, 173-177 (1995)

Laser Cooling of a Bunched Beam in a Synchrotron Storage Ring

J. S. Hangst, J. S. Nielsen, O. Poulsen, P. Shi, and J. P. Schiffer

Phys. Rev. Lett. 74, 4432-4435 (1995)

Effects of Pair Correlations in the Statistical $\gamma$-Decay Spectrum of a Nucleus

T. Dфssing, T. L. Khoo, T. Lauritsen, I. Ahmad, D. Blumenthal, M. P. Carpenter,

B. Crowell, D. Gassmann, R. G. Henry, R. V. F. Janssens, and D. Nisius

Phys. Rev. Lett. 75, 1276-1279 (1995)

A Harmonic Two Phonon $\gamma$-Vibrational State in Neutron-Rich $106 \mathrm{Mo}$

A. Guessous, N. Schulz, W. R. Phillips, I. Ahmad, M. Bentaleb, J. L. Durell, M. A. Jones,

M. Leddy, E. Lubkiewicz, L. R. Morss, R. Piepenbring, A. G. Smith, W. Urban, and

B. J. Varley

Phys. Rev. Lett. 75, 2280-2283 (1995) 
Search for Narrow Sum-Energy Lines in Electron-Positron Pair Emission from Heavy-Ion Collisions near the Coulomb Barrier

I. Ahmad, S. M. Austin, B. B. Back, R. R. Betts, F. P. Calaprice, K. C. Chan, A. Chishti, P. Chowdhury, C. Conner, R. W. Dunford, J. D. Fox, S. J. Freedman, M. Freer, S. B. Gazes, A. L. Hallin, T. Happ, D. Henderson, N. I. Kaloskamis, E. Kashy, W. Kutschera, J. Last, C. J. Lister, M. Liu, M. R. Maier, D. J. Mercer, D. Mikolas, P. A. A. Perera, M. D. Rhein, D. E. Roa, J. P. Schiffer, T. A. Trainor, P. Wilt, J. S. Winfield, M. Wolanski, F. L. H. Wolfs, A. H. Wuosmaa, G. Xu, A. Young, and J. E. Yurkon

Phys. Rev. Lett. 75, 2658-2661 (1995)

Proton Decay of an Intruder State in $185 \mathrm{Bi}$

C. N. Davids, P. J. Woods, H. T. Penttilä, J. C. Batchelder, C. R. Bingham,

D. J. Blumenthal, L. T. Brown, B. C. Busse, L. F. Conticchio, T. Davinson,

D. J. Henderson, R. J. Irvine, D. Seweryniak, K. S. Toth, W. B. Walters, and

B. E. Zimmerman

Phys. Rev. Lett. 76, 592-595 (1996)

Time Scale of Quasifission from Giant Dipole Resonance $\boldsymbol{\gamma}$-Ray Yield

J. Nestler, B. B. Back, K. S. Drese, D. J. Hofman, S. Schadmand, R. Varma, and P. Paul

Phys. Rev. C $\underline{51}$, 2218-2219 (1995)

$\beta$-Delayed Neutron Decay of ${ }^{14} \mathrm{Be}$

M. D. Belbot, J. J. Kolata, K. Lamkin, R. J. Tighe, M. Zahar, R. Harkewicz,

D. J. Morrissey, N. A. Orr, R. M. Ronningen, B. M. Sherrill, J. A. Winger, and $M$. Carpenter

Phys. Rev. C 1ㅣ, 2372-2380 (1995)

Identification of the Unfavored $\mathrm{N}=7$ Superdeformed Band in ${ }^{191} \mathrm{Hg}$

M. P. Carpenter, R. V. F. Janssens, B. Cederwall, B. Crowell, I. Ahmad, J. A. Becker,

M. J. Brinkman, M. A. Deleplanque, R. M. Diamond, P. Fallon, L. P. Farris, U. Garg,

D. Gassmann, E. A. Henry, R. G. Henry, J. R. Hughes, T. L. Khoo, T. Lauritsen,

I. Y. Lee, A. O. Machiavelli, E. F. Moore, D. Nisius, and F. S. Stephens

Phys. Rev. C $\underline{51}, 2400-2405$ (1995)

Rapid Increase in Prescission Giant-Dipole-Resonance $\gamma$-ray Emission with Bombarding Energy

D. J. Hofman, B. B. Back, and P. Paul

Phys. Rev. C 51, 2597-2605 (1995)

$\alpha$ Decay of a New Isotope, ${ }^{204} \mathrm{Ra}$

M. J. Leddy, S. J. Freeman, J. L. Durell, A. G. Smith, S. J. Warburton, D. J. Blumenthal,

C. N. Davids, C. J. Lister, and H. T. Penttilä

Phys. Rev. C 51, R1047-R1051 (1995)

Superdeformed Band in ${ }^{154} \mathrm{Dy}$

D. Nisius, R. V. F. Janssens, T. L. Khoo, I. Ahmad, D. Blumenthal, M. P. Carpenter,

B. Crowell, D. Gassmann, T. Lauritsen, W. C. Ma, J. H. Hamilton, A. V. Ramayya,

P. Bhattacharyya, C. T. Zhang, P. J. Daly, Z. W. Grabowski, and R. H. Mayer

Phys. Rev. C 51, R1061-R1064 (1995) 
Relative Spins and Excitation Energies of Superdeformed Bands in $190 \mathrm{Hg}$ : Further Evidence for Octupole Vibration

B. Crowell, M. P. Carpenter, R. V. F. Janssens, D. J. Blumenthal, J. Timar, A. N. Wilson,

J. F. Sharpey-Schafer, T. Nakatsukasa, I. Ahmad, A. Astier, F. Azaiez, L. du Croix,

B. J. P. Gall, F. Hannachi, T. L. Khoo, A. Korichi, T. Lauritsen, A. Lopez-Martens,

M. Meyer, D. Nisius, E. S. Paul, M. G. Porquet, and N. Redon

Phys. Rev. C 51, R1599-R1603 (1995)

Quasiparticle Excitations in Superdeformed ${ }^{192} \mathrm{Hg}$

P. Fallon, T. Lauritsen, I. Ahmad, M. P. Carpenter, B. Cederwall, R. M. Clark, B. Crowell,

M. A. Deleplanque, R. M. Diamond, B. Gall, F. Hannachi, R. G. Henry, R. V. F. Janssens,

T. L. Khoo, A. Korichi, I. Y. Lee, A. O. Macchiavelli, C. Schuck, and F. S. Stephens

Phys. Rev. C 51, R1609-R1612 (1995)

Proton and Neutron Excitations in Superdeformed ${ }^{150} \mathrm{~Tb}$

P. Fallon, C. W. Beausang, S. Asztalos, D. Nisius, R. V. F. Janssens, M. Bergstrom,

M. Carpenter, B. Cederwall, S. Clarke, B. Crowell, M. A. Deleplanque, R. M. Diamond,

R. G. Henry, T. L. Khoo, T. Lauritsen, I. Y. Lee, A. O. Macchiavelli, F. S. Stephens, and P. J. Twin

Phys. Rev. C 52, 93-98 (1995)

Onset of Collectivity in Neutron Deficient 196,198 Po

L. A. Bernstein, J. A. Cizewski, H.-Q. Jin, W. Younes, R. G. Henry, L. P. Farris,

A. Charos, M. P. Carpenter, R. V. F. Janssens, T. L. Khoo, T. Lauritsen, I. G. Bearden,

D. Ye, J. A. Becker, E. A. Henry, M. J. Brinkman, J. R. Hughes, A. Kuhnert, T. F. Wang,

M. A. Stoyer, R. M. Diamond, F. S. Stephens, M. A. Deleplanque, A. O. Macchiavelli,

I. Y. Lee, B. Cederwall, J. R. B. Oliveira, J. Burde, P. Fallon, C. Duyar, J. E. Draper,

E. Rubel, and D. T. Vo

Phys. Rev. C $\underline{52}, 621-627$ (1995)

Conversion Electron Spectroscopy at the Fragment Mass Analyzer Focal Plane: Studies of Isomeric Decays Near the Proton Drip Line

D. Nisius, R. V. F. Janssens, I. .G. Bearden, R. H. Mayer, I. Ahmad, P. Bhattacharyya,

B. Crowell, M. P. Carpenter, P. J. Daly, C. N. Davids, Z. W. Grabowski, D. J. Henderson,

R. G. Henry, R. Hermann, T. L. Khoo, T. Lauritsen, H. T. Penttilä, L. Ciszewski, and

C. T. Zhang

Phys. Rev. C $\underline{52}$, 1355-1360 (1995)

Half-lives of Isomeric States in ${ }^{57} \mathrm{Fe}$ and ${ }^{83} \mathrm{Kr}$

I. Ahmad, K. E. Rehm, E. P. Kanter, W. Kutschera, W. R. Phillips, and A. R. Barnett

Phys. Rev. C $\underline{52}$, 2240-2241 (1995)

Study of the ${ }^{18} \mathrm{~F}(\mathrm{p}, \alpha)^{15} \mathrm{O}$ Reaction at Astrophysical Energies Using a ${ }^{18} \mathrm{~F}$ Beam

K. E. Rehm, M. Paul, A. D. Roberts, D. J. Blumenthal, J. Gehring, D. Henderson,

C. L. Jiang, N. Nickles, J. Nolen, R. C. Pardo, J. P. Schiffer, and R. E. Segel

Phys. Rev. C 52, R460-R463 (1995)

Spectroscopy of ${ }^{194} \mathrm{Po}$

W. Younes, J. A. Cizewski, H.-Q. Jin, L. A. Bernstein, D. P. McNabb, D. N. Davids,

R. V. F. Janssens, T. L. Khoo, C. J. Lister, D. J. Blumenthal, M. P. Carpenter,

D. Henderson, R. G. Henry, T. Lauritsen, D. T. Nisius, H. T. Penttilä, and M. W. Drigert

Phys. Rev. C 52, R1723-R1726 (1995) 
Pairing Strength in Neutron Rich Isotopes of $\mathrm{Zr}$

J. L. Durell, W. R. Phillips, C. J. Pearson, J. A. Shannon, W. Urban, B. J. Varley,

N. Rowley, K. Jain, I. Ahmad, C. J. Lister, L. R. Morss, K. L. Nash, C. W. Williams,

N. Schulz, E. Lubkiewicz, and M. Bentaleb

Phys. Rev. C 2ㅡ, R2306-R2309 (1995)

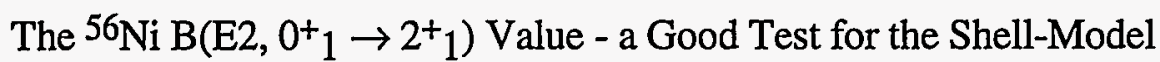

G. Kraus, P. Egelhof, C. Fischer, H. Geissel, A. Himmler, F. Nickel, G. Münzenberg,

W. Schwab, A. Weiss, J. Friese, A. Gillitzer, H. J. Körner, M. Peter, W. Henning,

J. P. Schiffer, B. A. Brown, J. V. Kratz, L. Chulkov, M. Golovkov, and A. Olgoblin

Physica Scripta T56, 114-119 (1995)

Gamma Rays from Fission Fragments

I. Ahmad and W. R. Phillips

Rep. Prog. Phys. 58, 1415-1463 (1995)

Ion Plasma Sputtering as a Method of Introducing Solid Material into an ECR Ion Source

R. Harkewicz, P. J. Billquist, J. P. Greene, J. A. Nolen, Jr., and R. C. Pardo

Rev. Sci. Instrum. 66, 2883-2887 (1995)

Ordered One-Component Plasmas: Phase Transitions, Normal Modes, Large Systems, and Experiments in a Storage Ring

J. P. Schiffer

Proceedings of the Nonneutral Plasma Workshop, Clark Kerr Campus, University of

California, Berkeley, July 17-20, 1994, eds. Joel Fajans and Daniel H. E. Dubin, AIP

Conference Proceedings 331 (AIP, New York 1995) pp. 191-203

Niobium Coaxial Quarter-Wave Cavities

K. W. Shepard, A. Roy, and P. N. Potukuchi

Proceedings of the 1994 International Linac Conference, Tsukuba, Japan, 21-26 August 1994, p. 287 (1995)

Construction of a Superconducting RFQ Structure

K. W. Shepard, J. Givens, and J. M. Potter

Proceedings of the 1994 International Linac Conference, Tsukuba, Japan, 21-26 August 1994 , p. 290 (1995)

Early Operational Experience with Uranium Beams at ATLAS

R. C. Pardo, J. A. Nolen, J. R. Specht, K. W. Shepard, P. J. Billquist, J. M. Bogaty,

B. E. Clifft, R. Harkewicz, F. H. Munson, and G. Zinkann

Proceedings of the 1994 International Linac Conference, Tsukuba, Japan, 21-27 August 1994, eds. K. Takata, Y. Yamazaki, and K. Nakahara, Vol. 2, pp 538-540 (1995)

Superconducting Heavy Ion Linacs

J. A. Nolen

Proceedings of the 1994 International Linac Conference, Tsukuba, Japan, 21-27 August 1994, eds. K. Takata, Y. Yamazaki, and K. Nakahara, Vol. 2, pp. 967-971 (1995)

Low-Energy Nuclear Physics with High-Segmentation Silicon Arrays

R. R. Betts

Conference Proceedings of the XXIV Zakopane School of Physics, Zakopane, Poland, September 5-14, 1994; Acta Physica Polonica B, Vol. 26, 487-501 (1995) 


\section{OPERATION AND DEVELOPMENT OF ATLAS}

Report from Working Group 2 - RFQs \& Linacs

J. A. Nolen

Particle Accelerators 47, 249-252 (1994)

Ion Plasma Sputtering as a Method of Introducing Solid Materials into an ECR Ion Source

R. Harkewicz, P. J. Billquist, J. P. Greene, J.A. Nolen, Jr., and R. C .Pardo

Rev. Sci. Instrum. 66, 2883-2887 (1995)

Review of Work Related to Ion Sources and Targets for Radiooactive Beams at Argonne

J. A. Nolen

Rev. Sci Instrum. 67, 935-937 (1996)

\section{MEDIUM-ENERGY NUCLEAR PHYSICS RESEARCH}

Measurement of Nuclear Transparencies from Exclusive $\rho^{0}$ Meson Production in Muon-Nucleus Scattering at $470 \mathrm{GeV}$

M. R. Adams et al. (Fermilab E665 Collaboration)

Phys. Rev. Lett. 74, 1525 (1995)

Exclusive Electron Scattering from Deuterium at High Momentum Transfer

H. J. Bulten, P. L. Anthony, R. G. Arnold, J. Arrington, E. J. Beise, E. Belz,

K. van Bibber, P. E. Bosted, J. F. van den Brand, M. S. Chapmsn, K. P. Coulter,

F. S. Dietrich, R. Ent, M. Epstein, B. W. Filippone, H. Gao, R. A. Gearhart,

D. F. Geesaman, J.-O. Hansen, R. J. Holt, H. E. Jackson, C. E. Jones, C. E. Keppel,

E. Kinney. S. E. Kuhn, K. Lee, W. Lorenzon, A. Lung, N. C. R. Makins, D. J. Margaziotis,

R. D. McKeown, R. G. Milner, B. Mueller, J. Napolitano, J. Nelson, T. G. O'Neill,

V. Papavassiliou, G. G. Petratos, D. H. Potterveld, S. E. Rock, M. Spengos, Z. M. Szalata,

L. H. Tao, J. L. White, and B. Zeidman

Phys. Rev. Lett. 74, 4775 (1995)

Nuclear Decay Following Deep Inelastic Scattering of $470 \mathrm{GeV}$ Muons

M. R. Adams et al. (Fermilab E665 Collaboration)

Phys. Rev. Lett. 74, 5198 (1995)

Extraction of the Ratio $\mathrm{F}_{2}^{\mathrm{n}} / \mathrm{F}_{2}^{\mathrm{p}}$ in Muon-Deuteron and Muon-Proton Scattering at Small $\mathrm{x}$ and $\mathrm{Q}^{2}$

M. R. Adams et al. (Fermilab E665 Collaboration)

Phys. Rev. Lett. $\underline{75}$, 1466-1470 (1995)

Measurement of the Spin-Dependent Asymmetry in ${ }^{3} \overrightarrow{\mathrm{H}}\left(\overrightarrow{\mathrm{e}}, \mathrm{e}^{\prime}\right)$ Inelastic Scattering at Low Energy Transfer

C. E. Jones, J. Arrington, E. J. Beise, B. Bray, R. W. Carr, B. W. Filippone, H. Gao,

A. F. Lung, R. D. McKeown, B. Mueller, M. L. Pitt, D. DeSchepper, G. Dodson, K. Dow,

R. Ent, M. Farkhondeh, J.-O. Hansen, W. Korsch, L. H. Kramer, K. Lee, N. C. R. Makins,

R. G. Milner, D. R. Tieger, T. P. Welch, E. Candell, J. Napolitano, C. Tripp,

B. B. Wojtsekhowski, and W. Lorenzon

Phys. Rev. C $\underline{52}$, 1520-1523 (1995)

Multiplicity Distributions from Central Collisions of $16 \mathrm{O}+\mathrm{Cu}$ at $14.6 \mathrm{~A} \mathrm{GeV} / \mathrm{c}$ and Intermittency

T. Abbott et al. (E-802 collaboration - BNL)

Phys. Rev. C 52, 2663-2678 (1995) 
Trigger Processor for the APEX Positron-Electron Spectrometer

M. Wolanski, S. J. Freedman, J. W. Dawson, W. N. Haberichter, K. C. Chan,

A. A. Chishti, N. I. Kolaskamis, and C. J. Lister

Nucl. Instrum. Methods A361, 326-337 (1995)

Laser-Driven Source of Spin-Polarized Atomic Hydrogen and Deuterium

M. Poelker, K. P. Coulter, R. J. Holt, C. E. Jones, R. S. Kowalczyk, L. Young,

B. Zeidman, and D. K. Toporkov

Nucl. Instrum. Methods A364, 58-69 (1995)

A-Dependence of Nuclear Transparency in Quasielastic A(e,e'p) at High $Q^{2}$

T. G. O'Neill, W. Lorenzon, P. L. Anthony, R. G. Arnold, J. Arrington, R. J. Beise,

J. E. Belz, P. E. Bosted, H.-J. Bulten, M. S. Chapman, K. P. Coulter, F. S. Dietrich,

R. Ent, M. Epstein, B. W. Filippone, H. Gao, R. A. Gearhart, D. F. Geesaman,

J.-O. Hansen, R. J. Holt, H. E. Jackson, C. E. Jones, C. E. Keppel, E. Kinney, S. E. Kuhn,

K. Lee, A. Lung, N. C. R. Makins, D. J. Margaziotis, R. D. McKeown, R. G. Milner,

B. Mueller, J. Napolitano, J. Nelson, V. Papavassiliou, G. G. Petratos, D. H. Potterveld,

S. E. Rock, M. Spengos, Z. M. Szalata, L. H. Tao, K. van Bibber, J. F. van den Brand,

J. L. White, and B. Zeidman

Phys. Lett. B351, 87 (1995)

The Nuclear EMC Effect

D. F. Geesaman, K. Saito, and A. W. Thomas

Ann. Rev. Nucl. and Part. Sci. $\underline{45}$, 337-390 (1995)

Shadowing in Inelastic Scattering of Muons on Carbon, Calcium and Lead at low $\mathrm{x}_{\mathrm{Bj}}$

M. R. Adams et al. (Fermilab E665 Collaboration)

Z. Phys. C 67, 403 (1995)

Two Body Photodisintegration of the Deuteron Up to $2.8 \mathrm{GeV}$

J. E. Belz et al.

Proceedings of the 5th Conference on the Intersections and Particle and

Nuclear Physics, St. Petersburg, FL, 31 May-6 June 1994, ed. Susan J. Seestrom, AIP Conference Proceedings $\underline{338}$ (AIP Press, Woodbury, NY 1995), pp. 678-681

Measurement of the Asymmetry in the Electro-Disintegration of Tensor Polarized Deuterons at the Novosibirsk VEPP-3 Ring

J. A. P. Theunissen, C. W. de Jager, J. J. van Leeuwe, G. A. Retzlaff, H. de Vries,

D. M. Nikolenko, S. G. Popov, I. A. Rachek, K. P. Coulter, R. Gilman, R. J. Holt,

C. E. Jones, E. R. Kinney, M. Poelker, D. H. Potterveld, and L. Young

Proceedings of the 14th International Conference on Few-Body Problems in Physics,

Williamsburg, VA, 26-31 May 1994, ed. Franz Gross, AIP Conference Proceedings 334

(New York, 1995) pp. 768-771

Laser-Driven Polarized Targets of Hydrogen and Deuterium

C. E. Jones, K. P. Coulter, R. J. Holt, R. S. Kowalczyk, T. G. O'Neill, M. Poelker, and

B. Zeidman

Proceedings of the 5th Conference on the Intersections and Particle and Nuclear Physics,

St. Petersburg, FL, 31 May-6 June 1994, ed. Susan J. Seestrom,

AIP Conference Proceedings 338 (AIP Press, Woodbury, NY 1995), pp. 357-360 
Measurement of the Neutron Magnetic Form Factor from Inclusive Quasielastic Scattering of Polarized Electrons from Polarized ${ }^{3} \mathrm{He}$

H. Gao, J. Arrington, E. J. Beise, B. Bray, R. W. Carr, B. W. Filippone, A. Lung,

R. D. McKeown, B. Mueller, M. L. Pitt, C. E. Jones, D. DeSchepper, G. Dodson, K. Dow,

R. Ent, M. Farkhondeh, J.-O. Hansen, W. Korsch, L. H. Kramer, K. Lee, N. Makins,

R. G. Milner, D. R. Tieger, T. P. Welch, E. Candell, J. Napolitano, B. B. Wojtsekhowski,

C. Tripp, and W. Lorenzon

Proceedings of the 5th Conference on Intersections Between Particle and Nuclear Physics, St. Petersburg, FL, 31 May-6 June 1994, ed. Susan J. Seestrom,

AIP Conference Proceedings $\underline{338}$ (AIP Press, Woodbury, NY 1995) pp. 704-707

Electron Scattering from Polarized Deuterium at VEPP-3

C. E. Jones, R. J. Holt, R. Kowalczyk, M. Poelker, D. H. Potterveld, L. Young,

S. I. Mishnev, D. M. Nikolenko, S. G. Popov, I. A. Rachek, A. Sukhonov,

D. K. Toporkov, E. P. Tsentalovich, A. Volosov, E. R. Kinney, K. P. Coulter,

C. W. deJager, G. Tetzlaff, J. Theunissen, H. deVries, R. Gilman, V. V. Nelyubin,

and V. N. Stibunov

Proceedings of the 5th Conference on Intersections Between Particle and Nuclear Physics, St. Petersburg, FL, 31 May-6 June 1994, ed. Susan J. Seestrom,

AIP Conference Proceedings 338 (AIP Press, Woodbury, NY 1995) pp. 713-716

\section{THEORETICAL PHYSICS}

Positive Parity States in ${ }^{11} \mathrm{Be}$

H. Esbensen, B. A. Brown, and H. Sagawa

Phys. Rev. C 1ㅣ, 1274-1279 (1995)

Proton-Proton Bremsstrahlung Below and Above Pion-Threshold: Influence of the $\Delta$ Isobar

F. de Jong, K. Nakayama, and T.-S. H. Lee

Phys. Rev. C 51, 2334-2346 (1995)

Variational Calculations of the $\Delta$-Separation Energy of the ${ }_{\Lambda}^{17} \mathrm{O}$ Hypernucleus

A. A. Usmani, Steven C. Pieper, and Q. N. Usmani

Phys. Rev. C 1, 2347-2355 (1995)

Three-Body Correlations in Few-Body Nuclei

A. Arriaga, V. R. Pandharipande, and R. B. Wiringa

Phys. Rev. C 52, 2362-2368 (1995)

Topology, Locality, and Aharonov-Bohm Effect with Neutrons

M. Peshkin and H. J. Lipkin

Phys. Rev. Lett. 74, 2847-2850 (1995)

Quantum Monte Carlo Calculations of A $\leq 6$ Nuclei

B. S. Pudliner, V. R. Pandharipande, J. Carlson, and R. B. Wiringa

Phys. Rev. Lett. 74, 4396-4399 (1995)

Octupole Deformations in the Very Extended Nuclear Shapes of the A 180 Region

R. R. Chasman and L. M. Robledo

Phys. Lett. B351, 18-23 (1995) 
Higher-Order Effects in Inclusive Electron-Nucleus Scattering

O. Benhar, A. Fabrocini, S. Fantoni, V. R. Pandharipande, S. C. Pieper, and I. Sick

Phys. Lett. B359, 8-12 (1995)

Interference Effects in the Coulomb Dissociation of ${ }^{8} \mathrm{~B}$

H. Esbensen and G. F. Bertsch

Phys. Lett. B359, 13-16 (1995)

The Off-Shell Axial Anomaly Via the $\gamma^{*} \pi^{0} \rightarrow \gamma$ Transition

M. R. Frank, K. L. Mitchell, C. D. Roberts, and P. C. Tandy

Phys. Lett. B $\underline{359}$, 17-22 (1995)

Very Extended Shapes in the A 156 Mass Region

R. R. Chasman

Phys. Lett. B364, 137-142 (1995)

Pion Loop Contribution to the Electromagnetic Pion Charge Radius

Reinhard Alkofer, Axel Bender, and Craig D. Roberts

Int. J. Mod. Phys. A 10, 3319-3342 (1995)

Model Gluon Propagator and Pion and p-Meson Observables

M. R. Frank and C. D. Roberts

Phys. Rev. C $\underline{53}$, 390-398 (1996)

Analytic Structure of the Quark Propagator in a Model with an Infrared Vanishing Gluon

Propagator

Axel Bender and Reinhard Alkofer

Phys. Rev. D $\underline{53}, 446-457$ (1996)

Calculation of the Anomalous $\gamma \pi^{*} \rightarrow \pi \pi$ Form Factor

Reinhard Alkofer and Craig D. Roberts

Phys. Lett. B369, 101-104 (1996)

Wave Function Calculations in Finite Nuclei

Steven C. Pieper

Proceedings of the 6th Workshop on Perspectives in Nuclear Physics at Intermediate

Energies, ICTP, Trieste, Italy, May 3-7, 1993, eds. Sigfrido Boffi, Claudio Ciofi degli

Atti, and Mauro Giannini (World Scientific, Singapore 1995) pp. 248-255

Final State Correlations in Realistic (e,e'p) Calculations

M. Radici, S. C. Pieper, S. Boffi, and V. R. Pandharipande

Proceedings of the 6th Workshop on Perspectives in Nuclear Physics at Intermediate

Energies, ICTP, Trieste, Italy, 3-7 May 1993, eds. Sigfido Boffi, Claudio Ciofi degli Atti, Mauro Giannini (World Scientific, Singapore 1994) pp. 256-265

What Did We Learn from the Aharonov-Bohm Effect? Is Spin 1/2 Different?

Murray Peshkin

Proceedings of the International Workshop on Quantum Systems, Minsk, Belarus, 23-29 May 1994, eds. A. O. Barut, I. D. Feranshuk, Ya. M. Shnir, and L. M. Tomichik (World Scientific, Singapore 1995) pp. 163-172 
Monte Carlo Approaches to the Few-Nucleon Continuum

R. Schiavilla, J. Carlson, and R. B. Wiringa

Proceedings of the 14th International Conference on Few-Body Problems in Physics,

Williamsburg, VA, 26-31 May 1994, ed. Franz Gross, AIP Conference Proceedings 334

(New York, 1995) pp. 79-97

On the Aharonov-Bohm Effect with Neutrons

Murray Peshkin

Proceedings of the Conference on Fundamental Problems in Quantum Theory:

A Conference Held in Honor of Professor John A. Wheeler, Baltimore, MD,

18-22 June 1994, eds. Daniel M. Greenberger and Anton Zeilinger,

Ann. NY Acad. Sci, Vol. 755, 1995, pp. 330-334

Pion Observables and QCD

Craig D. Roberts

Proceedings of the Workshop on Chiral Dynamics: Theory and Experiment, MIT, Cambridge, MA, 25-29 July 1994, eds. Aron M. Bernstein and Barry R. Holstein (Springer-Verlag Heidelberg 1995) Lecture Notes in Physics 452, pp 68-77

Pion Observables and the Quark-Quark Interaction in the Infrared

Craig Roberts

Proceedings of the Workshop on Chiral Dynamics: Theory and Experiment, MIT,

Cambridge, MA, 25-29 July 1994, eds. Aron M. Bernstein and Barry R. Holstein

(Springer-Verlag Heidelberg 1995) Lecture Notes in Physics 452, pp. 112-113

Time-Dependent and Temperature-Dependent Aspects of Electron Distribution Functions: $\mathrm{H}, \mathrm{Ar}$, and Cs Atomic Gases

I. Krajcar-Bronic, M. Kimura, and M. Inokuti

J. Chem. Phys. 102, 6552-6558 (1995)

Ionization Cross Sections of Gases for Protons at Kinetic Energies between $20 \mathrm{MeV}$ and $385 \mathrm{GeV}$, and Applications to Vacuum Gauges in Superconducting Accelerators

H. Ishimaru, S. Shibata, and M. Inokuti

Phys. Rev. A51, 4631-4639 (1995)

Remarks on the Stopping Power: Its Connections with Particle Transport and with the Electronic Structure of Matter

M. Inokuti

Int. J. Quantum Chem. 57, 173-182 (1996)

Atoms as Many-Body Systems

M. Ya. Amusia

"Harmony in Physics" International Conference, Philadelphia, PA, 9-14 May 1994;

Physics Reports 264, 7-26 (1996)

Foreword

M. Inokuti

to Atomic and Molecular Data for Radiotherapy and Radiation Research. Final Report of a Co-ordinated Research Programme of the International Atomic Energy Agency, IAEA-TECDOC-799, Vienna, p. 3 (1995) 
Photoabsorption, Photoionization, and Photodissociation Cross Sections

Y. Hatano and $M$. Inokuti

in Atomic and Molecular Data for Radiotherapy and Radiation Research. Final Report of a Co-ordinated Research Programme of the International Atomic Energy Agency, IAEA-TECDOC-799, Vienna, pp. 331-369 (1995)

Yields of Ionization and Excitation in Irradiated Matter Photoabsorption, Photoionization, and Photodissociation Cross Sections

D. Srdoc, M. Inokuti, and I. Krajcar-Bronic in Atomic and Molecular Data for Radiotherapy and Radiation Research. Final Report of a Co-ordinated Research Programme of the International Atomic Energy Agency, IAEA-TECDOC-799, Vienna, pp. 547-631 (1995)

\section{ATOMIC AND MOLECULAR PHYSICS RESEARCH}

Fine-Structure Energies for the $1 \mathrm{~s} 2 \mathrm{~s}^{3} \mathrm{~S}-1 \mathrm{~s} 2 \mathrm{p}^{3} \mathrm{P}$ Transition in Heliumlike $\mathrm{Ar}^{16+}$

K. W. Kukla, A. E. Livingston, F. G. Serpa, H. G. Berry, R. W. Dunford, D. S. Gemmell,

E. P. Kanter, R. Ali, J. Suleiman, S. Cheng, and L. J. Curtis

Phys. Rev. A51, 1905 (1995)

Positron Production in Heavy-Ion Collisions: Current Status of the Problem

APEX Collaboration

Nucl. Phys. A583, 247 (1995)

Transition Selective Investigation of Resonant Transfer and Excitation in $\mathrm{U}^{90+} \rightarrow \mathrm{C}$ Collisions

T. Kandler, P. H. Mokler, T. Stöhlker, H. Geissel, H. Irnich, C. Kozhuharov,

A. Kriessbach, M. Kucharski, G. Münzenberg, F. Nickel, P. Rymuza, C. Scheidenberger,

Z. Stachura, T. Suzuki, A. Warczak, D. Dauvergne, and R. W. Dunford

Phys. Lett. A204, 274 (1995)

The Interactions of High-Energy, Highly-Charged Xe Ions with Fullerenes

T. LeBrun, H. G. Berry, S. Cheng, R. W. Dunford, H. Esbensen, D. S. Gemmell,

E. P. Kanter, and W. Bauer

Nucl. Instrum. Methods B98, 479-481 (1995)

Spectral Distribution of the Two-Photon Decay of He-Like Krypton

R. Ali, I. Ahmad, H. G. Berry, R. W. Dunford, D. S. Gemmell, E. P. Kanter, P. H. Mokler,

A. E. Livingston, S. Cheng, and L. J. Curtis

Nucl. Instrum. Methods B98, 69-73 (1995)

The Interactions of High-Energy, Highly-Charged Xe Ions with Buckyballs

R. Ali, H. G. Berry, S. Cheng, R. W. Dunford, H. Esbensen, D. S. Gemmell, E. P. Kanter,

T. LeBrun, L. Young, and W. Bauer

Nucl. Instrum. Methods B96, 545-549 (1995)

Fine Structure Energies for the $1 \mathrm{~s} 2 \mathrm{~s}^{3} \mathrm{~S}-1 \mathrm{~s} 2 \mathrm{p}{ }^{3} \mathrm{P}$ Transition in Helium-Like Ions

A. E. Livingston, K. W. Kukla, C. M. Vogel Vogt, H. G. Berry, R. W. Dunford,

D. S. Gemmell, E. P. Kanter, J. Suleiman, R. Ali, S. Cheng, and L. J. Curtis

Nucl. Instrum. Methods B98, 28 (1995) 
Search for Narrow Sum-Energy Lines in Electron-Positron Pair Emission from Heavy-Ion Collisions near the Coulomb Barrier

APEX Collaboration

Phys. Rev. Lett. 75, 2658 (1995)

Hyperfine Structure Studies of Nb II: Experimental and Relativistic Configuration-Interaction Results

L. Young, S. Hasegawa, C. Kurtz, Debasis Datta, and Donald R. Beck

Phys. Rev. A51, 3534-3540 (1995)

Non-Dipolar Asymmetries of Photoelectron Angular Distributions

B. Krässig, M. Jung, D. S. Gemmell, E. P. Kanter,'T. LeBrun, S. H. Southworth, and

L. Young

Phys. Rev. Lett. 75, 4736-4739 (1995)

Evolution of X-Ray Resonance Raman Scattering into X-Ray Fluorescence from the Excitation of

Xenon near the $\mathrm{L}_{3}$ Edge

M. A. MacDonald, S. H. Southworth, J. C. Levin, A. Henins, R. D. Deslattes, T. LeBrun,

Y. Azuma, P. L Cowan, and B. A. Karlin

Phys. Rev. A51, 3598 (1995)

Anisotropy of Si $\mathrm{K} \beta$ Emission: Interference of Fluorescence X-Rays

Y. Ma, K. E. Miyano, P. L. Cowan, Y. Aglitzkiy, and B. A. Karlin

Phys. Rev. Lett. 74, 478 (1995)

Ion Charge-State Production and Photoionization near the K-Edge in Argon and Potassium

H. G. Berry, Y. Azuma, P. L. Cowan, D. S. Gemmell, T. LeBrun, and M. Ya. Amusia Proceedings of the $7^{\text {th }}$ International Conference on the Physics of Highly Charged Ions, Vienna, Austria, 19-23 September 1994; Nucl. Instrum. Methods B98, 25-27 (1995)

A Comparison of Radiative and Non-Radiative Resonant Raman Studies of the Inner-Shell Electronic Structure of Atoms

T. LeBrun, S. H. Southworth, M. A. MacDonald, Y. Azuma

Proceedings of the Oji International Seminar on Atomic and Molecular Photoionization,

A. Yagashita and T. Sasaki, eds., (Universal Academy Press, Tokyo) in press 